\title{
30th International Conference on
}

\section{Information Modelling and Knowledge Bases}

Eds.

\author{
Bernhard Thalheim \\ Marina Tropmann-Frick \\ Hannu Jaakkola \\ Yasushi Kiyoki
}

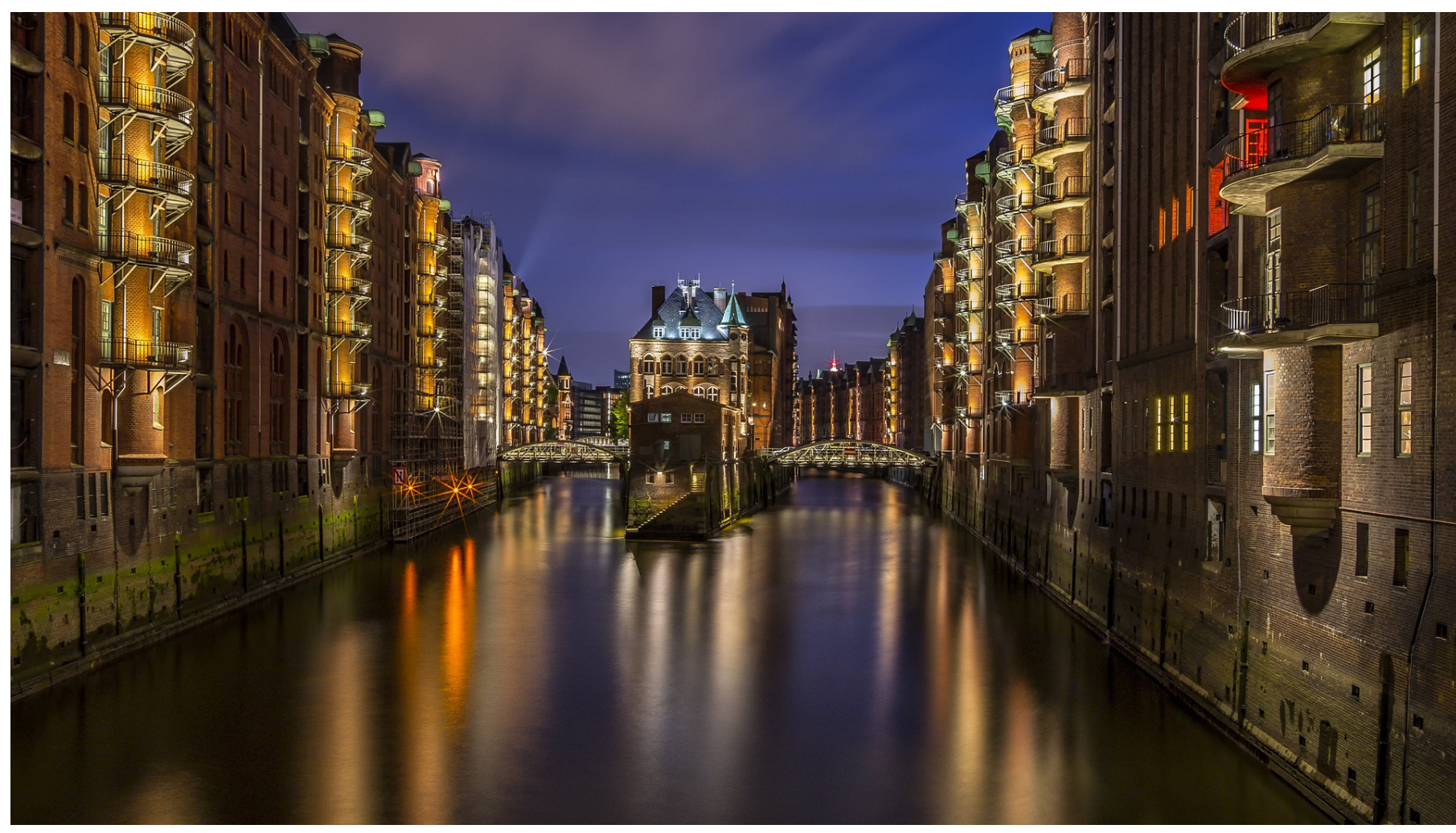

2020/1

Kiel Computer Science Series 


\title{
30th International Conference on Information Modelling and Knowledge Bases \\ EJC 2020
}

\author{
Bernhard Thalheim \\ Marina Tropmann-Frick \\ Hannu Jaakkola \\ Yasushi Kiyoki
}

June 8-9, 2020, Hamburg, Germany 


\section{Impressum}

\section{Editors}

Bernhard Thalheim

Information Systems Engineering

Dept. of Computer Science

Christian-Albrechts-University Kiel

24118 Kiel, Germany

Hannu Jaakkola

(Prof. Emeritus)

Information Technology and Communication

Sciences

Tampere University, Pori

P.O.Box 300, FIN-28101 Pori, Finland
Marina Tropmann-Frick

Fakultät Technik und Informatik

Department Informatik

University of Applied Sciences Hamburg

Berliner Tor 5

20099 Hamburg, Germany

Yasushi Kiyoki

Graduate School of Media and Governance

Keio University

P.O.Box 300, 5322 Endoh

Fujisawa, Kanagawa

Japan, 252-0882

\section{Kiel Computer Science Series}

The "Kiel Computer Science Series" (KCSS) is published by the Department of Computer Science of the Faculty of Engineering at Kiel University. The scope of this open access publication series includes conference proceedings, dissertation theses, habilitation theses, and text books in computer science.

Kiel Computer Science Series (KCSS) 2020/1

dated June 7, 2020

ISSN 2193-6781 (print version)

9 (electronic version)

$10.21941 / \mathrm{kcss} / 2020 / 1$

Electronic version and errata available via https://www.informatik.uni-kiel.de/kcss

No updates after 2020-06-07

The editor of KCSS can be contacted via http://www.mip.informatik.uni-kiel.de

Published by the Department of Computer Science, Kiel University Information Systems Engineering

Please cite as:

B. Thalheim, M. Tropmann-Frick, H. Jaakkola, Y. Kiyoki (eds). Proceedings of the 30th International Conference on Information Modelling and Knowledge Bases (EJC 2020), June 8-9, 2020, Hamburg, Germany.

Number 2020/1 in Kiel Computer Science Series.

Department of Computer Science, Faculty of Engineering, Kiel University.

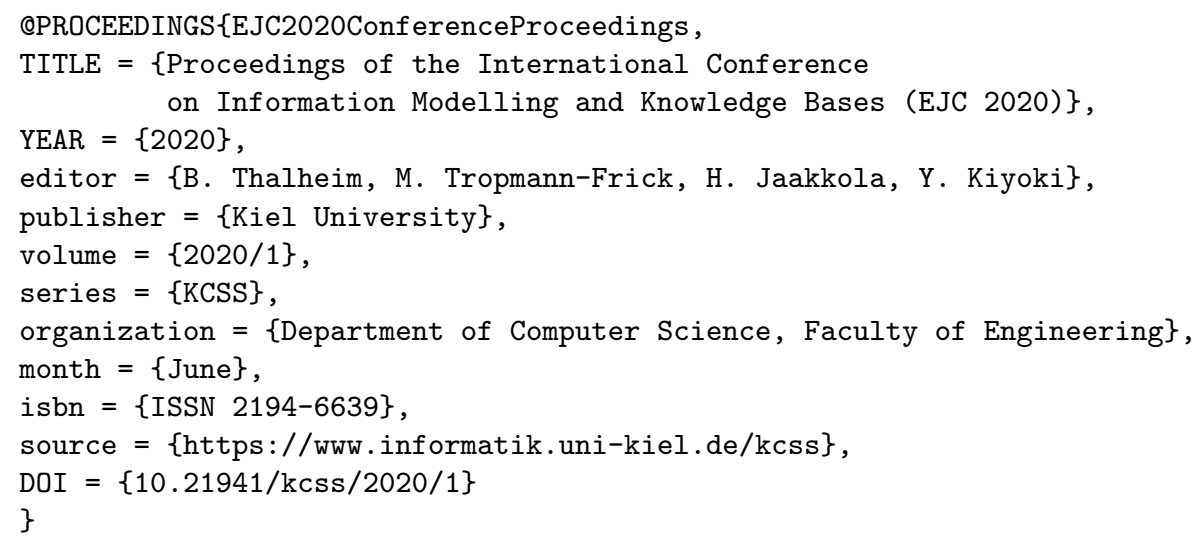

(c) Bernhard Thalheim, Marina Tropmann-Frick, Hannu Jaakkola, Yasushi Kiyoki 
DAY1: June 8, 9:00-12:00 (Central European Time (CET)) (16:00- Japanese Time)

\section{Session Chairs: Bernhard Thalheim (Program), Marina Tropmann-Frick (Technical)}

(0) EJC2020 Opening

Yasushi Kiyoki (Program Committee Co-Chairs)

Bernhard Thalheim (Program Committee Co-Chairs)

Hannu Jaakkola (General Organizing Chair)

(1) Conceptual Modeling of Hiking Trail Networks with Logical for Consistent Signage Planning and Management (\# 2003)

András J. Molnár

(2) A Concept for Control and Program Based on the Semantic Space Model (\# 2014)

Yukio Chen, Maimai Prayongrat, Yashushi Kiyoki

(3) Open Routed Energy Distribution Network Simulation for Maui Village Case Study (\# 2016)

Alexander Dudko, Tatiana Endrjukaite, Leon Roose

(4) Trends and Future of Data Modelling (\# 2002)

Hannu Jaakkola, Bernhard Thalheim

(5) A Global \& Environmental Coral Analysis System with SPA-based Semantic Computing for Integrating and Visualizing Ocean-Phenomena with "5-Dimensional World-Map" (\# 2019)

Yasushi Kiyoki, Petchporn Chawakitchareon, Sompop Rungsupa, Xing Chen,

Kittiya Samlansin

(6) Recognizing Human-Object Interaction in Multi-Camera Environments (\# 2027)

Marina Tropmann-Frick, Thien Phuc Tran

(7) Modeling the Software Prototyping Process in a Research Context (\# 2009)

Mika Saari, Jari Soini, Jere Grönman, Petri Rantanen, Timo Mäkinen, Pekka Sillberg

(8) A Natural Logic System for Large Knowledge Bases (\# 2024)

Troels Andreasen, Henrik Bulskov, Jørgen Fischer Nilsson

(9) Global \& Geographical Mapping and Visualization Method for Personal/Collective Health Data with 5D World Map System (\# 2025)

Shiori Sasaki, Koji Murakami, Yasushi Kiyoki, Asako Uraki

(10) XML Schema support in knowledge management (\# 2028)

Botjan Šumak, Marjan Heričko, Maja Pušni

(11) Digital Intelligence Banking of Adaptive Digital Marketing with Life Needs Control (\# 2022) Ryosuke Konishi, Fumito Nakamura, Yasushi Kiyoki

(12) Defects Recognition on Wafer Maps using Multilayer Feed-forward Neural Network (\# 2005)

Radoslav Štrba, Daniela Bordencea 
DAY2: June 9, 9:00-12:00 (Central European Time (CET)) (16:00- Japanese Time)

\section{Session Chairs: Bernhard Thalheim (Program), Marina Tropmann-Frick (Technical)}

(1) Modelling Temporal Scales in (Pre-/Proto-)History (\# 2020)

Yannic Kropp, Bernhard Thalheim

(2) Application of a Heterogeneous Correlation Integration Method to a Context Cube Semantic Network Model for Railway Passengers

Motoki Yokoyama, Yasushi Kiyoki, Tetsuya Mita

(3) Privacy by Design in EU's GDPR (\# 2026)

Bostjan Brumen

(4) Search for Appropriate Textual Information Sources (\# 2021)

Marie Duzi, Marek Mensik, Adam Albert, Vojtech Patschka, Miroslav Pajr

(5) Contentual Characteristics of Concepts (\# 2015)

Jari Palomäki

(6) Smart Cities through the Lens of News: A Comparative Content Analysis of Japanese and Slovenian Newspapers (\# 2010)

Yukiko Sato, Stefan Brückner, Maja Pušnik

(7) Visitor Journey Application Development For Omni-Channels (\# 2008)

Bakhtiyor Esanov, Ajantha Dahanayake

(8) Augmented Reality for Human Needs: an Ontology (\# 2004)

Manal Yahya, Ajantha Dahanayake

(9) Cross-Cultural Religious Tourism with Impression Distance Search System (\# 2017)

Piyaporn Nurarak, Yasushi Kiyoki, Shiori Sasaki, Irene Erlyn Wina Rachmawan

(10) Conceptualization of Aviation Cabin Crews Fatigue Risk Assessment (\# 2001)

rem evik, Bibek Bam, Ajantha Dahanayake, Kalle Elfvengren

(11) A Mental Health Database Creation Method with Neuroscience-inspired Search Functions (\# 2013)

Venera Raneva, Yasushi Kiyoki

(12) Q-Learning as Failure (\# 2012)

Kei Takahata, Takao Miura 


\section{Preface}

Information Modeling and Knowledge bases have become an important technology contributor for the 21st centurys academic and industry research that address the complexities of modeling in digital transformation and digital innovation reaching beyond the traditional boarders of information systems and computer science academic research. The amount and complexity of information itself, the number of abstraction levels of information, and the size of databases and knowledge bases are continuously growing. Conceptual modelling is one of the sub-areas of information modelling. The aim of this conference is to bring together experts from different areas of computer science and other disciplines, who have a common interest in understanding and solving problems on information modelling and knowledge bases, as well as applying the results of research to practice. We also aim to recognize and study new areas on modelling and knowledge bases to which more attention should be paid. Therefore philosophy and logic, cognitive science, knowledge management, linguistics and management science are relevant areas, too. In the conference, there will be three categories of presentations, i.e. full papers, short papers and position papers.

The international conference on information modelling and knowledge bases originated from the co-operation between Japan and Finland in 1982 as the European Japanese conference (EJC). Then professor Ohsuga in Japan and Professors Hannu Kangassalo and Hannu Jaakkola from Finland (Nordic countries) did the pioneering work for this long tradition of academic collaboration. Over the years, the organization extended to include European countries as well as many other countries. In 2014, with this expanded geographical scope the European Japanese part in the title was replaced by International. The conference characteristics include opening with the appetizer session that allows participants to introduce their topic in a three minute short presentation followed by presentation sessions with enough time for discussions. The limited number of participants is typical for this conference.

The 30th International conference on Information Modeling and Knowledge Bases (EJC2019) held at Hamburg, Germany constitutes a research forum exchanging of scientific results and experiences drawing academics and practitioners dealing with information and knowledge.

The main topics of EJC2020 cover a wide range of themes extending the knowledge discovery through Conceptual Modelling, Knowledge and Information Modelling and Discovery, Linguistic Modelling, CrossCultural Communication and Social Computing, Environmental Modeling and Engineering, and Multimedia Data Modelling and Systems extending into complex scientific problem solving. The themes of the conference presentation sessions; Learning and Linguistics, Systems and Processes, Data and Knowledge Representation, Models and Interfaces, Formalizations and reasoning, Models and Modelling, Machine Learning, Models and Programing, Environment and Predictions, Emotion Modeling and Social Networks reflected the coverage of those main themes of the conference.

The contributions of this proceeding of the 30th International Conference of Information Modeling and Knowledge bases feature twenty-six reviewed, selected, and upgraded contributions that are the result of presentations, comments, and discussions during the conference. Suggested topics of the call for papers include, but are not limited to:

Conceptual modelling: Modelling and specification languages; Domain-specific conceptual modelling; Concepts, concept theories and ontologies; Conceptual modelling of large and heterogeneous systems; Conceptual modelling of spatial, temporal and biological data; Methods for developing, validating and communicating conceptual models.

Knowledge and information modelling and discovery: Knowledge discovery, knowledge representation and knowledge management; Advanced data mining and analysis methods; Conceptions of knowledge and information; Modelling information requirements; Intelligent information systems; Information recognition and information modelling.

Linguistic modelling: Models of HCI; Information delivery to users; Intelligent informal querying; Linguistic foundation of information and knowledge; Fuzzy linguistic models; Philosophical and linguistic foundations of conceptual models.

Cross-cultural communication and social computing: Cross-cultural support systems; Integration, evolution and migration of systems; Collaborative societies; Multicultural web-based software systems; Intercultural collaboration and support systems; Social computing, behavioral modeling and prediction.

Environmental modelling and engineering: Environmental information systems (architecture); Spatial, temporal and observational information systems; Large-scale environmental systems; Collaborative knowledge base systems; Agent concepts and conceptualisation; Hazard prediction, prevention and steering systems.

Multimedia data modelling and systems: Modelling multimedia information and knowledge; Contentbased multimedia data management; Content-based multimedia retrieval; Privacy and context enhancing technologies; Semantics and pragmatics of multimedia data; Metadata for multimedia information systems. 
The EJC2020 will be held online and hosted by the Department Informatik of University of Applied Sciences Hamburg, Germany. Due to regulations caused by Corona virus this year conference is transformed to into virtual event, held on June 8th and 9th at 9-12 German time. Presentations, prepared according to the instructions, have 10 minutes time slot. Virtual conference is organized with Zoom platform.

Please follow the guidelines below for participation and presentation:

1. Prepare your presentation material on your computer (or device).

2. Join the Zoom conference via link (will be sent per e-mail by organizer).

3. Mute your microphone and turn your camera off.

4. Please actively enter questions regarding the presentation. Specifically, address questions to All participants in the chat window. This will help the session chair in responding to questions after the presentation.

5. After the permission of Chair, unmute the microphone and enable your camera.

- Share your presentation material and start presentation. Click on the green "Share Screen" icon. You will see a pop-up window where you select your desktop or application.

- If you have some videos in your slides, you may select "Optimize for full-screen video clip". Click the check box in the lower left that says "Share computer sound". Now when you are in screenshare mode it will play all computer sounds to the audience.

- After finishing your presentation, please answer questions from the session chair or participants.

- You have 10 min. including Q\&A.

- After finishing Q\&A, please stop sharing the contents from the menu above the screen.

6. If there is a discussion, please speak in a way that other participants can understand.

- Start your statements indicating whether it is a "Question" or "Comment".

- State your name and affiliation each time you speak.

- When you speak, ensure your environment is quiet before un-muting your microphone (to avoid transmitting noises around you to other participants).

We thank all colleagues for their support in making this conference successful, especially the program committee, organization committee, and the program coordination team, especially Naofumi Yoshida who maintains the paper submission and reviewing systems and compiled the files for this book.

The Editors

Bernhard Thalheim

Marina Tropmann-Frick

Hannu Jaakkola

Yasushi Kiyoki 


\title{
Conceptual Modeling of Hiking Trail Networks with Logical Rules for Consistent Signage Planning and Management
}

\author{
András J. MOLNÁR \\ Christian-Albrechts-University Kiel, Department of Computer Science, D-24098 Kiel, \\ Germany; E-mail: ajm@informatik.uni-kiel.de
}

\begin{abstract}
.
Trail route networks provide an infrastructure for touristic and recreational walking activities worldwide. They can have a variety of layouts, signage systems, development and management patterns, involving multiple stakeholders and contributors, and tend to be determined by various interests on different levels and dynamically changing circumstances. This paper aims to develop the skeleton of TRAILSIGNER, a sound geospatial conceptual data model suite of trail networks, waymarked routes and their signage systems and assets, which can be used as a basis for creating an information system for the effective, organic and consistent planning, management, maintenance and presentation of trails and their signage. This reduces potential confusion, mistrust and danger for visitors caused by information mismatches including incomplete, incoherent or inconsistent route signposting. To ensure consistency of incrementally planned signposts with each other and with the (possibly changing) underlying trail network, a systematic, set-based approach is developed using generative logical rules and incorporated into the conceptual model suite as signpost logics. The paper also defines a reference ruleset for it. This approach may further be generalized, personalized and adapted to other fields or applications having similar requirements or phenomena.
\end{abstract}

\section{Keywords.}

conceptual modeling, model suite, tourism and recreation, hiking trails, route network, signpost system, spatial data modeling, spatial information system, knowledge and information modeling, knowledge representation and management, collaborative information system, planning and facility management, change management, logical representation and reasoning

\section{Introduction and Outline}

Trail networks provide an infrastructure for touristic and recreational walking (hiking, rambling, trekking, mountaineering, etc.), biking or running activities, mainly in natural but also in built-up areas. Routes are based on publicly passable paths and road sections in the field [1, Walking Routes]. Waymarking, signposting on the field and presentation of the routes on maps, in apps and other media can effectively, reliably and safely support 
the visitors in planning their trips and their orientation on the terrain, if done properly. In this paper, we consider walking routes only, but the observations can be generalized for trails of other activities.

Hiking and walking trails have been developed in the last two centuries in many countries, and a substantial growth in the recent decades can be observed all around the world. New countries and regions are developing their trails, and existing networks face challenges of a higher demand for visitor management and effective maintenance. Slow tourism and outdoor recreation contributes to well-being and sustainability in many ways. With new target groups being involved with less expertise, proper information provision becomes more and more crucial. Community mapping has been growing in the last decades, websites and apps about trails are almost uncountable, and the first integrated platforms for trail network and facility management have been appearing on the market in the these years.

Even in our digital age, when navigation systems are in widespread use, the on-field information provided in various forms such as waymarks, signposts or info-panels, as well as off-line maps, guidebooks and other media seem to be essential. It provides a ceratin safety against possible technical failures or limitations (e.g. precision or battery power). More importantly, 'switching-off' and 'detoxication' from digital devices is one of the significant motivations for going out to trails $[13,6]$. The parallel development of digital tools with off-line information assets raises challenges not only towards the effective and direct support of trail users, but also the systematic and consistent management of trail facilities themselves and their related off-line information assets.

A recreational and touristic trail network is a complex reality, and different terminologies co-exist in different regions around the world, or even among visitors and professionals with different backgrounds in the same region (forestries, national park or other conservation authorities, tourism agencies, hiking or mountaineering associations, etc). Routes and their networks may have various layouts and signage systems. In many areas, routes or even networks with different thematics co-exist in parallel, lacking a unified network concept or signage system.

Trail development and management has its challenges, caused by various ownership, stewardship, institutional and societal patterns, and reflects many aspects of the local, regional and national context and its recent evolution. Actions are not always systematic, but often ad-hoc or step-by-step, initiated and implemented by some of stakeholders not necessarily considering the aspects of the whole network. Resources are often limited and a large-scale systematic development phase is not always followed by a long-term, reliable maintenance and update phase, with a proper change management. Such circumstances often cause mismatch, incompleteness or inconsistency in the information provided through various assets, including waymarks and signposts on the field, digital and printed maps. Improper information provision leads to confusion, mistrust and may lead to dangerous situations for the visitor.

More and more trail-related platforms and applications are available, including crowdsorcing, for various audiences and stakeholders, such as trail information and navigation for visitors, and facility management for trail stakeholders.

The aim of this paper is to develop the basis of TRAILSIGNER, a sound geospatial conceptual data model suite for managing waymarked trail networks and their signage assets, that can go beyond the capabilities of existing systems, and consistently provide various view interfaces for different actors as they need for their tasks, especially sup- 
porting composition of trips for visitors, and proper signage planning and management for providers over dynamically changing networks in a partially automatized, incremental way. This work can be treated as a proposal for future development of existing systems, and ultimately, as a potential basis of an envisioned, collaborative, integrated trail information system, that can support the effective, organic and consistent planning, management and maintenance of trails and their signage, and provide rich touristic content for the visitors in a flexible way.

The used methodology is to observe and analyze the application domain, consider typical actors and their general needs, look at typical real-life static and dynamic phenomena related to trail networks and their management, identify the challenges these impose on data modeling and management, considering these in the light of the data model of current systems, especially the conventions of OpenStreetMap, and develop an integrated data model suite where each (sub-)model constitutes a specific part or aspect and has a well-defined role and function. For supporting signage management, both the actually installed signs and the needed, planned or recommended signs (changes) should be modeled, with semantic relationships to the trail routes and network, and this way, existing inconsistencies can be revealed, and any effects of network changes on signage can be generated and with the necessary user (signage planner) input, turned into actions of implementation (for the facility management).

The paper is organized as follows: Sect. 2 gives a systematic overview about the application domain with references, sets up a common terminology for further use and reveals some general requirements for modeling. Existing information systems and related data modeling issues are presented in Sect. 3. Sect. 4, 5 and 6 propose a unified conceptual model suite composed of sub-models, that can be a basis of an integrated information system for trail network and signage design, management, maintenance and presentation; and shows its capabilities through solutions to particular real-life issues. Signpost logics will be constructed in Sect. 6 and the Appendix, supporting one of the main challenges addressed: incremental and consistent signage planning over a dynamically changing trail route network. Sect. 7 concludes and adds some future prospects.

\section{The Application Domain: Trails and Networks}

\subsection{A Running Example with Modeling Considerations}

In order to introduce and illustrate the concepts and phenomena being discussed thorughout the paper, consider the example of a trail network shown on Fig. 1. The network consists of four designated routes which are marked with different colors (both on maps and on the terrain with waymarks) and denoted with small letters. A route has a continuous linear geometry defined over already existing ways: paths, walkways, paved and unpaved roads of various types. ${ }^{1}$ Named locations, denoted by capital letters, are places or localities along the trails, which are identifiable in the landscape, usually displayed on maps and have significance for visitors as possible destinations or anchor points in their orientation and trip planning. Pictograms denote types of features (including facilities) based on relevant selected points of interest (POI) next to the trails. They may be related to named locations, but not necessarily. Black nodes denote trail network nodes, which are starting

\footnotetext{
${ }^{1}$ This is being named simple route in our modeling, while complex routes will be a collection of simple routes.
} 


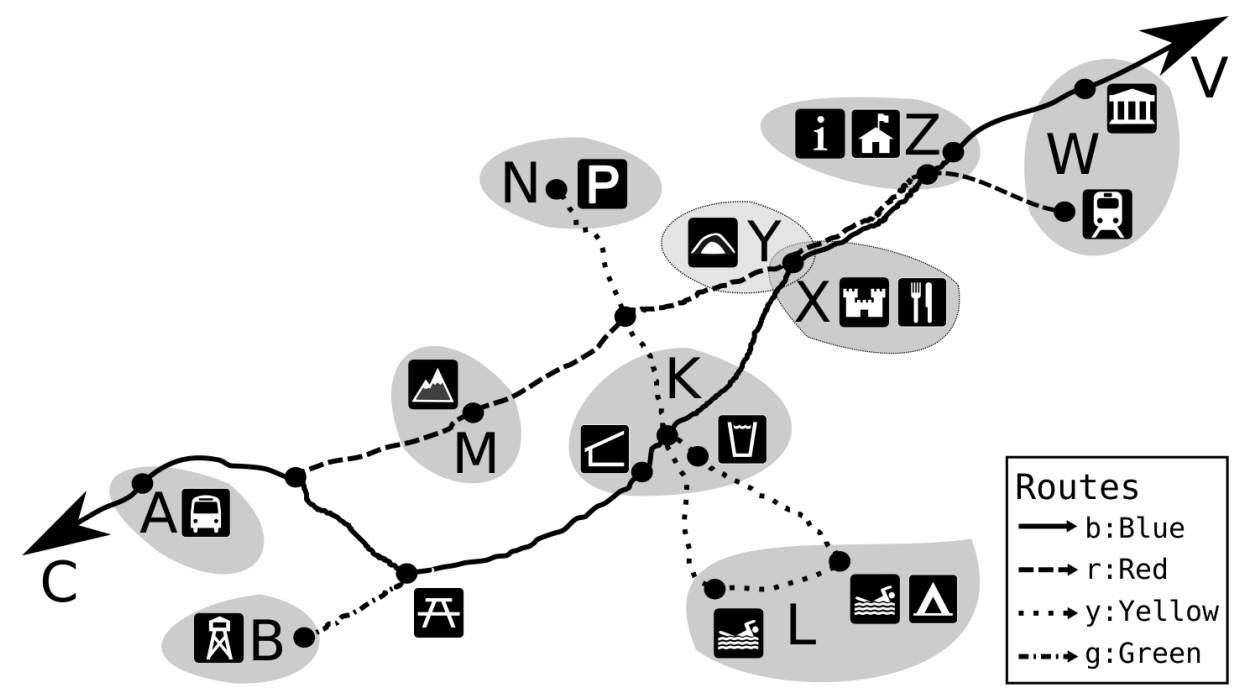

Figure 1. Hiking trail route network example

and ending points, junctions of routes, or places with similar or higher significance (aka. pseudo-junctions, selected points along the trails for anchoring locations, signpost positions and POI features). We call a part of a route between two neighboring nodes a trail section, so routes are composed of subsequent sections. In this example, we treat each route as bi-directional, and declare west-to-east as the primary direction of each. ${ }^{2}$

A high-level description of a route can be a route location list, i.e. a listing of selected subsequent locations passed-by; e.g. the blue route $b$ on Fig. 1 can be described by the list $b:(C, A, K, X, Z, W, V)$. If another trail crosses or branches at an unnamed junction (such as the green route here), the name of the other trail can be used instead of the location: $b:(C, A, g, K, X, Z, W, V)$. This description is not a unique characterisation but rather provides a first simple overview for the users in guidebooks or other media. Any part of the route between locations or explicitly referred unnamed junctions is called a route stage. For longer (complex) routes, multiple levels of route location lists can be specified in a hierarchy.

In general, some relationships in trail-related data are based on pure geographical proximity (such as to collect all POIs along a route). Other relationships are, however, semantical, intentional, and up to the trail managers' decisions as part of touristic presentation or interpretation (to present the trail to the intended audience, e.g. a cultural route, educational trail, etc., or textual descriptions with selected media content such as photos). Explicit selection and edition adds high value of the presented information, and proper data modeling must provide a schema for doing it.

The network of Fig. 1 has the following characteristics and anomalies that occur in real-life situations, which must be considered by proper modeling (supposed the network itself is given as it is and changing or refining anything is not directly possible):

\footnotetext{
${ }^{2}$ By default, a route will have a primary direction in our model, and if it is marked bi-directionally, its reversed variant will a related route.
} 
- Some routes are long-distance trails which extends towards locations outside the scope of the regional network (such as route $b$ towards $C, V$ ).

- Names correspond to locations that can be clusters of nearby POIs and trail junctions (such as $K$ or $Z$ ), or larger geographical entities such as towns (here: $W$ ) or lakesides $(L) .^{3}$

- A route may pass multiple times by the same location $(y:(N, r, K, L, L, K))$, different routes may lead to different points of the same location $(W)$, and different routes may pass through the same location but at different POI features $(K)$, or not even passing by the location's 'main' point where the facilities can be actually found (such as route $r$ at $Z$ ). In the latter case, route $r$ may still be described as passing through the location $Z$, but the signposting should ensure visitors can find the related facilities without accidentally passing-by.

- In some cases the POI feature(s) can extend the location name for unambigous identification and information (e.g. $W_{\text {railway }}, K_{\text {shelter }}$ ), or sub-locations should be defined and related hierarchically to the larger location entity (see later on Fig.3). This may become a particular issue when new routes are created, since there would be no ambiguity if only one of the routes $b$ or $r$ existed yet.

- Different location names can refer to the same place, and can be specific to one or more POIs found at the same (or nearby) place (see $X$ and $Y$ ). Depending on the route thematics, one or another can be referred by specific routes (e.g. if the route $b$ is more cultural-oriented, $X$ with the castle will be referred in the location list of the route, while route $r$ may be more nature-oriented, and the cave named $Y$ will be used instead).

- Not all trail junctions or POIs are named, not even some starting or ending points of certain routes if they merge with other routes (see the example: $r$ : $(b, M, y, Y, Z, W), g:(b, B))$.

- Some routes may be one-directional, others are marked in both directions (we assume all routes in the example are marked in both directions, and use the notation $b^{\prime}$ for reversing a route $b$, such as $\left.b^{\prime}:(V, W, Z, X, K, g, A, C)\right)$.

- Two or more routes may share the same section (such as $b$ and $r$ between $X / Y$ and $Z$ ).

- A longer route may be technically split into shorter stages ( $b$ may be split at $X$, for example: $\left.b_{1}:(C, A, g, K, X), b_{2}:(X, W, V)\right)$. These shorter stages as simple routes should explicitly be related to form a complex route. The reversed version of a route should be split at the same points as its original. The split is not necessarily visible to the user of the complex route, or only as an additional information.

- The network or signage concept may define relationships among the routes (such as $g$ is a side-trail of $b$, or $r$ is a variant of $b$ ).

- Routes are mostly linear but there are circular routes or routes with loops (here: y). Circular routes which end at their starting point can be properly handled, but identifying directions at loop junctions where a trail diverts from itself can be

\footnotetext{
${ }^{3}$ Modeling of locations related to POIs, trail nodes and routes is non-trivial. They can be defined by the support of geospatial proximities or clustering, but due to the many types of locations and possible special cases related to route network situations, it is a problem of its own and can be taken as a future issue. What this paper aims is, to show some of the typical, non-trivial issues and to create a flexible data model that can handle these. But how to identify and define the named locations themselves, is considered as manual work by now and out of the scope of this paper.
} 
problematic (e.g. $y$ at $K$ ). A general recommendation is to split the route into two stages when modeled, so a (one-way) direction can be uniquely identified as the route continuation at each point or junction: $y_{1}:\left(N, r, K, L_{\text {camp }}\right), y_{2}$ : $\left(L_{\text {camp,swim }}, L_{\text {swim }}, K\right)$. A complex route is then formed by linking these simple routes together.

\subsection{Trails and Signage Systems}

Structuring, presentation and on-field marking of a trail network is determined by its signage system. Countries and regions have developed their own systems during the last century [4], based on different network structuring principles. A general data model must be able to cope with such differences and give the opportunity to effectively and flexibly utilize the information inherently contained by the network and its signage system. [11] defines two following ground types, and lists the advantages and drawbacks of each.

Junction-based system (Knotenleitsystem) No routes are defined explicitly by the trail signage. The network graph (see Fig. 7 later as an example) is taken as it is, and each trail section is classically waymarked with a single, unified symbol. Multiple types of waymarks, if used, can denote properties of trail sections (e.g. difficulty by color or allowed transport modality by pictograms). Each trail network node is identifiable with its unique name. Directional information at each node must be provided towards the next nodes, and to some further, important destinations. It is up to the visitor to combine his/her own tour itinerary by route planning along the graph, and recommended trips can be given in form of trail node listing.

Route-based system (Routenleitsystem) The trail system is a collection of independent routes marked with their unique symbols (colors, logos or numbers), and these routes are self-contained, recommended trip itineraries, with their own names, thematics, and without specific attention to their connections with each other. Route information is usually provided using textual and visual media content (e.g. guidebooks), route-specific waymarks ensure the route can be followed at every junction and all along its way. Signposts are linked to routes and can provide auxilliary information, such as the distances of places directly located along the route.

The above ground types are rarely used in their pure form in practice. Most trail signage systems and network concepts are based on a combined approach [4]. A junctionbased system can be enhanced with specific routes or a route-based system can be enhanced with side trails, route variants or connecting trails, using a standard set of waymark symbols or colors for local route identification. Routes can be properly aligned to each other to form a connected network, including routes which do not form a self-contained trip itinerary by themselves, but can be reached by other routes. Our running example is of this type. A specific mixture of route- and junction-based system is when the routes are numbered, and these numbers appear with standard waymark symbols at junctions (or even with destination signposting), and a standard, simplified waymark without route numbers is used along the trail sections between junctions.

Routes are marked with the symbol or color determined by the applied network concept and signage system. Some systems feature local distinguishability of routes by not allowing two routes of the same color or symbol cross at one place, while other systems use colors to determine the route type, difficulty or role in the network instead (in our 
running example, routes are distinguishable by their colors, however, the yellow route has a loop).

\subsection{The Trail Signage Stack}

The trail network is based on existing ways (roads or paths) accessible to the public. It is assumed that the routes are marked on the terrain with simple waymarks such as painted blazes with their respective colors (in case of a classical junction-based system, a route equals to a trail seciton), so they can be followed by visitors relying on the waymarks [3] (in both directions, unless explicitly stated otherwise). At trail nodes, properly designed signposting can be built on top of the basic waymarking signage, which inform visitors about directions and distances to named destinations along the routes, or reachable by their combinations. To construct a unified data model for signage, being able to describe the different types of above mentioned signage systems, we define the signage stack first, where signage facilities are layered, built on top of each other and must form a coherent and consistent system, all together with the digital information provided through various channels:

- Passable paths must exist which can be technically and legally designated as a waymarked trail, and made part of the network. It must be maintained.

- The path must be continuously followable by subsequent waymarks placed along it (usually painted trail blazes, or more generally, trail markers which may contain more information), especially at open spaces and junctions with other (not necessarily marked) ways. These marks are usually painted blazes or some simple signs on trees or objects next to the trail, and marking is considered more likely as a linear feature related to way sections ('the section is waymarked') rather than managing each single waymark as a point feature (which is also possible if needed).

- The route (and/or trail type) should be identifiable by identification elements in the signage, at least at certain points (junctions with other trails, start/end points). This can be a number, a logo, either painted, mounted as part of another sign or separately. We call it a route sign. The trail marker may already have this role.

- The route network should be navigable: Places along the route are recognizable using place marks (location signs, usually displaying the altitude of the place above sea level) wherever it is meaningful. Route directions with distances or walking times should be pointed at at junctions and trailheads, in form of route destination signs, which can contain pictograms of relevant POI features found at the displayed destination. Directional sign boards (such as Fig. 2) mounted on guideposts display one or more route destination signs, usually in the order of distance. They display the route or waymark information (color, name, number and/or logo), or a combination of routes to reach the displayed destination(s). ${ }^{4}$

- The network should be overviewable using maps, displayed on info boards at trailheads or touristic centres, and takeaway-maps in paper form or by digital media.

Distance or walking time, as well as difficulty (if displayed) must be computed in an unified way given the track of the path to the signposted destination. Walking time can mostly be determined by a formula based on an aggregation of lengths and altitude

\footnotetext{
${ }^{4}$ These are also called signposts, but we use this term in a more general meaning: either as signposting a route (adding location and route destination signs) or as any single item of the route signposting.
} 


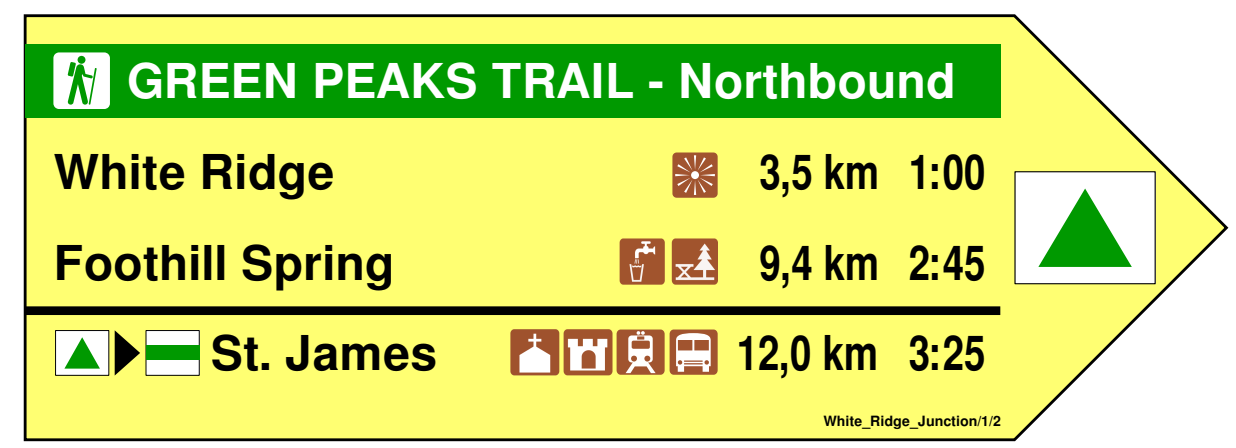

Figure 2. A sample sign board (signpost) with a route sign at its top and three route destination signs with location features, distances and walking times. The common trail mark of the route is conceptually part of the route destination signs, but by the actual design of the sign board, it is displayed only once (in the arrow part) except for the last, combined destination sign indicating an indirect route connection.

profiles of involved trail sections (such as in [15]) with a reasonable rounding principle. However, some sections may need manual update due to specific path conditions. Difficulty may be aggregated depending on what exactly it means (c.f. [33]): the aggregated technical difficulty is the maximum difficulty level of the involved sections, while the necessary effort must depend on a sum of sections. These parameters should be computed the same way for each information asset (including signposts, maps, digital touristic information provided) and therefore, should be managed centrally on the route network level and queried for any presentation interface.

Additional signage, such as interpretative, educative, warning and regulatory signs can also be considered, but as they are not contributing to the orientation and navigation tasks, they are out of the scope of this paper.

\subsection{Actors and Dynamics}

Different actors have their own view of the same reality, and the key of an integrated data model is to unify these properly.

A visitor who is planning the trip needs only an overview, with appealing media content, and aggregated information (e.g. sum of kms or altitude gain, max-min altitude, max of technical difficulty, max of steepness). Simplified route representations and descriptions are sufficient, with the ability to take a route or combine their sections into an itinerary. On the field, the visitor may need detailed topographical and navigational information about trail sections and their junctions, and the exact places of POI features at specific locations.

A trail crew needs to implement changes or reparations and has to know on the field exactly where to do what, based on a detailed network plan, and data of existing and planned facilities. A trail network designer needs to know the existing segments and suggest extensions or changes, new routes or re-routing, and will give input to touristic content editors to extend or adapt the presentation of trails for visitors. The signage system designer needs to know the rotes and his/her task is to propose what content should be displayed at which places conceptually (ideally, in coherence with the touristic content being provided, e.g. when defining route stages), while the signage implementor needs to have all this information compiled, enhanced with precise data, and has the task to design 


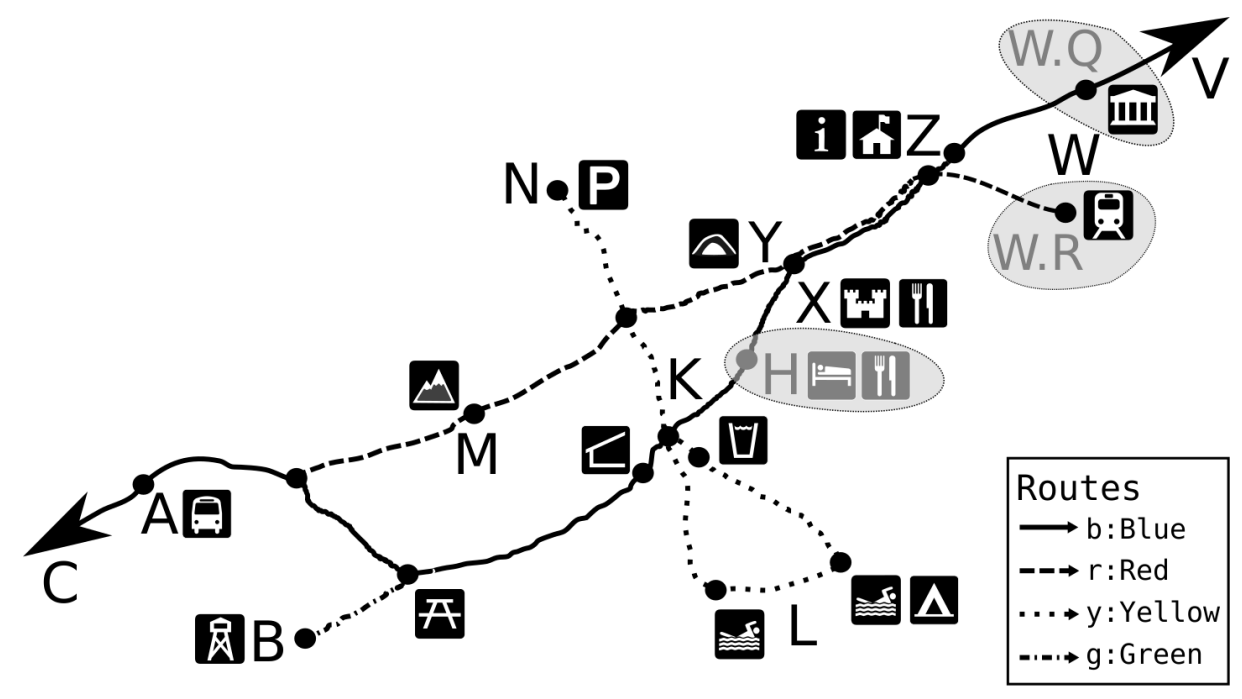

Figure 3. Hiking trail route network example with refined and added locations

and produce each signpost and pass to the trail crew for installation. Certainly, different role settings are possible, but these tasks are to be suported.

Trail routes or networks and their management patterns are often developed incrementally and organically, and change dynamically over time. They usually involve bottom-up civil and volunteer engagement and enthusiasm, and at the same time, are dependant on governmental authorities, must obey certain policies and regulations, and must take into account interests of private and public land owners as well. Development of trails is often determined by interests and activities of a multitude of stakeholders and contributors on multiple levels, and is usually incremental and organic, providing the user a 'local-as-design/global-as-view' ambiente. Sometimes there is no unified organization behind a trail or network, but only contributors. In other cases, network design and trail management is driven by strong public or private interests, and is more like a top-down process, involving less number of stakeholders and have a solid organizational structure. In such cases, it becomes similar to a 'global-as-design/local-as-view' system for visitors.

Trail networks are more versatile than public road systems. For example, new attractions or facilities are created along the routes, which may have substantial effect on the network layout and signage. Courses of paths may change for various reasons, sections or routes may be added to the network, temporarily or permanently closed, and routes may need to be re-routed. Signage is often developed incrementally, but the system should support its consistency. These processes include user decision and input, but some parts can be automatized. Example scenarios inculde:

- A new hotel is being built along one of the trails. It needs to be incorporated into the signage system and touristic contents provided (such as location $H$ on Fig.3).

- A village can support installation of exactly 3 guideposts. Where to put them and what to indicate on them?

- A trail section is closed or modified and a route must be re-routed. Which sigposts have to be updated, moved or newly installed?

- One of the routes need full signposting at each junction. Plan it, implement it. 
- Location names need to be refined in relation to route info and signage to aviod confusion (such as $W$ on Fig. 3 where two routes arrive at different parts of the town $W$ ).

\subsection{Signage Planning and Management}

One of the major aims is to support the systematic and consistent design and maintenance of trail signage. Signposting is not always complete and up-to-date, and may be at different development stages for each route or network part. Revealing inconsistencies and generating actions for necessities and recommendations is an important task that can be supported, especially for complex networks.

Actual signposts (sign boards mounted on guideposts) can be composed of multiple route destination signs (c.f. Fig. 2), but for simplicity, we use the term signpost in this section as an abbreviation for route destination signs, and do not consider how they are actually presented on sign boards, but only what locations need to be signposted at which places via which routes.

Signposts are not independent of each other, they must be treated systematically, as a set of them along the routes. If a signpost is already placed, it will imply other signs we must place along the route(s), to ensure consistency and the continuous, unambigous followability of the signposted routes and directions. The European Ramblers' Association (ERA) has created common guidelines for the application of signage elements [3]. For signposts, it contains the following rules. These may be extended by other guidelines, specific to the locally applied signage system.

(ERA-8) 'Directional panels or fingerposts indicating the route of the marked path should be placed at the starting/ending points of the route, at junctions and at other necessary places along the route.'

(ERA-9) 'Fingerposts and directional panels should indicate one or more destination along the marked route and the distance in $\mathrm{km}$ (miles) or hours. If a destination is shown on a fingerpost or panel, it must be shown on all other subsequent fingerposts or panels until the destination itself is reached.'

Another, reasonable guideline can be that the locations need to be identifiable as they are reached: if a destination is signposted anywhere to be reachable along a route, a location sign of some form must be installed at the point where it is (meant to be) reached. It can be an explicit planning decision because named locations often do not correspond to a single trail junction or POI (c.f. locations $K, L, Z, W$ on Fig. 1 and their connections to different routes). If main facilities are not trivial to find at a location, local signposts must be added.

Fig. 4 shows a signposting example for Fig. 1, which is neither complete for any route or the network, but is logically closed and consistent. It shows the implications of placing specific signposts at point $A$, towards $Z$ along $b$ and towards $M$ along $b$ followed by $r$ : The latter one implies an extra signpost towards $M$ to be placed at the next junction along $r$, and the existence of this one together with the previous one at $A$ towards $Z$ implies a sign towards $Z$ via $b$ (if rule (ERA-9) is taken strictly). Furthermore, location signs are implied as follow-up signs by signposts direcly pointing towards any destination to confirm the destinations are reached. We make the following observations for consideration: 


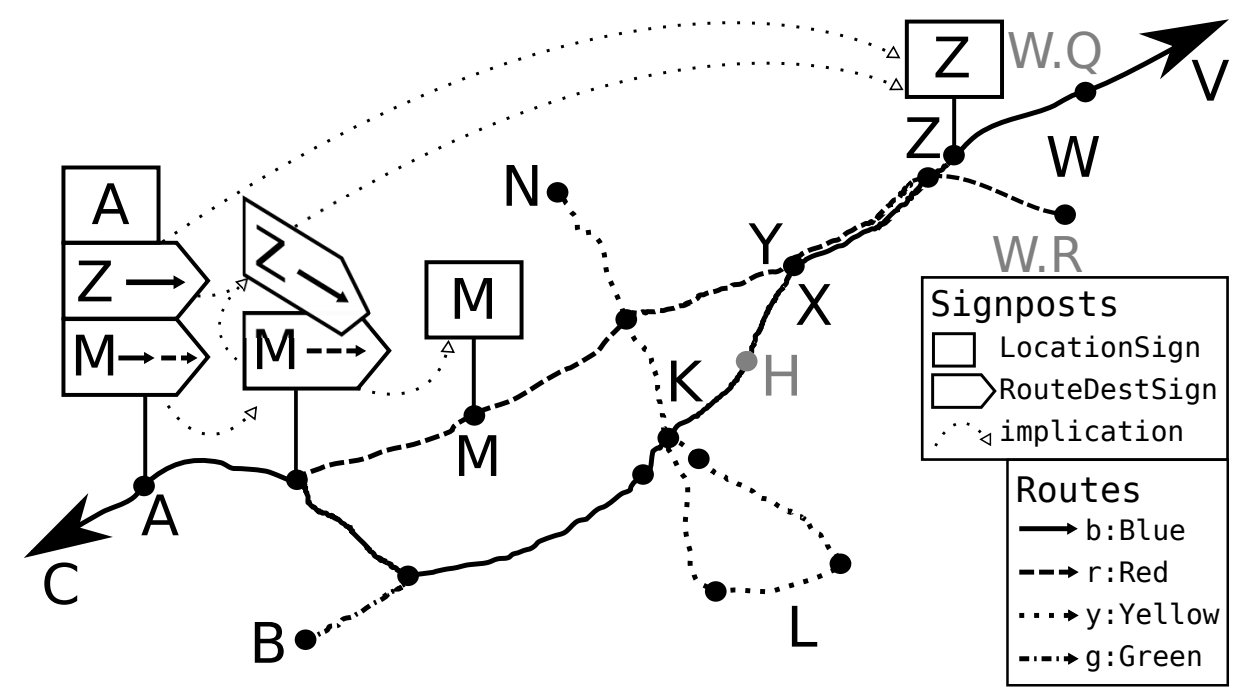

Figure 4. Signposting example

- Signposts do not necessarily mean the shortest possible way towards the displayed destination, and there might be different options, so the data model needs to handle it by explicitly or implicitly adding the whole course of the pathway meant to reach the displayed destination from the signpost (e.g. route $r$ takes to $Z$ as well via $M$, and it can be signposted additionally, as an alternative direction).

- If we placed another signpost at place $K$ showing $X$ is reachable via route $b$ from there, it may cause confusion unless $Z$ is repeatedly signposted there.

- In a route-based signage system, signposts primarily designed for the routes and display destinations directly reachable along routes (without changing to another route). There might be important destinations that fall outside of these, so they can be pre-announced by a signpost with a notice that the visitor must change for another route at some later point. With this flexibility, some of the strengths of a junction-based signage system can be adapted into a route-based system. This is the case of the mountain $M$ along the red route, for example. It causes interdependence between the signposting of multiple routes.

- Regarding to what destination should appear on a signpost at location $B$ (since route $g$ does not end at a named or significant location), it is reasonable to choose a further location along route $b$ (or its reversed $b^{\prime}$ ). Existing or already planned signposts of routes $b, b^{\prime}$ at the end node of $g$ (e.g. $A, X, Z$ or $K$ ) impose a suggestion or possible choice for a planned signposting at location $B$ for route $g$.

A common practice of systematic signpost planning is the route destination table (or route signposting table) method [14], where subsequent trail network nodes along the route are listed in a column as potential signpost places, and the destinations to be signposted are written in the rows towards each direction (forward and backward). The number of signposed destinations at each place in each route direction is limited by the used network signage system (usually 2 or 3 , and they are actually displayed on a single signpost per route, in the order of reachability along the route). Fig. 5 shows an example for route $b$ as an extension of the signposting at Fig. 4. Trail network nodes along the 


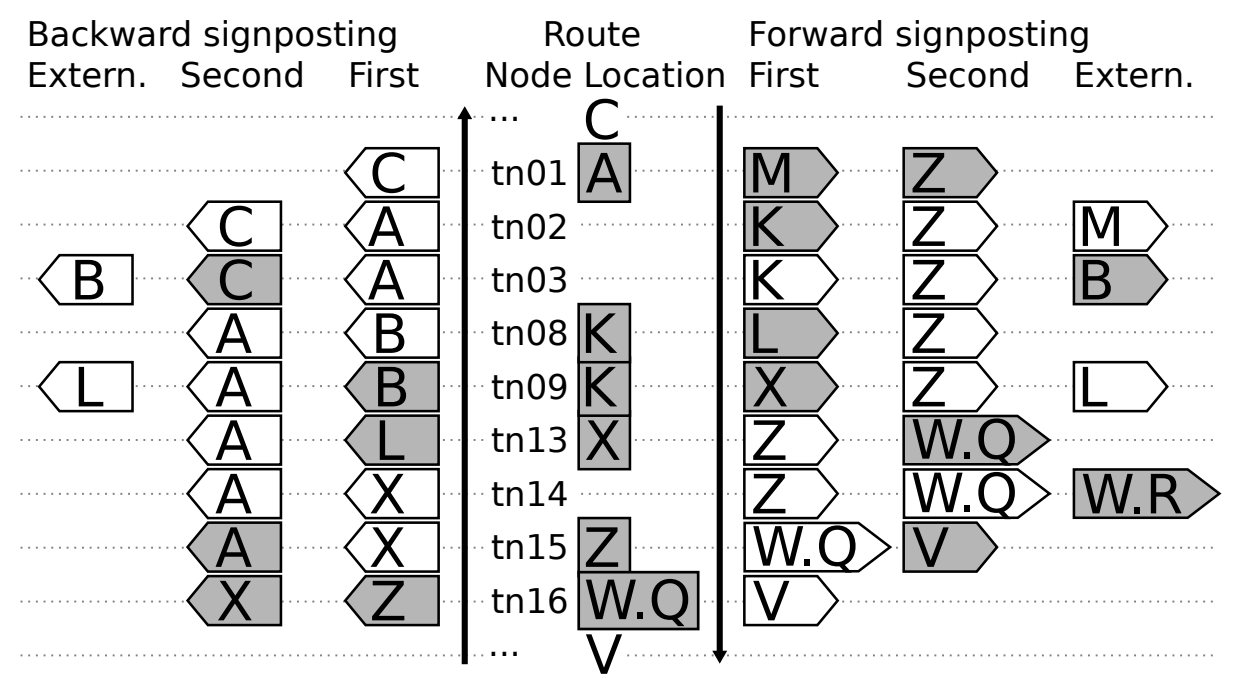

Figure 5. A full route signposting table example of route $b$ (Blue) in both directions. Location signs are planned based on user-chosen route stages. Grey signposts are chosen by the user (signpost system designer). Signposts with white backrounds are implied by the already chosen grey ones. Note that some signposted destinations are outside of the route $b$ and therefore, this route table is interrelated with signpost plans of other routes (through the Extern. columns). Some Extern. signs are duplicates as they appear in both directions.

route are listed in the middle column, and in each row, the right side contains planned signposts in forward direction (top-down), and the left side in reversed direction (bottomup, it is actually the signpost table of route $b^{\prime}$ ). At most two destination signs are planned for each possible signposting point per route and direction. Implications are shown with different backgrounds, but the exact places of location signs must be explicitly decided since there may be more nodes fall into the same location (e.g. at $K$ ). After all route signposting table is completed, the planned signposts are collected for each place (node) from all routes, and put onto a special form for each location (Standortformular, see [14]) where the precise content and arrangement of signposts and their planned installation is designed, based on the layout of the junction.

Note that some destinations fall outside of the actual route $b$, and they imply external signposts (pointing towards another route). The signposting table of a route has implications on the signposting table of another route. If the network is dense, and there are many routes, it becomes difficult to ensure consistency when designing the signposting system by hand, and might be more difficult to handle if changes occur in the network. So far, it is not known if any trail facility management software supports this functionality.

\section{Trail Information Systems and Modeling}

\subsection{Models and Features of Current Systems}

Many applications, websites and map tools provide information about trails and their networks, completely different trail systems exist around the world, related to various fields and levels of profession (tourism, transport, conservation, sport, forestry, etc), each having their own approaches. Technical documentation is in many cases not available, 
non-public, insufficient or not internationalized. This heterogenity makes it difficult to give a well-founded overview of the situation, but some visible trends can be outlined.

Apps or websites for visitors usually have two basic options for trip selection or planning: choosing a recommended, pre-compiled trip itinerary from a catalogue and take it as a whole trip or outing, or perform a totally flexible routing along a network graph and generate a trip this way.

In the latter case, most of the additional information provided for the pre-defined trips (tours) cannot be utilized (except for POI information if generated based on proximity) and the user is usually facing a low-level (too detailed) representation of the planned trip. Multi-level modeling has been existing already for a long time for road networks, such as $[34,16]$ but haven't been adapted for walking/hiking trails. Even if it might not be needed for effective way finding, it seems to be reasonable for suitable information presentation and to establish a unified and semantically enhanced signage management.

Most touristic apps presenting trails do not handle networks, and the pre-compiled tour itineraries don't have any flexibility. An exception is the GuideAtHand app [21] which allows content providers to plan some optional detours or variations into its walks, and the system ask the user on the field which variant to take further. It is still far from trip plannig and adaptive walking-through by allowing a dynamic combination of precompiled partial trips forming a network, which might be a better option for users searching for some variation but feeling somewhat 'lost' with a totally flexible network routing in a complex network, and would utilize more linear-based, pre-compiled touristic information such as a route description and people's feedbacks on trail sections.

[15] mentions the lack of standard data description models for pedestrians and so, for hiking routes and their networks. It identifies various information requirements and introduces the hikingtrack concept as an extension in schema.org modeling. Not even difficulty rating is standardized: it defines 3 different types (effort, technical, pshycological) which should co-exist. Hiking time computation are based on various methods (such as [33] and others). Some of these are proprietary.

Trail and network planning and facility management can be supported by multiple GIS and cartographical tools, including general and custom solutions. Current trail managers most likely use custom solutions (if any), because there has been only recent widespread publicity on trail facility management systems. [5] presents Komoot, NatursportPlaner, OutdoorActive and RealityMaps as 'digital trail mangement software'. Natursportplaner [6] is generally used for planning, while the platform of OutdoorActive integrates trail information provision for visitors with facility management functionalities $[19,32]$. The publicly available data model of OutdoorActive [24] contains various point features (POI), a tour as a linear feature (corresponds to a trip in our terminology) and a region type (all originated from a common supertype object of interest) with categories applicable to them.

Another, newly developed platform is OuterSpatial [7,12], which provides information for visitors as well as for trail managers and partners, and an interface for community contribution with user feedbacks directly channeled into facility management. Its data model is based on areas, various types of locations/POIs, trails and outings, with map and media content in PDF.

Such platforms usually support the management of field assets, including signposts, based on their location, status, type, and can store the printable image of the signposts for (re)production. Some tools may support the computation of distance or walking time 
based on manual selection of displayed destinations on signposts. Since no public documentation is available for most systems, it is difficult to assess, but features are not known which would semantically link the signposts to each other and support their systematic design and change management in the way has been presented here. [9] reports a custom design process for a large-scale, long-distance trail signage design, but it does not consider the operational management phase, and no official feedbacks or follow-up information is available.

Besides commercial products, custom solutions and platforms provided by trail or tourism organizations, there is a wide variety of community-based map tools and applications, primarily based on OpenStreetMap ([1, Comparison of Android applications] shows more than a hundred of Android apps - not only related to trails, while [1, Hiking Maps] compares 9 different map services/platforms especially for hiking). Wikiloc [2] is also extensive and popular (featuring almost 15 million of trails at the time of writing this paper), but its functionality is limited to precompiled, recommended trips as trails.

A multitude of related work exists on surveying hiking preferences (such as [13]). In the recent years, analyzing user behaviour, personalized recommendations became widespread in general, based on tracking or social network data for mobility patterns (such as [10]) and some works especially for hiking and walking have recently been appeared (such as [20,26]). Personalized trip recommendations for flexible rambling is a promising future direction, and should be supported by proper modeling.

\subsection{OpenStreetMap and its Trails Data Model}

OpenStreetMap is likely to be the most widely used community mapping platform with a multitude of related services, websites and apps. Its data model is based on flexible tagging and conventions rather than strict structures or standards. Conventional tagging schemata of features related to hiking is documented in its Wiki [1, Hiking] and a substantal amount of hiking routes and facilities are already mapped.

It uses a route-based approach to model trail networks [1, Walking Routes], with the option to define short routes with similar signage, so a junction-based or mixed system can be simulated. There is a hierarchy of routes defining international, national, regional and local walking routes, and hyperrelations allow to create a hierarchic long-distance route with daily stages. There are no unified conventions for further semantic connections among routes.

Trail routes are so called relations composed from an arbitrary choice of elementary way segments. Direction of the route is not specified, and route geometries are not necessarily continuous linear features. Flexible trip planning is provided with general routing, which does not take into account the waymarked routes (services are currently limited for planning along waymarked trails). Trail and signage-related tagging varies by countries or regions, as the signage systems differ. Way segments do not generally match trail network sections as we define it (and our definition includes user decisions as well, by the pseudo-junctions - however we did not say anything about their actual implementation), they are usually shorter and not necessarily cut at trail junctions, but the route planner can work on partial way segments as well.

Modeling of routes on OpenStreetmap is lacking the explicit assignment of locations [1, Walking Routes], as providing intentional touristic content is out of the scope of that system. POIs or other features can be implicitly collected to a route based on geographic 
proxmity. Route descriptions with starting and ending points or important intermediary locations often appear in the textual description or name of the route. However, they have no explicit semantic relationship to the geographic features they refer to.

For our purposes, the basis of the route network can be extracted from OpenStreetMap, together with specified types of POI and other geographic features in proximity of waymarked routes, as well as manually selected non-marked way connections. [27] shows a method for road networks guided by a conceptual schema. Post-processing may include checking route geometries, cutting dangling geometries (each route should have a single continuous line course), making route directions explicit (generate reversed routes as necessary), and assigning named locations.

For signposting, there exists a tagging scheme for modeling route destination signs [1, Destination Sign], to aid navigation software when displaying the actual directions to follow (e.g. instead of just 'turn right', 'turn right in the direction signposted towards $\mathrm{X}^{\prime}$ ). It is a relation containing an exact location, a junction or way reference point, the direction where the arrow points (in form of a way starting at the junction), the name of the destination, distance and/or time in hours written on the signpost, and a route reference. It is a purely formal representation of what the signpost is, without semantic relationships to the shown destination or the actual route or pathway meant to reach it. It may contain more than one sign items (route destination signs in our terminology) at once.

This tagging scheme may be enhanced to form an extended data model, where the signs of destination directions displayed on signposts contain actual references to the destinations they show, and to the pathway along the network of routes to reach it. This way, the systematic design and also the maintenance of signage can be supported, so that the displayed distances can be computed automatically, the validity of actually existing signposts can be checked, and it can give an input to the community mappers to map missing parts of routes referenced by signposts.

\subsection{Related Work on Modeling}

Considering what has been presented so far, the following works (tools, concepts and methods) seem to be especially relevant and useful for our modeling case. Their particular application should be elaborated in more details in the future.

The aimed integrated information system must provide multiple facets to various actors, who need to see the same reality from different viewpoints (actually in different models), also depending on their task and current situation (e.g. whether a visitor is at home and on the field). It assumes a complex database setting whose way of operation may also change during the phases of network or organizational development, but the operations should be well-defined. Therefore, model suites presented in [28] seem to be a proper means of specification in the long term as they can provide a framework for multi-level data settings, and coherent, but flexible exchange and collaboration patterns.

For conceptual modeling of data structuring, the higher-order entity relationship notation (HERM) is especially convenient [30,29] as it is well-founded and can compactly express complex settings. Traditional ER or UML class diagram could be also used.

Specification of interaction and functionality should be based on flexibly combinable, but self-contained (mini-)workflows where user interaction can be combined with system automatisms. For that, the concept of mini-stories for generic workflow presented in [31] could be a suitable framework. The interaction space can be specified in a flexible way, but specific stories can be featured and translated to workflows [23]. 


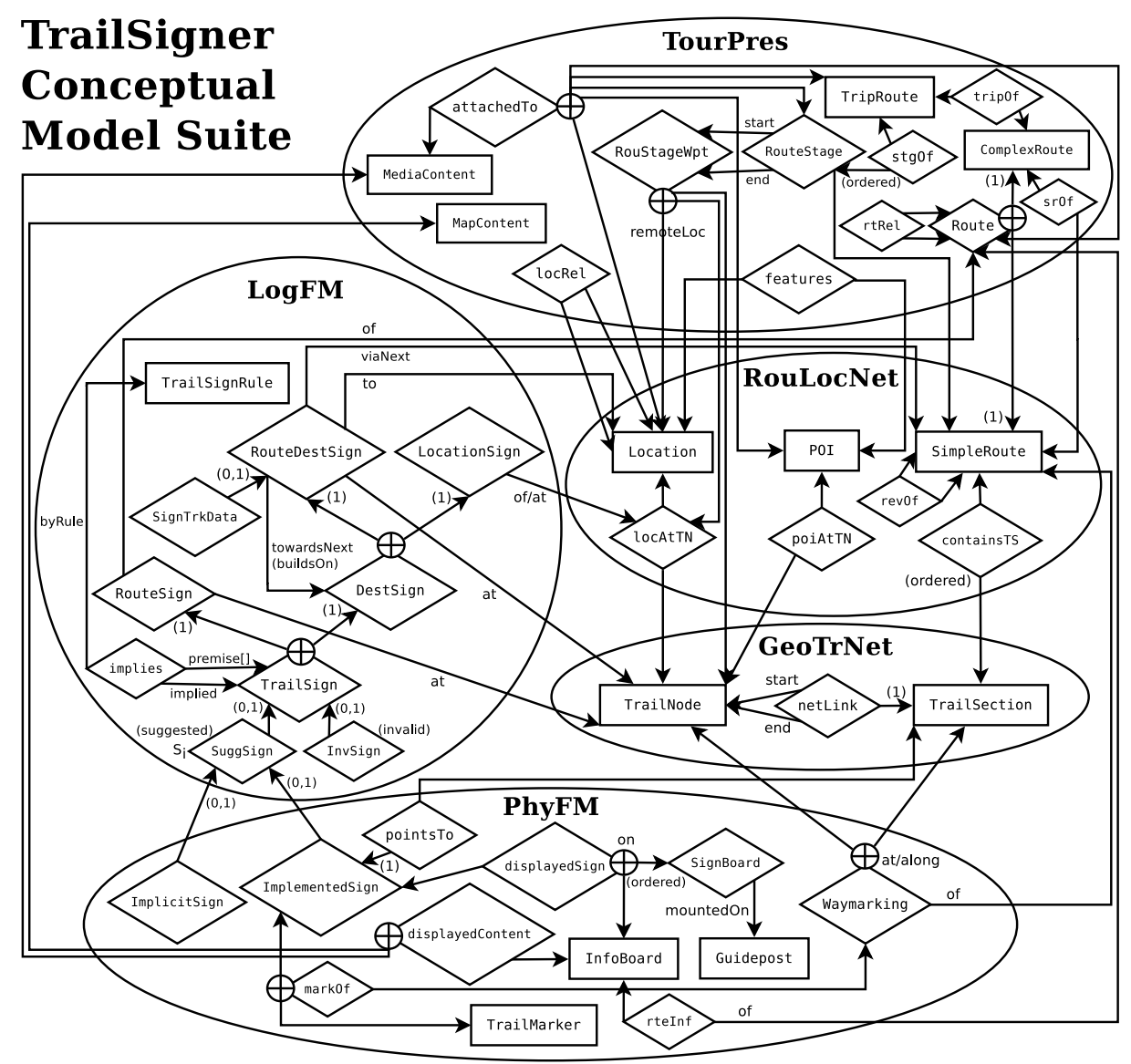

Figure 6. The TRAILSIGNER HERM model suite of trail systems. Cardinalities - wherever constrained - are shown in the participation notation. Attributes and some derived relationships are not shown, for simplicity. Some of them are subject to model variations as well.

Modeling data constraints and derived attributes or relationships presupposes the use of active database concepts [25]. Implementation of signage dynamics and implication can be either based on triggers and database constraints in a classical relational RDBMS architecture, or this part of the system can be oragnized separately, using a logic-based data platform, with the relevant considerations kept in mind (such as in [8]). From the user perspective, systematic signage design should involve some way of graphical and spreadsheet reasoning, as presented in $[18,17]$ for the schema level, but here on the instance level (c.f. the route signposting table method).

\section{Overview of the Proposed Model Suite}

We propose the skeleton of TRAILSIGNER, a unified conceptual model on Fig. 6, in higher-order ER [30,29]. It can be defined as a model suite [28], in which consistency criteria and collaboration styles may be flexibly declared among its (sub-)models. Its (sub-) models and their functions are described briefly. 


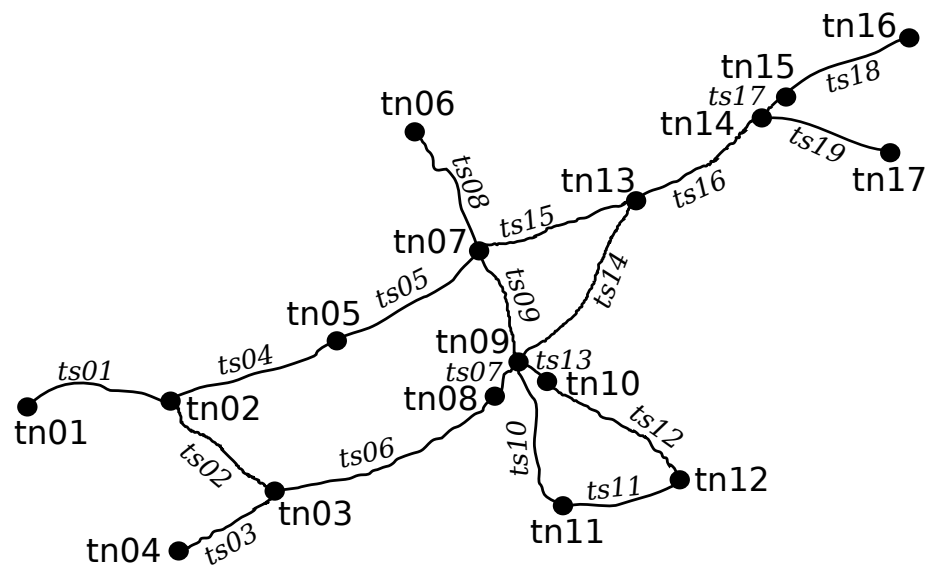

Figure 7. Hiking trail network graph

Implementation details are not subject of this paper and are left open for further refinement of the model, but a general versioning facility is in any case needed for tracking historical states and to explicitly publishing network changes. It is a conceptual model and different implementation schemata can be constructed to it. Some of the model constructs have more possible translations into logical database schemata. Some of the relationship types can be (at least partially) derived, based on geolocations and other relationships. The actual implementation may depend on the workflow or policy of the organization(s) as well. However, to assess the feasibility of it, a detailed version of this schema is being published by the author with one possible implementation as an on-going project under [22]. Nevertheless, the following sections briefly discuss how the model in its current level can cope with the challenges described in the previous sections.

\section{Modeling Trail Route Network and Touristic Content}

Each of the following three (sub-)models are generally a different view of the same reality for different purposes (with specific enhancements as their own data), and therefore, must be kept consistent. A refined definition of the model suite may further specify their relationships and implementation.

\subsection{The Trail Network Graph (GeoTrNet)}

The trail network model forms a classical geospatial routable graph structure. Its function is to represent the exact trail courses as they are on the terrain, mainly for trail work and on-site navigation (including the altitude dimension). Fig. 7 depicts a possible underlying local graph of routes of Fig. 1 with technical identifiers of trail nodes and trail sections connecting them. Note that this graph can basically be generated from the geometries of routes and their intersections, by splitting route geometries and adding a node to each route junction and endpoint, but must be refined by adding extra nodes at pseudojunctions (facility locations). The graph is generally bi-directional, except for explicitly defined one-way trail sections. Technical difficulty, computed (and in special cases refined) walking time can be stored or computed on-the fly for each section, together with 
distance and elevation gain/loss derived from geometries. Status of a trail section may indicate a planned or a closed section and influence its permitted operations and visitibility to different actors. Changes of the network, new related locations or features, including sigposts may imply sections to be formally split. For such cases, update mechanisms must be defined for each of the relationships of trail sections if the split is implemented as the cut of the line (other possibilities also exist by linear referencing, but here we speak about a conceptual model and it is the question of the implementation schema). Minor changes of trail sections, which does not change the network structure might have effect on signposts, or stay below the rounding tolerancy of displayed data.

\subsection{Routes, Locations and POI (RouLocNet)}

Trail routes are represented with their full geometries (mutual consistency must be ensured with GeoTrNet, either by defining a master-slave data exchange pattern in either direction, restricting the modification of geometries in one of the models, or allowing any modifications supported by a two-way update mechanism). Its function is for network designers, managers and visitors, to represent the routes and how the locations and facilities are connected by them. Any trail section has to be covered by a route, as waymarked routes define the network (trail sections without an assigned route are considered to be planned or closed). Signposts and touristic content can be assigned to routes and not to trail sections directly. In a junction-based system, each trail section defines a route, or routes can be arbitrarily chosen to cover the (junction-based part of the) network. If there is no actual waymarked route along a section, and the connection is important, a pseudoroute can be generated, making the section as part of the trail network on the route level as well. Routes may have no or partial actual geometries, either in planning phase, or when a long-distance route spans beyond the regional scope of the system, in the latter case, derived data may be imported from elsewhere for the missing sections and locations, to enable seamless signposting and (long-)distance computation along routes. Routes have simple continuous geometries and do not intersect themselves, nor repeat or lead back on the same section. More complex routes must be split into simple routes and connected as a complex route on a higher level, in the TourPres model.

If a new route is added or its course is changed, the updated network must be generated with possibly new nodes (split of existing sections, their derived data recomputed) and it may imply changes to the signposting and touristic contents (see below). Route status and versioning is necessary for an integrated system which actually contains planned trails and historical data.

Routes are taken as one-directional. Bidirectional routes must be duplicated, inverted and related to their primary variant ( $r e v O f$ ). This controlled redundancy has the advantage of simpler handling of waymarking, signposting assets, and touristic information.

Relations in this model are completely generated by actual geometrical relationships, but trail-related, relevant named locations and POIs must be defined or selected explcitily.

A location may be defined by a single POI or a cluster of nearby POIs (such as $M$ or $K$ on Fig. 1, respectively), or a geographic object with an extent, such as a village $(W)$, lake or lakeside $(L)$. A location can also represent a geographic place name without any specific POI feature type, or not originating from the POI(s) actually displayed as trail features (e.g. the bus stop at $A$ may have got its name from a farm there called $A$, which itself is not relevant as POI for the trails, but gives the location name). Modifications of locations impose an update on the network and related entities. 


\subsection{Touristic Content and Presentation (TourPres)}

Its function is to give a higher-level overview of the trails, their features and touristic interpretation to the visitors, making the trail network overseeable and browseable, and provide a platform for media content input (images, descriptions attached to locations, POIs, routes, or parts of them). Trip routes can be composed from parts of network routes (either simple or complex - the latter may have variants, or may be a multi-day trail with day sections defined as simple routes) and offered to the visitor, and the visitors can themselves assemble their own itineraries. Routes may be divided into longer stages, based on explicitly defined route location lists (possibly multiple sections, either defined by an unnamed node, a node of a location or a remote location not part of the local(ly stored) network). Stages are the basis of linear touristic content management, and visitors can select or combine their trip routes from them. Location priorities along routes can be added to the TourPres model by defining hierarchically nested route stages (location lists), for example. This gives a flexibility to assign content in route views of different details.

This part can be integrated into a content management system. Actual geometrical relationships can support the relationships in this model (by constraints and suggestions), but all of them are actually determined by the content editor. At this level, structural or semantic relationships can be defined between locations (e.g. hierarchy), locations and POIs (selected relevant features of places), and routes (e.g. reversed, variant, continuation of another route). If a new feature is added to the system and assigned to routes, a new trail node may be necessarily generated.

This model part acts as the space of personalized user content as well (future issue).

\section{Models for Facility Management and Signpost Logics}

\subsection{Physical Facility Management (PhyFM)}

The Physical FM (signpost and waymarking) model represents the inventory of the actual trail signage assets as they are situated on the field, and those signage elements that have been decided to be implemented and are under production and installation. Waymarking of a route is assigned to trail nodes and sections, while location, route and destination signs can be nested into info boards or sign boards mounted on guideposts (c.f. the Signage stack in Sect. 2). Status of the signs can be stored here (either valid or invalid; existing, lost or planned) together with facility management actions (not indicated for simplicity). Signs may be implemented (actually existing) or implicit (expressed by other signs, and therefore, not explicitly needed). Besides the actual graphical and textual content of each signage element, semantical references are also kept (e.g. not just the displayed name of a location, but the foreign key of the referenced location itself as well), so that the validity of signs can be checked against the actual (potentially changing) trail network data.

\subsection{Logical Facility Management (LogFM)}

The Logical FM (signpost) model is the most novel part of the proposed model suite. It has the function of supporting incremental and consistent signpost planning and change management. It is a platform for conceptual set-based signpost planning and a mediator for change management between trail routes and their effect on signposts in the physical 
model. An instance of it is a partly generated, partly intentionally selected set of ideal sign elements in form of logical facts with different priority levels, which are always consistent with each other and with the actual version of the trail route network. A logical location sign at a node of a named location indicates a need for placing a sign indicating the location name there. A logical route sign refers to the need of displaying the route identification and continuation at a node. A logical route destination sign is an expression that the direction of a specific destination should be signposted at a node via a route or route combination. The exact meaning of such a logical sign is modeled in a linked-list-like fashion, always pointing towards another, follow-up sign: if the signposted destination is meant to be reached by following a single route, then the follow-up sign is a location sign at the destination. If the visitor has to change routes at a later point, then the follow-up sign will be a logical route destination sign at the change point towards the destination (see the chain of signs towards $M$ on Fig. 4). Such signs are composed by structural recursion, always having a location sign at the end (finiteness, without circles). This construction is modeled by a cluster type DestSign, which is a generalization of location and route destination signs. The chain of follow-up signs is part of the identification of route destination signs, thus forming a complex natural key domain. The sign track data can be computed from the trail network and route data using aggregation (e.g. total distance, computed walking time, max difficulty, trailmark(s) to show).

The above composition mechanism can be used to create possible logical signs that can be implemented in the physical FM model. Since we do not store any data such as route or location names, waymarks, distances etc. explicitly in the core sign data types of the logical FM model, only references to the actual network objects, an actual view of the logical signs is always consistent with the network (some logical signs may not be valid after a network change, though, as they will refer to obsolete versions of trail routes, locations, etc). Attached SignTrkData is always (re-)generated or validated automatically.

The signage planner user may take decisions on the logical level regarding to which signs should be implemented. These must be consistent, e.g. if a sign is to be implemented, then a follow-up sign is required too. Similar guidelines for signage application (for recommendations and obligations) can be formulated as TrailSignRules. Each time a logical sign is added, removed or changed, the set of signs will be updated as the logical closure generated by these rules. This way, consistency and signage guideline compliance can easily be checked and the needed signs or updates can be selected for implementation. Different levels of implementation priority (necessity, primary or secondary recommendations) can be defined by the rules. Implication is tracked for logical signs to distinguish between explicitly added and implied ones. The implied ones can always be re-generated.

A formal definition of signpost logics is presented in the Appendix.It defines a series of predicates $S_{i}$ that indicate the necessity levels of logical trail signs (it can be implemented either as attributes or database relations in the LogFM model). In a planning process, the signpost planner user decides on initial (existing or initially planned) signs, and sets them as $S_{0}$, and optionally adds lower priority signs to $S_{1}, S_{2}$, etc. Then an iterative logical implication step is made using the rules to generate further necessary signs into $S_{0}$ and recommended signs with different priorities $S_{i}, i>0$. The signpost planner then makes one or more choices and moves some signs from $S_{i}, i>0$ to $S_{0}$ and a next implication is being performed for further necessary and recommended signs. Although there is no direct formal conceptual relationship between LogFM and TourPres, the signpost 
planner should consider harmonizing the signposting with the touristic content provided (route stages), and the system can give respective recommendations.

If the signs are settled down in $S_{0}$, they can be put into planned stage, which means the sign track data is computed and it becomes ready for implementation (graphical design, production and installation). This is performed in the PhyFM database. The signs marked for implementation are not always identical to the actual signs placed on the field: some physical signs can be obsolete, and not all of the logical signs are actually implemented as physical signs (ImplicitSigns, whose information is expressed in other ways). The actual situation may differ from the ideal and the difference induce necessary actions, which can be represented by signpost statuses in PhyFM. To handle this properly, versioning of the trail data is needed and the historical data kept in the database.

If the network changes, logical signs are checked first as some of them may become invalid after a structural change in the network. They are backed up (marked as obsolete and become inactive for the signpost logics) and the status of their implemented signs is changed in PhyFM as deprecated (and a maintenance action is generated for them to be removed from the field). After that, all the implemented physical signs are checked for validity in PhyFM (including a recomputation of the sign track data), and any mismatches between the ideal and the actual data are reported as update-or-remove requests for maintenance. The signpost planner can add or remove any logical signs or change their necessity level $\left(S_{i}\right)$. The logical closure of the set of logical signs is, however, always re-generated after any changes and the signs eventually are in $S_{0}$ will be then marked as (to be) implemented signs. The sign track data is generated for them and they are ready for the signpost designer to craft them onto real sign and information boards, and the maintenance staff to organize their production and installation.

If such a sign is to be installed outside existing nodes, a new trail node in the network must be added. The system should implement it as a seamless way, similarly to how a new (pseudo-)junction is added, so users are not bothered with extra operations.

Implementation of the logical rules can be based on database constraints and triggers. The rules are safe as they have no implied negations, and finiteness of closure is ensured by: the finiteness of the underlying nodes and routes, the limitation of $i$ for $S_{i}$ and the fact that the only rules generate structurally new logical signs imply them into $S_{i}$ 's with higher $i$ as of their preconditions.

A further characterization of allowed logical rules, or defining a more sophisticated logics (e.g. considering to frame it as a proper modal logic) and their implementation schemes is a future issue, but the aim is to make a generic system which is flexible for multiple signage systems with different signpost policies.

\section{Conclusion and Future Work}

In this paper, we have introduced a terminology and the skeleton of TRAILSIGNER, a conceptual model suite for hiking trail networks of various signage system types, which goes beyond the particular models of current platforms and can handle special or complex situations in an integrated way. It can be refined and implemented as a basis of an integrated, collaborative information system for multiple stakeholders and visitors for the consistent planning, promotion, management and maintenance of trails and their signage assets. This will reduce potential confusion, mistrust and danger for visitors caused by 
information mismatches including incomplete, incoherent or inconsistent trail route information or signposting.

To ensure consistency of incrementally planned signposts with each other and with the (possibly changing) underlying trail network, a novel, systematic, set-based approach has been developed with generative rules for managing logical trail signs, which mediate the physical implementation and trail signage facility management. An extensive example ruleset is developed for signpost logics, and incorporated into the conceptual model suite.

The next steps can be the refinement of the model, defining model suite connections and collaboration patterns for different phases of the network developments, and interaction modeling with storyboarding and mini-stories for possible event scenarios. Creating a reference implementation and testing with an existing complex regional trail network and signpost system is an on-going work [22].

Future work may include personalization features for different visitors and situations (virtual signposts, adaptive route recommendation, feature engineering for ML methords, etc), designing an easy-to-use GUI, investigating on automatized location generation and poi assignments, enhancing the OpenStreetMap data model and implementing an on-line tool for checking the consistency of existing signposts and/or recommending trip routes, and ultimately, to develop a fully-fledged pilot system implementation with complex trail networks of different types. This approach may further be generalized, personalized and adapted to other application fields having similar requirements or phenomena.

\section{References}

[1] OpenStreetMap Wiki. https ://wiki.openstreetmap.org, [Online; accessed 25-Jan-2020]

[2] Wikiloc - trails of the world, https : //www.wikiloc.com/, [Online; accessed 26-Jan-2020]

[3] General principles for signing and marking footpaths. http://www.era-ewv-ferp.com/ fileadmin/user_upload/dokumenter/Walking_in_Europe/Waymarking_in_Europe/ General_principles__waymarking.pdf (2015), [Online; accessed 20-Jan-2020]

[4] Waymarking in Europe. http://www.era-ewv-ferp.com/fileadmin/user_upload/ dokumenter/Walking_in_Europe/Waymarking_in_Europe/ENG_WAYMARKING_2017_01.pdf (2017), [Online; accessed 20-Jan-2020]

[5] Digitales wegemanagement - wege. Ein pfad voller daten. Tourismus Digital (2018)

[6] Online-software NatursportPlaner. digitales wegemanagement für reale erlebnisse. https: //wanderverband.de/conpresso/_data/PM_33_Wandern_Digital.pdf (12 2018), [Online; accessed 20-Jan-2020]

[7] OuterSpatial - a stewardship-first approach to data, maps and apps for recreation (2019), http:// www.alaska-trails.org/2019-trails-conference-presentations.html, [Online; accessed 25-Jan-2020]

[8] Abiteboul, S., Vianu, V.: Datalog extensions for database queries and updates. Journal of Computer and System Sciences 43, 62-124 (08 1991). https://doi.org/10.1016/0022-0000(91)90032-Z

[9] Anikó, K., Dávid, M., Dénes, M., Ferenc, P., Gábor, T.: Az Országos Kékkör táblarendszerének kialakítása térinformatikai eszközökkel. In: Theory Meets Practice in GIS VI. - Térinformatikai Konferencia és Szakkiállítás. Debreceni Egyetem (2015), http://giskonferencia.unideb.hu/arch/ GIS_Konf_kotet_2015.pdf

[10] Ayala-Gómez, F., Daróczy, B., Mathioudakis, M., Benczúr, A., Gionis, A.: Where could we go? recommendations for groups in location-based social networks. In: Proceedings of the 2017 ACM on Web Science Conference. pp. 93-102. WebSci '17, Association for Computing Machinery, New York, NY, USA (2017)

[11] Brämer, R.: Heimliche revolution. Was bringen die neuen wanderleitsysteme? https://www . wanderforschung.de/files/wanleitneu1233090989.pdf (05 2003), [Online; acc. 20-Jan-2020] 
[12] Brancifort, R., Likins, Z.T., O’Connor, M., Kleinknecht, S., Nuñez, D.: Connecting 10 million people to the outdoors in Los Angeles County - Trails LA County mobile app (2019), https://www . americantrails.org/resources/connecting-10-million-people-to-theoutdoors-in-los-angeles-county, [Online; accessed 25-Jan-2020]

[13] Bühler, G., Hermann, M., Lambertus, M.: Sehnsuchtsort natur in der digitalen leistungsgesellschaft. https://sotomo.ch/site/sehnsuchtsort-natur (05 2019), [Online; accessed 20-Jan-2020]

[14] Bundesamt für Strassen (ASTRA), Schweizer Wanderwege, Bern: Signalisation Wanderwege (2013), [Online; accessed 22-Jan-2020]

[15] Calbimonte, J.P., Martin, S., Calvaresi, D., Zappelaz, N., Cotting, A.: Semantic Data Models for Hiking Trail Difficulty Assessment, pp. 295-306 (01 2020)

[16] Car, A., Frank, A.: Hierarchical street networks as a conceptual model for efficient way finding. Proceedings of the 4th European Conference on Geographical Information Systems 1 (02 1970)

[17] Demetrovics, J., Molnár, A., Thalheim, B.: Graphical reasoning for sets of functional dependencies. In: Conceptual Modeling - ER 2004, 23rd International Conference on Conceptual Modeling, Shanghai, China, November 2004, Proceedings. pp. 166-179 (11 2004)

[18] Demetrovics, J., Molnár, A., Thalheim, B.: Relationship design using spreadsheet reasoning for sets of functional dependencies. In: Advances in Databases and Information Systems, 10th East European Conference, ADBIS 2006, Thessaloniki, Greece, September 3-7, 2006, Proceedings. pp. 108-123 (09 2006)

[19] Gramer, E.: Outdooractive Facility: Der wanderhimmel wird digital. https://corporate. outdooractive.com/oa-blog/outdooractive-facility-der-wanderhimmel-wirddigital (06 2016), [Online; accessed 20-Jan-2020]

[20] Lera, I., Pérez, T., Guerrero, C., Eguíluz, V.M., Juiz, C.: Analysing human mobility patterns of hiking activities through complex network theory. In: PloS one (2017)

[21] Márkus, Z., Wagner, B.: GUIDE@HAND: digital GPS based audio guide that brings the past to life. In: Pavlov, R., Stanchev, P. (eds.) Digital Preservation and Presentation of Cultural and Scientific Heritage. pp. 15-25. Bulgarian Academy of Sciences, Sofia (2011), http://eprints.sztaki.hu/6712/

[22] Molnár, A.: The TrailSigner project repository on Github. https://github.com/zarandras/ trailsigner, [Online; updated 15-May-2020]

[23] Molnár, A.J., Thalheim, B.: Usage models mapped to programs. In: New Trends in Databases and Information Systems. pp. 163-175. Springer International Publishing, Cham (2019)

[24] Outdooractive: Outdooractive - Data Model, [Online; accessed 20-Jan-2020]

[25] Paton, N.W., Díaz, O.: Active database systems. ACM Comput. Surv. 31(1), 63-103 (Mar 1999)

[26] Semenov, A., Zelentsov, V., Pimanov, I.: Application suggesting attractive walking routes for pedestrians using an example of saint-petersburg city. Procedia Computer Science 156, 319-326 (2019), 8th International Young Scientists Conference on Computational Science, YSC2019, 24-28 June 2019, Heraklion, Greece

[27] Steinmetz, D., Dyballa, D., Ma, H., Hartmann, S.: Using a conceptual model to transform road networks from OpenStreetMap to a graph database. In: Trujillo, J.C., Davis, K.C., Du, X., Li, Z., Ling, T.W., Li, G., Lee, M.L. (eds.) Conceptual Modeling. pp. 301-315. Springer International Publishing, Cham (2018)

[28] Thalheim, B.: Model suites for multi-layered database modelling. In: Information Modelling and Knowledge Bases XXI, volume 206 of Frontiers in Artificial Intelligence and Applications. pp. 116-134. IOS Press (2010)

[29] Thalheim, B.: Foundations of entity-relationship modeling. Annals of Mathematics and Artificial Intelligence 7, 197-256 (03 1993). https://doi.org/10.1007/BF01556354

[30] Thalheim, B.: Entity-Relationship Modeling: Foundations of Database Technology. Springer (2000)

[31] Tropmann, M., Thalheim, B.: Mini story composition for generic workflows in support of disaster management. In: DEXA 2013. pp. 36-40. IEEE Computer Society (2013)

[32] Wimmer, H.: Outdooractive Facility oder Natursportplaner? https ://corporate.outdooractive. com/oa-blog/outdooractive-facility-oder-natursportplaner (04 2019), [Online; accessed 20-Jan-2020]

[33] Witt, P.J.: The development of a predictive hiking travel time model accounting for terrain variations. In: T. Jekel, A. Car, J. Strobl, \& G. Griesebner (Eds.), GI_Forum 2012: Geovisualization, Society and Learning. pp. 102-112. Salzburg (2012)

[34] Zhi-ren, S., Min, H., Hai-bin, W.: A conceptual multi-level data model for road networks. In: Fifth International Conference on Intelligent Computation Technology and Automation. pp. 712-715 (Jan 2012) 


\section{A. Appendix: A Reference Axiomatization for Signpost Logics}

\section{A.1. General Notations, Atoms, Predicates and Functions}

N1. Letters $q, r, \ldots$ denote (simple) trail routes.

N2. Capital letters $M, N, \ldots$ denote trail nodes.

N3. Location $(N, D)$ : Node $N$ is at named location $D$ (by RouLocNet and GeoTrNet).

N4. Route predicates (based on RouLocNet and GeoTrNet):

Route $(N, r, M)$ : Node $N$ is (not necessarily directly) followed by $M$ along $r$.

Is Endpoint $(r, M): M$ is the end node of $r$.

Is Reversed $(r, q): r$ is the reversed version of $q$ (by RouRel in TourPres).

Has I dentical W aymark $(r, q)$ : routes having the same waymark (causes ambiguous junctions without direct identification)

N5. Sign types:

$\operatorname{loc} \operatorname{Sign}(N, D)$ : location sign denoting $D$ at node $N$,

$r d \operatorname{Sign}(N, r, \sigma)$ : route destination sign placed at $N$ showing direction towards the destination of the follow-up sign $\sigma$ (being either a locSign or another $r d t$ Sign, by structural recursion) via route $r$, $r d t \operatorname{Sign}(N, r, \mathrm{~T})$ : a symbolic sign atom that means route $r$ should be signposted at $N$, i.e. a sign $r d \operatorname{Sign}(N, r, \sigma)$ with some destination should be added by the signage planner if there is no one yet. If no destinations are added, it is interpreted as a route sign (only a route identification showing its continuation).

N6. Place of a sign - the node where it is located:

$\operatorname{at}(\operatorname{loc} \operatorname{Sign}(N, D))=N$, and $D$ is the name of the location: $\operatorname{Location}(N, D)$

$\operatorname{at}(r d t \operatorname{Sign}(N, r, \sigma))=N$ and $\sigma$ is either a loc Sign or a $r d t \operatorname{Sign}$ (by structural recursion) or the generic T symbol.

N7. Destination of a sign - the location where it points to: $\operatorname{to}(\operatorname{loc} \operatorname{Sigh}(N, D))=D$, where $\operatorname{Location}(N, D)$. $\operatorname{to}(r d t \operatorname{Sign}(N, r, \sigma))=\operatorname{to}(\sigma)$ and $\sigma$ is either a locSign or a $r d t \operatorname{Sign}$ (by structural recursion).

N8. Necessity (implementation priority) level of a sign: $S_{0}(\sigma)$ : planned/existing/necessary (exists or is going to be implemented), $S_{i}(\sigma)$ : recommended with priority $i=1,2, \ldots, d$ (the lower the more necessary), where $d$ is a small defined natural constant.

N9. Invalidity of a sign: $\operatorname{Inv}(\sigma)$ : the sign is invalid (it was generated earlier and does not match the current network data) and must be removed from the set of current logical facts.

\section{A.2. Checking Rules}

If a checking rule fails, the corresponding sign gets an invalid status and must be inactivated.

C1. True location principle: location signs have to be placed faithfully (checking rule): $\neg\left(S_{i}(\operatorname{loc} \operatorname{Sign}(N, D)) \rightarrow \operatorname{Location}(N, D)\right) \Rightarrow \operatorname{Inv}(\operatorname{loc} \operatorname{Sign}(N, D))$

C2. Pointing forward principle: signposts show destinations along routes in their direction (checking rule): $\neg\left(S_{i}(r d t \operatorname{Sign}(N, r, \sigma)) \wedge \sigma \neq \top \rightarrow \operatorname{Route}(N, r, \operatorname{at}(\sigma))\right) \Rightarrow \operatorname{Inv}(r d t \operatorname{Sign}(N, r, \sigma))$ $\neg\left(S_{i}(r d t \operatorname{Sign}(N, r, \top)) \rightarrow \exists M: \operatorname{Route}(N, r, M)\right) \Rightarrow \operatorname{Inv}(r d t \operatorname{Sign}(N, r, \top))$

C3. Propagation of invalidity by structural recursion: $\operatorname{Inv}(\sigma) \wedge S_{i}(r d t \operatorname{Sign}(N, r, \sigma)) \Rightarrow \operatorname{Inv}(r d t \operatorname{Sign}(N, r, \sigma))$

\section{A.3. Generative Rules}

Note: every rule has an implicit pre-requisite that the referenced signs are valid $(\forall \sigma$ in the rule body: $\neg \operatorname{Inv}(\sigma))$.

G1. Signposted location identification: Arrival to a previously signposted destination must be confirmed by a location sign:

$S_{i}(r d t \operatorname{Sign}(N, r, \operatorname{loc} \operatorname{Sign}(M, D))) \Rightarrow S_{i}(\operatorname{loc} \operatorname{Sign}(M, D))$

G2. Waymark disambiguation: if two different routes with the same waymark meet and continue diverted, both need signposting:

$\operatorname{Route}(N, r, M) \wedge \operatorname{Route}(N, q, L) \wedge r \neq q \wedge M \neq L \wedge \neg I$ SReversed $(r, q) \wedge$

HasIdenticalWaymark $(r, q) \Rightarrow S_{0}(r d t \operatorname{Sign}(N, r, \top)) \wedge S_{0}(r d t \operatorname{Sign}(N, q, \top))$ 
G3. Related recommendations: if a route is signposted then recommendations on the next priority level should be generated:

$S_{i}(r d t \operatorname{Sign}(N, r, \sigma)) \wedge \sigma \neq \top \Rightarrow S_{i+1}(r d t \operatorname{Sign}(N, r, \mathrm{~T})), i<d$

G4. Formal completeness: if a route is specifically signposted at a point, it is also generally signposted: $S_{i}(r d t \operatorname{Sign}(N, r, \sigma)) \Rightarrow S_{i}(r d t \operatorname{Sign}(N, r, \top))$

G5. Location identification recommendation: if a route is signposted at a named location then a location sign is also recommended (note that a node may belong to multiple locations, and in this case, an explicit decision is possible): $S_{i}(\operatorname{rdt} \operatorname{Sign}(N, r, \sigma)) \wedge \operatorname{Location}(N, D) \Rightarrow S_{i+1}(\operatorname{loc} \operatorname{Sign}(N, D)), i<d$

G6. Completeness of routes at a signposted location: (only as recommendation on the next priority level, to make the system more flexible, but can be strengthened):

$S_{i}(r d t \operatorname{Sign}(N, r, \mathrm{~T})) \wedge \operatorname{Route}(N, q, M) \Rightarrow S_{i+1}(\operatorname{rdt} \operatorname{Sign}(N, q, \mathrm{~T})), i<d$

Note: This will imply the derived necessary signposts for route $q$ generated as $S_{i+1}$ and the related recommentations will be $S_{i+2}$

G7. Traversing: when a signed destination is out of the first followed route, the destination must be signposted at the place of route transfer: $S_{i}(r d t \operatorname{Sign}(N, r, r d t \operatorname{Sign}(M, q, \sigma))) \Rightarrow S_{i}(r d t \operatorname{Sign}(M, q, \sigma))$

G8. Forward-continuity: if any destination has been signposted along a route, it must be repeated at each signposting place where any destination is signposted for the same route, until the destination is reached or the route is changed:

$S_{i}(r d t \operatorname{Sign}(N, r, \sigma)) \wedge S_{i}(r d t \operatorname{Sign}(M, r, \top)) \wedge$

$\operatorname{Route}(N, r, M) \wedge \operatorname{Route}(M, r, \operatorname{at}(\sigma)) \Rightarrow S_{i}(r d t \operatorname{Sign}(M, r, \sigma))$

G9. Recommendation of subsequent place: If signposting is planned at a place, then it is suggested to choose the next signpost location (if named) or named location as a destination (note: for avoiding indetermininsm caused by this rule, all implications for level $S_{i}$ must be derived before any implication for $S_{i+1}$ is generated):

$S_{i}(r d t \operatorname{Sign}(N, r, \top)) \wedge \operatorname{Route}(N, r, M) \wedge \operatorname{Location}(M, D) \wedge$ $(N$ extSignpost At $(N, M, r) \vee N o N$ amed LocBtw $(N, M, r))$ $\Rightarrow S_{i+1}(r d t \operatorname{Sign}(N, r, \operatorname{loc} \operatorname{Sign}(M, D))), i<d$ where:

(a) no signpost is along $r$ between $N$ and $M$ :

NextSignpost At $t_{i}(N, M, r)=S_{i}(r d t \operatorname{Sign}(M, q, \top)) \wedge$

$\nexists L, j:\left(\operatorname{Route}(N, r, L) \wedge \operatorname{Route}(L, r, M) \wedge S_{j}(r d t \operatorname{Sign}(L, r, \top)) \wedge j \leq i\right)$

(b) no named location is along $r$ between $N$ and $M$ :

$N o N$ amed LocBtw $(N, M, r)=\nexists L, E: \operatorname{Location}(L, E) \wedge \operatorname{Route}(N, r, L) \wedge \operatorname{Route}(L, r, M)$

G10. Recommendation of destinations signposted at subsequent place(s): If signposting is planned at a place, then it is suggested to choose the destination dispayed on the next signpost(s) along the route(s) (continued below in multiple variants):

$S_{i}(r d t \operatorname{Sign}(N, r, T)) \wedge \operatorname{Route}(N, r, M) \wedge N$ extSignpost At $t_{i}(N, M, r) \ldots$

(a) along the same route (primarily):

$\ldots \wedge S_{j}(r d t \operatorname{Sign}(M, r, \sigma)) \Rightarrow S_{\max (i, j)+1}(r d t \operatorname{Sign}(N, r, \sigma)), i, j<d$

(b) along a different route (secondarily):

$\ldots \wedge S_{j}(r d t \operatorname{Sign}(M, q, \sigma)) \wedge q \neq r \wedge \neg I$ sReversed $(r, q)$

$\Rightarrow S_{\max (i, j)+2}(r d t \operatorname{Sign}(N, r, r d t \operatorname{Sign}(M, q, \sigma))), i, j<d-1$

(c) along a different route if the next sign is at the end of the route, which is not a named locaction (primarily):

$\ldots \wedge S_{j}(r d t \operatorname{Sign}(M, q, \sigma)) \wedge q \neq r \wedge \neg I$ SReversed $(r, q) \wedge I s$ Endpoint $(r, M) \wedge$

$\neg \exists D: \operatorname{Location}(M, D) \Rightarrow S_{\max (i, j)+1}(r d t \operatorname{Sign}(N, r, r d t \operatorname{Sign}(M, q, \sigma))), i, j<d$

G11. Recommendation of route endpoint: if a route is being signposted at a place, and the endpoint of the route is a named location, then it is recommended (secondarily) to be chosen for signposting: $S_{i}(r d t \operatorname{Sign}(N, r, \top)) \wedge \operatorname{Route}(N, r, M) \wedge \operatorname{Location}(M, D) \wedge I \operatorname{ISEndpoint}(r, M)$

$\Rightarrow S_{i+2}(r d t \operatorname{Sign}(N, r, \operatorname{loc} \operatorname{Sign}(M, D)))$ 


\title{
A Concept for Control and Program Based on the Semantic Space Model
}

\author{
Xing Chen ${ }^{1}$, Maimai Prayongrat ${ }^{2}$ and Yasushi Kiyoki ${ }^{3}$ \\ ${ }^{1}$ Department of Information \& Computer Sciences \\ Kanagawa Institute of Technology, Japan \\ ${ }^{2}$ Mechanical Engineering, Chulalongkorn University, Thailand \\ ${ }^{3}$ Graduate School of Media and Governance, Keio University, Japan
}

\begin{abstract}
The most important mechanism of the computer is that various functions are implemented based on programs stored in it. Programs are developed by program languages implementing functions of models. One of the efficient methods to construct a model is to construct it by semantic computation models. Using semantic computation models, we can construct a model in a semantic space. In this paper, we present a mechanism to execute models presented by the semantic spaces. We have presented a mechanism to implement combinational and sequential logic computations based on the semantic space model. The combinational and sequential logic computations are the basic functions in computer systems. However, we still need a control mechanism like that in computers. In this paper, we present a control mechanism based on the semantic space model and some of execution examples. The most important contribution of this paper is that we first present a concept for control and program based on the semantic space model. In order to demonstrate the efficiency of the proposed mechanism, we performed a demonstration experiment. In the experiment, an agent is constructed for unmanned ground vehicle control with the control mechanism. A video camera is used to determine the position of the vehicle and obstacles on the road. The control signals, including "turn left," "turn right," "go ahead" and "stop" outputted from the agent are used to demonstrate the efficiency of the mechanism
\end{abstract}

Keywords. Artificial intelligent, semantic space, semantic computation, unmanned control

\section{Introduction}

Control mechanism is a basic required mechanism for automatic control systems. In a computer system, the control unit controls the arithmetic and logic calculation to implement the basic functions of the computer according to programs stored in the memory. Programs are developed by program languages implementing functions of models. One of the efficient methods to construct a model is to construct it by semantic computation models $[1,2,3,4]$. The semantic computation models are based on multiple matrix calculation which is also utilized for implementing artificial neural networks and deep-learning [5]. By using semantic computation models, we can create human understandable models.

\footnotetext{
${ }^{1}$ Xing Chen, 1030 Simo-Ogino, Atsugi-shi, Kanagawa 243-0292, Japan; chen@ic.kanagawa-it.ac.jp
} 
The combinational and sequential logic computations are the basic functions in computer systems. We have presented a mechanism to implement combinational and sequential logic computations based on the semantic space model [6]. In our semantic computing models, data are presented as points in semantic orthogonal spaces $[1,2,3$, 4], and the semantic calculation is transmitted to calculate Euclidean distances of those points. For example, in the case for implementing semantic query, a query data set is mapped into a semantic space and summarized as a point in the space. Retrieval candidate data are also mapped into the semantic space and summarized as other points. Euclidean distance is calculated between the query point and each retrieval candidate point. When the distance of a retrieval candidate is shorter than a given threshold, its relative retrieval candidate is extracted as the query output.

Mapping matrixes are used to map input into the semantic space. Different mapping matrixes are required when the semantic space model is applied in different application areas [7- 15]. Therefore, we developed many methods to create mapping matrixes and apply the model in the areas of semantic information retrieving $[10,11$, 15], semantic information classifying [12], semantic information extracting [13], and semantic information analyzing on reason and results [15], etc. We furtherly developed a method to create the mapping matrixes through deep-learning [16].

In order to apply the semantic computation model for implementing the combinational and sequential logic computations, mapping matrixes are created according to truth tables and state transition tables. Each rows of the tables are multiplied with the mapping matrixes and mapped into the semantic spaces. In this way, the logical computation is implemented as calculating Euclidean distances of mapped points in a semantic space. Thus, we obtained the basic functions for implement the intelligent systems. However, we still need a control mechanism like that in computers. In this paper, we present a control mechanism based on the semantic space model and some of execution examples. The most important contribution of this paper is that we first present a concept for control and program based on the semantic space model. In order to demonstrate the efficiency of the proposed mechanism, we performed a demonstration experiment. In the experiment, an agent is constructed for unmanned ground vehicle control with the control mechanism. A video camera is used to determine the position of the vehicle and obstacles on the road. The control signals, including "turn left", "turn right", "go ahead", "stop", etc., outputted from the agent are used to demonstrate the efficiency of the mechanism.

In the following, we first briefly review the semantic space model in Section 2. In Section 3, we review the concept of the combinational and sequential logic computations implemented based on the semantic space model. After that, we present the control mechanism and execution examples in Section 4. This mechanism addresses the concept of time, clock, state memory and subspace creation. In section 5, we present the demonstrate experiment and the experimental results. Finally, we will present our conclusions in section 6 .

\section{The semantic space model}

\subsection{Semantic Feature Extracting Model (SFEM)}

In the semantic space model, we create a semantic space by a pre-selected training data set into several clusters. In SFEM model $[1,2]$, a data set is used where each of the 
clusters has a feature that some common features of data frequently appear among the data sets in the same cluster but rarely appear in the data sets of the other clusters. The common features which frequently appear in a cluster $C_{i}$ are referred to as $C_{i}$ 's key features. By using the training data clusters, we construct a matrix, which is referred to as $K-C$ matrix. In the $K-C$ matrix, each of the rows corresponds to a key feature set $K_{i}$, and each of the columns corresponds to a cluster. The $i j^{\text {th }}$ entry of the matrix is the number of the key features in the set $K_{i}$ appearing in the cluster $C_{j}$. Because key features in the set $K_{i}$ only appear in the cluster $C_{i}$, therefore, if $i$ is not equal to $j, i \neq j$, the value of the $i j^{\text {th }}$ entry is 0 . Therefore, the $K-C$ matrix is a diagonal matrix. That is to say that an orthogonal space is created. The value of the $i t^{\text {th }}$ entry of the matrix is the number of the elements of the set $K_{i},\left|K_{i}\right|$.

Next step in the semantic space model is to map data into the semantic space. By using the $K$ - $C$ matrix, each cluster is represented as a $q$ dimensional vector. We use $\mathbf{C}_{i}$ to represent the vector of the cluster $C_{i}$. We use a unit vector $\mathbf{c}_{\mathbf{i}}$, where its norm is 1 $\left(\left|\mathbf{c}_{\mathrm{i}}\right|=1\right)$, to represent the cluster vector $\mathbf{C}_{i}$ as $\mathbf{C}_{i}=\left|\mathrm{K}_{i}\right| \mathbf{c}_{i}$. When the data are classified into $q$ clusters, we obtain $q$ cluster vectors. Therefore, we define $q$ unit vectors $\mathbf{c}_{1}, \mathbf{c}_{2}, \ldots, \mathbf{c}_{q}$, to represent the $q$ cluster vectors. We refer the vector space constructed by the $q$ unit vectors to as the "space". Because the inner product of two different unit vector is 0 , $\left(\mathbf{c}_{i} \cdot \mathbf{c}_{j}\right)=0, i \neq j$, and there are $q$ unit vectors, the space is a $q$ dimensional orthogonal space.

When data $d_{j}$, is vectorized according to the key features, the count of the occurrences of the $C_{i}$ 's key features in the data $d_{j}$ is defined to $e_{i, j}$. We set the value of $e_{i, j}$ based on the following rule: when a key feature in the key feature set $K_{i}$ appearing in the data $d_{j}$, it is counted only once. If the number of the key feature sets is $q$, the data $d_{j}$ is vectorized to a $q$ dimensional vector. We represent the vector of the data $d_{j}$ as $\mathbf{d}_{j}$ :

$$
\mathbf{d}_{j}=\left[\begin{array}{c}
e_{1, j} \\
e_{2, j} \\
\vdots \\
e_{q, j}
\end{array}\right]
$$

We use $v_{t}$ to represent the counted value of a key feature $t$. In the following, we use the expression $\sum_{t \in K_{i}}\left\{v_{t}\right\}$ to represent the sum of $v_{t_{1}}+v_{t_{2}}+\cdots+v_{t_{a}}$, where, $t_{1}, t_{2} \ldots$ $t_{a}$ are the elements of the key feature set $K_{i}$ :

$$
\sum_{t \in K_{i}}\left\{v_{t}\right\}=\left\{v_{t_{1}}+v_{t_{2}}+\cdots+v_{t_{a}} \mid t_{1} \in K_{i}, t_{2} \in K_{i}, \cdots, t_{a} \in K_{i}\right\} .
$$

In this way, the calculation for $e_{i, j}$ is represented by the following formula:

$$
e_{i, j}=\sum_{t \in K_{i}}\left\{v_{t} \mid \text { if } t \in d_{j} v_{t}=1 \text { else } v_{t}=0\right\},
$$

where, $K_{i}$ is the set of the $C_{i}$ 's key features and $\mathrm{v}_{t}$ is the counted value of one of the $\mathrm{C}_{i}$ 's key feature $t$. If the data $d_{j}$ contains the key feature $t, v_{t}$ is set to 1 . If the data $d_{j}$ does not contain the key feature $t, \mathrm{v}_{t}$ is set to 0 .

With the definition of the retrieval space, we express the data vector $\mathbf{d}_{\mathrm{j}}$ as 


$$
\mathbf{d}_{j}=\sum_{i=1}^{q} e_{i, j} \mathbf{c}_{i} .
$$

In this way, data are mapped onto the $q$ dimensional space.

The third step is to calculate Euclidean distances for data retrieval, classification or recognition. Take the data query as an example. In the processing of data query, the retrieval candidates are mapped in the semantic space and summarized as retrieval candidate points. A query is also mapped in the semantic space and summarized as a query point. We use two methods to implement the Euclidean calculation. The first method is to calculate the distances between the retrieval candidate points and query point. The second method is to is to select a subspace by a given query and mapped the query into the original point of the subspace and calculate the length of each retrieval candidate points from the original point. That is to calculate the norms of the retrieval candidate points. By ranking the retrieval candidates based on the norms of their relative points, the query result is obtained.

When a subspace is selected from the semantic space based on queries, the subspace is a $v$-dimensional space which is a part of the $q$-dimensional space, where $v$ is smaller than $q$. The $v$-dimensional subspace correlates to $v$ clusters. The subspace is selected by the following steps.

(1) When a query $Q$ is given, the data which contain the same features in the query is searched. Data that have the same feature as those in the query are extracted.

(2) From all the component items of the selected data vector, the cluster vector $\mathbf{c}_{\mathrm{i}}$ is extracted where the related component item $\left|e_{i, j} \mathbf{c}_{i}\right|$ has the maximum value.

$$
\left|e_{i, j} \mathbf{c}_{i}\right|=\operatorname{MAX}\left(e_{1, j}, e_{2, j}, \cdots, e_{q, j}\right)
$$

We use a vector $\mathbf{q}$ referred to as the query vector to represent the extracted clusters. We add the extracted cluster vector $\mathbf{c}_{i}$ to the vector $\mathbf{q}$,

$$
\mathbf{q}=\mathbf{q}+\mathbf{c}_{\mathbf{i}} \text {; where the initial value of } \mathbf{q} \text { is } \mathbf{0} \text {. }
$$

(3) A retrieval subspace $S$ corresponding to the query is selected from the entire retrieval space by calculating the inner product of $\mathbf{c}_{i}$ and $\mathbf{q}$. If the value of the inner product $\mathbf{c}_{i} \cdot \mathbf{q}$ is greater than or equal to a threshold $\varepsilon$, which is referred to as the subspace selection threshold, $\mathbf{c}_{\mathrm{i}}$ is added to $S$.

These steps (2) and (3) are repeated for each extracted data vector.

When the subspace $S$ is obtained, the data vector on the subspace is projected and represented as

$$
\mathbf{d}_{j}=\sum_{i=1}^{q}\left\{e_{i, j} \mathbf{c}_{i} \mid \mathbf{c}_{i} \in S\right\} .
$$

In the second step, the data are ranked by calculating the norms of the data vectors on the selected subspace:

$$
\left|\mathbf{d}_{j}\right|=\left|\sum_{i=1}^{q}\left\{e_{i, j} \mathbf{c}_{i} \mid \mathbf{c}_{i} \in S\right\}\right| .
$$




\subsection{Mathematical Model of Meaning (MMM)}

In MMM, the semantic interpretation is performed as projections of the semantic space dynamically, according to contexts, as shown in Figure 1.

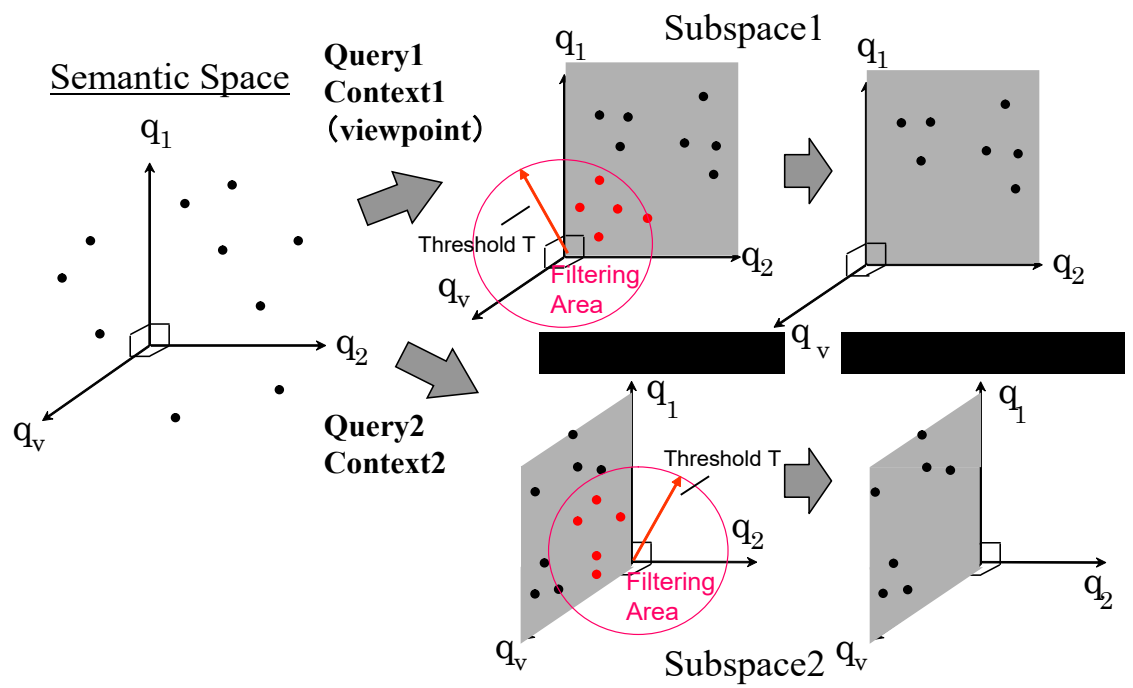

Figure 1. Semantic interpretation according to contexts in MMM

In the Mathematical Model of Meaning (MMM) [4, 7], an orthogonal semantic space is created for semantic associative search. Retrieval candidates and queries are mapped onto the semantic space. The semantic associative search is performed by calculating the correlation of the retrieval semantic space.

In MMM, the acquisition of information or knowledge is performed by semantic computations. Context-dependent interpretation means that information is dynamically extracted by a semantic computation with context-recognition. The method realizes the computational machinery for recognizing the meaning of contexts and obtaining the semantically related information to the given context. MMM is essentially different from those methods. The essential difference is that this method provides dynamic recognition of the context. That is, the "context-dependent interpretation" is realized by dynamically selecting a certain subspace from the entire semantic space. The other methods do not provide the context dependent interpretation, that is, their space is fixed and static. The outline of MMM [4, 7] is summarized as the following:

The semantic associative computing algorithm is extended to include a deeplearning process in the MMM semantic space in the following steps:

(1) A set of $m$ words is given, and each word is characterized by $n$ features. That is, an $m$ by $n$ matrix $\boldsymbol{M}$ is given as the data matrix.

(2) "Context words" and "image" are characterized as "context" by using the $n$ features and representing them as $n$-dimensional vectors.

(3) The context words and "image" are mapped into the orthogonal semantic space by computing the Fourier expansion for the $n$-dimensional vectors. 
(4) A set of all the projections from the orthogonal semantic space to the invariant subspaces (eigen spaces) is defined. Each subspace represents a phase of meaning, and it corresponds to "context."

(5) A subspace of the orthogonal semantic space is selected according to the given "context" expressed in $n$-dimensional vectors, which are given as "context" represented by "a sequence of words" and "image."

(6) The most correlated information resources to the given "context" are extracted as the selected subspace by applying the metric defined in the semantic space.

\section{Implementing combination logic calculation by multiple matrix computation}

\subsection{The combinational logic computations implemented based on the semantic space} model

Logical design is performed based on truth tables. In the truth table, all output values are given to all possible input values. The input data values and output data vaudes are Boolean values. That is, the value can only be ' 1 ' or ' 0 '. For example, if there are two logical input data, $\mathrm{x}_{1}$ and $\mathrm{x}_{2}$, all the possible input data values of the input data $\mathrm{x}_{1}$ and $\mathrm{x}_{2}$ are $(0,0),(0,1),(1,0)$ and $(1,1)$, in which $(a, b)$ means the value of $x_{1}$ is ' $a$ ' and the value of $x_{2}$ is ' $b$ '. As shown in Table 1, each input data pair is given a corresponding output value in the truth table. The output values ' 0 ', ' 0 ', ' 0 ' and ' 1 ' are given to the input values $(0,0),(0,1),(1,0)$ and $(1,1)$ in the "and" logic truth table. Based on the truth table, logical formulas are derived. For example, for the "and" logic, an equation

$$
\mathrm{y}=\mathrm{x}_{1} * \mathrm{x}_{2} \text {, }
$$

is derived, where '*' presents logic "and". In the same way, an equation

$$
\mathrm{y}=\sim \mathrm{x}_{1} * \mathrm{x}_{2}+\mathrm{x}_{1}{ }^{* \sim} \mathrm{x}_{2}
$$

is also derived for the "xor" logic, where ‘*', ‘+' and ' ’ present logic "and", "or" and

\begin{tabular}{|c|c|c|c|c|c|c|c|c|}
\hline \multicolumn{3}{|c|}{ "and" logic } & \multicolumn{3}{|c|}{ "or" logic } & \multicolumn{3}{|c|}{ "xor" logic } \\
\hline$x_{1}$ & $x_{2}$ & $y$ & $x_{1}$ & $x_{2}$ & $y$ & $x_{1}$ & $x_{2}$ & y \\
\hline 0 & 0 & 0 & 0 & 0 & 0 & 0 & 0 & 0 \\
\hline 0 & 1 & 0 & 0 & 1 & 1 & 0 & 1 & 1 \\
\hline 1 & 0 & 0 & 1 & 0 & 1 & 1 & 0 & 1 \\
\hline 1 & 1 & 1 & 1 & 1 & 1 & 1 & 1 & 0 \\
\hline
\end{tabular}
"not", respectively.

A logical system is constructed by the derived formulas from the truth table. Boolean algebra is applied to simplify the derived formulas in order to reduce the complexity of the designed system. For example, the formula

can be simplyfied as

$$
a^{*} b+a * c+\sim a^{* \sim} c+b * c
$$

$$
\mathrm{b}+\mathrm{a}^{*} \mathrm{c}+\mathrm{a}^{* \sim} \mathrm{c} .
$$


In the following, we use an example to illustrate how to implement "and," "or" and "xor" combination logical calculation based on the semantic calculation model. First we present a data set with two inputs $\mathrm{x} 1$ and $\mathrm{x} 2$ and three outputs corresponding to "and," "or" and "xor," as shown in Table 2.

Table 2. State transition table

\begin{tabular}{ll||lll}
$\mathrm{X}_{1}$ & $\mathrm{X}_{2}$ & $Y_{\text {and }}$ & $Y_{\text {or }}$ & $Y_{\text {xor }}$ \\
\hline 0.2 & 0.1 & 0.1 & 0.2 & 0.1 \\
0.1 & 0.9 & 0.2 & 0.8 & 0.9 \\
0.9 & 0.1 & 0.1 & 0.9 & 0.8 \\
0.9 & 0.8 & 0.9 & 0.8 & 0.2
\end{tabular}

The values in data set are set as: when a value is close to 0 , it might be logic ' 0 ;' when a value is close to 1 , it might be logic ' 1 .' If a data value is 0.5 , it might be logic ' 0 ' or logic ' 1 .' For the given data set, $\mathrm{x}_{1}=0.9$ and $\mathrm{x}_{2}=0.1$, it means that the input might be $\mathrm{x}_{1}=1$ and $\mathrm{x}_{2}=0$. The "and" output of it might be ' 0 .' The "and" output of it might be ' 0 .' The "or" output of it might be ' 1 ,' and the "xor" output might be ' 1 .'

Set a data set as a matrix M. A well-known method of the principal component analysis is the Singular Value Decomposition (SVD), which is a matrix computation widely used in spectral analysis, eigenvector decomposition and factor analysis. The computation is performed on a matrix with different entities on the rows and the columns. When SVD is performed on the matrix M, this matrix is decomposed into three other matrixes that contain "singular vectors" and "singular values". We call these three matrixes as $\mathrm{U}, \mathrm{S}$ and $\mathrm{V}$ :

$$
\mathrm{M}=\mathrm{U}^{*} \mathrm{~S} * \mathrm{~V},
$$

where, $\mathrm{S}$ is a diagonal matrix that contains singular values, matrixes $\mathrm{U}$ and $\mathrm{V}$ are left and right matrix of $\mathrm{S}$, respectively. $\mathrm{V}^{\prime}$ is the transposed matrix of $\mathrm{V}$. The matrix $\mathrm{V}$ has orthonormal columns, that is

where I is the identity matrix.

$$
\mathrm{V} * \mathrm{~V}=\mathrm{I}
$$

We call the space $\mathrm{U}^{*} \mathrm{~S}$ is the semantic space created by the matrix $\mathrm{M}$. As

$$
\begin{gathered}
\mathrm{M}=\mathrm{U}^{*} \mathrm{~S}^{*} \mathrm{~V}^{\prime} \\
\mathrm{M}^{*} \mathrm{~V}=\mathrm{U}^{*} \mathrm{~S}^{*} \mathrm{~V}^{*} \mathrm{~V} \\
\mathrm{M}^{*} \mathrm{~V}=\mathrm{U}^{*} \mathrm{~S}^{*} \mathrm{I} \\
\mathrm{M}^{*} \mathrm{~V}=\mathrm{U}^{*} \mathrm{~S},
\end{gathered}
$$

we call the matrix $\mathrm{V}$ as the space mapping matrix. That is, any matrixes of data sets which have the same number of columns of the matrix $M$ can be mapped to the semantic space through the mapping matrix V.

When SVD is performed to the matrix of the given data set,

$$
M=\left[\begin{array}{lllll}
0.2 & 0.1 & 0.1 & 0.2 & 0.1 \\
0.1 & 0.9 & 0.2 & 0.8 & 0.9 \\
0.9 & 0.1 & 0.1 & 0.9 & 0.8 \\
0.9 & 0.8 & 0.9 & 0.8 & 0.2
\end{array}\right]
$$

we get three matrixes: 


$$
\begin{array}{llll}
U= & S= \\
{\left[\begin{array}{rrrrr}
-0.1 & -0.1 & 0.1 & -1.0 \\
-0.5 & 0.6 & -0.6 & 0.0 \\
-0.5 & 0.3 & 0.8 & 0.1 \\
-0.6 & -0.7 & -0.2 & 0.1
\end{array}\right] \quad\left[\begin{array}{lllll}
2.5 & 0.0 & 0.0 & 0.0 & 0.0 \\
0.0 & 0.9 & 0.0 & 0.0 & 0.0 \\
0.0 & 0.0 & 0.8 & 0.0 & 0.0 \\
0.0 & 0.0 & 0.0 & 0.0 & 0.0
\end{array}\right] \quad\left[\begin{array}{rrrrr}
-0.5 & -0.4 & 0.6 & 0.1 & -0.5 \\
-0.4 & 0.0 & -0.7 & -0.2 & -0.5 \\
-0.3 & -0.6 & -0.3 & 0.5 & 0.5 \\
-0.6 & 0.1 & 0.1 & -0.6 & 0.5 \\
-0.4 & 0.7 & 0.1 & 0.6 & 0.0
\end{array}\right] .}
\end{array}
$$

The semantic space $\mathrm{U}^{*} \mathrm{~S}$ is

$$
\left[\begin{array}{rrrrr}
-0.3 & 0.0 & 0.1 & 0.0 & 0.0 \\
-1.3 & 0.6 & -0.5 & 0.0 & 0.0 \\
-1.3 & 0.2 & 0.6 & 0.0 & 0.0 \\
-1.6 & -0.7 & -0.2 & 0.0 & 0.0
\end{array}\right]
$$

This is a five dimensional space. Each row of the matrix represents a mapping point of the data set. As it is a four rows matrix, that means four points are mapped to the semantic space. It is worth to notice that the values of the last two columns of the matrix is zero, therefore, we remove the last two rows and get a new matrix $P$,

$$
\left[\begin{array}{rrr}
-0.3 & 0.0 & 0.1 \\
-1.3 & 0.6 & -0.5 \\
-1.3 & 0.2 & 0.6 \\
-1.6 & -0.7 & -0.2
\end{array}\right]
$$

The meaning of removing the last two rows of the matrix is that the semantic space is compressed from a five dimensional space to a three dimensional space.

Strictly speaking, that is each of the value of the last two rows is smaller than a threshold. In this example, the absolute value of the threshold is set to 0.003 .

As $\mathrm{U}^{\prime} * \mathrm{U}=\mathrm{I}$, that is

$$
\begin{aligned}
& \mathrm{U}^{\prime * \mathrm{U}=} \\
& {\left[\begin{array}{llll}
1.0 & 0.0 & 0.0 & 0.0 \\
0.0 & 1.0 & 0.0 & 0.0 \\
0.0 & 0.0 & 1.0 & 0.0 \\
0.0 & 0.0 & 0.0 & 1.0
\end{array}\right]}
\end{aligned}
$$

the data set is mapped to an orthogonal space. This orthogonal characteristic is not changed to the compressed space:

$$
\begin{aligned}
P^{\prime * P} & =\left[\begin{array}{rrrr}
-0.3 & -1.3 & -1.3 & -1.6 \\
0.0 & 0.6 & 0.2 & -0.7 \\
0.1 & -0.5 & 0.6 & -0.2
\end{array}\right] *\left[\begin{array}{rrr}
-0.3 & 0.0 & 0.1 \\
-1.3 & 0.6 & -0.5 \\
-1.3 & 0.2 & 0.6 \\
-1.6 & -0.7 & -0.2
\end{array}\right] \\
= & {\left[\begin{array}{lll}
6.2 & 0.0 & 0.0 \\
0.0 & 0.8 & 0.0 \\
0.0 & 0.0 & 0.6
\end{array}\right] . }
\end{aligned}
$$


As the data set is mapped to an orthogonal space, Euclidean distance calculation can be applied to calculate the distance of a new mapped point to the points already in the space.

For example, give a new input set $\mathrm{x}_{1}=0.7, \mathrm{x}_{2}=0.3$. As is different from the given data set, we should calculate four outputs $Y_{\text {and }}, Y_{\text {or }}$ and $Y_{x o r}$ for the given input data set. As the output value is not known, we set the values of the three outputs as 0.5 , that means it might be logic ' 0 ' or logic ' 1 .' Thus we get a vector

$$
\left[\begin{array}{lllll}
0.7 & 0.3 & 0.5 & 0.5 & 0.5
\end{array}\right] \text {. }
$$

Mapping the vector to the semantic space,

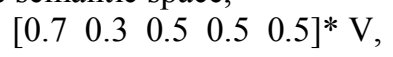

we get a mapping point $\mathrm{p}_{\mathrm{x}}$ represented as a three dimensional vector,

$$
\mathrm{p}_{\mathrm{x}}-\left[\begin{array}{lll}
-1.1 & -0.2 & 0.2
\end{array}\right] \text {. }
$$

Dividing each rows of matrix $\mathrm{P}$, we get four mapping points of the given data set in the semantic space, $\mathrm{p}_{1}, \mathrm{p}_{2}, \mathrm{p}_{3}$ and $\mathrm{p}_{4}$, represented as four vectors:

$$
\begin{aligned}
& \mathrm{p}_{1}=\left[\begin{array}{lll}
-0.3 & 0.0 & 0.1
\end{array}\right], \\
& \mathrm{p}_{2}=\left[\begin{array}{lll}
-1.3 & 0.6 & -0.5
\end{array}\right] \text {, } \\
& \mathrm{p}_{3}=\left[\begin{array}{lll}
-1.3 & 0.2 & 0.6
\end{array}\right] \text {, } \\
& \mathrm{p}_{4}=\left[\begin{array}{lll}
-1.6 & -0.7 & -0.2
\end{array}\right]
\end{aligned}
$$

Calculating the Euclidean distances of $\mathrm{p}_{\mathrm{x}}$ to $\mathrm{p}_{1}, \mathrm{p}_{2}, \mathrm{p}_{3}$ and $\mathrm{p}_{4}$, we get four values: 0.64 , $1.01,0.44$ and 0.58 . Among them, the smallest value is the third one, 0.44 . That is, point $\mathrm{p}_{\mathrm{x}}$ is most close to the point $\mathrm{p}_{3}$. The value of $\mathrm{p} 3$ in the data set is

$$
\left[\begin{array}{lllll}
0.9 & 0.1 & 0.1 & 0.9 & 0.8
\end{array}\right]
$$

thus we set the output value as $\mathrm{Y}_{\text {and }}=0.1, \mathrm{Y}_{\text {or }}=0.9$ and $\mathrm{Y}_{\mathrm{xor}}=0.8$ for the input value $\mathrm{x}_{1}$ $=0.7$ and $\mathrm{x}_{2}=0.3$.

\subsection{The sequential logic computations implemented based on the semantic space model}

Sequential logic calculation is required for Spatio-temporal data processing. The Spatio-temporal data processing includes the processing of text understanding, sound recognition and motion processing, etc. We use an example case to illustrate the mechanism. In the example, we have two values $s_{0}$ and $s_{1}$ to present a state $\left(s_{0}, s_{1}\right)$. When the state $\left(s_{0}, s_{1}\right)$ is $(0,0)$, its next state is $(0,1)$. When the state is $(0,1)$, its next state is $(1,0)$. When the state is $(1,0)$, its next state goes back to $(1,0)$. The output in the state $(1,0)$ is 1 . The output is 0 in the states $(0,0)$ and $(0,1)$. This is illustrated in Figure 2 and summarized in Table 3.

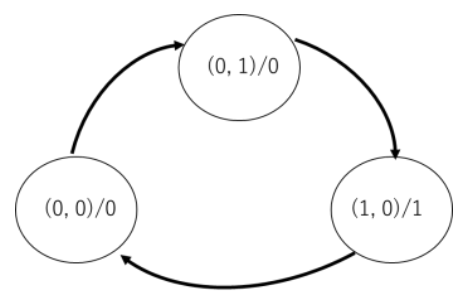

Figure 2. State transition diagram 


\begin{tabular}{|c|c|c|c|c|}
\hline$s_{1}$ & $\mathrm{~s}_{0}$ & next $\mathrm{S}_{1}$ & & output \\
\hline 0 & 0 & 0 & 1 & 0 \\
\hline & 1 & 1 & 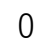 & \\
\hline & 0 & 0 & 0 & \\
\hline
\end{tabular}

From Table 3, we get a matrix M,

$$
\begin{aligned}
& \mathrm{M}= \\
& {\left[\begin{array}{lllll}
0 & 0 & 0 & 1 & 0 \\
0 & 1 & 1 & 0 & 0 \\
1 & 0 & 0 & 0 & 1
\end{array}\right]}
\end{aligned}
$$

When SVD is performed to the matrix of the given data set, we get three matrixes:

$$
\begin{aligned}
& \mathrm{U}= \\
& {\left[\begin{array}{rrr}
0 & 0 & -1 \\
-1 & 0 & 0 \\
0 & -1 & 0
\end{array}\right] \quad\left[\begin{array}{rrrrr}
1.41 & 0 & 0 & 0 & 0 \\
0 & 1.41 & 0 & 0 & 0 \\
0 & 0 & 1 & 0 & 0
\end{array}\right]}
\end{aligned}
$$$$
\begin{aligned}
& V= \\
& {\left[\begin{array}{rrrrr}
0 & -0.7 & 0 & -0.5 & -0.5 \\
-0.7 & 0 & 0 & -0.5 & 0.5 \\
-0.7 & 0 & 0 & 0.5 & -0.5 \\
0 & 0 & -1 & 0 & 0 \\
0 & -0.7 & 0 & 0.5 & 0.5
\end{array}\right]}
\end{aligned}
$$

The semantic space $\mathrm{U}^{*} \mathrm{~S}$ is that

$$
\mathrm{U}^{*} \mathrm{~S}=\left[\begin{array}{rrrrr}
0 & 0 & -1 & 0 & 0 \\
-1.4 & 0 & 0 & 0 & 0 \\
0 & -1.4 & 0 & 0 & 0
\end{array}\right]
$$

This is a five dimensional space. As the values of the last two columns of the matrix is zero, we compress the semantic space by removing the last two rows and get a new matrix $P$,

$$
\left[\begin{array}{rrr}
0 & 0 & -1 \\
-1.4 & 0 & 0 \\
0 & -1.4 & 0
\end{array}\right]
$$

In this example, the absolute value of the threshold for the semantic space compressing is set to 0.001 .

Dividing each rows of matrix $\mathrm{P}$, we get three mapping points of the state table in the semantic space, $\mathrm{p}_{1}, \mathrm{p}_{2}, \mathrm{p}_{3}$ and $\mathrm{p}_{4}$, represented as three vectors:

$$
\begin{aligned}
& \mathrm{p}_{1}=\left[\begin{array}{lll}
0 & 0 & -1
\end{array}\right], \\
& \mathrm{p}_{2}=\left[\begin{array}{lll}
-1.4 & 0 & 0
\end{array}\right], \\
& \mathrm{p}_{3}=\left[\begin{array}{lll}
0 & -1.4 & 0
\end{array}\right] .
\end{aligned}
$$

During the processing of text understanding, sound recognition and motion processing, etc., tt is common that input data are not logical data. Suppose that the input data set is $\mathrm{s} 0=0.2$ and $\mathrm{s} 1=0.9$, we set the input vector as

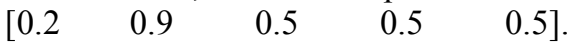


This vector is mapped to the semantic space by multiply the vector V. the Euclidean distances of the input vector and p1, p2, p3 are 1.2, 0.8 and 1.4, respectively. The input vector is closest to p2. Therefore, we get out vector as [ $\left[\begin{array}{lllll}0 & 1 & 1 & 0 & 0\end{array}\right]$. That is, the processing state now is $(0,1)$. Its next state is $(1,0)$ and its output is 0 .

\section{The control mechanism and execution examples}

In this section, we use a case study to illustrate the control mechanism. We suppose that there is an agent with a control unit created based on the control mechanism. The agent moves on a plane surface with obstacles. We set a start point and an end point for the agent. We define a path to as the traces of the agent moved from the start point to the end point and a matrix $\mathbf{X}$ to represent the paths and obstacles on the surface.

$$
\mathbf{X}=\left[\begin{array}{llll}
0 & 0 & 0 & 0 \\
S & 1 & 1 & 1 \\
0 & 1 & 0 & 1 \\
0 & 1 & 1 & G
\end{array}\right]
$$

This matrix $\mathbf{X}$ represents that the plane surface is divided into 16 areas. Each element of the matrix $\mathbf{X}$ represents an area. When an area is on the path, the relative value of the matrix $\mathbf{X}$ is set to ' 1 '. If there is obstacle in the area, that is the agent cannot path through the area, the relative element of $\mathbf{X}$ is set to ' 0 '. The area with the start point is set to ' $\mathrm{S}$ ' and that with goal point to ' $\mathrm{G}$ '. In this example, the value of $\mathrm{X}(2,1)$ is ' $\mathrm{S}$ ', and that of $\mathrm{X}(4,4)$ is ' $G$ '. All the ' 1 ' value elements are $\mathrm{X}(2,2), \mathrm{X}(2,3)$, $\mathrm{X}(2,4), \mathrm{X}(3,2), \mathrm{X}(3,4), \mathrm{X}(4,2)$ and $\mathrm{X}(4,3)$.

We design a control unit with a state memory access function, a clock function, and a control signal memory access function. The state memory access function is used to access the state memory. The control unit works based on the clock function. The outputs of the clock function are execution step signals, Step-1, Step-2, Step-3, ... The control signal memory access function is used to access the control signal memory. Along with the step signals, the state memory access function and the control memory signal access function are executed. The control unit works as follows:

Step-1: Execute the state memory accessing function to obtain the next step state matrix and control signal matrix. Execute the clock function and go to Step-2.

Step-2: Execute the state interpretation function to obtain the control signals. Execute the clock function and go to Step-3.

Step-3: Executing the sate memory accessing function to store states into the state memory. Execute the clock function and go to Step-4.

Step-4: Execute the clock function. The output of this function is the next step signal. If the output is Step-1, go to Step-1. If the output is Step-4, stay in this step.

The clock function detects the states of the control unit. If a state is changed, this function will output a next step signal. Otherwise, the next step signal will be the same one of this time. This is based on concept of time in our mechanism. Here we use an example to illustrate this concept. Suppose that we have one state and a timer in a system. The time expressed by the timer in the system is $t 1, \mathrm{t} 2, \mathrm{t} 3, \ldots$ We use $\mathrm{T} 1, \mathrm{~T} 2$, $\mathrm{T} 3, \ldots$, to express the time in the outside of the system. If we observe the state from the 
outside of the system, and we find that state is change at T3, but inside of the system, the time is lasted from $t 1$ to $t 2$. That is, the times inside and outside of the system are different. The time lasts speed depends on the detection of state changes.

We use matrixes to present states. Suppose an agent with the control mechanism moves on the path described by equation (2).

$$
\left[\begin{array}{llll}
0 & 0 & 0 & 0 \\
S & 1 & 1 & 1 \\
0 & 0 & 0 & 1 \\
0 & 0 & 0 & G
\end{array}\right]
$$

If the positions of the agent on the surface are defined as states, the states $s_{1}, s_{2}, s_{3}, s_{4}, s_{5}$ and $\mathrm{s}_{6}$ are defined as:

$$
\begin{gathered}
s_{1}=\left[\begin{array}{llll}
0 & 0 & 0 & 0 \\
1 & 0 & 0 & 0 \\
0 & 0 & 0 & 0 \\
0 & 0 & 0 & 0
\end{array}\right], s_{2}=\left[\begin{array}{llll}
0 & 0 & 0 & 0 \\
0 & 1 & 0 & 0 \\
0 & 0 & 0 & 0 \\
0 & 0 & 0 & 0
\end{array}\right], s_{3}=\left[\begin{array}{llll}
0 & 0 & 0 & 0 \\
0 & 0 & 1 & 0 \\
0 & 0 & 0 & 0 \\
0 & 0 & 0 & 0
\end{array}\right] \\
s_{4}=\left[\begin{array}{llll}
0 & 0 & 0 & 0 \\
0 & 0 & 0 & 1 \\
0 & 0 & 0 & 0 \\
0 & 0 & 0 & 0
\end{array}\right], s_{5}=\left[\begin{array}{llll}
0 & 0 & 0 & 0 \\
0 & 0 & 0 & 0 \\
0 & 0 & 0 & 1 \\
0 & 0 & 0 & 0
\end{array}\right], s_{6}=\left[\begin{array}{llll}
0 & 0 & 0 & 0 \\
0 & 0 & 0 & 0 \\
0 & 0 & 0 & 0 \\
0 & 0 & 0 & 1
\end{array}\right] .
\end{gathered}
$$

If the traces of the agent on the surface are defined as states, the states $s_{1}, s_{2}, s_{3}, s_{4}, s_{5}$ and $\mathrm{s}_{6}$ are defined as:

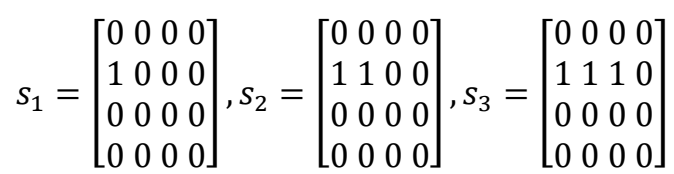

$$
\begin{aligned}
& s_{4}=\left[\begin{array}{llll}
0 & 0 & 0 & 0 \\
1 & 1 & 1 & 1 \\
0 & 0 & 0 & 0 \\
0 & 0 & 0 & 0
\end{array}\right], s_{5}=\left[\begin{array}{llll}
0 & 0 & 0 & 0 \\
1 & 1 & 1 & 1 \\
0 & 0 & 0 & 1 \\
0 & 0 & 0 & 0
\end{array}\right], s_{6}=\left[\begin{array}{llll}
0 & 0 & 0 & 0 \\
1 & 1 & 1 & 1 \\
0 & 0 & 0 & 1 \\
0 & 0 & 0 & 1
\end{array}\right] .
\end{aligned}
$$

When we put the agent at the begin point, the state $s_{1}$ appears. This triggers the clock and the control unit in the agent starts to go to the Step-1 processing. Suppose that the state memory and the control signal memory are empty at the beginning. In this processing, a semantic control subspace, $s p_{1}$ is created. We call it a subspace because we refer to the space mapped all the states as the full space. On the subspace, the movement of the agent from the position $(2,1)$ to $(2,2)$ as shown in the equation (2) is presented as a point $p_{R}$. If we define four movement actions, "Left," "Right," "Up" and "Down" as four points in the subspace, the point $\mathrm{p}_{R}$ that presents the movement from the position $(2,1)$ to $(2,2)$ is interpreted as "move right" action and set close to the point "Right," as shown in Figure 3. The agent cannot move from the position $(2,1)$ to $(1,1)$. The reason of this is interpreted as "Block" on the subspace and a point $\mathrm{p}_{U}$ is set close to it. In the same reason, the movement from the position $(2,1)$ to $(3,1)$ is also interpreted as the "Block" and a point $\mathrm{p}_{D}$ is set close to it. The agent cannot move from 
the start point to the "Left" direction because the start position is at edge of the plain. Thus, it is interpreted as "Forbidden" and a point $\mathrm{p}_{l}$ presenting this movement is set close it.

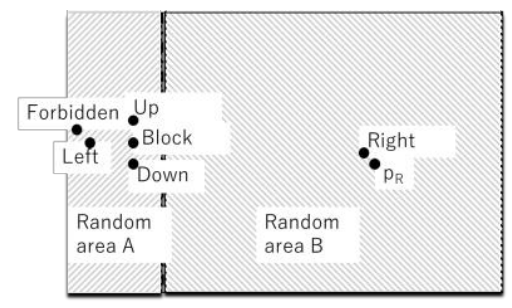

Figure 3. The semantic control subspace with interpretations and actions

During the processing of Step-1, if the state memory is not empty, the semantic control subspaces are read out from the memory. When the processing of this step is finished, the processing of Step-2 is started. In this processing, four passible action signals presenting "move left," "move up," "move right" and "move down", are randomly generated and mapped to the semantic control subspace. In the case of presented in Figure 3, "move left" is mapped close to "Forbidden," therefore, it is interpreted as to disable action. Same as that, both "move up" and "move down" are interpreted as to disable action. Only "move right" is an effective action, therefore "move right" signal is outputted. In this step, if a random generated action signal is a disable action, the action signal generation is continuing to perform until an effective action signal is generated.

The random action signal generation processing is performed based on the "random area" as shown in Figure 3. As shown in Figure 3, the area of "Random area A" is smaller that of "Random area B", the occurrence probability of the actions in "Random area A" is lower than that of in "Random area B". That is, in this example, the signal of "move right" has a high probability to be generated. In the case that the agent is at the position $(2,2)$ as shown in equation (1), it can move to two positions, $(2,3)$ or $(3,2)$ mapped as $p_{R}$ and $p_{D}$ on the semantic subspace. In this case, the "random area" for each movement has the same area "Random area B" and "Random area C" as shown in Figure 4. Thus, the agent has the same possibility to go "right" or go "down."

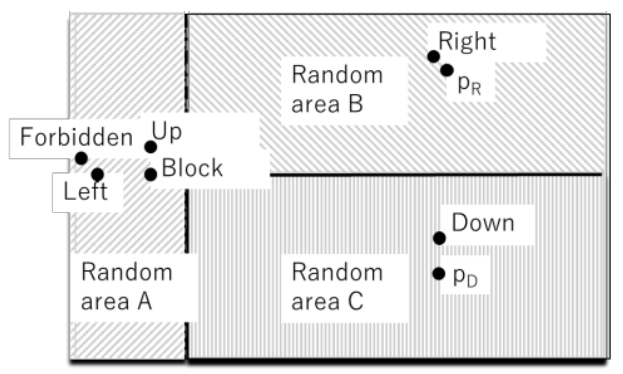

Figure 4. The semantic control subspace with two same size random areas 
After the processing Step-2 is finished, the processing of Step-3 is started. In this step, the new generated semantic control subspace is stored together with the state $s_{1}$ is into the state memory.

It will be occurred that a path is blocked by some accident. For example, the path, presented by the points $(2,1),(2,2),(3,2),(4,2),(4,3),(4,4)$ is blocked by an accident that an obstacle is appeared at place $(4,2)$, as shown by equation $(3)$.

$$
\left[\begin{array}{llll}
0 & 0 & 0 & 0 \\
S & 1 & 1 & 1 \\
0 & 1 & 0 & 1 \\
0 & 0 & 1 & G
\end{array}\right]
$$

When the agent happened moves to the position $(3,2)$, it cannot move to the position $(4,2)$ because there is an obstacle there. A new semantic control subspace is created as shown in Figure 5 (b). In Figure 5 (a), the semantic control subspace shows that the agent can go down from the position $(3,2)$ to $(4,2)$. In Figure 5 (b), it shows that the agent must be go back, that is go up from the position $(3,2)$ to $(2,2)$ because an action is happened that position $(4,3)$ is blocked.

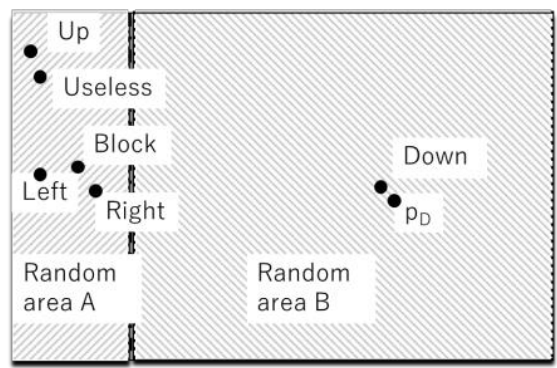

(a) Possible to move from $(3,2)$ to $(4,2)$

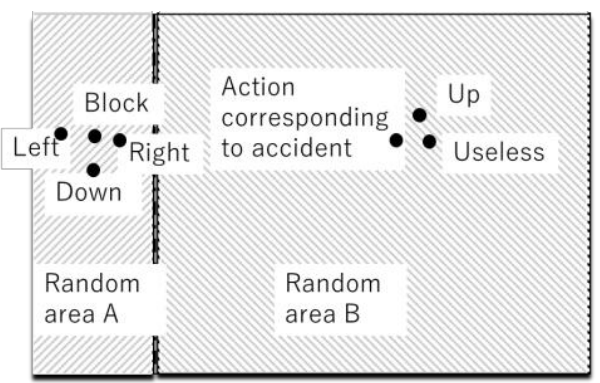

(b) Impossible to move from $(3,2)$ to $(4,2)$

Figure 5. The semantic control subspace when an accident happened

When the agent goes back to the position $(2,2)$, a new semantic control subspace is created as shown in Figure 6. We can create two kinds of the subspace as shown in Figure 6 (a) and (b).

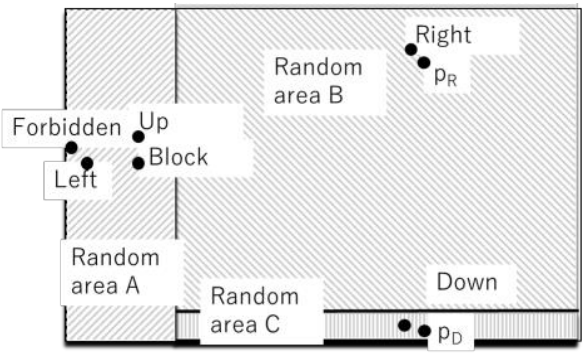

(a) Setting a small size of "random area C"

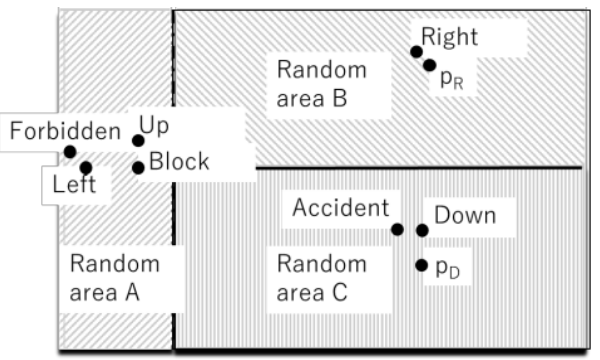

(b) Adding "Accident" concept close to the point "Down"

Figure 6. The semantic control subspace of $s_{2}$ when an accident happened 
The first one is to set a small size of "Random area C" as shown in Figure 6 (a). In this way, the agent will move to the "right" direction in high possibility and in small possibility try to go to the "down" direction testing if the obstacle is removed out or not. The other one is to add "Accident" concept and map this concept as a point close to the point of "Down". If a "move down" signal is generated, it will be interpreted as a useless action because it is close to the "Accident" point.

\section{The demonstrate experiment and the experimental results}

In the demonstrate experiment, we construct an agent for unmanned ground vehicle control with the control mechanism. We set a video camera in a car driven at a driving school. The video camera is used to determine the position of the vehicle and obstacles on the road. In our experiment, we use recorded video as inputs of the agent. The control signals, including "turn left," "turn right," "go ahead" and "stop," etc., outputted from the agent are used to demonstrate the efficiency of the mechanism.

State are constructed by several basic parts, "go right sign," "straight," "forward arrow," "lane," "oncoming lane," "no entry sign," "one way sign" and "obstacle", etc., as shown in Figure 7. We constructed a convolutional neural network (CNN) to catch these parts from the recorded video. As the car moved along the lane, different parts are caught and the states are changed as the new parts are caught.

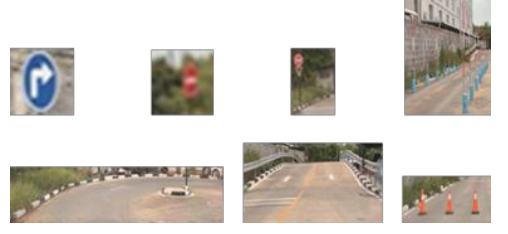

Figure 7. Parts for constructing states

The control subspaces are constructed with actions, "turn right curve," "turn left curve," "go ahead," "turn left," "turn right," "back" and "stop," and concepts same as that of the state parts. Actions and concepts are set on the control subspaces based on the driver's actions at each state. Figure 8 shows an example.

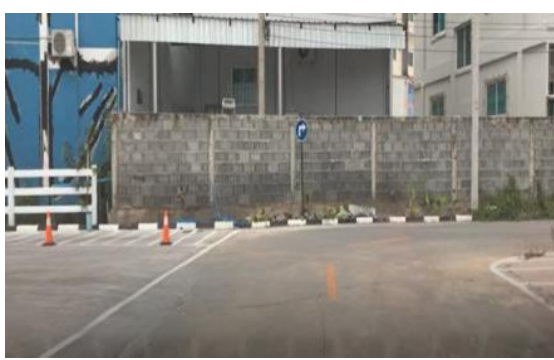

(a) A video scene before right curve

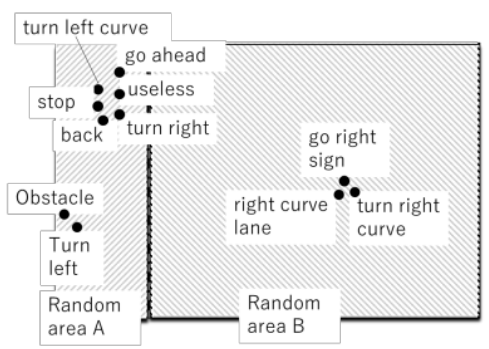

(b) Control subspace for turn right

Figure 8. An example of video scene and control subspace 
Figure 8 (a) shows the scene when the car arrives at the right curve. Therefore, "turn right curve" signal should be outputted. The created control subspace is shown in Figure 8 (b). As shown in the figure, "turn right curve" signal is close "right curve lane" and "go right sign".

The state with the parts "right curve lane" and "go right sign" are used as the teacher data. Recorded video is used to train the CNN network with the teacher data. After the training, when the recorded video is played again, states are generated by the network with the video as its input. The scenes used for training and playing are different. The scene extracted from the video used for training is not used during the playing as shown in Figure 9. Figure 9 (a) is the scene used for training and (b) is the scene used in the playing.

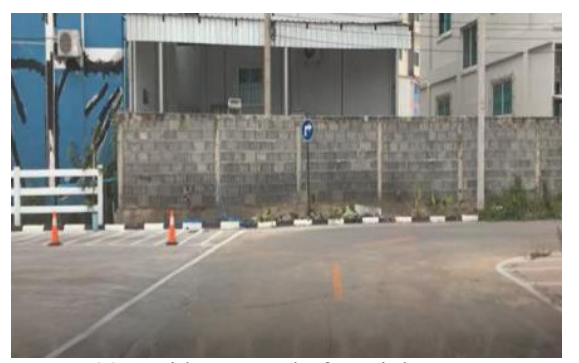

(a) A video scene before right curve

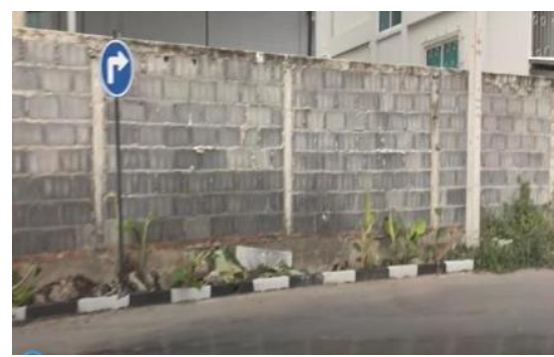

(b) Control subspace for turn right

Figure 9. Parts for constructing states

We use different scene as the input of the agent to imitate it moving. We exam the output control signals of the agent to demonstrate the efficiency of the mechanism proposed in this paper. In the following, we present part of experimental results.

As shown in Figure 10 (a), there is an obstacle on the road. The agent must avoid the obstacle by turning right. It should go ahead through the "oncoming lane." Therefore, we expected that the output signal at Figure 10 (a) position is "turn right" and that at Figure 10 (b) is "go ahead." The agent correctly outputs the two signals at these two positions.

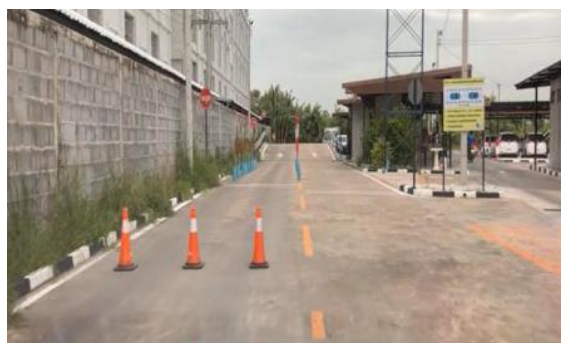

(a) The position to output "turn right"

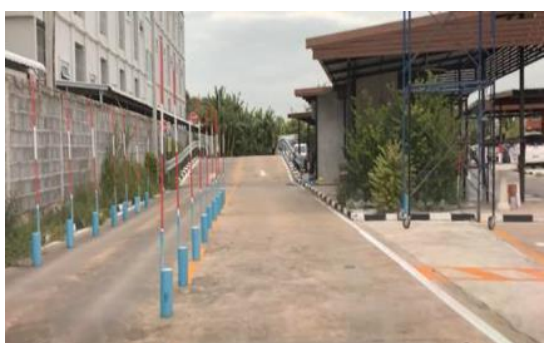

(b) The position to output "go ahead"

Figure 10. Check the output signals "turn right" and "go ahead"

In Figure 11 (a), the scene shows that the agent is in the lane of "oncoming lane." The agent must go back to the correct lane. Therefore, we expected that the output signal at Figure 11 (a) position is "turn left." In Figure 11 (b), the scene shows that there is an obstacle on the lane of "oncoming lane." As the obstacle is not on the lane 
that the agent will go through, "go ahead" signal is expected. The agent correctly outputs the two signals at these two positions.

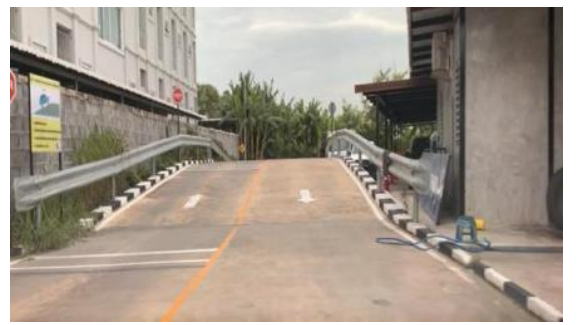

(a) The position to output "turn left"

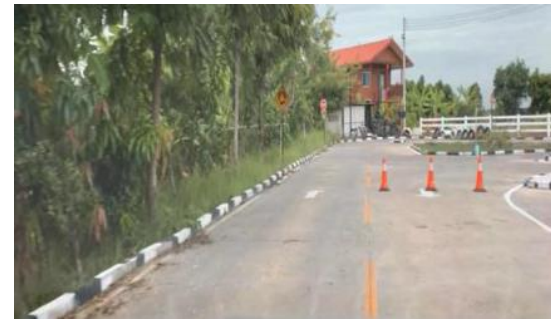

(b) The position to output "go ahead"

Figure 11. Check the output signals "turn left" and "go ahead"

The next experimental results are shown in Figure 12, in which the agent correctly outputted two signals, "turn right curve" and "stop." In Figure 12 (a), a state is shown that the agent is in front of a right curve. In Figure 12 (b), it is shown that the road is blocked.

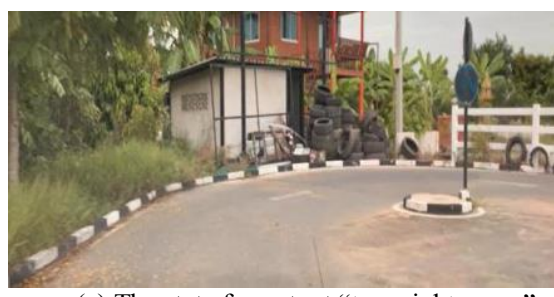

(a) The state for output "turn right curve"

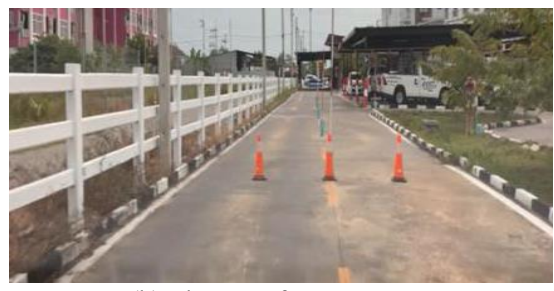

(b) The state for output "stop"

Figure 12. Check the output signals "turn left" and "go ahead"

In Figure 13, we present our experiment to test if the agent can correct its action from an error state. In Figure 13 (a), it can be find that at right side, there is a "no entry sign." We set the agent in a state trying to enter the right side road. We expect that a "turn left" signal can be outputted from the agent. In our experiment, we obtained the expected result.

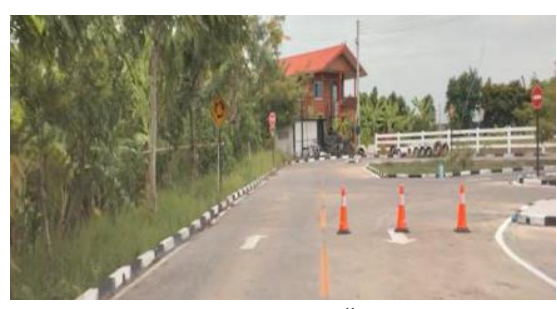

(a) At right side, there is a "no entry sign".

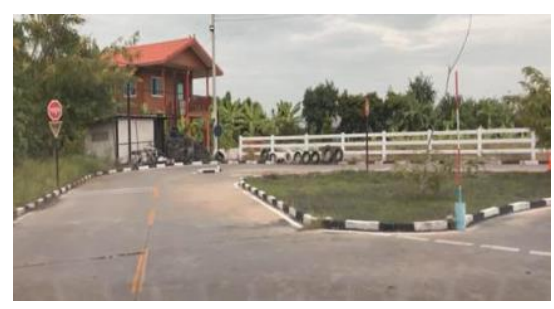

(b) A "turn left" signal is outputted.

Figure 13. Check the output signals "turn left" and "go ahead"

When the agent arrives to the goal, we set a series actions, "slow down" and "softbreak". The "slow down" relates the action of "easing the accelerator." The "slow down" actions relates the action of "stepping on the brake lightly." This action will 
perform several times till the agent arrived at goal point. During our experiment, the expected signals are corrected outputted from the agent.

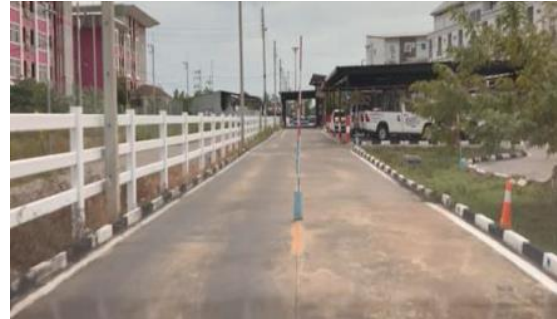

(a) A signal "slow down" is outputted.

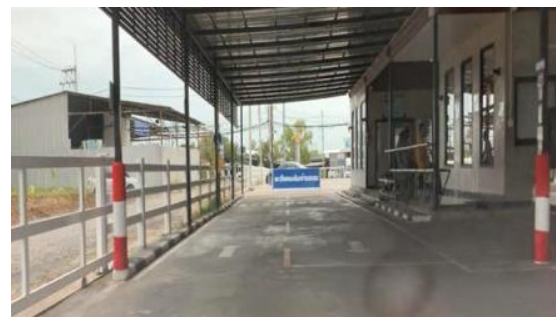

(b) A "soft-break" signal is outputted.

Figure 14. Actions before and reaching "goal"

Based on the above presented experimental results we demonstrate the efficiency of the proposed mechanism.

\section{Conclusion and future work}

In this paper, we presented control mechanism based on the semantic space model. This mechanism is the base for implementing the programs presented by the semantic spaces, referred to as the control subspaces. It is the most important contribution of this paper. In the paper, we presented design idea of the mechanisms. A state memory is designed for storing states and control subspaces. In the mechanism, states are the input and the control subspaces are the output of the memory. Like the programs stored in the memory of computers, the control subspaces implement the required functions. We use examples to illustrate the basic idea on the control subspace construction. Another contribution of the paper is the idea of indicating control signals on the semantic space instead of coding program with program language. Using the semantic space to indicate control signals makes it possible to add both the reason and the explanation for the control signals on the space. As all those are presented as points on the space, we can implement semantic interpretation for the control signals by Euclidean distance calculation. This is the third contribution of this paper that we presented a method for constructing artificial intelligence systems based on the semantic and knowledge. We furtherly introduced the concept, time, and presented the clock mechanism for the execution flow of this mechanism. In order to demonstrate the efficiency of the proposed mechanism, we performed a demonstration experiment. In the experiment, an agent is constructed for imitating an unmanned ground vehicle control with the control mechanism. We use recorded video as inputs of the agent. The control signals, like "turn left," "turn right," "go ahead" and "stop" outputted from the agent are used to demonstrate the efficiency of the mechanism. As all the expected control signals are obtained at proper times, we confirmed the efficiency of the proposed mechanism. As our future work, we will use this mechanism to implement some artificial intelligence systems to furtherly confirm the effectiveness of these mechanisms in practice. 


\section{Acknowledgment}

We are particularly grateful for the assistance given by Sorawit Sirimongkol in taking video clips used in our experiments.

\section{References}

[1] Chen, X. and Kiyoki, Y., "A query-meaning recognition method with a learning mechanism for document information retrieval," Information Modelling and Knowledge Bases XV, IOS Press, Vol. 105, pp.37-54, 2004.

[2] Chen, X. and Kiyoki, Y., "A dynamic retrieval space creation method for semantic information retrieval," Information Modelling and Knowledge Bases XVI, IOS Press, Vol. 121, pp.46-63, 2005.

[3] Kiyoki, Y. and Kitagawa, T., "A semantic associative search method for knowledge acquisition," Information Modelling and Knowledge Bases, IOS Press, Vol. VI, pp.121-130, 1995.

[4] Kitagawa, T. and Kiyoki, Y., "A mathematical model of meaning and its application to multidatabase systems," Proc. 3rd IEEE International Workshop on Research Issues on Data Engineering: Interoperability in Multidatabase Systems, pp.130-135, April 1993.

[5] Negnevitsky, M., "Artificial Intelligence: A Guide to Intelligent Systems," Pearson Education, ISBN 0321-20466-2, Second edition published 2005

[6] Chen, X. and Kiyoki, Y., "On Logic Calculation with Semantic Space and Machine Learning," Information Modelling and Knowledge Bases XXXI, IOS Press, Vol. 321, pp.324-343, 2019.

[7] Chen, X., Kiyoki, Y. and Kitagawa, T., "A multi-language oriented intelligent information retrieval system utilizing a semantic associative search method," Proceedings of the 17th IASTED International Conference on Applied Informatics, pp.135-140, 1999.

[8] Chen, X., Kiyoki, Y. and Kitagawa, T., "A semantic metadata-translation method for multilingual crosslanguage information retrieval," Information Modelling and Knowledge Bases XII, IOS Press, Vol. 67, pp.299-315, 2001

[9] Kiyoki, Y., Kitagawa, T. and Hitomi, Y., "A fundamental framework for realizing semantic interoperability in a multidatabase environment, " International Journal of Integrated Computer-Aided Engineering, Vol.2, No.1(Special Issue on Multidatabase and Interoperable Systems), pp.3-20, John Wiley \& Sons, Jan. 1995.

[10] Kiyoki, Y., Kitagawa, T. and Hayama, T., "A metadatabase system for semantic image search by a mathematical model of meaning, " ACM SIGMOD Record, Vol.23, No. 4, pp.34-41, Dec. 1994

[11] Kiyoki, Y, Chen, X. and Kitagawa, T., " $A W W W$ Intelligent Information Retrieval System Utilizing a Semantic Associative Search Method," APWeb'98, $1^{\text {st }}$ Asia Pacific Web Conference on Web Technologies and Applications, pp. 93-102, 1998.

[12] Ijichi, A. and Kiyoki, Y.: "A Kansei metadata generation method for music data dealing with dramatic interpretation," Information Modelling and Knowledge Bases, Vol.XVI, IOS Press, pp. 170-182, May, 2005.

[13] Kiyoki, Y., Chen, X. and Ohashi, H.: "A semantic spectrum analyzer for realizing semantic learning in a semantic associative search space," Information Modelling and Knowledge Bases, Vol.XVII, IOS Press, pp.50-67, May 2006.

[14] Takano, K. and Kiyoki, Y.: "A causality computation retrieval method with context dependent dynamics and causal-route search functions," Information Modelling and Knowledge Bases, ISO Press, Vol.XVIII, pp.186-205, May 2007.

[15] Chen, X. and Kiyoki, Y.: "A visual and semantic image retrieval method based on similarity computing with query-context recognition," Information Modelling and Knowledge Bases, IOS Press, Vol.XVIII, pp.245-252, May 2007.

[16] Nitta T, "Resolution of singularities introduced by hierarchical structure in deep neural networks," IEEE Trans Neural Netw Learn Syst., Vol.28, No.10, pp.2282-2293Oct. 2017.

[17] Wiatowski, T. and Bölcskei, H., "A Mathematical Theory of Deep Convolutional Neural Networks for Feature Extraction," IEEE Transactions on Information Theory, PP(99) · Dec. 2015.

[18] Hochreiter, S., Bengio, Y., Frasconi, P. and Schmidhuber, J. "Gradient flow in recurrent nets: the difficulty of learning long-term dependencies. In Kremer, S. C. and Kolen, J. F. (eds.), A Field Guide to Dynamical Recurrent Neural Networks," IEEE Press, 2001.

[19] Hochreiter, S. and Schmidhuber, J., “Long short-term memory,". Neural computation, Vol.9, No.8, pp.1735-1780, 1997.

[20] Kalchbrenner, N., Danihelka, I. and Graves, “A. Grid long short-term memory,” CoRR, abs/1507.01526, 2015 . 


\title{
Open Routed Energy Distribution Network Simulation for Maui Village Case Study
}

\author{
Alexander DUDKO ${ }^{\mathrm{a}}$, Tatiana ENDRJUKAITE ${ }^{\mathrm{b}}$, Leon ROOSE $^{\mathrm{c}}$ \\ ${ }^{a}$ KEIO University, Graduate School of Media and Governance, Kanagawa, Japan \\ ${ }^{\mathrm{b}}$ Transport and Telecommunication Institute, Research Department, Riga, Latvia \\ ${ }^{\mathrm{c}}$ Hawaiian Natural Energy Institute, University of Hawaii, Honolulu, US
}

\begin{abstract}
As the electricity generation is shifting to renewable energy sources (RES), the grid infrastructure faces multiple challenges, such as intermittency and volatility of a wide range RES. A high penetration of renewables requires profound changes to the current energy distribution system. The conventional grid is increasingly becoming a bottleneck for expanding the share of RES because of its rigid architecture, which is built around centralized energy source. We propose a new energy exchange model for a routed energy distribution system, which can perform electricity routing based on smart routing algorithms and presented protocols. We utilize a concept of an energy router device that controls energy flows and utilizes protocols stack to smartly route the energy between houses in the grid. This paper describes current results with experimental network of Maui village with multiple houses interconnected through energy routers.
\end{abstract}

Keywords: Energy distribution system, RES, Smart Grid, Energy Router.

\section{Introduction}

To support increasingly high penetration of intermittent renewables, such as solar and wind power generation, and distributed generation (DG), many regions are planning to add new high capacity transmission lines. These additional transmission lines strengthen grid synchronization but will also increase the grid's operation equipment requirements and protection complexity, and furthermore will be very costly.

The conventional approach of providing electricity from the point of generation, such as power plant, deals with a high power and therefore requires very powerful equipment. Every sub-station is responsible for providing power to designated region or area. If a sub-station goes down, the area is left without energy supply. Such case is highly undesirable, that is why to ensure energy supply there should be reserve substations or more complex fault-tolerant equipment. Modern energy grid has been developed with extensive interconnections with the purpose to improve reliability through redundancy. However, this interconnection increases the risk of wide area failures because any imbalance can be propagated quickly over an ever-widening area [1].

As the electricity generation is shifting to renewable sources, the grid infrastructure faces multiple challenges: intermittency and variability of a wide range of renewable 
sources, geographically distributed generation, bi-directional power flow and a need for energy storage systems (ESS) for meeting demand response requirements [2, 3, 4]. A high penetration of renewables requires profound changes to the current energy distribution system. The conventional grid is increasingly becoming a bottleneck for expanding the share of renewable energy sources (RES), because of its rigid architecture, which is built around centralized energy source.

Modern solutions such as renewables based distributed generation (DG) units, energy storage systems (ESSs), and advanced control strategies based on information and communication technologies have made possible for energy engineers and researchers to redesign the conventional power systems. However, not all these redesigning procedures are accomplished until now, as more researches are needed to make such advanced systems a reality $[5,6]$.

Modern world needs more than a set of new improvements. The need of a revolution of the energy generation and distribution is essentially important. The new system has to be redesigned from a scratch using all achievements in the field and to have a target to meet all the needs of today's and foreseeable future.

The direction of the future energy system needs a way to standardize the area to some extent and contribute to projects cooperation to move to the common goal. The new standard needs to be based on the common concepts so that different systems could be easily integrated together but be open for extensions, so that it does not limit the possibilities. And researches do not need to invent entire architecture complexity with all the details but focus only on specific aspects without the need to prove and explain all possible implementation variety.

This paper presents an alternative way of energy distribution. It is based on our recent research paper where we proposed a new energy exchange model for a routed energy distribution system, which can perform electricity routing based on smart routing algorithm and protocols [12]. We utilized a concept of an energy router device that uses energy as an input and protocols stack to smartly route an energy between houses in the grid.

It is a smart grid where electrical energy flows are controlled by routers. Such network is a combination of both power system and information communication system networks. Energy distribution and management system by means of the new concept of the energy routing aims to make a significant step forward into the next generation of electric grid and smart energy management.

This paper is organized as follows: Related work is presented in Section 2. Section 3 describes flows calculation approach. Experiments results are presented in Section 4. Section 5 presents conclusions of this work.

\section{Related Work}

To the best of our knowledge, there is not much work on the next generation energy smart grids in the world today that radically shifts the vision of electric grids and significantly changes the topology of the energy network.

However, during the last two decades researchers started to explore alternatives ways for energy systems. Several attempts have been done by researchers to propose distributed energy generation concepts for RES and new architectures for the energy distribution. Some researches try to reuse most of the existing infrastructure and simplify 
the transition into a new architecture [1,9], others require complete rebuild from the ground up but with integration option into existing electrical grid [10, 11].

Moreover, there are some ongoing research projects, like in Okinawa Island in Japan. There is a direct current (DC) based Open Energy System (DCOES) joint research project. This project is researching on a DC-based, bottom-up system that generates, stores, and shares electrical energy. Every house is equipped with PV panels, batteries, and an energy exchange system. These houses are interconnected via DC networks. It is designed to use the combination of various renewable energy sources and is expected to be deployed in various types of communities [7].

Annette Werth et al. analyzed a new type of DC-based, distributed interconnection of DC nanogrids. In her paper she proposed a new concept, both in terms of hardware and software architecture and showed the benefits on four-node simulations using physical model. Werth noted that the research is ongoing and some parts of the concepts still need to be studied [8].

DC power network with the concept of micro grid (MG) can provide power supply only to a restricted area, such as a small village, or building, for example a university campus. However, it is impossible to shift from AC to DC network globally and make DC power network a standard for commercial energy systems as it was done with the AC power network. Nowadays there are centralized power stations and electricity has to be transmitted over long distances.

Moreover, currently RES have installations limitations in the modern power grid due to two main reasons. First, backfeeding energy at peak times can break transformer and other equipment in the grid, when generated energy amounts are too high. Second, if baseline power plant generation goes too low when RES provide enough energy it becomes a risk, because power plant ramp rate might be not enough to quickly provide high power; for example when clouds covers all photovoltaic (PV) panels in the grid and RES generation is suddenly stopped.

\section{Approach}

This chapter describes the flows calculation approach and discusses the convergence of the entire system by continuously adapting the behavior of routers individually. A simple model simulation and results represent the important feature of the system to automatically reorganize the flows in case of broken transmission lines.

\subsection{Flows calculation}

The proposed energy exchange model is built on an idea of storage units and flows between them where energy routers represent a virtual storage unit and electrical lines in the network carry the flows. Every router $R_{i}$ has its level $L_{i}$, input and output flows $F\left(R_{i}, R_{j}\right)$ with connected router $R_{j}$. Directly connected routers have an energy flow to equalize their levels as shown in (1), where $\mathrm{k}$ is a flow sharing coefficient. Differential equation (2) shows how the router level depends on flows connected to router $R_{i}$.

$$
\begin{gathered}
F\left(R_{i}, R_{j}\right)=k \times\left(L_{i}-L_{j}\right) \\
d L_{i}=\sum_{j=1}^{N} F\left(R_{i}, R_{j}\right)
\end{gathered}
$$


Energy flow $F\left(R_{a}, R_{b}\right)$ is positive if energy is flowing from router $R_{a}$ to router $R_{b}$ and is negative if the direction is opposite, so the $F\left(R_{a}, R_{b}\right)=-F\left(R_{b}, R_{a}\right)$.

For a more realistic case the energy flow has to be limited according to physical constraints of the wire which links the two routers, so the flow equation becomes as shown in equations (3.1) and (3.2).

$$
\begin{gathered}
F\left(R_{i}, R_{j}\right)=g\left(k \times\left(L_{i}-L_{j}\right), F_{\text {max }}\left(R_{i}, R_{j}\right)\right) \\
g(x, m)=\left\{\begin{array}{cc}
-m, & x<-m \\
x, & -m \leq x \leq m \\
m, & x>m
\end{array}\right.
\end{gathered}
$$

\subsection{System Convergence}

The entire system is converging to satisfy all demands. The router levels $L$ do not become equal. Instead, the levels get values different to amount of flows between routers, and the system keep dynamically adjusting to the changing energy demands.

System convergence speed depends on the network topology, number of routers and links between them, as well as depends of the demand values from the customers. On the other side, the model has a flow sharing coefficient $k$ which can be chosen specifically for a given static configuration of the network. The lower is the value of $\mathrm{k}$, the slower the system gets to the convergence. If the coefficient is chosen too high, the model may not get to the convergence. Figure 1 shows how model reaches convergence given the different values of the $k$.

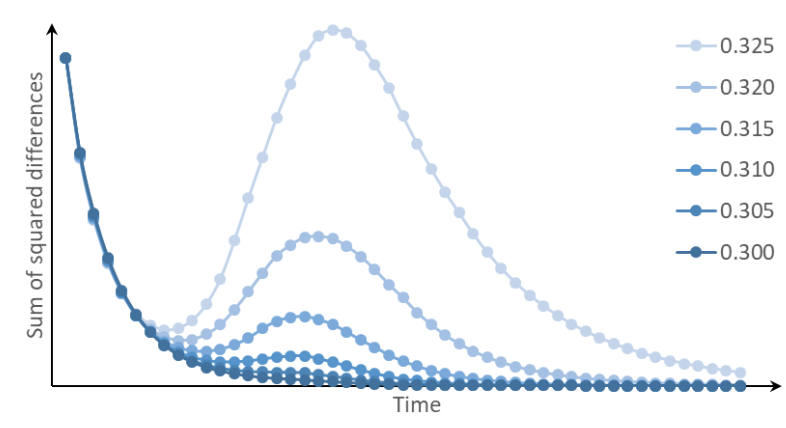

Figure 1. Convergence of the system depending on different flow sharing coefficient $\mathrm{k}$.

For a case of a relatively small network with multiple routers where consumers are located many hops away from energy generators a notable delay can appear between the moment of demand and the moment when energy is supplied. It happens because the demand gradually propagates through routers path to the generator and then gradually the energy supply makes its way back to the consumer.

In a more populated network the energy flow is constantly high, so individual consumers' power demand variations are small compared to overall network demand and they can be supplied immediately at the cost of little "power drop" for all neighbors for a short period until the system converges again. 
Convergence factor is a changeable parameter and it has to maintain the balance between the responsiveness and stability. On one side it specifies the convergence speed, on the other side it prevents energy to travel between routers in arbitrary way due to quick demand fluctuations.

\subsection{Grid Model and Simulation}

The considered case with 20 houses in a village and one power station connected to a routed smart grid was chosen as a model for simulation and experiments. In the basic simulation every house demands $5 \mathrm{~kW}$ of energy. The goal of the simulation was to determine the energy flows between routers (R1-R7), houses, and the power station S. Figure 22 shows the simulation result. Arrows in the figure show flows directions and amounts in $\mathrm{kW}$.

Table 1 shows a simulation result for the experimental model. All houses are receiving the demanded energy flows from connected routers. The second part of the experiment shows what happens when a single connection line breaks. The results after switching down line S-R5 is shown in Table 2. The system adapted to deliver energy to customers through new paths passing the broken line.

Table 1. Energy flows in experimental village model.

\begin{tabular}{|c|c|c|c|c|c|c|c|c|}
\hline $\mathrm{S}$ & R1 & $\mathrm{R} 2$ & R3 & R4 & R5 & R6 & R7 & $\mathrm{H}$ \\
\hline S & 0.00 & 0.00 & 0.00 & 26.14 & 27.05 & 21.92 & 24.64 & \\
\hline 0.00 & & -2.26 & -1.96 & -10.73 & 0.00 & 0.00 & 0.00 & 15.0 \\
\hline 0.00 & 2.26 & & 0.30 & 0.00 & -7.55 & 0.00 & -9.97 & 15.0 \\
\hline 0.00 & 1.96 & -0.30 & & -8.76 & -7.85 & 0.00 & 0.00 & 15.0 \\
\hline-26.14 & 10.73 & 0.00 & 8.76 & & 0.91 & -4.22 & 0.00 & 10.0 \\
\hline-27.05 & 0.00 & 7.55 & 7.85 & -0.91 & & 0.00 & -2.41 & 15.0 \\
\hline-21.92 & 0.00 & 0.00 & 0.00 & 4.22 & 0.00 & & 2.72 & 15.0 \\
\hline-24.64 & 0.00 & 9.97 & 0.00 & 0.00 & 2.41 & -2.72 & & 15.0 \\
\hline $\mathrm{H}$ & -15.0 & -15.0 & -15.0 & -10.0 & -15.0 & -15.0 & -15.0 & \\
\hline
\end{tabular}

Table 2. Village model with changes after disabling line between $\mathrm{S}$ and R5.

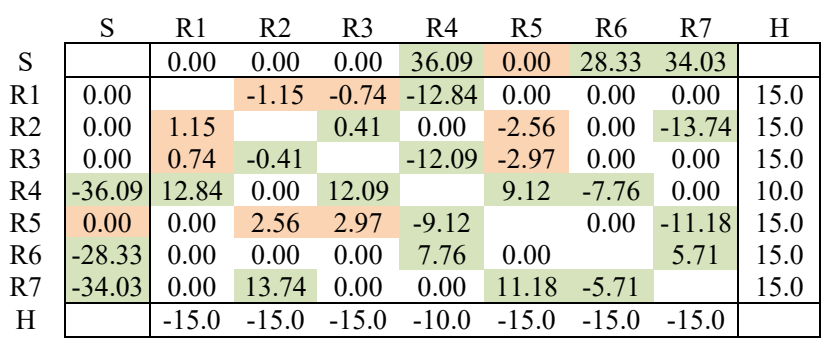




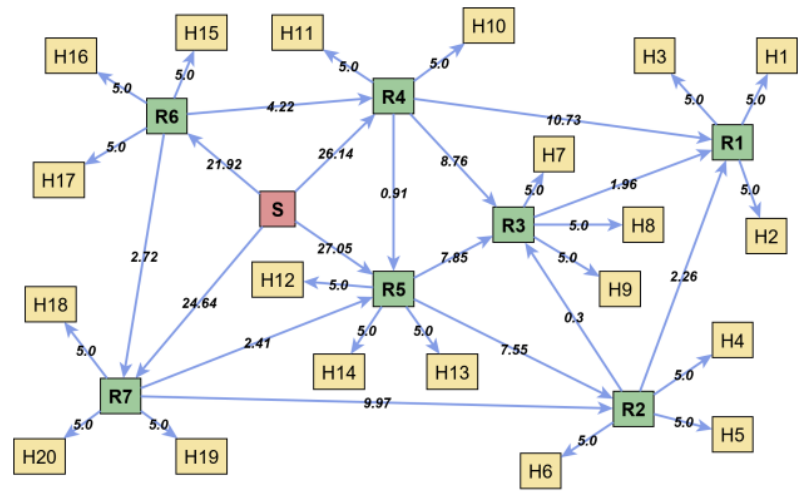

Figure 2. Model simulation result for experiment 1.

From the result we can see that all consumers receive demanded energy $5 \mathrm{~kW}$, total output flow from the power station $\mathrm{S}$ is equal to the total consumed power by all consumers and is $100 \mathrm{~kW}$. What is important to mention is that the energy distribution configuration was not calculated in a single place, but instead every router was performing local simple calculations and none of the routers has information about the network topology, size, or any other configuration. So, the solution for a complex system is getting found through a swarm intelligence (SI) where every individual performs simple operations and calculations.

\section{Experiments}

In this chapter we describe experiment of the proposed routed energy distribution network concept in a part of Maui village, located in Hawaii islands, USA. The village part of 37 houses is connected to utility power line station was chosen as a model for simulation and experiments. For the simulation every house demand was measured for 24 hours with discretization by 1 second.

\subsection{Existing Power Lines}

The considered case is based on a part of village in Maui, an isolated island in Hawaii, USA. The village part of 37 houses connected to utility power line station was chosen as a model for simulation and experiments. Every house demand was measured for 24 hours with discretization by 1 second. Figure 2 shows the existing power lines provided by the electrical utility company and connections to houses. 


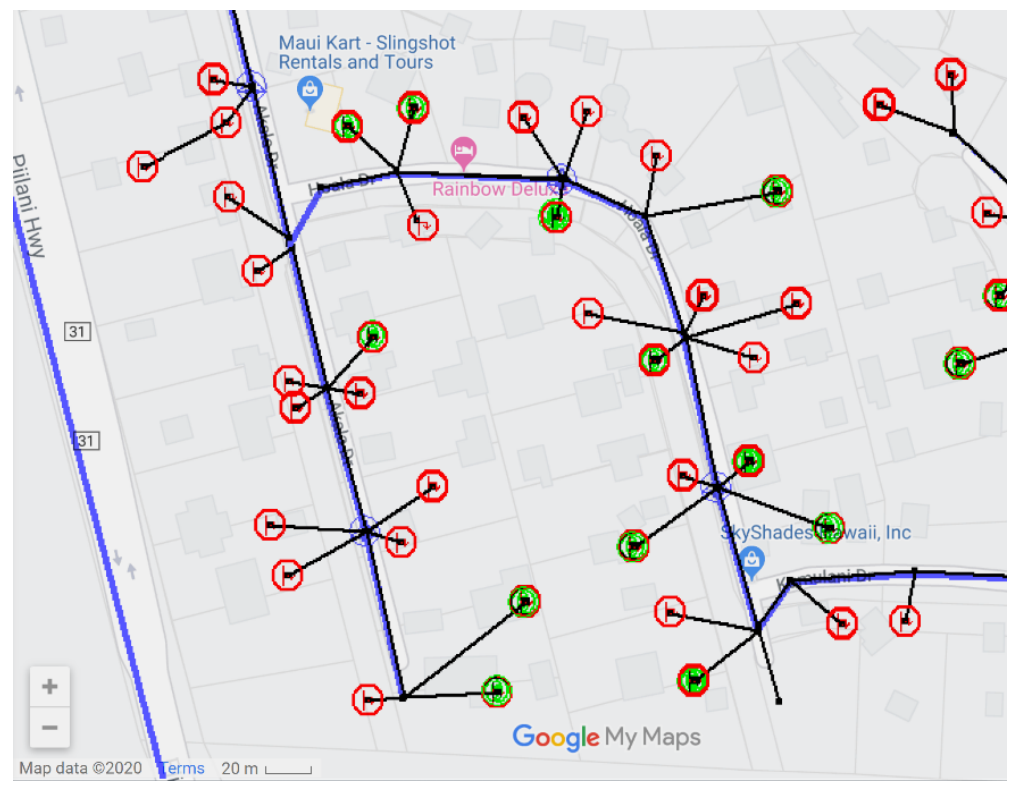

Figure 3. Existing power lines.

\subsection{Maui Village Model}

In this experiment a village model was created based on the existing power lines and houses connection to closely simulate a real usage scenario. The goal of the simulation was to show the operation of the system throughout the 24 hours and to determine the energy flows between routers R1-R10, houses H1-H37, and the power station presented as a generator G1. The model is shown in Figure 4. 


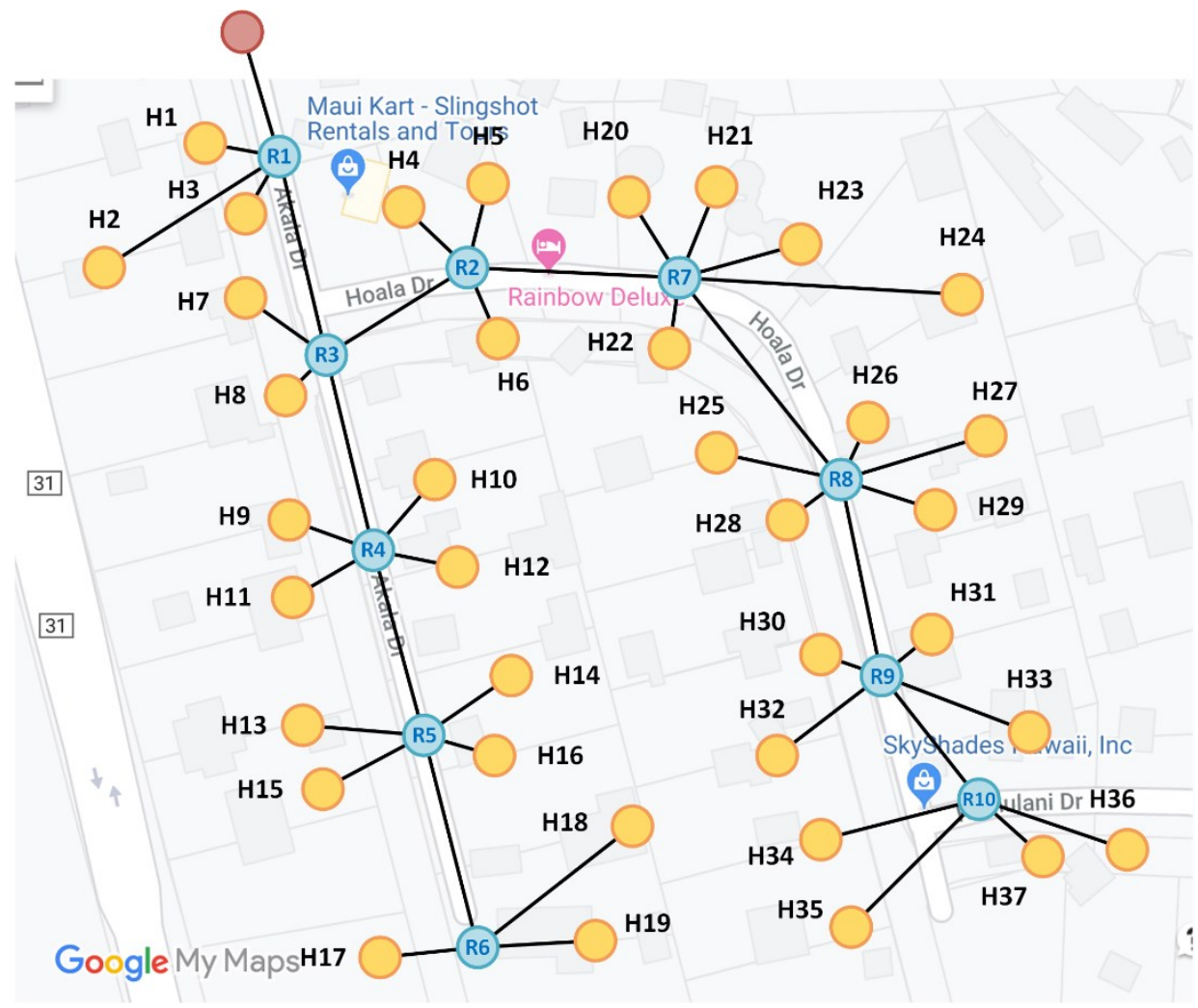

Figure 4. Maui village model.

Figure 5 shows a typical house load data (a) and its breakdown into components which shows own consumer load (b) with a periodic component (c), and high frequency fluctuations (d) which are observable equal for all houses within a network.

(a)

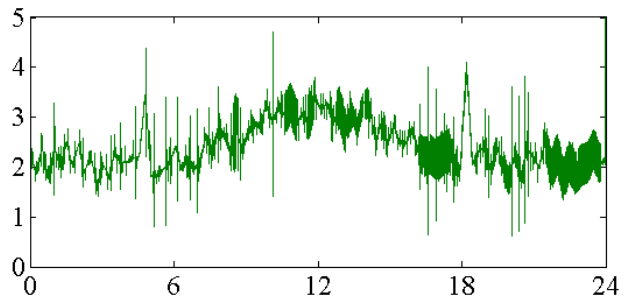

(b)

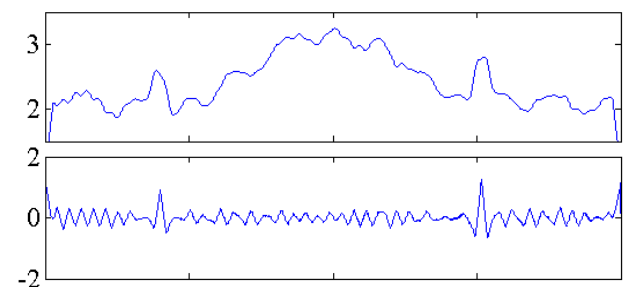


(d)

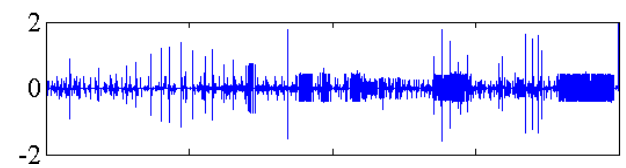

Figure 5. Breakdown of a load signal from a real world consumer.

Figure 6 shows 5-minute fragment of the simulation result which shows how the network reacts to high-frequency fluctuations in the houses loads. The model shows that fast fluctuations and jitter are not propagating much with a network affecting only local segments. Network smooth out any quick fluctuations which makes the system stable and resistant to noise.

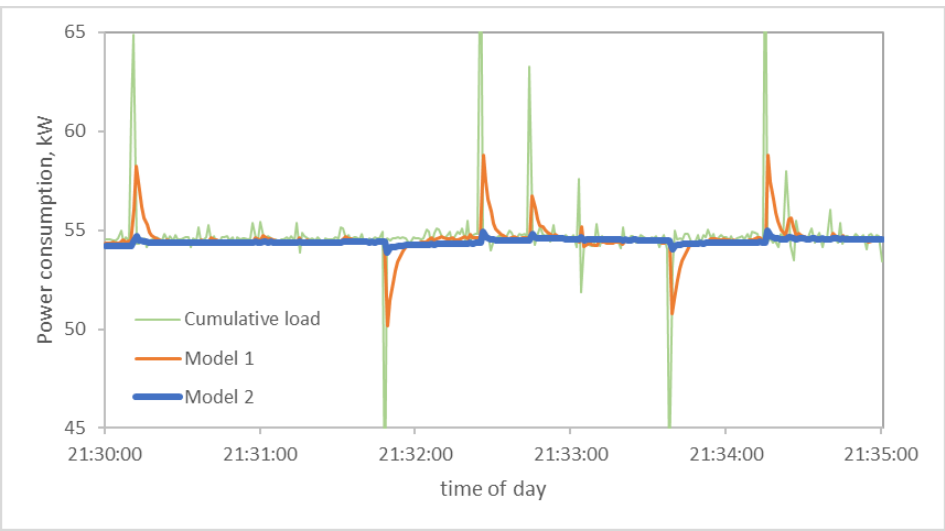

Figure 6. 5-minute fragment of the simulation with high-frequency fluctuations.

Table 3 shows total amount of energy passed by connection lines in $\mathrm{kWh}$ for simulation period of 24 hours. We can clearly see that full amount of energy is passing through the router R1 which is the only path between the village and the power plant substation. Every further line down passes less and less energy delivering it houses down the line.

Table 3. Total amount of energy flows between routers and houses of the network in $\mathrm{kWh}$.

\begin{tabular}{|c|c|c|c|c|c|c|c|c|c|c|c|c|}
\hline & G1 & R1 & $\mathrm{R} 2$ & R3 & R4 & R5 & R6 & $\mathrm{R} 7$ & $\mathrm{R} 8$ & R9 & $\mathrm{R} 10$ & $\mathrm{H}$ \\
\hline G1 & & 965 & 0 & 0 & 0 & 0 & 0 & 0 & 0 & 0 & 0 & \\
\hline $\mathrm{R} 1$ & -965 & & 0 & 832 & 0 & 0 & 0 & 0 & 0 & 0 & 0 & 133 \\
\hline $\mathrm{R} 2$ & 0 & 0 & & -517 & 0 & 0 & 0 & 444 & 0 & 0 & 0 & 73 \\
\hline R3 & 0 & -832 & 517 & & 274 & 0 & 0 & 0 & 0 & 0 & 0 & 41 \\
\hline $\mathrm{R} 4$ & 0 & 0 & 0 & -274 & & 109 & 0 & 0 & 0 & 0 & 0 & 165 \\
\hline R5 & 0 & 0 & 0 & 0 & -109 & & 48 & 0 & 0 & 0 & 0 & 62 \\
\hline R6 & 0 & 0 & 0 & 0 & 0 & -48 & & 0 & 0 & 0 & 0 & 48 \\
\hline R7 & 0 & 0 & -444 & 0 & 0 & 0 & 0 & & 381 & 0 & 0 & 63 \\
\hline R8 & 0 & 0 & 0 & 0 & 0 & 0 & 0 & -381 & & 242 & 0 & 139 \\
\hline R9 & 0 & 0 & 0 & 0 & 0 & 0 & 0 & 0 & -242 & & 147 & 96 \\
\hline R10 & 0 & 0 & 0 & 0 & 0 & 0 & 0 & 0 & 0 & -147 & & 147 \\
\hline $\mathrm{H}$ & & -133 & -73 & -41 & -165 & -62 & -48 & -63 & -139 & -96 & -147 & \\
\hline
\end{tabular}




\subsection{Distributed Generation}

Since the penetration of renewable energy resources increases, the need to effectively manage these resources in distribution networks also increases. By means of Energy Routers more renewables can be installed compared to the conventional networks. Customers are not limited in the power of renewable energy generation units and their amount in the network. The house owners are able to use own generated energy, and the energy router is spreading the remaining energy between other routers as much as they can accept.

This experiment includes 7 photovoltaic (PV) generators in the model very close to how these are installed in the existing houses. PV panels generation was taken based on measured values for 24 hours data with discretization of 1 value per second. Figure 7 shows locations of PV panels and new network configuration for total of 55 nodes.

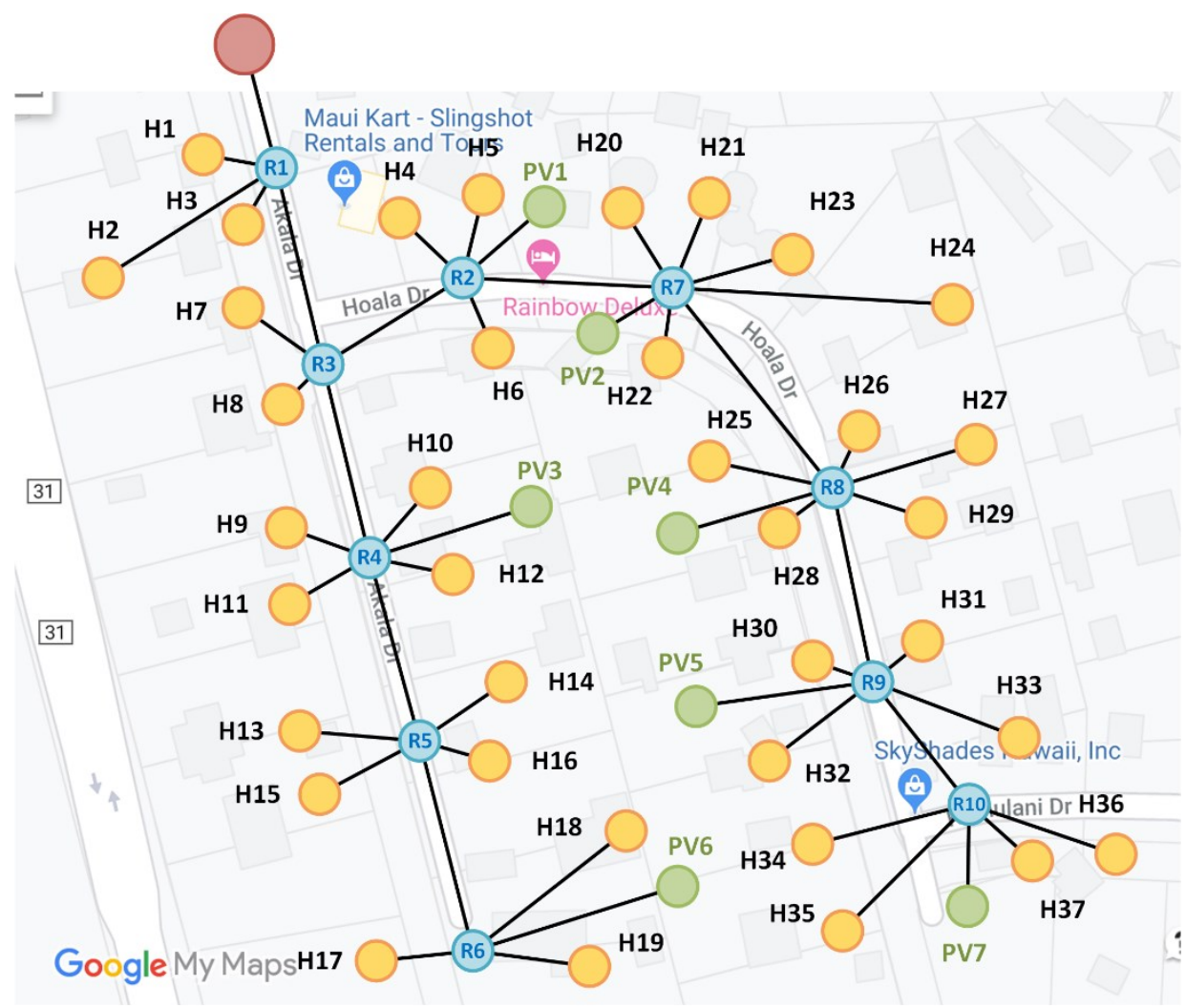

Figure 7. New network configuration with PV.

The results of simulation are shown on Table 4 . The values are much lower compared to previous experiment shown in Table 3, because the energy was mostly consumed locally, therefore reducing the high energy flows throughout the entire network. 
Table 4. Total amount of energy flows between routers and houses of the network in kWh with PV.

\begin{tabular}{|c|c|c|c|c|c|c|c|c|c|c|c|c|c|}
\hline & G1 & R1 & $\mathrm{R} 2$ & $\mathrm{R} 3$ & $\mathrm{R} 4$ & R5 & R6 & R7 & $\mathrm{R} 8$ & R9 & $\mathrm{R} 10$ & $\mathrm{H}$ & PV \\
\hline G1 & & 141 & 0 & 0 & 0 & 0 & 0 & 0 & 0 & 0 & 0 & & \\
\hline $\mathrm{R} 1$ & -141 & 0 & 0 & 8.1 & 0 & 0 & 0 & 0 & 0 & 0 & 0 & 133 & 0 \\
\hline $\mathrm{R} 2$ & 0 & 0 & 0 & 43 & 0 & 0 & 0 & -11 & 0 & 0 & 0 & 73 & -105 \\
\hline $\mathrm{R} 3$ & 0 & -8.1 & -43 & 0 & 10 & 0 & 0 & 0 & 0 & 0 & 0 & 41 & 0 \\
\hline $\mathrm{R} 4$ & 0 & 0 & 0 & -10 & 0 & 4.2 & 0 & 0 & 0 & 0 & 0 & 165 & -159 \\
\hline R5 & 0 & 0 & 0 & 0 & -4.2 & 0 & -58 & 0 & 0 & 0 & 0 & 62 & 0 \\
\hline R6 & 0 & 0 & 0 & 0 & 0 & 58 & 0 & 0 & 0 & 0 & 0 & 48 & -105 \\
\hline R7 & 0 & 0 & 11 & 0 & 0 & 0 & 0 & 0 & 21 & 0 & 0 & 63 & -95 \\
\hline R8 & 0 & 0 & 0 & 0 & 0 & 0 & 0 & -21 & 0 & -2 & 0 & 139 & -116 \\
\hline R9 & 0 & 0 & 0 & 0 & 0 & 0 & 0 & 0 & 2 & 0 & 16 & 96 & -114 \\
\hline R10 & 0 & 0 & 0 & 0 & 0 & 0 & 0 & 0 & 0 & -16 & 0 & 147 & -130 \\
\hline $\mathrm{H}$ & & -133 & -73 & -41 & -165 & -62 & -48 & -63 & -139 & -96 & -147 & & \\
\hline V & & 0 & 105 & 0 & 159 & 0 & 105 & 95 & 116 & 114 & 130 & & \\
\hline
\end{tabular}

Ultimately, the more people install own energy sources such as PV panels the less network depends on the power station energy. The area or a village with many RES generators and ESSs becomes more independent in this case. Central power station becomes less important and less used. Instead the whole network becomes a decentralized virtual power plant (VPP) with multiple small power sources which together can cover needs of the whole network.

\section{Conclusions}

In this paper we introduced a novel approach of energy distribution based on routing. The proposed Routed Smart Grid system has a lot of advantages compared to conventional energy systems widely used nowadays.

More stable source load. When we are using energy routers the network has a potential to have a more stable network load. Energy is not just flowing from the source to customers, it is smartly routed between photovoltaic panels, houses and other routers. Especially effective stabilization can be achieved when batteries are added to the system and distributed throughout the grid, so that routers can store and manage generated energy. In the future work we are planning to extend the proposed routed energy smart grid model with batteries, which will further stabilize the load in the network.

Greater usage of renewable energy sources. By means of energy routers, we can install more renewables compared to the system without routers. Customers are not limited in any way in the power of solar panels they install on the roofs. The owner will be able to use generated energy, and the energy router near the house will be spreading the remaining energy to the neighboring routers as much as they can accept. Furthermore, the network will not be damaged because excesses are not sent to the network. Moreover, to minimize energy losing in the case of very powerful PV, the neighboring routers and wires to them can be replaced with a slightly more powerful ones, and the house can install additional battery.

Higher reliability of the system and better fault tolerance. Since there are many ways how energy can be provided to the every consumer, the damage of routers or wires will not cause the network to stop operate and not provide the energy to clients. What is 
also very important, the bigger is the network, the higher is the reliability, because bigger networks have more energy sources and more links that can be used to deliver energy.

Routers give smart control over energy flows. We can easily measure, route, and limit the amounts of energy for any node in the system.

Better scalability. Conventional energy distribution networks are not flexible in terms of scaling. Equipment need to be changed when the network grows bigger and bigger. On the other hand, proposed Routed Smart Grid system easily scales by simply connecting and disconnecting nodes anywhere in the network.

Finally, energy distribution system which is based on presented energy routers, opens new possibilities in building less expensive, stable, scalable and reliable energy networks with ability of measuring, controlling, and mining energy flows. Based on that, we can bring energy networks to the next generation of energy management.

\section{Acknowledgement}

This work was financially supported by the specific support objective activity 1.1.1.2. "Post-doctoral Research Aid" (Project id. N. 1.1.1.2/16/I/001) of the Republic of Latvia, funded by the European Regional Development Fund. Tatiana Endrjukaite research project No. 1.1.1.2/VIAA/1/16/095 "Integrated Model for Energy Generation, Distribution and Management".

\section{References}

[1] R. Abe, H. Taoka, D. McQuilkin. Digital grid: communicative electrical grids of the future. IEEE Transactions on Smart Grid 2011; 2(2): 399-410.

[2] A. Werth, N. Kitamura, M. Tokoro, K. Tanaka. Evaluation model for multi-microgrid with autonomous DC energy exchange. IEEJ Transactions on Electrical and Electronic Engineering 2017 12(5): 676-682.

[3] H. Farhangi, The path of the smart grid. IEEE Power and Energy Magazine 2010; 8: 18-28.

[4] P. Khayyer and U. Ozguner. Decentralized Control of Large-Scale Storage-Based Renewable Energy Systems. IEEE Transactions on Smart Grid 2014; 5: 1300-1307.

[5] J. Justo, F. Mwasilu, J. Lee, J. Jung. AC-microgrids versus DC-microgrids with distributed energy resources: A review. 2013. Renewable and Sustainable Energy Reviews, vol. 24, (2013), 387-405.

[6] P. Asmus. 2010. Microgrid, virtual power plants and our distributed energy future. The Electricity Journal, vol. 23, no. 10 (2010), 72-82.

[7] M. Tokoro. 2014. Sony CSL-OIST DC-based open energy system (DCOES). In Proc. of the 1st International Symposium on Open Energy Systems (Okinawa, Japan, 2014), 64-67.

[8] A. Werth, N. Kitamura, K. Tanaka. 2015. Conceptual Study for Open Energy Systems: Distributed Energy Network Using Interconnected DC Nanogrids. IEEE Transactions on Smart Grid, vol. 6, no. 4, (Jul. 2015), $1621-1630$.

[9] T. Nguyen-Van, R. Abe. K. Tanaka. MPPT and SPPT Control for PV-Connected Inverters Using Digital Adaptive Hysteresis Current Control. Energies 2018, 11, 2075.

[10] T. Kaipia, P. Salonen, J. Lassila, J. Partanen. Application of low voltage DC-distribution system: a techno-economical study. In Proc. of 19th Int. Conf. on Electricity Distribution, Vienna, Austria, 2007, $1-4$.

[11] T. Sakagami et al. Performance of a DC-based microgrid system in Okinawa. In Proc. of International Conference on Renewable Energy Research and Applications 2015.

[12] T. Endrjukaite, A. Dudko, L.Roose. Energy Exchange Model in Routed Energy Distribution Network. In Proc. of the 6th ACM International Conference on Systems for Energy-Efficient Buildings, Cities, and Transportation (ACM BuildSys 2019), New York, US, November 2019, 393-394. 


\title{
Trends and Future of Data Modelling
}

\author{
Hannu JAAKKOLA ${ }^{\mathrm{a}, 1}$ and Bernhard THALHEIM ${ }^{\mathrm{b}, 2}$ \\ ${ }^{a}$ Tampere University, P.O.Box 300, FI-28101 Pori, Finland \\ ${ }^{\mathrm{b}}$ Christian-Albrechts-University Kiel, Computer Science Institute, 24098 Kiel, \\ Germany
}

\begin{abstract}
Data (conceptual, data, information, knowledge) modelling is still the work of an artisan, i.e. an art in the best case, made by humans, because of the need for human intelligence. Data modelling is an essential part of Information System (IS) design, specifying how data is implemented as part of an IS. The principles of data modelling follow the evolution of IS development paradigms, and these in turn follow the progress of technological changes in computing. Although technology has changed a lot during the decades of commercial use of computers - since the early 1950s to now, close to 70 years - data modelling is still based on the same basic principles as decades ago. Or is it really so? Finding the answer to this question was the main motivation to start writing this paper. Since the future is more interesting than the past, we set our research problem to be "What are the challenges for data modelling in the future?". The reason for this is that we see some significant changes in the future in the data modelling sector which we wanted to examine. However, the future is a continuum of the past. The future cannot be fully understood without understanding the past. Humans also tend to forget the details of the past. Even the most remarkable innovations from the past have become part of the new normal. Consequently, at the beginning of our paper we look shortly at the progress of data modelling during the era of commercial computing. Our focus is on the recent past and we look at the technological changes that have been of key importance in data modelling in the role of triggers and enablers. To find the answer to our research question, we retrieved some recent studies handling the future of data modelling and analyse the challenges found in these sources. The paper is concluded by some future paradigms. In general, the big changes seem to be the growing importance of Artificial Intelligence (AI) and machine learning (ML) as its fuel. AI not only conducts algorithmic rule-based routines, it has learning capability, which makes it more intelligent and adaptable, and able to compete with human intelligence, even in data management tasks.
\end{abstract}

Keywords. data modelling, information modelling, information system modelling, information system design, data base

\section{Introduction}

Data Modelling defines the principles of how to create data models (physical, logical, conceptual, etc.) related to an information system (IS). It covers defining and determining the data needs of the target organization, and the goals of using the data. It is an essential part of IS design and creates the foundation of the whole development life cycle. Since the 1950s - the early phase of the commercial use of computers - the paradigms and

\footnotetext{
${ }^{1}$ hannu.jaakkola@iki.fi

2 thalheim@is.informatik.uni-kiel.de
} 
technologies of IS development have changed radically. The same goes for the principles of data modelling.

A variety of terms - conceptual modelling, data modelling, information modelling, etc. - are used to describe the same basic element of IS design from different points of view and abstraction levels. In our paper we have adopted the term "data modelling" to cover the use of modelling techniques in different parts of the IS development lifecycle; thus it is an umbrella term covering conceptual modelling as well as data-oriented aspects in IS design at all levels of abstraction. The development of the model reflects the principles of the development paradigm. The final manifestation of the conceptual model (after several modifications during the development life cycle) can be seen in the data structure (database, software data structure) and functionality of the application.

The aim of this paper is to consider the changes in data (conceptual, information, ...) modelling over the decades, with the main aim to look at the current situation and the future. We look backwards to a paper from the late 1990s, which the second author of this paper co-wrote (Chen, Thalheim \& Wong in 1999) [3]. If we examine the era of databases and the conceptual modelling related to it, that paper was written in the growth era (technology was maturing towards the growing use of relational databases) of database management systems (DBMS). It is reviewed briefly in Section 2 of this paper to provide an opportunity to compare "how the future was seen in 1999", "what really happened," and how we see the future today.

What has been the progress of data modelling over the decades? Foote's paper in 2017 [7] answers this question; it is slightly modified in the following according to the authors' subjective interpretations. It separates four (overlapping) phases covering the era of systematic data modelling from the 1960 s to 2020 . These are briefly introduced in the following paragraphs.

The development of DataBase Management Systems - DBMS (from roughly the 1960 s to 1999). In this period a variety of implementation architectures existed: hierarchical, inverted list, network, and at the end of the period the idea of object-oriented database management systems - OODBMS. The last mentioned emerged in parallel with the coming of the object-oriented programming paradigm and Smalltalk. The first commercial database system, the integrated data store (IDS), was introduced in 1964 by Charles Bachman. Most of the "OODBMSs" were implemented on top of an existing architecture without having the pure properties of an object model; these extensions were also typical in the following era (RDBMS).

The birth of Relational DBMS - RDBMS (started at the end of the 1980s/early 90s). Edgard F. Codd introduced his data model based on relational calculus in 1969/1970 ${ }^{3}$. At the beginning, it was the focus of a wide researcher community, and the first experimental implementations occurred quite quickly. Although the first "commercial" implementations (Multics) was available in the late 1970s, RDBMSs were not adopted for real commercial use until the 1990s. In addition to the logical structure of databases it brought a user-friendly way to define database contents and to implement queries in it articles:

${ }^{3}$ The starting point of relational model and RDBMS is based on the following two

Codd, E.F (1969). Derivability, Redundancy, and Consistency of Relations Stored in Large Data Banks, Research Report, IBM.

Codd, E.F (1970). A Relational Model of Data for Large Shared Data Banks. Communications of the ACM. Classics. 13 (6): 377-87. doi:10.1145/362384.362685. Archived from the original on 2007-06-12. 
the Structured English QUery Language (SEQUEL), which was later abbreviated to the more commonly known SQL. This also gave rise to the commonly used name for this era: SQL Databases. In IS industry practices this era is continuing, but to an increasing extent it is mixed with approaches providing additional functionalities for data handling.

Specialized DBMSs and their use in data analysis (started in 1990s and continuing in its varying forms). Foote refers to this era as the support for online analytical processing (OLAP) but in practice it covers a variety of forms to apply managed data for business intelligence applications, data mining and a variety of (big) data analytics. OLAP itself is an approach that allows multi-dimensional handling of data. To allow powerful processing the data must be organized to support this (multidimensional) method of data handling. In practice these are different applications of relational and NoSQL? databases. Currently the frontier technologies support effective handling of mixed structured and non-structured data in (big) data analytics. One of the frontier technologies today is Apache Hadoop ${ }^{4}$, which consists of a data storage part (distributed file system (HDFS)) and a processing part (MapReduce programming model) supporting parallel data processing. We see this as a continuum of the progress that began with more simple "intelligent" data analysis, despite it being considered as the start of a new era of (specialized) data processing.

NoSQL (started in late 2000). The progress of the NoSQL data model was accelerated by the growing need for big data analytics. NoSQL provides a mechanism for handling data that no longer follows the structure of relational databases. The source of such data may be in large masses of documents and in the data streams (pipelines) of real-time WWW applications. In this kind of context, the role of data modelling faces new challenges; traditionally the data model is based on a structure - with components and the relations between them presented in a selected framework. In NoSQL data modelling, with such data stores, the model is embedded in the code, instead of traditional data models.

From this, we continue in the following sections of this paper by providing a look at the current situation and the future. The purpose and the role of data modelling have varied over the eras presented above. There is a clear link between the models, the $I S$ development paradigms and the tools used in different life cycle phases. The evolution of programming languages has had a strong influence on shaping the practices of using these languages, as well as on the tools used to build the IS models. An additional aspect worth noticing is the capabilities of computing environments. Lack of processing power, computer memory capacity, the structure and capacity of mass memory are typical reasons for delays in adopting new technologies. What is the reason for RDBMS becoming a commercial success some 20 years after its theoretical foundations were introduced? According to Jaakkola et al. in 2017 [15], computer performance (memory, processing), mass memory capacity and networking (technology, speed) are triggers for adopting new paradigms and technologies. Progress in storing and handling data, as well as in data modelling, has had the same change triggers.

The aim of this paper is to consider the changes in data modelling over the decades, with the focus on the future. The history and path of the progress were dealt with above. We continue in Section 2 by mirroring the current situation with a view from 1999. Section 3 reports our findings related to the future of data modelling based on a "literature mapping" study. In Section 4 two future "paradigms" are discussed. Section 5 concludes the paper.

\footnotetext{
${ }^{4}$ Apache Hadoop - see https://en.wikipedia.org/wiki/Apache_Hadoop.
} 
The research problem of this paper can be crystallized as follows:

What are the challenges for data modelling in the future?

\section{How the future was seen in 1999}

\subsection{View of the future from the past}

The work of Chen, Thalheim \& Wong in 1999) [3] summarizes and abstracts the papers of a 1997 workshop published in a post-proceedings form. The papers in this volume were written by several researchers, mainly from the conceptual modelling community and especially by contributors to ER conferences. The paper we reconsider summarizes the entire post-proceedings, which were the basis for subsequent compilations by various authors, e.g. Embley \& Thalheim in 2011 [6] and Thalheim in 2000 [25]. Here, however, we will use the summary from 1999 and then discuss the differences with the current state of the art.

The future and important directions are divided into the following in this paper:

- Active modelling: Modelling should become a continuous process. Once a model has been implemented within a system, the improvement of the system and the database code should be accompanied by a revision of the model. Models need continuous improvement due to changes in the applications, changes in the technology, integration of systems, extension to areas in which databases can also be successfully used, the quality of data in use, modernization of DBMS, and size of code and models.

- Relationship between natural languages and conceptual modelling: The semiotics of natural languages has great potential for improvement of modelling languages. At the same time, a sophisticated methodology similar to literal would improve modelling. Modelling languages should become as powerful as natural languages.

- Conceptual framework for sharable information services: Technology may enable the development of global information services that can be called in a variety of exchange facilities and protocols. The information explosion has to be met by the provision of small and effective services for the needs and demands of users.

- Relationships between the real world and the software world: From the viewpoint of normal users, computer systems are mainly software systems since hardware has become encapsulated and interfaced by software. Models of the software world and its activities allow us to handle, understand and evolve it properly according to the needs of the real world. These models should also support the cross-infusion of the software world and the information systems world.

- Conceptual model as the basis for interoperability: Systems should be transitioned into harmonized systems that run in a consistent and seamless manner instead of using a "let's-glue-pieces-together" approach. Models and conceptual models might be a top-down solution.

- Conceptual modelling as the first step for application engineering: Systems are continuously evolving. New users need their own interfaces which should be based on proper understanding of the entire metadata. Modelling should 
become a continuous activity which includes management of change, extension of scope, and utilization as well as integration into existing infrastructures.

- Global communication: While global data sharing is going to take place, sharing knowledge and high-quality information remains a key concern. Aspects of information and knowledge management have to be included.

- Human knowledge integration: Human knowledge can be understood from three perspectives: normative, situational and existential. Its integration is the ultimate goal for information technology development. Such systems must support inference, justification, question proliferation and answer generation according to the profile of human beings.

- Pragmatics of conceptual modelling: Modelling languages should be extended in such a way that they allow the development of a conceptual data model at a high-quality level. At the same time, they should correspond to improved object-relational database management systems.

The list of trends remained incomplete at that time. It would never be possible to provide an exhaustive list. However, it is a survey of most of the major trends as seen in 1999.

\subsection{Continuing - Some trends since 1999}

The architecture of information systems became separated into a frontend system and a backend system. The frontend system can use several data model paradigms. The backend system is typically built as an object-relational system. The systems around 1999 followed a global-as-design approach with a global data model for the backend and derivable views defined on this global model for the backend. With the sophistication of the frontend, this decision has been revised, especially for web architectures and distributed databases. Data models then became model suites (by Thalheim in 2008) [26]. This model suite approach also allows the co-designing of data models with business process models.

The experience gained with the maturation of classical applications has been summarized, generalized and abstracted in reference models. These reference models can be used in new projects as a starting point. Universal applications use such generalized data models for the generation of a specific model

Information systems became the kernel of data-intensive applications with the advent of web infrastructure. These systems are distributed and integrate a number of viewpoints. They use a variety of data models and are continuously revised and modernized. The two main branches of programming (in the small and in the large) have been extended to programming in the web by partners that only partially follow a common strategy. Typical programming in the web is not governed by any guru. Projects co-evolve without tight coordination, integration or harmonization.

Data modelling has become an issue for almost all disciplines of science and engineering. While in the past databases were developed by specialists, laymen have now become systems developers. At the same time development and research have become more interdisciplinary. Collaborations that use common data are not yet supported by the co-development of the corresponding databases.

Databases have become huge in some cases. At the same time, data models have become more complex. Big databases are highly distributed and run in parallel. Data replication has enabled controlled redundancy. 


\subsection{Analysis and the state of the art compared to 1999}

Let us now structure our trend observations and experience gained in many projects into three groups: directions that have resulted in almost completed research, directions that are still research tasks but achievable, and directions that proved to be unachievable:

(1) Successful research

- The linguistic foundation of modelling has become a common framework in data modelling.

- Modelling methodologies have reached maturity at ISO 33001 level 2 or 3.

- Distributed and federated database systems have become well-supported by modelling.

- Ontologies can be used for harmonization of viewpoints for business users.

- Service architectures and provision have become the standard.

- Question-answer forms combined with input-output forms provide an initial solution to the global communication problem.

(2) Issues for continuing research:

- Data modelling is still an art and has not become a culture. Each research and development group follow their own approach. The integration and harmonization of the variety of approaches hinder data integration in such collaboration projects.

- Model transformation and active modelling has not yet become state-of-the-art.

- Co-evolution of database systems and models is nowadays tackled by modelsas-programs.

- Continuous modelling in dependence on changes is still a major lacuna.

(3) Tasks that have not been continued:

- Modelling languages will not achieve the expressivity of natural languages.

- Pragmatics of modelling did not receive common treatment in dependence of users.

- Research on object-oriented database systems became the source for objectrelational system technology.

- Interoperability of data-intensive systems and applications can only be provided for greenfield application development and cannot be supported in migration and evolution scenarios.

- Knowledge and information management inside a database system is not supported.

We will return to these topics to some extent in the following sections.

\section{How the future is seen in 2020}

\subsection{Challenges to data modelling in its current form}

We know of more than two dozen data modelling languages. For instance, the handbook of conceptual modelling by Embley \& Thalheim in 2011 [6] discusses almost a dozen of them. Half a dozen of these languages have found their way into practice with the most

${ }^{5}$ Obtained from a large reference list for this body of knowledge and research. 
prominent UML class diagrams and various extensions of entity-relationship modelling languages. Data modelling based on such languages faces several challenges:

- The challenge of neglected foundations: Most languages are purely syntactically defined. Some of them exist only as graphical languages. UML class diagrams have several interpretations. Data modelling languages mainly provide notions with one lexical meaning of a word in a given culture and organization, e.g. the term "Person Name" has many different meanings. Part of the foundation is thus to enhance a data model with concepts from a conception space. The syntax orientation of constructs limits semantics to local semantics for each construct. Full semantics of a data model are not derivable.

- The challenge of model usage beyond the description purpose: So far, data modelling has eroded to descriptive modelling. Data models are generally considered to be inspiration models for database system developers. The model will not be used as a prescription for development since many essential elements that are needed for development can be interpreted in various ways or are missing. The model will become an historic document for system documentation that cannot be and will not be maintained or modernized with respect to changes.

- The challenge of non-agreement in the community of practice: The (conceptual) data modelling communities have not agreed on a common notion of the (conceptual) data model in 45 years of its investigation in conferences, international bodies and projects. There are more than threescore different notions (Thalheim in 2018) [27]. Three ER conferences $(2017,2018$ and 2019) incorporated workshops for the central issue of developing a commonly accepted notion of the conceptual model.

- The challenge of language simplicity: The entity-relationship model in its current widely used form uses rather simple language constructs. The main sentence constructor follows the SPO paradigm, i.e. two or more kinds of object are associated by a relationship type. English language has 25 sentence patterns and is thus far richer. The ER language is not cognitively complete. It allows only representation of three out of six cognition dimensions. Advanced extensions of the ER modelling language struggle with the sixth dimension.

- The challenge of the logical separation of syntax and semantics: Natural languages use wording that have a syntax, semantics and pragmatics. A word field combines all three semiotic dimensions. Computer science uses rigid layering and omission: syntax first, semantics later on the basis of the syntax, and pragmatics is not of interest. This separation leads to flat models, i.e. models whose constructs do not have an inner structure and an inner semantics, e.g. "Person_Name" with its specific nature.

- The challenge of concentration on one abstraction level for data: Data often come from various sources with their quality, i.e. micro-data, cleansed meso-data, aggregated macro-data. OLAP cube applications suggest that it might be essential to drill down from more abstract data to less abstract or to roll up from fine granular data to less granular. Moving though data abstraction layers requires understanding of the meaning of the data and of the attributes at their abstraction layers (Molnar \& Thalheim in 2007) [22].

- The challenge of structure-behaviour separation: A common understanding of a data model is that a data model defines the "data structures, data operations, and so forth, that together make up the abstract machine with which the user interacts" (Date in 2005) [5]. Data models thus concentrate on the data structure, constraints defined on 
those structures and, to a certain extent, operations which can be compiled from higher-order functions in order to support computing on these structures. Structures and constraints may however be dynamic (Thalheim in 2000) [25]. Furthermore, performance support requires auxiliary structures, application-governed management of the data and structuring. The theory of normal form which is today outdated has been developed for data restructuring according to performance and behaviour.

- The challenge of M0-M1-M2 layering: Natural languages allow definitions and construction of complex items inside the same language. They are somehow "flat". OMG proposes to separate things at the M0 layer from their representation at the M1 layer (termed the model layer), to separate representation from the language layer (M2, called the "meta"model layer), and to separate the language layer from the foundations layer (M3, called the "meta" "meta" layer). This leads to a difficult treatment of IsA hierarchies together with class hierarchies ('a student is a person', 'the class of student is a sub-set of the class of persons'). It is often inappropriate, e.g. the difference of various variants Is-A and of Is-Instance associations is not clear. Multilevel data modelling (Frank in 2014) [8] crosses these layers.

- The challenge of global-as-design: Data modelling assumes that a global schema is specified at the conceptual and implementation layers (which can be separated into a logical and a physical layer). The external layer concentrates on defining views which represent the viewpoints of business users. The three-layer architecture was appropriate for mainframe systems. Local viewpoints have to be represented by views. Co-design of data and business processes becomes very difficult since the business processes are based on a local-as-design paradigm. It results essentially in an impedance mismatch between the two extreme modelling strategies.

Despite these challenges there are many other lacunas, unexplored scientific issues and hidden culture problems.

\subsection{First steps from the past to the present}

At the end of section 1 we defined the research problem of this paper: What are the challenges for data modelling in the future". We also concluded that data modelling principles cannot be distinguished from the general trends in IS development, which in turn influences the IS development environment, including modelling and implementation tools. We also emphasized that progress in tools and environments depends significantly on progress in the triggering technologies. What are the leading technologies defining the trends in IS development? Some of these are listed here, referring to a variety of sources: virtualization and cloud, XaaS (something as a service), opportunity to use parallel (cluster based) processing, growing importance of system interoperability and commonly used open interfacing solutions (APIs, e.g. REST), integrating (big) data analytics to legacy ISs, the transition from plan-driven development towards agile development processes, distributed development and DevOps, the growing importance of application development for affordable devices (mobile terminals) using development platforms for them, the scalability of IS to be used in varying platforms, the use of the internet as a delivery mechanism, new kinds of revenue logics (transfer from licenses to pay-as-you-go), replacement of the customer by a faceless user (loose, if any, supplier-customer relationship), the importance of openly available components and (sub)solutions, growing value of the (external) data, "every product is a software product" - the era of IoT and embedded software. This progress 
was examined from the point of view of the software business in a paper by Yrjönkoski et al. in 2019 [29].

Going back to history, the basic principles of conceptual modelling (e.g. ER models) were developed in the period of very traditional IS development based on the waterfall type of life cycle, a strong client-customer interface and the use of IS in a customermanaged operating environment. The progress in modelling has gradually followed the progress in the characteristics of ISs and their development process by adapting to the changes. One remarkable step towards the real needs of the ICT sector was the unification of modelling practices (languages) by introducing the unified modelling language (UML) ${ }^{6}$ in the middle of the 1990s. Closely related to this, an iterative software development process - the rational unified process (RUP) ${ }^{7}$ - was introduced to replace the traditional waterfall model. An important step in the adoption of UML was introduced by Kruchten in his $4+1$ view software architecture model ${ }^{8}$. It integrates five views of IS models (based on UML) and highlights the principle of multiple (consistent) models in the modelling of IS during its development life cycle. Where traditional ER modelling focused on the static structure of the IS (concepts and their relations) leaving modelling of its dynamics (behaviour) to be done separately, UML highlighted and supported the need to model IS's dynamics as an integrated part of the whole. UML replaced the scattered set of models with an integrated set of modelling languages and transferred IS development from the era of structured development to the object-oriented (OO) era.

When analysing ER conference proceedings since 2010, we observe an increasing number of papers that solve some of the modern application problems by developing enhancements for the current technology. The data models proposed are essentially extensions of classical data models. Typical data modelling examples are proposals oriented to application challenges such as the following: (big) data analytics, data warehouses and data marts, data exchange among distributed systems, heterogeneous data ensembles, weakly structured massive data, evolving and migrating systems, clients such as search clients, web-based systems, support of the entire development cycle starting with requirements, graph-based computation, support for advanced systems such as geographic information systems, XML representation, challenging applications such as genomics and life sciences, enterprise data integration, service systems, research collaboration, support of model-driven approaches, privacy, not-only-SQL (NoSQL) systems, and the integration and collaboration of systems. At the same time, ontologies have become widely accepted as a modelling technique.

${ }^{6}$ OMG (1997). UML Specification version 1.1 (OMG document ad/97-08-11)". Retrieved from https://www.omg.org/cgi-bin/doc?ad/97-08-11 on January $29^{\text {th }}, 2020$.

7 Wikipedia (2020). Rational Unified Process. Retrieved from https://en.wikipedia.org/wiki/Rational_Unified_Process on January 29, 2020.

${ }^{8}$ Kruchten, Philippe (1995). ViewModel of Software Architecture. IEEE Software 12 (6), November 1995, pp. 42-50. Openly available and retrieved from https://www.cs.ubc.ca/ gregor/teaching/papers/4+1view-architecture.pdf on January $29^{\text {th }}, 2020$. 


\subsection{The current trends - being prepared for future needs}

We approached this topic by conducting a simple literature study, implemented as an Internet search, applying (freely) the principles of the mapping study approach by Pettersen in 2008 [23]. From the results we selected a dozen articles for further investigation. The study gave evidence of our expectations: this topic has not been dealt with widely by researchers in scientific writings, but more by well-known analyst companies (Gartner, Forbes, McKinsey etc.) in their studies. In turn, these have been analysed in numerous articles, which were finally selected as the sources of our summarizing analyses. This was done in a critical manner augmented with interpretations / concluding remarks / subjective experience-based opinions.

The article by Ghosh in 2019 [13] names Artificial Intelligence (AI) and Machine Learning (ML) as the key factors of the current dynamic in data modelling. She lists some remarkable changes that are discussed in the following paragraphs - interpreted and integrated by the authors.

Data Science. Significant changes will occur in the division of jobs and duties. Ghosh refers to the study of Gartner in 2019 [12], which predicts 40 per cent automation in data science tasks (augmented analytics, with augmented data analytics as part of it). Model analytics decreases the opportunity for human error and increases the quality of models, whereas automatic model generation decreases human work. Data analysis tasks and traditional practices in master data management (MDM), metadata management and data governance can be automated by AI/ML. More power in (business) data modelling will be offered to citizen data scientists; analytics will be driven by automated ML models. Two types of data models (views of the same data) are needed - one for professionals and one for citizen users on a plug-and-play basis to execute quick solution type tasks.

Non-structured data and pipeline-driven data integration. The growing importance of non-structured data is a reality. New database technologies - NoSQL/non-relational databases, data lakes - are becoming part of ISs. In applications, instead of on the traditional ETL (extract, transform, load) basis, data is transferred in a pipeline-driven manner as a continuous stream from a variety of sources (IoT, social network data streams, etc.), which is not common in traditional ISs.

Traditional DB technologies are not disappearing - instead there is a hybrid mix of technologies. In spite of the rise of non-relational databases, the hybrid mix of database technologies maintains the importance of traditional data modelling - relational systems are not disappearing and will have a more important role than ever as part of the data infrastructure. Although "algorithmic intelligence, self-describing data formats and standardized models" have taken some of the labour out of data modelling, the newer database technologies have initiated a whole new set of challenges for data modellers. These hybrid systems are challenging for data modellers, who must be familiar with the beneficial use of opportunities that AI and ML provide for their traditional tasks.

Robotic Process Automation (RPA): The rise of RPA gives an increasing role to business process modelling. Combined with the cloud trend - SaaS (software as a service) solutions and ML (MLaaS - machine learning as a service) -, this creates challenges in data governance issues, which may remain external to the organization (externalization).

Transition? from problem specific to problem area specific solutions. In data modelling there is a transition from problem specific to problem area specific instruments. This is analogous to the progress of ISs, which are no longer single systems but complicated systems of systems; see the conference Paper of Boehm in 2006 [1]. At 
the conceptual level we can also create an analogy as the transition towards concept systems instead of concepts per se - a kind of hierarchy. Interoperability-related requirements will of necessity move towards commonly used standardized data structures and models. Modern systems are to an increasing extent built with the guidance of existing frameworks - platforms and ecosystems, which leads to the higher abstraction of the models. The role of the ecosystem owner may create links and dependences that are not fully under the control of an individual organization. The performance expectations of modelling are expanding to new areas - to the ability to manage complex IS network structures, their interfaces and interactions, for example. Data governance is becoming more problematic - there are an increasing number of unknown data governance issues, due to cloud-based (XaaS) solutions. Cloud dominance affects data structures and needs support from a complicated data management ecosystem.

In summary, it is easy to see the growth of modelling complexity, the transfer of data (modelling) related tasks from professionals to end users and AI to support human work. At a basic level, data modelling remains as it has always been, but in practice a lot of new challenges are appearing.

The paper by Knight in 2020 [21] emphasizes that in the future (and now): "Modeling will need to do more, faster, with less". Faster means speed, more refers to the growth of the amount and complexity of data, and less indicates the need to lower the costs. Information must be processed faster, the volumes handled are greater, having their source in a multitude of systems, and a certain dynamic (readiness to react quickly to changing needs) is expected. AI will have a growing role in automating business tasks, even in the data management sector. The work of data modellers will transition to solving a decreasing amount of complex business problems in a short timeframe. The aim of companies is to optimize the data modelling activities to be able to manage them at lower cost; this includes the adoption of $\mathrm{AI}$ and the ML within it. Knight emphasizes four important trends in data modelling in 2020: just-in-time data modelling, better automation and machine learning, more widespread use of data modelling, and focused data modelling. These are briefly explained in the following paragraphs.

Just-in-time data modelling. This refers to changes in the IS development process. In agile development, even agile data modelling is expected. Because the agile philosophy is based on minimal (sufficient) design, even modelling activities are based on the on-the-fly principle. Just-in-time data modelling deals well with varying mixed (hybrid) data structures - relational, NoSQL, dimensional ${ }^{9}$ (data warehouse), master data $^{10}$, graph database ${ }^{11}$, etc. The basic work of a data modeller remains the same documenting relational (and other) structures. The work will happen to an increasing extent in the cloud because of its easy scalability, availability and low cost. This will also encourage use of larger (and more complicated) applications. Thanks to accelerating data transmission, data modelling is becoming "portable" - higher volumes of data from a wider range of systems and easy access to data models from anywhere by the relevant stakeholders.

Better automation and machine learning. Knight follows the same theme as Ghosh (discussed above) a year earlier. A lot of tasks will be automated based on the AI and ML built in the tools and development environments. The tasks following predefined

\footnotetext{
${ }^{9} \mathrm{https}: / /$ en.wikipedia.org/wiki/Dimension_(data_warehouse)

${ }^{10} \mathrm{https} / / /$ en.wikipedia.org/wiki/Master_data

${ }^{11}$ https://en.wikipedia.org/wiki/Graph_database
} 
processes will be replaced / supported by RPA-type solutions, which transfer human routine work to machines. Therefore, the duties of data scientists / data modellers will be changed to more complex (non-routine, non-algorithmic) tasks.

More widespread use of data modelling. This confirms the trend already discussed above. Put simply - the role of citizen data scientists is growing because of the higher intelligence of the plug-and-play type (flexible) analysis and modelling tools.

Focused data modelling. Data modelling will become more focused; this is because less comprehensive general-purpose data models are needed for use with hybrids of DB architectures and data streams. The scope of the data models will cover larger amounts of enterprise knowledge and technical details. According to the IDC study and prediction $^{12}$, unstructured data will account for $80 \%$ of enterprise knowledge by 2025 . The focus of data management will move to a higher level of abstraction, the management of knowledge. The models will be used by an expanding audience of stakeholders and must be customized to cover the needs of specific purposes. Data models must be organized by multi-level concerns covering business level, solution level (logical level concerns) and implementation, in some cases providing additional viewpoints.

Related to the other papers selected for our analysis, the findings seem to repeat the topics handled above. This is evidence of penetration (as a stop rule in the literature study) in the coverage of the topics handled. Therefore we will satisfy ourselves with considering these papers and articles briefly in the following paragraphs, by highlighting some new findings.

Frisendal (in 2020) [10]. This paper points out the importance of semantic graphbased modelling techniques and graph databases as an important category of NoSQL databases. Semantic graphs allow the modelling of semantic relationships between concepts. The ISO/IEC JTC1 SC32 WG3 database languages committee (SQL committee) has decided to add support for property graph queries within SQL. Graph databases represent data and its relations as a set of nodes; this "semantic network" supports data analysis type tasks. Frisendal also refers to traditional "semantic web" technologies (RDF, OWL etc.), which support the knowledge graph idea and have been adopted by some vendors.

Cagle (in 2018) [2]. This article focuses on large-scale enterprise models with large vocabularies to manage. He provides $\mathrm{RDF}^{13}$ (resource description framework; semantic web) as a tool for such enterprise-level modelling.

Roussopoulos \& Karagiannis (in 2009) [24]. This paper provides an interesting historical perspective on the progress of conceptual modelling. The last section of the paper focuses on the future. Conceptual modelling is seen as a continuous process. The web environment is seen as a constantly changing environment. The user must have the opportunity to adapt the conceptual schema and to track his interactions with the Web and SOA (service oriented architecture). This approach is called "schema-during (SD)", in which the process of conceptual modelling is on a continuum with the operations in the database. Maintenance of the model needs a user-friendly click-language tool for end-user conceptual modelling. This approach is convergent with the growing

12 https:/www.datanami.com/2019/08/23/enterprise-search-in-2020-and-beyond-5trends-to-watch/; See also https://solutionsreview.com/data-management/80-percent-ofyour-data-will-be-unstructured-in-five-years/.

${ }^{13}$ https://www.w3.org/RDF/ 
importance of citizen data analysts - more responsibility is being transferred from professionals to end users.

Harper (in 2019) [14]. This article emphasizes the change from relational database towards heterogeneous data sources, which are purpose-built for real-time use (IoT, data from a variety of sources based on pipelining). Adaptable data modelling practices accelerate the modelling process, especially when including diverse sources. It is based on the use of tools supporting codeless models (model-driven techniques) and visual model building, which can be used for collaborative data management, especially when the data comes from a wide variety of different sources.

Wells (in 2016) [28]. This article provides an interesting view of data pipelines, which lead to reverse (backward) data modelling. The traditional model is built on a topdown basis - conceptual modelling -> logical design -> physical model - to get the structure to store the data. In pipeline-based data sources, the data already exists and is stored without the opportunity to change its physical structure. The logical model is then deduced in a reverse modelling process by starting with fields, then trying to deduce the items that they describe and finally the relationships between those items. ER models are not able to handle many constructs that are typical of NoSQL databases. Wells lists the following: many-to-many relationships, multi-valued attributes, embedded arrays, associations implemented without foreign key relationships.

Guess (in 2020) [11]. This article handles the role of low-code programming. Although this phenomenon is a little beyond the scope of the debate on data modelling, it confirms the transfer of work from professionals to end users, as in the "citizen data analyst" and "no-code" data modelling discussed above. Low-code development is supported by a platform that provides an environment for the programmer to use when developing an application using a graphical user interface. The platform typically supports application area-oriented development and may need additional coding for special situations. Typical applications suitable for low-code development cover databases, automation of business processes, user interfaces and web applications.

David (in 2017) [4]. This paper largely follows the same approach as the one we followed at the beginning of this paper. It lists milestones that have had / will have high importance in IS development, including aspects related to data modelling. All the aspects included in the paper have already been covered in the discussion above.

Kiyoki et al. handle in their paper [20] semantic modelling. It is based on the Mathematical Model of Meaning (MMM), in which an orthogonal multidimensional semantic space is created, and used for semantic associative search. Retrieval candidates and queries are modelled - mapped - onto the semantic space, and the semantic associative search is performed by calculating the correlation of the retrieval semantic space. In [20] the method is applied in the multi-spectrum images used for environmental analysis, covering spatial, temporal and semantic axis in the analysis and modelling.

We have analysed a set of articles / papers dealing with data modelling, and a slightly wider scope of IS modelling. The concluding message is that the future of modelling work - already visible in the present - faces a lot of challenges caused by the breakthrough of new types of data sources. On the one hand automation helps the work, but on the other hand the remaining work needs new updated skills. The role of the end user will grow in IS development and in the use of data sources. The essence of data is changing towards built-in semantics and characteristics of knowledge. 


\subsection{Summarizing the findings - discussion}

We apply the categorization of technological changes introduced by Freeman \& Perez in 1988 [9] in the classification of the changes in data modelling; the changes are divided into four categories (discussed in detail by Jaakkola et al. in 2017 [16; 17]):

- Incremental changes appear continuously in existing products and services (continuing the existing trend); these accelerate the existing change in the existing trend.

- Radical changes appear when new research findings are applied in products to transfer their properties or performance to a new step or cycle; at a certain moment there is an upward shift (break in the trend) caused by the innovation.

- Changes in technological systems are caused by combinations of several incremental and radical innovations in societal and organizational systems; these changes provide the means for increasing competitiveness and fast growth of productivity for early adopters.

- Changes in paradigms are revolutionary and lead to pervasive effects throughout the target system under discussion. They indicate permanent changes in societal systems, daily ways of operating and business models that replace the old ones.

Although the ideas of Freeman \& Perez were developed to analyse technological changes in society, the principle can be applied in the case of a single technology, i.e. data modelling (as one of the technologies in IS design). We have included one additional category in our analysis below - triggering and enabling technologies. These are changes that do not have a direct connection to data modelling itself, but act as an enabler to make the changes necessary or possible.

In technology analysis it is worth noting that the situation does not remain stable. New change-driven innovative technologies tend to become the new normal over the course of time. This has happened for instance to UML and the object-oriented approach. These were revolutionary (radical, system change, paradigm change) at the time of their appearance, but no longer cause any significant changes.

In sub-section 3.1 we discussed the challenges related to the practices of data modelling and the languages used. The source of the challenges lies in the expression power of the modelling tools (languages), which typically are semi-formal by nature. They have high (but limited) expression power, but lack precision, which leaves space for interpretation. The beneficial aspect is high (visual, graphical) readability from the point of view of a heterogenous set of interest groups. The challenges have solutions, which are incremental, and are at most radical in character.

Sub-section 3.2 focused on the near-past phenomena that mainly have characteristics of triggering and enabling technologies and radical changes. ISs are transitioning quickly and powerfully towards cloud-based implementation and service architectures (XaaS, SOA). This has caused remarkable changes in data modelling. Modelling tasks are "outsourced" to the responsibility of the cloud data management and to an increasing extent an individual organization has to adopt the dominance of "external" data models and adapt their own solutions within these. We listed the following trends, which belong to the category of changes in technological systems:

- the growing importance of system interoperability and interfaces (API),

- transfer from plan-driven to agile development processes, 
- the use of distributed collaborative (concurrent) development environments (Devops),

- transition towards platform and ecosystem dominance, and

- the growing value of external (non-modellable, non-structured) data produced by IoT and a variety of other external sources.

We are also losing the power of direct supplier-customer communication, which is being replaced by the development paradigm "faceless supplier to faceless client" caused by the growing demand for service-based systems, the use of web-based and ecosystemdominated applications, and openly available components. This makes requirements engineering and elicitation of requirements of an IS more demanding.

Sub-section 3.3 focused on the "future" of data modelling. The future is partially already here as a continuum of the current movement towards the more mature solution models and technologies of the future. These are long-term changes in data modelling. Consequently, we have included these in the system changes category. Almost all the papers we analysed underlined $A I$ and $M L$ as the key change factors in data modelling. Undoubtedly, these have characteristics of triggering / enabling technologies. We do not want to separate these from their application context: on their own they are not important but, when connected to a variety of application contexts, they are. AI supports the work of data analysts, which is partially automated. Part of the work is also transferred to the stakeholders in their other roles - as citizen data scientists, low code programmers, end users etc. Data modelling must support the heterogenous needs of all these groups - the transition to multilevel concerns covering a variety of abstraction levels is a reality. Adaptable schemas, adaptable data modelling and visual model building are needed to support the needs of end users. In addition, the agile development process needs an onthe-fly type of modelling. IS complexity is growing. Consequently, models are growing, and focus must be placed on a multitude of systems instead of only one. Data management solves complex business problems rather than less complex IS problems. In data modelling the role of external data (pipeline-based, having the source in IoT nodes, sensors and a variety of data streams) is growing. Challenges in managing mixed (hybrid) data formats in one application make data modelling complex. External pipeline data requires reverse modelling without the opportunity to affect its structure. Platform and ecosystem based IS development supports the transition from specific data models to less comprehensive, problem area specific and general-purpose data models that support interoperability between independent systems. $R P A$ transfers the focus in modelling from IS level to business process modelling.

In section 4, we will build a view of the future by presenting ideas about paradigm changes in data modelling.

\section{Future paradigms in data modelling}

\subsection{Data modelling will become database programming}

Data models are widely used in most data-intensive applications. Data models could be reduced to a database systems construction. There are, however, also other scenarios for the usage of a data model: communication and negotiation, description and conceptualization, documentation, explanation and discovery for applications, 
knowledge discovery and experience propagation, and explanation and discovery for systems.

For system construction, we can see the first generation of data modelling: Models are used as a mediator and blueprint for database realization, for the description of the data in an application, for negotiation etc. They allow reasoning on database systems, i.e. model-based development and reasoning. This generation can be characterized as datamodelling-enabling-data-programming.

Jaakkola and Thalheim in 2020 [18] have also envisioned a second generation of data modelling: Model development and the modelling infrastructure have reached a maturity that allows the derivation of database structuring and supporting programs (or their essential parts) from data models or data model suites. Models will thus be the source code for executable programs. We characterize this generation as data-modellingas-programming.

The next generation can be characterized as data-models-as-database-programs. Data models can be directly translated into database dictionaries and database programs, i.e. database programming can be performed almost entirely through modelling. The third generation of data modelling is then the true fifth generation database programming, which essentially frees the database developer from writing database programs and host programs with third or fourth generation languages. Everybody, including database laymen, who can specify data models will become a database programmer. Data models will then be model suites.

\subsection{Data modelling must change along with application changes and technology evolution}

Modern database applications are becoming more voluminous, have higher velocity, use a variety of models, are often hampered by data quality and veracity, are becoming more viable, have to cope with variations, use highly heterogeneous data, are challenged by viability, and must provide tangible added value. An appropriate technology has still not been found. To some extent, big data machines can solve some of the problems. It seems, however, that conventional technology must also be revised. Already very large data sets frighten us and result in the avoidance of complex operations beyond $\mathrm{n} \cdot \log (n)$ complexity. Big data should be supported by technology with operations far below data size (i.e. n) complexity. Proper data modelling approaches that could meet these challenges are under investigation. So far, modelling research has not led to any convincing approaches.

The classical database systems have been based on OLTP-web_frontend, distributed database, OLTP-OLAP, OLTP-data_warehouse-data_mart, or the micro_datamacro_data-analysis_data layered database architecture. They result in an increase in data size and computational problems. We already know solutions for modelling through model suites, i.e. a collection of coherent and sufficiently tightly associated models where each of the data models reflects some of the aspects in these architectures. Kiyoki \& Thalheim in 2013 [19] revised the layered architecture for a real big data application to a highly distributed data network with some data collaboration styles and patterns, e.g. publish-subscribe or master-slave. Each node in this network could use its data model. In the given case, each node used an internal data model, an import data model and an export data model where the last two were essentially views of the first model. 
The application world will result in a number of rapid changes to data modelling as well as the capability of modern systems. All these changes will initiate revision modernization of data modelling. Let us consider some of them:

- Flexible model transformation without information loss: Models represent a specific application viewpoint that coexists with a larger variety of viewpoints in other application fields. At the same time, partners essentially operate using the same data. Therefore, we need data models that can be transferred to each other without loss of data and structure information. The notion of infomorphism is a starting point.

- Large data models: Already legacy (or rather heritage) applications use models that have been developed by generations of developers and have become "mannered and stilted cathedrals". With the wide spread of database technology, data models become larger and larger. Their management is becoming an obstacle for applications.

- Weakly structured data: Data might be weakly structured and can be enhanced at a later stage with refined structuring. Data models must be sufficiently robust for this kind of evolution or complete modernization.

- Data models reflecting provenance and quality: Data is seldom 100\% correct. This observation was already true in 1999. Nowadays, such data is widely used. We use data that is partially corrected and cleansed together with data that needs improvement. Data might also be changed especially for biased usage with decreasing quality. Engineering has learned how to cope with errors inside its systems without changing the system. Such kind of inner quality correcting data models? allow to become robust.

- Toughening the data model: A data model might be a starting point that is continuously improved during exploitation. The model has a higher quality within the quality portfolio of the application. The classic 'code-that-smells' repair approaches will be replaced by the co-evolution of models and their data.

- Adaptable and self-adapting data models: Data structures and applications continuously evolve. This evolution can be mastered by a real artisan. It is far better to integrate the evolution strategy and the adaptation tactics into the data model.

- Heritage extraction models: Many applications have journeyed through data models that have evolved over time. This journey is badly documented. It is also hampered by very special implementation issues. We will fall into the Excel macro trap if we are unable to recover the models in such a form that the current status of data is captured.

- Just-in-time data models: Data models will be developed wherever data occurs in an application. The initial data model will often be proprietary and not harmonized with existing ones. A typical case is large research clusters (e.g. within the German Excellence Programme) without controlled data integration.

- Data models reflecting storage are separated from data models supporting computation: It is not necessary to keep all the data together with the data processing facilities. Instead, we might use a universal data store with all the data together with local data stores that neatly correspond to the local application. The binding mechanism could be a sophisticated export/import view management.

- Special data models for special tasks: Data mining and analysis also target the detection of patterns within the data. These patterns are the kernel of explanation models. The data used is empirical data and is thus often of problematic quality. We might use data model pipelines that reflect this mining and analysis framework. 
Similarly, many applications need their specific domain-oriented modelling language which is well embedded into the application domain. Enterprise data models must thus be different from social data models.

- Layman data models: Data models are often developed by users that do not have sufficient experience and knowledge of modelling. Models reflect their disciplinary, education and business context without explicitly stating it. Models are local. Their integration is only possible if the full context is known.

- Harmonization of data modelling languages with computation features: Big data challenges current technology and will force us to redevelop current database technology. We envision that only those operations that perform well in a given architecture and in a given application world are going to be realized in systems. Similar to the past when we co-developed algorithms with their own data structure, we need data modelling languages that support high-performance computation for challenging data volumes.

This list is at its best only a starting point. It should be enhanced by the trends discussed above in order to meet the challenges discussed above. One solution path to better data models is standardization. We start with high-quality models as components of the models. Model composition follows approved composition approaches. Mechanical engineering and industrialization have widely used this approach. Another solution is development through generic models. These models are supported by sophisticated refinement strategies and tactics for their improvement. Generic models are also widely used in engineering for production moulds or forms.

\section{Conclusions}

At the beginning of this paper we set our research problem: "What are the challenges for data modelling in the future?". We did not dissect it in the form of research questions but followed a systematic path that started with an analysis of the progress path of data modelling, covering the decades from the early phases of commercial computing until the present day. After that we made a "forecast of the past" by benchmarking a future forecast from 1999 - over twenty years ago. It provided us with an opportunity to compare the state of the art of today to the expected progress. Understanding the future is not possible without understanding the past - everything is a kind of continuum from the past to today and on into the future. The future analysis of the paper is based on the "literature" mapping study. We collected expert opinions from several sources about future visions in data modelling. These findings were then classified and analysed. No clear future path can be defined, but a set of dominant factors can be gathered - the growing complexity of models, increasing tool intelligence, growing importance of external (non-modellable) data, heterogeneity of stakeholders in modelling and technology as a change driver - just to mention some of the findings. The paper concludes with two future paradigms.

\section{References}

[1] Boehm, B. (2006). A view of 20th and 21st Century Software Engineering. Paper presented at the Proceedings of the 28th International Conference on Software Engineering (ICSE), Shanghai, China. 
[2] Cagle, Kurt (2018). Why Data Modelling is Important (and Why It's Not). Retrieved from https:/www.forbes.com/sites/cognitiveworld/2018/11/05/why-data-modeling-is-important-and-whyits-not/\#1 e9cc98e50cb on January 31st, 2020.

[3] Chen, P. P., Thalheim, B., \& Wong, L. Y. (1999). Future Directions of Conceptual Modeling. In G. Goos, J. Hartmanis, J. van Leeuwen, P. P. Chen, J. Akoka, H. Kangassalu, \& B. Thalheim (Eds.), Conceptual Modeling: Current Issues and Future Directions (pp. 287-301). Berlin, Heidelberg: Springer Berlin Heidelberg. Available also from https://www.researchgate.net/publication/2847577_Future_Directions of_Conceptual_Modeling on January 31st, 2020.

[4] David, Amos (2017). Current trend in data modeling and information systems. International Conference on Applied Information and Communication Technology. Lead City University, Ibadan, Nigeria. Retrieved from https://www.researchgate.net/publication/327120249 Current trend in data modeling_and_information_systems on January 31st, 2020.

[5] Date C.J. (2005). Database in depth: Relational theory for practitioners. O'Reilly, Sebastopol.

[6] Embley D. and B. Thalheim, editors (2011). The Handbook of Conceptual Modeling: Its Usage and Its Challenges. Springer.

[7] Foote, Keith D. (2017). A Brief History of Data Modeling. Retrieved from https://www.dataversity.net/ brief-history-data-modeling/ on January 29th, 2020.

[8] Frank U. (2014). Multilevel modeling - toward a new paradigm of conceptual modeling and information systems design. Business \& Information Systems Engineering, 6(6):319-337, 2014

[9] Freeman C., Perez C. (1988). Structural Crises of Adjustment, Business Cycles and Investment Behavior. In Dodi G., Freeman C., Nelson R., Silverberg G. and L. Soete L. (Eds), Technical Change and Economic Theory. Pinter Publishers, London.

[10] Frisendal, Thomas (2020), Ten 2020 Visions for Data Modelers. Retrieved from https://www.dataversity.net/ten-2020-visions-for-data-modelers/ on January 31st, 2020.

[11] Guess A.R. (2020). Low-Code 20/20: A Clear Vision for Solving the Software Crisis. Retrieved from

[12] Gartner (2019). To 10 Strategic Technology Trends for 2019. Retrieved from https://datavizblog.com/ 2018/11/18/gartner-top-10-strategic-technology-trends-for-2019/ on January 29 ${ }^{\text {th }}, 2020$.

[13] Ghosh, Paramita. (2019). Data Modeling Trends in 2019. Retrieved from https://www.dataversity.net/data-modeling-trends-in-2019/ on January $29^{\text {th }}, 2020$

[14] Harper, Jelani (2019). 2019 Trends in Data Modeling: Real-Time Integration for Cognitive Computing. Retrieved from https:/aibusiness.com/2019-trends-data-modeling/ on January 31st, 2020.

[15] Jaakkola, H., Henno, J., \& Mäkelä, J. (2017). Technology and the Reincarnation Cycles of Software. In Z. Budimac (Ed.), SQAMIA 2017 - Proceedings of the Sixth Workshop on Software Quality Analysis, Monitoring, Improvement, and Applications. Belgrade, Serbia, September 11-13, 2017. (Vol. Vol-1938, pp. 5:1-10). Belgrade, Serbia: CEUR Workshop Proceedings.

[16] Jaakkola, H., Henno, J., Mäkelä, J., \& Thalheim, B. (2017a). Today is the Future of Yesterday, What is the Future of Today? In P. Biljanović (Ed.), MIPRO 2017 - Proceedings of the 40th Jubilee International Convention. May 22-26, 2017, Opatija, Croatia. (pp. 741-749). Opatija, Croatia: Mipro and IEEE.

[17] Jaakkola, H., Henno, J., Thalheim, B., \& Mäkelä, J. (2017b). The educators' telescope to the future of technology. In P. Biljanović (Ed.), MIPRO 2017 - Proceedings of the 40th Jubilee International Convention. May 22-26, 2017, Opatija, Croatia. (pp. 766-771). Opatija, Croatia: Mipro and IEEE.

[18] Jaakkola H. and Thalheim, B. (2020). Model-based fifth generation programming. In Information Modelling and Knowledge Bases Vol. XXXI, Frontiers in Artificial Intelligence and Applications, 312 , pp. 377-396. IOS Press.

[19] Kiyoki Y. and Thalheim, B. (2013). Analysis-driven data collection, integration and preparation for visualisation. In Information Modelling and Knowledge Bases, volume XXIV, pp. 142-160. IOS Press.

[20] Kiyoki, Y., Chen, X., Sasaki, S. and Koppåipat, C., Multi-Dimensional Semantic Computing with Spatial-Temporal and Semantic Axes for Multi-spectrum Images in Environment Analysis. In Welzer, T., Jaakkola, H., Thalheim, B., Kiyoki, Y., Yoshida, N. (Eds.), Information Modelling and Knowledge Bases XXVII. IOS Press, 2016, pp. 14-31.

[21] Knight, Michelle (2020). Data Modeling Trends in 2020: A Year of Optimization. Retrieved from https://www.dataversity.net/data-modeling-trends-in-2020-a-year-of-optimization/ on January 31, 2020.

[22] Molnar A. and B. Thalheim (2007). Conceptual development of OLAP applications. In Business Intelligence: Methods and Applications, pp. 27 - 38. Klöden-Verlag, 2007.

[23] Pettersson F., Ivarsson M., Gorschek T., Öhman P. (2008). A practitioner's guide to lightweight software process assessment and improvement planning. Journal of Systems and Software 81, 6 (June 2008), 972 995. DOI:https://doi.org/10.1016/j.jss.2007.08.032.

[24] Roussopoulos N., Karagiannis D. (2009). Conceptual Modeling: Past, Present and the Continuum of the Future. In: Borgida A.T., Chaudhri V.K., Giorgini P., Yu E.S. (eds) Conceptual Modeling: Foundations and Applications. Lecture Notes in Computer Science, vol 5600. Springer, Berlin, Heidelberg

[25] Thalheim B. (2000) Entity-relationship modeling - Foundations of database technology. Springer, Berlin. 
[26] Thalheim, B. (2008). Model suites. In H. Jaakkola, editor, Selected Topics on Distributed Disaster Management: Towards Collaborative Knowledge Clusters., pp. 108 - 128. Tampere University Press, Pori unit.

[27] Thalheim B. (2018) Conceptual model notions - a matter of controversy; conceptual modelling and its lacunas. EMISA International Journal on Conceptual Modeling, February, pp. 9-27.

[28] Wells, Dave (2016). Big Changes in the World of Data Modeling. Retrieved from https://tdan.com/big changes-in-the-world-of-data-modeling/19578 on January 31st, 2020.

[29] Yrjönkoski, K., Jaakkola, H., Mikkonen, T., Systa, K., \& Henno, J. (2019). SQAMIA: Software business: a short history and trends for the future. In Z. Budimac \& B. Koteska (Eds.), 8th Workshop on Software Quality Analysis, Monitoring, Improvement and Applications, SQAMIA 2019 Proceedings (Vol. Vol2508, pp. 18:11-18:18): CEUR Workshop Proceedings. 


\title{
A Global \& Environmental Coral Analysis System with SPA-based Semantic Computing for Integrating and Visualizing Ocean-Phenomena with "5-Dimensional World-Map"
}

\author{
Yasushi Kiyoki ${ }^{1}$, Petchporn Chawakitchareon ${ }^{2}$, Sompop Rungsupa ${ }^{3}$, Xing $\mathrm{Chen}^{4}$ and \\ Kittiya Samlansin ${ }^{2}$ \\ ${ }^{1}$ Graduate School of Media and Governance, Keio University, Japan \\ kiyoki@sfc.keio.ac.jp \\ ${ }^{2}$ Environmental Engineering Department, Chulalongkorn University, Bangkok, \\ Thailand; petchporn.c@chula.ac.th \\ ${ }^{3}$ Aquatic Resources Research Institute, Chulalongkorn University, Bangkok, Thailand \\ ${ }^{4}$ Department of Information \& Computer Sciences Kanagawa Institute of Technology, \\ Japan; chen@ic.kanagawa-it.ac.jp
}

\begin{abstract}
Semantic computing is essentially significant for realizing the semantic interpretation of natural and social phenomena and analyzes the changes of various environmental situations. The 5D World Map (5DWM) System [4,6,8] has introduced the concept of "SPA (Sensing, Processing and Analytical Actuation Functions)" for global environmental system integrations [1-4], as a global environmental knowledge sharing, analysis and integration system Environmental knowledge base creation with 5D World Map is realized for sharing, analyzing and visualizing various information resources to the map which can visualize and facilitate the comparisons in multidimensional axes.

The 5DWM system and its architecture have been implemented as a knowledge sharing, analyzing, searching, integrating and visualizing system with multidimensional control mechanisms. This system integrates and visualizes various environmental information resources, related to local natural and social phenomena to the multi-dimensional world map to be globally shared and analyzed with multidimensional axes.

5D World Map System is globally utilized as a Global Environmental Semantic Computing System, in SDGs 9, 11, 14, United-Nations-ESCAP: (https://sdghelpdesk.unescap.org/toolboxes), as a KEIO-MDBL-UN-ESCAP Joint system for disaster, natural phenomena, ocean-water analysis with local and global multimedia data resources. This paper proposes a new semantic computing method as an important and promising approach to semantic analysis for various environmental phenomena and changes in real world. This method realizes "SelfContained-Knowledge-Base-Image" \& "Contextual-Semantic-Interpretation" as a new concept of "Coral-Health-level Analysis in Semantic-Space for Oceanenvironment" for global ocean-environmental analysis $[8,9,12,18]$. This computing method is applied to automatic database creation with coral-health-level analysis sensors for analyzing and interpreting environmental phenomena and changes occurring in the oceans in the world. We have focused on "Coral-Health-level
\end{abstract}

\footnotetext{
${ }^{1}$ Graduate School of Media and Governance, Keio University, Japan; kiyoki@sfc.keio.ac.jp
} 


\begin{abstract}
Analysis in Semantic-Space for Ocean-environment", as an experimental study for creating "Coral-Health-level Analysis Semantic-Space for Ocean-environment" $[8,9,12,18]$. This method realizes new semantic interpretation for coral health-level with "coral-images and coral-health-level knowledge-chart".

Keywords. Global Environmental Analysis, Semantic Computing, SPA, 5Dimensional World Map System, Coral Nanalysis, Environmental Data Mining
\end{abstract}

\title{
1. Introduction
}

5D World Map System and its applications create new analytical research environments with the SPA concept (Sensing, Processing and Analytical Actuation) for searching, sharing, analyzing and visualizing natural and social environmental aspects, as shown in Fig. 1. This system realizes "environmental analysis and situation-recognition" which will be essential for finding out solutions for global environmental issues. The 5D World Map System collects and facilitates a lot of environmental information resources which are characteristics of ocean species, disasters, water-quality and deforestation.

As conceptual modeling for making appropriate and urgent solutions to global environment changes in terms of short and long-term changes, "six functional-pillars" are essentially important with "environmental knowledge-base creation" for searching, sharing, analyzing and visualizing various environmental phenomena and changes in a real world.

(1) Cyber \& Physical Space Integration,

(2) SPA-function,

(3) Spatio-Temporal computing,

(4) Semantic computing,

(5) World map-based visualization,

(6) Warning message propagation

As an actual implementation of the SPA architecture, 5D World Map System Project in Figure 1 has presented a new concept of "Water-quality Analysis Semantic-Space for Ocean-environment" for realizing global water-environmental analysis [8,9,18]. The semantic space and the computing method have been implemented with knowledge-base creation with water-quality-analysis sensors for analyzing and interpreting environmental phenomena and changes occurring in the oceans in the world. We have focused on sea-water quality data, as an experimental study for creating "Water-quality Analysis Semantic-Space for Ocean-environment" [8,9]. 


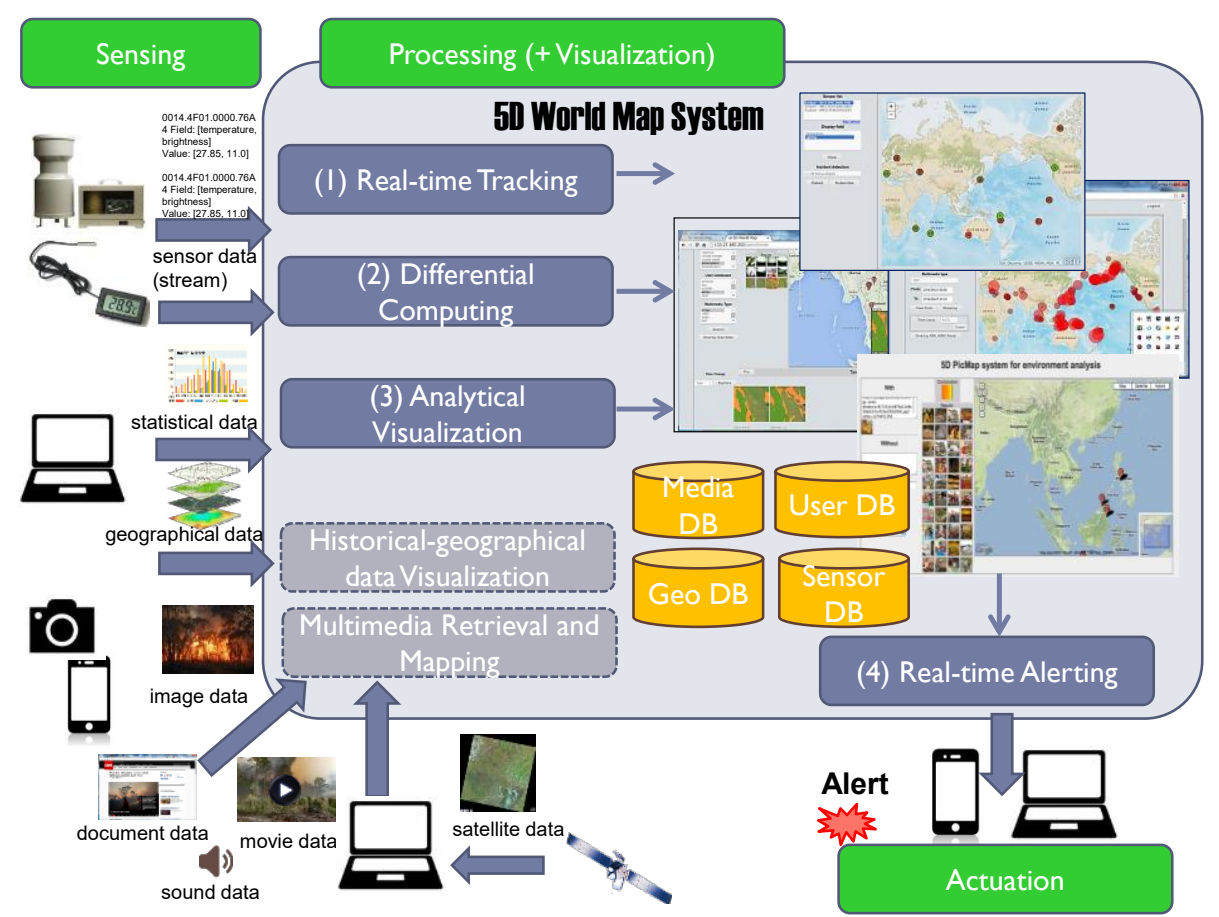

Figure 1. Basic SPA functions in 5D World Map System

We have introduced the architecture of a multi-visualized and dynamic knowledge representation system "5D World Map System [4,6,8]," applied to environmental analysis and semantic computing. The basic space of this system consists of a temporal $\left(1^{\text {st }}\right.$ dimension$)$, spatial $\left(2^{\text {nd }}, 3^{\text {rd }}\right.$ and $4^{\text {th }}$ dimensions $)$ and semantic dimensions $\left(5^{\text {th }}\right.$ dimension, representing a large-scale and multiple-dimensional semantic space that is based on our semantic associative computing system (MMM). This space memorizes and recalls various multimedia information resources with temporal, spatial and semantic correlation computing functions, and realizes a 5D World Map for dynamically creating spatio-temporal and semantic multiple views applied for various "environmental multimedia information resources."

\section{Semantic Computing in MMM and 5D World Map System}

We apply the dynamic evaluation and mapping functions of multiple views of spatiotemporal metrics and integrate the results of semantic evaluation to analyze environmental multimedia information resources. The MMM is a fundamental semantic computing model for the 5D World Map System, as a semantic associative search method [1,2,3] for realizing the concept that "semantics" and "impressions" of environmental multimedia information resources, according to the "context". The main feature of this system is to create world-wide global maps and views of environmental situations expressed in multimedia information resources (image, sound, text and video) dynamically, according to user's viewpoints. Spatially, temporally, semantically and impressionably evaluated and analyzed environmental multimedia information resources are mapped onto a 5D time-series multi-geographical space. The basic system structure 
of the MMM and 5D World Map System is shown in Figure.2 and Figure. 3. The 5D World Map system applied to environmental multimedia computing visualizes worldwide and global relations among different areas and times in environmental aspects, by using dynamic mapping functions with temporal, spatial, semantic and impression-based computations $[4,5,6,7,8]$.

\subsection{Semantic Computing in 5D World Map System}

We have introduced the architecture of a multi-visualized and dynamic knowledge representation system "5D World Map System," applied to environmental analysis and semantic computing in Fig.2 and Fig. 3. The basic space of this system consists of a temporal (1st dimension), spatial (2nd, 3rd and 4th dimensions) and semantic dimensions (5th dimension, representing a large-scale and multiple-dimensional semantic space that is based on our semantic associative computing system (MMM). This space memorizes and recalls various multimedia information resources with temporal, spatial and semantic correlation computing functions, and realizes a 5D World Map for dynamically creating temporal-spatial and semantic multiple views applied for various "environmental multimedia information resources."

\section{The concept of 5D World Map}

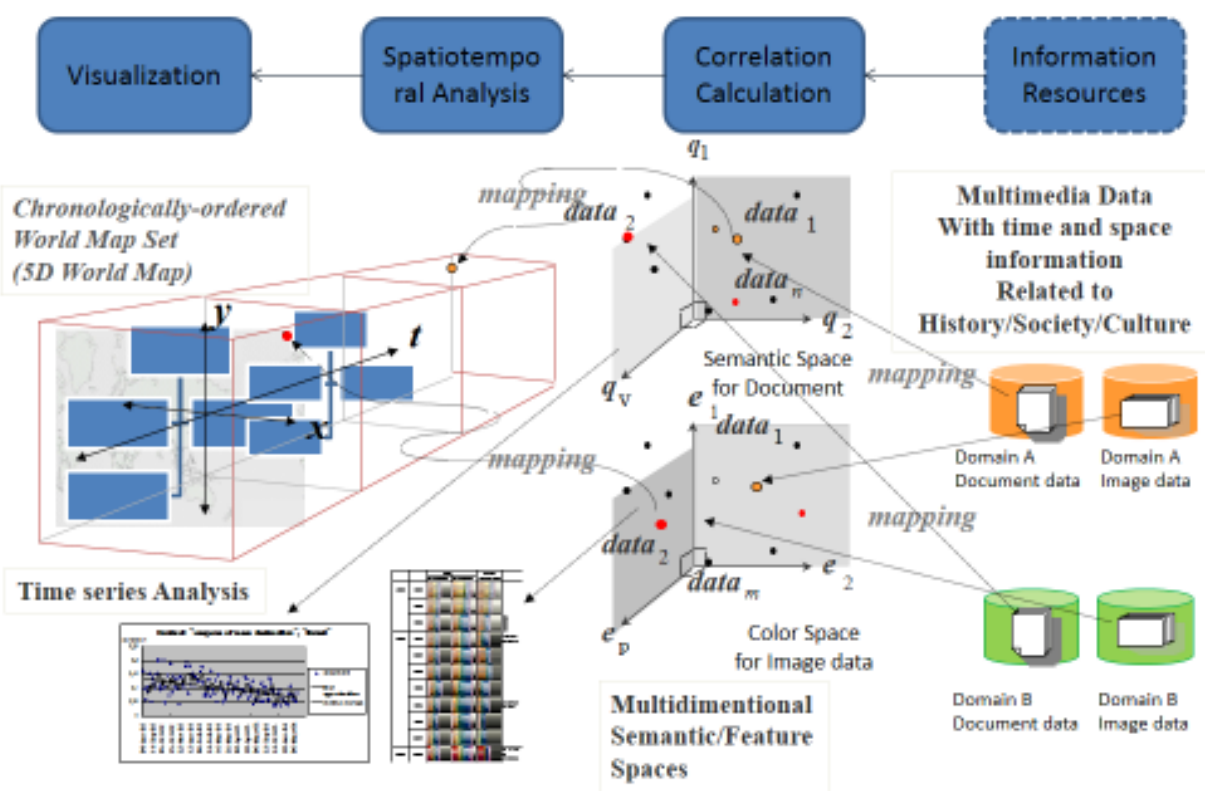

Figure 2. 5D World Map System for world-wide viewing for Global Environmental Analysis 


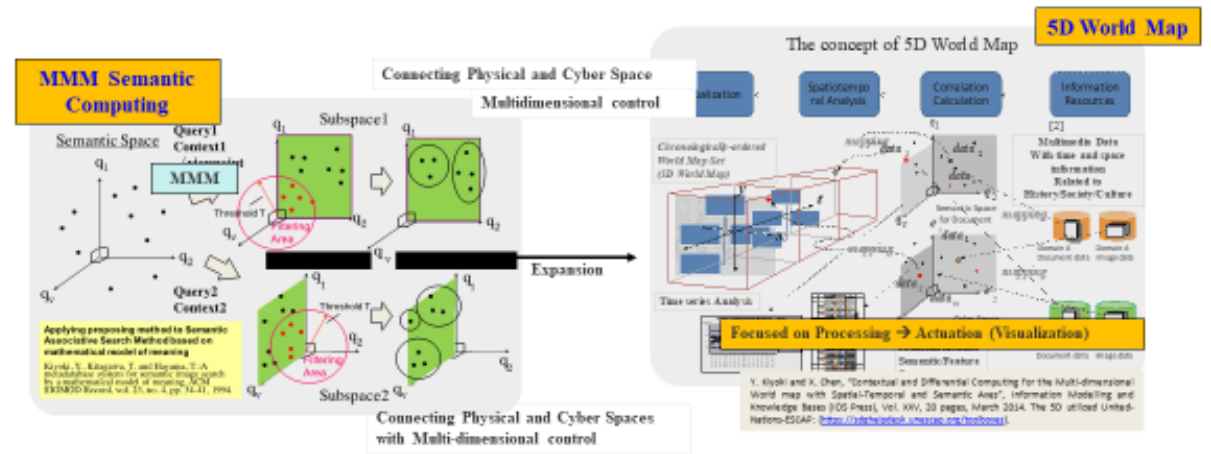

Figure 3. The system structure of MMM Semantic-Computing and 5D World Map System

\subsection{SPA: Sensing, Processing and Analytical Actuation Functions in 5D World Map}

"SPA" is a fundamental concept for realizing environmental system with three basic functions of "Sensing, Processing and Analytical Actuation" to design an environmental system with Physical-Cyber integration. "SPA" realizes a significant function to detect environmental phenomena as real data resources in a physical-space (real space) in in 5D World Map, map them to cyber-space to make analytical and semantic computing, and actuate the analytically computed results to the real space by visualization for expressing environmental phenomena with causalities and influence. This concept is applied to global water-quality and coral-analysis with our semantic computing in 5D World Map System, as shown in Fig. 4, 5 and 6.

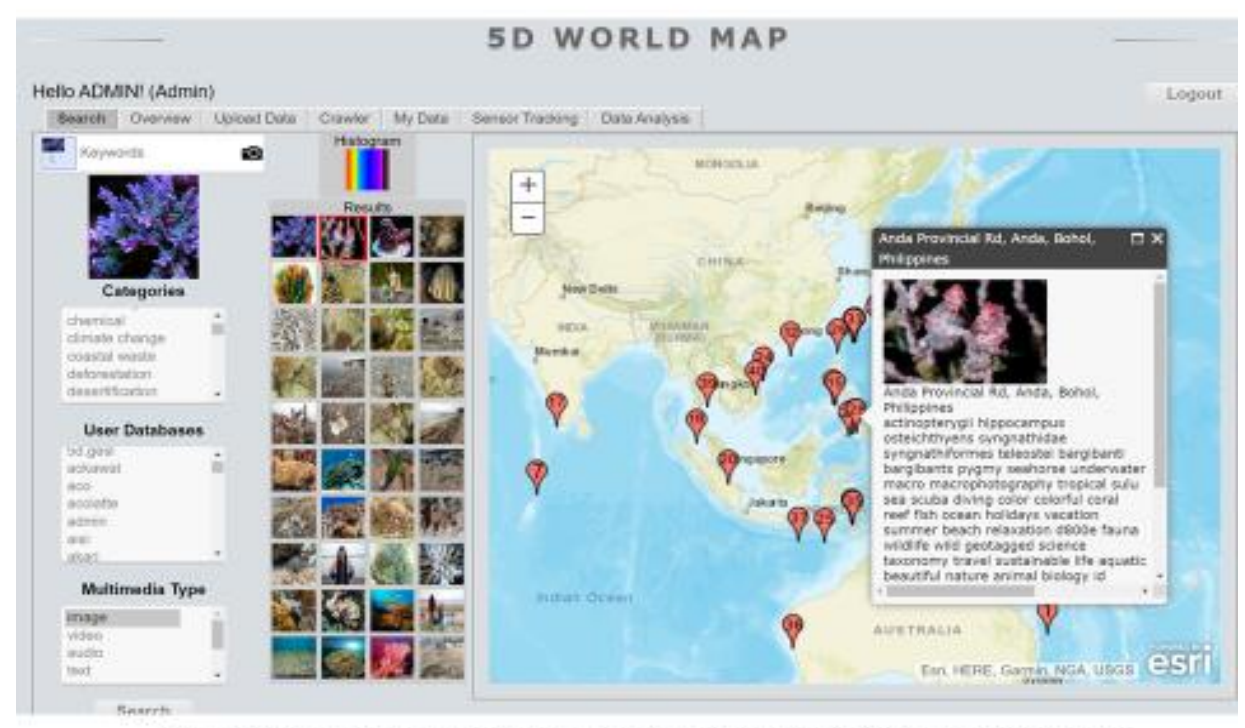

Input image = purple coral, Categories = coastal waste, SDG14, ocean pollution

Figure 4. Global Environmental Analysis and Visualization of "Coral" in "5D World Map System 


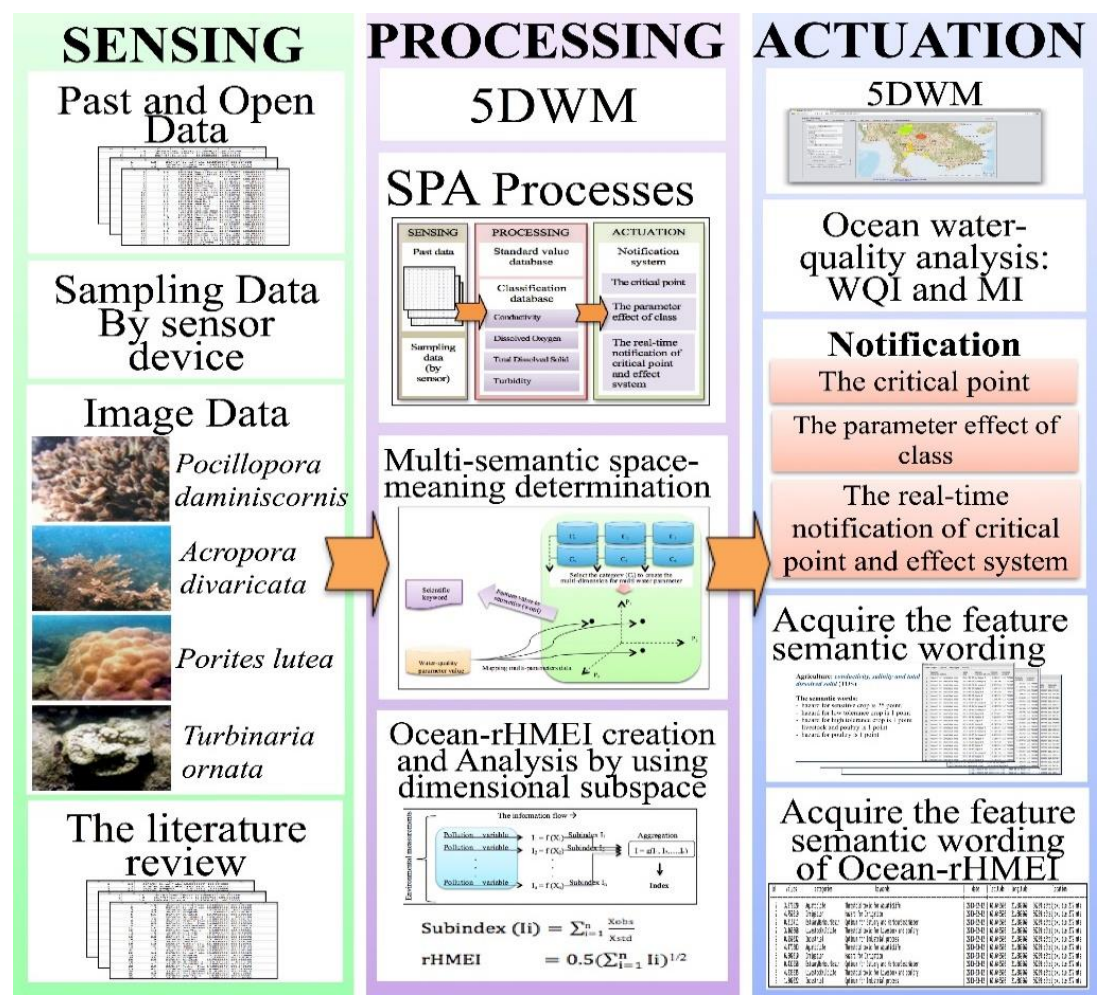

Figure 5. SPA functions for Ocean Environment-analysis with multi-dimensional control in 5D World Map System $[9,18]$

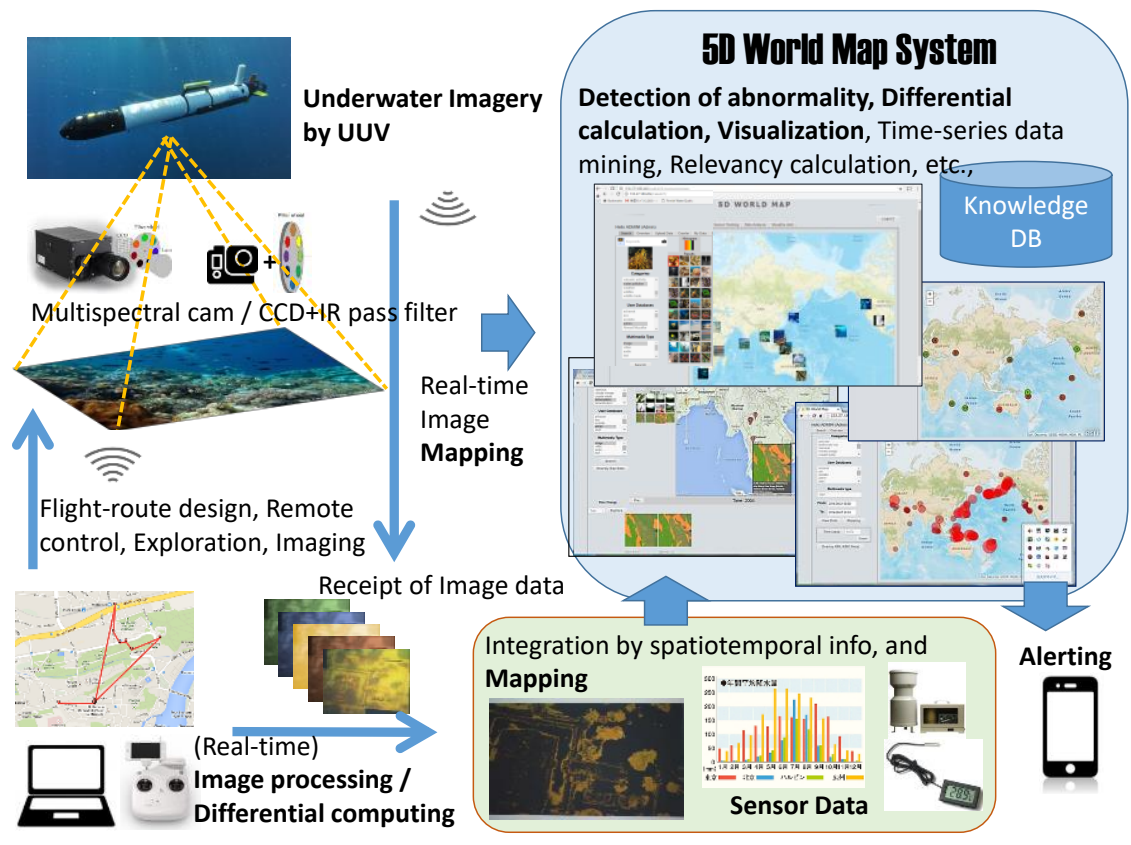

Figure 6. SPA functions for Underwater Environment-Images in 5D World Map System 


\section{Semantic Computing for Coral-health-analysis in MMM}

A MMM-based semantic computing method [1,2,3] is applied to coral health-level analysis with a knowledge base in a coral image. This method realizes automatic semantic interpretations of Coral health-level with a coral-image containing the knowledge base of coral-health. We use "Coral-health-chart [26]" as an example, to express color-health-level correspondences

This method consists of three essential elements.

(1) Coral image

(2) "Coral-health-level-Color-correspondence chart" as a knowledge-base

(3) Semantic distance computing between "Coral-color" and "Coral-health-levelColor-correspondence chart"

The overview of this method is shown in Figure 7, as "Self-contained-KnowledgeBase-Image" \& "Contextual-Semantic-Interpretation Algorithm"

\subsection{Data structure}

The basic data structure of this method is defined as follows:

(Data-structure-1) "Coral-Image-with-chart" with "Target-Coral" and "CoralChart"

(Data-structure-2) "Target-Coral": Coral-color table (81-pixels-coral-color vector (3-color-elements (RGB) between "coral-pixel-id" and its "coral-color vector"

(Data-structure-3) "Semantic Interpretation Knowledge Base": (pairs of "color and its meaning") :

Chart-color table (24 chart-color vectors (3-color-elements (RGB) ) between "chart-color" and its "chart-color vector" with meanings)

\subsection{Basic Functions and "Contextual-Semantic-Interpretation Algorithm"}

The two basic functions are defined as follows:

(1) "Semantic-Distance-calculation" between "coral-color vector" and each of "chart-color vectors" to "semantic-distances"

(2) Ranking-calculation in correlations among "semantic-distances" as "semanticordering" (ascending-order or descending-order)

"Contextual-Semantic-Interpretation Algorithm" for "Self-Contained-KnowledgeBase-Image" is defined as the following procedure.

"Contextual-Semantic-Interpretation Algorithm" for "Self-Contained-KnowledgeBase-Image":

(Step-1) It receives "Coral-Image-with-chart" including "Target-Coral" and "CoralChart" as a single-image (Self-Contained-Knowledge-Base-Image) is as Figure 7.

(Step-2) Coral-color analysis:

(Step-2-1) It searches for "Target-Coral" in "Coral-Image-with-chart", and describes horizontal and vertical lines for specifying "coral-pixel-id", as Figure 7.

(Step-2-2) it accesses to each coral-pixel, reads and makes the pixel values (ex, R, G, B or H, S, V) as a "coral-color-vector", 
(Step-2-3) It creates "Coral-color table" consisting of "coral-pixel-id" and "coralcolor-vector" for every coral-pixel

(Step-3) Chart-color analysis:

(Step-3-1) It searches for "Coral-Chart" in "Coral-Image-with-chart", and describes horizontal and vertical lines for specifying "chart-color" and its "chart-color vector" with meanings.

(Step-3-2) It accesses to each chart-color, reads and makes the chart-color values as a "chart-color-vector",

(Step-3-3) It creates "Chart-color table" consisting of "chart-color" and "chart-colorvector" with meanings for every chart-color

(Step-4) Semantic interpretation with "semantic-distances" between "coral-color vector" in Step-2-3 and each of "chart-color vectors" in Step-3-3

(Step-4-1) For each of "coral-pixel-id", it calculates distances between "coral-color vector" and each of "chart-color vectors" as "semantic-distances" of the "coral-pixel-id"

(Step-5) Ranking-calculation among "semantic-distances" as "semantic-ordering" (ascending-order or descending-order) (Step-5 assigns a "meaning" to each "coral-pixelid", as the result of semantic-interpretation for "Self-Contained-Knowledge-BaseImage")

(Step-5-1) For each of "coral-pixel-ids", it calculates a ranking of chart-colors among "semantic-distances", as "semantic-ordering" (ascending-order or descending-order), and extracts the corresponding meaning to the top-ranked "chart-color",

(Step-6) Aggregation Function for meanings of coral-pixels

(Step-6-1) It integrates all the calculation results of "semantic-distances" for each "coral-pixel-id" to each chart-color obtained in Step-4, as an integrated "coral-pixel-id \& chart-color table with semantic-distances".

(Step-6-2) For each chart-color in "coral-pixel-id \& chart-color table", it computes "max-value", "min-value" and "average-value" for all the semantic distances to every coral-pixel-id, and makes ranking of chart-colors (each of them has meanings) according to "max-value", "min-value" or "average-value", as the ranking of meanings interpreted for all the coral-pixel-ids. That is, the top-ranked meaning is interpreted as the meaning of the target-coral

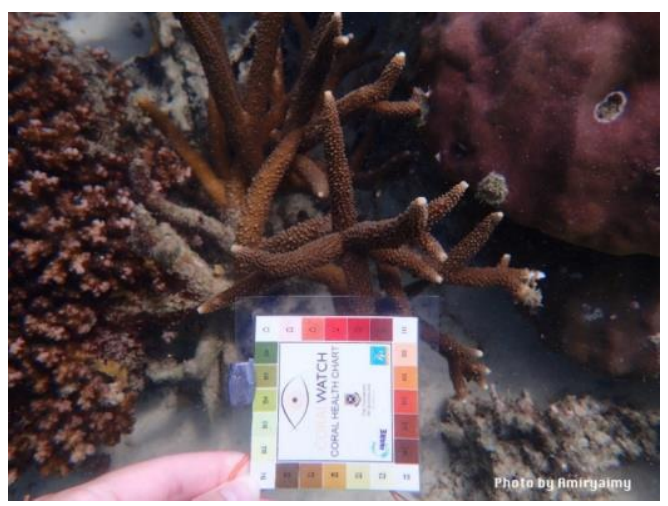

Figure 7. "Self-contained-Knowledge-Base-Image" \& "Contextual-Semantic-Interpretation" 


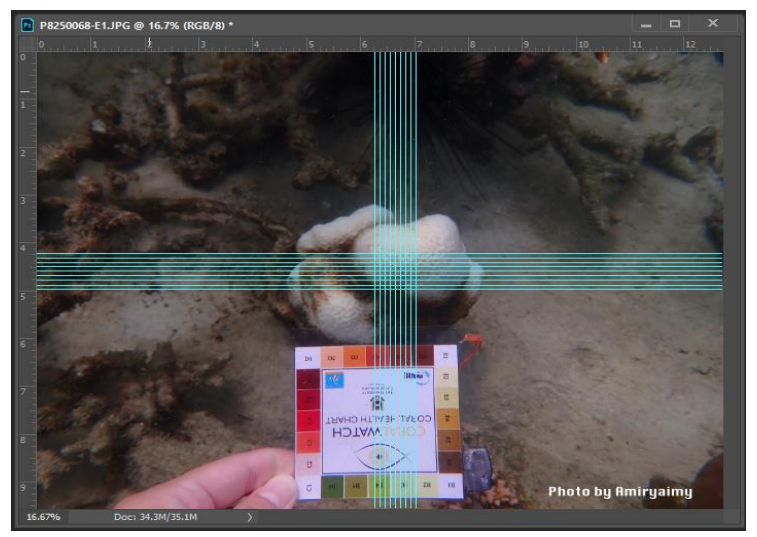

Figure 8. "Self-contained-Knowledge-Base-Image" \& "Contextual-Semantic-Interpretation" with extracting Coral-color and "Coral-Chart" pixels

The execution result of "Self-contained-Knowledge-Base-Image" \& "ContextualSemantic-Interpretation" with extracting coral-color and "coral-chart pixels" is shown in Fig. 9. This result gives the meaning of the coral health-level as "E2" (corresponding to "damaged-coral in health-level"), according to the distance ordering of "minimal value" between coral-color and the closed color, "Color-E2("damaged-coral in health-level") in the coral-chart. This method realizes the automatic interpretation of the health-level of Coral with the "Self-contained-Knowledge-Base-Image" \& "Contextual-SemanticInterpretation algorithm."

\begin{tabular}{clll}
\hline color & \multicolumn{1}{c}{ max-value } & \multicolumn{1}{c}{ “min-value" } & average-value \\
\hline E2 & 199.173291382153 & 5.3851648071345 & 92.8795932168451 \\
\hline B2 & 180.549162279973 & 8.30662386291807 & 78.8662052947991 \\
\hline B3 & 157.676884799263 & 14.7309198626562 & 68.5533171328608 \\
\hline B6 & 211.011848008589 & 16.0312195418814 & 115.848366433731 \\
\hline C2 & 189.886808388577 & 20.6639783197718 & 89.7862646632142 \\
\hline E3 & 149.435604860421 & 22.0907220343745 & 71.4354194997922 \\
\hline B5 & 179.724789609002 & 36.7287353444139 & 96.229974266913 \\
\hline C1 & 229.150605497782 & 36.8374809127877 & 125.545628555143 \\
\hline B1 & 219.64289198606 & 38.7556447501522 & 119.715940857096 \\
\hline D1 & 224.784341091634 & 39.6610640301039 & 123.489598143384 \\
\hline D2 & 152.558185621093 & 42.0119030752 & 83.2472631277141 \\
\hline E1 & 233.096546520964 & 42.8602379834737 & 130.364768299334 \\
\hline B4 & 136.97810043945 & 43.3820239269677 & 81.6315504391287 \\
\hline E6 & 240.459975879563 & 45.3872228716409 & 145.859388823329 \\
\hline E5 & 198.597583066864 & 56.4446631666803 & 117.369279848312 \\
\hline D6 & 249.713836220583 & 63.7573525171803 & 157.66160136726 \\
\hline C6 & 248.233760798164 & 67.705243519243 & 157.742461708311 \\
\hline E4 & 155.058053644433 & 69.9857128276908 & 103.590703682212 \\
\hline D5 & 232.036635038521 & 73.3552997403732 & 148.155810217487 \\
\hline C5 & 224.385828429516 & 90.27181176868 & 148.719759483493 \\
\hline D3 & 151.479371532892 & 98.9393753770459 & 123.909610409952 \\
\hline D4 & 209.461213593352 & 107.060730429042 & 146.596739253442 \\
\hline C3 & 188.430358488222 & 107.429046351534 & 137.932225797416 \\
\hline C4 & 222.117086240568 & 127.334205930692 & 164.516483142756 \\
\hline E & & &
\end{tabular}

Figure 9. “Contextual-Semantic-Interpretation" of "Self-contained-Knowledge-Base-Image" \& "Contextual-Semantic-Interpretation" with extracting Coral-color and "Coral-Chart" pixels

There was some limitation in this method by using 3-color-elements or RGB color model as misinterpretation in damaged coral. The damaged coral represents as white coral at an unhealthy level which is easily disturbed in coral color by environmental light 
underwater. Under this light situation, creating shadow, highlight, and reflection on either coral or chart or both. Therefore, leading to misinterpretation in the result of damaged coral.

By our new finding, the effectiveness of this method can be increased by partly adjusting in 3-color-element. HSV (hue, saturation, value) color model was applied instead of RGB color model. HSV is the most used in computer graphics researcher [1316] and is closer to humans perceive color than RGB. In order to convert RGB to HSV, it can manually calculate by using HSV conversion formula [17] or using online color converters which are optionally convenient.

The result of damaged coral shows as E2 at coral health-level in Fig 10. The result of RGB shows in Fig 11, the distance between coral-color and the closed color in coralchart is "Color-B6" according to minimal value while the result of HSV in Fig 12 shows "Color-E2" in minimal value. Then, applying HSV shows closer in the result than RGB, in case of misinterpretation in RGB. In summary, differences in the color system (RGB or HSV) can assuredly affect how closely the result.

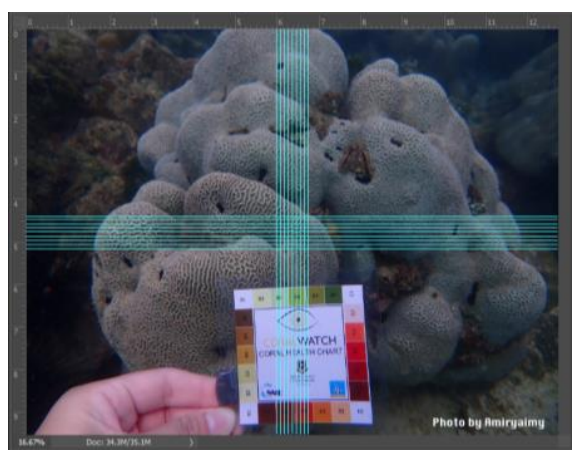

Figure 10. Damaged coral in coral health level at E2

\begin{tabular}{clll}
\hline color & \multicolumn{1}{c}{ max-value } & \multicolumn{1}{c}{ "min-value" } & average-value \\
\hline B6 & 143.436397054583 & 15.6843871413581 & 53.9530082764298 \\
\hline E6 & 113.141504320917 & 23.5372045918796 & 52.2947725655229 \\
\hline D2 & 179.783202774898 & 26.229754097208 & 105.050060069544 \\
\hline D6 & 155.206314304541 & 32.0312347560939 & 80.9509797660195 \\
\hline E3 & 194.887146831185 & 36.7695526217005 & 116.012734824335 \\
\hline B5 & 101.764433865668 & 41.1096095821889 & 60.5440201042771 \\
\hline E5 & 105.995282913911 & 44.1134900002256 & 66.7361943931025 \\
\hline C6 & 167.406690427832 & 50.0699510684802 & 97.4038646615865 \\
\hline E4 & 141.495582969929 & 50.1697119784437 & 85.572961931423 \\
\hline B4 & 152.072351201657 & 51.7397332811061 & 88.153219620497 \\
\hline C3 & 173.856262469892 & 54.4609952167604 & 127.88716462633 \\
\hline D5 & 149.361976419703 & 60.6382717431821 & 88.5311896581875 \\
\hline B3 & 226.192395981828 & 65.00769185258 & 146.684142614392 \\
\hline E2 & 229.322916430086 & 65.9545297913646 & 149.154457736076 \\
\hline D3 & 141.911944528993 & 70.3491293478462 & 107.061472382264 \\
\hline D4 & 142.323574997258 & 93.3005894943864 & 109.566790703382 \\
\hline C5 & 166.72432336045 & 93.8775798580257 & 115.962469146496 \\
\hline B2 & 258.149181676022 & 95.1104620953973 & 177.993696839244 \\
\hline C2 & 264.049237832644 & 103.213371226794 & 184.514200836705 \\
\hline D1 & 261.218299512113 & 107.694010975541 & 184.072133009289 \\
\hline C4 & 170.393661853955 & 116.172285851661 & 137.423837778023 \\
\hline E1 & 274.901800648886 & 118.110118110177 & 196.728808812628 \\
\hline C1 & 301.15776596329 & 142.256810030311 & 222.318845971243 \\
\hline B1 & 311.117341207461 & 151.297719744879 & 231.960252595281 \\
\hline & & &
\end{tabular}

Figure 11. The result of RGB color model 


\begin{tabular}{clll}
\hline color & \multicolumn{1}{c}{ max-value } & \multicolumn{1}{c}{ "min-value" } & average-value \\
\hline E2 & 17.5569615257933 & 2.10612650549263 & 6.31762959664565 \\
\hline B6 & 12.5808920997929 & 2.19201593116456 & 6.39178314184013 \\
\hline E6 & 19.2400953511202 & 2.59927273045414 & 12.7081295615523 \\
\hline C3 & 19.911280661172 & 2.67169663296457 & 9.83780652698295 \\
\hline E3 & 16.6462076525158 & 3.07620746204729 & 10.4944454715758 \\
\hline D2 & 18.614161557258 & 3.10368116171445 & 12.8329250384822 \\
\hline B3 & 12.8418180694141 & 3.19913668044159 & 7.34225025413962 \\
\hline B2 & 12.2167221280738 & 3.50272344440962 & 7.0180543996998 \\
\hline C2 & 14.5340397117224 & 3.66093731078246 & 5.9251992689127 \\
\hline D4 & 20.1898764369652 & 4.08300378180018 & 10.0474629296558 \\
\hline E5 & 18.0692860479916 & 4.45330238797583 & 12.4111880101652 \\
\hline E1 & 13.5860454292085 & 4.63171603361099 & 6.63766241940873 \\
\hline D5 & 19.833681264057 & 4.63542752720645 & 9.23234830474871 \\
\hline D1 & 13.3733878102211 & 4.77153983025864 & 6.63191130593785 \\
\hline B5 & 16.0475570072356 & 4.81755900775598 & 10.1462423314754 \\
\hline D6 & 18.948684513604 & 4.81893534182752 & 8.02499275731741 \\
\hline C1 & 13.5621368550884 & 5.03598786866439 & 7.04735755271535 \\
\hline E4 & 17.5214441479184 & 5.07105939170588 & 12.09928801166 \\
\hline B1 & 13.7793964600946 & 5.07241033263328 & 7.14802014322634 \\
\hline C4 & 20.5014728780713 & 5.23527274022288 & 11.1633815397469 \\
\hline B4 & 15.0575130567888 & 5.4354499776699 & 9.70072280774479 \\
\hline C5 & 20.4880430672202 & 6.01199655098454 & 10.9362854879886 \\
\hline C6 & 19.8877963039989 & 6.01212213159579 & 9.82187295447358 \\
\hline D3 & 19.0145659234063 & 6.96028363526647 & 13.7586714234836 \\
\hline & & &
\end{tabular}

Figure 12. The result of HSV color model

\section{Global and Environmental Coral-Analysis and Visualization in 5D World Map}

This section focuses on semantic computing for Coral analysis with a "SemanticInterpretation method". We realized the experimental system using SPA function in 5D World Map and applied the coral-image data. Coral-leaf analysis has been done in a lot of coral-leaf research projects, as discussed in [18-25]. One of the important applications of the semantic computing system is "Global Environment-Analysis," which aims to evaluate various influences caused by natural disasters in global environments. Our experimental results have shown the feasibility and effectiveness of our semantic associative computing system based on "MMM" in global environmental analysis. We have constructed "SPA-based Global-Environment-Analysis System" for sharing and analyzing environmental situations with MMM functions applied to "environmental multimedia data sharing," as a new platform of collaborative environment analysis [1-5]. This platform enables to create a remote, interactive and realtime environmental and academic research exchange among different areas. We visualized the important factor off water-quality for coral on 5DWM which it shown in Fig. 13 - Fig. 16. 


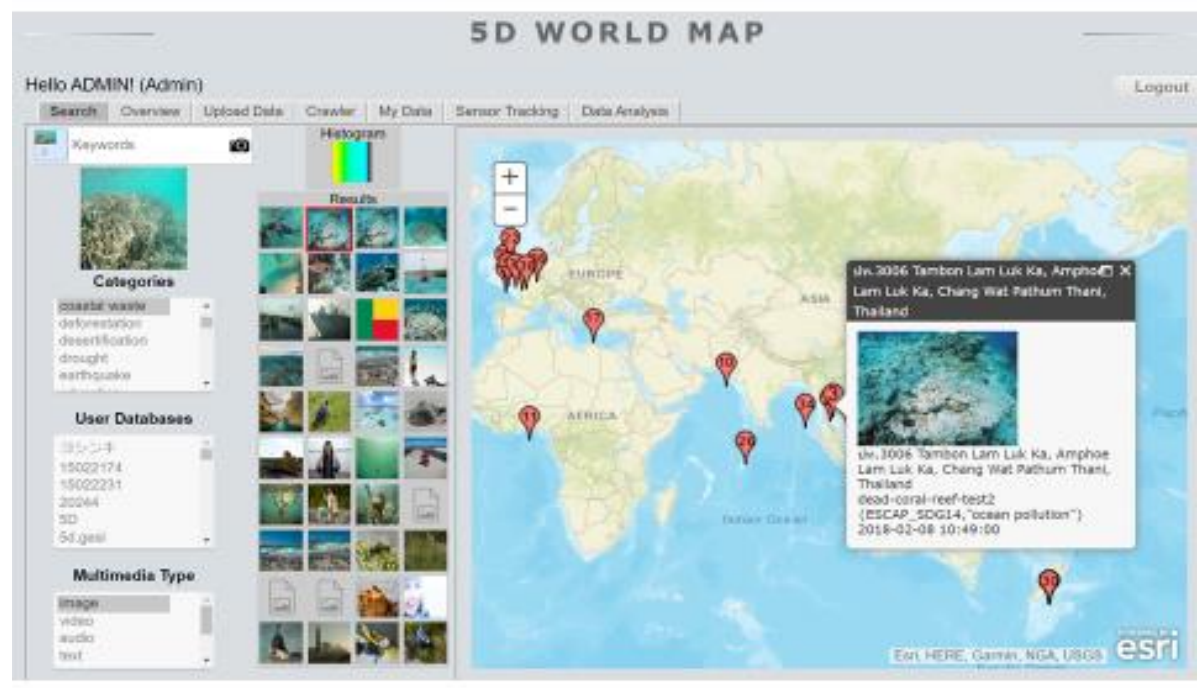

Input image $=$ dead coral, Categories $=$ coastal waste, SDG14, ocean pollution

Figure 13. Global Environmental Analysis of "Coral" in "5D World Map System: (a) Image search by color information, (b) Spatiotemporal analysis (global overview of geographical distribution and the time-series change), and (c) Example of image data collection

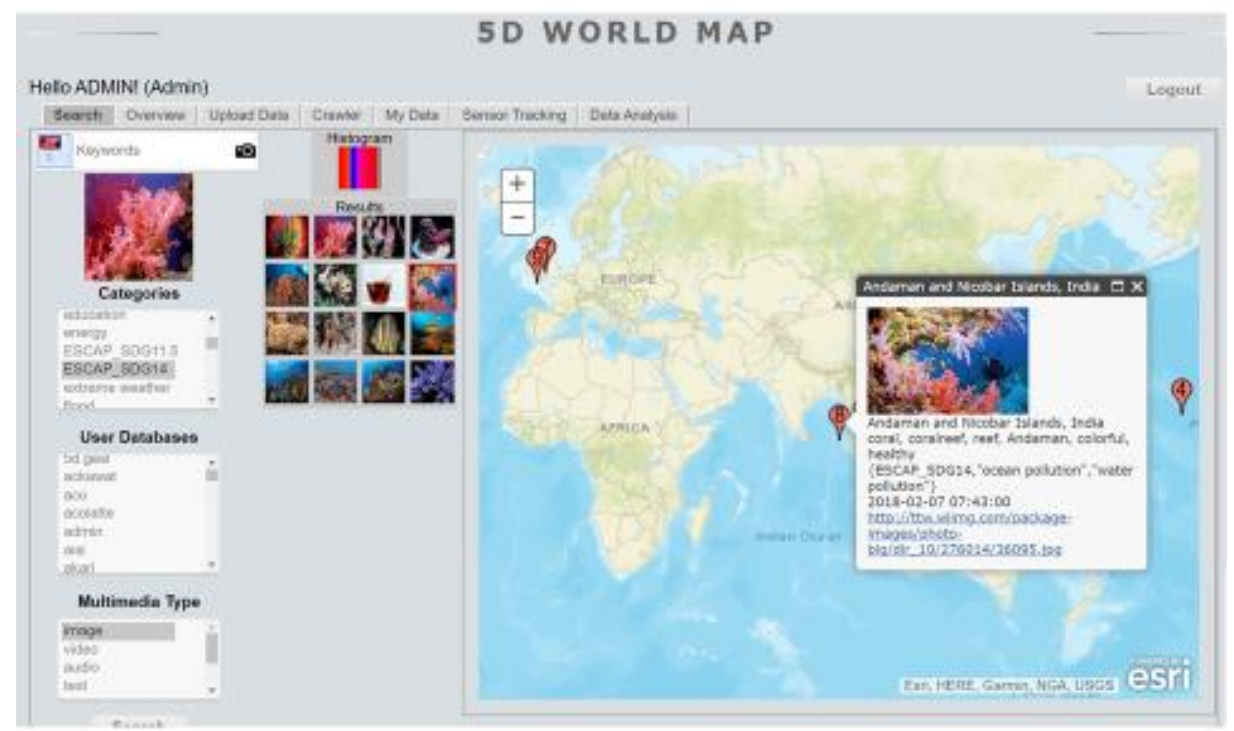

Input image $=$ colorful coral, Categories $=$ coastal waste, SDG14, ocean pollution

Figure 14. Global Environmental Analysis of "Coral" in "5D World Map System: (a) Image search by color information, (b) Spatiotemporal mapping in the global overview of geographical distribution 


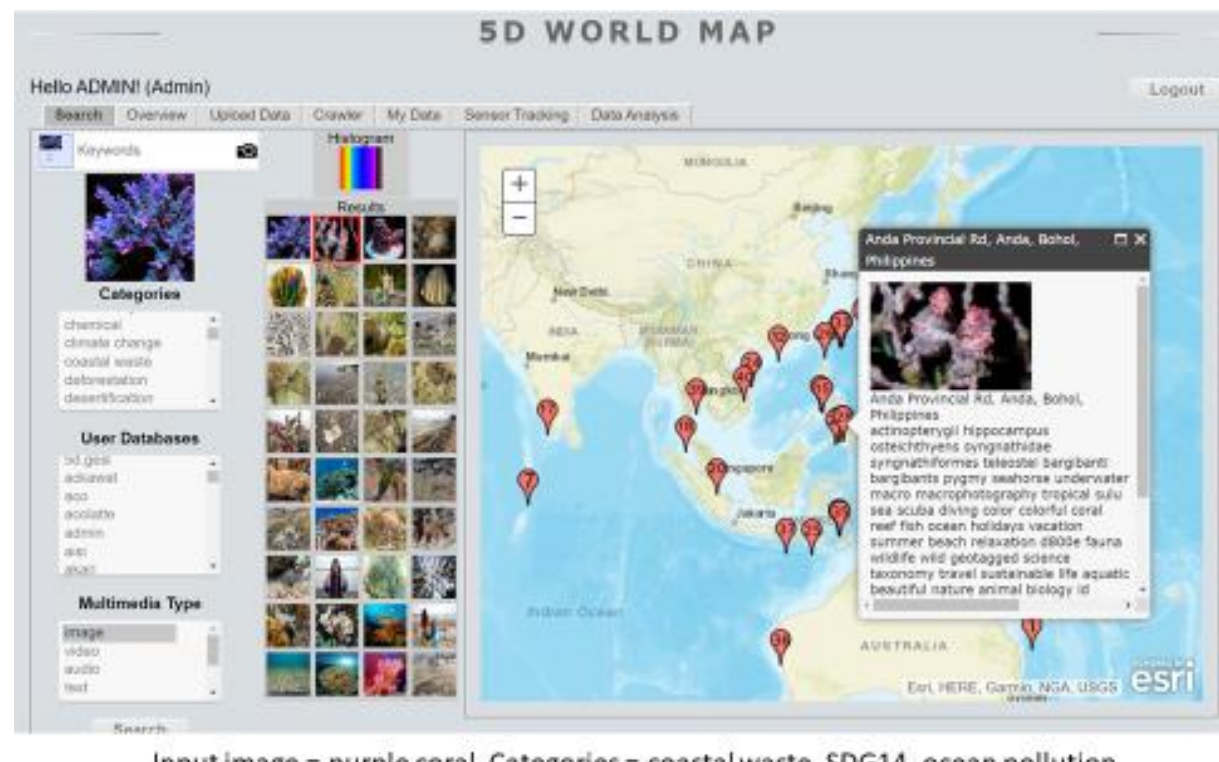

Input image $=$ purple coral, Categories $=$ coastal waste, SDG14, ocean pollution

Figure 15. Global Environmental Analysis of "Coral" in "5D World Map System: (a) Image search by color information, (b) Spatiotemporal analysis (global overview of geographical distribution

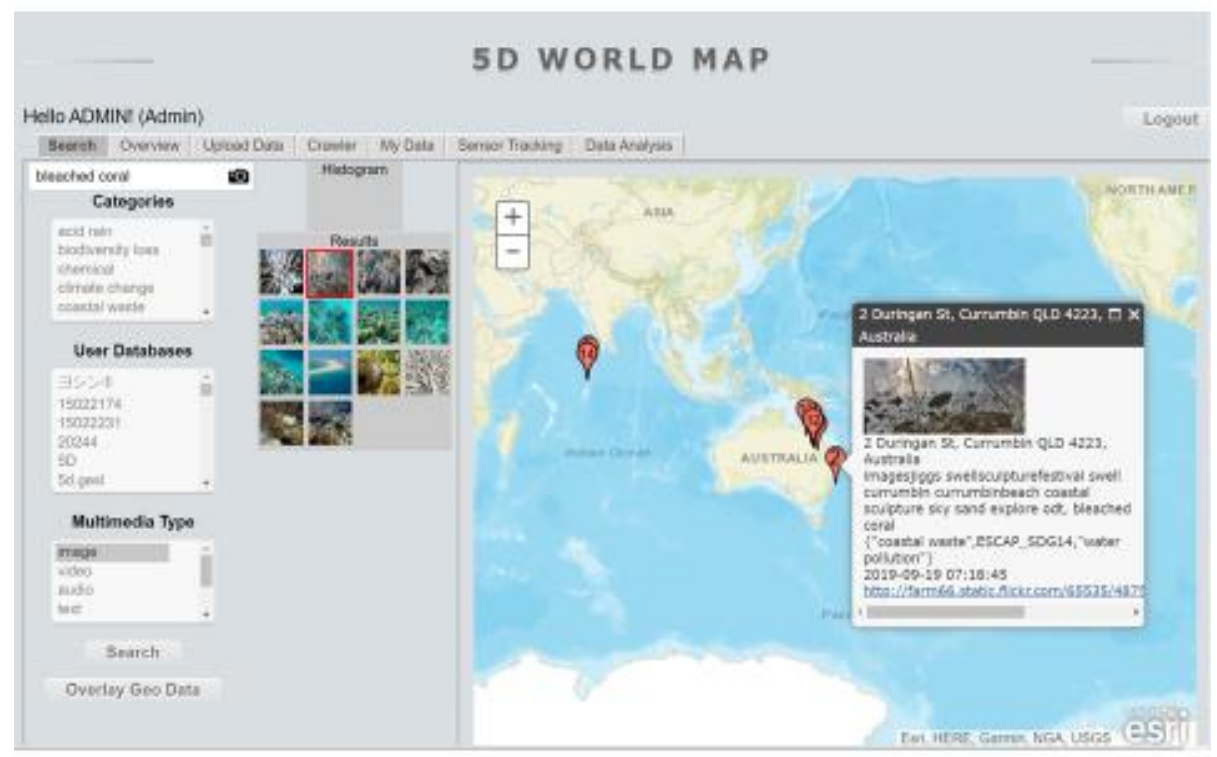

Input keyword = "bleached coral", Categories = all (no specification)

Figure 16. Global Environmental Analysis of "Coral" in " $5 D$ World Map System: (a) Image search by color information, (b) Spatiotemporal analysis (global overview of geographical distribution) 
We have realized several experiments of SPA Function in 5D World Map for SeaWater Quality Data in "Coral Areas" of "Si chang-Islands (http://www.arri.chula.ac.th/). We realized the experimental system using SPA function in 5D World Map and applied the collected seawater quality data from points Si chang-Islands [11-12]. The major issues of seawater quality in Si chang-Islands is an effect of seawater pollution to coral life. For the major issue, we integrate the following data sources from Si chang-Islands, and water quality data period from 2011 - 2015, which it collected from Aquatic Resources Research Institute, Chulalongkorn University, Thailand. We upload them to $5 \mathrm{D}$ as text data with spatial and temporal data. For the result of effect of seawater pollution to coral life, we found that temperature, $\mathrm{pH}$, Suspended Solid, Ammonia, Nitrate and Phosphorus are an important factor in term of an essential nutrient, quantities and equilibrium supported of coral life.

5D World Map memorizes these seawater quality data in combination of environmental coral-image data to make correlation analysis of Ocean environment in this area with context-oriented semantic computing.

\section{Conclusion}

We have presented a new environmental-semantic computing system for coral-analysis in water-quality and multi-spectral image spaces with "Multi-Dimensional World Map." The main feature of our system is to realize integration and semantic-search among environmental-semantic spaces with water-quality and image databases. This system is based on the concept of "Coral-Analysis space" for realizing global environmental analysis. In our system, semantic associative search is realized in the multiple dimensional orthogonal semantic space with semantic projection functions. This space is created for dynamically computing semantic equivalence or similarity between waterquality data resources. We have applied this system to Coral-image data analysis in the experiments in the Pacific Ocean Area.

This system realizes a "global-semantic analysis and observation for environmental issues" with the integration of remote, interactive and real-time environmental and academic research knowledge and information resources existing in our planet. We have also presented Environmental Multimedia Computing system and the 5D World Map System, as an international and environmental research platform with Spatio-temporal and semantic analysers."

As our future work, we will extend our multimedia computing system to new international and collaborative research and education for realizing mutual understanding and knowledge sharing on global environmental issues in the world-wide scope.

\section{Acknowledgment}

We would like to appreciate Dr. Shiori Sasaki, Dr. Chalisa Veesommai, Dr. Irene Erlyn Wina RACHMAWAN, and Ms. Jinmika Wijitdechakul for their significant experimental studies and discussions on environmental knowledge base creations in 5D World Map system. We also would like to thank Ms.Veranuch Chawakitchareon for coral pictures taken at Samaesarn using for coral health analysis in MMM. 


\section{References}

[1] Yasushi Kiyoki, Takashi Kitagawa, and Takanari Hayama. "A metadatabase system for semantic image search by a mathematical model of meaning of meaning," ACM SIGMOD Record, vol. 23, no. 4, pp. 3441, 1994.

[2] Yasushi Kiyoki, Takashi Kitagawa and Takanari Hayama. "A metadatabase system for semantic image search by a mathematical model of meaning of meaning," Multimedia Data Management -- using metadata to integrate and apply digital media--, McGrawHill(book), A. Sheth and W. Klas(editors), Chapter 7, 1998.

[3] Yasushi Kiyoki and Saeko Ishihara. "A Semantic Search Space Integration Method for Meta-level Knowledge Acquisition from Heterogeneous Databases," Information Modeling and Knowledge Bases (IOS Press), Vol. 14, pp.86-103, May 2002.

[4] Yasushi Kiyoki, Shiori Sasaki, Nhung Nguyen Trang and Nguyen Thi Ngoc Diep. "Cross-cultural Multimedia Computing with Impression-based Semantic Spaces," Conceptual Modelling and Its Theoretical Foundations, Lecture Notes in Computer Science, Springer, pp.316-328, March 2012.

[5] Yasushi Kiyoki. “A "Kansei: Multimedia Computing System for Environmental Analysis and CrossCultural Communication," 7th IEEE International Conference on Semantic Computing, keynote speech, Sept. 2013.

[6] Shiori Sasaki, Yusuke Takahashi and Yasushi Kiyoki. "The 4D World Map System with Semantic and Spatiotemporal Analyzers," Information Modelling and Knowledge Bases, Vol.XXI, IOS Press, 18 pages, 2010.

[7] Totok Suhardijanto, Yasushi Kiyoki and Ali Ridho Barakbah. "A Term-based Cross-Cultural Computing System for Cultural Semantics Analysis with Phonological-Semantic Vector Spaces," Information Modelling and Knowledge Bases XXIII, IOS Press, pp. 20-38, 2012.

[8] Yasushi Kiyoki, Xing Chen, Shiori Sasaki and Chawan Koopipat. "Multi-Dimensional Semantic Computing with Spatial-Temporal and Semantic Axes for Multi-spectrum Images in Environment Analysis," Information Modelling and Knowledge Bases XXVII, IOS Press, Vol. XXVI, 20 pages, March 2016.

[9] Chalisa Veesommai, Yasushi Kiyoki, Shiori Sasaki and Petchporn Chawakitchareon. "Wide-Area RiverWater Quality Analysis and Visualization with 5D World Map System" Information Modelling and Knowledge Bases XXVII, IOS Press, pp.31-41, 2016.

[10] Khoumkham Ladsavong, Petchporn Chawakitchareon, Yasushi Kiyoki and Shiori Sasaki. "Preliminary Visualization of Surface Water Quality by 5D World Map System” International Transaction Journal of Engineering, Management, \& Applied Sciences \& Technologies," Vol 9, No.1 ISSN 2228-9860 eISSN 1906-9642, pp. 49-58, 2018. Available online: http://TUENGR.COM/V09/049.pdf.

[11] Petchporn Chawakitchareon, Khoumkham Ladsavong, Yasushi Kiyoki, Shiori Sasaki and Sompop Rungsupa. "Global Sharing Analysis and Visualization of Water Quality by 5D World Map: A Case Study at Sichang Island, Thailand,", Information Modelling and Knowledge Base XXIX, IOS Press, Vol. 301, pp. 216-227, 2018.

[12] Jinmika Wijitdechakul, Yasushi Kiyoki and Chawan Koopipat. "An environmental-semantic computing system of multispectral imagery for coral health monitoring and analysis," Information Modelling and Knowledge Bases XXX, Vol. 312, pp. 293 - 311, IOS Press, 2019.

[13] Poonthalir Ganesan, V. Rajini, B. S. Sathish, Khamar Basha Shaik. "HSV color space based segmentation of region of interest in satellite images," International conference on control, instrumentation, communication and computational technologies, IEEE, pp. 101-105, 2014.

[14] Chunxue Liu, Xiaobo Lu, Saiping Ji and Wei Geng. "A fog level detection method based on image HSV color histogram," International conference on progress in informatics and computing (PIC), IEEE, pp. 373-377, 2014.

[15] Ankit Chaudhary and Ankur Gupta. “Automated switching system for skin pixel segmentation in varied lighting," 19th International conference mechatronics and machine vision in practice, IEEE, pp. 26-31, 2012.

[16] George Paschos. "Perceptually uniform color spaces for color texture analysis: an empirical evaluation," IEEE Trans Image Process, 10 (6), pp. 932-937, 2001.

[17] Alvy Ray Smith. "Color gamut transform pairs. ACM siggraph computer graphics,” ACM, Vol. 12, pp. 12-19, 1978.

[18] Yasushi Kiyoki, Xing Chen, Chalisa Veesommai, Shiori Sasaki, Asako Uraki, Chawan Koopipat, Petchporn Chawakitchareon and Aran Hansuebsai. "An Environmental-Semantic Computing System for Coral-Analysis in Water-Quality and Multi-Spectral Image Spaces with "Multi-Dimensional World Map," Information Modelling and Knowledge Bases XXIX, IOS Press, 2018.

[19] Toby N. Carlson and David A. Ripley. "On the relation between NDVI, fractional vegetation cover, and leaf area index," Remote Sensing of the Environment 62(3):241-252, 1997. 
[20] McFeeters, S. K. "The use of Normalized Difference Water Index (NDWI) in the delineation of open water features," International Journal of Remote Sensing, 17(7):1425-1432, 1996.

[21] Chou, L. M., Sudara, S., Manthachitra, V., Moordee, R., Snidvongs, A., and Yeemin, Y. "Temporal variation in a coral reef community at Pattaya Bay, Gulf of Thailand," Environmental Monitoring and Assessment 19:295-307, 1991.

[22] Katharina Fabricius, Glenn Death, Laurence Mccook, Emre Turak and David M. Williams. "Changes in algal, coral and fish assemblages along water quality gradients on the inshore Great Barrier Reef," Marine Pollution Bulletin 51:384-398, 2005.

[23] Eric J. Hochberg, Marlin J. Atkinson, Amy Apprill and Serge Andrefouet. "Spectral reflectance of coral," Coral Reefs, 23: 84-95, 2004.

[24] Heather M. Holden and Ellsworth F. LeDrew. "Spectral Discrimination of Healthy and Non- Healthy Corals Based on Cluster Analysis, Principal Components Analysis, and Derivative Spectroscopy, and Derivative Spectroscopy," Remote Sensing of Environment, Vol. 65, pp. 217-224, 1998.

[25] Ian Leiper, Stuart Phinn, and Arnold G. Dekker. "Spectral reflectance of coral reef benthos and substrate assemblages on Heron Reef, Australia," International Journal of Remote Sensing, Vol. 33, No. 12, pp. 3946-3965, 2012.

[26] Mahshid Oladi, Mohammad Reza Shokri and Hassan Rajabi-Maham. "Application of the coral health chart to determine bleaching status of Acropora downingi in a subtropical coral reef," Ocean Science Journal, Vol. 52, pp. 267-275, 2017. 


\title{
Recognizing Human-Object Interaction in Multi-Camera Environments
}

\author{
Marina TROPMANN-FRICK ${ }^{\mathrm{a}, 1}$, Thien Phuc TRAN ${ }^{\mathrm{b}}$ \\ ${ }^{a}$ University of Applied Sciences Hamburg, Berliner Tor 7, 20099 Hamburg, Germany. \\ marina.tropmann-frick@haw-hamburg.de \\ ${ }^{\mathrm{b}}$ thienphuc.tran@haw-hamburg.de
}

\begin{abstract}
This work introduces Multi-Fusion Network for human-object interaction detection with multiple cameras. We present a concept and implementation of the architecture for a beverage refrigerator with multiple cameras as proofof-concept. We also introduce an effective approach for minimizing the required amount of training data for the network as well as reducing the risk of overfitting, especially when dealing with a small data set that is commonly recorded by a person or small organization.

The model achieved high test accuracy and comparable results in a real-world scenario at the Event Solutions in Hamburg 2019. Multi-Fusion Network is easy to scale due to shared learnable parameters. It is also lightweight, hence suitable to run on small devices with average computation capability. Furthermore, it can be used for smart home applications, gaming experiences, or mixed reality applications.
\end{abstract}

Keywords. Deep learning, computer vision, human-object interaction detection

\section{Introduction}

Nowadays, in the era of Internet-of-Things, computers or computational units can be used on many devices or things, also for personal usage. Computers cannot anticipate or recognize human activities without users giving them a hint or somehow telling them about their intent. Human-object interaction detection tries to make computers understand the visual world by learning how humans and objects in the real-world are interacting with each other. There is no common approach to tackle this problem yet, though almost all state-of-the-art methods are deep learning models trained in supervised manner.

There has been a big hype around deep learning in the last decade, especially in the area of computer vision. Many solutions in this area are shifting from statistical approaches to neural networks because of the enormous advantages of convolutional neural networks. State-of-the-art methods for human-object-interaction detection such as HAKE [3] and InteractNet [4] are using convolutional neural networks with special architecture as well. Besides, HAKE and InteractNet integrate human action embedding and region proposal networks, see Figure 1. Such complex approaches require high computational capability.

\footnotetext{
${ }^{1}$ Corresponding Author: Marina Tropmann-Frick, University of Applied Sciences Hamburg, Berliner Tor 7, 20099 Hamburg, Germany; E-mail: marina.tropmann-frick@haw-hamburg.de.
} 


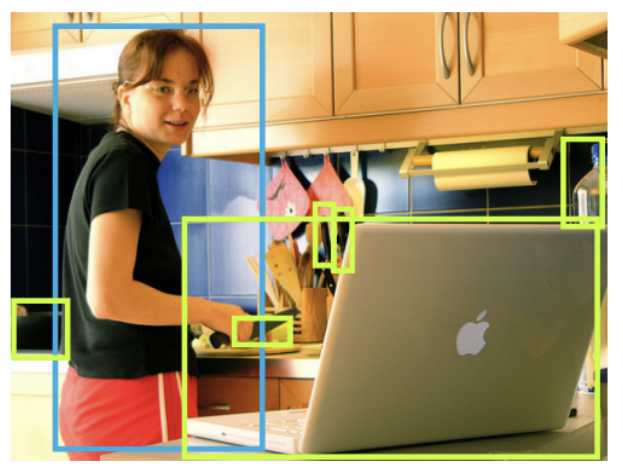

Figure 1. Region proposal network [4]

Therefore, they are mostly inappropriate for real-world use cases. For instance, when there can be only one person interacting with the system at once and only some human body parts are to be seen.

A monitoring system that uses a single camera could struggle to work reliably because of the dead zones, blind spots of the camera setup or occlusions that occur, when an object is hidden behind another object, see Figure 2. For that reason, many systems use multiple cameras to be able to cover the monitoring area fully.

However, an autonomous monitoring system using multiple cameras from different view angles and positions encounters other difficulties. Namely, additional computational burden and the necessity for merging observed information from all cameras into a final one stream. Development of such algorithms is highly difficult and time consuming. Furthermore, physical and mathematical approaches to solve this problem may strongly depend on cameras relative positions and hence require camera calibration or strict camera installation procedures, which may lead to further problems.

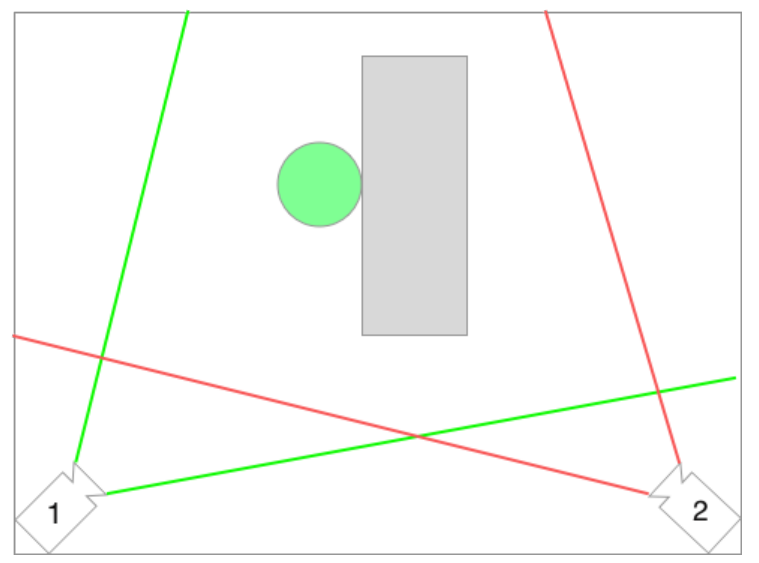

Figure 2. The occlusion problem: Only the camera 1 can see the object

Human perception enables us to understand actions regardless of view angles. Because we have been seeing and learning them since we were born. If a person cannot 
tell which action is being performed, he or she would try to move around and to look at the action from different view angles. In consideration of this intuition, deep learning is well-suited for solving this kind of problems without requiring any calibration or having to follow any installation rule.

The main contributions of this work are:

1. We introduce an architecture for human-object interaction detection: MultiFusion Network, which is simpler than state-of-the-art methods but is better suited for simpler use cases: when there is only one single subject to be observed. More importantly, the architecture supports both, single and multi-camera environments.

2. We show the implementation process for a beverage refrigerator with multiple cameras as a proof-of-concept. The cameras in the refrigerator should recognize in real-time, what the user is doing with objects inside the fridge and which object is being interacted with.

\section{Multi-Fusion network}

Multi-Fusion network follows the divide-and-conquer principle. It firstly divides the task of recognizing human-object interaction into two smaller ones: action recognition and object classification. This approach helps to use the data set more effectively and makes the models intuitive and more simple to construct.

Action recognition with multiple cameras is broken down into movement recognition with single-camera subtasks, the recognized movements from all cameras are then aggregated, forming a final action. The same approach is applied for object classification.

\subsection{Architecture}

The architecture of the Multi-Fusion network consists of two sub-models, see Figure 3. The action classification model is responsible for predictions on each video, the object classification model is responsible for prediction on every single video frame instead.

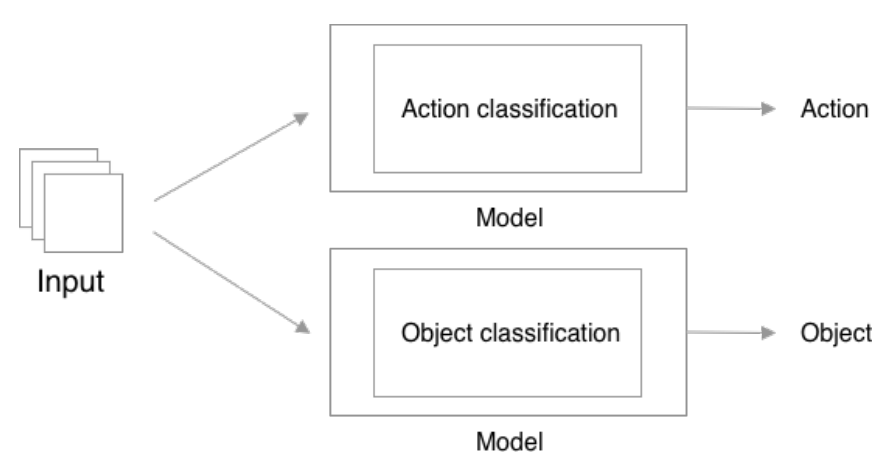

Figure 3. Illustration of using two sub-models 
Each pair of action-object should be treated as a single class, however, we lose the ability of generalization by doing so. The separation of action and object not only increases generalization ability, but also helps to use data efficiently, since using actionobject pair could make the number of classes explode rapidly.

This approach still encounters another issue when building the data set. Not all video frames of, e.g., "taking an apple" can be used for object class "apple", because there are also moments at which the hand is not holding anything when it is visible or even moves. Some pre-processing techniques can solve this problem, which will be discussed in a later chapter.

\subsection{Temporal fusion}

In order to detect the performed movements as actions we use the concept of temporal fusion in our approach. The temporal fusion block can be simply a fully connected layer or a more complex block that consumes an image sequence and produces an encoded information. Many scientific papers have proposed various approaches for video understanding such as 3D Convolutional Network, Inflated 3D Convolutional Network [5], CNN-RNN and especially Temporal Relation Network (TRN) [6].

We applied the idea of TRN for temporal fusion block (see Figure 4), which can learn to reason relations between changes of entities along the time axis. Rather than using optical flow to learn movement patterns, it only recognizes state changes in time and anticipates what is happening or reasons what happened.

Spatial information can contribute a lot to many action recognition tasks. However, actions in this concrete use case of the beverage refrigerator can only be distinguished primarily using temporal information, namely, hand movements and changes in the presence of an object. Therefore, this use case can evoke the potential of TRN, helping to achieve good performance with low computational costs. Although TRN can be imple-

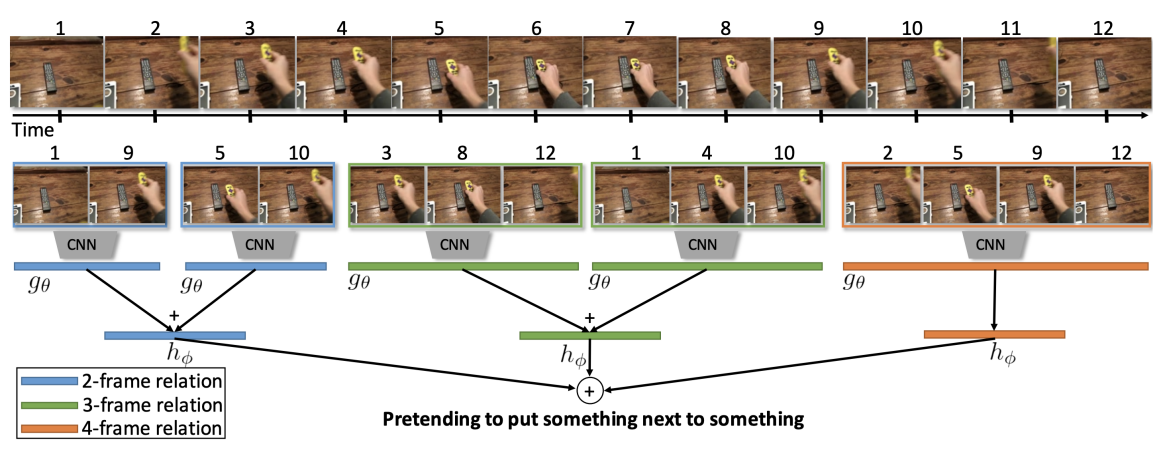

Figure 4. How Temporal Relation Network works [6]

mented with multi-scale time relations, in our approach single scale time relation is used. The reason for that is the fact that multi-scale time relations bring no significant gain for this use case after some experiments.

Instead of random sampling, as in the original paper, frames are sampled so that they are equally distributed across the time axis. This type of sampling ensures that the representative positions of movement are completely captured, because every hand position is 
crucial for understanding actions. In this case, a skipped snippet can also lead to a change in the meaning of the action.

After extracting information from sampled frames, the model needs to perform temporal fusion to connect information from many positions in time. In this work, the acquired information is fed into a GRU (Gated Recurrent Unit), which works as an encoder. The final hidden state of the GRU is then passed for further processing. This approach is introduced in the paper "Temporal Reasoning in Videos using Convolutional Gated Recurrent Units" [7].

\subsection{View fusion}

Due to the limitations of camera perspectives, a camera may not always be able to see an object, leading to wrong classifications. Multiple cameras can co-operate with each other to produce the best result. The main idea of multi-view fusion is to find a consensus among all the cameras, see Figure 5. The ideal mapping of temporal fusion block (before

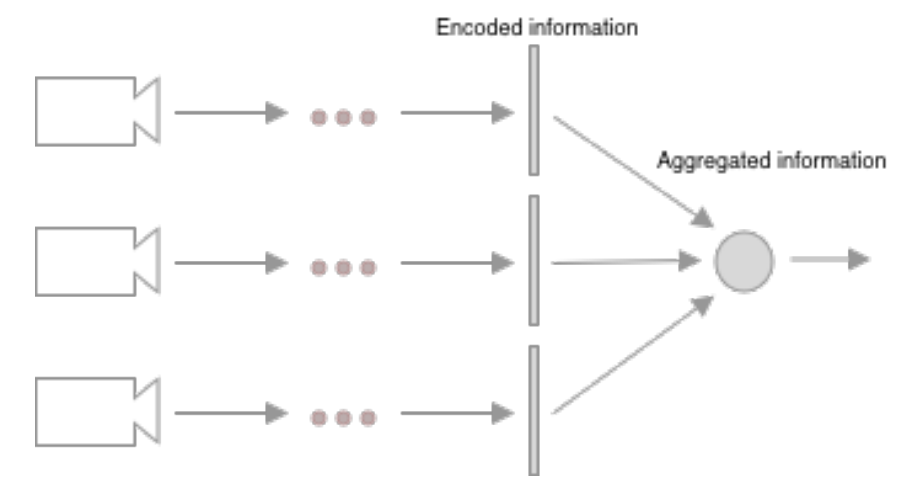

Figure 5. The illustration of the view fusion

the view fusion) is signal strength of informative movement patterns that each individual camera can observe despite their different view angles so that combining that information from all cameras can help to make the best decision. Therefore, instead of using another fully connected layer to merge all into one, the mean values of these signals from all cameras are calculated.

\subsection{Architecture overview}

The pre-processing blocks contain pre-processing operations such as inactivity removal, background subtraction, and density-based cropping, which clean the input and prepare it for further processing, as we will show in a later chapter. Video frames from the preprocessed input are then sampled and fed into the feature extractor. The extracted visual features are fused together by an encoder in the temporal fusion step. The encoded information from different cameras is aggregated into one, which is then finally passed to the classifier. The overview over the architecture is shown in the Figure 6.

Simultaneously, with action recognition, all pre-processed frames are also passed to the object classifier. The object classifier performs prediction on every frame that it receives from the pre-processing block. All object predictions, therefore, also need to reach a con- 


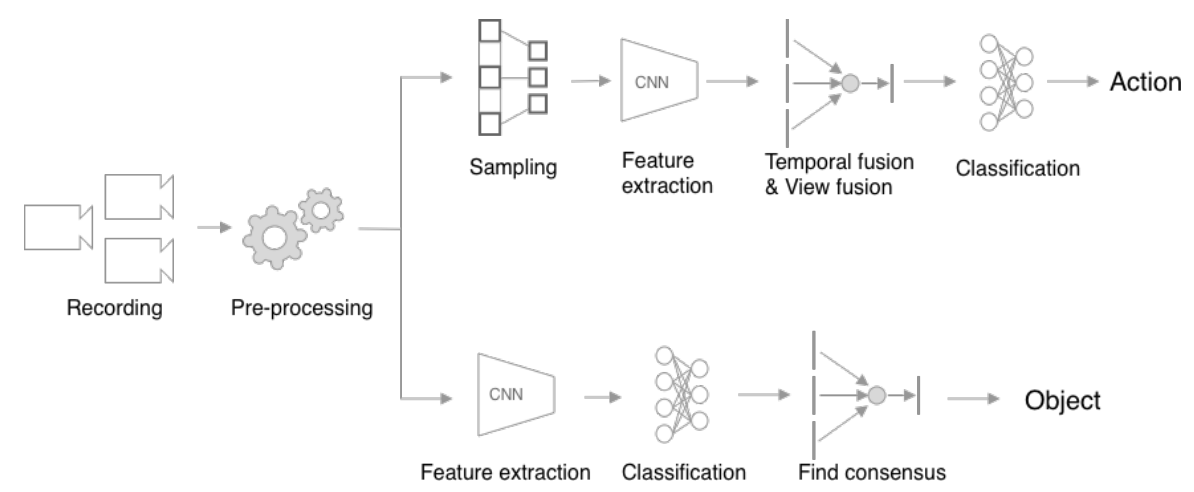

Figure 6. Architecture overview

sensus. Different policies can be applied to this action. The most frequently classified object (with a high confidence score) is chosen for this work.

\subsection{Shared weights}

Despite multiple input sources, a single feature extractor with shared weights is used for extracting visual features in each frame, so that it can learn to see an object from different points of view and also keep the number of parameters as low as possible.

The extracted features are then passed to corresponding temporal fusion blocks to recognize movement patterns. The GRU cell in temporal fusion block is shared since it should also learn to see actions from different angles. The hidden state of the GRU cell is reset after completing a computation task for a single camera.

All learnable parameters in feature extraction and temporal fusion stage are shared; only a mean calculation is performed in view fusion stage. Furthermore, the Multi-Fusion network architecture is scalable because increasing the number of cameras does not require an increase in the amount of parameters.

\section{Data set}

The data set for the beverage refrigerator contains seven object classes and three actions, captured by MQ013CG-E2 cameras (1.3 Megapixel) with wide-angle lenses at 20 FPS, see Figure 7. Some of the chosen objects possess different shapes and colors, which helps the model to distinguish between them easily. However, some pairs have the same shape or color in order to find out how well the model performs. An object can be taken or put, or nothing can also be done with the object (in each video sequence), see Table 1.

Four cameras are used to capture actions from different angles, preventing objects from being fully covered by hands or other objects. Two cameras are mounted at the front top left and right corners, pointing inwards. If an object is being taken by the left hand, the left camera may not be able to see that object, but the other front camera will. The third camera is mounted at the rear top left corner, pointing outwards, and the fourth is mounted on top of the refrigerator in order to provide more information.

No camera is mounted at the top center position (pointing downwards), because it would only be able to see bottle caps, and the model may have no chance to classify when 
M. Tropmann-Frick, T. P. Tran / Recognizing human-object interaction in multi-camera environments

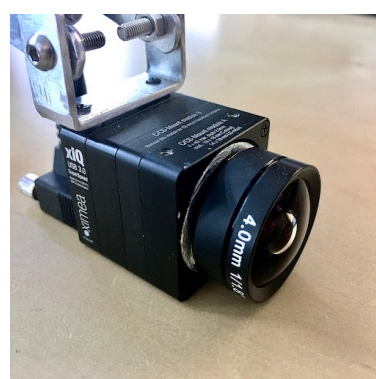

Figure 7. The camera and lens used to record the dataset

\begin{tabular}{|c|c|}
\hline Objects & Actions \\
\hline Apple & \\
Banana & \\
Lemonaid+ Blutorange (Bottle) & Nothing \\
Lemonaid+ Limette (Bottle) & Take \\
Club-Mate (Bottle) & Put \\
Granini Orange (Bottle) & \\
Veltins beer (Can) & \\
\hline
\end{tabular}

Table 1. Objects and actions in the dataset

working with low-resolution images correctly.

In the beginning, each camera recorded 1818 video frames. However, the model trained
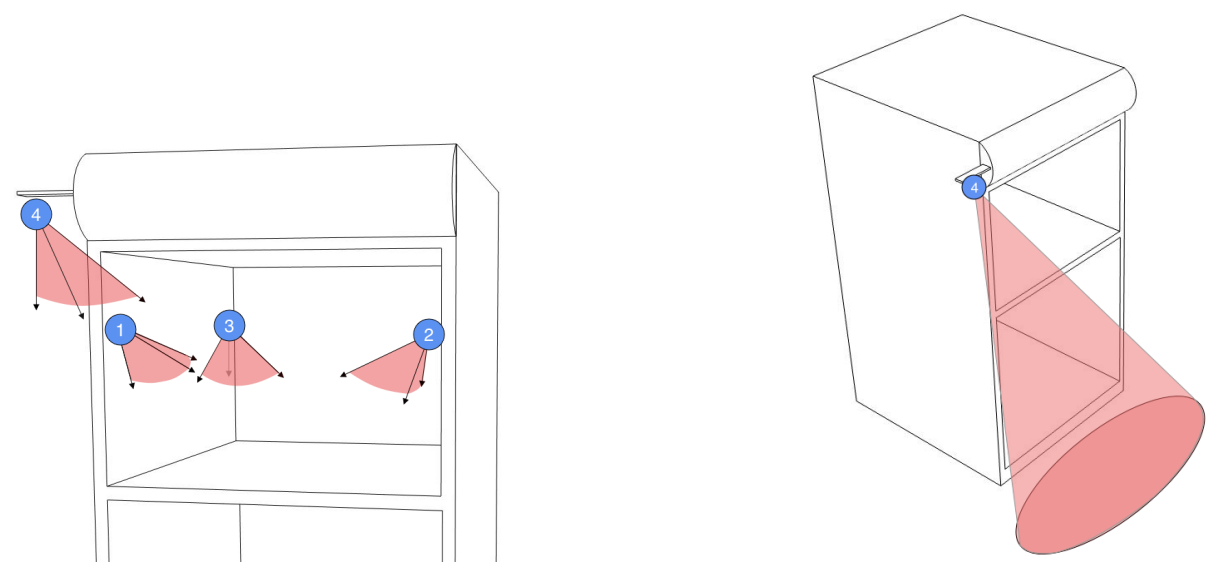

Figure 8. Camera positions

with those videos was strongly overfitted regardless of how hyperparameters were configured. The model performed very well during training; accuracy increased up to $99 \%$, but made random guess during the validation phase. After some investigations, it turned out to be caused by the lack of diversity in the data set.

Only four persons participated in the recording phase. Although there was an effort of changing clothes as well as shuffling object positions frequently, it was still insufficient 
for the model to generalize. Besides, the background in the third and fourth cameras rarely changes. The evaluation was performed on a model with base architecture, i.e.,
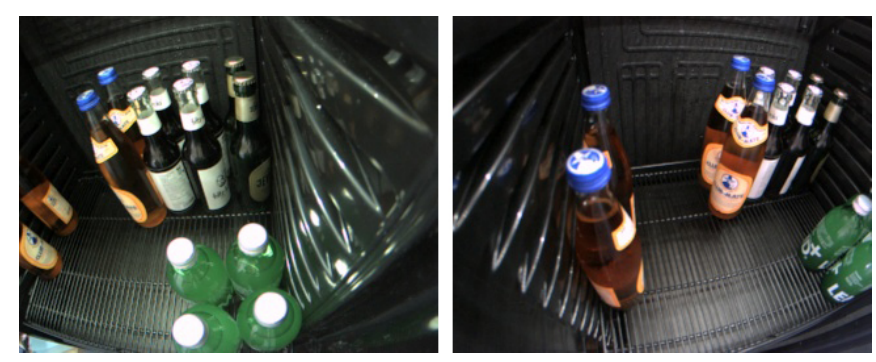

Figure 9. Images captured by camera 1 and 2
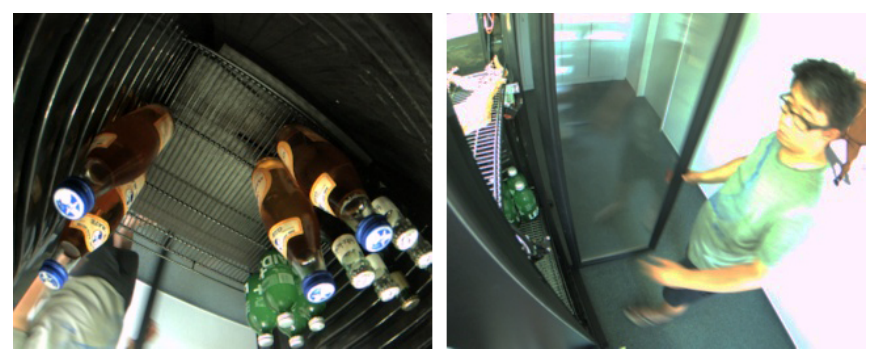

Figure 10. Images captured by camera 3 and 4

without additional pre-processing blocks. Due to the overfitting problem, the third and fourth cameras were removed. The model did show significant improvement by making reasonable classifications. Accuracy increased slowly over time and stopped at about $50 \%$ and about $30 \%$ for action classifier and object classifier, respectively.

\section{Preprocessing techniques}

The data sets were recorded several times, each time with minor changes in the camera's field of view. At first, various models were trained with different numbers of segments. The fact that the data sets are small and were recorded by four persons on the same day at the same location led to overfitting of all models, despite many regularization techniques such as reducing the number of hidden units, dropout, and adding regularization terms. The root cause of this overfitting problem is highly likely to be the lack of variance among samples in the data set. Background, surrounding objects, and clothing were infrequently changed. Since it is very labor-intensive to record such a data set that has many different backgrounds and users, some techniques should be applied in order not only to overcome overfitting by ignoring irrelevant information in video frames, but also to keep the amount of training data needed as small as possible. 


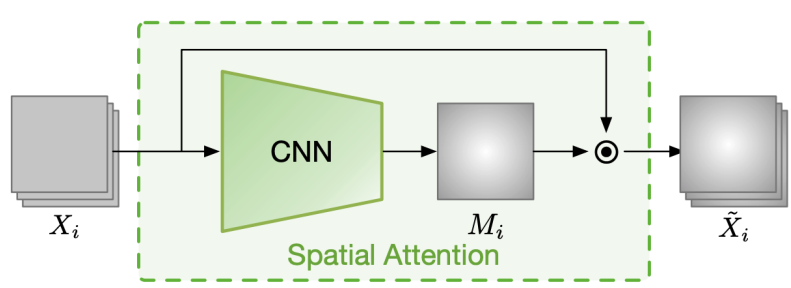

Figure 11. Spatial attention block [1]

\subsection{Finding the region of interest}

The fundamental idea of attention in video action recognition is that humans only need to look at parts of a video at each instance of time in order to understand which action is being taken. The ability to know where and when to pay attention to grasp the happening has been acquired since humans were born. Therefore, attention is nowadays widely applied in many machine learning fields in general and in action recognition tasks in particular. This work only makes use of spatial attention (where to look), see Figure 11; temporal attention is neglected to let the action classifier learn to understand the video by reasoning action sequences.

\section{Spatial attention}

A soft attention mechanism [1] was inserted before the feature extraction model and is responsible for blacking-out irrelevant parts of input to minimize their impacts on prediction. The spatial attention block consists of a convolutional network with the 'same' padding in all layers that learns to produce an importance mask for each input image, which is then multiplied element-wise with the original input image. Same padding means the width and height of output will be the same as those of input image. This block is designed to be plugged-in in any existing network easily. The input image will be fed to the spatial attention block, whose output will be then passed to the classifier.

However, the spatial attention block failed to produce reasonable importance masks in practice, and the training loss did not converge. Insufficient variation in images could be again the main reason for this failure.

\section{Background subtraction as an attention mechanism}

Background subtraction is a technique used for segmentation of the foreground objects from the background. In other words, background subtraction detects moving objects and is mostly used for traffic monitoring tasks such as detecting and tracking vehicles, pedestrians, etc.

Traditional methods, e.g., Frame Differencing, produce good results when the camera is stationary. Otherwise, every pixel in the image would change, and the background estimation algorithm fails. Many deep learning approaches developed in the last few years are, however, computationally too expensive for being used in a real-time application due to their convolutional encoder-decoder architecture.

Fortunately, in our approach, all of the cameras are stationarily mounted in the refrigerator in this specified use case. Thus, traditional algorithms could be applied to generate 


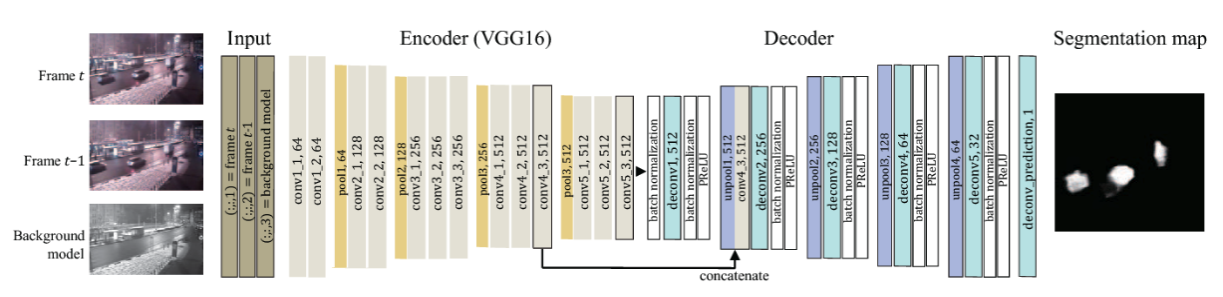

Figure 12. Encoder-decoder architecture for foreground detection [8]

foreground masks, which effectively works as a spatial attention mechanism.

Andrews Sobral implemented various algorithms and introduced BGSLibrary for foreground detection and background estimation in his work [9]. Although the library was written in $\mathrm{C}++$, BGSLibrary can also be used in Python, Java, and MATLAB with specific wrappers.

BGSLibrary supports different OpenCV versions, but the number of available algorithms does differ at the time of this experiment. BGSLibrary compiled with OpenCV 3, OpenCV 4 has 41 and 15 algorithms, respectively. Therefore, BGSLibrary used for this work was compiled with OpenCV 3.4.1 on Ubuntu 16.04 LTS.

41 available algorithms were benchmarked to find which is the best-suited candidate for this problem. Benchmarking criteria are computational complexity, noise level in the mask, intersection over union (Jaccard-index) of the detected moving object and ground truth.

According to the criteria mentioned above, the algorithms fall into three main groups (see Figure 13):

1. Fast, low mask quality

2. Slow, high mask quality

3. Average speed, average mask quality

Frame Differencing, SuBSENSE [11], and Local Binary Pattern with Markov Random Field (LBP-MRF) [10] are the representative algorithms for group 1, 2, and 3, respectively.

There is also a trade-off between speed and quality. Slow algorithms are not suitable for real-time requirements. Fast algorithms produce low-quality masks with a high noise level, which makes the relevant features in images barely recognizable. Eventually, LBPMRF is selected due to its sufficient speed to fulfill the real-time requirements, and due to its more than acceptable mask quality. More precisely, the density of positive pixels in the area of the moving objects is here much higher than that in the background area. The density difference between those two areas plays an important role in enhancing input quality, which will be further discussed in the next section.

\subsection{Zooming to the region of interest}

Input video frames usually contain black regions after multiplication with their masks. The average percentage of area that contains a moving object in the recorded data set is only about $10 \%$, i.e., $90 \%$ of data the neural network processes contains no information. Furthermore, most convolutional neural networks for extracting features are built in such a way that small, simple patterns in small areas are detected by the first filters; 
M. Tropmann-Frick, T. P. Tran / Recognizing human-object interaction in multi-camera environments

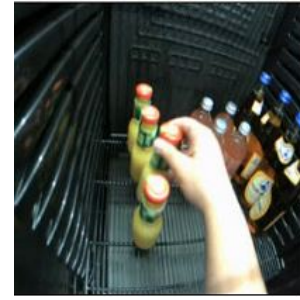

(a) Input image

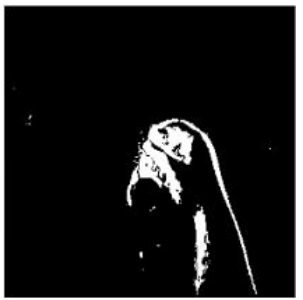

(b) Low quality

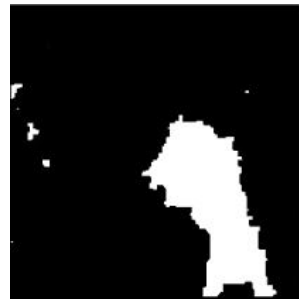

(c) Average quality

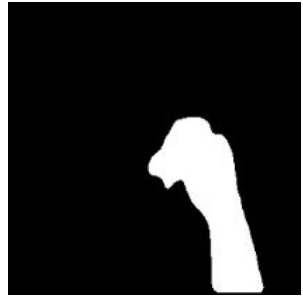

(d) High quality

Figure 13. Different mask qualities with a wide range of noise level and coverness

dimension of the input is step-wise reduced, complex patterns formed by simple ones are then detected by the last filters. Consequently, complex patterns in small areas are hardly detectable. Zooming to the area of the moving object helps to reduce wasted computation, to make patterns in images easier to detect by enlarging them, though it is also challenging to do so without affecting the real-time capability.

\section{Spatial Transformer}

Spatial Transformer [2] was introduced by Google DeepMind and became popular by its ability of learning rotation, translation, scale, etc., to help simplify classification tasks. The principle of STN is to use convolutional layers (or fully-connected layers) followed by a last fully-connected layer with 6 perceptrons to look at input image and produce an appropriate affine transformation matrix $\theta=\left[\begin{array}{lll}\theta_{11} & \theta_{12} & \theta_{13} \\ \theta_{21} & \theta_{22} & \theta_{23}\end{array}\right]$. The weights of the last layer are zero-initialized and its biases are initialized so that its output is an identity transformation matrix $\left[\begin{array}{lll}1 & 0 & 0 \\ 0 & 1 & 0\end{array}\right]$. The affine transformation is then applied to a sampling grid $G$. Because, we only need to zoom into the region of interest, only translation and

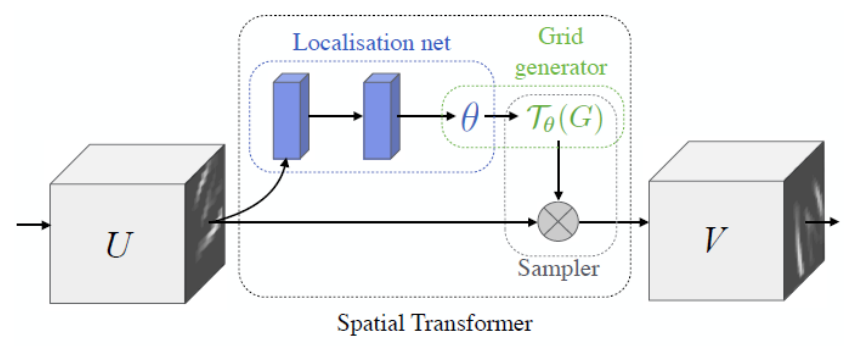

Figure 14. Spatial Transformer Network [2]

scale are needed for our approach. The number of parameters for affine transformation can be reduced from 6 to 4 , meaning $\theta=\left[\begin{array}{ccc}\theta_{s x} & 0 & \theta_{t x} \\ 0 & \theta_{s y} & \theta_{t y}\end{array}\right]$.

Spatial Transformer works as a plugin to many classification tasks on such data sets as MNIST, Street View House Numbers, German Traffic Signs, etc. However, the zooming effect of STN in this specific data set is not remarkable and reliable. Although it did zoom 


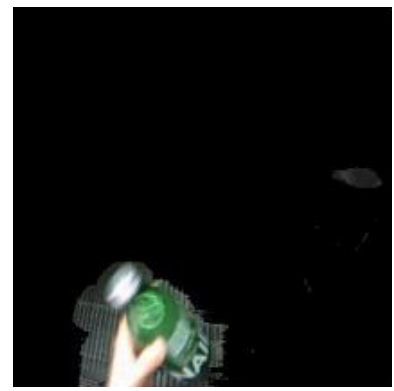

(a) Input image

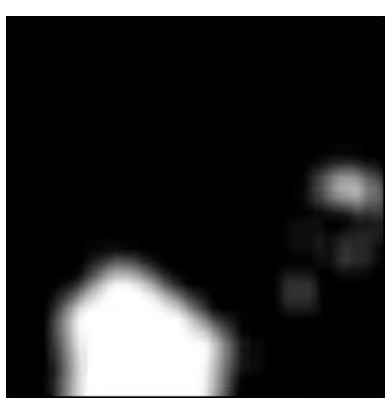

(b) After mean filtering

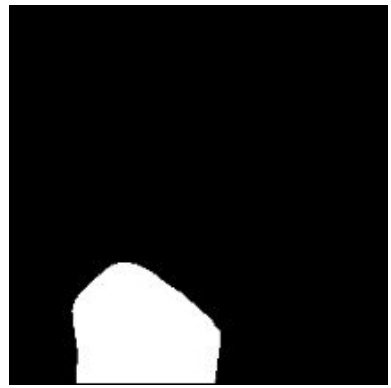

(c) After thresholding

Figure 15. Illustration of density-based cropping

into moving objects successfully, sometimes input images were squeezed or translated until they completely disappeared.

\section{Density-based cropping}

To utilize the fact that there is nothing else visible than the moving object in every frame, density-based cropping is introduced based on the following assumption. Density of positive pixels in an area that contains the moving object is significantly higher than that in an area solely containing background.

Firstly, a mean filter is utilized to reduce noise by computing the percentage of positive pixels in fixed-size areas, resulting in a blurry grayscale version of the input image. The area containing the moving object will still be white due to its high pixel density, while noise areas will fade to gray. Image thresholding is then applied to remove noise pixels completely, see Figure 15.

The cropping procedure is simple; all it needs to do is to slice the image, so that every row/column contains at least one white pixel. Hence, choosing the right threshold value plays a crucial role in producing reasonable results.

The image size for this use case is $96 \times 96$, the averaging kernel size is $5 \times 5$, and the threshold value is set to 0.7 . Density-based cropping has proved its ability in practice by producing desired results in most cases. Sometimes, input image is not well cropped because noises were not well eliminated; even one tiny noise in a corner that passed the thresholding stage could affect the results seriously, see Figure 16.

Unlike spatial transformer, density-based cropping may also fail to output good results as expected due to incorrect parameters (kernel size, threshold value). Moving objects are still visible in failure cases.

\section{Evaluation of Multi-Fusion Network}

The models are trained on a single GTX 1080Ti for maximal 50 iterations (epochs) with SGD optimizer. The batch size for the action model and object model is 16 and 64, respectively. Both action and object classifiers have a pre-trained ImageNet ResNet-18 as the base feature extractor. All layers of the base model ResNet except the last convolutional block are frozen, allowing them to learn blurry features that appear due to hand 


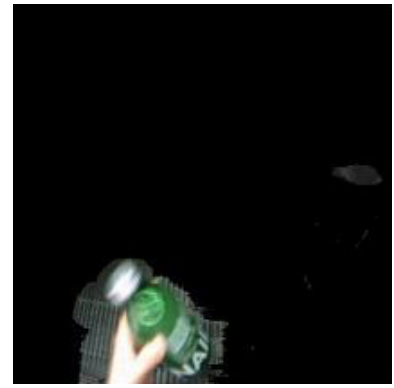

(a) Input image

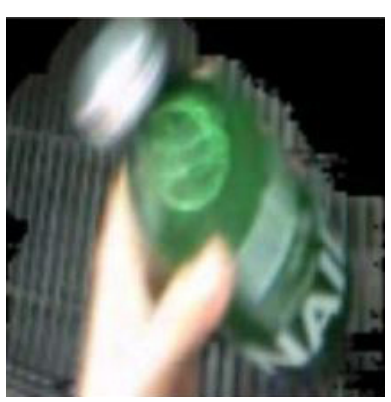

(b) Good result

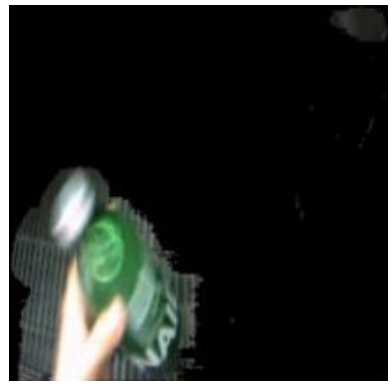

(c) Bad result

Figure 16. Success and failure of density-based cropping

and object motion. If this last block was frozen, the model could not achieve high accuracy.

\begin{tabular}{|l|l|l|}
\hline Bottleneck features & Temporal units & Accuracy (\%) \\
\hline 256 & 64 & 88.048 \\
\hline 128 & 64 & 90.438 \\
\hline $\mathbf{1 2 8}$ & $\mathbf{3 2}$ & $\mathbf{9 1 . 2 3 5}$ \\
\hline 128 & 16 & 86.065 \\
\hline 64 & 16 & 89.243 \\
\hline
\end{tabular}

Table 2. The result table of the action model

The number of features in this work is much smaller than that of the ImageNet data set. Shapes and colors of hands, bottles, cans, etc. are the most important features for this specific use case. Logically, a 512-dimensional output of ResNet is superabundant. A dropout layer with a rate of 0.7 is applied, followed by a bottleneck layer for honing common visual features into specific ones for this approach. 16 frames are sparsely sampled from every video in the same manner. The action classification model achieved $91.235 \%$ accuracy with 128 bottleneck features and 32 temporal units. The object classification model is trained for 41 epochs and achieved $98.954 \%$ accuracy.

A practical test is performed to ensure the accuracy of the trained models. The accuracy of both models in practice is just slightly lower than that in the validation step. The actions (put, take, nothing) are correctly classified in most cases, except some special cases where the object couldn't be seen or fully detected in the background subtraction stage. The action classifier performed well in the test, but it still can make false decisions, especially when unseen artifacts appear (e.g., different sleeve colors) or when other objects are also seen in the foreground (bad background subtraction).

The refrigerator is equipped with an external display for showing recognition results, see Figure 17. It was placed at the Event Solutions in Hamburg 2019 and Sommerfest 2019 for real-worl use case scenarios. The models performed well even at the event, where many other light sources could have had a negative impact on the results.

The experiments serve as proof-of-concept, and they have confirmed the feasibility and efficiency of the proposed architecture. The model performed its tasks correctly in practice, although it was trained on a tiny data set. However, more quantitative and qualita- 


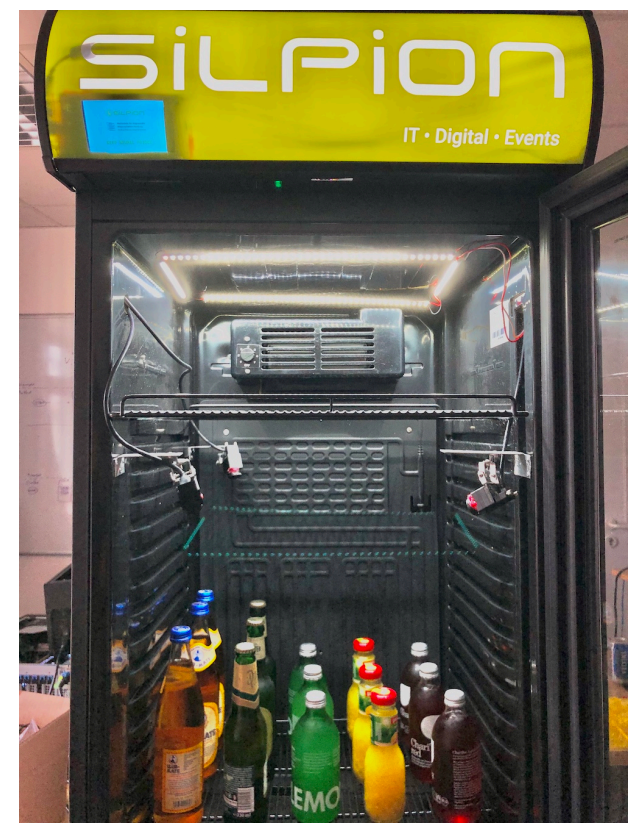

Figure 17. A photo of the beverage refrigerator

tive data, numerous classes recorded in various domains, are needed in order to fully and fairly evaluate the Multi-Fusion network architecture's effectiveness.

\section{Conclusion}

Supervised learning tasks are data-consuming giants, and it is extremely labor-intensive to make a large data set with a diversity of features. Nevertheless, it may be possible to minimize the amount of required data by analyzing project requirements, data set, then applying some techniques to reduce dimensionality. An important result of this work shows that decisions strongly depend on the presence/absence of an object currently in motion. Attention mechanism bring remarkable contribution to reducing the amount of data if it can focus on the moving object. Using background subtraction as a strong attention mechanism can also help to reduce overfitting. However, which background subtraction algorithm should be used depends on specific characteristics of the use case. Bad choice of background subtraction can lead to loss of relevant information and, therefore, to performance degradation.

Data recorded by a small organization could contain not enough variations for the model to be able to recognize and extract relevant features, which leads to the problem of overfitting. The presented Multi-Fusion network reduces the amount of training data and, therefore, also reduces the required effort and the risk of overfitting.

A lot of unexpected cases in real-world experiments are inevitable. Input standardization and noise removal are much more crucial in practice. This work utilizes density-based cropping and inactivity trimming in the pre-processing step for achieving the best results. Multi-Fusion network has proved its potential by achieving $91.235 \%$ accuracy on the test 
data set with only hundreds of training samples.

Potential of Multi-Fusion network could be also indoor activity recognition for gaming experiences, smart home applications, or augmented / mixed reality devices. This architecture could be used in embedded systems with average computational capability or small toolkits such as Google Coral or NVIDIA Jetson due to its lightweight and scalable architecture.

\section{References}

[1] Meng, Lili, et al. 'Interpretable spatio-temporal attention for video action recognition." Proceedings of the IEEE International Conference on Computer Vision Workshops. 2019.

[2] Jaderberg, Max, Karen Simonyan, and Andrew Zisserman. "Spatial transformer networks." Advances in neural information processing systems. 2015.

[3] Li, Yong-Lu, et al. "HAKE: Human Activity Knowledge Engine." arXiv preprint arXiv:1904.06539 (2019).

[4] Gkioxari, Georgia, et al. "Detecting and recognizing human-object interactions." Proceedings of the IEEE Conference on Computer Vision and Pattern Recognition. 2018.

[5] Carreira, Joao, and Andrew Zisserman. "Quo vadis, action recognition? a new model and the kinetics dataset." proceedings of the IEEE Conference on Computer Vision and Pattern Recognition. 2017.

[6] Zhou, Bolei, et al. 'Temporal relational reasoning in videos." Proceedings of the European Conference on Computer Vision (ECCV). 2018

[7] Dwibedi, Debidatta, Pierre Sermanet, and Jonathan Tompson. "Temporal reasoning in videos using convolutional gated recurrent units." Proceedings of the IEEE Conference on Computer Vision and Pattern Recognition Workshops. 2018.

[8] Lim, Kyungsun, Won-Dong Jang, and Chang-Su Kim. "Background subtraction using encoder-decoder structured convolutional neural network." 2017 14th IEEE International Conference on Advanced Video and Signal Based Surveillance (AVSS). IEEE, 2017.

[9] Sobral, Andrews. "BGSLibrary: An opencv c++ background subtraction library." IX Workshop de Visao Computacional. Vol. 27. 2013.

[10] Kertsz, Csaba, and Vincit Oy. "Texture-based foreground detection." (2011).

[11] St-Charles, Pierre-Luc, Guillaume-Alexandre Bilodeau, and Robert Bergevin. "Subsense: A universal change detection method with local adaptive sensitivity." IEEE Transactions on Image Processing 24.1 (2014): 359-373. 


\title{
Modeling the Software Prototyping Process in a Research Context
}

\author{
Mika SAARI, Jari SOINI, Jere GRÖNMAN, Petri RANTANEN, Timo MÄKINEN \\ and Pekka SILLBERG \\ Tampere University, Faculty of Information Technology and Communication Sciences, \\ Pori, Finland
}

\begin{abstract}
The paper examines the Third Mission of universities from the point of view of company collaboration in the prototype development process. The paper presents an implementation of university-enterprise collaboration in prototype development described by means of process modeling notation. In this article, the focus is on modeling the software prototyping process in a research context. This research paper introduces prototype development in a university environment. The prototypes are made in collaboration with companies, which offered real-world use cases. The prototype development process is introduced by a modeling procedure with four example prototype cases. The research method used is an eight-step process modeling approach. The goal was to find instances of activity, artifact, resource, and role. The results of modeling are presented using textual and graphical notation. This paper describes the data elicitation, where the process knowledge is collected using stickers-on-the-wall technique, and the creation of the model is described. Finally, the shortcomings found in our existing practices and possibilities for improving our prototype development processes and practices are discussed.
\end{abstract}

Keywords. Software process, process modeling, knowledge management, modeling methods, prototyping, modeling

\section{Introduction}

It is a common conception that the modern university serves three main purposes: teaching, research, and a broader social function. The latter of these functions, commonly dubbed "The Third Mission" [1-4], is considered to include measures contributing to social influencing and interaction. Nevertheless, multiple views in terms of defining the Third Mission exist, and Henry Mugabi [5] for example, compiled a selection of the varying definitions present in recent research literature in his dissertation. Moreover, the concept has been increasingly integrated into university strategies as well as operations pertaining to regional development [6]. Universities serve to produce and share knowledge and technological expertise, contributing in their part to the modernization and success of enterprises, and the "Third Mission" is often associated with tech-driven collaboration between the university and enterprises in addition to its social function.

The social significance of the Third Mission is widely acknowledged, and an international evaluation indicator conducted by UNESCO [7] places Finland among the top countries regarding collaboration between university and enterprises. In Finland, the most common manifestations of this kind of collaboration are various research and development projects that are often funded externally, examples of which include 
contributions by the Academy of Finland, Business Finland, various foundations, the European Union, and other international sources. For small and medium-sized enterprises (SMEs) [8] in particular, collaborative projects with a university offer significantly better opportunities to participate in R\&D activities for instance, as opposed to relying on internal funding and expertise only.

This paper introduces the model of prototype development practices that have been applied in research projects between university and enterprises (mostly SMEs) in Finland. The practices have been applied for many years in several collaborative projects between Tampere University, Pori unit, and regional enterprises. In this paper, the model is presented with the help of a few cases serving as concrete examples. Promoting and contributing to the Third Mission of the university from the perspective of regional development, the format introduced in the present paper is but one example of collaboration between the university and enterprises.

The paper is structured as follows: In Section 2, background information along with work related to the subject are presented. The research approach in terms of the applied model is presented in Section 3. Section 4 introduces the process knowledge with the help of examples, after which Section 5 describes the creation of the Prototype Development Process (PDP) model in selected cases. Section 6 discusses the observations and challenges confronted during the use of the model. Finally, Section 7 summarizes the paper.

\section{Related studies on the subject}

This section describes some points of view, factors, and experiences, which relate to the collaboration context between universities and enterprises. Earlier research results in this field are examined below.

In their research, Basili et al. propose guidelines on how collaborative research could succeed between public sector research and industry. In [9], a couple of arguments are present, which are worthy of examination. First, they say that there is not enough research considering real development contexts. Second, they suggest that software engineering research needs to foster context-driven research if it seeks to evolve towards a brighter future. The main contextual factors are human, organizational, and domain-related. In [9], they believe that practical software engineering, the big picture, and suitable solutions are mostly born from bottom-up research and a succession of case studies rather than from top-down research.

In context-driven research it is necessary to have intense collaboration between industry and academia. There need to be cycles of model building, experimentation, and learning in software engineering research. Usually researchers face some problems in identifying the challenges of collaborating organizations. After overcoming these challenges, the gap between the state of the art and the requirements for a solution must be assessed. In developing a solution, it is important to clearly define working assumptions in order to achieve applicability and scalability in context [9].

Another major part of this collaboration relates to funding. There are a couple of ways to start funding a research project between industry and academia. Companies can give grants to students/researchers for a specific project, build collaborative projects with academia in short- or long-term existing relationships, and there is an opportunity to build up a wider network of partners between industry and academia [10]. Also, governments are encouraging this kind of collaboration because it improves innovation 
efficiency and thereby enhances wealth creation [11]. As a result, several countries have already put innovative programs in place [9].

Industry-academia collaboration benefits those organizations that do not have their own R\&D facilities. Companies can utilize the knowledge of academic resources to understand their modern-day software engineering problems. Industry has noted that it can support innovation and development processes when collaborating with researchers [9].

Companies are increasingly investing in software development, although their core areas of expertise are defined around business areas and systems rather than software. However, most companies do not have the necessary resources and know-how to develop effective solutions to software engineering problems. This makes collaboration between industry and academia very useful [9].

The aim of a study by Wohlin [12] was to gather experiences and lessons learned from successful collaboration between industry and academia in two different environments. First, it was performed in Sweden and included a six-year collaborative project. The collaboration partners were five different-sized companies from various sectors and the Blekinge Institute of Technology. Later the study was replicated in Australia. Industry roles included product managers, project managers, developers, and testers. Academic roles included professors, researchers, and students at different levels.

The results from Wohlin's study [12] were that support from company management is crucial. There must be a champion at the company who argues for the cause, and not only a person assigned the responsibility for the project. There are different levels of understanding between different categories of people (for example, people in industry, senior researchers, and students). Social skills are particularly important in long-term collaboration.

In the study by Carver [10], there is an example of industry-academia collaboration. The background of the study was that the challenges faced by the companies were too labor intensive, lacked context-specific validation, or were not embedded into existing tools or design processes. Collaboration between industry and academia produces successful results when there is a good connection between academic and industry partners, there are the right collaborators on both sides, the timing of the interaction fits the requirements on both sides, and it is understood that the process from research prototypes and an academic publication to a deployed solution takes time.

In [13], the author interviewed researchers to understand their needs and problems in research-based projects. University research was more concerned with community issues, and companies had clients that were constrained by formal agreements. The industry groups had defined roles and responsibilities; the research teams were more dynamic. Industrial companies used formal development methods, but most university projects did not. The milestones for industrial projects were set by the customer, while the milestones for university projects were usually set by the funding partners. After the interviews, the author decided, for a number of reasons, including the uncertainty about the research objectives, that university researchers were unable to use a well-defined software development method.

The above-described examples provide the research background for the context that is discussed in this paper. The following section concentrates on explaining the basis of the process modeling, which is relevant background information for the description later, in Section 4, of the implementation of the university-enterprise collaboration with the help of the process modeling notation. 


\section{Research approach - An eight-step model}

This section deals with software process modeling in a research context. Modeling is an approach for analyzing and understanding a complex phenomenon resulting in a model, which is a simple and familiar structure that can be used to interpret some part of reality [14]. When the phenomenon to be analyzed is a software process, information is captured and classified into a model with the help of a process-modeling schema [15], i.e., a metamodel specifying the concepts, relationships, and rules [16] used when modeling processes. The basic concepts related to the software process include activity, artifact, resource, and role [17].

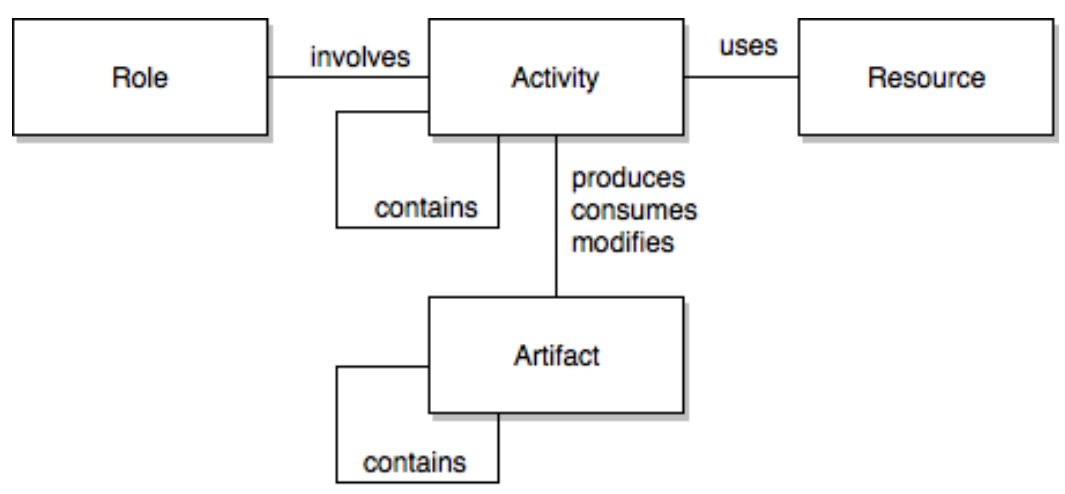

Figure 1. The basic concepts related to software processes.

The results of modeling are presented using a textual or graphical notation. There are several approaches for eliciting information for process models [15] such as interviews and artifact analyses. Process modeling can be prescriptive or descriptive. While a Prescriptive Process Model (PPM) describes how a process should be performed, a Descriptive Process Model (DPM) describes how it actually is performed [18].

In this study our aim is to model software development practices performed in an academic context. We follow the DPM approach proposed by Becker \& al. [19]. The approach consists of eight steps grouped into two phases:

Set-up phase

1. Objectives and Scope

2. Define Schema

3. Select Language

4. Select and Tailor Tools

Execution phase

5. Elicitation

6. Create Model

7. Check Model

8. Check Process 
We will apply the DPM approach in the following way: The data for the models is collected through interviews with the developers involved in the processes. The schema shown in Figure 1 guides the data collection, the results of which are shown on stickers on the wall during the work (Figure 2 in Section 4). In the resulting models, the activities are represented as rectangles with rounded corners. Stick figures represent roles and different icons represent resources. Artifacts appear as parallelograms, cylinders, and document symbols. The associations between activities and artifacts are represented by continuous arrows and the links between activities and roles and resources are dashed. Gray symbols and dashed rectangles represent aggregations (Figures 3-6 in Section 4). Graphical representations of the models are produced by a free online diagram software, draw.io. (DPM Steps 2-4).

The objectives and scope of the modeling are presented in the introduction of this paper (Step 1). The following section provides an example of data elicitation (Step 5) including the modeling results (Step 6). After that, the possibilities for improving the modeled processes are discussed (steps 7-8).

\section{Process elicitation and resulting models}

This section describes the steps 5 and 6 of the DPM approach. Process knowledge is highlighted in this section, which introduces four different prototype development processes (PDP 1-4).

Information for the process models is collected from four cases:

PDP 1 - Verification of customer complaints related to bus routes [20].

PDP 2 - Verification of customer complaints related to garbage collection. [20]. PDP 3 - Data collection in a public indoor swimming pool [21, 22].

PDP 4 - Passenger counting in a free-to-ride bus [23].

Common to all of these example cases is the software development resulting in a working prototype. The development process starts from the idea of collecting certain data with certain equipment. Then the idea is validated - can it be viably implemented? If the answer is yes, the implementation phase produces the first working prototype. Usually prototype implementation includes software coding and the implementation of hardware from off-the-shelf devices. The working prototype is tested in a laboratory and if the device displays sufficient reliability, the device is moved to real-world testing. The development processes and testing phases usually produce data. The overall outcome from these prototype development processes has been academic output such as a research paper. Knowledge of the prototyping process is visualized as illustrated in Figure 2. 


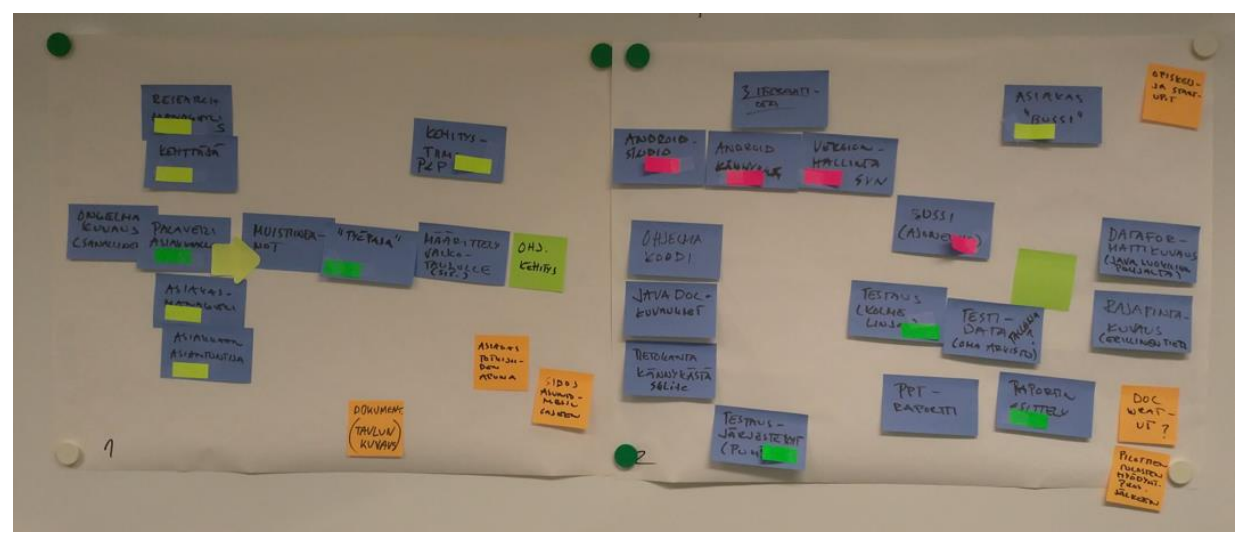

Figure 2. Whiteboard and notes.

This whiteboard and the Post-It notes are one way to highlight development process practices [24]. The notes are color-coded: Yellow markers are roles, green denotes activity, red is for resources, and the blue notes are artifacts. The orange notes describe issues and improvement ideas that came up during the data elicitation process. Knowledge of the development process is collected by means of this whiteboard and note notation (stickers-on-the-wall technique).

Further, this section presents the creation of a model for each of the PDPs. First we introduce the developed model for PDP 1 and 2. Figure 3 presents the university company interaction. The model includes six steps which have been identified from the development process. The steps start from requirements definition and end with the publication of results. The gray boxes - the software development step and the development artifacts - are discussed more in the subsections on the bus case and the garbage collection case.

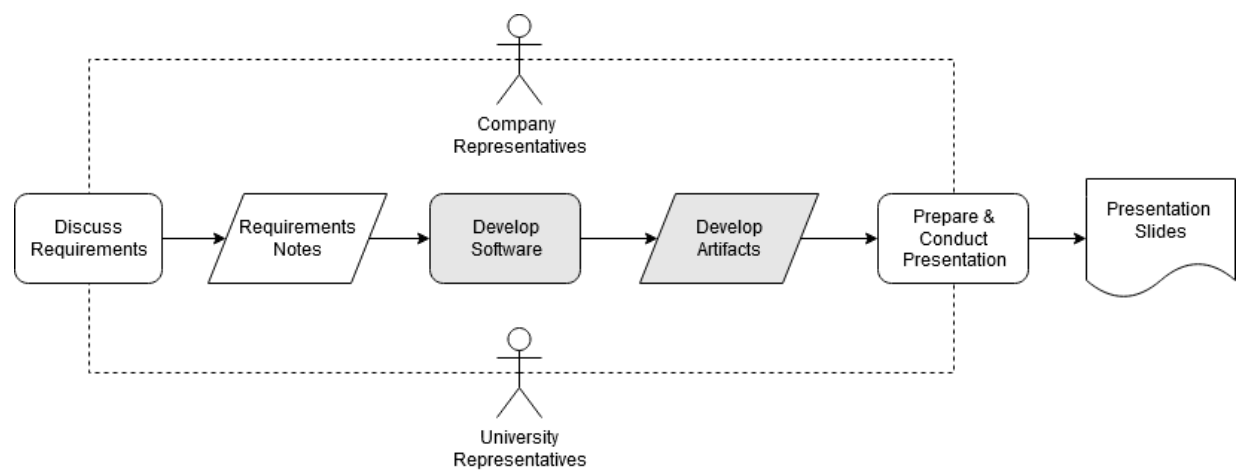

Figure 3. The illustration of university - company interaction during development (common to all PDPs).

The model includes all the main factors. The university representatives are the research group including the project manager and software/hardware developers. This group has the main responsibility for the prototype development. The company representatives are involved in the development process in the role of instructor. In the 
presented PDPs 1-4, the company representatives are active at the starting and ending points: at the start with the definition of requirements and at the end where the results are presented to them. They also provide the testing environment if the testing is done at their company. However, they are not involved in the development process itself.

\subsection{Verification of customer complaints related to bus routes (PDP 1)}

The bus case (PDP 1) was established for handling customer complaints. The bus company had received complaints from customers that the buses did not stop to pick up customers or did not come at all. The prototype was developed to collect photos with time stamps at defined bus stops. This prototype was implemented in mobile phones and the main idea of prototype was to collect photos of the bus stops as the bus approached. The project group at the university developed a working prototype, which was then tested in the buses [20].

The development process is illustrated in Figure 4, expanding the previously presented steps "Develop Software" and "Develop artifacts". The development group in this process consists of only university personnel. The development process starts with a design discussion - the first activity, which produces the first artifact: the whiteboard sketch. The results are then used in the software construction phase. This activity produces the second artifact: software code with documentation. After that the test activity starts, which produces the collected data artifact. The coding and testing activities could be iterated several times. Figure 4 also includes the resources used during the development process: programming language, test device with GPS, camera and network, and the testing environment - the bus itself.

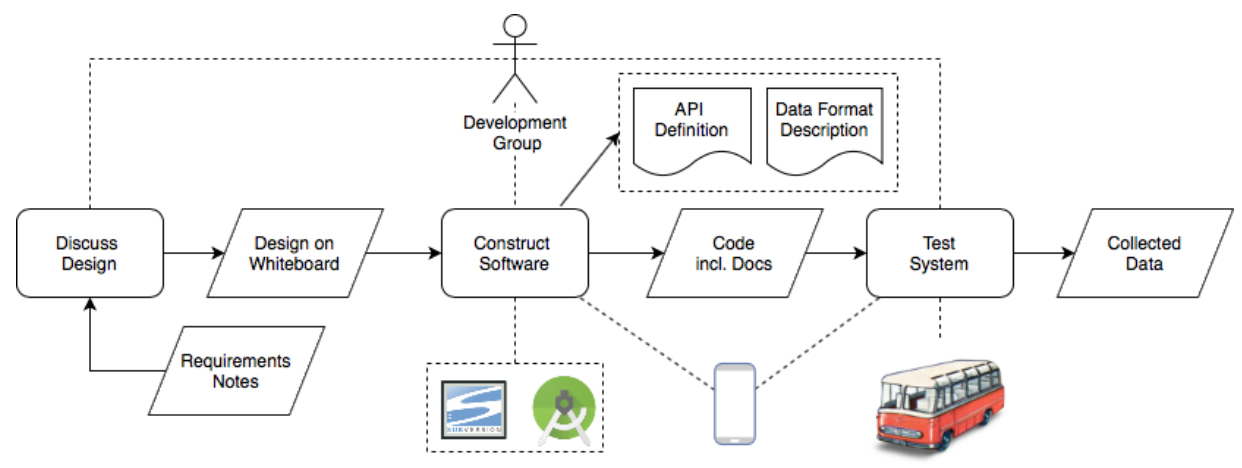

Figure 4. The development process of the bus case.

The presented development process produces a working prototype. In this case, the prototype was introduced to the customer - the bus company.

\subsection{Verification of customer complaints related to garbage collection (PDP 2)}

The garbage collection case (PDP 2) was similar to PDP 1. The use case was also intended for managing customer complaints. The garbage collection company had 
received complaints from their customers that the trashcans had not been emptied. In most cases the reason was a vehicle blocking the garbage collection truck or similarly that the truck was unable to empty the trashcan. The prototype solution in this case used the same idea as in the bus case: namely, put the camera in the truck and take pictures when the GPS registers the right location [20].

Figure 5 shows the activities and artifacts. The biggest difference was in the resources: the cellphone was replaced by a Raspberry Pi with a camera, GPS, and network device. The data collector resource was the MySQL server instead of the phone. In addition, the testing environment was the garbage collection truck itself.

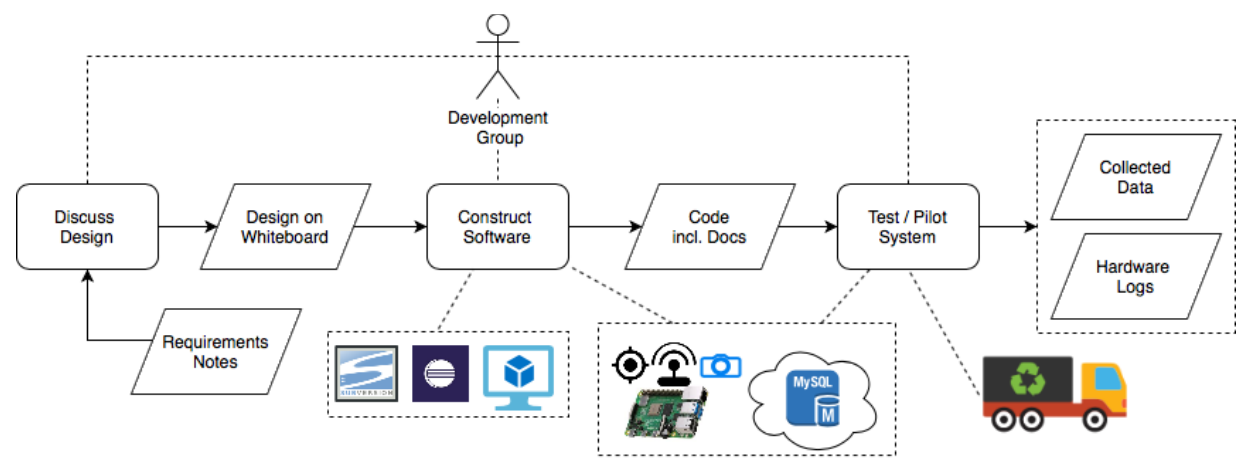

Figure 5. The development process of garbage collection case.

The developed prototype worked and the piloting phase in the garbage truck lasted several weeks. The development process captured data, which in this case were photos. In addition, the device - a Raspberry Pi, generated a test log during the pilot phase.

\subsection{Combined model of bus and garbage collection cases}

PDP 1 and PDP 2 should be presented together because the second prototype - the garbage truck camera system - utilized the definitions and results of the first prototype. They were also implemented very close to each other in time.

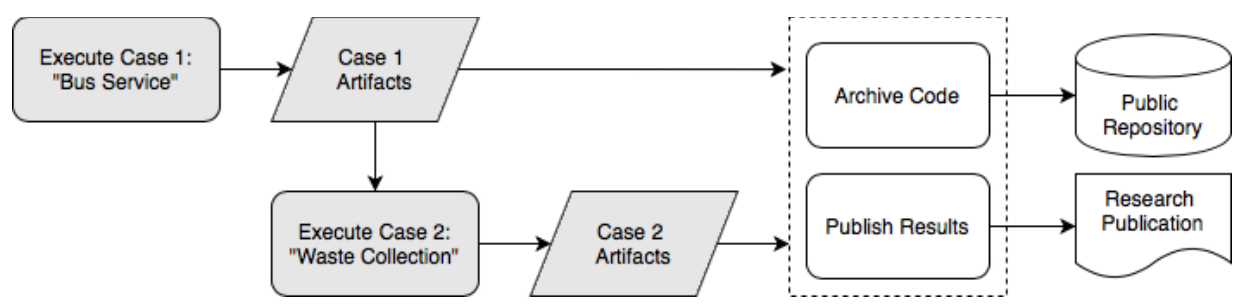

Figure 6. The combined model of the bus and garbage collection cases.

The outcomes of these PDPs were a public repository and research publication [20]. The public repository includes all the developed software code. 


\subsection{Data collection in a public indoor swimming pool (PDP 3)}

PDP 3 handled a prototype system with the purpose of improving the quality of manually collected data. The prototype was a mobile application that the maintenance personnel used to collect and store data from several different meters in a public swimming pool. More information on the prototype can be found in the research articles by Soini [21, 22].

The Prototype Development Process was similar to the one shown in Figure 3. Activities included the discussion of requirements, software coding, and the presentation of the results. In addition, the artifacts were similar: discussion notes, software, and a research publication. The biggest difference to the other cases was that the implemented prototype remained in use after the pilot phase. This "extended piloting" period is handled in the research paper by Soini [22] along with aspects of software evaluation. Also, long-term piloting is examined from the point of view of system developers, administrators (maintenance), and end users [22].

\subsection{Passenger counting in a free-to-ride bus (PDP 4)}

PDP 4 handled a prototype system with the aim of counting passengers on a free-to-ride shuttle bus. Unlike an ordinary people counter, the customer wanted more information on where and when passengers got on and off [23].

The Prototype Development Process was similar to the one shown in Figure 3. Activities included the discussion of requirements, software coding, and the presentation of the results. Again, the artifacts were similar: discussion notes, software, and a research publication. The difference compared to PDP 1-3 was that this prototype was developed for a real-life use case of collecting statistics about bus passengers on a free-to-ride shuttle bus route at a large public event in the summer of 2018 in Pori, Finland. In this case, the development process ended in the one-month pilot. The outcome of the pilot was the presentation of the pilot results to the customer.

\section{Discussion}

The process introduced and modeled in this paper has been used to create multiple prototypes and pilot experiments during recent years. Thus, based on the observed results, it can be considered to be successful and fit the needs of our development cases. However, while discussing past projects with the team members several challenges did come up. Furthermore, while collecting data for the model, notes were made of issues that the team members pointed out (the orange notes on the whiteboard in Figure 2).

The first issue was the documentation of intermediate specifications described on whiteboards. The funding or goals of the projects do not especially require extensive intermediate documentation, and in practice, only very rarely has there been the need to study the intermediate specifications created during the process. The low requirements for documentation have probably been one of the main reasons for the bad habits in documentation practices. Generally, the decision of whether to prepare any documentation has been based on the developer teams' "gut feeling" about how complex the specification was. In other words, "proper" documentation has been created for more complex intermediate specifications, but simpler specifications and drawings have not been documented in any way. Nowadays, it is quite simple to use a smartphone to capture 
the information on whiteboards, so in the future, it might be better to document everything systematically.

The second issue was the interaction with the customers (e.g., companies) - or the lack of it - during the actual prototype development process. The interaction has often been limited to the use case definition phase, to the organization of practical arrangements of pilots (e.g., agreements on which bus lines could be used for testing prototypes), and to the presentation of the research findings. In the final presentation meetings, the companies have never indicated that they would have liked to be more involved in the process. The feedback from the companies has mainly been related to the research findings, and the developed prototypes, and not to the development process itself. In our case, the companies have often not been software-oriented, which could have had an effect on their interest in the process, and it could also have limited the advantages achievable by involving them further in the process. In addition, the companies did not (directly) invest any resources (money or personnel) in the projects. This might have further reduced their interest in participating more deeply in the development process. Furthermore, as the companies had their actual business to run, there could have been challenges in creating a common schedule for meetings for all the parties involved. Of course, depending on the outcome of the research and pilots the companies can obtain knowledge, business ideas, or even working software to use in their actual business, but during the development these results may be too abstract to evoke deeper interest. In addition, the fact that the issue was not brought up in the meetings does not necessarily mean that there is a lack of interest in deeper involvement from the company side. As the university team did not especially raise the issue, it might be that the companies felt that they simply did not want to interfere in the university practices. Thus, to improve our model, the actual company interest in the prototype development process should be further studied.

Third, the subject of the usability of the project results came up. In principle, anyone can use the results because the codes and documentation are published as open source, but no studies have been performed on how or if the results are actually used. In general, after the projects (and thus, funding) has ended, the results have been left "as is" without maintenance, bug fixes, or feature improvements. The purpose of the projects was not to create "end products", and usually productization is not one of the project goals, leaving the created software and hardware applications in a state that would require further development into an end-user quality product. Also, it can be slightly challenging to find the material from, for example, the GitHub repository if one does not know exactly what one is looking for. Internally, the published codes and specifications have been reused in future projects when applicable. One potential future direction for research would be a study on how the results of university projects should be published to be most useful for outside parties, and what the crucial elements are that should be published — or are the elements practically the same as in any other prototype development project?

Finally, the participation of university students was discussed. The advantage of involving the students more deeply would be to give the students more meaningful task assignments (for example, for programming or other software engineering courses), but in practice, in the past the participation of students has been rare, and has mainly been limited to PhD students who have been hired by the university or worked at the university on their own funding. In the future, the model introduced in this paper could also be expanded to describe the involvement of students. 


\section{Summary}

The paper examined the Third Mission of universities from the point of view of company collaboration in the prototype development process. The paper presented an implementation of university-enterprise collaboration in prototype development described by means of process modeling notation. The process introduced and modeled in this paper has been used to create multiple prototypes and pilot experiments over recent years. The prototypes were made in collaboration with companies, which offered real-world application problems.

The prototype development process was introduced by a modeling procedure with four example prototype cases. The research method used and presented here was an eight-step process modeling schema. The basic concepts relating to the software process included four factors: activity, artifact, resource, and role. The results of the modeling were presented using textual and graphical notation, and the elicitation of process knowledge the creation of the model were described. Furthermore, certain shortcomings in our existing practices were discovered. Based on the findings the model as well as our prototype development process and practices could be further improved in the future.

In summary, the PDP model provides one concrete and systematic example of how university-enterprise collaboration can be executed in practice. Moreover, the model presented is a real-life indication of how the Third Mission task set for universities can be successfully implemented. Thus, based on the observed results the model can be considered to be successful and fit the needs of our development cases.

\section{References}

[1] F. Schutte, and P. C. van der Sijde, “The University and its region”. Examples of regional development from the European Consortium of Innovative Universities. Twente University Press, Enschede, Netherlands. 2000

[2] T. Vorley, and J. Nelles, "Building Entrepreneurial Architectures: a conceptual interpretation of the Third Mission", Policy Futures in Education, Vol. 7, No. 3, pp. 284-296, 2009.

[3] I. Niiniluoto, "Yliopistot ja ammattikorkeakoulut yhteiskunnallisina vaikuttajina", In the publication Vastuullinen ja vaikuttava. Opetus- ja kulttuuriministeriön julkaisuja 2015:3, pp. 11-30, 2015.

[4] K. Kankaala, E. Kaukonen, P. Kutinlahti, T. Lemola and M. Nieminen (2004), Yliopistojen kolmas tehtävä ?, Edita Publishing Oy. Helsinki, pp.15-42, 2004.

[5] H. Mugabi, Institutionalisation of the "Third Mission" of the University. The case of Makerere University. Academic Dissertation, Tampere University Press, Finland 2014.

[6] A. Zomer, and P. Benneworth, "The Rise of the University's Third Mission", Reform of Higher Education in Europe, (eds.) Enders, J., de Boer H.F. ja Westerheijden, D.F., Sense Publishers. Netherlands, pp. 81 $102,2011$.

[7] UNESCO (2015), UNESCO science report: Towards 2030, p. 74, https://unesdoc.unesco.org/ark:/48223/pf0000235406/PDF/235406eng.pdf.multi (Retrieved 4/1/2020 World Wide Web)

[8] M. Marchesnay, "Fifty years of entrepreneurship and SME: A personal view". Journal of Small Business and Enterprise Development, Vol. 18, No. 2, pp. 352-365, 2011.

[9] V. Basili, L. Briand, D. Bianculli, S. Nejati, F. Pastore and M. Sabetzadeh, "Software Engineering Research and Industry: A Symbiotic Relationship to Foster Impact," in IEEE Software, Vol. 35, No. 5, pp. 44-49, 2018.

[10] J. C. Carver and R. Prikladnicki, "Industry-Academia Collaboration in Software Engineering," in IEEE Software, Vol. 35, No. 5, pp. 120-124, 2018

[11] T. Barnes, I. Pashby, A. Gibbons, "Effective University - Industry Interaction: A Multi-case Evaluation of Collaborative R\&D Projects,” in European Management Journal, Vol. 20, Issue 3, pp. 272-285, 2002. 
[12] C. Wohlin, A. Aurum, L. Angelis, L. Phillips, Y. Dittrich, T. Goschek, H. Grahn, K. Henningsson, S.Kagstrom, G. Low, P. Rovegard, P. Tomaszewski, C. van Toorn and J. Winter, "The Success Factors Powering Industry-Academia Collaboration," in IEEE Software, Vol. 29, No. 2, pp. 67-73, 2012.

[13] D. M. P. Dias, "Managing research based software product development in Sri Lankan universities", University of Colombo School of Computing, 2016.

[14] M. Boman, J. Bubenko, P. Johannesson and B. Wangler, "Conceptual Modeling". Prentice Hall, 1997.

[15] U. Becker-Kornstaedt, "Prospect: a Method for Systematic Elicitation of Software Processes". PhD dissertation. Technische Universität Kaiserslautern, 2004.

[16] ISO/IEC 24744:2006. Software Engineering - Metamodel for Development Methodologies. Draft International Standard ISO/IEC FDIS 24744:2006(E), ISO/IEC JTC1/SC7, Montréal, Québec, Canada.

[17] U. Becker-Kornstaedt and R. Webby, "A Comprehensive Schema Integrating Software Process Modeling and Software Measurement”. Research Report 047.99/E, Fraunhofer IESE, Kaiserslautern, Germany, 1999.

[18] C. Jensen and W. Scacchi, "Discovering, Modeling, and Re-enacting Open Source Software Development Processes: A Case Study". In Silvia T Acuña, S. T. and Sánchez-Segura, M. I. (eds.). New Trends in Software Process Modeling. World Scientific Pub. Co., 2006.

[19] U. Becker, D. Hamann, D and M. Verlage, "Descriptive Modeling of Software Process". Research Report ESE-Report, 045.97/E, Fraunhofer IESE, Kaiserslautern, Germany, 1997.

[20] J. Grönman, P. Rantanen, M. Saari, P. Sillberg, and H. Jaakkola, "Lessons Learned from Developing Prototypes for Customer Complaint Validation", Software Quality Analysis, Monitoring, Improvement, and Applications (SQAMIA), Novi Sad, Serbia, August 27-30, 2018

[21] J. Soini, P. Sillberg, and P. Rantanen, "Prototype System for Improving Manually Collected Data Quality," in Proceedings of the 3rd Workshop on Software Quality Analysis, Monitoring, Improvement, and Applications, SQAMIA 2014, Lovran, Croatia, pp. 99-106, 2014

[22] J. Soini, M. Kuusisto, P. Rantanen, M. Saari and P. Sillberg, "A Study on an Evolution of a Data Collection System for Knowledge Representation", EJC 2019: Proceedings of the 29th International Conference on Information Modelling and Knowledge Bases, 2019.

[23] J. Grönman, P. Sillberg, P. Rantanen and M. Saari, "People Counting in a Public Event-Use Case: Freeto-Ride Bus", 2019 42th International Convention on Information and Communication Technology, Electronics and Microelectronics (MIPRO), Opatija, Croatia, 2019.

[24] A. Raninen, J. J. Ahonen, H.-M. Sihvonen, P. Savolainen, and S. Beecham, "LAPPI: A light-weight technique to practical process modeling and improvement target identification," Journal of Software Evolution and Process, Vol. 25, No. 9, pp. 915-933, Sep. 2013. 


\title{
A Natural Logic System for Large Knowledge Bases
}

\author{
Troels ANDREASEN ${ }^{\mathrm{a}}$, Henrik BULSKOV ${ }^{\mathrm{a}}$ and Jørgen FISCHER NILSSON ${ }^{\mathrm{b}}$ \\ ${ }^{a}$ Computer Science, Roskilde University, Denmark, \{troels, bulskov\}@ruc.dk \\ ${ }^{\mathrm{b}}$ Mathematics and Computer Science, Technical University of Denmark, \\ Denmark,jfni@dtu.dk
}

\begin{abstract}
This paper describes principles and structure for a software system that implements a dialect of natural logic for knowledge bases. Natural logics are formal logics that resemble stylized natural language fragments, and whose reasoning rules reflect common-sense reasoning. Natural logics may be seen as forms of extended syllogistic logic. The paper proposes and describes realization of deductive querying functionalities using a previously specified natural logic dialect called NATURALOG. In focus here is the engineering of an inference engine employing as a key feature relational database operations. Thereby the inference steps are subjected to computation in bulk for scaling-up to large knowledge bases. Accordingly, the system eventually is to be realized as a generalpurpose database application package with the database being turned logical knowledge base.
\end{abstract}

Keywords. Natural Logic, Deductive Querying, Large Logical Knowledge Bases, Relational Data Base Operations

\section{Introduction}

In a number of papers $[1,2,3,4,5,6]$ we have proposed and analyzed a dialect of natural logic intended for knowledge base use. Natural logics are forms of formal logic that appear as stylized fragments of natural language. Therefore, a knowledge base consisting of natural logic sentences can be read and understood right away by domain specialists without understanding of predicate logic, description logic or logic programming.

In the applied dialect, called NATURALOG, one can state implicitly quantified relationships between introduced classes. In particular one can specify formal ontologies by means of the subclass relationship and partonomies by means of parthood relations. Moreover, one can introduce relations ad libitum. The natural logic NATURALOG basically covers a fragment of predicate logic with respect to expressivity, see also $[7,8,9,10,11,12]$. Natural logics are further distinguished by their appealing to high level "natural" reasoning rules reminiscent of and departuring from the elementary reasoning rules known from syllogistic logic.

This paper focusses on the engineering of an appropriate inference engine serving diverse forms of deductive reasoning and querying. In order to address the 
scaling-up problem encountered as computational intractability this paper proposes an inference engine using relational database operations such as selection and equi-join. Thereby logical consequences of knowledge base sentences are computed and recorded en masse rather than in a conventional stepwise fashion. We envisage applications e.g within the life-sciences employing quantitative complex models with thousands of classes structured into ontologies supplemented with a variety of class-class relations.

The paper is organized as follows: After having introduced NATURALoG in section 2, section 3 explains how sentences are encoded in a relational data base relation, and section 4 describes realization of the inference engine by computing and storing of relevant parts of the deductive closure using relational database query operations. Section 5 covers implementation and section 6 covers query issues.

\section{The NAturaLog Natural Logic}

Our natural logic comprises sentences that specify relationships between stated classes of not further described entities. Here we describe a somewhat simplified version of NATURALoG. For the currently full version we refer to [12]. Classes of not further described entities are introduced by common nouns such as cell, betacell, insulin, hormone. In the case of substances such as insulin the entities of the class may be conceived as portions of such substances.

The key class-class relationship is the well-known subclass relationship isa. Thus one can form sentences such as betacell isa cell and insulin isa hormone. The pertinent sentence form $C$ isa $D$ is actually a shorthand of every $C$ isa $D$. This is reminiscent of affirmative categorical sentences in syllogistic logic, cf. [2].

This sentence form is actually a special case of the more general NATURALOG key sentence form

$$
\text { every } C R D
$$

where $R$ is a linguistically transitive verb (or more generally a verb form) designating a binary relation. This is exemplified by the sentence every betacell produce insulin. Again, the determiner every is optional: betacell produce insulin is shorthand of every betacell produce insulin.

There is also the NATURALog form

$$
\text { some } C R D
$$

in which presence of the determiner some is made mandatory.

These natural logic sentence forms have predicate logical construals: [ every ] betacell produce insulin in predicate logic becomes the rather incomprehensible $\forall x($ betacell $(x) \rightarrow \exists y(\operatorname{produces}(x, y) \wedge$ insulin $(y)))$, and in description-logical becomes the obscure betacell $\sqsubseteq \exists$ produces.insulin. The sentence insulin isa hormone is explicated simply as $\forall x(\operatorname{insulin}(x) \rightarrow$ hormone $(x))$. The subclass relation isa is equipped with built-in rules yielding reflexivity and transitivity.

NATURALog adopts existential import, meaning that for each mentioned class $C$ there is implicitly what in a predicate logical construal appears as the existential statement $\exists x C(x)$, see for instance $[12,2,12]$. As a consequence, there is no notion of empty class in our natural logic. Two classes are assumed disjoint 
if they have no common subclass. Introduction of a common subclass makes the two classes overlapping, since their common subclass is bound to be non-empty. Thus classes at the outset are disjoint. This is in accord with the common default principle for scientific taxonomies and formal ontologies. We refer to [2, 12] for further discussion of the rationale.

NATURALog in addition to simple classes designated by common nouns importantly affords compound terms comprising modifiers that restrict classes to subclasses. For instance, the sentence [ every ] cell that produce hormone reside-in gland comprises the compound term cell that produce hormone consisting of the class cell attributed the modifier that produce hormone linguistically in the form of a restrictive relative clause that $R D$. The compound term cell that produce hormone thereby designates a subclass of cell. This conforms with the notion of generative ontologies introduced in [13] reflecting the recursive structure of the attached modifiers for generating evermore specialized subclasses.

To this end let us specify the recursively defined syntactic notion of compound concept terms:

A concept term $C$ term is a class name $C$ as introduced above, optionally affixed a modifier taking form of either

- a restrictive relative clause that $R$ Cterm, where $R$ is a verb form, or

- a prepositional phrase $R$ Cterm, where $R$ is a preposition here also designating a binary relation.

Compound modifiers may further be combined by means of logical conjunction in NaturaLog as introduced in [12].

An essential aspect of natural logic is the provision of inference rules reflecting direct "natural" reasoning on the linguistic forms in sentences. It is important to stress that the computational reasoning enabling deductive querying is accomplished directly on natural logic forms and thus are not conducted by reduction to predicate logic. This is detailed in section 4 . As a preliminary step we explain how NATURALog sentences are represented in a database relation.

\section{Encoding of Natural Logic in Data Base Relations}

The NATURALog sentences are to be represented in a database relation in the knowledge base. In the simple cases with no compound terms this is done straightforwardly in principle with tuples such as,

$\mathrm{kb}(\ldots$, , every, betacell, produce, insulin)

for the sentence [ every] betacell produce insulin. The tuple is stored in a relation $\mathrm{kb}$ and specifies values for attributes QUANT, SUB, REL, OBJ. However, to distinguish given sentences from derived and to separate propositions from definitions two additional attributes MODE and TYPE are added such that the full schema for the $\mathrm{kb}$ relation becomes:

kb(MODE, TYPE, QUANT, SUB, REL, OBJ)

where MODE can take the values given and deriv, TYPE can take the values prop and defin and QUANT is either every or some.

Sentences with compound terms are decomposed into simple sentences following the methods detailed in $[2,6,12]$, that is, by introduction of appropri- 
ate auxiliary class names representing the compound terms. As an example, the above sentence cell that produce hormone reside-in gland gives rise to the auxiliary class name cell-that-produce-hormone which has to be defined by two additional propositions, labeled "defin", giving altogether three propositions, that is, three database tuples

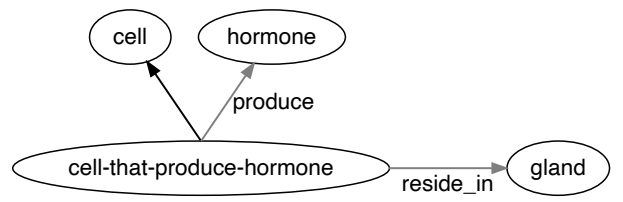

Figure 1. Example graph including three sentences

The sentences included in the graph in figure 1 will be represented by the following tuples in the kb-relation:

$$
\begin{aligned}
& \mathrm{kb} \text { (given, defin, every, cell-that-produce-hormone, isa, cell) } \\
& \mathrm{kb} \text { (given, defin, every, cell-that-produce-hormone, produce, hormone) } \\
& \mathrm{kb} \text { (given, prop, every, cell-that-produce-hormone, reside-in, gland) }
\end{aligned}
$$

Notice that in the graph notation unlabelled arrows represent isa. The definitional contributions are logically explicated as "if-and-only-if":

cell-that-produce-hormone $(x) \leftrightarrow \operatorname{cell}(x) \wedge$ producehormone $(x)$

This reduction of recursive compound terms serves easing and streamlining of the computation of inference and query answers.

\section{Inference through Data Base Query Operations}

Let us begin considering a tiny natural logic knowledge base containing solely the two sentences betacell produce insulin and insulin isa hormone. Suppose we wish to know which kind of cells that produce hormone giving this information. To this end we might form the parameterized "query" sentence X produce hormone, aiming at obtaining answers as instantiation of the parameter variable $\mathrm{X}$. However, no answer is obtained. This is because the common-sense expected answer $\mathrm{X}=$ betacell necessitates the introduction of inference rules capable of combining information in sentences according to logical consequence principles in the computation.

In $[6,12]$ we devised a collection of deductive inference rules for decomposed and encoded NATURALog sentences in a knowledge base. The rules are specified in DATAlog. This means that DATALOG functions as a metalogic for NATURALOG, where also various forms of deductive querying are stated. Here, instead of DATALOG we apply an ordinary relational database query language. The derived sentences are added to the database relation $\mathrm{kb}$. In this way the query-relevant part of the deductive closure is materialized in a iterative pre-compilation involving the knowledge base in its entirety. In the course of this computation new compound terms being potentially relevant to queries are generated. This implies that subsequent deductive query answer computations reduce to mere sentence 
retrieval from the knowledge base now being extended with materialized inferred propositions. For instance, for the above example with just two sentences, the inference engine adds the encoded form of the deduced sentence betacell produce hormone using one of the inference rules described below.

This approach to computation replaces the common goal-driven top-down approach to derivations, known e.g. from logic programming, with a bottom-up pre-computation with caching drawing on the entire knowledge base. Thereby, backtracking computation is avoided at the expense of deriving sentences being irrelevant to the queries at hand. Thus time-consuming query reasoning is traded for compile-time computation of the deductive closure and storage use.

As indicated above, the inference engine is a mechanism that iteratively materializes (adds) new tuples to the kb relation by application of the inference rules. The NATURALog inference rules are presented below along with examples of inferred propositions, that is, tuples that can be inferred from other tuples in the $\mathrm{kb}$ relation. The result of adding all propositions that can be inferred is a limited deductive closure of the knowledge base. For practical purposes this computation should not be performed tuple by tuple. In section 5 examples of how materialization of inferred tuples can be performed using SQL bulk insertions.

\subsection{Weakening and Dual_Relationship}

As mentioned in section 2, all classes are non-empty (due to existential import), and therefore we have that any default every proposition can be weakened to a some proposition. This is expressed in the weakening rule:

$$
\frac{\text { every } C R D}{\text { some } C R D}
$$

allowing to make derivations such as:

$$
\frac{\mathrm{kb} \text { (given, prop, every, betacell, produce, insulin) }}{\mathrm{kb}(\text { deriv, prop, some, betacell, produce, insulin) }}
$$

Mathematically, each relation $R$ possesses an inverse relation $R^{-1}$. Therefore, for strictly logical reasons and given our pervasive principle of non-empty concepts, for $C R D$ (i.e. every $C R$ some $D$ ) implicitly, as explained in chapter [12], we also have the dual

$$
\text { some } D R^{-1} \text { some } C
$$

Dual relations need to be explicitly specified in the knowledge base. For this purpose we introduce a database relation inv with the schema:

$$
\operatorname{inv}\left(R, R_{\text {inv }}\right)
$$

Using this we can e.g. specify produced_by to be the inverse of produce:

$$
\text { inv(produce, produced_by) }
$$

The rule expressing the existence of an inverse is the following:

$$
\frac{\text { some } C R D}{\text { some } D R_{i n v} C}
$$

where $R_{\text {inv }}$ is the inverse relation to $R$. As an example we have: 
$\mathrm{kb}$ (given, prop, some, betacell, produce, insulin)

$\mathrm{kb}($ deriv, prop, some, insulin, produce_by, betacell)

Notice that relation inversion corresponds to active to passive voice switching as covered in more detail in [12].

The weakening (1) and the dual relationship (2) rule in combination implies:

$$
\frac{C R D}{\text { some } D R_{i n v} C}
$$

An example of application of this rule is:

$\mathrm{kb}$ (given, prop, every, betacell, produce, insulin)

$\mathrm{kb}$ (deriv, prop, some, insulin, produce_by, betacell)

Observe that for the copula isa we have inv(isa, isa).

\subsection{Monotonicity Deduction Rules}

(a)

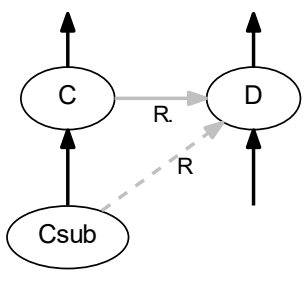

(b)

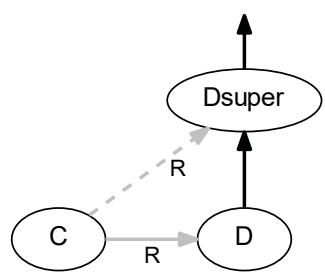

Figure 2. Monotonicity rules: (a) inheritance and (b) generalization. Dashed relations are inferred.

A query task appeals implicitly to appropriate deduction rules. This is because a query normally involves propositions that are deducible from the ones given explicitly in the knowledge base. As an example, given the knowledge base propositions insulin isa hormone and betacell produce insulin, the query

\section{$X$ produce hormone}

would intuitively yield $X=$ betacell (with multiple answers to be expected in a more comprehensive knowledge base). This is achieved by means of a pair of logical deduction rules known as monotonicity rules in natural logic [7], the inheritance and the generalization rule, which can be stated as follows:

$$
\begin{gathered}
\frac{C_{\text {sub }} \text { isa } C \quad C R D}{C_{\text {sub }} R D} \\
\frac{C R D \quad D \text { isa } D_{\text {super }}}{C R D_{\text {super }}}
\end{gathered}
$$


examples of application of these rules are:

$$
\begin{aligned}
& \mathrm{kb} \text { (given, prop, every, pancreas, isa, gland) } \\
& \frac{\mathrm{kb} \text { (given, prop, every, gland, produce, hormone) }}{\mathrm{kb}(\text { deriv, prop, every, pancreas, produce, hormone) }} \\
& \frac{\mathrm{kb} \text { (given, prop, every, betacell, produce, insulin) }}{\mathrm{kb}(\text { given, prop, every, insulin, isa, hormone) }} \\
& \frac{\mathrm{kb}(\text { deriv, prop, every, betacell, produce, hormone) }}{}
\end{aligned}
$$

The rules are illustrated in figure 2 . The inheritance rule provides inheritance to all sub-concepts of a concept $\mathrm{C}$, while the generalization rule admits generalization of an ascribed property. A special case, the monotonicity rules provide transitivity of isa with the relation $R$ being isa.

The two monotonicity rules above apply to the form every $C R D$. The logic calls for corresponding rules to the form some $C R D$.

Recalling existential import, we get an overlap rule:

$$
\frac{\text { every } C \text { isa } D_{1} \quad \text { some } C \text { isa } D_{2}}{\text { some } D_{1} \text { isa } D_{2}}
$$

as well as negative sentences:

$$
\frac{\not \forall \text { some } C R D}{\text { no } C R D}
$$

As a special case we get the non-overlap rule where $R=$ isa:

$$
\frac{\nvdash \text { some } C \text { isa } D}{\text { no } C \text { isa } D}
$$

The conclusions obtained in rules 7 and 8 by means of negation by non-provability are not added to knowledge base.

\subsection{Subsumption}

The presence of if-and-only-if definitions in the knowledge base, indicated by the use of definitions for compound terms, calls for the following subsumption rule:

$$
\frac{D \text { isa } D 1 \otimes D R D 2 \quad C \text { isa } D 1 \quad C R D 2}{C \text { isa } D}
$$

where $\otimes$ indicates the compound term definition as in the decomposition of the form $D$ isa $D 1$ that $R D 2$. The rule is shown in graph form in figure 3(a). This subsumption rule yields a derived concept inclusion proposition depicted as a dashed arc in figure 3. Due to the if-and-only-if definition of D, it holds that for any concept $\mathrm{X}$ such that $\mathrm{X}$ isa $\mathrm{D} 1$ and $\mathrm{X}$ is $\mathrm{R}$ related to $\mathrm{D} 2$ we must have that $\mathrm{X}$ isa D. 

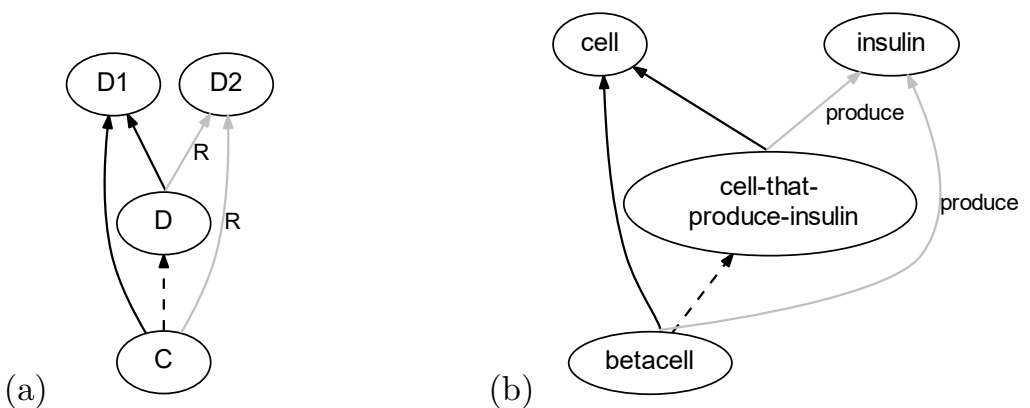

Figure 3. The subsumption rule with an example. Dashed relations are inferred.

An example of application of this rule is :

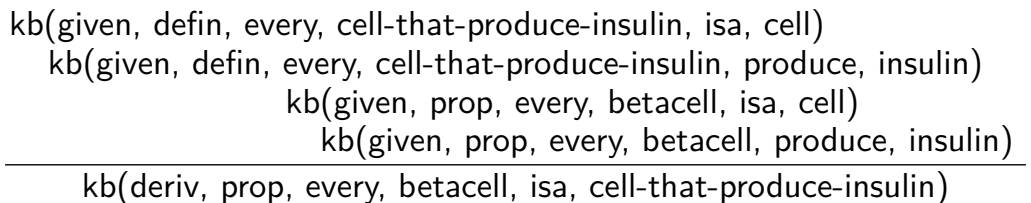

this example is also shown in graph form in figure 3(b)

\subsection{Materialization}

All concepts potentially contributing to the answer of a query are to be made explicit in the knowledge base. To achieve this, we now introduce materialization inference rules for integrating new concepts. For instance, from cell and produce and hormone we can construct the constant cell-that-produce-hormone. We distinguish two materialization cases for pairs of definitional arcs and for pairs of non-definitional arcs. The following rule take care of pairs of definitional arcs.

$$
\frac{A \text { isa } B \otimes A R C \quad C \text { isa } D}{\langle B \text {-that- } R \text { - } D\rangle \text { isa } B \otimes\langle B \text {-that- } R-D\rangle R D \quad A \text { isa }\langle B \text {-that- } R \text { - } D\rangle}
$$

Where $B$-that- $R$ - $D$ is a new concept positioned as illustrated in figure 4 that also includes a concrete example where the materialized concept is cell-that-producehormone. Observe that in the graph rendition pairs of definitional arcs, unlike non-definitional arcs, meet in their starting points.

An example of tuples inferred by materialization for definitional arcs is the following:

$$
\begin{gathered}
\mathrm{kb}(\text { given, defin, every, betacell, isa, cell) } \\
\mathrm{kb}(\text { given, defin, every, betacell, produce, insulin) } \\
\mathrm{kb}(\text { given, prop, every, insulin, isa, hormone) } \\
\frac{\mathrm{kb}(\text { deriv, defin, every, cell-that-produce-hormone, isa, cell) }}{\mathrm{kb}(\text { deriv, defin, every, cell-that-produce-hormone, produce, hormone) }} \\
\mathrm{kb}(\text { deriv, prop, every, betacell, isa, cell-that-produce-hormone) }
\end{gathered}
$$

For the case of non-definitional arcs of concepts we have the following rule. 
(a)

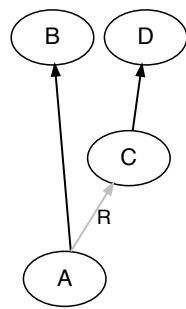

(b)

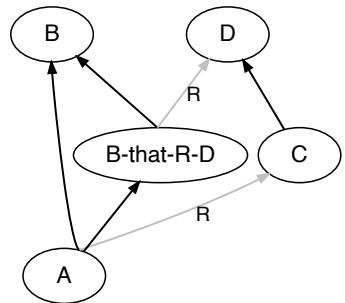

(c)

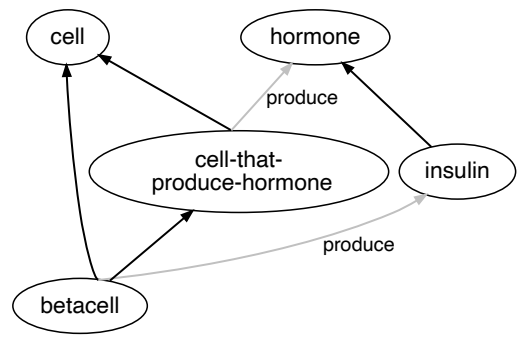

Figure 4. Materialization from definitional arcs with example. New concepts B-that-R-D and cell-that-produce-hormone

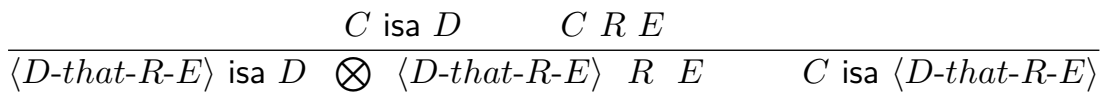

The rule is shown in graph form in figure 5, that also includes an example. The materialized concepts in the figure are D-that-R-E and cell-that-produce-insulin respectively. The example in figure 5 in inferred database tuple form is the following.

$$
\begin{aligned}
& \text { kb(given, prop, every, betacell, isa, cell) } \\
& \text { kb(given, prop, every, betacell, produce, insulin) } \\
& \frac{\mathrm{kb}(\text { deriv, defin, every, cell-that-produce-insulin, isa, cell) }}{\mathrm{kb}(\text { deriv, defin, every, cell-that-produce-insulin, produce, insulin) }} \\
& \mathrm{kb} \text { (deriv, prop, every, betacell, isa, cell-that-produce-insulin) }
\end{aligned}
$$

(a)

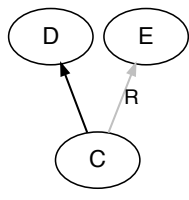

(b)

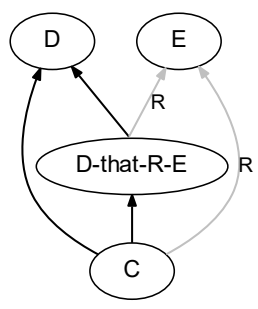

(c)

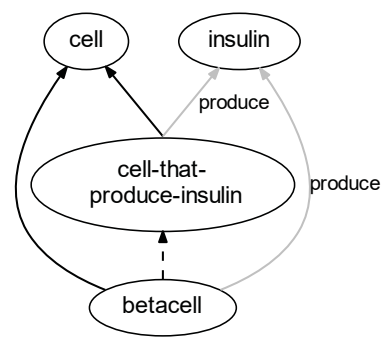

Figure 5. Materialization from non-definitional arcs with example. New concepts are D-that-R-E and cell-that- produce-hormone

\section{Implementation of the Inference rules by means of Data Base Querying}

Execution of queries in a system consisting of a knowledge base and a set of inference rules as presented in the previous section, can be prohibitively ineffective if these rules must be applied while evaluating the queries. Instead, as a preprocess before querying, the knowledge base can be extended with the relevant deductive closure, i.e. assertion of all the expressions that can be inferred from the set of inference rules over that base. 
Given the following SQL definition of the database schema to represent the knowledge base $\mathrm{kb}$ and the inverse relations inv

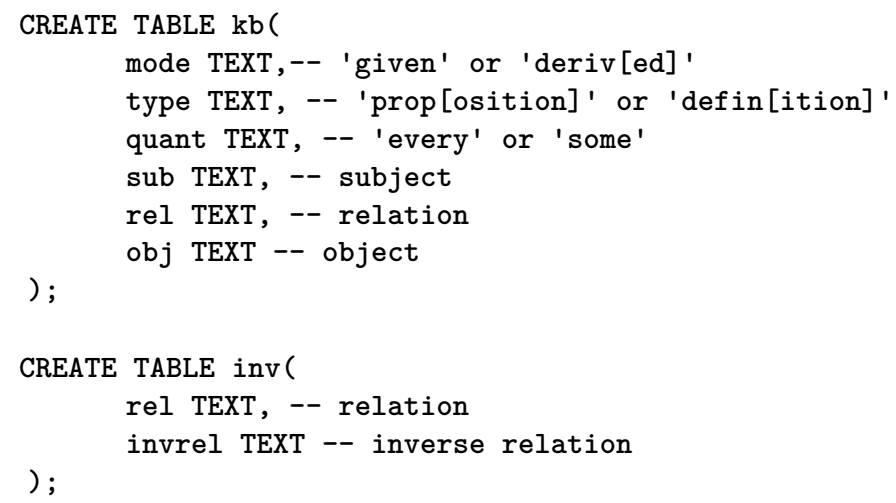

the deductive closure of the knowledge base $\mathrm{kb}$ can be achieved by insertion of all the new tuples that can be inferred from existing tuples using the inference rules, as shown in the previous section.

For the monotonicity rule (4)

$$
\frac{C_{\text {sub }} \text { isa } C \quad C R D}{C_{\text {sub }} R D}
$$

the following SQL statement inserts all tuples inferred from the tuples in $\mathrm{kb}$

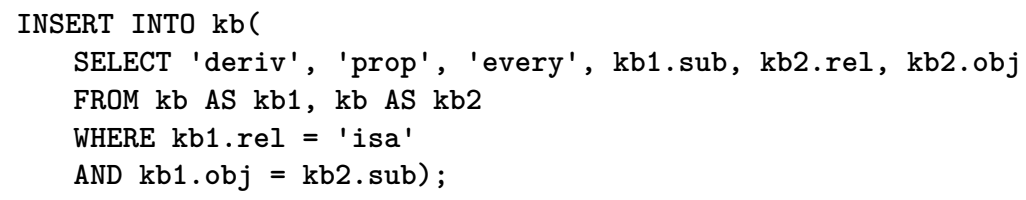

And for the subsumption rule (9)

$$
\frac{D \text { isa } D 1 \otimes D R D 2 \quad C \text { isa } D 1 \quad C R D 2}{C \text { isa } D}
$$

the following SQL statement inserts all tuples inferred from the tuples in kb excluding the cases where $D$ is equal to $C$ and $D 1$ is equal to $D 2$. 


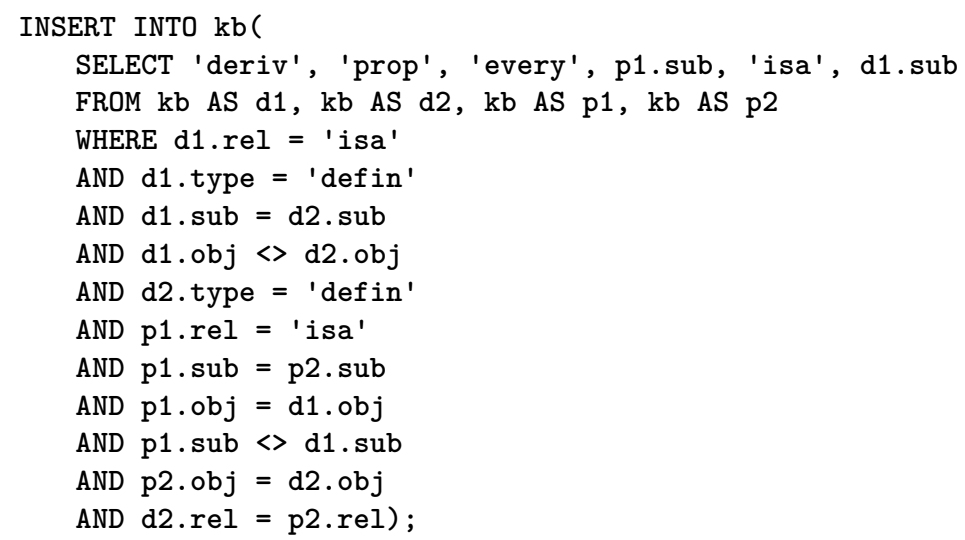

The remaning inference rules can likewise be expressed as insertion statements. Note, that the insert statements also must take into account not adding tuples that are already present in $\mathrm{kb}$, which is left out here for clarity.

Assuming a set of functions that extends the knowledge base if the inference rules can be applied, for instance, the function monotonicity_rules() to apply the two monotonicity rules. An algorithm applying all the inference rules until all possible inferences are asserted into the knowledge base would look:

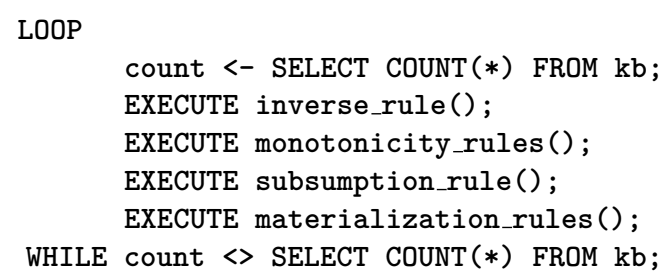

Applying this algorithm on the knowledge base in figure 6 will recursively assert all inferred tuples. The first rule to be executed in the algorithm is the inverse rule which add all the inverse edges to the knowledge base as shown in figure 7 , where the dotted lines represent the inverted relations in the form some $D R^{-1}$ some $C$ to distinguish from the every form. In the rest of the example figures in this section the inverted edges are omitted for clarity.

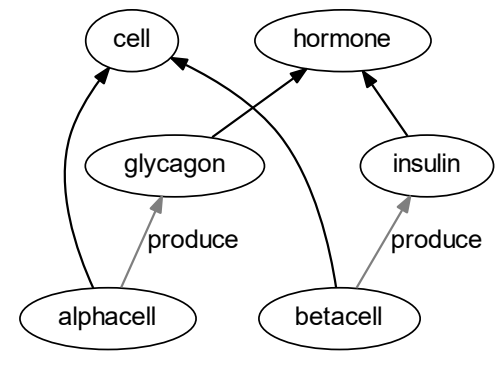

Figure 6. Knowledge base with given propositions

Figure 8 shows the result of running one iteration of the algorithm (omitting dual propositions) where the monotonicity rules add the two tuples 


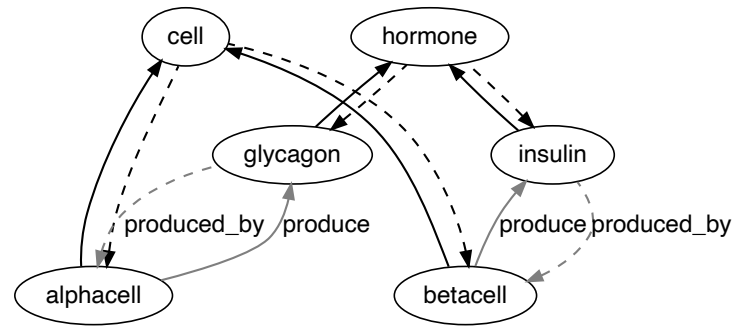

Figure 7. Applying the dual relationship rule on the knowledge base from figure 6

$\mathrm{kb}$ (given, prop, every, alphacell, produce, hormone)

$\mathrm{kb}$ (given, prop, every, betacell, produce, hormone)

and the materialization rules the following tuples

$\mathrm{kb}$ (given, prop, every, cell-that-produce-glycagon, produce, glycagon)

$\mathrm{kb}$ (given, prop, every, cell-that-produce-glycagon, isa, cell)

$\mathrm{kb}$ (given, prop, every, alphacell, isa, cell-that-produce-glycagon)

$\mathrm{kb}$ (given, prop, every, cell-that-produce-insulin, produce, insulin)

$\mathrm{kb}$ (given, prop, every, cell-that-produce-insulin, isa, cell)

$\mathrm{kb}$ (given, prop, every, betacell, isa, cell-that-produce-insulin)

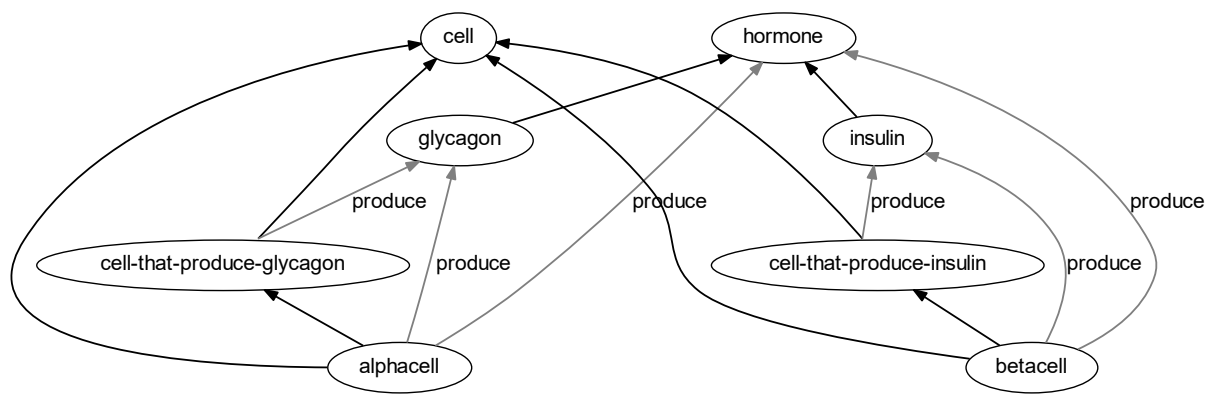

Figure 8. The first iteration of the algorithm (dual propositions omitted)

The first iteration adds in total eight new tuples (plus all the inverted edges) to the knowledge base and thus the algorithm will continue with another iteration. In this next iteration the monotonicity rule will add

$\mathrm{kb}$ (given, prop, every, cell-that-produce-insulin, produce, hormone)

$\mathrm{kb}$ (given, prop, every, cell-that-produce-glycagon, produce, hormone)

and the materialization rules will add one new concept leading to the following tuples

$\mathrm{kb}$ (given, prop, every, cell-that-produce-hormone, isa, cell)

$\mathrm{kb}$ (given, prop, every, cell-that-produce-hormone, produce, hormone)

$\mathrm{kb}$ (given, prop, every, cell-that-produce-insulin, isa, cell-that-produce-hormone)

$\mathrm{kb}$ (given, prop, every, cell-that-produce-glycagon, isa, cell-that-produce-hormone)

Here we have shown the first steps of the deductive closure, but the algorithm will continue executing all the inference rules on the knowledge base until no more tuples are added. 


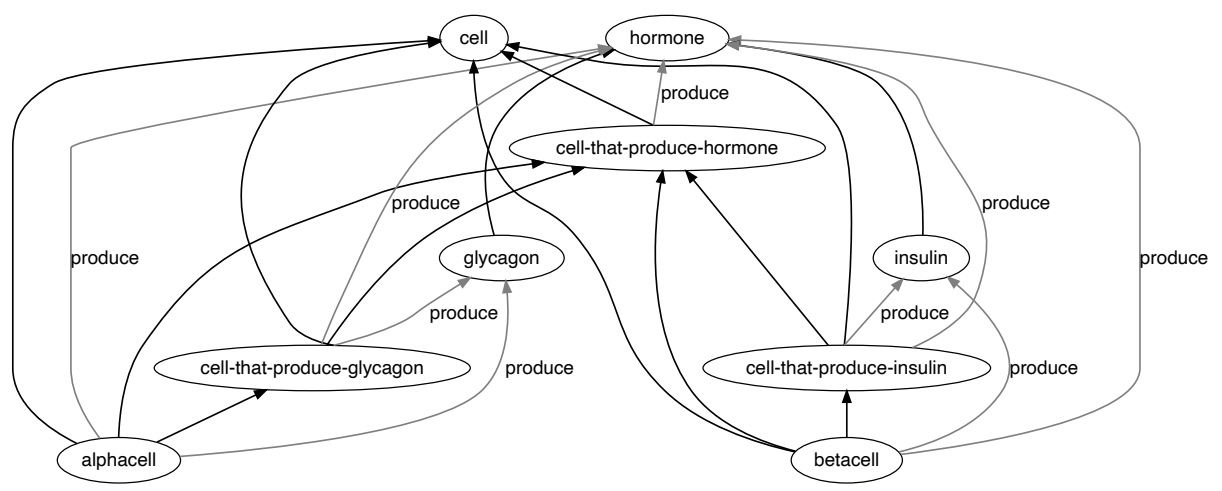

Figure 9. The second iteration of the algorithm (dual propositions omitted)

\section{Deductive Querying}

Section 4 introduced to the inference rules that apply to a NATURALOG knowledge base and gave examples of how the rules apply to specific tuples. Section 5 exemplified how deductive closure corresponding to the given inference rules can be derived through database updates that iteratively extends the database relation $\mathrm{kb}$ by adding new inferred tuples. Below we introduce different types of queries and describe how these can be evaluated by accessing the kb relation.

\subsection{Concept querying}

A basic query form is an open proposition with one or more free query variables. To query e.g. "what produce insulin" the following parameterized NATURALOG sentence can be used:

\section{$X$ produce insulin}

For the knowledge base shown in figure 9 this query would yield \{betacell, cell-thatproduce-insulin $\}$ as possible instantiations for the variable $X$, while the query $X$ produce hormone would lead to the answer \{alphacell, cell-that-produce-alphacell, betacell, cellthat-produce-insulin, cell-that-produce-hormone\}, and the query betacell produce $Y$ would lead to the answer \{insulin, hormone\}. Using a variable in the position of the relation provides similarly the possible instantiations. For instance, the query betacell $\mathrm{R}$ hormone yields \{produce\}, while $\mathrm{X} \mathrm{R}$ hormone leads to the answer \{(glycagon, isa), (cellthat-produce-hormone, produce), (cell-that-produce-glycagon, produce), (cell-that-produceinsulin, produce), (insulin, isa), (alphacell, produce), (betacell, produce)\}.

Expressions for such concept queries with one or more free variables in SQL are straightforwardly obtained from the proposition form: The first and the last of the queries mentioned above can be expressed in SQL as follows.

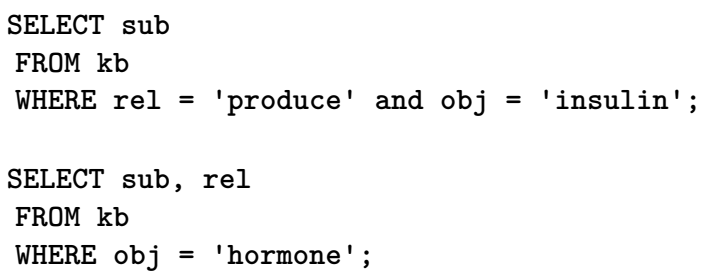


In the examples above we consider the default proposition form every $C R D$. As noticed, the indicated closures shown in figures 6,8 and 9 does not include propositions derived from the dual relationship rule that take the form some $D R^{-1} C$. This is to avoid cluttering and maintain readability especially in the two last of these figures. Figure 7, however, includes duals derived from the given propositions in figure 6 and indicates how the knowledge base expands when closuring also with respect to dual relationship. For instance the query $X \mathrm{R}$ insulin to the knowledge base in figure 7 would yield, explicating also the quantifier: $\{$ (every, betacell, produce), (some, hormone, isa) $\}$. The SQL expression to retrieve this would simply be:

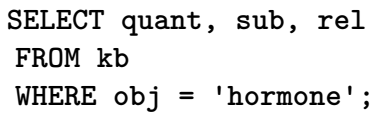

\subsection{Commonality querying}

Among the more sophisticated query forms that NATURALOG enables there is commonality querying. The commonality for a pair of stated concepts $C$ and $D$ are the properties they have in common. Considering for instance alphacell and betacell in figure 9 the commonality would be $\{$ (produce, hormone), (isa, cell), (isa, cell-that-produce-hormone) $\}$. This can be retrieved by the simple SQL expression:

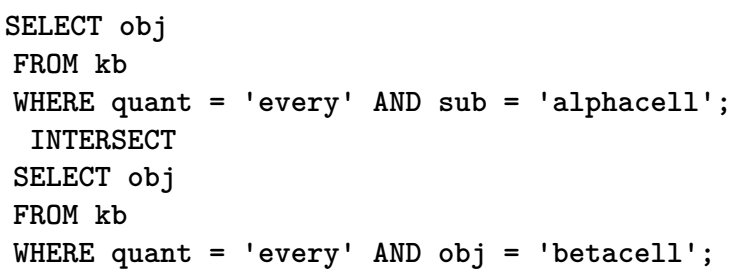

However, the most interesting contribution to the answer in this case would be the most specific part, that is, $\{(($ isa, cell-that-produce-hormone $)\}$. This can also be expressed in SQL in a straight manner, such as:

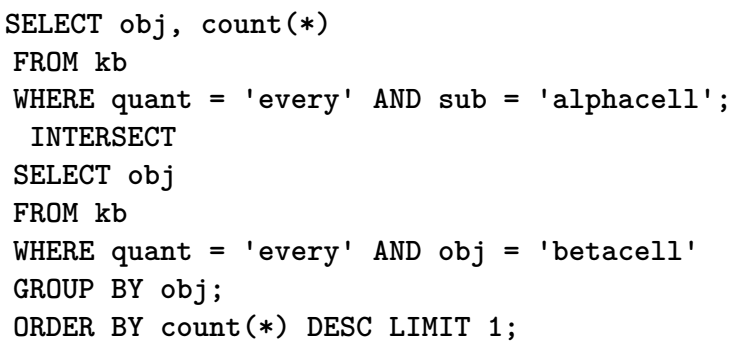

\subsection{Pathway querying}

The entire knowledge base graph forms a road map between all the applied concepts. The introduction of a universal concept at the top of the ontology ensures that all concepts are connected. This concept map can be queried by means of dedicated rules searching pathways in the graph between two stated concepts in the knowledge base. 
The pathway querying applies the given sentences suplemented with their duals, ignoring other derived propositions. A simple example is given in figure 7 .

\section{Conclusion}

In this paper we have described principles and structure for a software system that implements a dialect of natural logic for knowledge bases. This has been done by explaining how the various inference rules can be realized by relational database query facilities. The derived sentences forming a deductive closure are stored so that querying in the natural logic is conducted as a mere retrieval process. Accordingly, the system is to be implemented as a general-purpose database application. It remains to be seen whether the devised database inference engine principles, is a viable approach when scaling-up knowledge bases. 


\section{References}

[1] Troels Andreasen, Henrik Bulskov, Per Anker Jensen, and J. Fischer Nilsson. Computing pathways in bio-models derived from bio-science text sources. In Proceedings of the IWBBIO International Work-Conference on Bioinformatics and Biomedical Engineering, Granada, April, pages 217-226, 2014.

[2] J. Fischer Nilsson. In pursuit of natural logics for ontology-structured knowledge bases. In The Seventh International Conference on Advanced Cognitive Technologies and Applications, 2015. ISBN 978-1-61208-390-2.

[3] Troels Andreasen, Henrik Bulskov, Per Anker Jensen, and J. Fischer Nilsson. A system for conceptual pathway finding and deductive querying. In Flexible Query Answering Systems 2015, pages 461-472. Springer, 2015.

[4] Troels Andreasen, Henrik Bulskov, Per Anker Jensen, and J. Fischer Nilsson. Partiality, Underspecification, and Natural Language Processing, chapter A Natural Logic for Natural-Language Knowledge Bases. Cambridge Scholars, 2017.

[5] Troels Andreasen, Henrik Bulskov, Per Anker Jensen, and J. Fischer Nilsson. Pathway computation in models derived from bio-science text sources. In Marzena Kryszkiewicz, Annalisa Appice, Dominik Slezak, Henryk Rybinski, Andrzej Skowron, and Zbigniew W. Ras, editors, Foundations of Intelligent Systems, pages 424-434, Cham, 2017. Springer International Publishing. ISBN 978-3-31960438-1.

[6] Troels Andreasen, Henrik Bulskov, Per Anker Jensen, and J. Fischer Nilsson. Deductive querying of natural logic bases. In Alfredo Cuzzocrea, Sergio Greco, Henrik Legind Larsen, Domenico Saccà, Troels Andreasen, and Henning Christiansen, editors, Flexible Query Answering Systems, pages 231-241, Cham, 2019. Springer International Publishing. ISBN 978-3-030-27629-4.

[7] Johan van Benthem. Essays in Logical Semantics, Volume 29 of Studies in Linguistics and Philosophy. D. Reidel, Dordrecht, Holland, 1986.

[8] J van Benthem. Natural logic, past and future. In Workshop on Natural Logic, Proof Theory, and Computational Semantics, 2011.

[9] Lawrence S. Moss. Syllogistic logics with verbs. J. Log. Comput., 20(4):947-967, 2010.

[10] Ian Pratt-Hartmann and Lawrence S. Moss. Logics for the relational syllogistic. Review of Symbolic Logic, 2(4):647-683, 2009.

[11] Gyula Klima. Natural logic, medieval logic and formal semantics. MAGYAR FILOZÃŞFIAI SZEMLE, 54 (4):58-75, 2010.

[12] Troels Andreasen, Henrik Bulskov, Per Anker Jensen, and J. Fischer Nilsson. Natural logic knowledge bases and their graph form. submitted to journal, 2020. 34 pages, submitted to journal (under review).

[13] Troels Andreasen and J. Fischer Nilsson. Grammatical specification of domain ontologies. Data Knowl. Eng., 48(2):221-230, 2004. URL http://dblp.uni-trier.de/db/journals/dke/dke48.html. 


\title{
Global \& Geographical Mapping and Visualization Method for Personal/Collective Health Data with 5D World Map System
}

\author{
Shiori Sasaki a,1, Koji Murakami ${ }^{\mathrm{b}}$, Yasushi Kiyoki ${ }^{\mathrm{a}}$, Asako Uraki ${ }^{\mathrm{a}}$, \\ ${ }^{a}$ Graduate School of Media and Governance, Keio University, Japan \\ ${ }^{\mathrm{b}}$ PreventScience Co.,Ltd
}

\begin{abstract}
This paper presents a new knowledge base creation method for personal/collective health data with knowledge of preemptive care and potential risk inspection with a global and geographical mapping and visualization functions of 5D World Map System. The final goal of this research project is a realization of a system to analyze the personal health/bio data and potential-risk inspection data and provide a set of appropriate coping strategies and alert with semantic computing technologies. The main feature of 5D World Map System is to provide a platform of collaborative work for users to perform a global analysis for sensing data in a physical space along with the related multimedia data in a cyber space, on a single view of time-series maps based on the spatiotemporal and semantic correlation calculations. In this application, the concrete target data for world-wide evaluation is (1) multi-parameter personal health/bio data such as blood pressure, blood glucose, BMI, uric acid level etc. and daily habit data such as food, smoking, drinking etc., for a health monitoring and (2) time-series multi-parameter collective health/bio data in the national/regional level for global analysis of potential cause of disease. This application realizes a new multidimensional data analysis and knowledge sharing for both a personal and global level health monitoring and disease analysis. The results are able to be analyzed by the time-series difference of the value of each spot, the differences between the values of multiple places in a focused area, and the time-series differences between the values of multiple locations to detect and predict a potential-risk of diseases.
\end{abstract}

Keywords. Semantic Search, Semantic Computing, Medicine, Medical Data, Big Data, Biographical Data, Vital Data, Sensing, AI, Cyber-Physical System, Visualization, Data Mining, Warning, SPA, Sensing, Processing, Actuation, SDGs, SDG3, UN, United Nation,

${ }^{1}$ Corresponding Author, Shiori Sasaki*, Keio University, Endo 5322, Fujisawa, Kanagawa, Japan,; Email:sashiori@sfc.keio.ac.jp.

\section{Introduction}

"Preemptive Care" is a preventive medicine that diagnoses, predicts, and intervenes of a subject's disease by checking biologic data such as blood pressure, blood glucose, blood protein, uric acid level, BMI, CT images, personal genes, mRNA, etc.. If 
preemptive care is realized, it is possible to prevent disease by detecting abnormal values of biologic data even in cases where an individual does not have serious symptoms but have mild symptoms. In addition to suppressing rising medical and nursing care costs in the aged society, it has attracted attention because it can improve treatment results and extend healthy life expectancy.

Although the survival rate of patients of adult-onset disease and infectious disease is improving due to the emergence of new medicines and the development of new treatments, the number of those patients will continue to increase depending on the trend of globalized society, which requires the information infrastructure to support constant healthcare treatments. It has been proven in many diseases that the early detection of the symptom and prevention will increase the survival rate.

This paper presents a new knowledge base creation method for personal/collective health data with knowledge of preemptive care and potential risk inspection with a global and geographical mapping and visualization methods of 5D World Map System. The final goal of this research project is a realization of a system to analyze the personal health/bio and potential-risk inspection data and provide a set of appropriate coping strategies and alert with semantic computing technologies.

The main feature of 5D World Map System is to provide a platform of collaborative work for users to perform a global analysis for sensing data in a physical space along with the related multimedia data in a cyber space, on a single view of time-series maps based on the spatiotemporal and semantic correlation calculations.

In this application, the concrete target data for world-wide evaluation is (1) multiparameter personal health/bio data such as blood pressure, blood glucose, BMI, uric acid level etc. and daily habit data such as food, smoking, drinking etc., for a health monitoring and (2) time-series multi-parameter collective health/bio data in the national/regional level for global analysis of potential cause of disease. This application of 5D World Map System realizes a new multidimensional data analysis and knowledge sharing for both a personal and a global level health monitoring and disease analysis. The results are able to be analyzed by the time-series difference of the value of each spot, the differences between the values of multiple places in a focused area, and the timeseries differences between the values of multiple locations to detect and predict a potential-risk of diseases.

The originality of this research is in the realization of the system to collect fragmented biologic data and knowledge of healthcare and set into the systematic Sensing-Processing-Actuation (SPA) process model to analyze, share and automate the SPA calculation model and realize the functions to extract the important and essential elements of SPA for prediction from fragmented knowledge systematically.

\section{Global and Geographical Visualization of Health Data with 5D WMS in SPA Process model}

5D World Map System [1][2][3] is a knowledge representation system that enables semantic, temporal and spatial analysis of multimedia data and integrates the analyzed results as 5-dimentional dynamic historical atlas (5D World Map). The composition elements of 5D World Map are a spatial dimension (3D), a temporal dimension (4D) and a semantic dimension (5D).

A semantic associative search method [4] is applied to this system for realizing the concept that "semantics" of words, documents, multimedia, events and phenomena vary 
according to the "context". The semantics of each target multimedia data regarding to any events, phenomena or topics are calculated on a multidimensional vector space and represented as one dimensional ranking on a time-series world map space. The main feature of this system is to create various context-dependent patterns of social stories according to user's viewpoints and the diversity of context in phenomena dynamically.

The main feature of this system is to create infinite patterns of stories or scenarios, according to user's viewpoints dynamically. Semantically evaluated and analyzed multimedia data are mapped onto a time-series multi-geographical space dynamically. When the users set up conditions and inquire them into the system, the system evaluates all the multimedia data connected to the system by means of spatial, temporal and semantic computation functions, and integrates these results of dynamic multi-contextual computation onto a set of chronologically-ordered world maps. The basic concept of the 5D World Map System is shown in Figure 1.

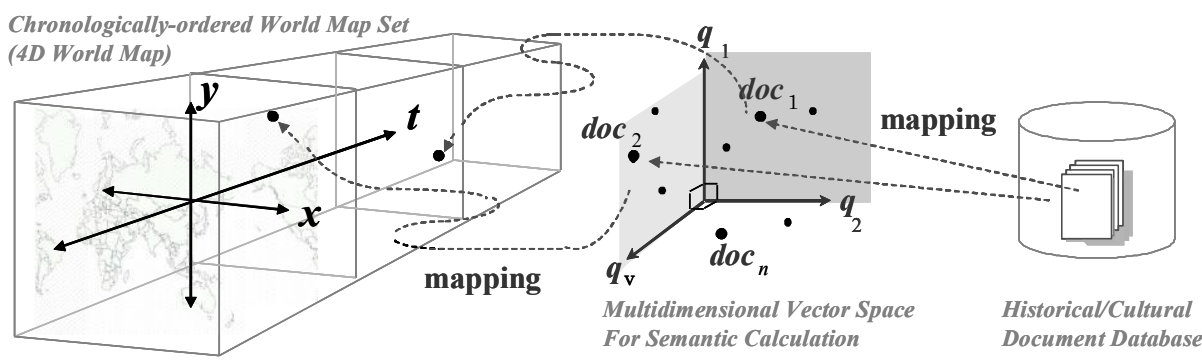

Figure 1. Conceptual Overview of 5D World Map System [1]

5D World Map System [1][2][3] has been providing various functionalities to share and visualize various types of multimedia data [5][6][7][8]. A combination of the analysis and visualization functions for multimedia and real-time sensing-data of 5D World Map System has been proposed to make environmental analysis much richer and deeper, which contributes to activities of collaborative environmental knowledge creation [5][6]. Also, a multi-dimensional and multi-layered visualization and Monitoring-Analysis-Warning functions of 5D World Map System for building disaster resilience has been proposed for monitoring Sustainable Development Goals in United Nations ESCAP [8].

In this project of preemptive care with 5D World Map System, we apply these analytical visualization functions in the SPA (Sensing-Processing-Actuation) process as shown in Figure 2. 


\section{Sensing}

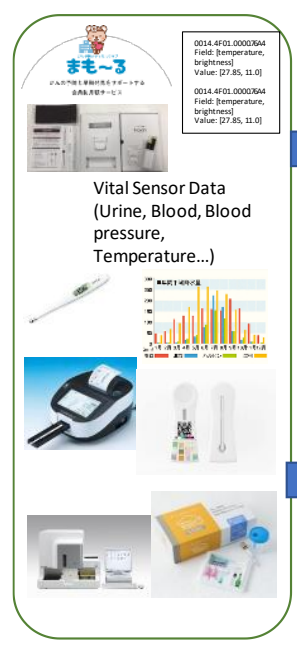

\section{Processing}

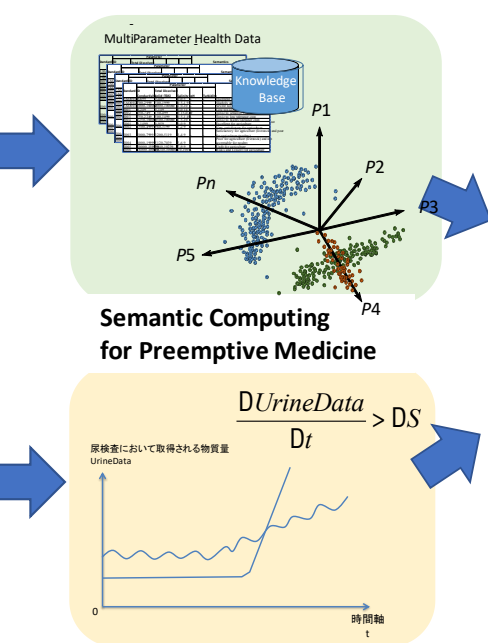

Differential Computing with Urine Data

\section{Actuation}

Geographical Mapping \& Visualization

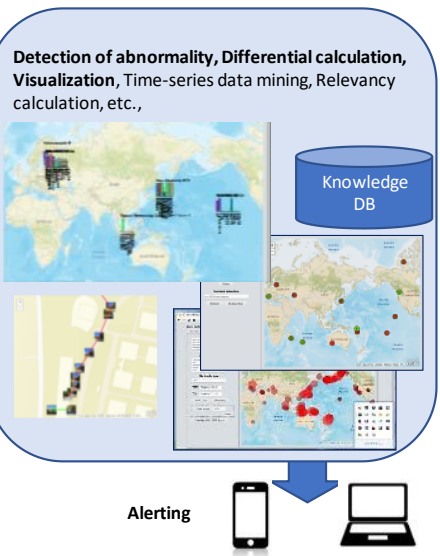

Figure 2. SPA process of 5D World Map System for Preemptive Care

\section{Implementation}

In this section, we introduce multiple applications of global and geographical mapping and visualization of personal/collective health/bio data with 5D World Map System to show the feasibility of the method. The applications are 1) Spatiotemporal Analysis with Single Parameter Visualization: the case of "Coronavirus disease (COVID-19)" infection spreading around the world (2020), 2) Spatial Analysis with Multiple Parameters Visualization: the case of "Oesophagus Cancer" - drinking habit relation around the world (2018), 3) Demographical and Seasonal Analysis with Multi-layered Visualization: the case of "Dengue Fever" in Surabaya, Indonesia (2007 - 2012), 4) Personal Action-History Analysis: the case of "Infectious-disease danger-zone" in Honolulu, Hawaii, 5) Spatiotemporal Document-based Analysis: the case of "Avian Influenza" around the world (2007-2017). By showing these multiple applications, we will examine the feasibility and applicability of 5D World Map System for Preemptive Care in the field of health care.

\subsection{Spatiotemporal Analysis with Single Parameter Visualization: "Coronavirus disease (COVID-19)" infection spreading around the world (2020)}

To examine the feasibility of applying spatiotemporal analysis with single parameter visualization function of 5D World Map System to analyze the time-series change of geographical distribution of infectious disease, we focused on the early stage of emergent disease "COVID-19" and collected the daily number of infected people around the world from Jan. to March 2020 from WHO [9], AFP BB [10] and Nikkei [11].

In the case of COVID-19 analysis in SPA process model, the important elements of SPA process in national level are defined as below. 
Sensing: the daily number of total confirmed cases, total confirmed new cases, total death, total new death, transmission classification, total PCR test, total antibody test, total antigen test, positive rate, effective reproduction number, etc.

Processing: calculation of increasing rate

Actuation: prediction of the next stage, visualization of the current stage

In this preliminary experiment, we tried to focus on the visualization of the early stage of COVID-19 spreading trend without Processing process. Figure 3 (a) - (h) shows the temporal change of the geographical distribution with the number of total confirmed cases of COVID-19 from Jan. 20 to March 14, 2020 by every 3 days.

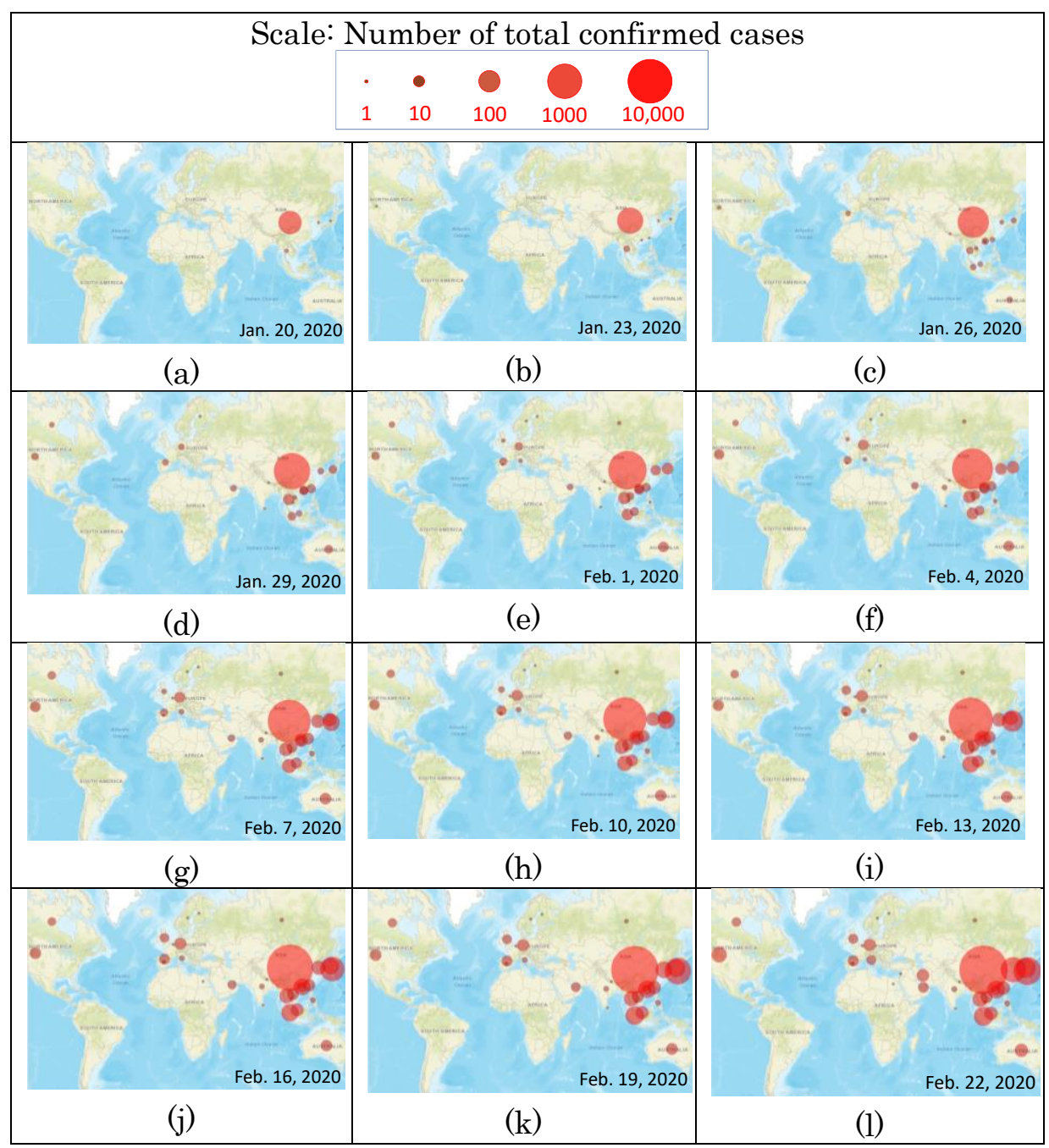




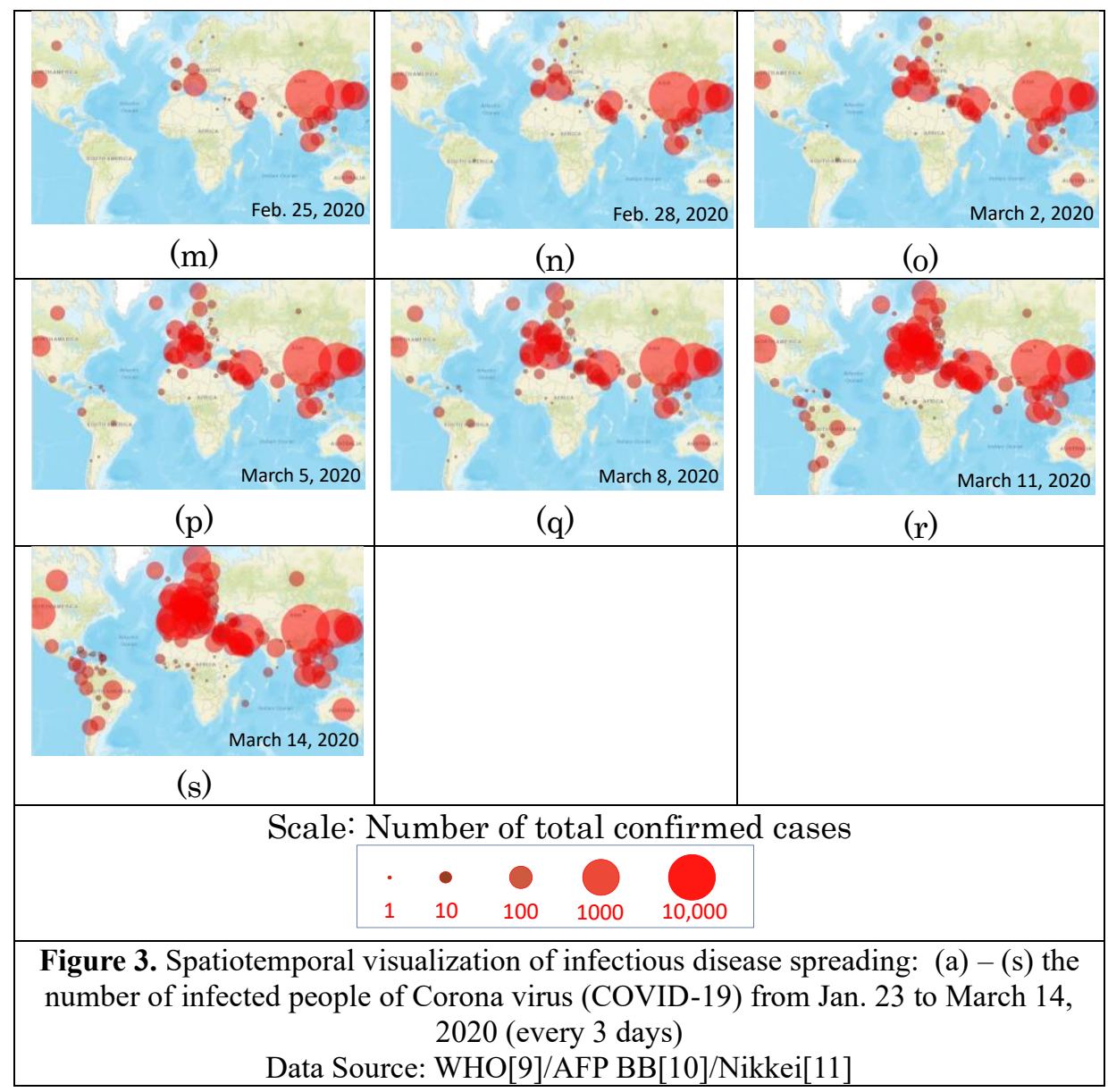

From Figure 3 (b) Jan. 23 and (c) Jan. 26, we can find that the number of confirmed cases of COVID-19 in China was increased from 571 to 2,744, and the cases outside mainland China was expanded to South-East Asia countries. From (d) Jan. 29, (e) Feb. 1 and (f) Feb. 4, we observe that the number of cases in China was increased from 7,711 to 20,438, and the cases outside China was spread to South Asia and Europe. From (g) Feb. 7, (h) Feb. 10 and (i) Feb. 13, it is observed that the number of cases in a cruise ship anchored off the shore of Yokohama Port in Japan was increased from 61 to 218 while that of other countries was not very much increased, and the number of cases in China was increased from 31,147 to 58,761 .

As shown in Figure 3 from (i) February 13th to (s) March 14th, it can be confirmed that the infected areas were spread to the Middle East, Central and South America, Africa, and Eastern Europe and Northern Europe. The characteristics are found not only the number of cases but also in the geographical spread. The number of countries and regions where new infections have been confirmed is increasing explosively.

In addition, the important point that can be discovered by these observations is not the grasp of the countries / regions that have undergone large changes such as the rapid increase in the number of confirmed cases, but the "danger of lurking in the countries / regions without changes of numbers" as a sign. It is easy to confirm by daily 
news reports and WHO reports for areas where the number of confirmed cases and death has increased sharply, whereas there are few reports about areas where the number of confirmed cases has little or no change in the early stage (E.g. Italy: Feb.1 =1, Feb. $7=$ 3, Feb. $22=9$, Russia: Feb. $22=2$, March $8=3$, March $11=11$, March $14=34$ )

From these results, in the case of COVID-19 analysis, there can be an assumption that the countries / regions where the number of infected people continues to increase at a constant rate, especially in developed countries, can be considered to be the countries / regions where the infection is controlled to some extent. On the contrary, it can be assumed that the developed nations, where there is no or almost no change in the number, suggests that the actual situation cannot be grasped accurately by the tests or inspection. This can be used to predict the trends of the second and third waves that may occur in near future.

\subsection{Spatial Analysis with Multiple Parameters Visualization: "Oesophagus Cancer" - Drinking habit relation around the world (2018)}

To examine the feasibility of applying spatial analysis with multiple parameters visualization function in the national/regional level to analyze the cause-effect relations between disease and daily habitat, we focused on the "Oesophagus Cancer".

It is known that there is a cause-effect relation between a smoking habit and lung/voice-box cancer. Also, it is said that there is cause-effect relation between a drinking and smoking habit and oesophagus/digestive system cancer, and Helicobacter pylori (HP) infection and stomach cancer.

In the case of the analysis of oesophagus/digestive system cancer in SPA process model, the important elements of SPA process are defined as below. In the case of stomach cancer analysis in regional/national level, the detection of HP (by urine/blood test) is added to Sensing process.

Sensing: alcohol consumption per person in the year, share of adults who drank alcohol in the year, share of drinkers who have had a heavy drinking session, share of adults who smoke, daily cigarette per smoker, etc. (Ref: [12])

Processing: calculation of correlation between sensed data and the confirmed/estimated disease cases, estimation of increasing rate

Actuation: prediction of the next stage, visualization of the current stage

In this preliminary experiment, we collected the share of drinkers who have had a heavy drinking session in past 30 days, 2010 from Our World in Data [12] and the estimated number of new oesophagus cancer cases in 2018, both sexes, all ages from WHO Cancer Today [13]. Figure 4 shows the geographical mapping and visualization results of the share of heavy drinkers and estimated number of new oesophagus cancer cases in national/regional level without Processing process.

Figure $4(\mathrm{a})(\mathrm{d})(\mathrm{g})(\mathrm{j})$ show the share of heavy drinkers of each country in Europe, South-East Asia, Africa and Latin America respectively by red circles, and Figure 4 $(b)(e)(h)(k)$ show the estimated number of oesophagus cancer cases in each country in each area by yellow circles. Then, Figure 4 (c)(f)(i)(l) show the layered visualization of both parameters of the share of heavy drinkers and estimated number of new oesophagus cancer cases. 


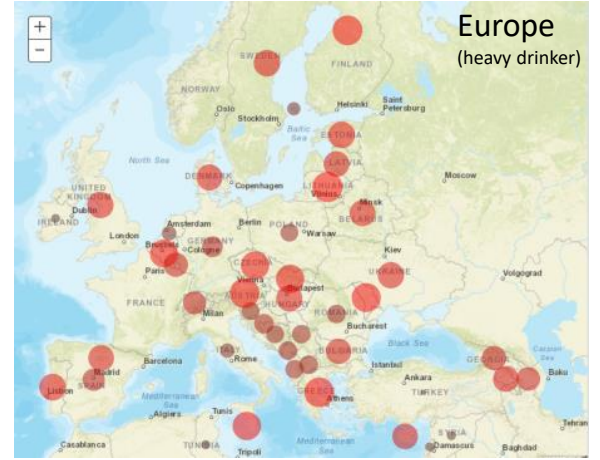

(a)

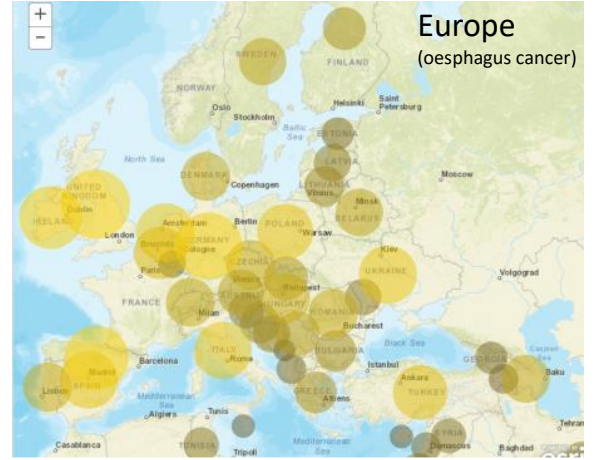

(b)

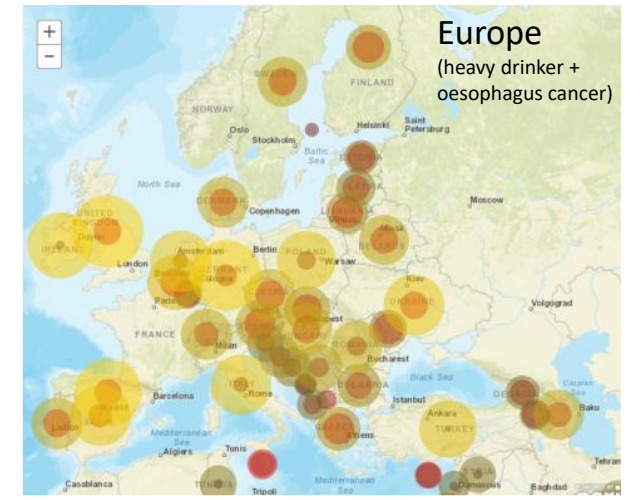

(c)

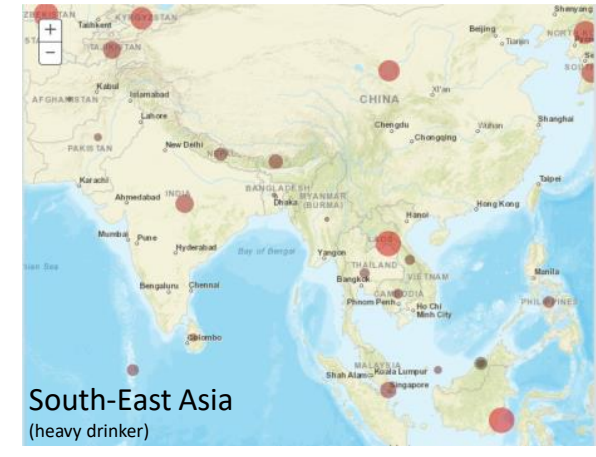

(d)

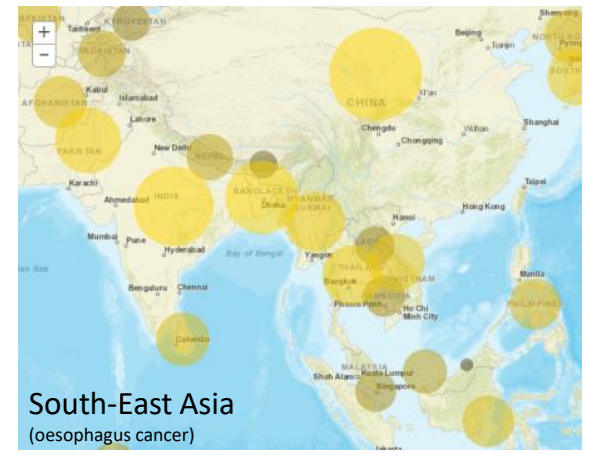

(e) 


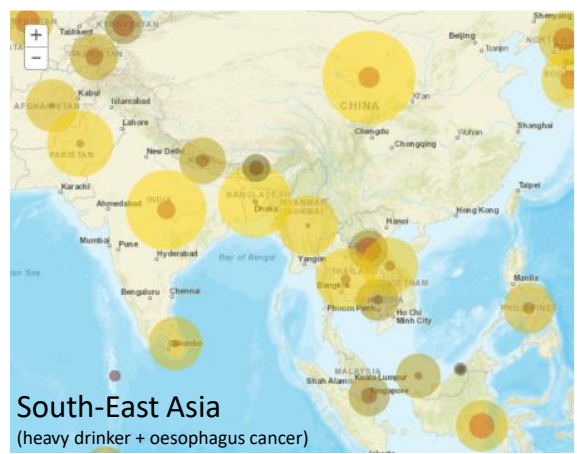

(f)
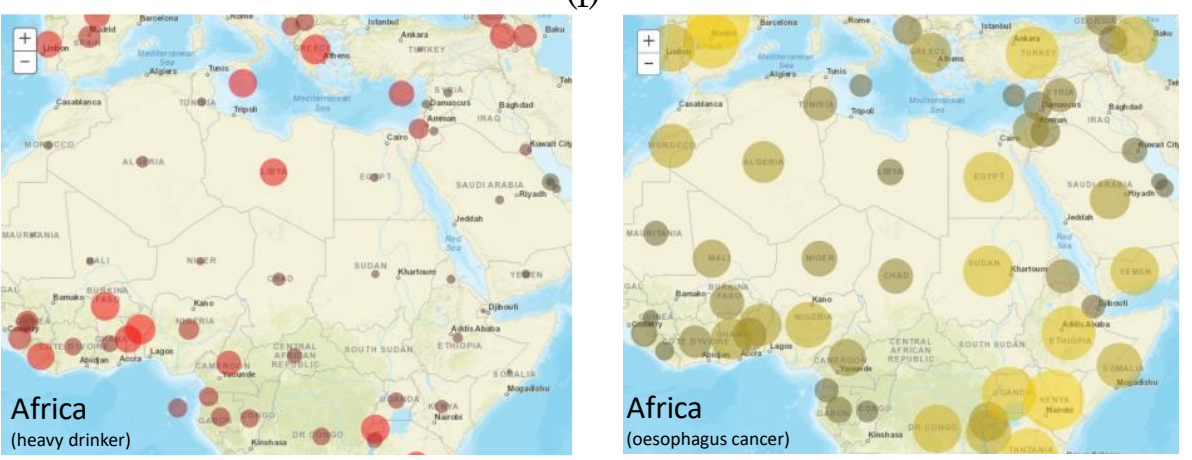

(g)

(h)

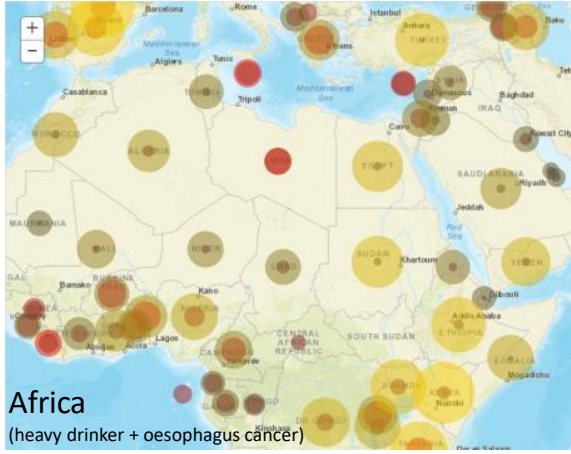

(i) 


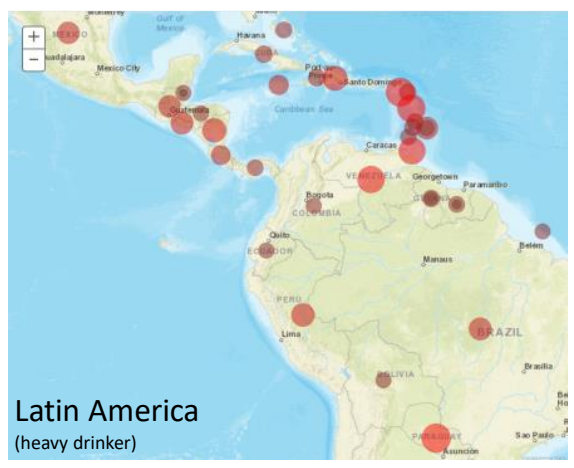

(j)

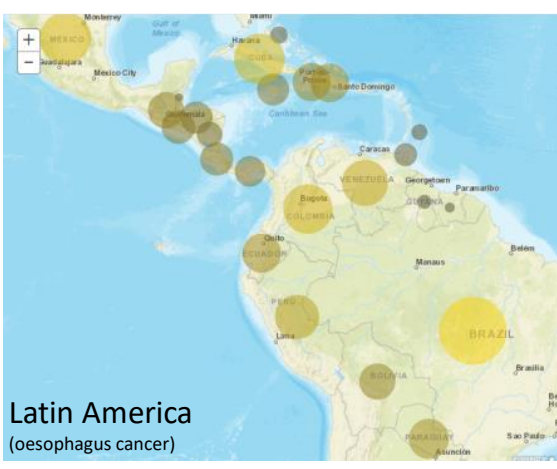

(k)

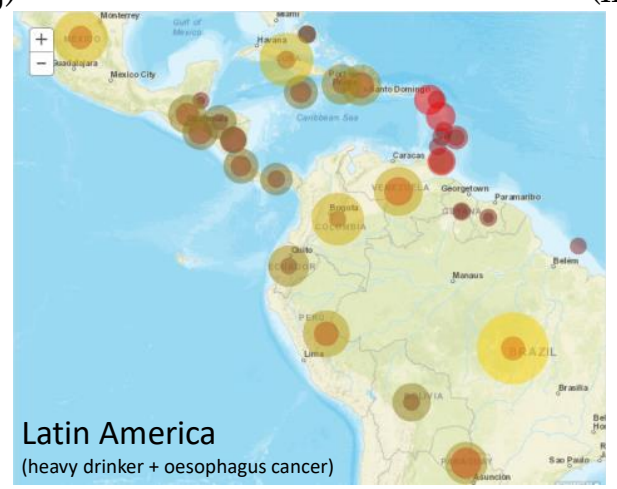

(l)

Figure 4. Geographical mapping and visualization results of the share of heavy drinkers and the estimated number of new oesophagus cancer cases in national/regional level: $(a)(d)(g)(j)=$ the share of heavy drinkers of each country in Europe, South-East Asia, Africa and Latin America respectively by red circles, (b) (e) (h) $(\mathrm{k})=$ the estimated number of oesophagus cancer cases in each country by yellow circles, and $(c)(f)(i)(1)=$ the layered visualization of both parameters.

Contrary to expectations, the results did not show a strong cause-effect relation between the share of heavy drinkers and the estimated number of oesophagus cancer cases as a whole. From Figure 4 (a)(b)(c) of Europe and (j)(k)(l) of Latin America, we can find some countries which have correlating trend of both parameters, whereas other countries do not have any correlations between them. For example, Poland, Germany, UK, Ireland, Italy, Spain in Europe and Brazil, Colombia, Cuba, Mexico have a high estimated number of oesophagus cancer cases although their shares of heavy drinkers are not so high. From Figure 4 (d)(e)(f) of South-East Asia and (g)(h)(i) of Africa, totally opposite trend is observed. The countries without high share of heavy drinkers such as China, India, Myanmar, Bangladesh, Pakistan, Afghanistan and the countries in the south-eastern Africa have high estimated number of oesophagus cancer cases.

From these results, we reaffirm that a cause-effect and correlation analysis of the disease cannot be done simply. We may need to consider and examine the other factors/parameters such as personal anamnestic history of other diseases and sociocultural daily habit such as dietary, smoking, fitness etc. from various contexts. 
3.3. Demographical and Seasonal Analysis with Multi-layered Visualization: "Dengue Fever" in Surabaya, Indonesia (2007 - 2012)

To show the feasibility of applying multiparameter visualization function to analyze the demographical and seasonal analysis of infectious-disease spreading phenomena in a local level, we introduce the mapping results of infectious disease "Dengue Fever" with multimedia, sensor data and demographical data collected from Surabaya local government and EEPIS-HDRC, Indonesia from 2007 to 2012 [14] .

In the case of dengue fever analysis in SPA process model, the important elements of SPA process in national/local level are defined as below.

Sensing: 1. Humidity, 2. Precipitation, 3. Number of media (mosquitoes), 4. Geolocation of places with high population density (open-air market, mall, school, hospital, etc.)

Processing: Calculation of area's risk degree of spreading by sensing data $1,2,3$ and 4 .

Actuation: Spatiotemporal visualization of high-risk area and geographical indication of high-risk spots, Prediction of annual trend of spreading area and season

In this preliminary experiment, we tried to focus on the geographical visualization of the number of infected cases in the local districts without Processing process.

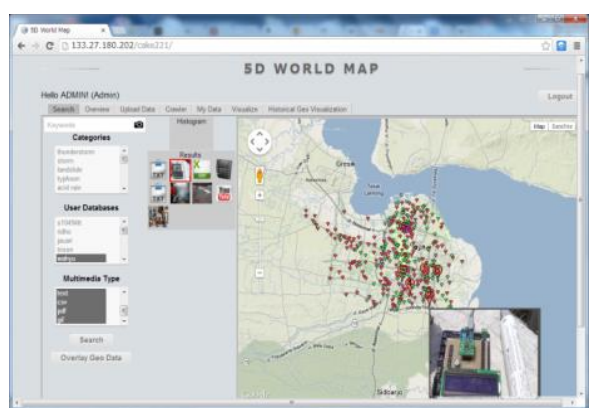

(a)

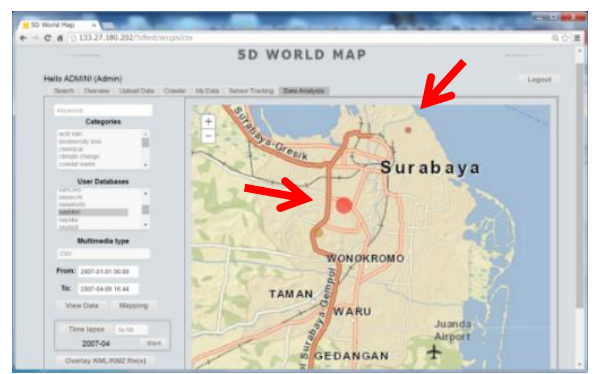

(c)

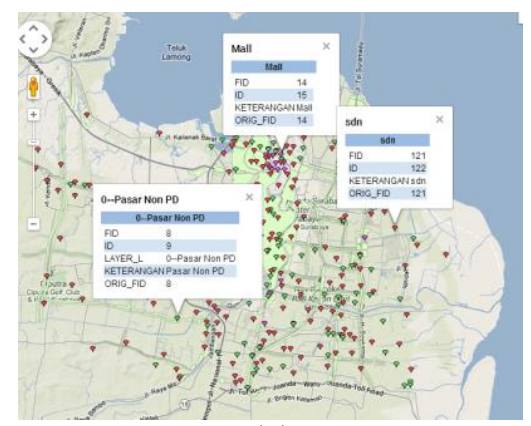

(b)

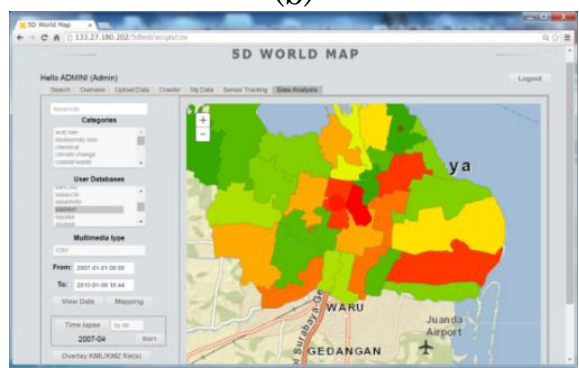

(d) 


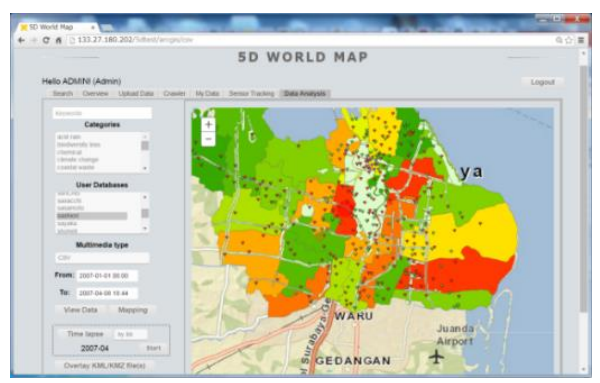

(e)

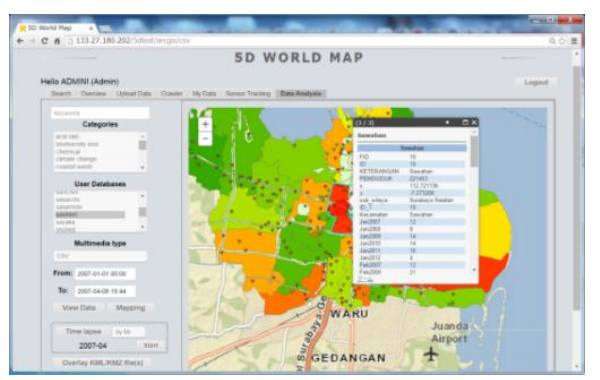

(f)

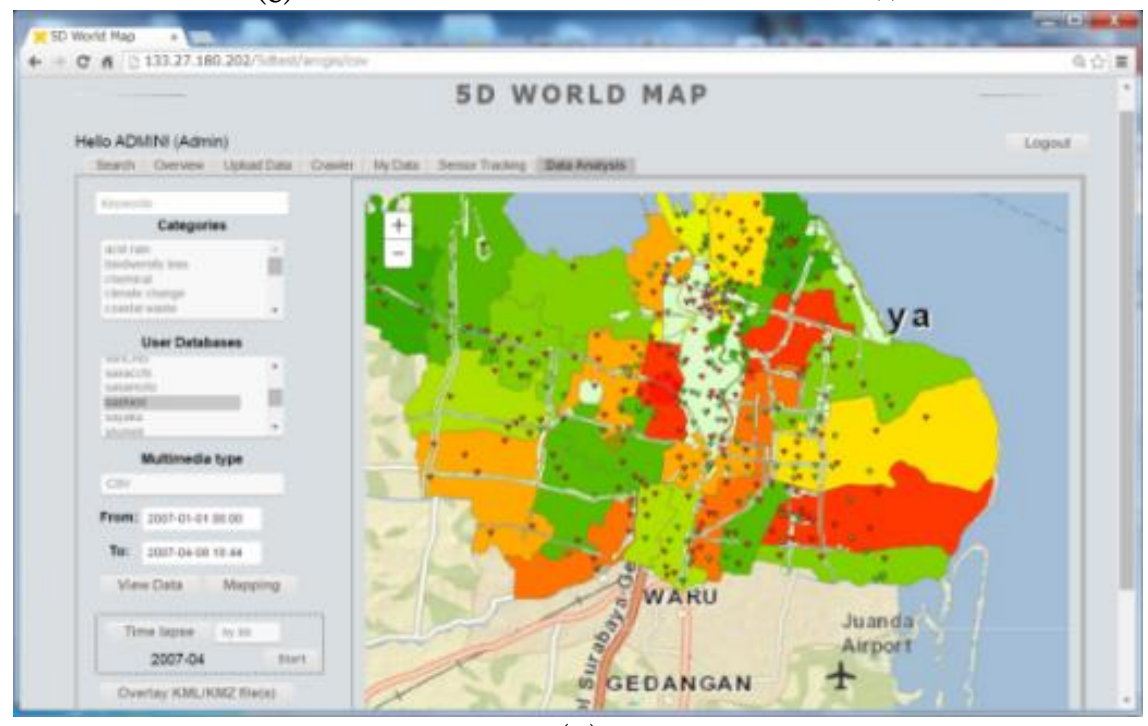

(e')

Number of infected cases

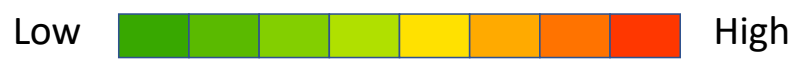

School (the place where young people get together)

$\square$ Outdoor Market (the place where people may contact with mosquitos)

$\square$ Shopping Mall (the place where a lot of people get together)

Figure 5. Mapping and overlaying results of statistical data: number of infected cases of dengue: (a) socio-cultural data, multimedia and sensor data related to dengue fever, and (b) geographical distribution of school, residence, hospital, and industrial districts, and the information of human contact, (c) the number of infected cases in each district with variable-size markers with continuous colors (Marker size $=$ number of infected cases, marker color: dark $=$ small number, bright $=$ large number), (d) the same infected cases' number data in KMZ files overlapped onto the map (District color: red=high density, yellow=medium, green=low density), (e) the

overlapping results of several KMZ files and a KML file of geographical and human-contact information, and (f) the interface to show the detailed information of each area by raw data [14]. 
Figure 5 (a) shows the mapping and overlying results of various types of data: sociocultural data, multimedia (image, text and movie) and sensor data, and the combination of image data about weather sensor and sociocultural information related to drive contagious among people. Figure 5 (b) shows different feature classes of the area. Points represent the buildings of school, market, mall, and hospital, and polygon represents the area of industry or resident. It is overlaid in shape file and converted to KML/KMZ (Keyhole Markup Language) file type for 5D mapping. Both (a) and (b) are the information infrastructure for the following spatiotemporal analysis of dengue spreading phenomena.

Figure 5 (c) shows the number of infected cases in each district in variable-size markers with continuous colors by the analytical visualization function of statistical and sensing data, and (d) shows the same data as a density of infected cases by KMZ files processed in the common GIS software, ArcGIS, and overlapped onto the map by the historical-geographical information visualization function. Figure 5 (e) shows the overlapping results of several KMZ files and a KML file of geographical and humancontact information, and (f) shows the interface to show the detailed information of each area by raw data.

From Figure 6 of monthly visualization of the number of infected cases, we can observe that the areas with many infected cases (yellow to red) are increasing from January to March, April, May, and then those are decreasing from June to December. This trend represents the relation between seasonal weather (dry season and rainy season) to the number of infected cases.

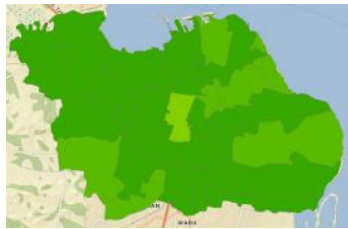

Jan. 2007

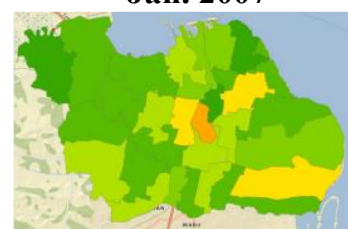

April 2007

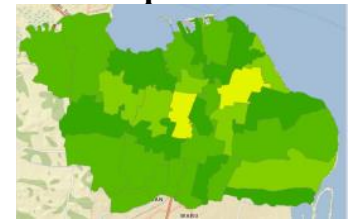

July 2007

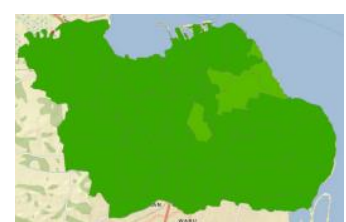

Oct. 2007

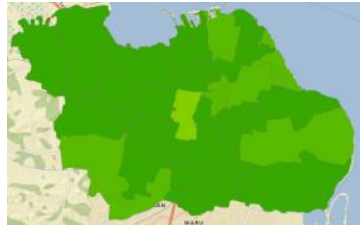

Feb. 2007

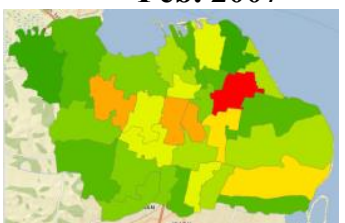

May 2007

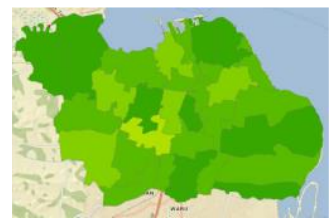

Aug. 2007

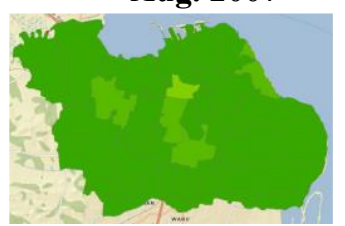

Nov. 2007

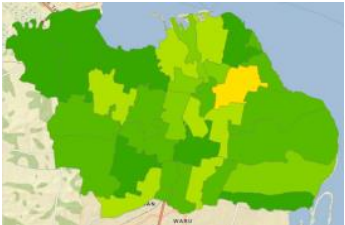

March 2007

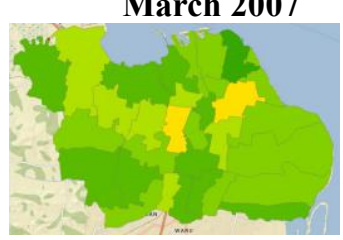

June 2007

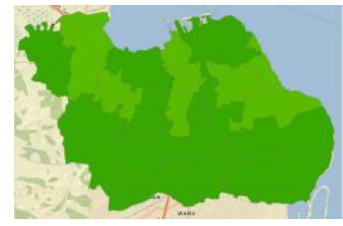

Sep. 2007

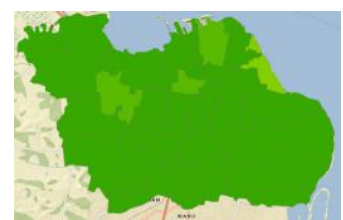

Dec. 2007 


\section{Low}

Figure 7. Visualization results of monthly change of the number of infected cases of Dengue fever in 2007 by KMZ mapping [14]

Next, we focused on the same seasons - April (rainy season) and November (dry season) - in every year. The results are shown in Figure 8. From the results, we can observe that there are not very many cases in both April and November in 2011 and 2012. We can presume that this phenomenon happened because in 2011 and 2012, dry season in Surabaya is longer than wet season. Water is media of mosquito breeding, if the quantity of rainfall is decrease, media of breeding will shrink. As a consequence, the population of mosquito also decreases [15]. Also, it is pointed that the government controlled dengue actively more by involving local communities to remain alert to the possibility of dengue outbreak [16].

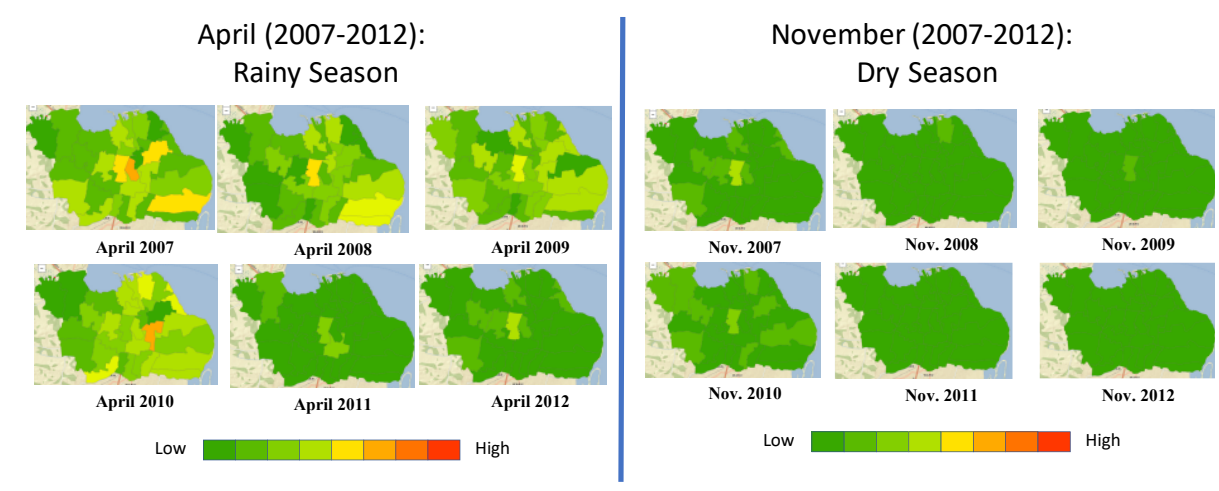

Figure 8. Comparison of the number of infected cases of dengue fever in Surabaya, Indonesia between rainy season and dry season for 2007-2012: visualization of the normalized values by KMZ [14]

Accessing and visualizing infectious disease-related data has been the challenge for awareness building. Data sources are not homogeneous and the data collection is not centralized. It is scattered across various platforms and databases at both the sub-national and national levels. The multi-dimensional and multiple contextual analysis of 5D World Map System will contribute to bring together data under one platform in order to understand the multiple factors/parameters/contexts in infectious disease analysis.

3.4. Personal Action-History Analysis: "Personal Infectious-disease danger-zone" in Honolulu, Hawaii 
In this section, we introduce our trial on mapping of personal action history to 5D World Map to examine the feasibility of health monitoring by daily habit tracking in individual level.

In the case of personal infectious-disease danger-zone analysis in SPA process model, the important elements of SPA process in individual level are defined as below.

Sensing: 1. Tracking of the route of personal movement, 2. geolocation of places where the infection occurred

Processing: Calculation of spatiotemporal overlap between 1 and 2 as a risk

Actuation: Geographical indication of personal danger-zone

In this preliminary experiment, we set two kinds of user, User A and User B and track their walking movement and the path by GPS without Processing process. Figure 9 shows the results of preliminary experiments in Honolulu, Hawaii.

In Figure 9, the walking path of User A is shown as blue line, and the walking path of User B is shown as green line. The yellow pin \#1 in the northern part of the map indicates a crowded shopping mall with high infection risks of influenza. The pin \#2 in the middle part indicates the points of a lot of mosquitos existing, and the pin \#3 in the southern part indicates the points of a lot of ants existing. From the results, we can assume that if User A stays long in the point of yellow pin \#1 and is infected to influenza, the point of yellow pin \#1 could be a point with high infection risk of influenza. In the same way, we can assume that if User B gets mosquito-borne disease and ant-borne allergy after this movement, the point of yellow pin \#2 and \#3 could be points of high infection risks of these diseases.

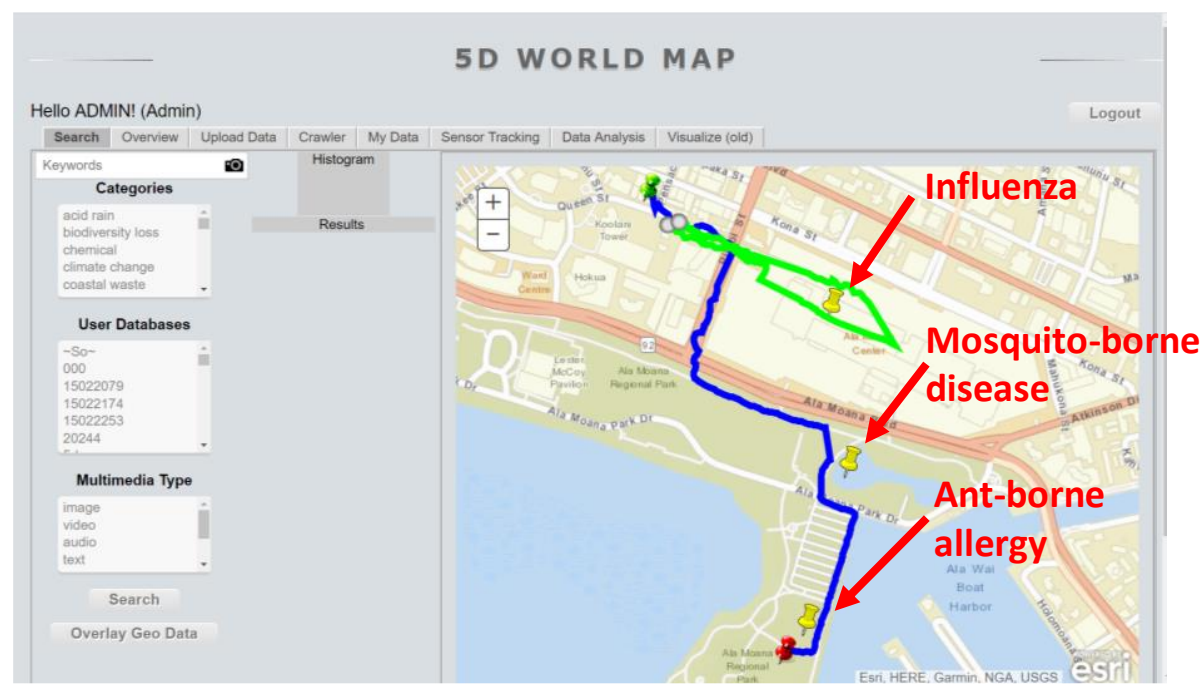

Figure 9. Visualization of personal movement tracking and the points of infection risks: Blue line $=$ walking path of user A, Green Line $=$ walking path of user B, Yellow Pins $=$ points of infection risks of \#1 influenza, \#2 mosquito-borne disease and \#3 Ant-borne allergy 


\subsection{Spatiotemporal Document-based Analysis: “Avian Influenza” around the world}

(2007-2017)

In this section, we introduce the document retrieval and visualization results [7] with 5,941 "global warming"-related news articles from 2007 to 2017 owned by Nikkei Newspaper [17] .

In the case of personal infectious-disease risk-hotspot analysis in SPA process model, the important elements of SPA process in individual level are defined as below.

Sensing: Extraction of Regional/National level (City/Country) geolocation and time of places where infection occurred

Processing: Extraction of important keywords (metadata) from newspaper articles, mapping the metadata to the semantic space of "Global Warming", and calculation of semantic correlation between the input keywords and the articles

Actuation: Spatiotemporal visualization

In this preliminary experiment, we examine the effectiveness of the spatiotemporal mapping and analytical visualization functions of 5D World Map System. Figure 10 and Figure 11 show the search and visualization results with a keyword "Avian Influenza"

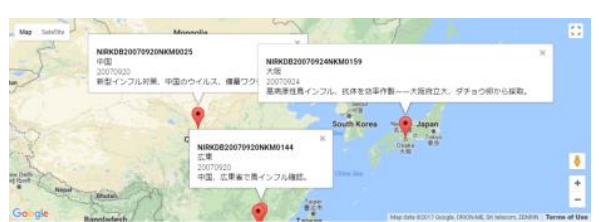

Sep., 2007: Infections were reported in China and Japan.

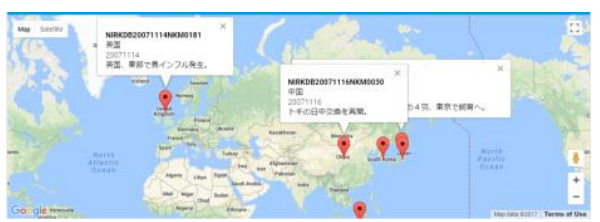

Nov., 2007: Infections were reported in Indonesia, China, Korea, Japan and Eastern UK.

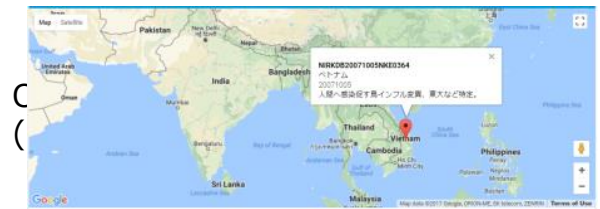

Oct., 2007: Infections were reported in Korea and Vietnam.

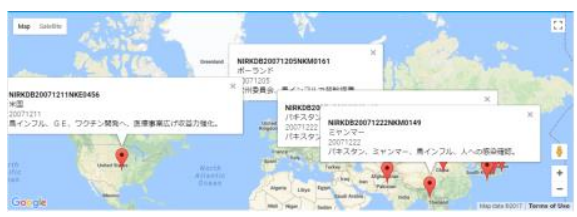

Dec., 2007: Infections were reported in Pakistan, Myanmar, China and Japan. EU decided embargo, and vaccine development was started in US.

Figure 10. Example of News articles retrieval and visualization results: Period = Sep. Dec., 2007 (Unit = Month), Keyword = “Avian Influenza” [7]

Figure 10 shows the document retrieval results for a group of articles of every month from September to December 2007. From these results, we can grasp how the infection phenomenon and the infection control strategies spread over the world: the first infection of the year was reported in China and Japan in September 2007, the infection spread to Southeast Asia and eastern United Kingdom from October to November. In December, the infection spread to South Asia, and in the EU, the European Commission decided an embargo on Dec. $5^{\text {th }}, 2007$. Finally, the vaccine development began in the United States on Dec. $11^{\text {th }}, 2007$. 


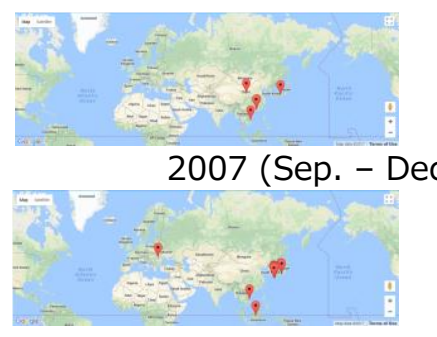

2010

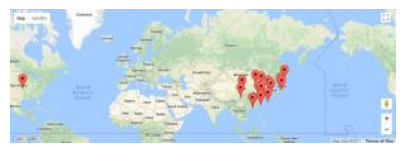

2013

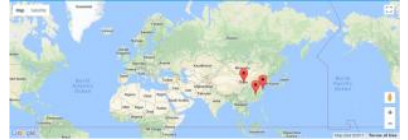

2016

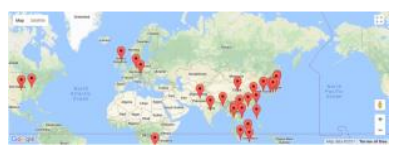

2008

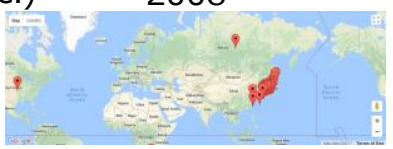

2011

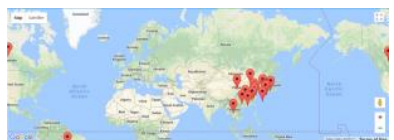

2014

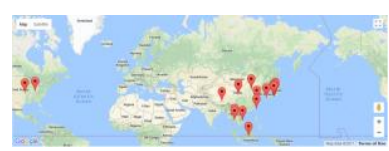

2009

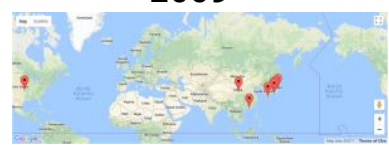

2012

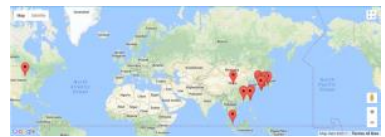

2015

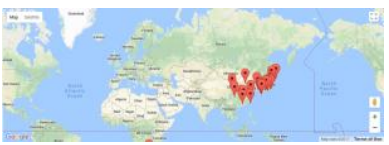

2017 (Jan. - July)

Figure 11. Example of News articles retrieval and visualization results: Period $=2007$ 2017 (Unit = Year), Keyword = "Avian Influenza" (The results indicate a trend or periodicity of the infection phenomena.)

Figure 11 shows the document retrieval and visualization results for a group of articles from 2007 to 2017 year-by-year. Based on these results, from the geographical distribution of articles and the trend of increase / decrease in number of articles per year, we can heuristically grasp the fact that the worldwide spread of infection is happened in 2008 first, and many infection cases in the East and Southeast Asia region were reported in 2011, 2013-2014 and 2017 periodically.

The results show the effectiveness of the spatiotemporal mapping and visualization functions of 5D World Map System as a tool of "Visual Datamining" as well as a verification tool of phenomena.

\section{Future Work}

In this paper, we have presented a new knowledge base creation method for personal/collective health/bio data with knowledge of preemptive care and potential risk inspection with a global and geographical mapping and visualization methods of 5D World Map System. This research project focuses on the realization of a system to analyze the personal health and potential-risk inspection data and provide a set of appropriate coping strategies with semantic computing technologies.

We have examined the feasibility and applicability of 5D World Map System with SPA process modelling for Preemptive Care by showing 5 applications: 1) Spatiotemporal Analysis with Single Parameter Visualization: the case of "Coronavirus disease (COVID-19)" infection spreading around the world (2020), 2) Spatial Analysis with Multiple Parameters Visualization: the case of "Oesophagus Cancer" - drinking habit relation around the world (2018), 3) Demographical and Seasonal Analysis with Multi-layered Visualization: the case of "Dengue Fever" in Surabaya, Indonesia (2007 - 
2012), 4) Personal Action-History Analysis: the case of "Infectious-disease danger-zone" in Honolulu, Hawaii, 5) Spatiotemporal Document-based Analysis: the case of "Avian Influenza" around the world (2007-2017).

As a next step of our project, we will develop a system to estimate a user's own health-condition, compares his/her own health condition with the selected data set to predict the risk of disease occurrence, and performs a context-dependent multiparameterized "Processing process" in the SPA architecture in 5D World Map System.

\section{Reference}

1. Sasaki, S., Takahashi, Y, Kiyoki, Y., "The 4D World Map System with Semantic and Spatiotemporal Analyzers," Information Modelling and Knowledge Bases, Vol.XXI, IOS Press, pp. 1 - 18, 2010

2. Kiyoki, Yasushi, and Xing Chen. "Contextual and Differential Computing for the MultiDimensional World Map with Context-Specific Spatial-Temporal and Semantic Axes." Information Modelling and Knowledge Bases XXV 260 (2014): 82.

3. Kiyoki, Y., Sasaki, S., Nguyen N. T., Nguyen, T. N. D., "Cross-cultural Multimedia Computing with Impression-based Semantic Spaces", Conceptual Modelling and Its Theoretical Foundations, Lecture Notes in Computer Science, Springer, pp.316-328, March 2012.

4. Kiyoki, Y., Kitagawa, T., A semantic associative search method for knowledge acquisition, Information Modelling and Knowledge Bases VI, H. Kangassalo et al eds. IOS Press 1995 pp. 121-130.

5. Sasaki, S. and Kiyoki, Y., "Real-time Sensing, Processing and Actuation Functions of 5D World Map System: A Collaborative Knowledge Sharing System for Environmental Analysis" Information Modelling and Knowledge Bases, Vol. XXVIII, IOS Press, pp. 220239, May 2016.

6. Shiori Sasaki, Yasushi Kiyoki, "Analytical Visualization Functions of 5D World Map System for Multi-Dimensional Sensing Data", Information Modelling and Knowledge Bases XXIX, IOS Press, pp.71 - 89, May 2017.

7. Shiori Sasaki, Yasushi Kiyoki, Hanako Fujioka, Toshihiro Watanabe, Kyohei Otsuka, Masayuki Ishii, "Application of 5D World Map System to Large News-Article Database for Realizing Context-Diversity-Responsive Semantic Associative Search,” Information Modelling and Knowledge Bases XXX, Proceedings of the 28th International Conference on Information Modelling and Knowledge Bases, IOS Press, pp. 276 - 292, May 2019.

8. Shiori Sasaki, Yasushi Kiyoki, Madhurima Sarkar-Swaisgood, Jinmika Wijitdechakul, Irene Erlyn Wina Rachmawan, Sanjay Srivastava, Rajib Shaw, Chalisa Veesommai, 5D World Map System for Disaster-Resilience Monitoring from Global to Local: Environmental AI System for Leading SDG 9 and 11, Information Modelling and Knowledge Bases XXXI, Proceedings of the 28th International Conference on Information Modelling and Knowledge Bases, EJC 2019, Rapperanta, Finland, 5-9 June 2019, pp. 306 - 323.

9. WHO https://www.who.int/

10. AFP BB News https://www.afpbb.com/

11. Nikkei https://vdata.nikkei.com/newsgraphics/coronavirus-world-map/

12. Our World in Data, Oxford University https://ourworldindata.org/

13. WHO Cancer Today http://gco.iarc.fr/today/

14. Sesulihatien, W. T, Sasaki, S, Kiyoki, Y, Harsono, T, Basuki, A, Safie, A, "Local-RegionalGlobal Analysis on Infectious Disease with Analytical Visualization Functions of 5D World Map System: A Case of Comparative Analysis on Dengue Fever Spreading in Indonesia and Malaysia," Malaysian Journal of Tropical Geography, June 2016.

15. Aziz Shafie, 2011, Evaluation of the Spatial Risk Factors for High Incidence of Dengue Fever and Dengue Hemorrhagic Fever Using GIS Application, Sains Malaysiana 40(8)(2011): 937-943 
16. Surachart Koyadun,2012. Ecologic and Sociodemographic Risk Determinants for DengueTransmission in Urban Areas in Thailand, Interdisciplinary Perspectives on Infectious Diseasese, Volume 2012

17. Nikkei Telecom: http://telecom.nikkei.co.jp/ 


\title{
XML Schema support in knowledge management
}

\author{
Boštjan ŠUMAK ${ }^{\mathrm{a}}$, Marjan HERIČKO ${ }^{\mathrm{a}}$ and Maja PUŠNIK ${ }^{\mathrm{a}, 1}$ \\ ${ }^{a}$ University of Maribor, Faculty of Electrical Engineering and Computer Science
}

\begin{abstract}
Well organized data contributes extensively to classification possibilities and quality of knowledge management. XML schemas play an important role in data organization activities and provide basic foundations for companies and organizations, dealing with large amounts of data. In times, where knowledge represents greatest advantage in a competitive economy and is relatively simple to find through different web providers, quality of internal data structures and efficient management of company's valuable information is of utmost importance. XML schemas are one of the mechanisms that can provide a data organization system in a qualitative manner and efficient knowledge management as soon as data is defined or accumulated. A good XML schema support is a way to increase competitiveness of an organization with ensuring structured data quality and simplifying the knowledge management process.
\end{abstract}

Keywords. Information management, Data management, Knowledge management, XML schema, XML document, Data quality

\section{Introduction}

Knowledge is a product of well-developed data and by definition, knowledge management $(\mathrm{KM})$ is an efficient handling of information and resources within an organization. It is the process of capturing, developing, sharing, and effectively using organizational knowledge [1] by making the best out of given data. It's an interdisciplinary field, an intersection of computer science and management, addressing benefits in organizational aspects of business sectors, however greatly relying on computer and information science. KM has been around since the mid-1990s and its attempts to collect, store, categorize, mine, and process data into knowledge [31], correspond to Big Data challenges (capturing data, data storage, data analysis, search, sharing, transfer, visualization, as well as querying, updating, information privacy and data source), in both cases dealing with large size data.

Managing large size data or knowledge within an organization is unimaginable without the use of advanced information systems; while the design and implementation of such systems demands great organization as well as presents several technical challenges. Another closely tied discipline is knowledge engineering, which refers to all technical, scientific and social aspects involved in building, maintaining and using knowledge-based systems, which is vital for enterprise interoperability and networking.

\footnotetext{
${ }^{1}$ Corresponding Author, Corresponding author, Book Department, IOS Press, Nieuwe Hemweg 6B, 1013 BG Amsterdam, The Netherlands; E-mail: bookproduction@iospress.nl.
} 
The paper focuses on advanced information and knowledge based systems, supporting KM. Furthermore, it is specifically focusing on the data part of IT systems, , containing the potential for creating knowledge. The data can originate in several forms, where common denominators are often XML documents and XML schemas, defining the structure of XML data. The quality of data structure is a precondition for the knowledge based system and the basis for many other disciplines such as machine learning, data mining and others. Difficulties and importance in building a qualitative data sets are addressed in this paper, focusing on XML schemas as de facto standard for data interchange [2] and an important part of information solutions, considered a prerequisite to knowledge management.

In time of e-business, data is exchanged based on XML documents, a mark-up language that defines a set of rules for encoding documents in a format that is both human-readable as well as machine-readable. Several other formats (MS Office, Open office), can be easily translated in XML files, which provide users several advantages, information and knowledge extraction being only one of them. Each XML document is defined by XML schema (https://www.w3.org/XML/Schema), the focus technology of this paper. The structure and contents of XML schema documents has a significant influence on the value of data, which indirectly influences data quality in general, presenting a challenge in the first levels of information and KM. A good XML schema leads to a higher efficiency of data usage, extraction and organization as well as simplification of information solutions, simplified maintenance and therefore higher quality of data, information and knowledge. The paper focuses on measuring quality of XML schemas and addresses it from structural and contents/data perspective, presenting data as the hart of today's economy [3] as well as the fundament of KM.

Companies use different databases, often including some sort of XML contents due to e-oriented business world, suitable for web presentation of data in various domains and digital libraries, which allow users to find anticipated search items [4]. Based on empirical research of 200 case studies of XML schema usage [5] (from various humanistic, natural and technical domains), XML schemas are often built in a manner, merely satisfying the minimum requirements of syntactic correctness and contents sufficiency (the effort however varies between previously mentioned domains). Often this is the problem with generated XML schemas or inappropriate (simplified) approach to XML schema building by non-experts. Difficulties occur when extending, reforming or reusing poorly designed XML schemas and consequently KM is less efficient.

Existing metrics for quality evaluation only partially address the problem; they base their metrics on existing solutions known in software engineering and do not address the problem of an objective quality evaluation. Dynamic creation and adaptation of XML schemas presents an additional research challenge that requires the use of new approaches and solutions. Additionally, while the structure rules are mostly acquired, a good data organization is often neglected and often insufficient, especially in case of large size data. For example in case of Big Data, where volume, velocity, and variety is emphasized (in addition to veracity, variability, visualization and value of gathered data), the quality of data organization is crucial, suitably addressing challenges specific to big data [25]. As the authors in [25] point out: big data is a lot of data, and with the IT support the user should be able to import and process data based on specific criteria defined by the user (in form of an XML schema or otherwise). The ability to handle different kinds of data sources in a systematic and controlled manner, with the goal of generating suitable information for organizing data, would provide the most value to the user. XML schemas and Big Data combined could provide possibilities to evaluate not only quality 
of data but also enable a quantitative analysis of attitudes, intentions and motivation of (XML schema) users, possibly providing insight about descriptive and predictive user behavior. In this paper, the quality of XML schemas is evaluated on a structural level of 200 examined examples, however an additional quantitative analysis (statistical modelling for good data structure practices identification) could immensely improve XML schema quality, KM support and also reduce risk of data misunderstanding. Based on [25] and [32], the hierarchy of data, information, knowledge and how it connects to XML technologies, is presented in Figure 1.

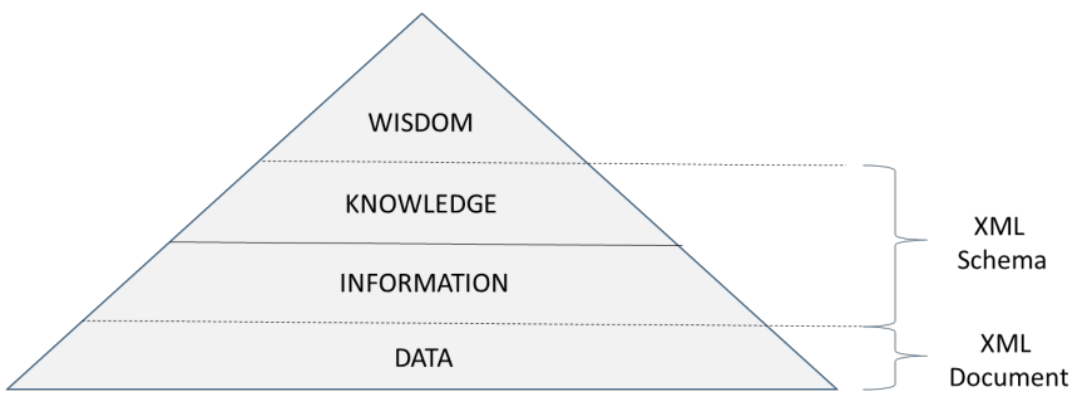

Figure 1 The hierarchy of data, information, knowledge and wisdom [11], connected to XML technologies

The aim of this paper is to focus on KM needs and ensure evaluation of XML data quality in business documents, supporting a better quality of data organization. Theoretical knowledge was tested on an expanded set of test data of already established XML schemas (for business documents) in various organizations with help of composite metrics. The paper's main research question investigates, how XML schema characteristics impact the quality of data, and influence knowledge management possibilities. To provide an answer, XML schema quality aspects were defined. A set of metrics for assessing different quality aspects of XML schemes was developed and results were combined with XML Schema Quality Index, a tool for reporting data quality of XML schemas [5]. In the following sections, those aspects are presented, indicating that quality of XML schemas address KM quality as well.

The structure of the paper is as follows: after introduction to the problem (section 1), the paper presents related work on XML schema quality research (section 2) and establishment of knowledge management connection with XML schemas is provided (section 3). Section 4 focuses on quality aspects and in section 5 results of analysis are provided. The conclusion (section 6) holds a summary of results and future research plans are given.

\section{Related work}

Several scientific and professional papers are addressing quality of XML schemas in software applications in general within the time scope of last 15 years. There have been few attempts to evaluate and measure XML schemas, however the number of evaluated XML schemas was low and the quality aspect of XML schemas was mostly onedimensional, focusing only on complexity. Several metrics are summed by Zhang in [8], and significantly related work was done by [6], [7] and [8], where attempts to measure 
XML schemas and software in general were made. Finding suitable metrics for XML technologies was addressed in other papers, however their backgrounds mainly being software metrics, not applicable to all data aspects of XML schema quality measurements, the main focus in this paper. Authors in [9] address metrics for evaluation of XML schema structure complexity, pointing out entropy. The proposed metric is empirically evaluated based on a case study. While applying the metric, the authors do not include the variety of preferences for XML schemas based on their purpose or data domain. The authors in [10] share the approach to measuring XML schema quality; however they consider structural aspect as well as aspect of contents. Their metric enables user modification of quality measurement settings. In our paper we take a step forward and include several other aspects of XML schema quality although not providing user modification possibilities.

Improving XML schema quality is the focus in [11], addressing the problem of changing existing XML documents (according to XML schema changes). To present the problems, each change has an assigned cost and preference weight. A scalar measurement is provided for each XML schema quality aspects, as proposed and further developed in this paper. Assigning properties and rules in XML schemas is addressed in [12], focusing on information system integration and the role of XML schemas in the integration process. Furthermore, the evaluation of standard quality in [12] is a simplified version of evaluation proposed in our paper. The complexity aspect is addressed in [13], based on internal structure of XML schemas and providing validation variables and a measurement method for calculating complexity. The above mentioned researches depict several variables, important for XML schema quality evaluation and measurement, however mostly stay on the data level and do not address the quality aspects, affecting the knowledge management based on XML schema defined documents. Main focus on the data level quality, respectively data bases and XML schemas is evident in papers [20] and [21] enforcing the important connection of XML schemas and data sources. According to [2], the integration of heterogeneous data sources has become a central problem of modern computing, especially in domains where data currently resides in many different formats. In addition to a relational database, XML data can be stored in an object-oriented database, or in a semi-structured system, providing more possibilities for KM activities.

A lot of effort and application of XML related technologies was addressed also within the EJC conference on all levels of IT development (from concepts, models, and data bases to higher logic layer and user interfaces, applying XML technologies as a format for data extraction). The usefulness and wide application of XML documents and schemas is addressed in [24] and the authors in [22] presented the widespread usability of XML, by describing a conceptual model for XML, dividing the modeling process to conceptual and structural level, using XML documents as well as XML schemas. The author in [23] developed a framework for modelling and implementing layered database applications, defining a database model for each of the layers and a facility for exchange of data throughout different layers in a coherent form, using XML as a hierarchical modelling language for internal, conceptual and external schema. The connection of (XML) data and knowledge is clearly addressed in [25] while XML application at all levels of development of IT solution is presented in [26], where the output of a developed algorithm is an XML file. XML is clearly infiltrated in all levels of IT architecture and the authors in [27] address the use of XML in modelling (used for communication between interest groups and inside development teams), revealing different modelling 
cultures and emphasizing the significance of data planning [28] [29] [30] and importance of XML supporting technologies in knowledge management.

\section{Knowledge management and XML schemas}

The amount of available knowledge has grown in the past years, bringing challenges for controlling and managing [14]. A well collected and organized data is easily summarized and analyzed, providing possibilities of synthesizing information into knowledge, enabling several advantages to organizations. Efficient classification of data for example, important for various fields, such as libraries science [15], is well supported by XML schemas through different restriction building blocks. In addition, a suitable use of XML schema building block can largely support multilingual data, often necessary in the information globalization process [16]. Low-level data collection process is a fundament for higher knowledge creation levels, indicating the importance of data quality, which is often received and stored in XML documents. Figure 2 includes basic transformation levels from data to knowledge and connects them with XML schema building from basic structures to advanced XML schema use, indicating the knowledge about data. In the last years knowledge has become an important wealth indicator and $\mathrm{KM}$ has become a critical factor for organizations and an important tool for international and local competition. The goal of KM is to make organization, classification and validation work more effective; it's not just about the knowledge itself but about how to use and spread the knowledge in the most effective and wide way. In order to be valuable, knowledge must be shared [14], while (quality) XML schemas provide support within the KM process.

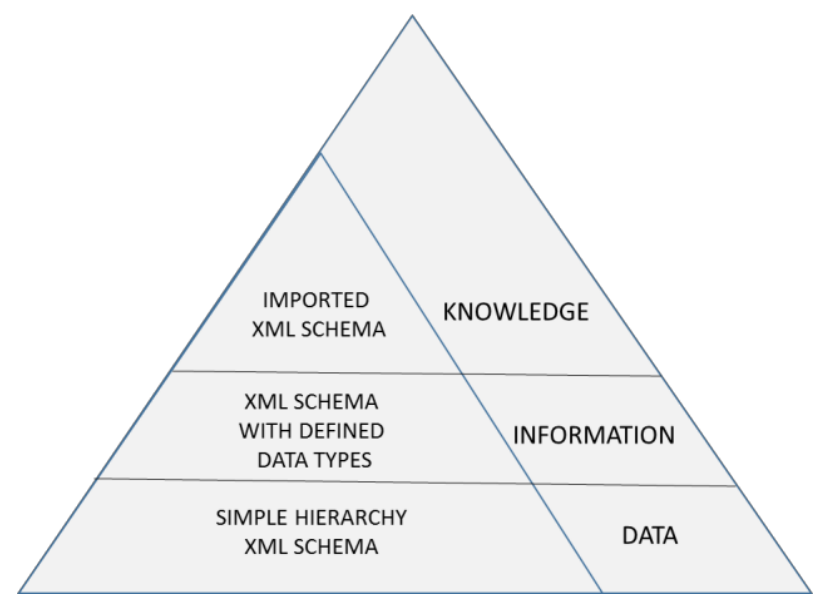

Figure 2. Data, information and knowledge management [1]

$\mathrm{KM}$ is the process of capturing, developing, sharing, and effectively using organizational knowledge [17]. On the level of using XML schemas, organization and classification are the most important aspects. The knowledge management methodology has been designed to ensure a proper, comprehensive, systematic and consistent approach and is in many ways coherent with XML schema development (respectively quality levels). It ensures proper attention is given to the critical success factors and quality aspects in 
successfully implementing knowledge management programs [18]. Several process steps are depicted in Figure 3, connected with XML schema quality levels. The XML schema building process, consists of the following levels:

- Simple hierarchy XML schema includes only element and attribute sequence and cardinality definitions,

- Local data type definitions add information to each element or attribute about its existing or newly defined data type,

- Global data type definitions provide globally defined building blocks for increased reuse, especially in cases of external XML schema use,

- Quality index indicates how advanced and controlled defined XML schemas actually are.

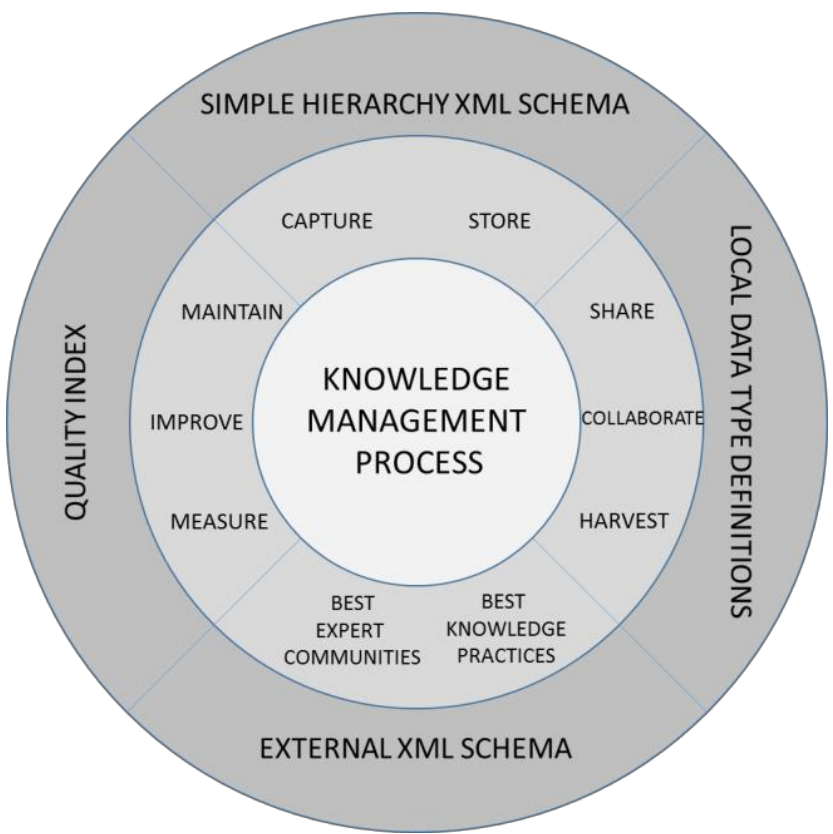

Figure 3. Knowledge management process and XML schemas [18]

Competitive advantage of organizations is gaining all possible knowledge to assimilate and learn faster. The need for knowledge management is infiltrated in several organizational frameworks using XML documents as well. Knowledge management in software industry deals with problematic of having specialists of a narrow sector, not always in tune with other knowledge intensive branches using their products [19]. Therefore, data refinement and information extraction is crucial for understanding and gathering knowledge. A quality XML schema simplifies this task, benefiting knowledge management in organizations as well as providing a metric, estimating quality of existing XML schemas. Main XML schema building blocks, influencing its quality will be indicated, providing a guideline to build more adapted XML schemas, suitable for data definition in the first level of knowledge management process. 


\section{Measuring quality of data in XML schemas}

XML schemas define the structure and content of all elements and attributes, storing valuable organizational data in XML documents, which is gaining popularity [4]. It provides a number of features that makes it suitable for the web based application. Its role is definition of all elements and attributes, organization of the hierarchy, sequence and cardinality of data elements. Each element or attribute is defined (its structure and contents) by a data type or integrated building blocks. Covering a wide range of data demands, organized and well-formed exchange of documents is essential in different domains of e-business (e-health, e-library, e-government etc.), web applications as well as data storage. XML schemas must therefore be extendable and flexible, suitable for future changes and have a rich set of building blocks

XML schema is a model, defining document structure and enabling a creation of an agreement regarding structure and contents, supported security via XML encryption. It enables a simplified creation of complex content models, focused on reuse of elements and attributes as well as data types, storing valuable data. One of most significant advantages of XML schemas is data type support, simplifying document contents description, validation of data correctness, defining data restrictions and data patterns. XML schemas are extendible and can be reused within other XML schemas. Within one XML document there is a possibility of holding several XML schemas (by include or import building block), spreading a set of possible types or/and elements. A well-shaped XML document is a document, adapted to all XML syntactical rules, as are XML declaration, one root element, suitable tag names, case- sensitivity, end tags, the hierarchy rules and attribute values within quotes. Although an XML document is well formed, errors can still exist, having serious consequences on data storage and system well-being. The problem is addressed by a suitable approach to building XML schemas, where good practices already exist.

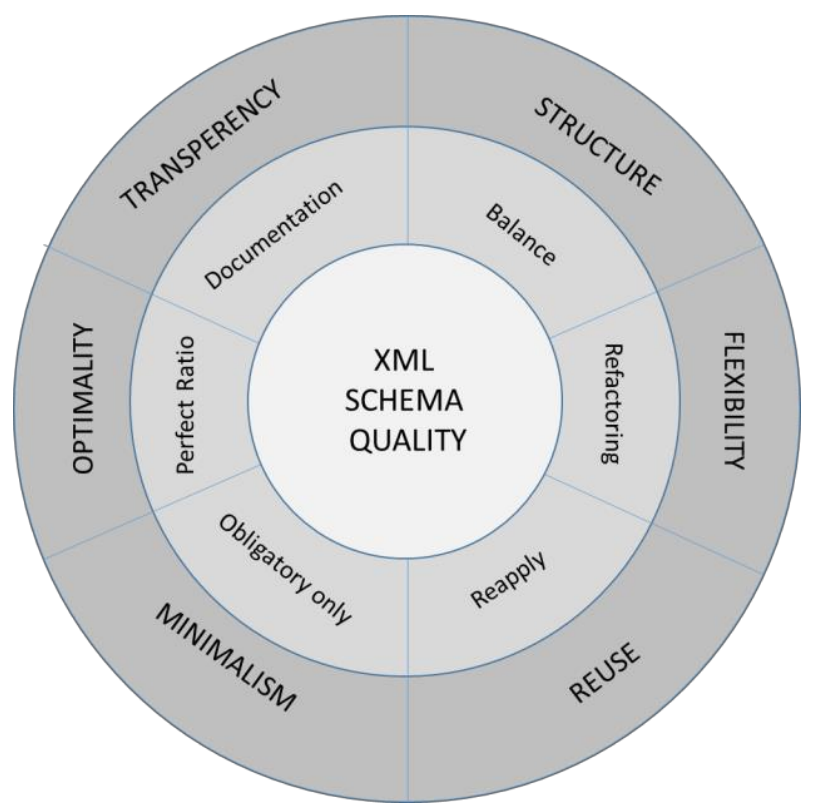

Figure 4. XML schema quality aspects [19] 
Literature review results of XML schema quality measurements field provided several metrics, historically linked to approaches and measurements methods in program engineering, focusing mostly on XML schemas structure and complexity. Based on definition of structure metrics, the paper includes other aspects of XML schema quality, important also for advanced knowledge management, summarized based on ISO/IEC 9126, theory of decision models and other papers. While choosing metrics with which we wish to improve XML schema quality, the paper includes parameters, effecting XML schema improvement (Figure 4):

Figure 4 presents a set of parameters, organized in 6 aspects. A composite metric is equally combining all six aspects (structure, clarity, optimality, minimalism, re-use, flexibility), presenting a general quality index, and defining a joint grade of quality.

\section{Results}

Two types of XML schemas were included: (1) XML schemas, designed through a standardization process of larger independent organizations (W3C, OASIS) and (2) XML schemas, defined within different companies and organizations for needs of ebusiness and knowledge management. Based on a data base of 200 XML schemas, found in the search process, all schemas were analyzed and measurable elements were extracted. The analysis was semi-manual with help of a tool, developed in Institute of Informatics, University of Maribor.

Table 1. Overview of positive and negative variables

\begin{tabular}{ll}
\hline \multicolumn{1}{c}{ Predictor } & Beta value \\
\hline Number of included XML schemas &, 164 \\
XML schema type &,- 384 \\
Number of local elements &,- 017 \\
Number of global complex elements &, 308 \\
Number of global simple elements &, 145 \\
Lines of code &,- 012 \\
Number of attribute groups &, 133 \\
Number of annotation &, 130 \\
Number of derived data types &, 047 \\
\hline
\end{tabular}

Analysis of data was conducted by regression analysis with help of a standard linear regression. Table 1 presents variables with significant impact on quality of XML schemas. The Beta indicator presents a value (positive or negative), indicating the addition or deduction of an XML schema quality in case an additional variable occurs in XML schema. For example with each additional external XML schema (Number of included XML schemas), the quality of XML schema goes higher for 0.1645 on a scale from 0 to 1 , indicating that in fact XML schema characteristics (measured parameters) impact it's quality and furthermore the possibilities of qualitative knowledge management

Overall findings about XML schema use produced the following results [33]: structure of XML schemas influences the quality of data application and further data management, however different domains require different XML schema types: economy related fields typically require a very non-flexible and strict structure, while technical and humanistic domains are much less strict. The most flexible XML schema structure is required in natural science domains, where possibly the pool of data varies the most. 
The general conclusion is that no XML schema type can be applied on all domains and needs, as well as the understanding of what makes a useful XML schema vary. Also, not all aspects can be equality satisfied, however there are some good practices, which can be used as general guidelines, presented in Table 2 (compared to average XML schemas):

Table 2. Good and bad characteristics of general XML schemas.

\begin{tabular}{|c|c|c|}
\hline & $\begin{array}{c}\text { Standardized properties of the } \\
\text { average XML schema }\end{array}$ & Good XML Schema Properties \\
\hline $\begin{array}{l}\text { Use of external } \\
\text { schemes }\end{array}$ & $\begin{array}{l}0.8 \text { use external schema per XML } \\
\text { schema (import or include) }\end{array}$ & $\begin{array}{l}\text { Minimum usage ( } 1 \text { or } 2) \text {, suitable for } \\
\text { separating data type definitions from } \\
\text { element and attribute definitions. }\end{array}$ \\
\hline Use of elements & $\begin{array}{l}3 \mathrm{x} \text { more local than global } \\
2 \mathrm{x} \text { more complex than simple }\end{array}$ & $\begin{array}{l}\text { Global 1, other local. It uses more in } \\
\text { exceptional cases when an XML schema } \\
\text { defines multiple XML documents. }\end{array}$ \\
\hline Use of attributes & $\begin{array}{l}\text { Typically local attributes, } 0.6 \text { attributes } \\
\text { per element, }\end{array}$ & Attributes minimum (0.05 per item). \\
\hline Use of groups & 4.4 groups per XML schema. & Not recommended \\
\hline Use of data types & 2.3 more simple data types & $\begin{array}{l}\text { At most as many global data types as } \\
\text { there are elements and attributes. Simple } \\
\text { are less than complex; a better complex } \\
\text { element with simple content that makes } \\
\text { it easier to modify the XML schema in } \\
\text { the future. }\end{array}$ \\
\hline Documentation & 1 annotation per complete scheme & $\begin{array}{l}\text { Annotation affects the quality of XML } \\
\text { schemas, but minimal documentation is } \\
\text { sufficient to improve it. }\end{array}$ \\
\hline Limits & $\begin{array}{l}1.3 \text { restrictions per individual building } \\
\text { block. }\end{array}$ & Each block should be restricted. \\
\hline
\end{tabular}

\section{Conclusion}

The paper addresses knowledge management issues through XML schemas, its characteristics, influence and contribution to knowledge organization and classification, focused on assessing the quality of XML schemas. The literature review reviled the importance of XML schemas within XML technologies and their importance in integration of information systems and knowledge management. In the paper we have analyzed demands of quality in XML schemas and the meaning of XML schemas in knowledge management organizations.

We have listed most important building blocks for quality evaluation by experimenting on 200 XML schemas of different origin. XML schemas were analyzed with regression analysis to establish which characteristics (building blocks) influence the quality of XML schemas the most, indicating critical quality aspect for future building of XML schemas.

Six aspects of quality were defined, combined into one value: the quality index. The quality aspects included (1) structure, (2) transparency, (3) optimality, (4) minimalism, (5) reuse and (6) integrability. All aspects are separately measured; a regression analysis was conducted, discovering nine variables; significantly (positively or negatively) influencing the final XML schema quality estimation. Results expose the following parameters as most significant: number of included external XML schemas, XML schema type, three variables connected to element use, number of attribute groups, lines of code, annotation usage and number of derived data types. All significant variables are 
in different forms included in metrics, addressing specific aspects of XML schema quality estimation, providing an answer to the research question: XML schema characteristics do impact quality of data, influencing knowledge management possibilities. More detailed impact of XML schema quality on knowledge management is yet to be empirically confirmed in the future work. The results in this paper provide possibilities for further scientific and applicative research in the field of XML schema quality, as well as in the field of XML data impact on knowledge management. Additional note: based on our research, $30 \%$ of XML schemas were not properly built and need adaptation mostly in fields of structure and transparency, therefore not providing enough support for knowledge management

The research within the paper also has limitations due to human error when analyzing XML schemas. To confirm validity of results, the research should be repeated. The future work will address the knowledge management efficiency connected with XML schema quality in more detail, as well as the influence of contents (data) on XML schema quality aspects. To efficiently address these aspects, new refined metrics will be introduced, tailored according to knowledge management specific needs. Additional future study will include measurement of higher architectural levels of IT solutions and how foundational XML schemas correlate with software quality, perceived usefulness of end product and user experience of creating, sharing, using and managing the knowledge and information to achieve organizational objectives by making the best use of data and knowledge. The possibilities of integrating Big Data concepts will also be examined.

\section{References}

[1] T.H. Davenport, Saving IT's Soul: Human Centered Information Management, Harvard Business Review 72 (1994), 119-131.

[2] S.K. Madria, XML schema and data management, Data \& Knowledge Engineering. 52 (2005), 181-183.

[3] A, Uden, Developing Data Analytics to Improve Services in a mechanical Engineering Company, Knowledge. Management. Organization (2014).

[4] S. Goswami, C. Kundu, XML based advanced distributed database: Implemented on library system, International Journal of Information Management 33 (2013), 28-31.

[5] M. Pusnik, M. Hericko, Z. Budimac, B. Sumak, XML schema metrics for quality evaluation, Computer Science and Information Systems 11 (2014), 1271-1290.

[6] B. Sumak, M. Hercko, M. Pusnik, Towards a Framework for Quality XML Schema Evaluation Keywords, Information Technology Interfaces (2007), 783-788.

[7] K.M.A.S.C. Yue, Analysis and Metrics of XML Schema, Proceedings of the International Conference on Software Engineering Research and Practice and Conference on Programming Languages and Compilers (2004), 538-544.

[8] V. L. Narasimhan, B. Hendradjaya, Detailed theoretical considerations for a suite of metrics for integration of software components, Advances in Systems, Computing Sciences and Software Engineering 177 (2006), 257-264.

[9] D. Basci, S. Misra, Entropy as a Measure of Quality of XML Schema Document, The International Arab Journal of Information Technology 8 (2010), 16-24.

[10] E. Pardede, T. Gaur, On the Development of a User-Defined Quality Measurement Tool for XML Documents, Information Systems Development, Springer New York (2011), 213-221.

[11] Z. Tan, L. Zhang, Improving XML Data Quality with Functional Dependencies, Database Systems for Advanced Applications (2011), 450-465.

[12] K.C. Morris, A framework for XML schema naming and design rules development tools, Computer Standard Interfaces 32 (2010), 179-184.

[13] D. Basci, S. Misra, Measuring and evaluating a design complexity metric for XML schema documents, Journal of Information Science and Engineering 25 (2009), 1405-1425.

[14] R. Suurla, Avauksia tietämyksen hallintaan, Helsinki, Oy Edita Ab. (2001), 190.

[15] U.A. Mokhtar, M.Y. Zawiyah, The requirement for developing functional records classification, International Journal of Information Management 35 (2015), 403-407. 
[16] J. Zhang, Y. Sun, A.J. Jara, Towards semantically linked multilingual corpus, International Journal of Information Management 35 (2015), 387-395.

[17] K. Management, E. Factors, Knowledge Management Enabler Factors and Competitive Advantage: An Empirical Study in Pharmaceutical Industry in Andhra Pradesh, India, The International Journal Of Business \& Management 2 (2014), 104-108.

[18] KM Consulting Methodology Overview, http://www.knowledge-management-online.com/KMconsulting-method-overview.html. (Accessed March $1^{\text {st }}$ 2020).

[19] Finland \& Knowledge Management, https://knowledgeandmanagement.wordpress.com/finlandknowledge-management/. (Accessed March $1^{\text {st }}$ 2020).

[20] E. Gallinucci, M. Golfarelli, S. Rizzi, Schema profiling of document-oriented databases, Information Systems 75 (2018), 13-25.

[21] Z. Brahmia, H. Hamrouni, R. Bouaziz, XML data manipulation in conventional and temporal XML databases: A survey, Computer Science Review 36 (2020).

[22] M. Necasky, J. Pokorny, Conceptual Modeling of IS-A hierarchies for XML. In: Proceedings of the 18th European Japanese ' Conference on Information Modelling and Knowledge Bases (2008), 65-84.

[23] B. Thalheim, Model suites for multi-layered database modelling. In: Proceedings of the 2010 conference on Information Modelling and Knowledge Bases XXI (2010), 116-134.

[24] S. Hartmann, S. Link, T. Trinh, Boolean Constraints for XML Modeling. In: Frontiers in Artificial Intelligence and Applications (2008), 85-104.

[25] P. Sillberg, Toward Manageable Data Sources. In: Proceedings of the 28th International Conference on Information Modelling and Knowledge Bases (2018), 485-494.

[26] M. Duží, M. Fait, Type checking algorithm for the TIL-Script language. In: Proceedings of the 28th International Conference on Information Modelling and Knowledge Bases (2018), 201-219.

[27] H. Jaakkola, B. Thalheim, Modelling Cultures. In: Proceedings of the 28th International Conference on Information Modelling and Knowledge Bases (2018), 33 - 52.

[28] G. Polančič, B. Šumak, M. Pušnik, A case-based analysis of process modeling for public administration system design. Information modelling and knowledge bases XXXI. Amsterdam: IOS Press (2020), 92104.

[29] B. Thalheim, H. Jaakkola, Models as Programs: The Envisioned and Principal Key to True Fifth Generation Programming. Information modelling and knowledge bases XXXI (2020).

[30] J. Musto, A. Dahanayake, Improving data quality, privacy and provenance in citizen science applications. Information modelling and knowledge bases XXXI (2020).

[31] S.Hijazi, Big Data and Knowledge Management: A Possible Course to Combine them Together. In: ASCUE Proceedings (2017).

[32] J. Rowley, The wisdom hierarchy: representations of the DIKW hierarchy. Journal of Information Science, 33 (2007).

[33] M. Pušnik, Quality evaluation of domain specific XML schemas. Doctoral dissertation. University of Maribor, Faculty of Electrical Engineering and Computer Science; 2014. 


\title{
Digital Intelligence Banking of Adaptive Digital Marketing with Life Needs Control
}

\author{
Ryosuke KONISHI ${ }^{\text {a }}$ Fumito NAKAMURA ${ }^{\text {a }}$ Yasushi KIYOKI $^{\mathrm{b}}$ \\ ${ }^{a}$ Generic Solution Corporation, Nampeidai-chou, Shibuya-ku, Tokyo, Japan \\ ${ }^{\mathrm{b}}$ Faculty of Environmental Information, KEIO University, Fujisawa, Kanagawa 252, \\ Japan
}

\begin{abstract}
While individuals benefit from the goods and services provided by companies that enrich their lives and that have adapted to a dynamic environment that is always changing, these companies pay a high communication cost to access opportunities to provide these goods and services and to seek a better understanding of individual customers' changing needs. Although vast amounts of information can be obtained, databases and machine learning are playing an increasingly important role in extracting meaning from this information, turning it into meaningful information assets that consider circumstances and contexts, and individualizing the economy of information. I propose an implementation method for providing information to enrich the profiles of individual customers by consolidating different data, calculating the individual customers' needs through the relationships between customers and products, evaluating the change in relationships between individual customers and products over time, and providing goods and services to suit different intervals of change to factors such as lifestyle and living environment. As there are different factors involved in estimating the incidence of needs, and different frequencies and rates at which they occur, based on the special characteristics of products, different data are required to estimate such needs. By profiling individuals over the long term, it is possible to build an information provision environment that is conducive to companies' customer acquisition.
\end{abstract}

Keywords. Recommendation system, Mathematical Model of Meaning, Logistic Regression Mixture Model, Local Variational Inference, Retail Application, Hawkes process,

\section{Introduction}

We live in an era in which digitalization technology, along with high-speed communication, large volumes of data, and advanced artificial intelligence technologies such as machine learning, can effectively support the use of information. Today, companies can not only send various types of messages to a massive number of customers and obtain their responses but also interact with them and get feedback on products and services through messaging. There is a growing expectation that further development of information digitalization will make it possible to store a huge amount of information in the form of databases that can be applied to digital marketing. In addition, the popularization of smartphones has increased the number of digital points of contact with customers, enabling the consideration of reachability, segmentation, perception, and recordability 
when evaluating such messaging media and recording their position, time, and response in more detail. Given the need to find meaning from information, convert it to a meaningful property that considers the situation and context, and personalize its economic efficiency, information technologies involving databases and machine learning are expected to be advanced further.

The era of digital marketing is forcing companies to change their business formats and break away from single channels with only brick-and-mortar stores. The same can be said about marketing channels, which require a paradigm shift to digital omni-channels and need to stop relying only on traditional distribution channels and sales methods. Thus, in this new era, adopting an omni-channel strategy is essential for survival. The essence of marketing is to generate and control customer needs for products and direct the flow of products and services from manufacturers to consumers and users by combining products, distribution, promotion, and prices. To this end, it is necessary to capture the behavior of each customer, record it as data, and monitor its trend for long periods.

The advertisement industry uses a technique that considers the processes of attention, interest, desire, memory, and action (AIDMA). For each of these processes, it identifies elements of perception, knowledge, feelings and emotions, intentions, and actions that form a consumer's attitude and measures advertising effectiveness by applying an attitude scale used in psychology. Companies are required to turn these processes into data and understand each process from the perspective of customers for all products and services. To ensure communication that encompasses the entire living environment of the dweller or consumer and stimulate these processes, as each person assigns a different value to information and requires it at different times, it is important for companies to specialize for each customer, design the value of information, record it, and use it to predict demand.

Response: Customer responses to messages Recommend: Recommendations made by companies based on analysis of customer responses Reach: Messages reach both companies and customers Relation: Sell products and services through relationship building Repeat: Multiple uses of products and services Retention: Maintain a customer base by transforming them into repeat purchasers Royalty: Win customer loyalty by gaining their trust

Several advertisement techniques are based on passive information, but users normally provide poor responses to information they did not request. Merely recorded information increases asymmetry, driving companies to implement massive promotion measures for marketing activities that explore the emergence of customer needs. Therefore, for companies to detect customer interest in products that emerges with life events, obtain information close to the timing of their purchase intention, and understand the customer's needs, they must evaluate the economic efficiency of the information through the use of a database.

When companies regard each customer as a human being, they can record marketing activities from the customer's human side, taking into consideration what kind of products and services would make their lives more comfortable, from birth till death. It can be said that understanding customers through communication is becoming an increasingly important element. In this context, companies are increasingly expected to implement digital marketing strategies that predict the timing of customer needs according to their life stages and events.

The expansion of contact points with customers faced limits. In face-to-face channels - because customer information, service history, and past suggestions are not shared 
across channels in real time-when collecting information regarding a first-time customer, it is necessary to interview them from the ground up; thus, the process from suggestion to purchase takes a considerable amount of time.

It is possible to identify the relationships among products from different backgrounds such as durable goods (e.g., cars, houses, furniture, household appliances), consumer goods (e.g., food, daily-use items), non-durable goods (e.g., eating out, cultural entertainment), and luxury consumer goods (e.g., travel, jewelry) as well as those among the methods of payment used to purchase such goods and financial products such as mortgages, fund management, and asset formation. Customers' opportunity and frequency of purchase of these products and services varies in their life. Companies are also expected to ensure consistency between customers' life events, evaluate their economic power including consumption capability (e.g., work and family history, asset status), analyze how it changes over time, and suggest products and services with adequate pricing. Financial institutions, particularly banks, possess a large number of information assets obtained through payment and deposit data for each customer. These information assets create opportunities for them and other companies to suggest financial products and offer services that suit customers' life events and style. Such use of information is expected to play an important role in the stimulation of processes through corporate marketing activities in the next years. While companies are working to stimulate marketing processes individually by offering products and services with different frequencies and cycles, banks and other financial institutions are expected to understand customers' global needs from their perspective and effectively support the stimulation of marketing processes. Banks record not only customers' attributes but also their purchase history and behavior through various points of contact, and, based on the potential needs identified, make suggestions from various channels through establishing contact in real time and immediately evaluate the causal effect and prediction accuracy of those suggestions. Based on the assessment results, they can quickly make further suggestions according to the customer needs identified. In such processes, the advancement of information technologies related to databases, machine learning, and big data analysis plays an essential role.

The advancement of such digital banking services will allow companies to build full profiles of their customers, enabling them to offer products and services according to their life events and styles and suggest related financial products. The effect of this process can be maximized if companies reduce the asymmetry of the information volume designed to learn about customers and make the information acquisition process more efficient. Targeting can play the most important role in maximizing the return on marketing investments.

\section{Related Works}

We have previously proposed a recommendation system that utilizes action and demand vector simultaneously and updates a recommendation list in a same cycle [1]. After this work, we have proposed another system that utilizes the action vector more frequently than the demand vector [2]. In this paper, we introduce a new concept life time that is longer interval to update than the action and the demand. 


\section{Basic Theories}

Here, we introduce the methodologies used in this study. Specifically, we use the mathematical model of meaning (MMM) and machine learning; Bayesian inference of a logistic regression mixture model (LRMM) and Hawkes process.

\subsection{Mathematical Model of Meaning}

The MMM was first used to extract semantic information behind data deterministically [3]. Let $X \in \mathbb{R}^{N \times M}$ be a data matrix, where $N$ is the number of data and $M$ is the number of features. We normalize each column of the matrix by the 2-norm, and denote the resulting normalized matrix by $\tilde{X}$, i.e. the $(i, j)$ th element of the matrix is

$$
\tilde{X}_{i, j}=\frac{X_{i, j}}{\sqrt{\sum_{k=1}^{N} X_{k, j}^{2}}} .
$$

This is referred to as the fundamental data matrix. For the product of $(1) \tilde{X} \tilde{X}^{\mathrm{T}} \tilde{X}$, we calculate the eigenvalues and eigenvectors. Let $\lambda_{1} \geq \lambda_{2} \geq \cdots \geq \lambda_{M} \geq 0$ be the eigenvalues and $v_{1}, v_{2}, \ldots, v_{M}$ the corresponding eigenvectors, $Y=\left[Y_{1}^{\mathrm{T}}, Y_{2}^{\mathrm{T}}, \ldots, Y_{t}^{\mathrm{T}}\right] \in \mathbb{R}^{t \times M} t \times M$ context matrix, and $Q=\left[v_{1}, v_{2}, \ldots, v_{M}\right] \in \mathbb{R}^{M \times M}$. Then, for a threshold $\epsilon$, MMM determines the index set of the chosen eigenvectors $\Lambda_{\epsilon}$ by

$$
\Lambda_{\epsilon}=\left\{j \in\{1,2, \ldots, M\} \mid 0<\epsilon<1, \frac{\left(Q_{Y}\right)_{j}}{\left\|Q_{Y}\right\|_{\infty}}>\epsilon\right\} .
$$

Here, $Q_{Y}=\sum_{i=1}^{t} Y_{i} Q,\left(Q_{Y}\right)_{j}$ is the $j$-th element of $Q_{Y}$, and $\left\|Q_{Y}\right\|_{\infty}=\max _{1 \leq j \leq M}\left|\left(Q_{Y}\right)_{j}\right|$. Arranging the chosen eigenvectors, we obtain the semantic projection

$$
P(Y)=\left[v_{j}^{\mathrm{T}} \mid j \in \Lambda_{\epsilon}\right] \in \mathbb{R}^{\left|\Lambda_{\epsilon}\right| \times M},
$$

where $\left|\Lambda_{\epsilon}\right|$ represents the cardinality of $\Lambda_{\epsilon}$. When we apply the projection to each datum $g \in \mathbb{R}^{M}$, the datum is regarded as an element of a semantic space, denoted by $P(Y) g$. In this semantic space, we can measure the distance between the datum and the semantic centroid $\bar{D}$ :

$$
\bar{D}=\frac{1}{\left\|P_{Y}\right\|_{\infty}} P_{Y}
$$

where $P_{Y}=\sum_{i=1}^{t} P(Y) Y_{i}$. Let $\bar{D}_{j}$ and $(P(Y) g)_{j}$ be the $j$-th elements of the vectors; the distance is calculated as the weighted Euclidean distance $\operatorname{dist}(\bar{D}, g)$,

$$
\operatorname{dist}(\bar{D}, g)=\sqrt{\sum_{j=1}^{\left|\Lambda_{\epsilon}\right|} c_{j}\left(\bar{D}_{j}-(P(Y) g)_{j}\right)^{2}},
$$

where $c_{j}=\frac{\left(P_{Y}\right)_{j}}{\left\|P_{Y}\right\|_{\infty}}$. 


\subsection{Logistic Regression Mixture Model and Local Variational Inference}

LRMM is a mixture of a logistic regression model, and used to solve classification problems like those found in marketing, clinical trials, and psychology [4,5,6]. This model represents the probability of a binary output $y$ given an input data $x$ as follows:

$$
p(y \mid x, w)=\sum_{k=1}^{K} a_{k} r\left(x, b_{k}\right)^{y}\left(1-r\left(x, b_{k}\right)\right)^{1-y},
$$

where $y \in\{0,1\}, x \in \mathbb{R}^{M}, b_{k} \in \mathbb{R}^{M}, 0 \leq a_{k} \leq 1$ for $1 \leq k \leq K, \sum_{k=1}^{K} a_{k}=1$, and $r(\cdot)$ is a sigmoid function $r\left(x, b_{k}\right)=1 /\left(1+\exp \left(-x^{\mathrm{T}} b_{k}\right)\right) . w=\left(a_{1}, a_{2}, \ldots, a_{K}, b_{1}, b_{2}, \ldots, b_{K}\right)$ is a parameter, and we must estimate it or the probability distribution itself from $n$ pairs of input and output $\left(x^{n}, y^{n}\right)=\left(x_{1}, x_{2}, \ldots, x_{n}, y_{1}, y_{2}, \ldots, y_{n}\right)$.

Several methods have been proposed to estimate the parameters $[7,8,9]$. In this study, we use the method proposed by [1] to estimate the probability distribution because it is more accurate than the other methods. Moreover, the method leverages mini-batch learning; thus it learns efficiently. The calculation is as follows. In the first step, we assume a prior and a posterior distribution of the parameter given the training data. Let $\varphi(w)$ be the prior distribution, and $p\left(y^{n} \mid x^{n}, w\right)$ the likelihood of (6); then, the posterior distribution of the parameter $p\left(w \mid x^{n}, y^{n}\right)$ is obtained as follows:

$$
p\left(w \mid x^{n}, y^{n}\right)=\frac{1}{Z} p\left(y^{n} \mid x^{n}, w\right) \varphi(w),
$$

where $Z=\int p\left(y^{n} \mid x^{n}, w\right) \varphi(w) d w$. By using the posterior distribution, we obtain the output distribution $p\left(y \mid x, x^{n}, y^{n}\right)$ that we want to predict, which we call the predictive distribution in this paper:

$$
p\left(y \mid x, x^{n}, y^{n}\right)=\int p(y \mid x, w) p\left(w \mid x^{n}, y^{n}\right) d w
$$

However, the normalization constant $Z$ of the posterior distribution is intractable. To avoid the problem, we leverage an approximated posterior distribution replacing (7). Local variational inference (LVI) with mini-batch learning can construct the approximated distribution efficiently and accurately. [1]. In mini-batch learning, we first divide the whole data $\left(x^{n}, y^{n}\right)$ into $T$ mini-batches of data:

$$
\left\{\left(x^{n}, y^{n}\right)\right\}=\left\{\left(x^{n_{1}}, y^{n_{1}}\right),\left(x^{n_{2}}, y^{n_{2}}\right), \ldots,\left(x^{n_{T}}, y^{n_{T}}\right)\right\} .
$$

Let the prior distribution $\varphi(w)$ be

$$
\varphi(w) \propto \prod_{k=1}^{K} a_{k}^{\alpha-1} \exp \left(-\frac{\beta}{2} b_{k}^{\mathrm{T}} b_{k}\right),
$$

where $\alpha, \beta \in \mathbb{R}_{+}$are hyperparameters that are tuning parameters. Then, the approximated posterior given the $t$-th partition $\left(x^{n_{t}}, y^{n_{t}}\right)=\left(\left(x_{1, t}, y_{1, t}\right), \ldots,\left(x_{n_{t}, t}, y_{n_{t}, t}\right)\right)$ is written as follows [1]: 


$$
\begin{aligned}
\hat{p}\left(w \mid x^{n_{t}}, y^{n_{t}}\right) & \propto \prod_{k=1}^{K} a_{k}^{\hat{a}_{k}^{t}-1} \exp \left(-\frac{1}{2}\left(b_{k}-\hat{\mu}_{k}^{t}\right)^{\mathrm{T}} \hat{\beta}_{k}^{t}\left(b_{k}-\hat{\mu}_{k}^{t}\right)\right) \\
\hat{\alpha}_{k}^{t} & =\hat{\alpha}_{k}^{t-1}+\sum_{i=1}^{n_{t}} u_{i k, t}(\xi), \\
\hat{\beta}_{k}^{t} & =\hat{\beta}_{k}^{t-1}-\sum_{i=1}^{n_{t}} 2 u_{i k, t}(\xi) v_{i k, t}(\eta) x_{i, t} x_{i, t}^{\mathrm{T}}, \\
\hat{\mu}_{k}^{t} & =\left(\hat{\beta}_{k}^{t}\right)^{-1} \hat{\kappa}_{k}^{t}, \\
\hat{\kappa}_{k}^{t} & =\hat{\kappa}_{k}^{t-1}+\sum_{i=1}^{n_{t}} u_{i k, t}(\xi)\left(y_{i, t}-0.5\right) x_{i} .
\end{aligned}
$$

For simplicity, we set the initial conditions as $\hat{\alpha}_{k}^{0}=\alpha, \hat{\beta}_{k}^{0}=\beta I_{M}$, and $\hat{\kappa}_{k}^{0}=0$. In (10)-(14), it is necessary to determine the local parameters $u_{i k}(\xi)$ and $v_{i k}(\eta)$. They are represented as follows:

$$
\begin{aligned}
& u_{i k, t}(\xi)=\frac{\exp \left(h_{i k, t}(\xi)\right)}{\sum_{l=1}^{K} \exp \left(h_{i l, t}(\xi)\right)} \\
& v_{i k, t}(\eta)=-\frac{\tanh \left(\frac{\sqrt{g_{i k, t}(\eta)}}{2}\right)}{4 \sqrt{g_{i k, t}(\eta)}}, \\
& h_{i k, t}(\xi)=\Psi\left(\hat{\alpha}_{k}^{t}\right)-\Psi\left(\sum_{k=1}^{K} \hat{\alpha}_{k}^{t}\right)+\left(y_{i, t}-\frac{1}{2}\right) x_{i, t}^{\mathrm{T}} \hat{\mu}_{k}^{t}-\log 2 \cosh \left(\frac{\sqrt{g_{i k, t}(\eta)}}{2}\right), \\
& g_{i k, t}(\eta)=x_{i, t}^{\mathrm{T}}\left(\hat{\mu}_{k}^{t}\left(\hat{\mu}_{k}^{t}\right)^{\mathrm{T}}+\left(\hat{\beta}_{k}^{t}\right)^{-1}\right) x_{i, t},
\end{aligned}
$$

where $\Psi(\cdot)$ is the digamma function, i.e. $\Psi(x)=\frac{d}{d x} \log \Gamma(x)$, where $\Gamma(\cdot)$ is the gamma function. Note that (10) - (14) and (15) - (18) include their opponent each other, thus by iterative update the method can attain a local minimum. Algorithm 1 outlines this approach.

The predictive distribution for the $\mathrm{t}$-th partition is approximated by replacing the obtained approximated posterior distribution with the posterior distribution used in (8), as follows:

$$
p\left(y \mid x, x^{n_{t}}, y^{n_{t}}\right) \approx \int p(y \mid x, w) \hat{p}\left(w \mid x^{n_{t}}, y^{n_{t}}\right) d w \approx\left\{\begin{array}{ll}
\sum_{k=1}^{K} \hat{a}_{k} \tilde{r}(x) & (y=1) \\
\sum_{k=1}^{K} \hat{a}_{k}(1-\tilde{r}(x)) & (y=0)
\end{array} .\right.
$$

Here, $\hat{a}_{k}^{t}=\hat{\alpha}_{k}^{t} / \sum_{l=1}^{K} \hat{\alpha}_{l}^{t}$ and $\tilde{r}(x)$ is

$$
\tilde{r}(x)=r\left(\frac{x^{\mathrm{T}} \hat{\mu}_{k}^{t}}{\sqrt{\left(1+\pi x^{\mathrm{T}}\left(\hat{\beta}_{k}^{t}\right)^{-1} x\right) / 8}}\right) .
$$




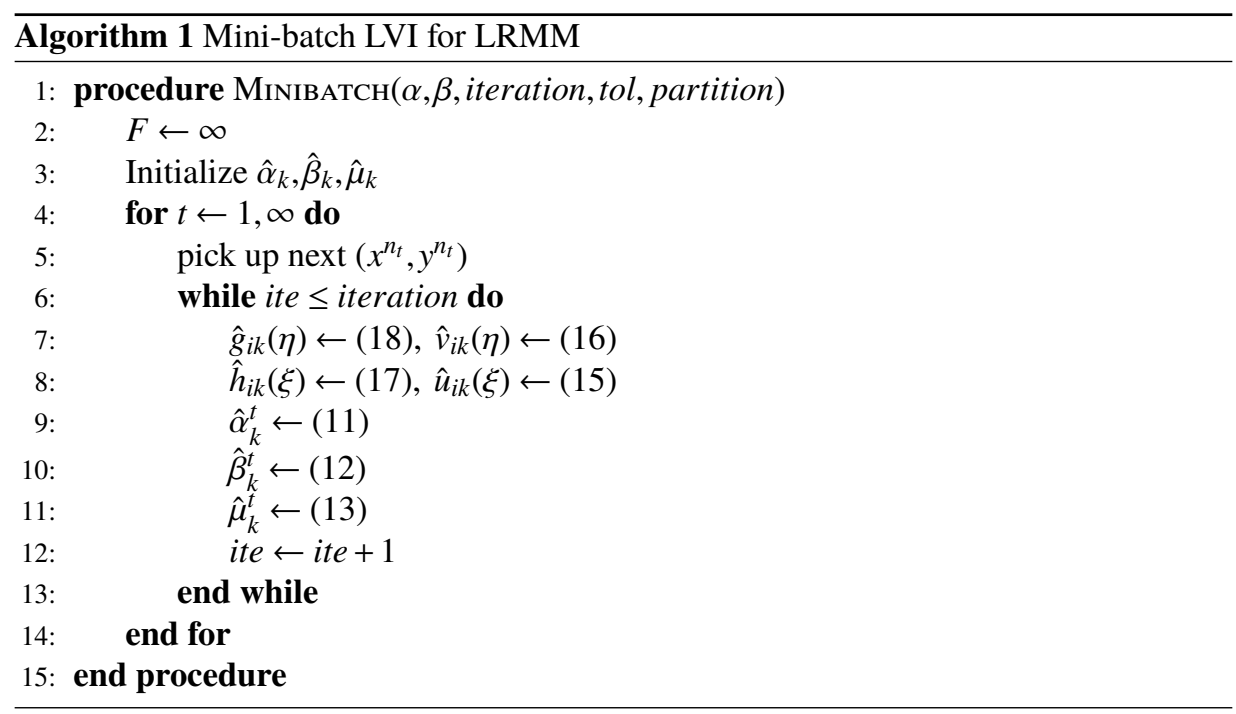

\subsection{Hawkes Process}

Hawkes process is one of a point process which is modeled to describe the timing of events like the timing of purchase, e-mail message, tweet, earthquake, and so on [10,11]. Especially, Hawkes process leverages past events, that is the more events happen in the past, the more likely they are to happen at the current time. We utilize the process to classify a current status of life event.

Let $\lambda(t)$ be an intensity function at time $t$, then the probability distribution of the number of event occurrence $N(t, t+\delta)$ between $t$ and $t+\delta$, where $\delta>0$ is a sufficiently small real number, is described as,

$$
\begin{aligned}
& p(N(t, t+\delta)>1)=o(\delta), \\
& p(N(t, t+\delta)=1)=\lambda(t) \delta+o(\delta), \\
& p(N(t, t+\delta)=0)=1-\lambda(t) \delta+o(\delta) .
\end{aligned}
$$

Here, $o(\delta)$ represents a term that converges to 0 faster than $\delta$. In particular, the Hawkes process consists of the following intensity function:

$$
\lambda(t)=\mu+\sum_{j \mid t_{j}<t} g\left(t-t_{j}\right),
$$

where $\mu \in \mathbb{R}_{+}$and $t_{j}$ is the timing of the event. $g\left(t-t_{j}\right)$ is non-negative real valued function and usually used the following exponential kernel:

$$
g\left(t-t_{j}\right)=\alpha \beta \exp \left(-\beta\left(t-t_{j}\right)\right)
$$

where $\alpha, \beta \in \mathbb{R}_{+}$. Figure 1 shows a intensity function of the Hawkes process and timing of events. The solid line shows the intensity function $\lambda(t)=10+\sum_{j \mid t_{j}<t} \exp \left(-\left(t-t_{j}\right)\right)$, and the cycle shows the timing. 


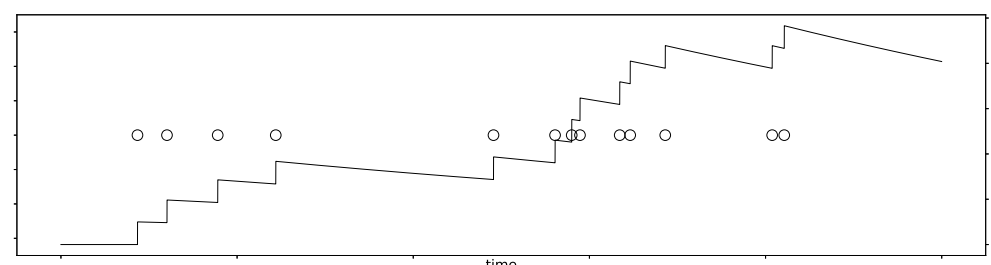

Figure 1. Example of Hawkes process with intensity function $\lambda(t)=10+\sum_{j \mid t_{j}<t} \exp \left(-\left(t-t_{j}\right)\right)$.

In practice, we should decide the parameters $\mu, \alpha$, and $\beta$ from the timing data $t^{n}=$ $\left(t_{1}, t_{2}, \ldots, t_{n}\right)$. Several methods to infer the parameters have been proposed $[12,11,13,14]$. In this paper, we optimize the following objective function: the function $L\left(\mu, \alpha, \beta \mid t^{n}\right)$ is formalized by negative log likelihood with a regular regularization term

$$
\begin{aligned}
L\left(\mu, \alpha, \beta \mid t^{n}\right)= & -\sum_{i=1} \log \left\{\mu+\sum_{j \mid j<i} \alpha \beta \exp \left(-\beta\left(t_{i}-t_{j}\right)\right)\right\} \\
& +\left\{\mu T+\sum_{i=1}^{n} \alpha\left(1-\exp \left(-\beta\left(T-t_{i}\right)\right)\right)\right\}+\frac{c_{1}}{2} \mu^{2}+\frac{c_{2}}{2} \alpha^{2}+\frac{c_{3}}{2} \beta^{2},
\end{aligned}
$$

where $T$ is total time that observes the events and $c_{1}, c_{2}, c_{3} \in \mathbb{R}_{+}$are hyperparameters. To minimize the objective function, we use quasi-newton method.

\section{Implementation for Architecture}

This section specifically shows the method to implement our recommendation system. Previous work [2] assumed that personalized recommendation system is necessary because the needs highly depend on each customer, and utilizes demand and action vectors to personalize the system. Additionally, we assume that the customer's behavior highly depends on their stage of life event, therefore we detect the status of the life event in this paper. Figure 2 shows a calculation flow that takes the above concept into account. In the term and cycle phase, the demand and action vector are updated respectively, and the stage of the life event is classified in the life time phase. The difference among the phases is their interval; the cycle phase is the shortest interval among the phases such as 1 hour, 30 minutes, 1 minutes, the term phase is the intermediate such as 1 week, 1 month, and the life time phase is the longest such as 1 year, 10 years. Note that we assume that the products information like assortment, sale products is updated in the term phase. We will explain each phase one by one.

\subsection{Initialization phase}

The system initializes the setting here. Since the system has no information about action vector at this phase, it utilizes the product information and the purchased information for each customer at the first cycle. The process comprises the following steps. 


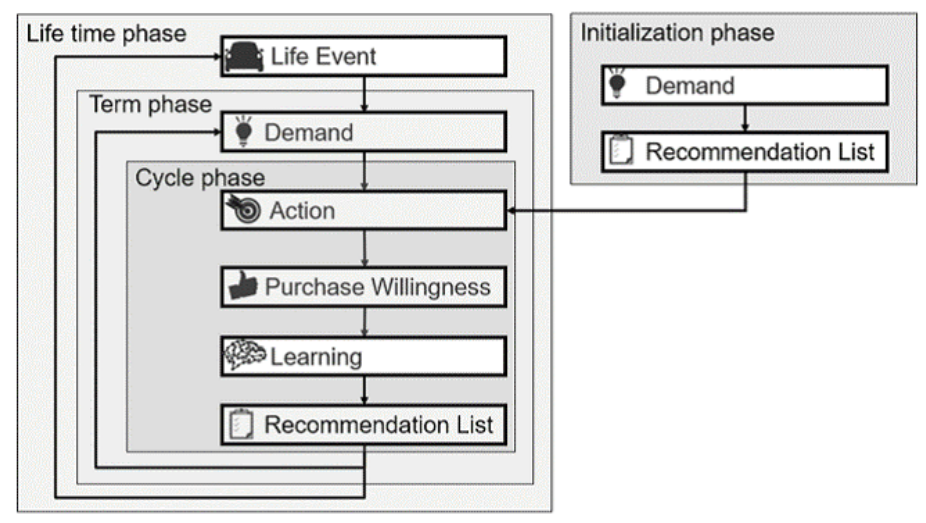

Figure 2. Calculation flow considered the above concept.

Step 1-1.

Determination for Recommendable products:

The system picks up products that can be recommended in the current term, and regard the normalized ones as a fundamental data matrix $\tilde{X} \in \mathbb{R}^{N \times M}$ as we explained at section 3.1. Here, $N$ is the number of possible products to recommend, and $M$ the number of their features.

Step 1-2.

Generation of a Demand Vector:

Let $Y_{j}$ be a context matrix that represents feature matrix of products that was purchased previously by the $j$-th customer, then the system can calculate the semantic projection $P\left(Y_{j}\right)$ in (3). Using the projection, the system projects a feature vector of a product onto the meaning space which we call the demand vector in this paper. For feature vector $g_{s} \in \mathbb{R}^{M}$ of an product $s$, the system projects it onto the demand vector $P\left(Y_{j}\right) g_{s}$.

Step 1-3.

Determination of Recommended Items:

The system calculates a weighted Euclidean distance $\operatorname{dist}\left(\bar{D}, g_{s}\right)$ in $(5)$ between the $s$-th demand vector $P\left(Y_{j}\right) g_{s}$ and the centroid of the meaning space $\bar{D}$ in (4), and recommends the products in ascending order of the distance by the number of possible indicating products $L$.

\subsection{Cycle phase}

After initialization phase, a customer's phase moves to the cycle phase. In the cycle phase, the system updates the recommendation list of customers that purchased something in the previous cycle. The process is the following steps.

Step 2-1.

Aggregating Customer's Action:

In this step, the system aggregates the customer's action log in the previous cycle.

Step 2-2.

Establishing Purchase Willingness: 
After aggregating the customer's action, the system calculates a purchase willingness for each product. The value of the purchase willingness $W_{s}$ for action vector $r_{s}$ of product $s$ is set as follows:

$$
W_{s}= \begin{cases}1 & \left(\operatorname{dist}\left(\bar{a}, r_{s}\right) \leq \epsilon_{r}\right) \\ 0 & (\text { otherwise }) .\end{cases}
$$

Here, $\bar{a}$ represents a centroid of action vectors that was purchased previously, and $\operatorname{dist}\left(\bar{a}, r_{s}\right)$ represents the Euclidean distance between $\bar{a}$ and $r_{s}$. The purchase willingness represents whether the action vector is close to the action vectors previously purchased or not.

\section{Step 2-3.}

\section{Updating Learning Model:}

In this step, the system adopts the mini-batch LVI for LRMM in section 3.2 to score the products. The demand and the action vectors of the products are the inputs $x^{n_{t}}$, and the purchase willingness of the products are the outputs $y^{n_{t}}$. Note that the learning model assumes that a dimension of the input does not change dynamically, thus the system initializes the model if the context is updated because the new semantic projection may have different dimensions or represent other demand even same dimensionality.

Step 2-4.

\section{Updating Recommendation Items:}

In this step, the system updates the recommendation products on the basis of predicted strength of the purchase willingness, which is obtained by $\hat{p}\left(y=1 \mid x, x^{n_{t}}, y^{n_{t}}\right)$ in (19). Since there is no information on which products will be purchased or reacted in the next cycle, the system substitutes the input of the next cycle for the current ones that was utilized for learning. The system recommends the products by ascending order of the strength $\hat{p}\left(y=1 \mid x, x^{n_{t}}, y^{n_{t}}\right)$ by the recommendation size $L$.

\subsection{Term phase}

After several cycle updates, the customer's phase moves to the term phase. In the term phase, products information is updated, therefore the system updates the demand vectors. The process is the following steps.

\section{Step 3-1.}

Updating Recommendable Products:

Like step 1-1, the system picks up the products matrix that that can be recommended in the current term, and normalizes it.

Step 3-2.

\section{Updating Demand Vector:}

In this step, the system updates the context for the customers that purchase something in the previous term. After that, the semantic projection is recalculated, and the demand vector for the current term is updated. 


\subsection{Life time phase}

After several term and cycle updates, the customer's phase moves to the life time phase. In the life time phase, the system classifies the stage of the life time, and the context is specialized on the basis of the life time stage. The process is the following steps.

\section{Step 4-1.}

\section{Defining Life Time Products:}

In this step, the system sets the life time products for each life event such as marriage, child care, education, and so on. For example, a marriage ring is in the marriage event, a house is in the child care event.

\section{Step 4-2.}

\section{Predicting Status of Life Event:}

In this step, the system detects the status of the life event. Let $\{1,2, \ldots, L\}$ be a category set of each life event $t_{l}^{n}=\left(t_{l}^{(1)}, \ldots, t_{l}^{\left(n_{l}\right)}\right)$ the timing of the life event $l$, where $l=1, \ldots, L$, then the estimated intensity function $\lambda_{l}(t)$ for the life event $l$ is obtained by minimizing (21). Since the intensity function is proportional to the event occurrence probability,

$$
\hat{l}=\underset{1 \leq l \leq L}{\arg \max } \lambda_{l}(t)
$$

is regarded as the label of the current life stage.

\section{Step 4-3.}

\section{Updating Context Based on Life Stage:}

In this step, when the estimated life stage $\hat{l}$ is different from the previous one, the system detects a change of life event. In that situation, the context is initialized on the basis of products that was previously purchased by other customers in the same life event.

\subsection{Algorithm}

Summarizing sections 4.1, 4.2, 4.3, 4.4, we can describe Algorithm 2 to implement them. Note that the sections $4.1,4.2,4.3$, and 4.4 are based on one customer, but in practice, the entire system needs to consider all customers, therefore a step of registration for customers to be calculated is appended in the algorithm.

\section{Conclusion}

In this study, we proposed a personalized recommendation system that not only consider about simple use of purchased information and action log, but also the purchased timing to detect specific life events. Compared to previous works, it is considered that our recommendation system may be proper for the area where products highly depend on the customer's life event such as finance. To validate our method and improve our model in tuning level, conducting numerical experiments based on synthetic and real data is our future work. 


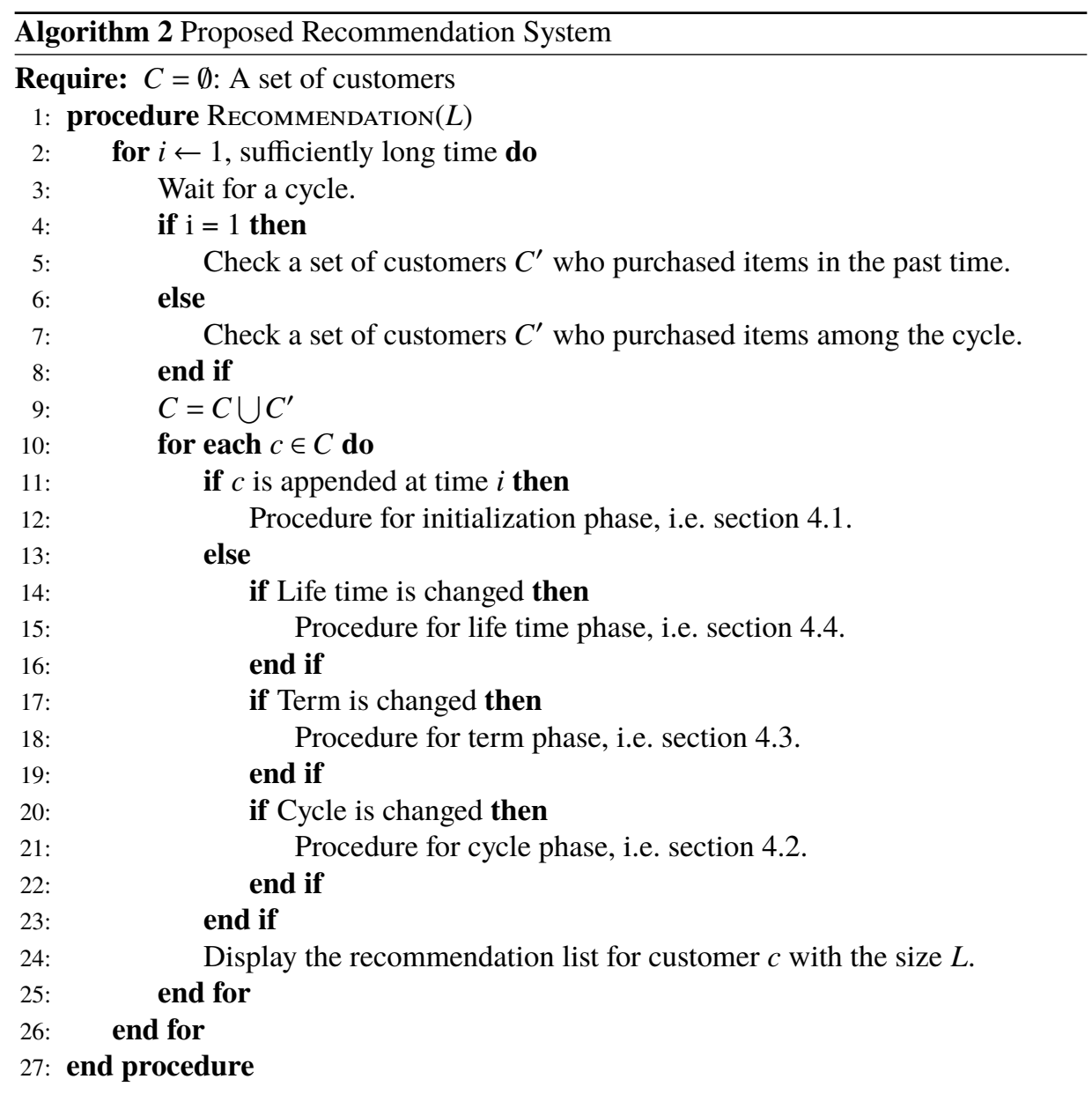

\section{References}

[1] R. Konishi, F. Nakamura, and Y. Kiyoki, "Estimating Adaptive Individual Interests and Needs Based on Online Local Variational Inference for a Logistic Regression Mixture Model," in Knowledge Creation and Intelligent Computing (IES-KCIC), 2018.

[2] R. Konishi, F. Nakamura, and Y. Kiyoki, "Responsive Calibrated Web Personalization System with Online Local Variational Inference for Logistic Regression Mixture Model.," in International Conference on Information Modeling and Knowledge Bases, pp. 94-110, 2019.

[3] T. Kitagawa and Y. Kiyoki, "A mathematical model of meaning and its application to multidatabase systems," in Research Issues in Data Engineering, 1993: Interoperability in Multidatabase Systems, 1993. Proceedings RIDE-IMS'93., Third International Workshop on, pp. 130-135, 1993.

[4] J. Rost, "Logistic Mixture Models," in Handbook of Modern Item Response Theory, pp. 449-463, New York, NY: Springer New York, 1997.

[5] J. Rounds, "Logistic Models with Missing Categorical Covariates," All Graduate Plan B and other Reports, 2009.

[6] M. Kaptein and P. Ketelaar, "Maximum likelihood estimation of a finite mixture of logistic regression models in a continuous data stream," arXiv preprint arXiv:1802.10529, 2018.

[7] P. Wang and M. L. Puterman, "Mixed Logistic Regression Models," Journal of Agricultural, Biological, and Environmental Statistics, vol. 3, no. 2, p. 175, 1998. 
[8] D. Flake, "Separation of Points and Interval Estimation in Mixed Dose-Response Curves with Selective Component Labeling," All Graduate Theses and Dissertations, 2016.

[9] F. Nakamura, R. Konishi, and Y. Kiyoki, "Inference for Logitistic Regression Mixture Model with Local Variational Approximation and Study for Variational Free Energy," in Information-Based Induction Science Workshop, pp. pp.29-36, 2018. (in Japanese).

[10] M. Miyake, T. Ikegami, M. Oka, and Y. Hashimoto, "Web data analysis with hawkes process and histogram bin-width optimization," The 31st Annual Conference of the Japanese Society for Artificial Intelligence, 2017.

[11] M. Rizoiu, Y. Lee, S. Mishra, and L. Xie, "A tutorial on hawkes processes for events in social media," arXiv preprint arXiv:1708.06401, 2017.

[12] T. Ozaki, "Maximum likelihood estimation of Hawkes' self-exciting point processes," Annals of the Institute of Statistical Mathematics, 1979.

[13] S. Linderman, Y. Wang, and D. Blei, "Bayesian inference for latent Hawkes processes," Advances in Neural Information Processing Systems, 2017.

[14] R. Zhang, C. Walder, and M. Rizoiu, "Sparse Gaussian Process Modulated Hawkes Process," arXiv preprint arXiv:1905.10496, 2019. 


\title{
Defects Recognition on Wafer Maps using Multilayer Feed-forward Neural Network
}

\author{
Radoslav ŠTRBA ${ }^{\mathrm{a}, 1}$ and Daniela BORDENCEA ${ }^{\mathrm{a}}$ \\ ${ }^{a}$ ON Semiconductor, 1. máje 2230, 75661 Rožnov pod Radhoštěm, Czech Republic
}

\begin{abstract}
Wafer-defect maps can provide important information about manufacturing defects. The information can help to identify bottlenecks in the semiconductor manufacturing process. The main goal is to recognize random versus patterned defects. A patterned defect shows that a step in the process is not performed correctly. If same defect occurs multiple times, then the yield can rapidly decrease. This article proposes a method for yield improvement and defect recognition by using a feed-forward neural network. The neural network classifies wafer-defect maps into classes. Each class represents certain defect on the map. The neural network was trained, tested and validated using a wafer-defect maps dataset containing real defects inspired from manufacturing process.
\end{abstract}

Keywords: wafer, semiconductor, yield, defects, neural network

\section{Introduction}

As semiconductor devices are being incorporated into more and more devices, the need of producing high-performance cost-effectively integrated circuits' (IC) increases. The semiconductor IC fabrication process is complex, containing various processing steps, among the most important being crystal growth, photolithography, etching, thermal oxidation, implant, deposition. The sand used for wafers growth has to be very clean. It is heated above its melting point and then a pure silicon seed crystal is placed into the molten sand bath. While being rotated, the seed is pulled out resulting an ingot. The ingot is sliced into very thin wafers. Wafers are polished till are very smooth and then they are going through other steps where new layers of material are added. During the flow, the wafers can be affected by adhesion of dust or particles, cracks, scratches, contaminants, process variations, operators or equipment errors that can cause defects.

Probing has an important role in the management of manufacturing process being also used for finding defects. Rapid defect learning and reduction is important in order to quickly find the processes where the failure appears. Defect reduction can be achieved by:

- defects detection

- defect classification

- $\quad$ source defects identification

- process correction in order to eliminate or reduce the defects

- monitoring the process for yield excursions.

\footnotetext{
${ }^{1}$ Corresponding Author
} 
Over the last period, the semiconductor companies are keenly seeking ways for elimination of the defects in order to improve the yield. Defect pattern recognition, clustering and classification is one of the well-investigated ways. Clustering defects refers to grouping the defects with closer relationship. Clustering may indicate an external surface damage, such as a scratch or also the wrong manipulation with the wafer.

Many studies were done by the researchers in this area. Young-Seon Jeong et al., in their paper [1], were showing a new methodology for classifying the defect patterns and also detecting the special autocorrelations using spatial correlogram. Their method was robust despite of the location and size of the defect.

Late in 2017, Kouta Nakata et al. [2] are presenting a way for monitoring the failure pattern, identifying the cause and also monitoring the failure recurrence by using machine learning and data mining algorithms. They are using:

- $\quad \mathrm{K}-\mathrm{Means}++$ for pattern monitoring [3]

- $\quad$ FPGrowth for identification [4]

- $\quad$ standard supervised learning approach for recurrence.

The recent paper of Takeshi Nakazawa [5] is focused on defect pattern classification and image retrieval using Convolutional Neural Networks (CNN). The image retrieval is using a binary code generated for each wafer map.

Another paper focused on same subject is [6]. It describes the classification of missed-type defect patterns using CNN. Using both simulated and also real examples, CNN performed significantly better even if mixed pattern defects are on a wafer with random defects. Moreover, the paper presents a detailed way of generating WBM data that mimics a real WBM dataset.

Single and mixed defects patterns using deep machine learning based approach were studied by [7]. At first, the random noise is filtered out with a spatial filter followed by the defect separation into single and mixed patterns. The single patterns are identified using randomized general regression network and the missed patterns are identified using deep structural convolutional network.

Clustering the defective patterns on wafers was also presented in [8]. The method used was based on the defect spatial dependence across all the wafers. The system was called DDPfinder and it uses the dominant defect as the base for clustering the patterns.

\subsection{Defect Patterns and Recognition}

The result of probing process is showed in wafer maps. Probing means testing of potential future chips while they are still part of the wafer. In Figure 1 can be seen the wafer map where the potential future chips represented as squares (also known as dice). A die can be pass (good die) or fail (bad die). In the figure, to a good die is associated the binary value 0 and to a bad die is associated the binary value 1 .

The detailed problem is described in Chapter 2. The main goal of the paper is to automatically recognize patterns in wafer maps after probing. For better understanding of defects and patters were created few examples. The first example is shown in Figure 1 and illustrates a wafer map. As can be seen, the bad dice are located and clustered in the top part of the wafer. It can be, for example, the incorrect manipulation with the wafer or the defect of the prober (probe tool). 


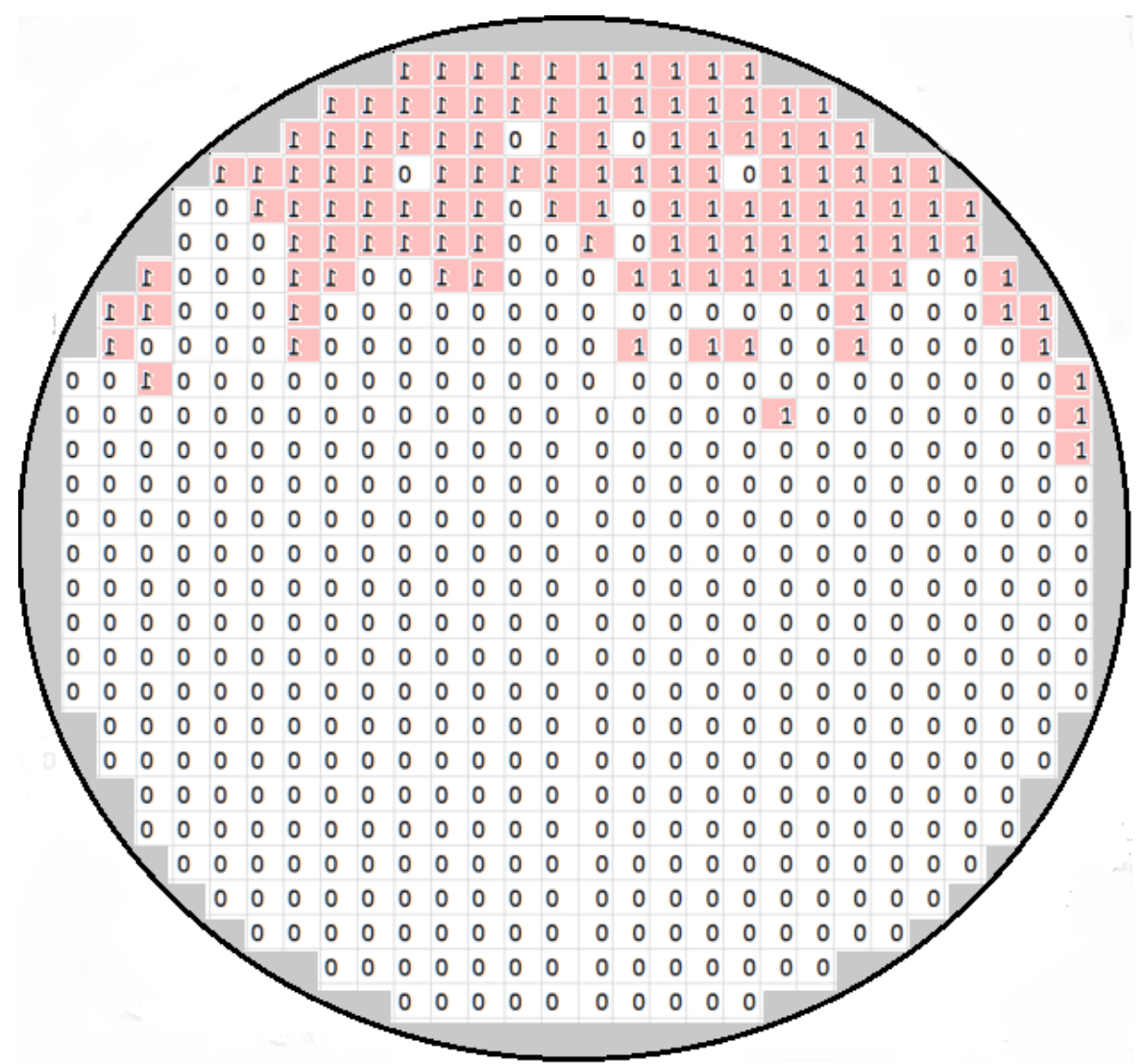

Figure 1. Example of the wafer map

\section{Defect types:}

- Random defects (Figure 2) - bad dice are located in random positions. If no pattern is visible then wafer map is not so helpful regarding bottlenecks identification in manufacturing process.
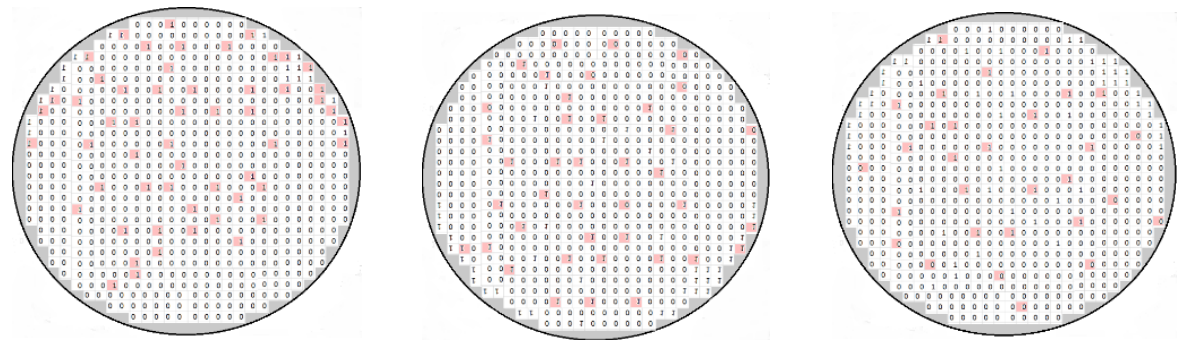

Figure 2. Example of random defects 
- $\quad$ Patterns in following Figure 3

a) RING_CENTER - bad dice are located around the center of the wafer.

b) RING_CENTER_FILLED - bad dice are located in the center.

c) TOP_CLUSTER - bad dice are clustered in the top half-part.

d) RIGH̄T_TOP_EDGE - bad dice are located in the right top edge.

e) SCRATCH - bad dice are showed as a line (straight or skewed).

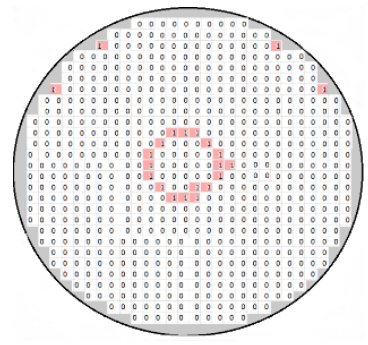

a)

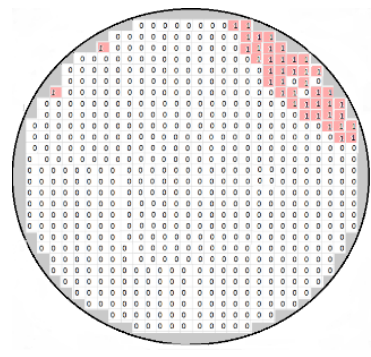

d)

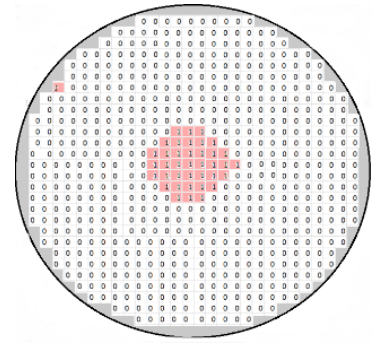

b)

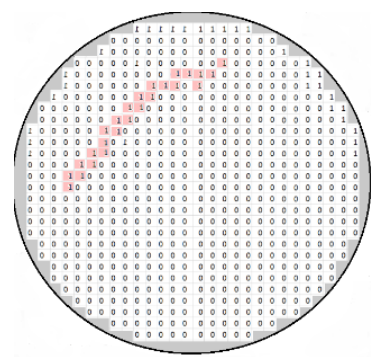

e)

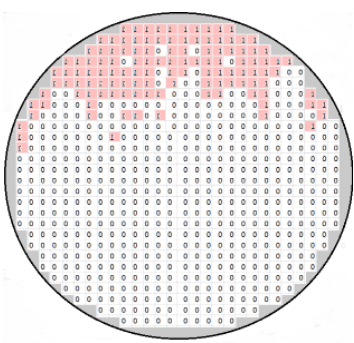

c)

Figure 3. Patterns a) Ring Center; b) Ring Center Filled; c) Top Cluster; d) Right top edge; e) Scratch

This paper is focusing on providing a method for improving the yield by finding the defect die using a feed-forward neural network. The article is structured as follows. In the first chapter, a brief overview of the manufacturing process flow is given, followed by the state of the art. The chapter continues with the description of defects and defects patterns. The second chapter describes the problem. The solution proposed and the experiments are presented in chapter 3 , respectively Chapter 4 . The paper ends with Chapter 5 - Conclusions. 


\section{Problem description}

The main problem during manufacturing process relates to high number of bad dice on the wafer that cause low yield. Let's say, on a wafer are 30 dice. If 3 of them are failed during the tests and 27 are passed, then it results in a $90 \%$ yield. Therefore, only $90 \%$ of the chips will be ready for shipping. This is called die yield, and it decreasing based on the number of total defective dice.

As already mentioned at the beginning of this chapter, the number of bad dice decrease yield. The paper main goal is to present a method for increasing the yield. Based on various reasons (for example: human error, machine error, combination of both), in most of the cases the yield will not reach $100 \%$. The die yield loss reasons can be random and non-random.

When a certain type of scratch appears regularly in a similar place on the wafer, it shows that it is probably caused by a non-random event. The scratches could be due to machine error and the defects on the wafer can caused by the operator. A machine can get damaged and scratch the wafers but also a person can manipulate the wafers incorrectly. The operators are using tools and equipment that can also damage the chips.

The yield, respectively productivity can be increased by identifying the bottlenecks. In manufacturing, from time to time you can meet a slow process. This is so called bottleneck and it refers to a process that accumulates the longest queue. A bottleneck can be automatically identified by:

- using mathematical computations (analytically)

- running a digital model for a period of time

- $\quad$ analyzing the process data.

By analyzing the data can be found diverse defect patterns on the wafer that can reveal important information about the abnormalities. One of the way of recognizing the defect patterns is by using machine learning techniques, such as:

- clustering algorithms using Bayesian interface

- using correlograms

- multi class support vector machine (SVM)

- $\mathrm{CNN}$

- fuzzy rules.

\section{Proposed method}

The method proposed in this article is focused on identification of process bottlenecks using pattern recognition. The method's steps are:

1. data preprocessing and transformation

2. configuration and training of a neural network

3. classification of new items (wafer defect maps)

4. identification of bottlenecks in a process. 


\subsection{Process Description}

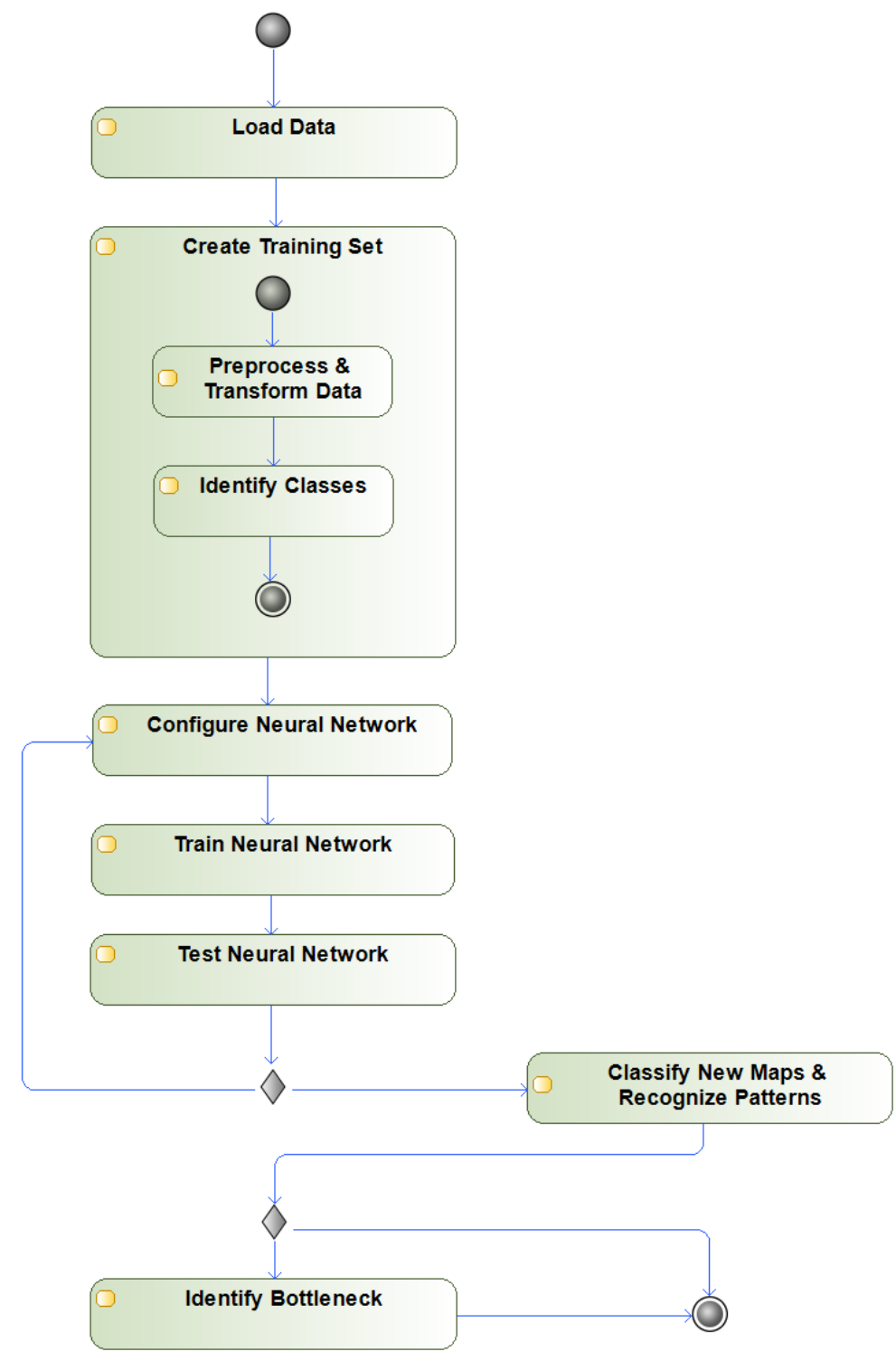

Figure 4. Activity Diagram.

\subsection{Load Data, Preprocessing and Transformation}

The classification accuracy mainly depends on the data quality. Low-quality data may lead to the training of over-fitting or not accurate classifiers. Thus, data preprocessing techniques are essential for data mining. Good preprocessing can improve the quality of the data, thereby helping to increase the accuracy and efficiency of the classifier. A series 
of data preprocessing techniques can be used, for example data cleaning or reduction. Cleaning means removal of the noisy data which can make the classifier mistaken. Reduction means subtraction of the data-size by aggregating and eliminating of the redundant or very similar (correlated) features. [9]

Each type of data requires different approach for preprocessing. Probe data from semiconductor manufacturing are stored in files generated by probers (probe tools) or retrieved from SQL databases. Therefore, all the available data from different sources should be considered for preprocessing. In this paper, preprocessing of data described is done by filtering the data, cleaning data and also performing transformations on those data (Figure 5).

The basic problem with creation of the training dataset is unequal sample-size. Therefore, the appropriate dataset for training of the classifier contains equal number of training data for each class. The dataset presented in this paper was generated from observation and replicating some real defects and patterns. This was done for having high-quality data and avoiding the imbalanced sample-sizes.

The transformation steps described below are shown to understand how the defect wafer maps represented as matrixes can be transformed to vectors. The top part Figure 5 is actually the top part of the wafer (first 3 rows) represented in the Figure 1 - Example of the wafer map. The maps are available for each wafer as binary matrixes, however the classifier presented in this article requires vectors instead of matrixes.

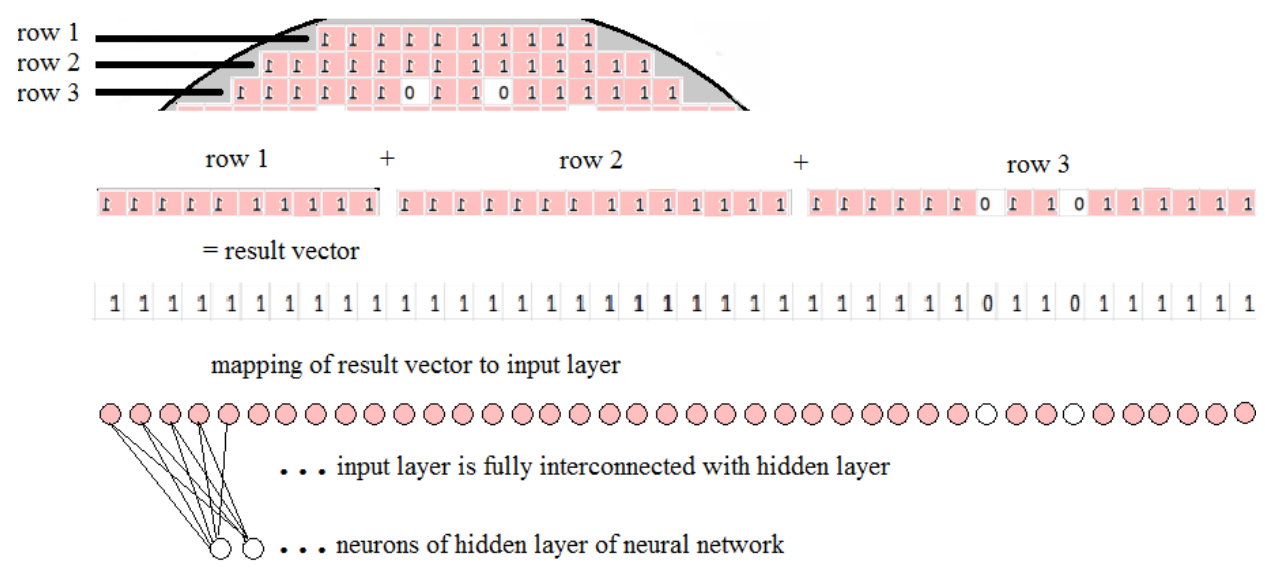

Figure 5. Example of the transformation step from a wafer map to a binary vector

Figure 5 shows the transformation steps from wafer map matrix to vector by joining all rows:

row $1+$ row $2+$ row $3+\ldots=$ result vector .

The result vector is interpreted as an array of binary values (the term array comes from programming languages). Each cell of the vector (array) contains 0 or 1 value. The number of input vector cells equals to the number of input neurons. All the neurons of the input layer (input neurons) are fully interconnected with all neurons of the first hidden layer of the neural network. Always when new item is provided to a neural network, it is provided to the input layer of this network. 


\subsection{The classification using Multilayer Feed-forward Neural Network}

The techniques for classification of the wafer maps can be also used as supportive techniques for defect recognition. The machine-learning methods help to classify the wafer maps and recognize the patterns, which are useful for identification of the bottlenecks in the manufacturing process. The classification is a process that is closely related to the pattern recognition. The neural network trained for classification is designed to take input samples and classify them into groups (classes) $[10,11]$. The set of the features given is represented by the cells of the binary values in the vector. The decision to be made is to which class number the value belongs. The given features can be filled into an input vector $\mathrm{x}$, also known as result vector in Figure 5. Being given a set of pattern samples, where each consists of a vector of the attribute values and the corresponding class label, the classifier will be trained to group the wafer maps. [12]

The Feed-forward is a common architecture for data classification. The Feedforward with more hidden layers is commonly used with the supervised training algorithm called Back-Propagation. "Back-Propagation training of the neural network searches for a set of weights and biases that most accurately predicts value from input samples. Once we have these weight and bias values, we could apply them to an upcoming dataset of items to classify them." $[13,14]$

The supervised training is deducted from the training dataset. [10] The labeled data transformed into vectors is necessary for training the neural network.

"The training of the neural network is the process of finding a set of weight and bias values. For a given set of inputs, the outputs produced by the neural network are very close to some target values." [13]

\subsection{Design and Configuration}

Nowadays, the deep learning is a popular term in the research community. Today, many neural networks are still based upon manual design and configuration due to practical and performance reasons. A person having knowledge about the specific application specifies a network architecture, configuration and activation dynamics. This is perhaps not surprising, given the fact that the general space of possible neural networks is large and complex. The automatically searching for an optimal network architecture may be computationally intractable or impractical for complex applications $[15,16]$. Feedforward network begins with input layer. The input layer is connected to hidden layers, which are connected to the output layer.

In this case the neural network is used to solve a classification problem. Items are classified into separate groups. Each output neuron represent specific pattern. [10] More information about the selection of the optimal neural network architecture and activation functions has been published in the paper named "Finding an Optimal Configuration of the Feed-forward Neural Network". [17]

The four-layered feed-forward network with hyperbolic-tangent activation function in hidden layer and soft-max activation function in the output layer neurons is trained using the scaled conjugate gradient back-propagation training algorithm. The training of the neural network automatically stops when generalization stops improving, as indicated by an increase in the cross-entropy error of the validation items. The example of designed neural network is showed in Figure 6. 


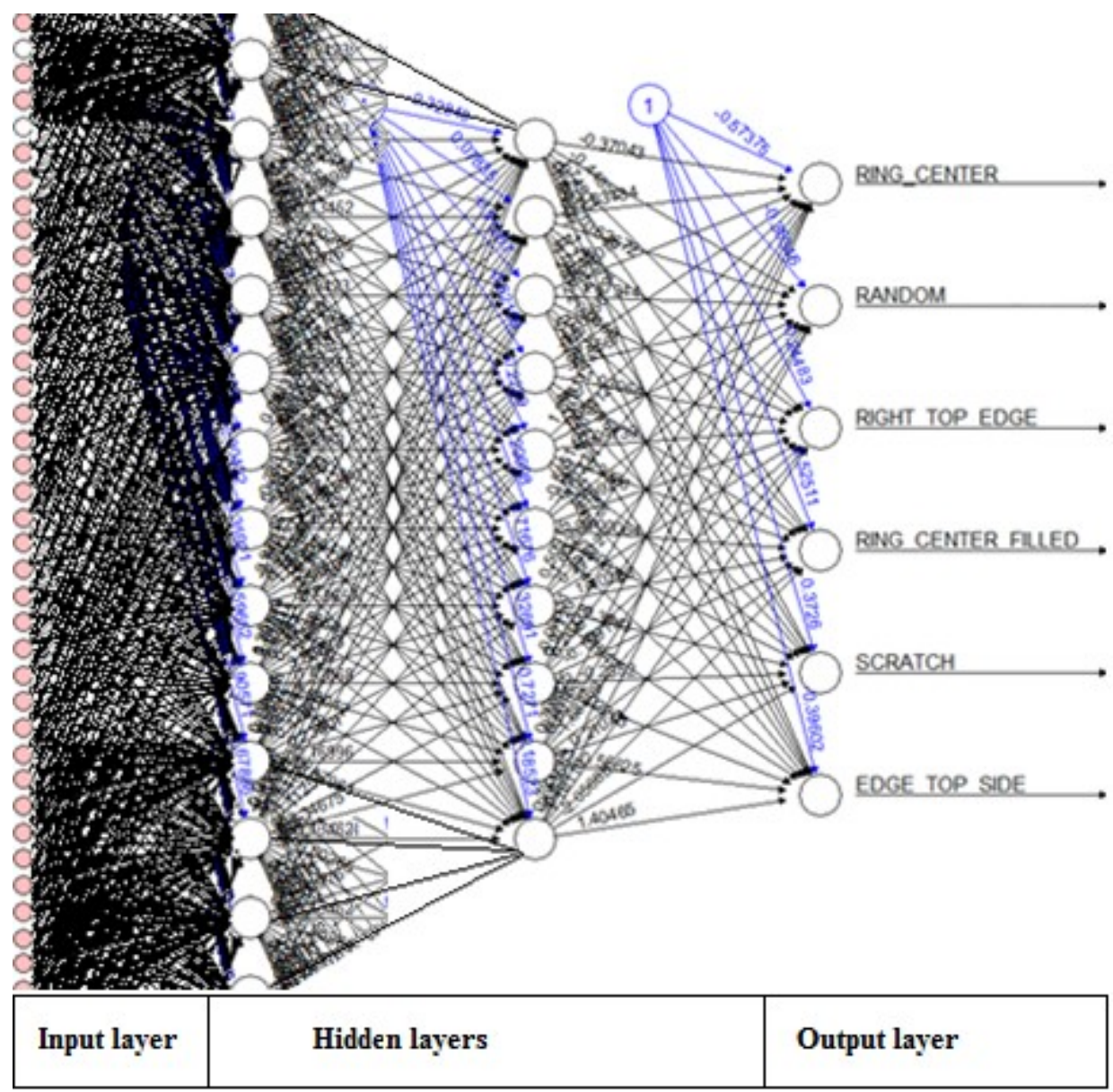

Figure 6. The designed Feed-forward multilayer neural network

The Table 1 below shows more detailed description of four layers included in the designed neural network from Figure 6.

Table 1. Description of layers.

\begin{tabular}{rcl}
\hline Layer & Number of Neurons & Activation Function \\
\hline Input & - dependent on wafer size - & \\
Hidden 1 & 35 & Hyperbolic tangent \\
Hidden 2 & 10 & Hyperbolic tangent \\
Output & 6 & Softmax \\
\hline
\end{tabular}

The hyperbolic tangent activation function (tanh) is used for neurons of the hidden layers. This activation function has been chosen for hidden layer because it returns both positive and negative values. When graphed, the hyperbolic tangent function looks quite similar to the log-sigmoid function. 


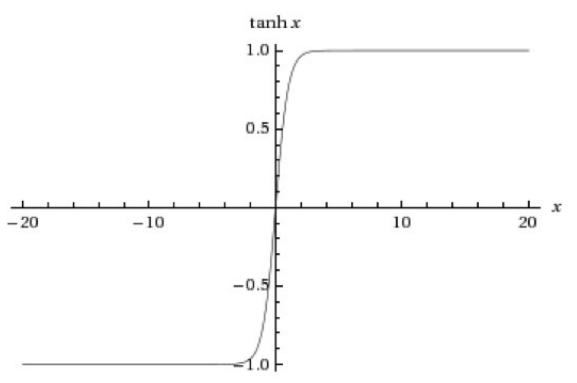

Figure 7. Hyperbolic tangent activation function.

The important difference between log-sigmoid and tanh is that tanh function returns a value between -1 and +1 instead of a value between 0 and 1 . The algebraic expression of the hyperbolic tangent activation function from the family of hyperbolic functions is illustrated bellow (1).

$$
\tanh x=\frac{\sinh \mathrm{x}}{\cosh x}=\frac{\mathrm{e}^{\mathrm{x}}-\mathrm{e}^{-\mathrm{x}}}{\mathrm{e}^{\mathrm{x}}+\mathrm{e}^{-\mathrm{x}}}=\frac{\mathrm{e}^{2 \mathrm{x}}-1}{\mathrm{e}^{2 \mathrm{x}}+1}=\frac{1-\mathrm{e}^{-2 \mathrm{x}}}{1+\mathrm{e}^{-2 \mathrm{x}}}
$$

The softmax activation function is popular as activation function for the output neurons of the feed-forward neural network. A softmax converts an arbitrary real-valued vector into a multinomial probability vector. It is used in the classification problems. This is the version of winner-take-all nonlinearity, in which maximum output is transformed to 1.0. The winner-neuron is activated and exactly the one neuron always wins.

$$
h(z)=\frac{e^{z}}{\sum_{i=1}^{n} e^{z_{i}}}
$$

\section{Experiment}

A neural network was designed and trained for classification of the wafer maps. Back-propagation as a supervised training has been used for the training of the neural network. [18], [19], [20] The experiment was performed in $\mathrm{R}$ environment by using $\mathrm{R}$ studio and MxNet library.

\subsection{Dataset Description}

The training dataset contains the row data (transformed wafer-defect maps) represented by binary parameters. The goal is to classify the items into 6 categories (patterns). These patterns have been created based on real wafer-defects observed during manufacturing process. The defects are divided into six groups. One group contains random defects, and next five groups contains patters, e.g. ring-center, ring-center filled, right top edge, edge top side, scratch. A whole dataset consists of 862 items. The dataset has been divided into two subsets. The first subset includes 518 items. It is used for 
computing the gradient and updating the network weights and biases. The second subset includes 344 test items. It is used for measurement of the neural network accuracy.

\subsection{Training, Validation and Results}

The four-layered feed-forward network with hyperbolic tangent hidden and softmax output neurons is trained using scaled conjugate gradient back-propagation training algorithm with a learning rate of 0.02 . The training of neural network automatically stops when generalization stops improving. It is indicated by an increase in the cross-entropy error of the validation items. The goal is to avoid the over-fitting of the classifier. Therefore, the neural network should be trained as accurate as possible but not overtrained.

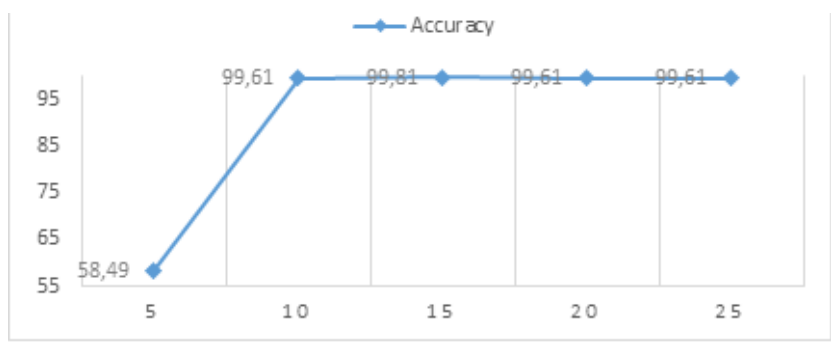

Figure 8. Part of the Training State Plot - Validation Checks

The plot with the validation performance (Figure 8) shows the results of the validation error during the training process. The $\mathrm{X}$-axis represents the trainings rounds and the Y-axis represents the training accuracy or error value. The best results of the validation are indicated at epoch 15 . The best validation performance at this epoch is when error value is 0.19 (validation accuracy is $99.81 \%$ ).

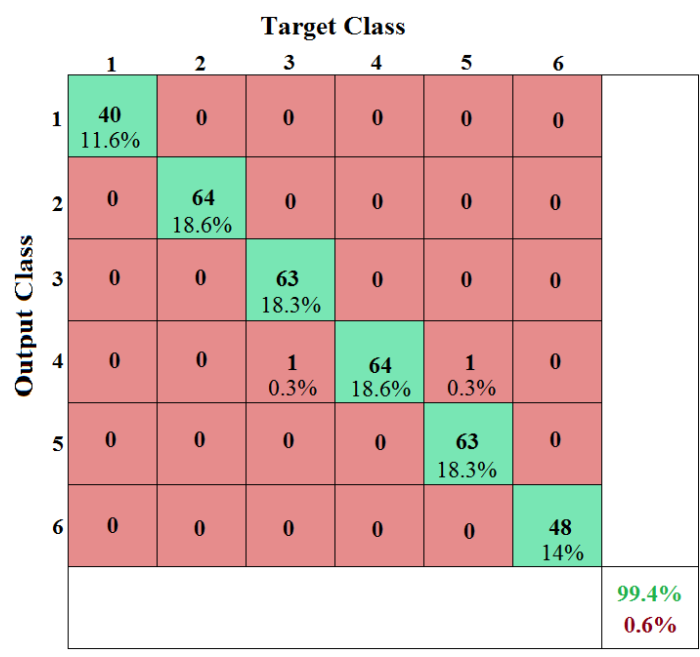

Figure 9. Test Confusion Matrix. 
The results of the classification testing accuracy are visualized using confusion matrix. The confusion matrix (Figure 9) shows the items number and its percentage classified correctly or misclassified to each class. Overall accuracy of designed classifier (feed-forward neural network) is $99.4 \%$ using independent testing dataset.

\section{Conclusion}

This paper shows a way of improving the yield using the machine-learning supportive technique called classification. The wafer-defect maps are classified, using a multilayer feed-forward neural network, into six groups: random defects, ring-center pattern, ringcenter filled pattern, right top edge pattern, edge top side pattern, scratch pattern. One group is non-pattern and five are pattern groups. The network is trained by backpropagation training algorithm.

The training dataset was composed by 862 items: 518 items are used for computing the gradient and updating the network weights and biases are 344 items are used for measuring the neural network accuracy. A four-layered feed-forward network with hyperbolic tangent activation function on hidden layer and with soft-max activation function on output layer was trained. The training algorithm used is scaled conjugate gradient back-propagation. It is shown that the lowest validation error value is 0.19 in the epoch 15 when the neural network stopped improving. Using the confusion matrix (Figure 8), it is shown that 342 items with percentage $99.4 \%$ were classified correctly to given groups and 2 items with percentage $0.6 \%$ were misclassified.

The overall accuracy of proposed method for improving of yield which is using custom designed classifier (feed-forward neural network) is $99.4 \%$. The accuracy was measured using an independent testing dataset. 


\section{References}

[1] Young-Seon Jeong, Seong-Jun Kim, and Myong K. Jeong, Automatic Identification of Defect Patterns in Semiconductor Wafer Maps Using Spatial Correlogram and Dynamic Time Warping, IEEE Transactions on Semiconductor Manufacturing vol 21, no. 4, 625 - 637, December 2008

[2] Kouta Nakata, Ryohei Orihara, Yoshiaki Mizuoka, and Kentaro Takagi, A Comprehensive Big-Data-Based Monitoring System for Yield Enhancement in Semiconductor Manufacturing, IEEE Transactions on Semiconductor Manufacturing, vol. 30, no. 4, November 2017

[3] B. Bahmani, B. Moseley, A. Vattani, R. Kumar, and S. Vassilvitskii, Scalable k-means++, Proc. VLDB Endowment, vol. 5, no. 7, pp. 622-633, 2012

[4] C. Borgelt, An implementation of the FP-growth algorithm, in Proc. 1st Int. Workshop Open Source Data Min. Frequent Pattern Min. Implement., Chicago, IL, USA, 2005, pp. 1-5

[5] Takeshi Nakazawa and Deepak V. Kulkarni, Wafer Map Defect Pattern Classification and Image Retrieval Using Convolutional Neural Network, IEEE Transactions on Semiconductor Manufacturing, vol. 31, no. 2, May 2018

[6] Kiryong Kyeong and Heeyoung Kim, Classification of Mixed-Type Defect Patterns in Wafer Bin Maps Using Convolutional Neural Networks, IEEE Transactions on Semiconductor Manufacturing, vol. 31, no. 3, August 2018

[7] Ghalia Tello, Omar Y. Al-Jarrah, Paul D. Yoo, Yousof Al-Hammadi, Sami Muhaidat, and Uihyoung Lee, Deep-Structured Machine Learning Model for the Recognition of Mixed-Defect Patterns in Semiconductor Fabrication Processes, IEEE Transactions on Semiconductor Manufacturing, vol. 31, No. 2, May 2018

[8] Kamal Taha, Khaled Salah, and Paul D. Yoo, Clustering the Dominant Defective Patterns in Semiconductor Wafer Maps, IEEE Transactions on Semiconductor Manufacturing, vol. 31, issue 1, February 2018, pp. 156-165

[9] D. M. Farid, L. Zhang, C. M. Rahman, M. a. Hossain, and R. Strachan, Hybrid decision tree and naïve Bayes classifiers for multi-class classification tasks, Expert Syst. Appl. (2014), vol. 41, no. 4 PART 2, 1937-1946.

[10] J. Heaton, Introduction to Neural Networks for Java, 2nd ed. Heaton Research, Inc., 2008. ISBN 1604390085.

[11] G. P. Zhang, "Neural Networks in Business Forecasting," Rev. Econ. Sci. (2004), vol. 6, pp. 161-176. Doi:10.4018/978-1-59140-176-6

[12] A. Holst, The Use of a Bayesian Neural Network Model for Classification Tasks. 1997. 110p. Dissertation Thesis at Department of Numerical Analysis and Computing Science, Stockholm Universidad.

[13] J. Mccaffrey, Neural Network Back-Propagation Using C\#. NEURAL NETWORK LAB [online]. (2013) [vid. 2015-12-02]. Available from: https:/visualstudiomagazine.com/articles/2013/08/01/neuralnetwork-back-propagation-using-c.aspx

[14] A. Azadeh, M. Saberi, and M. Anvari, An integrated artificial neural network algorithm for performance assessment and optimization of decision making units, Expert Syst. Appl. (2010), vol. 37, no. 8, 56885697. ISSN 09574174. doi:10.1016/j.eswa.2010.02.041

[15] S. Das. Elements of Artificial Neural Networks. IEEE Transactions on Neural Networks. (1998), Vol 9(1), 234-235.

[16] J. Jung, J. Reggia, The Automated Design of Artificial Neural Networks Using Evolutionary Computation. Success in Evolutionary Computation. (2008), vol 41(2), 19-41. Doi: 10.1007/978-3-54076286-7 2

[17] R. Štrba, S. Štolfa, J. Štolfa, Finding an Optimal Configuration of the Feed-forward Neural Network. Information Modelling and Knowledge Bases XXVII, Frontiers in Artificial Intelligence and Applications. 25th International Conference on Information Modelling and Knowledge Bases (EJC) (2015), Vol 280, 199 - 206. doi: 10.3233/978-1-61499-611-8-199

[18] Zhang, G.P.: Neural Networks in Business Forecasting. Rev. Econ. Sci. 6, 161-176 (2004).

[19] Ghiassi, M., Nangoy, S.: A dynamic artificial neural network model for forecasting nonlinear processes. Comput. Ind. Eng. 57, 287-297 (2009).

[20] Sharma, P., Kaur, M.: Classification in Pattern Recognition: A Review. Int. J. Adv. Res. Comput. Sci. Softw. Eng. 3, 298-306 (2013). 


\title{
Modelling Temporal Scales in (Pre-/Proto-)History
}

\author{
Yannic Ole KROPP ${ }^{\mathrm{a}, 1}$ and Bernhard THALHEIM ${ }^{\mathrm{a}, 2}$ \\ ${ }^{a}$ Christian Albrechts University Kiel, Department of Computer Science, D-24098 Kiel, \\ Germany
}

\begin{abstract}
This paper deals with the comparison of (heterogeneous) temporal datings in pre- and protohistory. It will present a first draft of a conceptual model for the description of the most common types of scales used in this context. The aim is to enable a system to compare objects according to their dating, regardless of the used method and scale. Thus temporally relevant objects can be selected by a query and do not need to be selected individually by an expert. Especially for larger data sets, automated computations, integrity checks, or pattern mining this is beneficial.
\end{abstract}

Keywords. conceptual modelling, archaeological dating, data selection, data filtering, data management

\section{Introduction}

Spatio-temporal analyses are an important tool in archaeology. ${ }^{3}$ Especially when examining transformations and processes of change the differences in spatial distributions over time are of high value. In this (short) paper we will focus on the temporal information needed to perform such analyses and deal with the task of managing data concerning archaeological datings.

Determining the age of archaeological objects is no uniform act. Depending on criteria like material, state of preservation, object-type, available funding and expertise different methods are applicable/available for the temporal classification. In effect the data is diverse regarding precision, accuracy, and robustness. Especially when the results of age determination map to different scales the comparison of datings is not trivial. Selecting adequate (data-)objects by their dating is therefore often done manually ${ }^{4}$ and thus time consuming. We want to ease this process by designing a (conceptual) model which enables the systematic description of 'temporal-dating-scales' 5 and their interlinkage in order to use these information for an automated comparison of dating instances regardless of the used scale.

For sure this approach cannot create more precision than given by the scales/methods and thus is itself often vague and unprecise. But this is typical for this context. An archealog-

\footnotetext{
${ }^{1}$ yk@is.informatik.uni-kiel.de

${ }^{2}$ thalheim@is.informatik.uni-kiel.de

${ }^{3}$ see for example [5], [4], [6]

${ }^{4}$ Even when a database is used, has the 'allowed' time frame to be defined (manually) for each scale.

${ }^{5}$ We will explicitly not compare or rate the methods, but try to match the scales.
} 
ical dating is more or less an 'educated guess' and just possible to a significant degree of (statistical) probability. ${ }^{6}$ In addition datings often refer to phases or periods and not to a single point in time. For example do buildings have a certain time between construction and destruction during which they just exist. Typically the datings consist of ranges which might span several years. These ranges can be cultural epochs like 'neolithic', ${ }^{7}$ but may also occur as variance in scientific measurements. ${ }^{8}$ Also the transitions between temporal phases are in most cases no sharp events, but fuzzy, stepwise and procedural. ${ }^{9}$ We are aware of those (and more) effects which make the comparison of datings not very exact. Anyhow this challenge is normal in the given context and this is the kind of data which is available. When dealing with processes and objects from 10.000 (or more) years ago a certain fuzziness is unavoidable (not only in regard to dating). ${ }^{10} \mathrm{We}$ thus do not aim for a full interoperability of dating-scales, but for enabling basic ordinal operations. ${ }^{11}$ Primary this includes just the binary operations 'equals' $(=, \neq)$ and

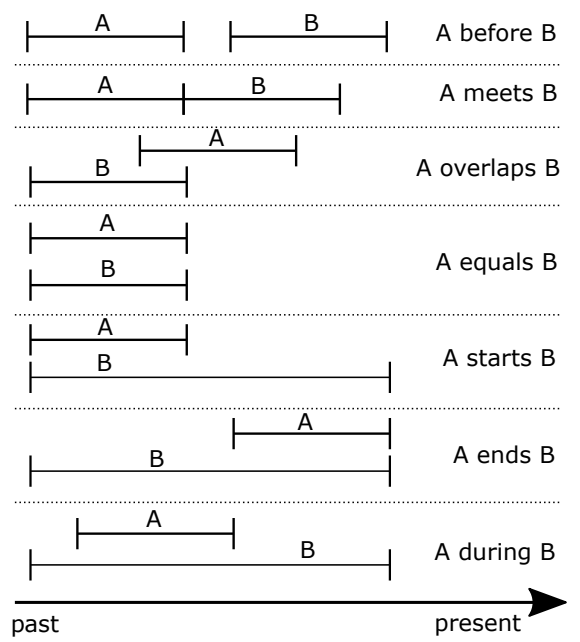

Figure 1. Possible relations between temporal periods according to [11]

'younger/older' $(<,>)$, but by representing periods by start and end times the basic relations of periods can also be used. Figure 1 shows these according to [11]. ${ }^{12}$

In effect the automated cross-scale comparison of datings won't be precise and exact, but will be sufficient for the purpose of identifying an initial set of potentially adequate objects by their dating. We will approach this objective by firstly examining and classi-

\footnotetext{
${ }^{6}$ The data is probabilistic but mostly not as sophisticated as in the probabilistic databases described in [13]. ${ }^{7}$ part of the stone age

${ }^{8}$ The 'hallstatt plateau' is a famous example for a systematic variance up to 400 years in the radiocarbon dating method.

9'Unfortunately' are often these transition phases the times which are scientifically most interesting.

${ }^{10}$ Archaeologists have certain methods to cope with it, see for example [10] or [9].

${ }^{11}$ For more information on ordinal scales (and other scales) see [12].

${ }^{12}$ As the datings are often not precise and a bit fuzzy, the relations which require an exact congruence in one point (meets, starts, ends, and maybe even equals) will probably in most cases result in FALSE, but especially the relations overlaps and during are very useful.
} 
fying the most common dating-scales in archaeology (section 2). ${ }^{13}$ Afterwards we will present a first draft of a conceptual model for the management of the dating-scales and actual dating-instances in section 3. Hereby we keep in mind, that the model shall enable ordinal comparisons of datings, regardless of the used scale. Finally section 4 will conclude our paper.

\section{Scales of Dating}

Temporal datings in archaeology are not only performed by archaeologists, but for example also by experts in botany, (geo-)physics, chemistry, biology/anthropology, climate, etc. For example belongs the popular radiocarbon dating-method to the academic field of chemistry. Depending on the objects and the material which shall be dated the applicable methods vary. The mentioned radiocarbon dating-method is for example only applicable on organic material. Anyhow the variety of methods is not the topic of this paper. We will focus on the dating-instances which result from applying the methods and take a look on the used scales. ${ }^{14}$

Traditionally there are two major types of datings in archaeology. On the one hand $a b$ solute datings which refer to absolute points in time and on the other hand relative datings which refer to some event or object which does not necessarily has to be dated absolute. ${ }^{15}$ As we deal with the scales we will additionally differentiate between textual (mostly ordinal) scales and numeric/quantifiable (mostly interval or ratio) scales.

\section{1. absolute numeric dating}

Absolute numeric datings are typically the ones which also 'normal' people understand easily. Basic example for such a scale would be the Gregorian calendar and datings like $10.000 \mathrm{BC}$ or $1066 \mathrm{AD}$. A more 'archaeological' example are calibrated radiocarbon dates basing on the analysis of ${ }^{14} C$-isotopes. These give the age of objects in (ranges of) years before present $(B P) .{ }^{16}$ Also (calibrated) dates computed by using other isotopes fit in this category.

In general do scales of this category have two main features:

- a fixed global reference point, often given by a specific (gregorian) year

- a steady numeric unit of measurement, like (earth-)years, enabling comparisons of ratio

\section{2. relative numeric dating}

Relative numeric datings do also have a steady numeric unit of measurement, but lack the globally fixed reference point. Examples are:

\footnotetext{
${ }^{13}$ As we are in involved in the research project $C R C 1266$, which explores transformations in pre- and protohistory, most of the presented scales are from that context.

${ }^{14}$ The information about the used methods are still valuable metadata and should not be disposed.

${ }^{15}$ Typically absolute datings are done by laboratories and are costly in terms of money and time and are thus not performed for all objects of an excavation. In addition is the (relative) on-site chronology often more important than the absolute dating of single objects (at least when dealing with just one/few site(s)).

16 'present' is in most cases assumed with the (gregorian) year 1950
} 
- dendrochronology (tree-ring dating) - As a tree at average gains one growth-ring per year and the pattern of the rings is roughly the same for all trees originating from the same region ${ }^{17}$ it is possible to compare the difference in age of wooden objects in years/seasons using these information.

- laminated sediments - Under specific conditions the seasonal (organic) sediments (leaves, pollen, etc.) are visible in the soil of (former) lakes as single layers. It is possible to count the seasons/years between two layers.

- written records - In some cases ancient texts exist which describe events and their dating by using other events. Thus statements of the form 'event A took place 14 years after event $B$ ' are used for temporal information.

In general this type of scaling provides a chronology and a quantified temporal sequence, but no fixed reference on an absolute scale. Still this kind of scale might be mapped to an absolute scale if some of its objects are dated absolute. But the precision of absolute dating is often worse than the relative connections and thus the relative information stay highly valuable.

\section{3. absolute textual dating}

Absolute textual scales refer to datings which use labels that are seen as synonyms for (more or less) specific absolute time periods. These temporal periods do not need to be equally long or uniform. In particular these scales mostly refer to cultural epochs like Neolithic or Iron Age. But also climatic/geological periods like Holocene or Boreal fit to this category, as well as the dynasties of ancient Egypt.

While climatic and geological periods are mainly global phenomena and the egyptian dynasties mainly concern Egypt, do cultural epochs have a strong spatial component. Actually they are not datings per se but describe a certain state of society and technology. The Neolithic for example is defined by the presence of settlements, agriculture, husbandry, ceramics and (flint-)tools. This cultural state has been reached in different regions at different (absolute) times. Thus 'Neolithic in Germany' is not equivalent to 'Neolithic in Japan'. Also the duration of such phases differs depending on the regions. In effect there is not one global scale for cultural epochs, but multiple scales for the different regions. Although there are some general patterns and hierarchies in the sequence of the epochs, in some regions certain epochs are skipped or left out. Figure 2 shows an excerpt of an overview about differing durations of cultural epochs within Germany.

In summary this kind of scales can be characterised as follows:

(hierarchical) sequence of textual values which are used as synonyms for absolute time periods, in some cases (especially cultural epochs) having a scope on specific spatial regions

\section{4. relative textual dating}

Relative textual dates have neither a fixed global reference point nor a steady numeric unit of measurement. They consist of qualitative ordinal relations between objects, events, etc. and are often basing on common sense and heuristics. Some examples:

\footnotetext{
${ }^{17}$ same seasonal weather conditions etc.
} 


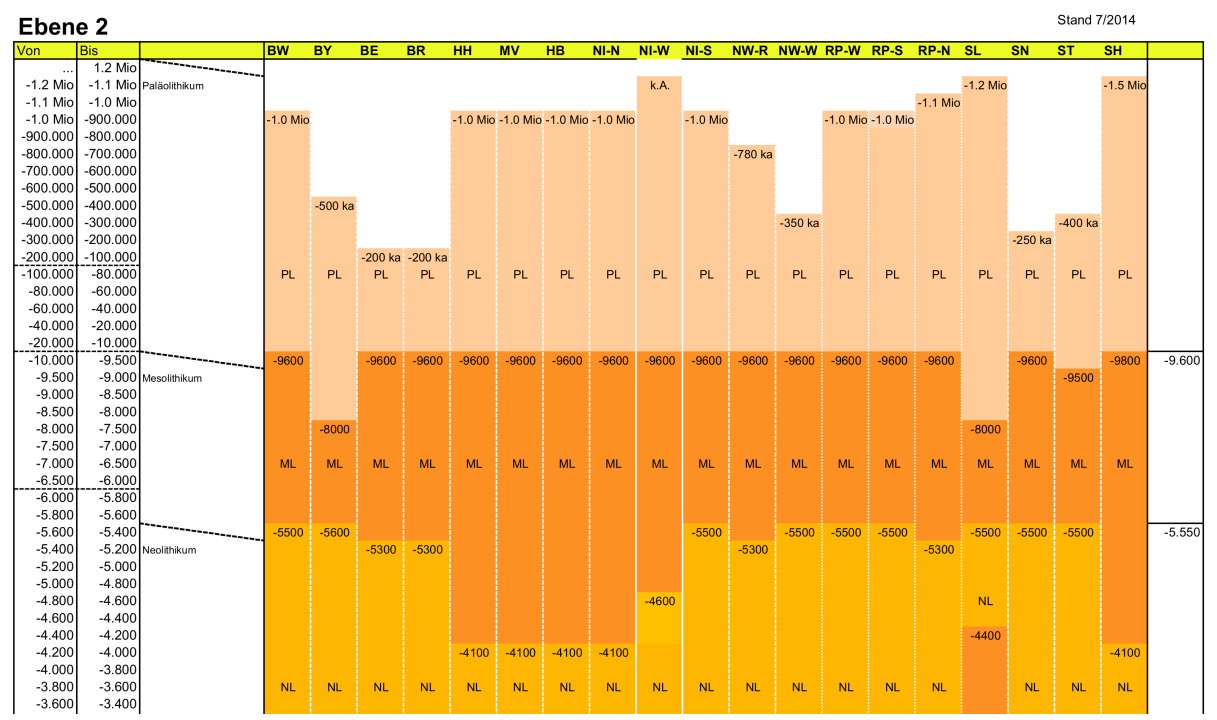

Figure 2. differing durations of cultural epochs within Germany, taken from [7]

- These artefacts originate from the same grave, they were probably deposited at the same time.

- This coin/text mentions a famous person, it must have been created after or while that person lived.

- Artefact A was (three layers) deeper in the ground than artefact B. A must be older than $B$.

Even if a referred object/event can be dated absolute ${ }^{18}$ there is just an indirect transitive connection to an absolute scale for the remaining objects/events, but no actual absolute dating for them. So this type of scale is more useful for (relative) chronologies and to determine ages when something was (definitely) not existing.

\section{Handling different 'temporal' Datings}

As section 2 described, are the common ways of temporal dating in archaeology not always equivalent, but might refer to different types of scales. In order to enable (computer) systems to compare 'incongruent' datings we use a straightforward approach:

- describe scales in a conceptual model

- add used scale to every dating instance

Figure 3 shows our initial draft of the conceptual model for temporal datings and datingscales. Basically the model contains elements for each type of scaling which was described in the previous section. The absolute scales are backed with information about

\footnotetext{
${ }^{18}$ If for example tephra is found within a (geological) layer it is mostly possible to determine the exact (dated) volcanic eruption. See [14].
} 


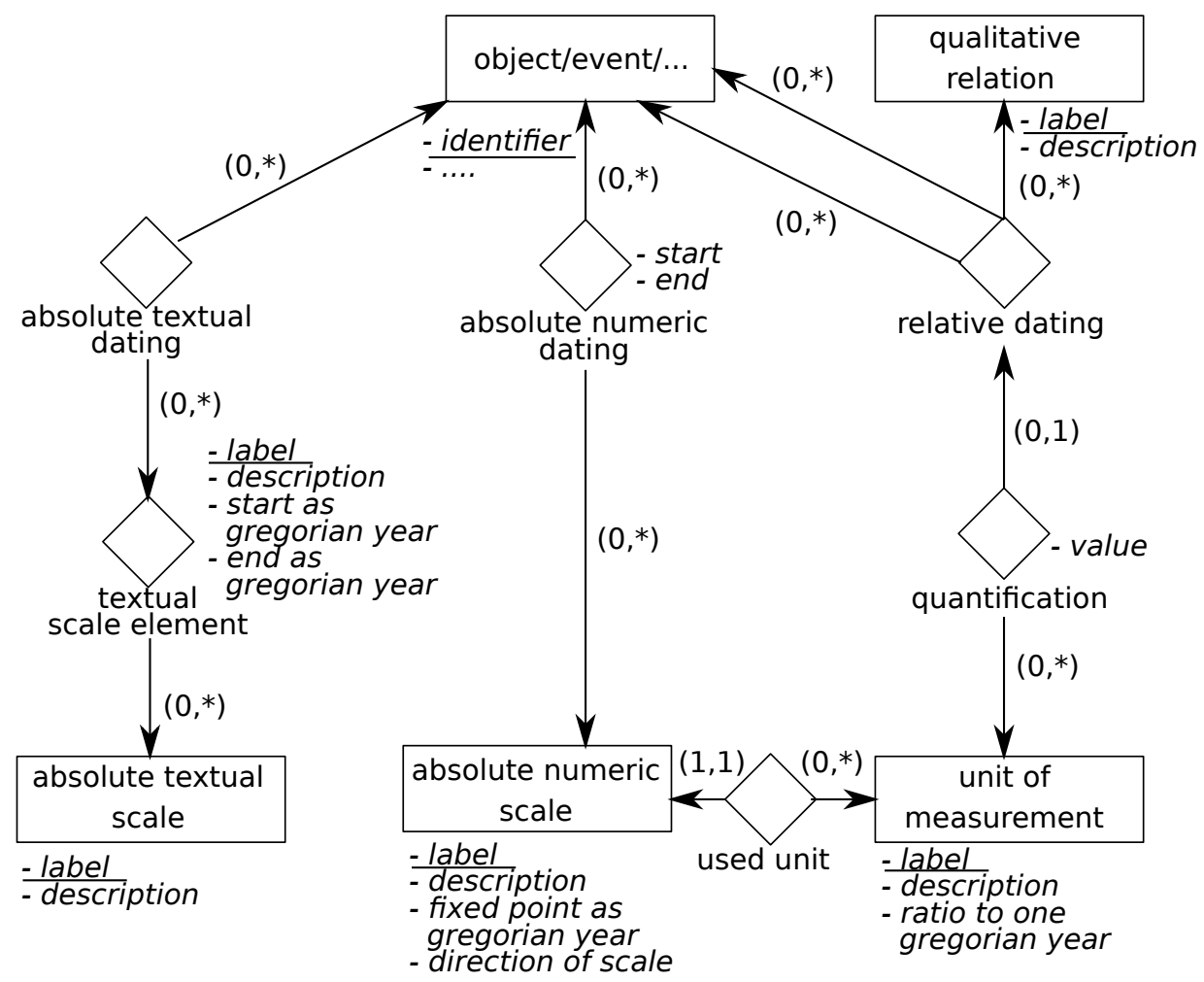

Figure 3. draft of a conceptual model for temporal datings and dating-scales

their orientation in the gregorian calendar. ${ }^{19}$ This information can be used (by automated functions) to check questions like:

Is 'Neolithic' on the northern-Europe scale the same like 'Neolithic' on the Mediterranean scale?

Is 4000 BC within the range of 'Neolithic' on the Mediterranean scale?

Is 4000 BC the same as $6050 \mathrm{BP}$ ?

etc.

In general the comparison of just absolute datings is quite unproblematic having the reference to the gregorian calendar as a 'mediating' scale. The comparison of relative datings or a relative dating and an absolute dating is more interesting. Especially when dealing with data from just one excavation-site, most of the objects will probably be initially dated with the same (unprecise) cultural period..$^{20}$ In such a context the relative relations of the artefacts and relative temporal sequences are highly valuable. Artificial phases or concrete events are used as reference to gain a temporal order. By explicitly considering such relations in the conceptual model we are not only able to store these information, but also to use them for (automated) logical reasoning. Some examples:

\footnotetext{
${ }^{19}$ having (sun-)years as the basic unit and a fixed starting point 2020 years ago

20 'This site, including all artefacts, is Neolithic.'
} 
- If $B$ is older than $A$ and $C$ is older than $B$ then must $C$ be older than $A$ and the sequence must be $C-B-A$. (due to transitivity of time)

- If $A$ is older than $B$ and $C$ is older than $B$, it is unclear if the sequence is $A-C-B, C$ $A-B$, or even $(A=C)-B$, but other sequences like $A-B-C, C-B-A$, etc. are definitely incorrect.

The ordinal position of two objects can apparently also be derived from absolute datings. And if an object is dated on more than one scale (especially on absolute and relative scales) objects which are (only) dated on different types of scales can (sometimes) be compared:

- $A$ is dated $4000 B C$ and $C$ is dated $4500 B C$ and $B$ is older than $C$ $\Longrightarrow C$ is older than $A$

But the direct temporal comparison of two objects is not always possible or (logically) derivable. If in the last example ' $C$ would have been older than $B$ ' the ordinal relation of $A$ and $C$ could not have been computed. ${ }^{21}$ Also the ordinal position of two objects which are both just dated by 'Mediterranean-Neolithic' is not clear, as this dating refers to a fairly long period.

Anyhow the result 'no ordinal relation is available/derivable' (due to lack of information) is acceptable, as an automated system will probably find this result faster than a person would and thus the work of archaeologists will still be eased. In addition the structure allows to (automatically) detect logical errors like circles, inconsistencies, contradictory values, etc. and to compute/check (feasible) chronological/ordinal sequences. ${ }^{22}$

\section{Conclusion \& Outlook}

In this (short) paper we deal with temporal datings of objects/artefacts in an archaeological context. We do not address the general concept of time (see [3]) or archaeological questions like 'What time-span is relevant for which phenomena?' (see [1], [2]), but restrict ourself to pure age determination. We though do not address challenges ${ }^{23}$ of the process 'dating' and its methods ${ }^{24}$ itself, but focus on data, scales, and comparability of object-datings. ${ }^{25}$

We characterised the different types of dating scales by the dimensions absolute-relative and textual-numeric. In general these types of scales are at least ordinal, but especially some of the numeric scales have interval- or even ratio-level. We introduced a draft for a conceptual model which allows the description of different scales and the storage of corresponding dating instances within one system.

The structure does also have the potential for enabling automated ordinal comparison of objects (in respect to temporal chronology) basing on the available datings regardless of the actual scales. Even if the available information is not sufficient for a meaning-

\footnotetext{
${ }^{21}$ At least if the relative relation of $A$ and $C$ wasn't quantified, like in ' $C$ is 30 years older than $B$ '.

${ }^{22}$ To perform this tasks the available ordinal information about objects can for example be viewed as mathematical transitive relations or directed graphs.

${ }^{23}$ age of object vs. age of material, different ages (of different parts) in one object, etc.

${ }^{24}$ The used method is a valuable metadatum, which should be captured in another system-component.

${ }^{25}$ Other temporal entities like time of excavation, age of data, etc. and can for example be handled by Kramers general time-component [8].
} 
ful result, the outcome that there's a lack of information is all right. Without sufficient information also a human expert could not perform better. In addition does the structure have potential for automated detection of contradictions and inconsistencies and for feasibility-tests of chronological sequences.

In summary does this (short) paper present initial ideas for handling temporal information across scales and a draft for the conceptual model, but no existing system. The systematic definition of rules, algorithms, and functions for (ordinal) comparisons, automated deduction, logical reasoning, etc. is part of future work.

Acknowledgement: This research was performed in the framework of the CRC $1266^{26}$ 'Scales of Transformation - Human-Environmental Interaction in Prehistoric and Archaic Societies' which is funded by the Deutsche Forschungsgemeinschaft (DFG, German Research Foundation - Projektnummer 2901391021 - SFB 1266) ${ }^{27}$. We thank both institutions for enabling this work.

\section{References}

[1] Geoff Bailey. Concepts, time scales and explanations in economic prehistory. 'Economic Archaeology', British Archaeological Reports, pages 97-117, 1981.

[2] Geoff Bailey. Time perspectives, palimpsests and the archaeology of time. Journal of anthropological archaeology, 26(2):198-223, 2007.

[3] Geoffrey N Bailey. Concepts of time in Quaternary prehistory. Annual review of anthropology, 12(1):165-192, 1983.

[4] Douglas James Cowie. Method and theory of archaeological spatio-temporal analysis. PhD thesis, University of Southampton, 2018.

[5] Centre for Baltic and Scandinavian Archaeology (ZBSA). EPHA - European prehistoric and historic atlas. http://www.zbsa.eu/zbsa/publikationen/open-access-datenmaterial/epha-european-prehistoric-andhistoric-atlas/.

[6] Daniel Knitter, Jan Piet Brozio, Wolfgang Hamer, Rainer Duttmann, Johannes Müller, and Oliver Nakoinz. Transformations and site locations from a landscape archaeological perspective: The case of Neolithic Wagrien, Schleswig-Holstein, Germany. Land, 8(4):68, 2019.

[7] Kommission Archäologie und Informationssysteme des Verbandes der Landesarchäologen (AG 'Thesauri'). Der 'Zeitstrahl' - Archäologische Datierungen in Deutschland. http://www.landesarchaeologen.de/verband/kommissionen/archaeologie-und-informationssysteme/, 2014.

[8] Frank Kramer. Ein allgemeiner Ansatz zur Metadaten-Verwaltung. Logos Verlag Berlin GmbH, 2019.

[9] Doris Mischka. Aoristische Analyse in der Archäologie. Archäologische Informationen, 27(2):233-243, 2004.

[10] Oliver Nakoinz. Datierungskodierung und chronologische Inferenz-Techniken zum Umgang mit unscharfen chronologischen Informationen. Praehistorische Zeitschrift, 87(1):189-207, 2012.

[11] Richard T Snodgrass. Developing time-oriented database applications in SQL. The Morgan Kaufmann series in data management series, 2000.

[12] S. S. Stevens. On the Theory of Scales of Measurement. Science, 103 2684:677-80, 1946.

[13] Dan Suciu, Dan Olteanu, Christopher Ré, and Christoph Koch. Probabilistic databases. Synthesis lectures on data management, 3(2):1-180, 2011.

[14] Robert Walter. Potassium-Argon/Argon-Argon Dating Methods, volume 2, pages 97-126. 011997.

\footnotetext{
${ }^{26}$ http://www.sfb1266.uni-kiel.de/en/

${ }^{27}$ http://www.dfg.de/en/index.jsp
} 


\title{
Application of a Heterogeneous Correlation Integration Method to a Context Cube Network Semantic Model for Railway Passengers
}

\author{
Motoki Yokoyama ${ }^{\mathrm{a}, 1}$, Yasushi KIYOKI ${ }^{\mathrm{b}}$ and Tetsuya MITA ${ }^{\mathrm{a}}$ \\ ${ }^{a}$ Research and Development Center of JR East Group, East Japan Railway Company \\ ${ }^{\mathrm{b}}$ Graduate School of Media and Governance, Keio University, Japan
}

\begin{abstract}
In recent years, with the development of information technology, many cyber-physical systems, in which real space and the information space are linked for data acquisition and analysis, have been constructed. The purpose of constructing a cyber-physical system is to solve and improve social and environmental problems. An important target is the railway space, which aims to provide safe and stable transportation services as part of the social infrastructure. In this paper, we propose a new data model, the "Context Cube Semantic Network", for the railway space and a metric method that employs an integrated scale based on heterogeneous correlations of purpose, sensibility, and distance for the railway space. Furthermore, we constructed a station guidance system that implements the proposed method and evaluates subjects at the station. As a result, we clarified the effectiveness and applicability of the system.
\end{abstract}

Keywords. Context Awareness, Semantic Computing, Data Modeling, Information Retrieval, Cyber-Physical System

\section{Introduction}

In recent years, with the development of information technology, many cyberphysical systems, in which the real space and information space are linked for data acquisition and analysis, have been constructed. The purpose of constructing a cyber physical system is to solve and improve social and environmental problems. An important target is the railway space, which aims to provide safe and stable transportation services as social infrastructure. An information provision system for railway users is important for improving the value of railways.

An important research field and technology for constructing cyber-physical systems is a database system that has functions of retrieving, combining and analyzing information on multimedia in various contexts. The basic function of this system is the application of the Mathematical Model of Meaning (MMM), which is a semantic computing model with a contextual analytical methodology [1]. Kiyoki et al. have proposed a mathematical model of meaning, which is a context-dependent semantic

\footnotetext{
${ }^{1}$ Corresponding Author, Corresponding author, Book Department, IOS Press, Nieuwe Hemweg 6B, 1013 BG Amsterdam, The Netherlands; E-mail: bookproduction@iospress.nl.
} 
associative memory model, and have conducted research on semantic and associative retrieval systems for various data $[2,3,4,5,6]$. Therefore, the application of these models to dynamically correlate various objects in railway space increases the feasibility of creating new services and business.

Today, railway operators have built information-providing systems for railway users to reduce the load of information acquisition [7,8]. Specific examples include a system that displays suspension/delay information on displays installed in stations and trains, a system that displays the positions of trains in real time, and a congestion degree visualization system that uses cameras installed in stations. In these systems, the information acquisition load of users has been improved with the development of cyberphysical systems.

Currently, the diversification of user values in Japan is progressing with the decrease in the birth rate, increase in foreign visitors, and rapid changes in the social environment. An actual problem is the difficulty of providing information to satisfy the user's needs in the conventional information provision for an unspecified majority. To solve this problem, we have proposed a method to dynamically measure the relevance between users and station facilities/stores according to their context. The basis of this approach is the Mathematical Model of Meaning that understands and measures the meaning of data according to the user's context. The features of the proposed method are to construct a spatial, temporal and semantic data model and measure the amount of correlation among the data models and dynamically measure the correlation among the data according to the user's context.

In this paper, we propose a metric method using an integrated scale that is based on heterogeneous correlations of purpose, sensibility, and distance for the railway space. In addition, a station guidance system will be built. The features of the system are described as follows.

(1) Spatial representation using a data model "context cube semantic network" for the railway space.

(2) Measurement and integration of the correlation among context cubes according to time, space, and meaning and the expression of this correlation in a one-dimensional space.

\section{Related Studies}

The information that satisfies the needs of individual passengers must reflect the context that represents the dynamic/static situation and dynamic/static intention of the passenger [9]. Abowd et al. [10] states "We define context as any information that can be used to characterize the situation of an entity, where an entity can be a person, place, or physical or computational object. We define context-awareness or context-aware computing as the use of context to provide task-relevant information and/or services to a user." Many information-providing systems that reflect the user context have been investigated. For example, a study explored the smartphone application search technique by using search keywords that represent the user status.

Yao et al. and Maroulis et al. aim to improve users by building context-aware location recommendation applications. Their method analyzes temporal, locational and social network data that are obtained via location-based social networks that are serviced 
by tensor factorization. This analysis contributes to higher accuracy in the recommendation of locations according to the context of the user. $[12,13]$

The proposed method in this paper is based on the human memory recall model and differs from methods that employ machine learning and neural networks. Studies have shown that humans have two intuitive and logical decision-making systems[14]. The proposed method in this paper is based on a memory recall model that corresponds to these systems.

\section{Approach}

\subsection{Data Model}

Our method can rank the services that satisfied passengers. In this paper, we classify the context of the passenger into the following eight types: Personal Static Situation (PSS), General Static Situation (GSS), Personal Dynamic Situation (PDS), General Dynamic Situation (GDS), Personal Static Intention (PSI), General Static Intention (GSI), Personal Dynamic Intention (PDI) and General Dynamic Intention (GDI). Personal contexts include personal information about passengers and information that is not disclosed by companies. The general contexts are information that is always shared, such as day, time, and weather information. These contexts are utilized to express railway space objects. The structure of each context is shown in Figure 1.

In this paper, we describe objects of railway space, such as passengers, services and facilities, with context cubes (Figure 2.).

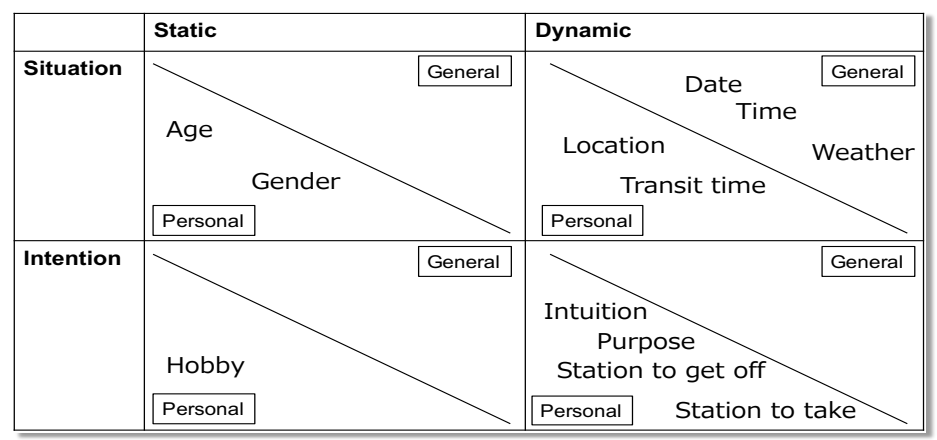

Figure 1. Example of a context 


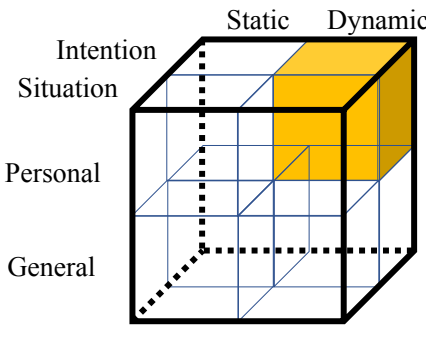

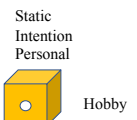
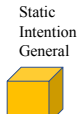

Static

Situa

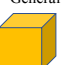

Static
Situation
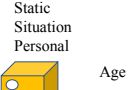

Figure 2. Structure of object context cub

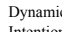

Intentio

0 Sensibility

purpose of move

Inntention

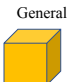

Dynamic

Situation

Location

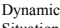

General Date

Date
Time

An object can be easily modeled by expressing it in a cube, and various objects can be expressed with the same data model. Both the side that accesses the cube and the cube can be designed to autonomously change its state. The cubes are composed of sub-cubes, which have elements that are contexts, such as passengers and services. The state of a cube changes depending on the state of the railway space and the dynamic interaction among the cubes. Private information can be concealed by target encapsulation.

We dynamically associate entities of the railway space by temporally, spatially and semantically filtering and ordering cubes. Semantic space should be constructed for each object; it will be the subject of weighing relationships, such as those of passengers, facilities and services. For example, in the case of providing information to passengers, a semantic space is constructed from meaningful elements based on passengers, and a cube is mapped to the space. By projecting to the subspace that corresponds to the context, its meaning is determined, and the semantic relation is determined by performing a distance calculation (Figure 3). Furthermore, depending on the temporal and spatial context, whether each cube is included in the ranking can be determined. Specific filtering factors include business hours and ticket gates inside and outside (Figure 4).

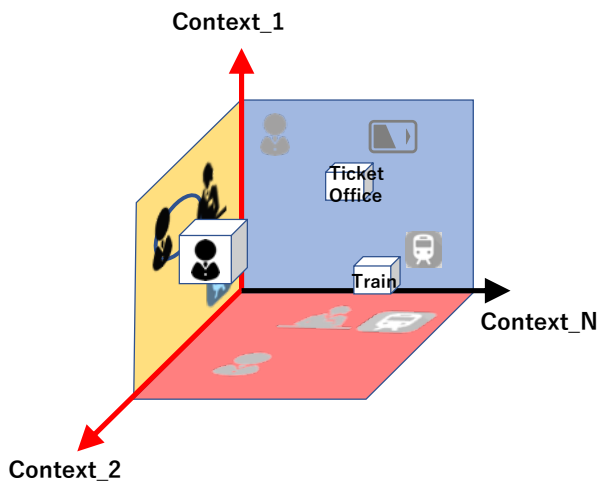

Figure 3.Correlation metric among cubes 


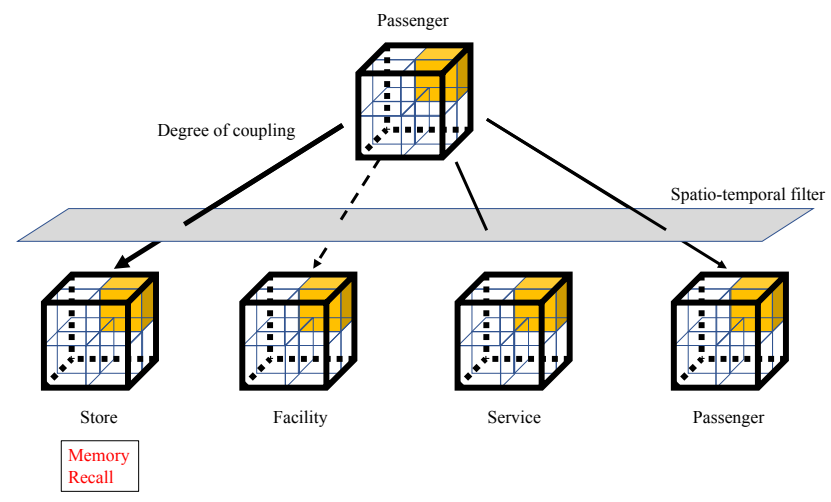

Figure 4. Memory recall by the context cube system

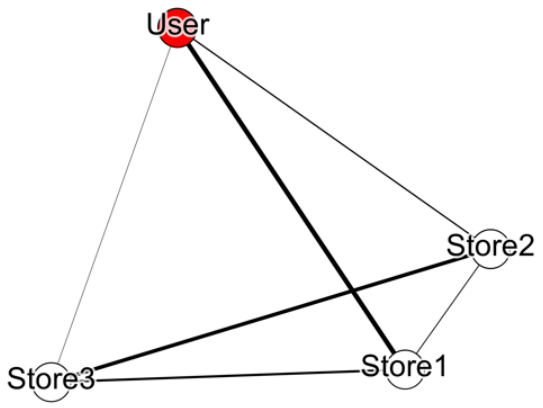

Figure 5. Context Cube Network in Railway Space

In this paper, we propose the Context Cube Semantic Network, which is a semantic network model of the Context Cube, and express the railway space. In this model, a node of a network is a context cube, and a link represents the correlation (weight) between among the context cubes. This model enables context-oriented semantic analysis of the railway space (Figure 5).

\subsection{Correlation Metric Method}

In this paper, we focus on the passenger and establish a correlation metric method among services to enable information provision in anticipation of passenger needs. The preliminary step of passenger needs in this paper is to select a subspace of semantic space based on the intention (purpose) and sense according to the context of passengers, estimate the passenger's context using the correlation measure results for passenger and service. The selection of a space refers to correlation amount weighing, which is an associative model that ignites memory recalls in contexts. The results of the memory recalls that are performed once in a context are different. The procedure of the method is described as follows: 
- $\quad$ Providing Intention Semantic Space $M_{I}$ and Sense Semantic Space Ms.

- Providing the Passenger's Context.

- Selecting the Semantic Subspace of MI and Ms.

- Correlation metric for Passenger's Semantic Subspace M' and Ms'.

- Semantic Correlation Integration in 1-dimension.

- $\quad$ Ranking

\subsection{Providing Semantic Space}

The Intention Semantic Space $\boldsymbol{M}_{\boldsymbol{I}}$ set of $m$ station services (e.g., ticket counter, travel center, etc.) and $\mathrm{n}$ features, in which context elements are utilized in the field of passenger service, is provided in the form of an $\mathrm{m} \times \mathrm{n}$ matrix $\boldsymbol{M}_{\boldsymbol{I}}$ (Figure 6.). Note that the Sense Semantic Space has the same structure as the Intention Semantic Space. In this example, the matrix represents several services that are accessed in the station. These services are characterized using 29 features. $\mathrm{C} 1$ to $\mathrm{C} 15$ in Table 1 are features of the Intention Semantic Space, and the other features pertain to the Sense Semantic Space. If the facility and feature are positively related, the matrix element is 1 ; if they are negatively related, the matrix element is -1 ; otherwise, it is 0 . We define the semantic space $\boldsymbol{M}_{\boldsymbol{I}}$ and $\boldsymbol{M}_{\boldsymbol{S}}$ as the span of the features in Table 1.

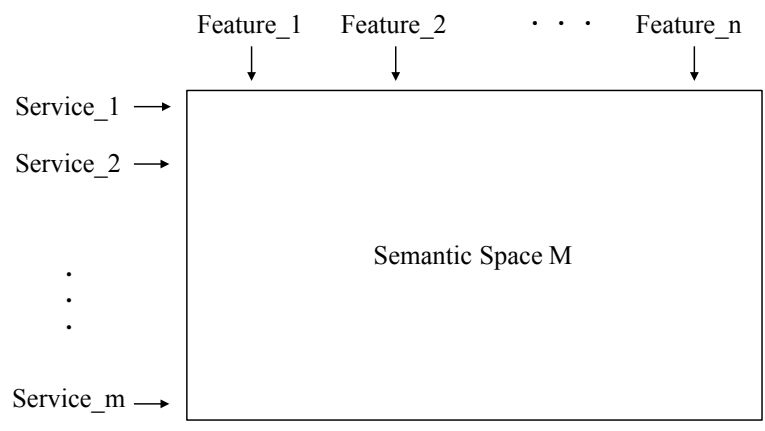

Figure 6. Structure of Semantic Space $\boldsymbol{M}$

Table 1. Abbreviations of context elements

\begin{tabular}{cl}
\hline Abbreviation & \multicolumn{1}{c}{ Feature } \\
\hline C1 & "Want to buy limited express ticket." \\
\hline C2 & "Want to buy fare tickets." \\
\hline C3 & "Want to move smoothly inside the station." \\
\hline C4 & "Want to take a rest." \\
\hline C5 & "Want to eat." \\
\hline C6 & "Want to know various information." \\
\hline C7 & "Want to use transportation that is not JR." \\
\hline C8 & "Want to meeting." \\
\hline C9 & "Want to do shopping." \\
\hline C10 & "Want to leave a luggage."
\end{tabular}




\begin{tabular}{ll}
\hline C11 & "Want to go to a safe and secure place." \\
\hline C12 & "Want to connect to the Internet." \\
\hline C13 & "Want to pass the time." \\
\hline C14 & "Want to use the Shinkansen." \\
\hline C15 16 & "Want to prepare a groomed appearance." \\
\hline C17 & "Speedy" \\
\hline C18 & "Feel free" \\
\hline C19 & "Elegance" \\
\hline C20 & "A relieved breath" \\
\hline C21 & "Recommended" \\
\hline C22 & "Profit" \\
\hline C23 & "Hint" \\
\hline C24 & "Familiar" \\
\hline C25 & "Fun" \\
\hline C26 & "Here, only" \\
\hline C27 & "Enchant" \\
\hline C28 & "Lively" \\
\hline C29 & "Family" \\
\hline
\end{tabular}

This matrix expresses the space formed by the axes that represent the presence or absence of a feature. Therefore, the services are represented as a vector of the semantic space $\boldsymbol{M}$, as shown in Eq. (1), (Figure 7).

$$
\vec{v}_{\text {service_i }}=\left\{x_{i 1}, \cdots, x_{i j}, \cdots, x_{i n} \mid x_{i j} \in \boldsymbol{M}\right\}
$$

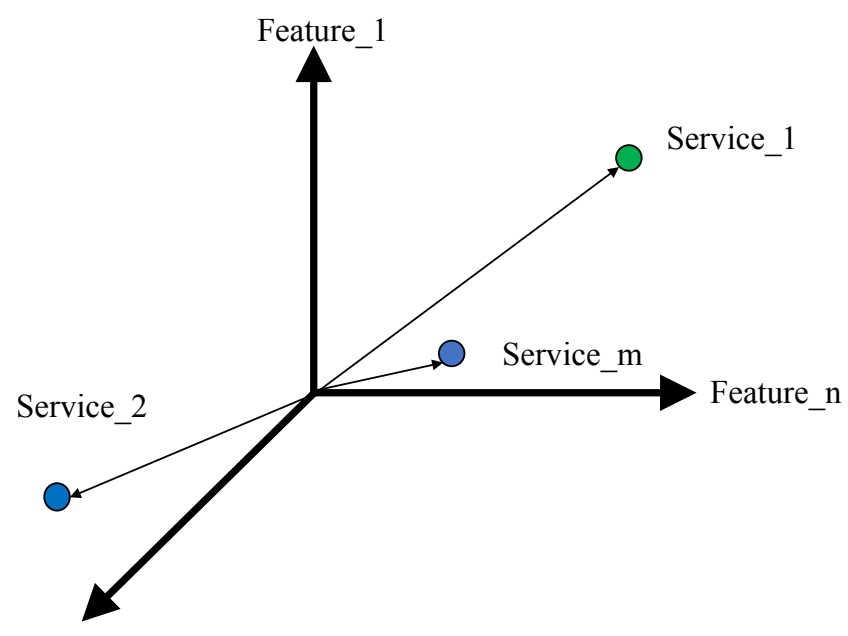

Feature 2

Figure 7. Semantic Space $\boldsymbol{M}$ 


\subsection{Providing the Passenger's Context}

The elements of a passenger's context are provided by smartphones or wearable sensors. In addition, any elements that cannot be acquired from the user are enabled to protect the user's privacy. In this paper, the context is an explicit input from the user interface, such as a button or a list in the smartphone or computer of the user. UC is the set of user context elements. Context elements include the user vector, user location ul, and time t, etc. The user vector is composed of the intention vector $\vec{v}_{u i}$ and sense vector $\vec{v}_{u s}$. Note that $\vec{v}_{u i}$ and $\vec{v}_{u s}$ have the same structure.

$$
\boldsymbol{U C}=\left\{\vec{v}_{u i}=\left\{y_{1}, \cdots, y_{.}\right\}, \vec{v}_{u s}=\left\{y_{1}, \cdots, y_{.}\right\}, u l, t\right\}
$$

\subsection{Selecting the Semantic Subspace}

The partial space $\boldsymbol{M}^{\prime}$ (semantic subspace) is selected by using features $\mathrm{q}_{i}$, which are beyond the passenger's context, element $y_{i}$, and threshold $\left|\varepsilon_{1}\right|$, in the context (Figure 8).

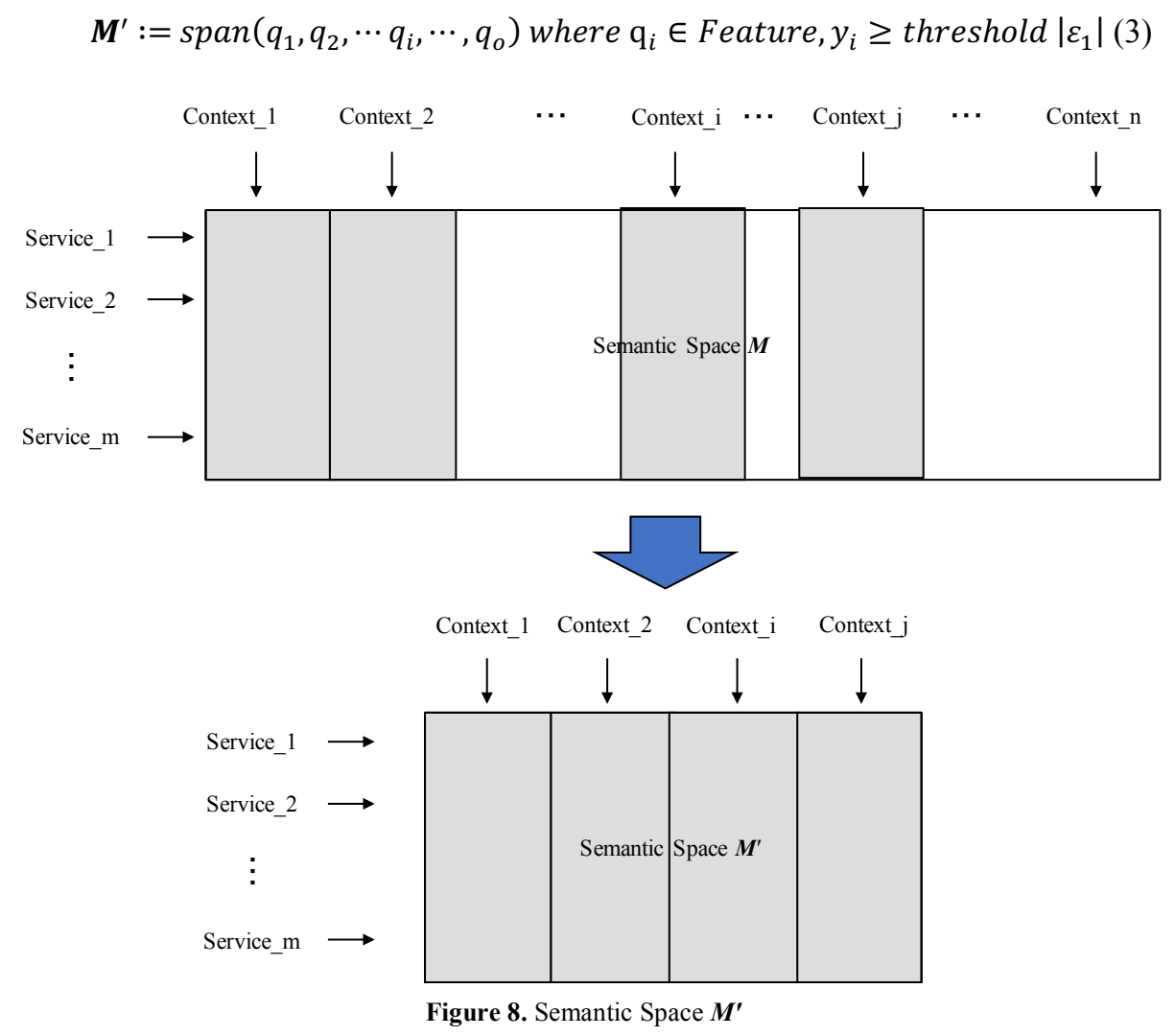




\subsection{Correlation metric on Passenger's Semantic Subspace $\mathbf{M}^{\prime}$}

The similarities of the passenger's context vector $\vec{v}_{u}$. and service vectors ${\overrightarrow{v^{\prime}}}_{\text {service } i}$ are computed as follows (cosine similarity) in the semantic subspace $\boldsymbol{M}^{\prime} \cdot{\overrightarrow{v^{\prime}}}_{u}$

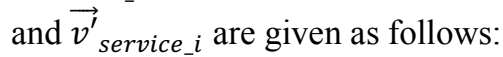

$$
\begin{gathered}
{\overrightarrow{v^{\prime}}}_{\text {service } i_{-}}=\left\{x_{i 1}, \cdots, x_{i j}, \cdots, x_{i \cdot} \mid x_{i j} \in M^{\prime}\right\} \\
{\overrightarrow{v^{\prime}}}_{u \cdot}=\left\{y_{1}, \cdots, y_{k}, \cdots, y_{.} \mid y_{k} \in \vec{v}_{u^{\prime},} y_{k} \geq \text { threshold }|\varepsilon|\right\}
\end{gathered}
$$

The similarity between $\vec{v}^{\prime}{ }_{u}$ and ${\overrightarrow{v^{\prime}}}_{\text {service_i } i}$ is computed as follows (cosine similarity):

$$
\operatorname{sim}\left({\overrightarrow{v^{\prime}}}_{u \cdot},{\overrightarrow{v^{\prime}}}_{\text {service }_{i}}\right)=\frac{\vec{v}_{u} \cdot \vec{v}_{\text {serivice_i } i}}{\left|\overrightarrow{v^{\prime} u} \cdot\right| \times\left|\vec{v}_{\text {service_i }}\right|}
$$

\subsection{Spatio correlation metric}

In this paper, we propose a method to measure the positional correlation between users and facilities/stores on the same floor. The measurement is obtained by filtering the area to be searched and measuring the physical distance. The filtering operation is realized by specifying the inside and outside of the ticket gate. The measurement of the physical distance sets the value range from 0 to 1 . For this purpose, the following formula is applied (7), where a represents the distance that exists in the same place as the user.

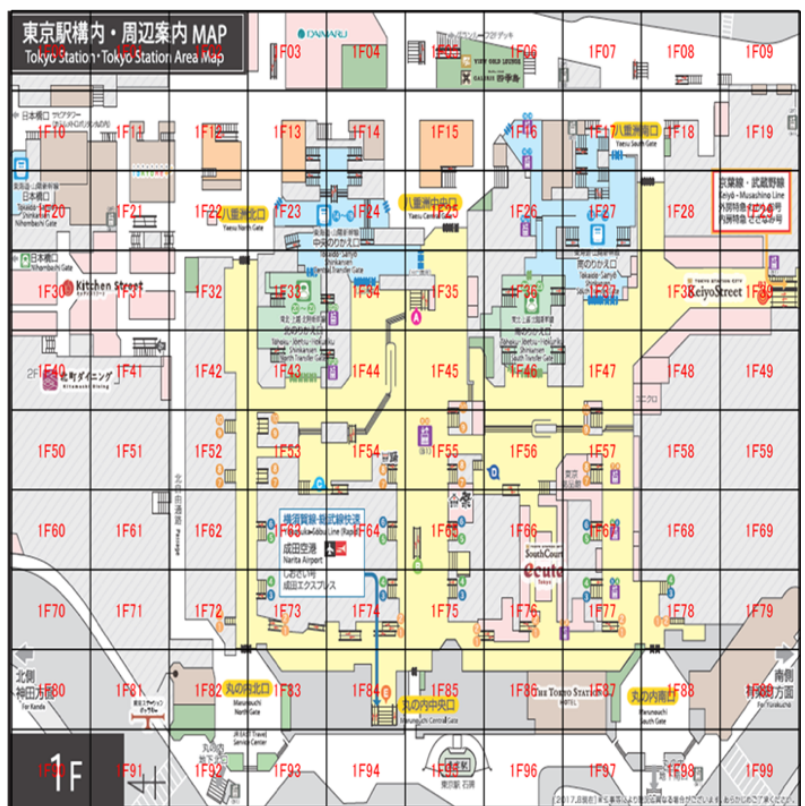

Figure 6. Spatio Mesh of Tokyo Station 


$$
\begin{aligned}
& \operatorname{sim}\left(u l_{\text {user }}, u l_{\text {service }_{i}}\right)=\frac{\log _{a}(a)}{\log _{a}\left(\left|u l_{\text {user }}-u l_{\text {service }_{i}}\right|\right)} \\
& \left|u l_{\text {user }}-u l_{\text {service }_{i}}\right|=\left\{\begin{aligned}
\left|u l_{\text {user }}-u l_{\text {service }_{i}}\right|, & \left|u l_{\text {user }}-u l_{\text {service }_{i}}\right| \geq a \\
a, & \left|u l_{\text {user }}-u l_{\text {service }_{i}}\right|<a
\end{aligned}(7)\right.
\end{aligned}
$$

\subsection{Semantic Anticipation of Needs}

In this paper, the integrated correlation (IC) among Intention, Sense, and Distance is defined by an expression that multiplies each weight.

$$
\text { IC }=\operatorname{sim}\left(\vec{v}_{u c}, \vec{v}_{\text {service }_{i}}\right) \times \operatorname{sim}\left(\vec{v}_{u_{s}}, \vec{v}_{\text {service }_{i}}\right) \times \operatorname{sim}\left(u l_{\text {user }}, u l_{\text {service }_{i}}\right)
$$

\subsection{Ranking}

We rank the services by the integrated correlation (IC). The rankings are displayed on a smartphone display and information display.

\section{System}

In this study, we constructed a system that provides facility/store information about the concourse on the first floor of Tokyo Station according to the context of the user. Figure 9, Figure 10, and Figure 11 show the system configuration diagram, class diagram, and ER diagram.

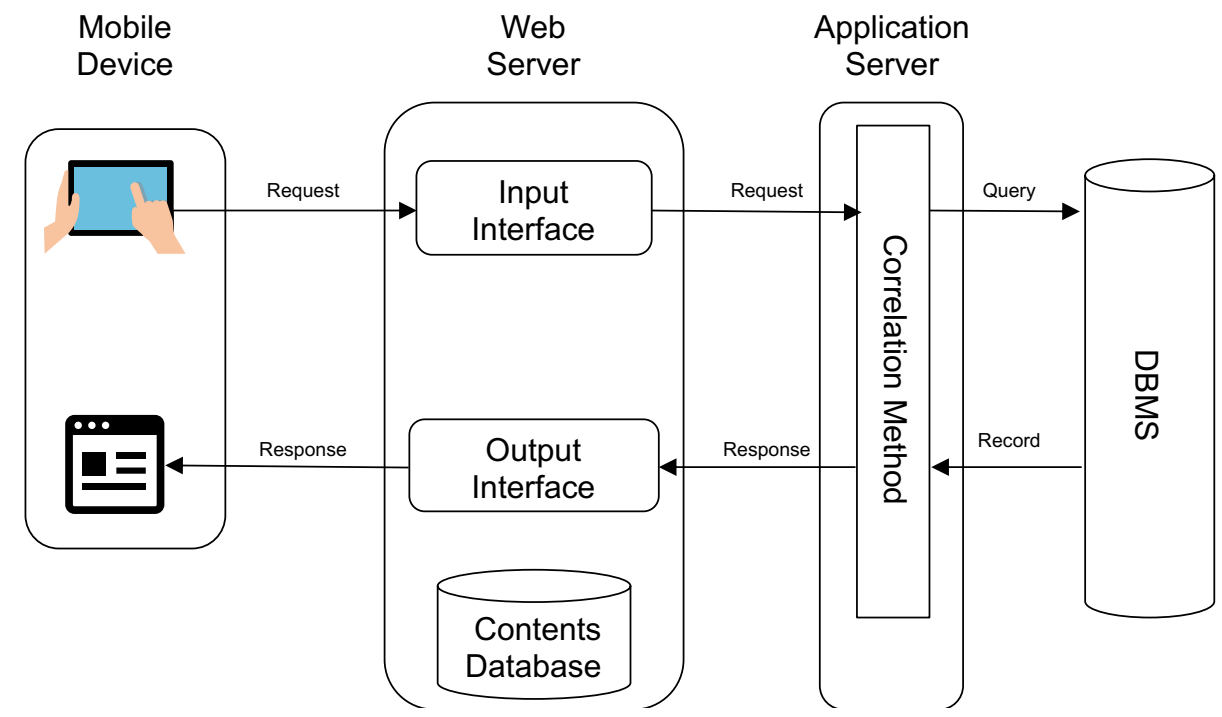

Figure 7. Architecture of Prototype System 


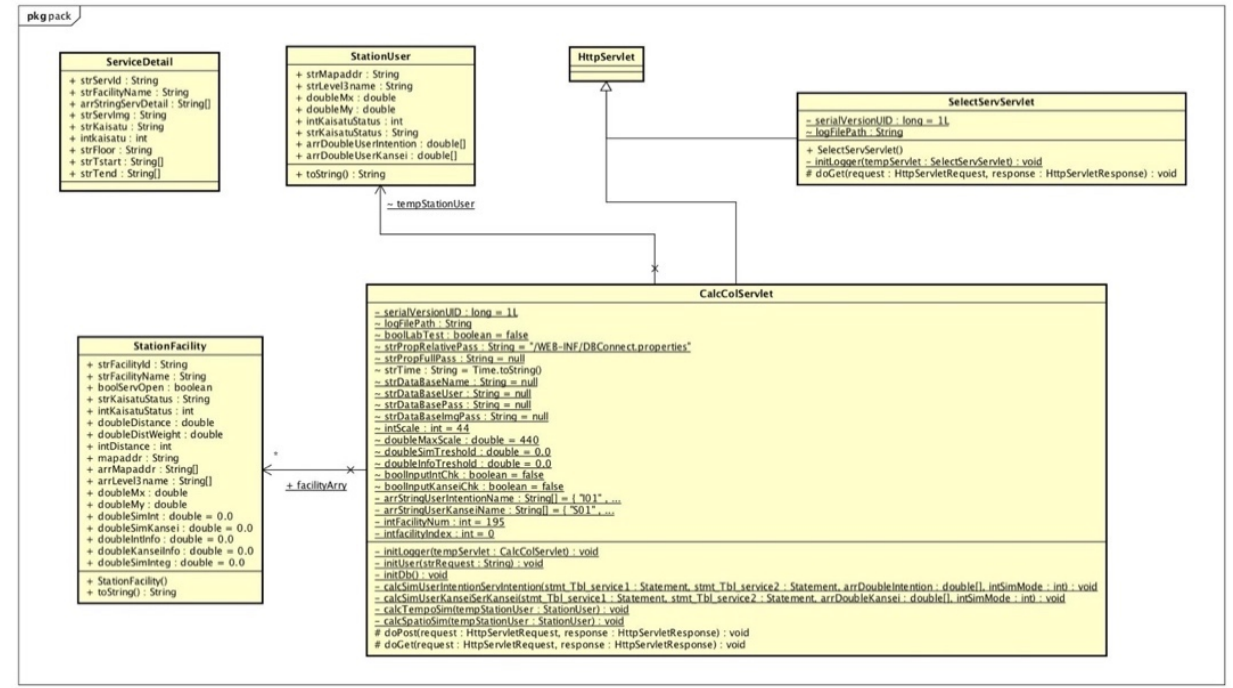

Figure 8. Class Diagram of the System

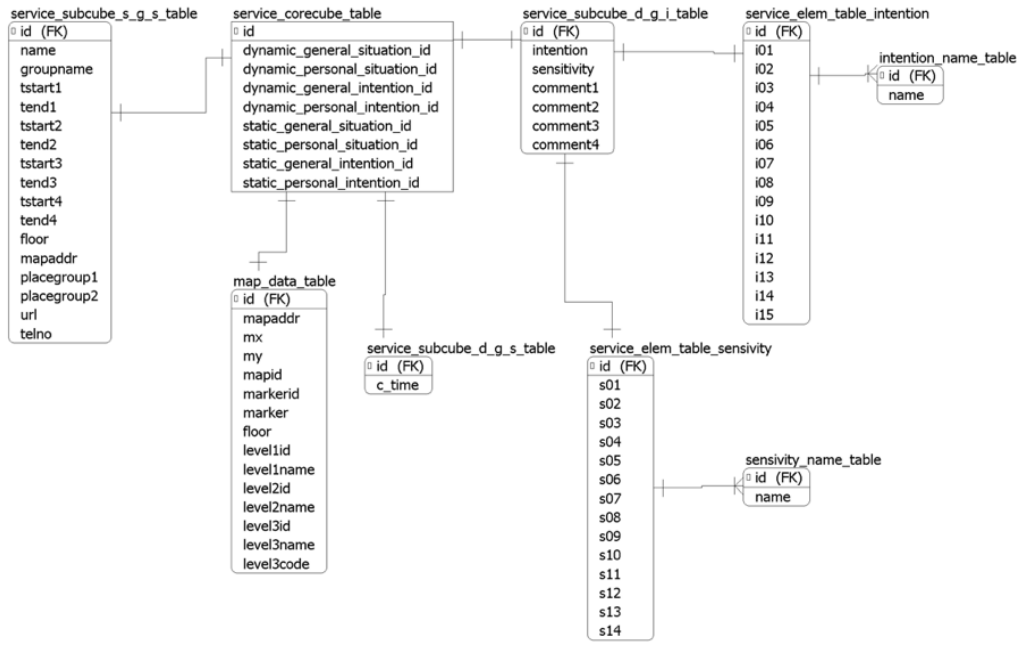

Figure 9. ER Diagram of the Database

The flow of use is listed as follows.

- The user selects his/her current position, search area (inside/outside the ticket gate), purpose, and sense in the input interface (Figure 12).

- The system computes the user input and the measurement of the correlation between stores and facilities. 
- The system displays a store/facility ranking list based on the correlation amount and a map that shows their locations on the output interface (Figure 13).

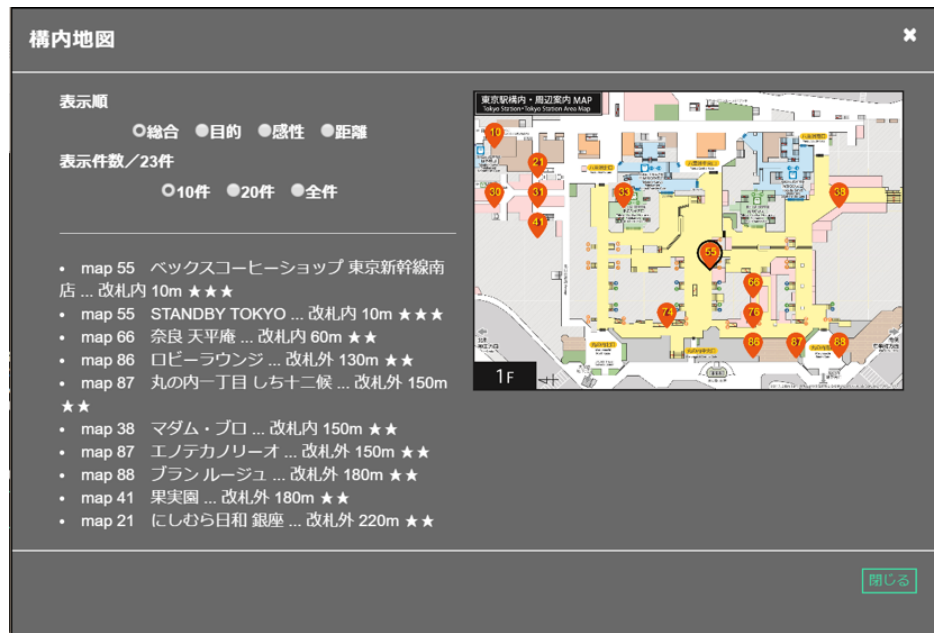

Figure 10. Input Interface

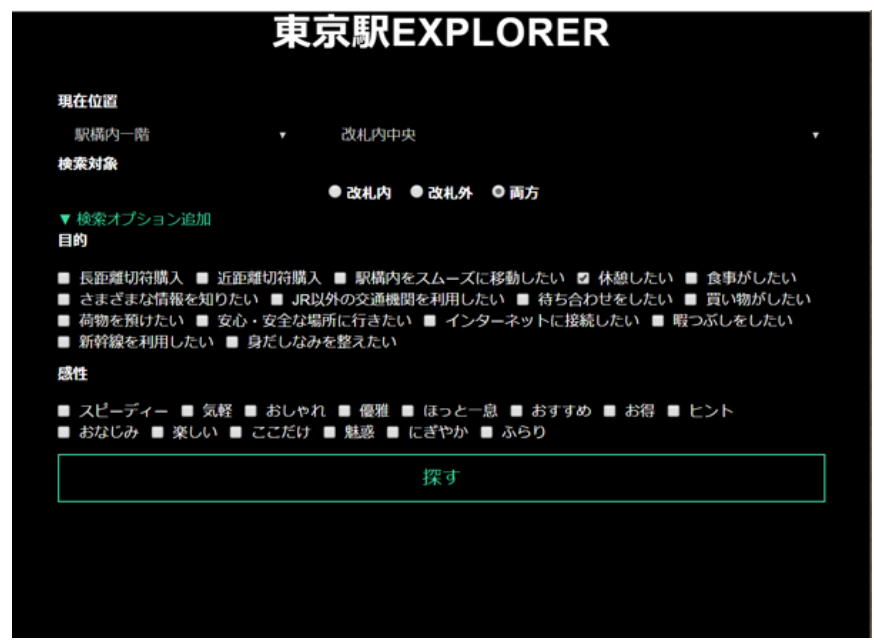

Figure 11. Output Interface

\section{Experiment}

We conduct an experiment to verify that the method of measuring the correlation between the user context and facilities/stores proposed in this study is effective for station guidance. Specifically, a prototype system is employed in Tokyo Station to evaluate the usability and information acquisition load. The details of the implementation are shown as follows:

- Implementation period

May 16, 2018-May 25, 2018 
- Implementation location

Three locations in Tokyo Station (near the central passage north ticket gate, near the Marunouchi north gate, and near the Marunouchi central gate)

- Number of subjects

18

- Evaluation method

The subject was presented with a usage scene of Tokyo Station and a search task, and an information search was performed. The subject was asked to answer questionnaires, such as the System Usability Scale and User Experience evaluation[15, 16].

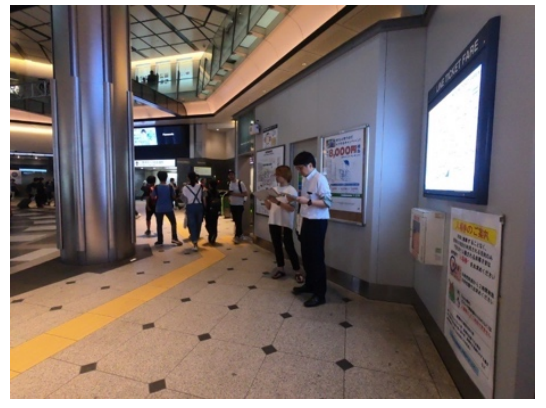

Figure 12. Experiment at Tokyo Station

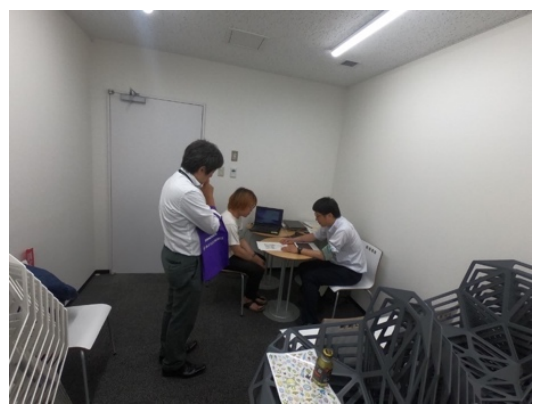

Figure 13. Questionnaire answer scene

\subsection{Correlation Calculation result}

The results of the correlation metric in the experiment are shown. The number of stores and facilities targeted by the constructed system is 300 , and those with a correlation of 0.1 or greater will be displayed in the ranking list.

\{"location":"1F38","intention": \{"I01":1\},"kansei": \{"S01":1,"S04":1\},"kaisatu ":"0","threshold":"0.1"\}

Figure 14 shows a parallel coordinate diagram of spatio correlation, intention correlation, and sensitive correlation. Figure 15 shows the integrated correlations of each store/facility. Figure 16 shows a visualized structure of the relationship between a user context and a store or a similar structure based on the integrated correlation amount. Gephi was employed as a visualization tool. In addition, a node arrangement algorithm is applied to understand the structure [17]. A user input example is provided as follows:

\{"location":"1F38","intention": $\{\mathrm{C} 1\}$,"sense": $\{\mathrm{C} 16\}$,"Gate": Inside and Outside”\} 


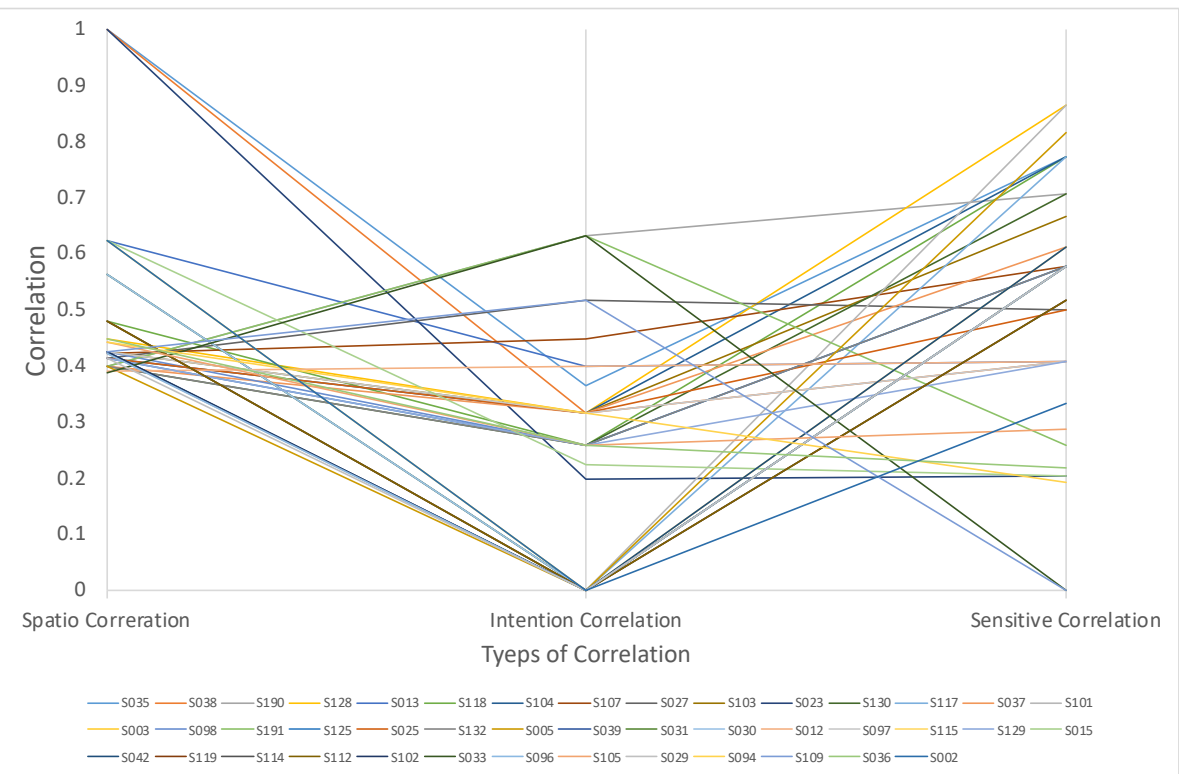

Figure 14. Parallel Coordinate Diagram

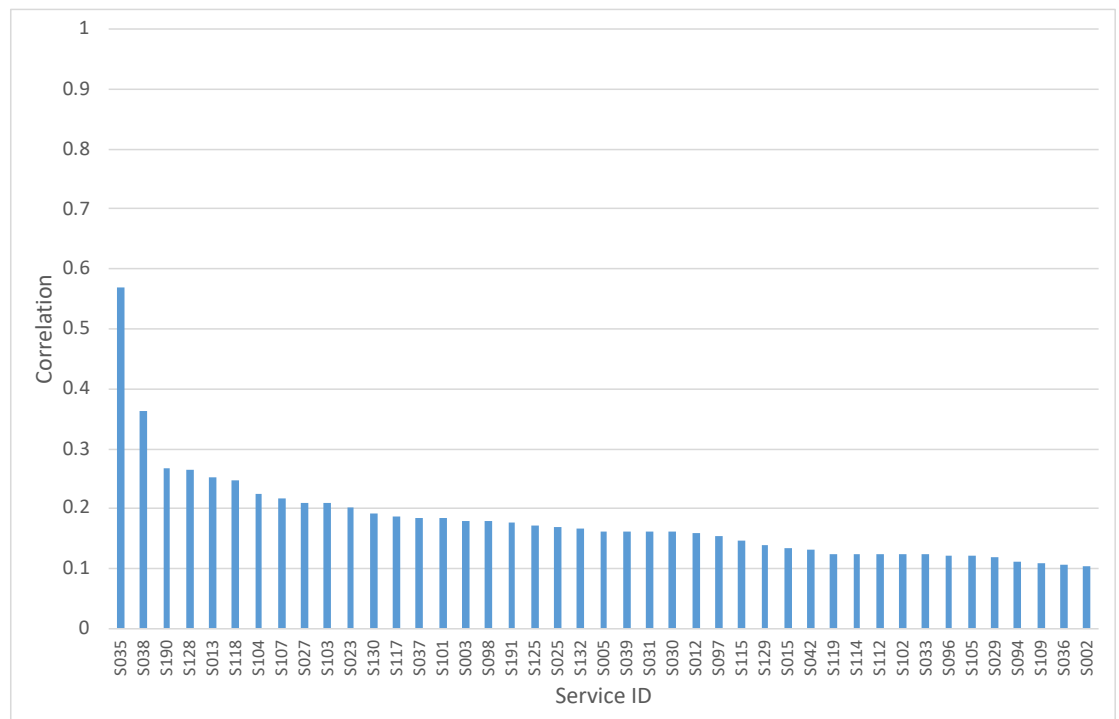

Figure 15. Total Correlation 


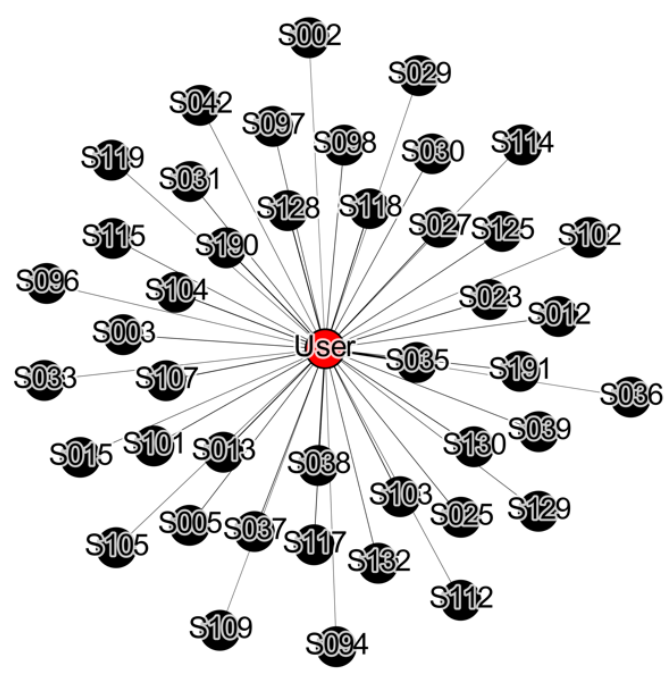

Figure 16. Context Cube Network of Correlation Results

\subsection{Usability evaluation questionnaire results}

Items of the questionnaire results and tabulation results are shown in Figure 17. The evaluation revealed that more than $70 \%$ of the participants wanted to use the prototype system, which confirms the effectiveness of the prototype system for information retrieval. More than $80 \%$ of users who utilize the system for the first time answered "I thought I need to learn a lot before using this system", "I do not think so or at all". This finding confirmed that the system was very effective.

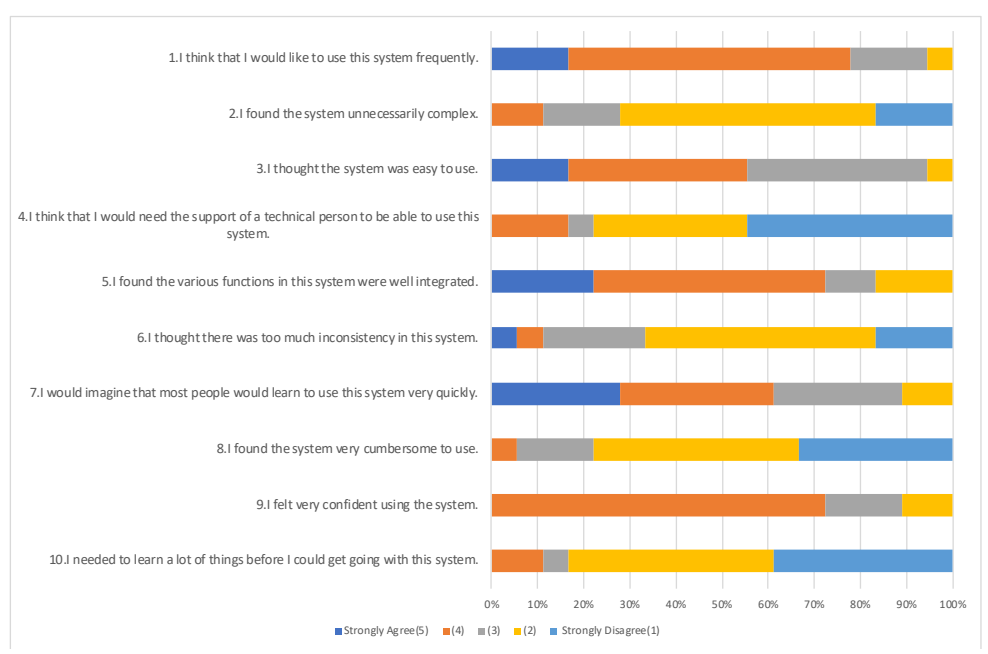

Figure 17. Evaluation results of usability (18 subjects) 


\subsection{Evaluation of purpose and emotional search method}

Figure 18 shows the evaluation of items related to purpose and sense search methods. For all items, "Strongly agree" and "Agree" exceeded $60 \%$, which indicates the effectiveness of the proposed method. In particular, the items of "This search method has potential" and "I want to use this search method in town" accounts for more than $80 \%$. The proposed method is novel, and discovering the service consumption of users is possible. In addition, more than $70 \%$ of participants respond "I would like to recommend this search method to my friends." and "I want to use this search method frequently", which confirms that the user's search needs can be satisfied.

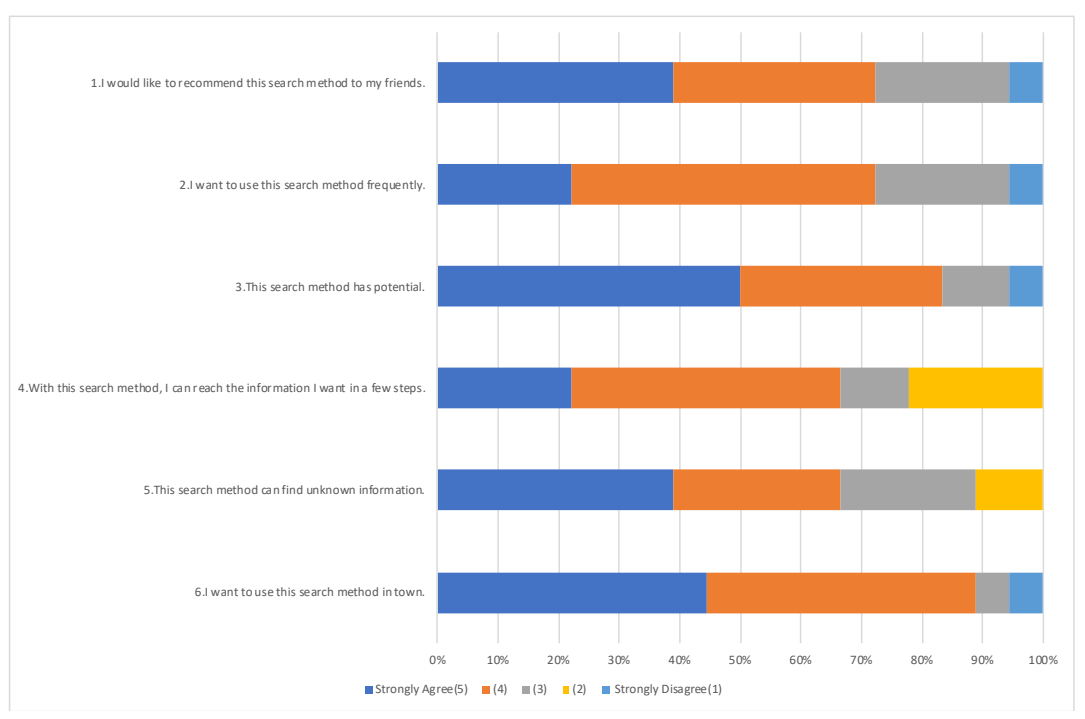

Figure 18. Evaluation of purpose and sense search method (18 subjects)

\section{Conclusions and Future Work}

In this study, we proposed a data model, the "Context Cube Semantic Network Model", that determines the user's context and facilities and stores in the station, and a measure that integrates the user's intention, sensitivity, and location with the correlation amount among the Context Cubes. We implemented a computing method for the station guidance system and allowed the subjects to use the system in the station to verify the effectiveness of the method.

For the usability evaluation, we obtained the evaluation value for usability and user experience. As a result, more than $60 \%$ of the participants answered positively about the ease of information acquisition, and more than half of the participants answered positively about the usability. Therefore, the proposed method was effective for information acquisition in the station yard. Since more than half of the subjects would like to introduce this search method to friends and others, communication to introduce the method will likely be induced. In addition, half of the participants indicated new 
discoveries. For this reason, we believe that more meaningful support for using the station premises is possible.

However, some subjects did not respond positively because they did not know which purpose/sensitivity item to choose and the ranking results did not show their desired store. Therefore, consider the function that supports the selection of items and the output of the ranking results that are more suited to the context of the user.

In the future, we will acquire a substantial amount of data about the situation, purpose, and sensibility when using the store and investigate the application of judgment algorithms, such as machine learning, to improve the user's station and railway experience.

\section{References}

[1] Yasushi Kiyoki, Takashi Kitagawa, Takanari Hayama, “A metadatabase system for semantic image search by a mathematical model of meaning," ACM SIGMOD Record, vol.23, no.4, pp.34-41, 1994.

[2] Yasushi Kiyoki, Shiori Sasaki, Nhung Nguyen Trang, Nguyen Thi Ngoc Diep, "Cross-Cultural Multimedia Computing with Impression-Based Semantic Spaces," Conceptual Modelling and Its Theoretical Foundations, Lecture Notes in Computer Science, Springer, March 2012, pp.316-328.

[3] Yasushi Kiyoki, Xing Chen, "Contextual and Differential Computing for the Multi-Dimensional World Map with Context-Specific Spatial-Temporal and Semantic Axes," Information Modelling and Knowledge Bases, vol.XXV, IOS Press, pp.82-97, 2014.

[4] Motoki Yokoyama, Yasushi Kiyoki, Tetsuya Mita, "Similarity-Ranking Method based on Semantic Computing for a Context-Aware System", The Fifth International Conference on Knowledge Creation and Intelligent Computing, Manado, Indonesia, November 15-17, 2016.

[5] Motoki Yokoyama, Yasushi Kiyoki, Tetsuya Mita, "A similarity-ranking method on semantic computing for providing information-services in Station-Concierge System", EMITTER International Journal of Engineering Technology, Vol.5 No1, June 2017.

[6] Motoki Yokoyama, Yasushi Kiyoki, Tetsuya Mita, "A Correlation Computing Method for Integrating Passengers and Services in Semantic Anticipation", INFORMATION MODELLING AND KNOWLEDGE BASES XXX, 2019.

[7] Mikiko Sakamoto, "Technical Innovation in Railway Service: The JR East App," JR East Technical Review, Report number: No. 28-Spring, 2014

[8] Takeshi Nakagawa, "ICT-Based Information Services for Customers," JR East Technical Review, Report number: No. 28-Spring, 2014

[9] Kaoru Mori, Shuichi Kurabayashi, Naoiki Ishibashi, Yasushi Kiyoki, "An Active Information Delivery Method with Dynamic Computation of Users' Information in Mobile Computing Environments," Proceedings of Data Engineering Workshop2004,1-A-04, 2004.

[10] Gregory D. Abowd, Anind K. Dey, Peter J. Brown, Nigel Davies, Mark Smith, Pete Steggles, "Towards a Better Understanding of Context and Context-Awareness," First International Symposium on Handheld and Ubiquitous Computing(HUC'99), Germany, pp. 304-307, 1999.

[11] Motoki Yano, Katsuhiko Kaji, Nobuo Kawaguchi, “App.Locky: Users' Context Collecting Platform for the Context-aware Service Recommendation," Journal of Information Processing ,52(12) 3274-3288, 2011.

[12] Lina Yao, Quan Z. Sheng, Yongrui Qin, Xianzhi Wang, Ali Shemshadi, and Qi He," Context-aware Point-of-Interest Recommendation Using Tensor Factorization with Social Regularization", SIGIR '15: Proceedings of the 38th International ACM SIGIR Conference on Research and Development in Information RetrievalAugust 2015 Pages 1007-1010.

[13] Stathis Maroulis, Ioannis Boutsis, Vana Kalogeraki,“Context-Aware Point of Interest Recommendation using Tensor Factorization",2016 IEEE International Conference on Big Data (Big Data).

[14] Daniel Kahneman, "Thinking, Fast and Slow”, Farrar, Straus \& Giroux Inc, 01 Sep 2017.

[15] BROOKE, John, "SUS'A quick and dirty' usability scale", Usability Evaluation in Industry, Taylor \& Francis, 1996

[16] Masaya Ando, "User Experience Design TextBook”, Maruzen Publishing Co.,LTD, 2016(Japanese).

[17] Tokyo station city, Tokyo station city, http://www.tokyostationcity.com/, Retrieved January $26^{\text {th }} 2018$.

[18] https://gephi.org/ 


\title{
Privacy by Design in EU's GDPR
}

\author{
Boštjan BRUMEN \\ University of Maribor (www.um.si), Faculty of Electrical Engineering and Computer \\ science, Smetanova 17, Si-2000 Maribor, Slovenia \\ bostjan.brumen@uni-mb.si
}

\begin{abstract}
Respect for privacy is not a modern phenomenon as it has been around for centuries. Recent advances in technologies led to the rise of awareness of the importance of privacy, and to development of principles for privacy protection to guide engineering of information systems on one side, and on using the principles to draft legal texts protecting privacy on the other side. In this paper we analyze how respect for privacy has been implemented in GDPR by automated comparison of similarity of GDPR's first 50 articles and text of seven principles of Privacy by Design. Our findings show that automated similarity comparison can highlight portions of legal texts where principles were observed. The results can support procedures of drafting legal texts to check whether important legal (or other) principles were adequately addressed.
\end{abstract}

Keywords. Privacy, GDPR, information system, privacy by design, similarity, semantics

\section{Introduction}

Privacy is not a new phenomenon. The existence of two areas, the public area of politics and political activity, the polis (gr. $\pi 0 \lambda 1 \zeta)$, and the private one of the family, the oikos (gr. oíko५), as two interdependent and sometimes conflicting areas, was well known in the times of Ancient Greek civilization [1, 2], and was reflected in classic dramas, e.g. in Sophocles' Antigone and Oedipus Rex. Interestingly, the New Order of the polis, despite its presumed weaknesses, reigns supreme at the end of the dramas [3].

Privacy was an important issue in medical profession as well: “...Whatever I see or hear in the lives of my patients, whether in connection with my professional practice or not, which ought not to be spoken of outside, I will keep secret, as considering all such things to be private. ..." [4] is the text from Hippocrates' oath that addresses privacy and instructs ancient doctors to keep private data - secret! Privacy and confidentiality are very important contemporary issues, especially in the Western world, and are not limited to the medical field only [5].

Privacy has re-emerged as an important issue post the wide spread of Internet and world wide web, a new ecosystem for data with all new challenges. Emergence of social networks has worsened the protection of private data. What users thought would remain private could and actually was used against their will and/or consent.

In light of preparations of European Union's new regulations on data protection in 2013, Mark Zukerberg, the founder of the most used social network Facebook, and Facebook's chief operation officer Sheryl Sandberg, stated that the privacy controls were centered at Facebook's core at all times [6, 7]. 
Then happened the Cambridge Analytica. Between 50 and 87 million Facebook user profiles, depending on the source ([8] and [9], respectively), were collected in a manner that users neither foresaw nor allowed. Previously, volunteers were analyzed using "OCEAN" psychological profile (openness, conscientiousness, extraversion, agreeableness, and neuroticism) and correlated it with their Facebook activity (likes and shares), demonstrating that Facebook profile data could be used instead of a formal psychographic instrument [9]. It then used the test results and Facebook data to build an algorithm that could analyze individual Facebook profiles and determine personality traits linked to voting behavior [8]. 50 million profiles at the time represented around a third of active North American Facebook users and nearly a quarter of potential US voters [8]. Displaying individualized, high impact messages to swing voters is sufficient to impact election results in a few states, especially in small ones with as few as a couple of hundred thousand voters $[9,10]$.

Facebook denied that harvesting of millions of profiles by Cambridge Analytica was a data breach and hence failed to report the regulators and individuals about the breach[8].

After two weeks the Cambridge Analytica scandal broke out, Facebook via Mark Zuckerberg apologized for a "breach of trust" in several US and UK newspapers adds [11]: "I'm sorry we didn't do more at the time. We're now taking steps to ensure this doesn't happen again."

Firstly, it was not only a breach of trust, it was a breach of privacy. Secondly, based on previous experiences, we can rest assured it will happen again.

Jim Isaak and Mina J. Hanna wrote: "It is clear that national governance institutions demonstrably lack the ability to anticipate technology's future impact on the rights and duties of its citizens, much less its impact on the structure of society, ideological divides, and political schisms among its citizens and the expansion of identity politics promoted by isolated social and news media echo chambers."

Cambridge Analytica scandal has firstly shown that there is a vast number of databases containing private data and they are readily available to be bought or exploited. Secondly, microtargeting of individuals is doable not only illegally, but (currently) also legally, without disclosure and informed consent, completely bypassing laws and regulations. Thirdly, the expenses for doing it are negligible and yields at high stakes. Lastly, corporations storing and processing the data are rarely held responsible and fined appropriately.

All this calls for changes on corporate and government levels. Corporations should anticipate legal changes and governments must ensure that private and/or personal data are protected so that individuals can best exercise their citizens' statutory and constitutional rights, such as due process, equal representation before the law, the right to appeal, freedom of expression, voting, and non-discrimination [9].

The laws of most developed countries impose obligations to respect informational privacy (e.g., confidentiality, anonymity, secrecy and data security); physical privacy (e.g., modesty and bodily integrity); associational privacy (e.g. intimate sharing of death, illness and recovery); proprietary privacy (e.g., self-ownership and control over personal identifiers, genetic data, and body tissues); and decisional privacy (e.g., autonomy and choice in personal relationships) $[12,13]$. It is the lack of respect of the laws or the incompleteness of these laws that privacy is not protected adequately. The lack of respect comes from either ignorance or deliberation. While deliberate acts will always happen - and need to be sanctioned appropriately -, the ignorance and incompleteness must be addressed. 
One way to address the issues is by following the already established principles on protecting the privacy and start protecting in the beginning of the processes - by following the privacy by design principles.

In this paper, the research question is how European regulation on data processing, the famous General Data Protection Regulation (GDPR) directive [14] - the Regulation (EU) 2016/679 of the European Parliament and of the Council of 27 April 2016 on the protection of natural persons with regard to the processing of personal data and on the free movement of such data - is addressing the privacy by using the privacy by design principles.

The rest of the paper is organized as follows. In Section 2, we give an overview of literature review dealing with privacy and privacy by design principle. In Section 3 we describe our research method and present the results. In Section 4 we conclude the paper with final remarks.

\section{Literature review}

"Privacy is a concept in disarray. Nobody can articulate what it means." is an interesting observation by D. Solove [15]. Nevertheless, privacy and confidentiality were well researched and addressed by philosophers and jurists alike, and later addressed by many technologies.

A systematic discussion on the concept of privacy has begun with the famous article by Samuel Warren and Louis Brandeis titled "The Right to Privacy" [16]. Citing "political, social, and economic changes" and a recognition of "the right to be let alone" they argued that existing law (i.e. the Constitution of the U.S.A.) afforded a way to protect the privacy of the individual, and they sought to explain the nature and extent of that protection. Focusing in large part on the press and publicity allowed by then "recent" inventions such as photography and newspapers, but referring as well to violations in other contexts, they emphasized the invasion of privacy brought about by public dissemination of details relating to a person's private life. Warren and Brandeis felt a variety of existing cases could be protected under a more general right to privacy which would protect the extent to which one's thoughts, sentiments, and emotions could be shared with others. Urging that they were not attempting to protect the items produced, or intellectual property, but rather the peace of mind attained with such protection, they said the right to privacy was based on a principle of "inviolate personality" which was part of a general right of immunity of the person, "the right to one's personality" [16]. Warren and Brandeis thus laid the legal foundation for a concept of privacy that has come to be known as control over information about oneself [17].

In 1960, Prosser systematically defined four different aspects of "privacy rights" being upheld in tort law: $[17,18]$ :

1. Intrusion upon a person's seclusion or solitude, or into his private affairs.

2. Public disclosure of embarrassing private facts about an individual.

3. Publicity placing one in a false light in the public eye.

4. Appropriation of one's likeness for the advantage of another [17].

Prosser noted that the intrusion in the first privacy right had expanded beyond physical intrusion and pointed out that Warren and Brandeis had been concerned primarily with the second privacy right. Nevertheless, Prosser felt that both real abuses and public demand had led to general acceptance of these four types of privacy 
invasions. Thomas Nagel, one of the America's top contemporary philosophers, gives a more contemporary (philosophical) discussion of privacy, concealment, publicity and exposure $[17,19]$.

As summarized by authors in [5], Adam Moore [20], building on the views of Ruth Gavison [21], Anita Allen [22], Sissela Bok [23] and others, offers a "control over access" account of privacy. According to Moore, privacy is a culturally and species relative right to a level of control over access to bodies or places and information. While defending the view that privacy is relative to species and culture, Moore argues that privacy is objectively valuable: human beings that do not obtain a certain level of control over access will suffer in various ways. Moore claims that privacy, like education, health, and maintaining social relationships, is an essential part of human flourishing or well-being [17].

In medical contexts, as viewed by Allen [13], the "privacy" at issue is very often "confidentiality" [24], specifically the confidentiality of patient-provider encounters (including the very fact that an encounter has taken place), along with the secrecy and security of information memorialized in physical, electronic and graphic records created as a consequence of patient-provider encounters [24]. Confidentiality is defined as restricting information to persons belonging to a set of specifically authorized recipients $[13,22,25,26]$. Confidentiality can be achieved either through professional silence, leaning on the moral aspect, or through secure data management [27], leaning on technologies and techniques.

The moral significance attached to privacy is reflected in data protection and security regulations adopted by local and national authorities around the world. One such regulation is European Union's General Data Protection Regulation.

\subsection{Privacy by design}

Literature presented above has shown that privacy has to be taken seriously as it addresses one of the basic human rights and has a special place in legal texts. It is explicitly stated under Article 12 of the 1948 Universal Declaration of Human Rights and protected by $1^{\text {st }}, 3^{\text {rd }}, 4^{\text {th }}$ and $5^{\text {th }}$ Amendment of the US Constitution [15]. In European Union, it is protected by Article 8(1) of the Charter of Fundamental Rights of the European Union and Article 16(1) of the Treaty on the Functioning of the European Union (TFEU), and several national constitutions [15].

The Ontario Privacy Commissioner Ann Cavoukian has developed a "Privacy by Design" $(\mathrm{PbD})$ framework [28-31] which is emphasizing the need to adopt a proactive rather than a reactive compliance approach to the protection of privacy. To safeguard privacy, legislation and regulation would no longer be sufficient; privacy needs to be proactively embedded directly into information technology, business practices, physical design, and networked infrastructures - making it the default [32]. Interestingly, the framework can be applied also when designing legal procedures [33, $34]$ and has become an international standard for assuring privacy in information era [32].

The framework relies on 7 principles [28], see Figure 1:

1. Proactive not Reactive; Preventative not Remedial

The meaning of the principle reads: "The Privacy by Design approach is characterized by proactive rather than reactive measures. It anticipates and prevents privacy invasive events before they happen. Privacy by Design does not wait for privacy risks to materialize, nor does it offer remedies for resolving privacy infractions 
once they have occurred - it aims to prevent them from occurring. In short, Privacy by Design comes before-the-fact, not after." [28]

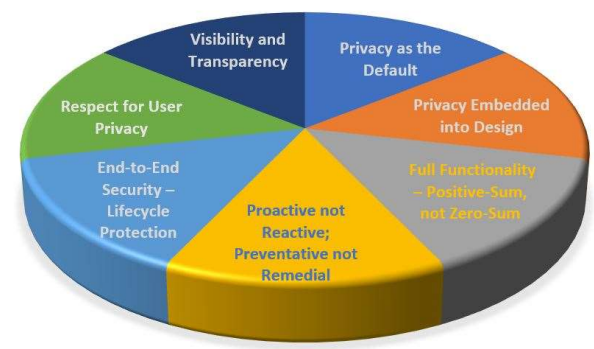

Figure 1: Seven principles of Privacy by Design

2. Privacy as the Default

"Privacy as the Default" principle reads: "We can all be certain of one thing - the default rules! Privacy by Design seeks to deliver the maximum degree of privacy by ensuring that personal data are automatically protected in any given IT system or business practice. If an individual does nothing, their privacy still remains intact. No action is required on the part of the individual to protect their privacy - it is built into the system, by default." [28]

3. Privacy Embedded into Design

"Privacy by Design is embedded into the design and architecture of IT systems and business practices. It is not bolted on as an add-on, after the fact. The result is that privacy becomes an essential component of the core functionality being delivered. Privacy is integral to the system, without diminishing functionality." [28]

4. Full Functionality - Positive-Sum, not Zero-Sum

"Privacy by Design seeks to accommodate all legitimate interests and objectives in a positive-sum win-win manner, not through a dated, zero-sum approach, where unnecessary trade-offs are made. Privacy by Design avoids the pretense of false dichotomies, such as privacy vs. security - demonstrating that it is possible to have both." [28]

\section{End-to-End Security - Lifecycle Protection}

"Privacy by Design, having been embedded into the system prior to the first element of information being collected, extends securely throughout the entire lifecycle of the data involved - strong security measures are essential to privacy, from start to finish. This ensures that all data are securely retained, and then securely destroyed at the end of the process, in a timely fashion. Thus, Privacy by Design ensures cradle to grave, secure lifecycle management of information, end-to-end." [28]

\section{Visibility and Transparency}

"Privacy by Design seeks to assure all stakeholders that whatever the business practice or technology involved, it is in fact, operating according to the stated promises and objectives, subject to independent verification. Its component parts and operations 
remain visible and transparent, to users and providers alike. Remember, trust but verify." [28]

\section{Respect for User Privacy}

"Above all, Privacy by Design requires architects and operators to protect the interests of the individual by offering such measures as strong privacy defaults, appropriate notice, and empowering user-friendly options. Keep it user-centric." [28]

The European GDPR was drafted with Privacy by Design as one of the guiding frameworks $[35,36]$. It is to notice that Article 25 of GDPR is titled "Data protection by design and by default", and that Recital 78 is mentioning the principles of data protection by design and by default.

In the next section, we will analyze the GDPR and answer the question, to what extent is GDPR, throughout its articles, addressing each of the 7 principles.

\section{Analysis of GDPR and seven Privacy by Design principles}

In this section we check how each of the principles is reflected, or addressed, in GDPR. For our study we selected the first fifty articles which represent the core of the GDPR and its intent to protect privacy; the remaining articles are provisions for establishment of independent supervisory authorities (e.g. privacy commissioners) and for remedies, liability and penalties.

We measure the extent to which these principles are reflected in GDPR's articles by using automated text similarities approach and the Universal Sentence Encoder (USE) [37]. USE is a pre-trained sentence encoder which encodes text paragraphs into high dimensional vectors that can be used for detecting semantic similarity (and other natural language tasks, such as text classification or clustering), see Figure 2. USE is typically pre-trained on a range of supervised and unsupervised tasks, in order to comprehend semantic information in texts [38]. It is learning from various data sources and on diverse tasks with the aim of dynamically accommodating a wide variety of natural language understanding tasks [37].

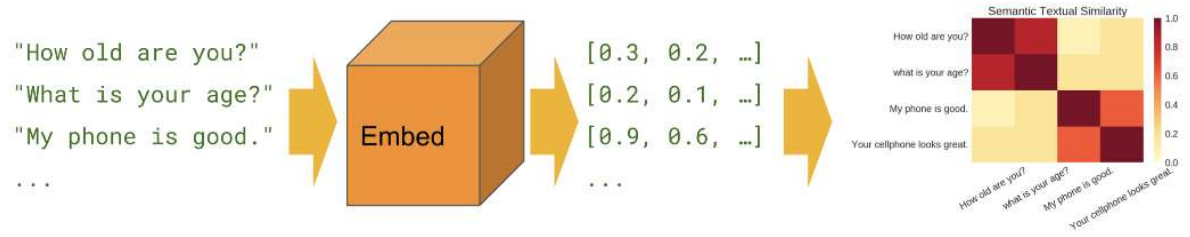

Figure 2: Process of embedding and comparing of different texts [38]

The input is variable length English text and the output is a 512-dimensional vector. The embeddings produced by the USE are approximately normalized. The semantic similarity of two sentences can be trivially computed as the inner product of the encodings.

We used the Google's Semantic Similarity with TF-Hub Universal Encoder online tool, available at https://colab.research.google.com/github/tensorflow/hub/blob/master/ examples/colab/semantic similarity with tf hub universal encoder.ipynb, and calculated the matrix of inner products between encodings of 7 principles' text, and 
encodings of each individual GDPR article's text. In the latter, we only removed numberings of paragraphs or sections (e.g. "1." and "(a)" were removed). Each article's text was joined into a single paragraph to be able to process it.

The automatically calculated semantic similarity was checked against the STS Benchmark [39] (http://ixa2.si.ehu.es/stswiki/index.php/STSbenchmark). It evaluates the degree to which similarity scores computed using USE are in line with human evaluation of similarity. The benchmark uses similarity scores for a diverse selection of sentence pairs. Pearson's R is calculated to estimate the relationship (quality) between the automatically generated similarity scores and to human evaluations. There is a strong, positive correlation between machine similarity scores and human evaluations, which is statistically significant $(\mathrm{R}=0.803, \mathrm{p}<0.005)$.

The similarity scores between the GDPR's first 50 articles and 7 principles of Privacy by Design are presented in Table 1 . A value of 0 represents no similarity, and value 1 represents perfect semantic similarity (equal texts).

Table 1: Similarity scores between GDPR's articles and 7 Privacy by Design principles

\begin{tabular}{|c|c|c|c|c|c|c|c|c|c|c|}
\hline Article & Principle 1 & Principle 2 & Principle 3 & Principle 4 & Principle 5 & Principle 6 & Principle 7 & Max & Min & Average \\
\hline 1 & 0.360347 & 0.491606 & 0.289023 & 0.338619 & 0.284758 & 0.260049 & 0.347171 & 0.491606 & 0.260049 & 0.338796 \\
\hline 2 & 0.236012 & 0.303697 & 0.234517 & 0.185280 & 0.238605 & 0.240943 & 0.183850 & 0.303697 & 0.183850 & 0.231843 \\
\hline 3 & 0.199190 & 0.275385 & 0.211665 & 0.156431 & 0.201384 & 0.288125 & 0.098190 & 0.288125 & 0.098190 & 0.204339 \\
\hline 4 & 0.277767 & 0.328543 & 0.234926 & 0.249763 & 0.255424 & 0.265395 & 0.183510 & 0.328543 & 0.183510 & 0.256475 \\
\hline 5 & 0.389129 & 0.432845 & 0.297043 & 0.425751 & 0.348858 & 0.344788 & 0.387155 & 0.432845 & 0.297043 & 0.375081 \\
\hline 6 & 0.254904 & 0.320956 & 0.217383 & 0.257224 & 0.251671 & 0.245881 & 0.224717 & 0.320956 & 0.217383 & 0.253248 \\
\hline 7 & 0.229825 & 0.350499 & 0.148846 & 0.216655 & 0.238838 & 0.234083 & 0.195935 & 0.350499 & 0.148846 & 0.230669 \\
\hline 8 & 0.206144 & 0.259325 & 0.103134 & 0.144194 & 0.133171 & 0.236139 & 0.095653 & 0.259325 & 0.095653 & 0.168252 \\
\hline 9 & 0.299966 & 0.328485 & 0.218131 & 0.273811 & 0.221169 & 0.219755 & 0.240247 & 0.328485 & 0.218131 & 0.257366 \\
\hline 10 & 0.288422 & 0.319372 & 0.196702 & 0.204103 & 0.249750 & 0.253504 & 0.194260 & 0.319372 & 0.194260 & 0.243731 \\
\hline 11 & 0.304790 & 0.368847 & 0.225721 & 0.227445 & 0.224513 & 0.250568 & 0.163251 & 0.368847 & 0.163251 & 0.252162 \\
\hline 12 & 0.221201 & 0.237019 & 0.104119 & 0.193814 & 0.166171 & 0.163481 & 0.148146 & 0.237019 & 0.104119 & 0.176279 \\
\hline 13 & 3057 & 0.362358 & 0.211481 & 0.254764 & 0.259486 & 8734 & 0.165719 & 0.362358 & 0.165719 & 0.243657 \\
\hline 14 & 9966 & 0.400297 & 912 & 0.297740 & 0.284296 & 0.238595 & 0.212792 & 0.400297 & 0.212792 & 0.286800 \\
\hline 15 & 0.238152 & 0.373431 & 0.185020 & 0.223043 & 0.235669 & 0.180948 & 0.188269 & 0.373431 & 0.180948 & 0.232076 \\
\hline 16 & 0.140253 & 0.337666 & 0.146838 & 0.187919 & 0.245466 & 0.119677 & 0.269672 & 0.337666 & 0.119677 & 0.206785 \\
\hline 17 & 0.307518 & 0.370307 & 0.267838 & 0.322436 & 0.247481 & 0.220210 & 0.207319 & 0.370307 & 0.207319 & 0.277587 \\
\hline 18 & 0.303190 & 0.385684 & 0.236388 & 0.318092 & 0.247065 & 7709 & 0.227209 & 0.385684 & 0.210709 & 0.275477 \\
\hline 19 & 0.241875 & 0.324473 & 0.152771 & 0.225434 & 0.172970 & 0.271217 & 0.191730 & 0.324473 & 0.152771 & 0.225781 \\
\hline 20 & 4367 & 0.374052 & 0.223007 & 1875 & 66 & 0.143427 & 0.161639 & 0.374052 & 0.143427 & 0.231391 \\
\hline 21 & 3663 & 0.439330 & 0.268501 & 0.315681 & 0.316107 & 0.252327 & 0.242396 & 0.439330 & 0.242396 & 0.306858 \\
\hline 22 & 0.278818 & 0.347352 & 0.223850 & 33086 & 0.242484 & 0.211744 & 0.215365 & 0.347352 & 0.211744 & 0.250385 \\
\hline 23 & 0.271151 & 0.253360 & 0.194426 & 0.246279 & 0.231250 & 0.225641 & 6124 & 0.271151 & 0.194426 & 0.235462 \\
\hline 24 & 0.125837 & 0.083455 & 0.062519 & 0.113094 & 3950 & 56676 & 3227 & 0.166676 & 0.062519 & 0.116965 \\
\hline 25 & 0.319180 & 0.389360 & 0.307548 & 0.295063 & 0.376919 & 0.324592 & 0.323794 & 0.389360 & 0.295063 & 0.333779 \\
\hline 26 & 0.079373 & 0.197641 & 0.124196 & 0.082077 & 0.142194 & 0.249562 & 0.178711 & 0.249562 & 0.079373 & 0.150536 \\
\hline 27 & 0.221496 & 0.243336 & 0.202414 & 0.174030 & 302 & 0.234979 & 0.151558 & 0.243336 & 0.149802 & 0.196802 \\
\hline 28 & 0.091010 & 0.121777 & 0.151472 & 0.080631 & 0.136006 & 0217 & 764 & 0.210217 & 0.068764 & 0.122840 \\
\hline 29 & 639 & 47 & & 227 & & 613 & & 613 & 227 & 0.146823 \\
\hline 30 & 0.108839 & 0.185647 & 882 & 0.084969 & 354 & 0.201238 & 208 & 0.201238 & 0.084969 & 0.136605 \\
\hline 31 & 0.000960 & 0.040138 & 0.003260 & 0.028368 & 0.023398 & 0.156040 & 0.125144 & 0.156040 & 0.000960 & 0.053901 \\
\hline 32 & 0.280560 & 0.306986 & 0.265131 & 0.256895 & 0.326574 & 0.310465 & 0.236674 & 0.326574 & 0.236674 & 0.283327 \\
\hline 33 & 0.428107 & 0.435881 & 0.266004 & 0.276108 & 0.322867 & 0.274054 & 0.285993 & 0.435881 & 0.266004 & 0.327002 \\
\hline 34 & 0.376568 & 0.466291 & 0.245363 & 0.322677 & 0.327622 & 0.284251 & 0.320097 & 0.466291 & 0.245363 & 0.334695 \\
\hline 35 & 0.291783 & 0.305647 & 0.205283 & 0.186971 & 0.279808 & 0.270116 & 0.260022 & 0.305647 & 0.186971 & 0.257090 \\
\hline 36 & 0.282082 & 0.237424 & 0.176635 & 0.142290 & 0.241672 & 0.233886 & 0.203028 & 0.282082 & 0.142290 & 0.216717 \\
\hline 37 & 0.175927 & 0.274563 & 0.204100 & 0.124045 & 0.227051 & 0.270106 & 0.228610 & 0.274563 & 0.124045 & 0.214914 \\
\hline 38 & 0.245430 & 0.326263 & 0.212728 & 0.197173 & 0.309278 & 0.336880 & 0.283499 & 0.336880 & 0.197173 & 0.273036 \\
\hline 39 & 0.144685 & 0.148925 & 0.095902 & 0.090626 & 0.172297 & 0.224215 & 0.164543 & 0.224215 & 0.090626 & 0.148742 \\
\hline 40 & 0.170306 & 0.145069 & & 0.081939 & 12100 & 0.164508 & 0.140494 & 0.170306 & 0.080152 & 0.127795 \\
\hline 41 & 0.204474 & 0.181865 & 0.193979 & 0.157812 & 0.166187 & 0.258832 & 0.146790 & 0.258832 & 0.146790 & 0.187134 \\
\hline 42 & 0.102236 & 0.086630 & 0.146038 & 0.016430 & 0.136060 & 0.214091 & 0.041492 & 0.214091 & 0.016430 & 0.106140 \\
\hline 43 & 0.087727 & 0.033069 & 0.097247 & 0.010421 & 0.075268 & 0.128426 & 0.007185 & 0.128426 & 0.007185 & 0.062763 \\
\hline 44 & 0.099720 & 0.208492 & 0.127479 & 0.086833 & 0.127452 & 0.121329 & 0.087357 & 0.208492 & 0.086833 & 0.122666 \\
\hline 45 & 0.153965 & 0.163503 & 0.136026 & 0.081461 & 0.150335 & 0.139639 & 0.078758 & 0.163503 & 0.078758 & 0.129098 \\
\hline 46 & 0.084423 & 0.115119 & 0.109503 & 0.039761 & 0.162764 & 0.085834 & 0.073094 & 0.162764 & 0.039761 & 0.095785 \\
\hline 47 & 0.234158 & 0.256798 & 0.183733 & 0.187696 & 0.240033 & 0.349676 & 0.202147 & 0.349676 & 0.183733 & 0.236320 \\
\hline 48 & 0.015412 & 0.123173 & 0.082316 & 0.079838 & 0.003679 & 0.073136 & 0.018383 & 0.123173 & 0.003679 & 0.056562 \\
\hline
\end{tabular}




\begin{tabular}{|l|r|r|r|r|r|r|r|r|r|r|}
\hline Article & Principle 1 & Principle 2 & Principle 3 & Principle 4 & Principle 5 & Principle 6 & Principle 7 & Max & Min & Average \\
\hline 49 & 0.218997 & 0.270453 & 0.177729 & 0.213976 & 0.202159 & 0.139674 & 0.120007 & 0.270453 & 0.120007 & 0.191856 \\
\hline S0 & 0.251524 & 0.326452 & 0.159450 & 0.186421 & 0.200024 & 0.100079 & 0.272655 & 0.326452 & 0.100079 & 0.213801 \\
\hline Average & 0.220783 & 0.277496 & 0.184460 & 0.187545 & 0.210880 & 0.220821 & 0.182603 & & & \\
\hline Max & 0.428107 & 0.491606 & 0.307548 & 0.425751 & 0.376919 & 0.349676 & 0.387155 & & & \\
\hline Min & 0.000960 & 0.033069 & 0.003260 & 0.010421 & 0.003679 & 0.073136 & 0.007185 & & & \\
\hline
\end{tabular}

Not surprisingly, the most similarity was found between $2^{\text {nd }}$ principle (Privacy as the Default) and GDPR's articles. $2^{\text {nd }}$ principle scored 32 maximum values (blue shaded cells of Table 1), followed by $6^{\text {th }}$ principle (Visibility and Transparency) with $13,1^{\text {st }}$ principle (Proactive not Reactive; Preventative not Remedial) with 3 and $5^{\text {th }}$ principle (End-to-End Security - Lifecycle Protection) with 2 maximum values.

Principles with most maximum values had also least minimum values. Least similarity was found between $7^{\text {th }}$ principle (Respect for User Privacy) with 16 minimum values (red shaded cells), followed by $3^{\text {rd }}, 4^{\text {th }}, 6^{\text {th }}, 1^{\text {st }}$ and $5^{\text {th }}$ principle with 12 , $11,7,2$ and 2 minimum values, respectively.

Principle 2 was not only in general most like GDPR's text, it was also most strongly similar. It had largest similarity index for articles $1,34,21,33,5,14,25,18$, $20,15,17,11,13,7,22,16,4,9$ and 50 , similarity values ranging from 0.491 to 0.326 .

Article 5 was most similar to all 7 principles with average similarity value of 0.375 , and Article 31 was least similar with average similarity value of 0.054 . Thus, automatic semantic encoder correctly identified the Article 5 that lists principles of GDPR; it's title is "Principles relating to processing of personal data". Article 31 's title is "Cooperation with the supervisory authority", requiring controller and processor to cooperate with the supervisory body; the material not covered by any of the seven principles, hence article's average similarity is extremely low.

Similarities between GDPR's articles and 7 principles can easily be seen in Figure 3. Most similarities are found between GDPR and $2^{\text {nd }}$ principle; $1^{\text {st }}$ and $5^{\text {th }}$ are quite similar too, especially in the first part of GDPR, up to article 25.

Additionally, more similarity is found between all principles and Articles 32-38. These articles deal with security of personal data an belong to Chapter IV titled "Controller and processor" [of personal data], Sections 2-4 titled "Security of personal data", "Data protection impact assessment and prior consultation" and "Data protection officer", respectively.

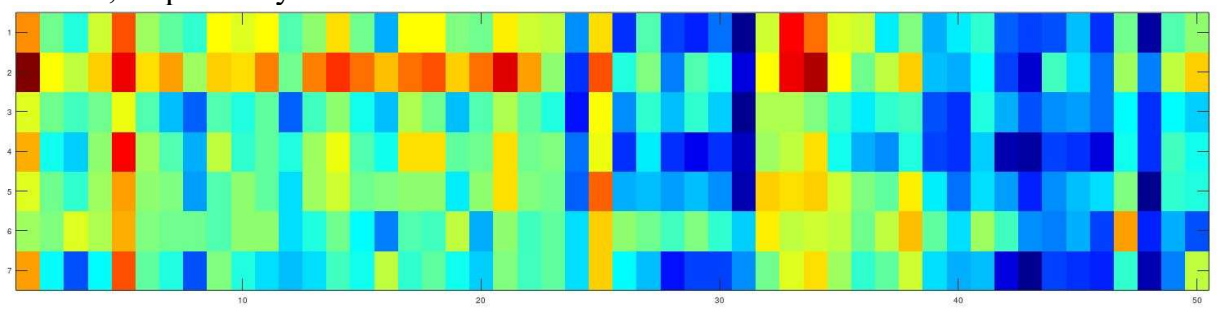

Figure 3: Heatmap of similarities between GDPR's articles (horizontal axis) and 7 Privacy by Design principles (vertical axis)

A low similarity can be found in Article 24, and 26-31. Article 24 deals with responsibilities of the controller, which is a general legal text. Articles 26-31 deal with joint controllers and processors of data.

There is relatively low similarity between 7 principles and GDPR's Articles from 38 onwards. These articles deal with tasks of the data protection officer (Article 39), with "Codes of conduct and certification" (Section 5 of Chapter IV, Articles 40-43) and 
with "Transfers of personal data to third countries or international organisations" (Chapter V, Articles 44-50).

\section{Discussion and conclusion}

In this paper, we checked how European regulation on data processing, the famous General Data Protection Regulation (GDPR) directive [14] - the Regulation (EU) 2016/679 of the European Parliament and of the Council of 27 April 2016 on the protection of natural persons with regard to the processing of personal data and on the free movement of such data - is addressing the privacy by using the Privacy by Design principles [28].

We used automated text similarities approach and the Universal Sentence Encoder to encode texts of the GDPR's articles and of the 7 Privacy by Design into vectors. Inner product of computed vectors represents the similarity measure among different pairs of texts.

We have found that among all principles, Principle 2 ("Privacy as the Default") was most similar, and it was also most strongly similar. Based on average similarity score principles 6 ("Visibility and Transparency"), 1 ("Proactive not Reactive; Preventative not Remedial") and 5 ("End-to-End Security - Lifecycle Protection") followed.

Least similar principles were $7^{\text {th }}$ ("Respect for User Privacy"), $3^{\text {rd }}$ ("Privacy Embedded into Design") and $4^{\text {th }}$ ("Full Functionality - Positive-Sum, not Zero-Sum").

From the order of the principles it is rather surprising that $7^{\text {th }}$ principle was least similar to GDPR text, despite the principle urging to build privacy around an individual Afterall, GDPR is protecting one of the basic human (individual) rights, the right to privacy. On the other hand, automatic semantic analysis has correctly identified Article 5 (describing the principles of GDPR) to be most similar to seven principles of Privacy by Design.

Our research has shown that automated text similarities approach can discover interesting similarities between legal texts and the underlying principles, not only in general, but in particular for each article-principle pair. The portions of texts where one or several principles prevail can easily be uncovered.

\section{Acknowledgement}

The author acknowledges the financial support from the Slovenian Research Agency (research core funding No. P2-0057, project funding No. V5-1725) and from University of Maribor (www.um.si, core funding).

\section{References}

1. Jowett B. Complete works of Aristotle. In: Barnes J, editor. Princeton, NJ: Princeton University Press; 1995.

2. Roy J. 'Polis' and 'Oikos' in Classical Athens. Greece \& Rome. 1999;46(1):1-18.

3. Shields JM. A Sacrifice to Athena: Oikos and Polis in Sophoclean Drama. Lewisburg, PA: Bucknell University, Department of Religion; 1991; Available from: 
http://www.facstaff.bucknell.edu/jms089/Z-Unpublished\%20Work/Athena.pdf. (Archived by

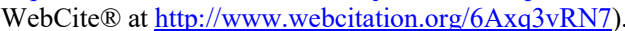

4. Post SG. Encyclopedia of Bioethics. New York, USA: Macmillan Reference; 2004. ISBN: 9780028657783

5. Brumen B, Heričko M, Sevčnikar A, Završnik J, Hölbl M. Outsourcing medical data analyses: can technology overcome legal, privacy, and confidentiality issues? J Med Internet Res. 2013 December 16, 2013;15(12):e283. PMID: 24342053. doi: 10.2196/jmir.2471.

6. Rooney B. Facebook Understands Europe's Privacy Fears Says Sandberg. The Wall Street Journal, TechEurope 2013-04-19.

7. Segall L. Facebook was 'the first innovator in privacy,' COO says. CNN Money. 2011-12-01

8. Cadwalladr C, Graham-Harrison E. Revealed: 50 million Facebook profiles harvested for Cambridge Analytica in major data breach. The guardian. 2018;17:22.

9. Isaak J, Hanna MJ. User Data Privacy: Facebook, Cambridge Analytica, and Privacy Protection. Computer. 2018;51(8):56-9. doi: 10.1109/MC.2018.3191268.

10. Bond RM, Fariss CJ, Jones JJ, Kramer ADI, Marlow C, Settle JE, et al. A 61-million-person experiment in social influence and political mobilization. Nature. 2012 2012/09/01;489(7415):295-8. doi: 10.1038/nature11421.

11. McKenzie S. Facebook's Mark Zuckerberg says sorry in full-page newspaper ads. CNN. 2018 March 25, 2018:Sect. Europe.

12. Allen AL. Privacy Law and Society. 1st ed: Thomson West; 2007 August 31, 2007. ISBN: 0314163581.

13. Allen AL. Privacy and Medicine. In: Zalta EN, editor. The Stanford Encyclopedia of Philosophy (Spring 2011 Edition). Stanford, CA, USA: Stanford University; 2011.

14. EU. Regulation (EU) 2016/679 of the European Parliament and of the Council of 27 April 2016 on the protection of natural persons with regard to the processing of personal data and on the free movement of such data. Official Journal of the European Union. 2016 4.5.2016;L:2016:119.

15. Solove DJ. A Taxonomy of Privacy. U Pa L Rev. 2006;154(3):477-564.

16. Warren SD, Brandeis LD. The Right to Privacy. Harv Law Rev. 1890;4(5):193-220.

17. DeCew JW. Privacy. In: Zalta EN, editor. The Stanford Encyclopedia of Philosophy. Stanford, CA, USA: Stanford University; 2008.

18. Prosser WL. Privacy. Calif Law Rev. 1960;48(3):383-423.

19. Nagel T. Concealment and exposure: and other essays. New York, USA: Oxford University Press; 2002. ISBN: 019515293X

20. Moore AD. Privacy: its meaning and value. American Philosophical Quarterly. 2003;40(3):215-27.

21. Gavison R. Privacy and the Limits of Law. Yale L J. 1980;89(3):421-71.

22. Allen AL. Uneasy access: Privacy for women in a free society. Totowa, NJ, USA: Rowman \& Littlefield Pub Inc; 1988. ISBN: 0847673286.

23. Bok S. Secrets: On the ethics of concealment and revelation. New York, USA: Vintage; 1989. ISBN: 0679724737.

24. DeCew JW. The priority of privacy for medical information. Soc Philos Policy. 2000;17(2):213-34.

25. Allen AL. Genetic Privacy: Emerging Concepts and Values. In: Rothstein MA, editor. Genetic secrets: Protecting privacy and confidentiality in the genetic era. New Haven: Yale University Press; 1997.

26. Kenny DJ. Confidentiality: the confusion continues. J Med Ethics. 1982 March 1, 1982;8(1):9-11. doi: 10.1136/jme.8.1.9. PMID: 7069738.

27. Sharpe VA. Privacy and Security for Electronic Health Records. Hastings Cent Rep. 2005;35(6):c3. doi: 10.1353/hcr.2005.0115. PMID: 16396204.

28. Cavoukian A. Privacy by design: The 7 foundational principles. Information and privacy commissioner of Ontario, Canada. 2009.

29. Cavoukian A. Big Data \& Privacy Together - It Is Achievable. Office of the Privacy Commissioner (Ontario): Ontario, Canada. Ontario, Canada: Office of the Privacy Commissioner (Ontario); 2013; Available from: http://www.privacybydesign.ca/index.php/big-data-privacy-together-is-achievable/. (Archived by WebCite ${ }^{\circledR}$ at http://www.webcitation.org/6GpVPLaXK).

30. Cavoukian A. 7 Foundational Principles of Privacy By Design. Ontario, Canada.: Office of the Privacy Commissioner (Ontario); 2013; Available from: http://www.privacybydesign.ca/index.php/about-pbd/7-foundational-principles/. (Archived by WebCite ${ }^{\circledR}$ at http://www.webcitation.org/6GqwiKldy).

31. Cavoukian A, Chanliau M. Privacy and Security by Design: A Convergence of Paradigms. Ontario, Canada: Office of the Privacy Commissioner (Ontario), 2013.

32. Cavoukian A. Privacy by design [leading edge]. IEEE Technology and Society Magazine. 2012;31(4):18-9. 
33. Cuijpers C, Purtova N, Kosta E. Data protection reform and the Internet: the draft Data Protection Regulation. Research Handbook on EU Internet Law: Edward Elgar Publishing; 2014.

34. van Dijk N, Gellert R, Rommetveit K. A risk to a right? Beyond data protection risk assessments. Computer Law \& Security Review. 2016;32(2):286-306.

35. EDPS. Opinion 5/2018: Preliminary Opinion on privacy by design. Brussels, Belgium: European Data Protection Supervisor, 2018.

36. Kuner C, Bygrave LA, Docksey C, editors. The EU General Data Protection Regulation (GDPR). A Commentary. Oxford, United Kingdom: Oxford University Press; 2020.

37. Cer D, Yang Y, Kong S-y, Hua N, Limtiaco N, John RS, et al., editors. Universal sentence encoder for English. Proceedings of the 2018 Conference on Empirical Methods in Natural Language Processing: System Demonstrations; 2018.

38. Sieg A. Text Similarities : Estimate the degree of similarity between two texts. 2018; Available from: https://medium.com/@adriensieg/text-similarities-da019229c894. Archived by archive.ph at http://archive.ph/VDtpY. Last accessed Feb/2020.

39. Cer D, Diab M, Agirre E, Lopez-Gazpio I, Specia L. SemEval-2017 Task 1: Semantic Textual Similarity Multilingual and Crosslingual Focused Evaluation. In: Bethard S, Carpuat M, Apidianaki M, Mohammad SM, Cer D, Jurgens D, editors. Proceedings of the 10th International Workshop on Semantic Evaluation (SemEval 2017); August 2017; Vancouver, Canada: Association for Computational Linguistics; 2017. doi: 10.18653/v1/S17-2001. 


\title{
Search for Appropriate Textual Information Sources
}

\author{
Adam ALBERT ${ }^{1}$, MARIE DUŽ I $^{1}$, Marek MENŠíK ${ }^{1}$, Miroslav PAJR ${ }^{2}$, Vojtěch PATSCHKA $^{1}$ \\ ${ }^{1}$ VSB-Technical University Ostrava, Department of Computer Science FEI \\ 17. listopadu 15, 70833 Ostrava, Czech Republic \\ ${ }^{2}$ Silesian University in Opava, Institute of Computer Science, \\ Bezručovo nám. 13, 74601 Opava, Czech Republic
}

\begin{abstract}
In this paper, we deal with the support in the search for appropriate textual sources. Users ask for an atomic concept that is explicated using machine learning methods applied to different textual sources. Next, we deal with the so-obtained explications to provide even more useful information. To this end, we apply the method of computing association rules. The method is one of the data-mining methods used for information retrieval. Our background theory is the system of Transparent Intensional Logic (TIL); all the concepts are formalised as TIL constructions.
\end{abstract}

Keywords. Machine learning; Transparent Intensional Logic; TIL; atomic concept; molecular concept; association rules; explication; natural language processing; information source recommendation

\section{Introduction}

We live in the era of globalisation, i.e. in times of interaction among people worldwide that has grown due to great advances in transportation, information and communication technologies. Though being a complex phenomenon, globalisation is usually characterised as a form of the integration of local economies into a global, unregulated market economy. Yet, the same phenomenon can be traced in other spheres of our lives, including science and research development. Globalisation has positive effects on the environment, culture, economic development, and in general human well-being in societies around the world. These are the upsides. However, some people complain that there are the downsides of globalisation as well because the gaps between rich and developing countries have grown. In 2000, the International Monetary Fund (IMF) identified four essential aspects of globalisation: trade and transactions, capital and investment movements, migration and movement of people, and the dissemination of knowledge. ${ }^{1}$

Here we are not going to deal with economic aspects of this phenomenon; rather, we are interested in the last issue mentioned, namely the increasing amount of knowledge, technology and information moving across international borders. Together with human innovation and progress in information technologies, these factors give rise to information overload'. True, there is a lot of knowledge around, spread in the vast amount of textual resources available on the Internet. Yet, there are also plenty of disinformation,

\footnotetext{
${ }^{1}$ For details, see [7].
} 
fake news, futile texts, canards, merely a lot of words, words, words. Many of our fellow researches certainly experienced the situation of googling for relevant, high-quality papers from reliable resources, only to the effect of obtaining a lot of futile results. The gist of the story is this. There is a need for an 'intelligent' question-answering system that would not only search by keywords but also evaluate the results, check for inconsistencies, derive their consequences logically entailed or just semantically associated with them, etc.

To this end, we decided to start a project on building such a system. Our background theory is the system of Transparent Intensional Logic (TIL) that makes it possible to formalise all the semantically salient features of natural language in a fine-grained way. ${ }^{2}$ Having formalised thousands of sentences in the form of TIL-constructions, we can now proceed in two closely interrelated ways. ${ }^{3}$ First, we have integrated special rules rooted in the rich semantics of natural language into a standard proof calculus of Genzen's natural deduction or general resolution in order to derive logical consequences of the results of a prior search. ${ }^{4}$ Second, by applying machine-learning methods adjusted to natural language processing in TIL, we explicate atomic concepts in order to 'understand' and manipulate them in the way human agents would do if only this task were not beyond their capacities. ${ }^{5}$ Here we mean Carnapian explication (see [2, pp. 7-8, §2]) that is transformation of a given more or less inexact concept (the explicandum) into an exact one (the explicatum). Our explicandum is usually an atomic concept such as 'cat', 'dog', 'lattice', 'group' to which a molecular concept is assigned that ontologically defines the objects falling under the explicandum. For instance, the concept 'cat' can be explicated by the biological definition of cats like 'a small domesticated carnivorous mammal of the family Felidae'. Having such a molecular concept, we can derive even more useful information from the vast number of textual corpora.

Explications of atomic concepts extracted from one textual document have been introduced in [12]. By applying a supervised machine-learning method to multiple textual resources, we obtain several different explications (see [10]). For instance, by applying the method to the atomic concept 'lattice', we obtain molecular concepts like 'a structure of crossed wooden or metal strips arranged to form a diagonal pattern of open spaces between the strips', 'a window, gate, or the like consisting of such a structure', or in physics 'the structure of fissionable and non-fissionable materials geometrically arranged within a nuclear reactor'. In mathematics, we can find two equivalent definitions of an abstract lattice structure, namely 'a partially ordered set in which every subset of two elements has a unique supremum and infimum' or 'an algebra with two binary operations meet and join that satisfy the axioms of commutativity, associativity and absorption'. In music, the same atomic concept can mean 'an organised grid model of pitch ratios.'

The question now arises how to evaluate these results so that to be able to recommend the most relevant, appropriate resources. There are several possibilities. We can check the results for inconsistencies or similarities, extract or generalise what they have

${ }^{2}$ See, for instance, [17].

${ }^{3}$ The text data are linguistically and logically processed so that TIL constructions are extracted by the Natural Language Logical Analyzer algorithm, see [9].

${ }^{4}$ For details, see [4], [5].

${ }^{5}$ The first application checking the possibilities of supervised machine learning methods adjusted to natural language processing has been introduced in [11] where the method has been applied to recognition of geometric figures. 
in common, etc. The goal of this paper is to introduce the algorithm based on associations between the data so that to compute and recommend the most relevant textual resources. For instance, concepts that are semantically associated with the above concept of the lattice are 'network', 'web', 'grid', 'structure', 'algebra', 'ordered set'. Yet, we are also interested in associations of concepts that follow from frequencies of their co-occurrence.

Depending on the amount of input data, users can obtain a huge number of different molecular concepts corresponding to the atomic concept that has been asked for. Now, the user can pick up one explication and the corresponding resource that seems to be relevant, but there can be other similar resources that are appropriate as well. Yet, due to a large amount of input textual data, the other proper documents can be overlooked and ignored. Thus, to prevent such a situation, we apply the method of discovering 'hidden' associations between the constituents of the resulting molecular concepts.

The whole process can be described as follows. First, a supervised machine learning method adjusted to TIL is applied to extract molecular concepts explicating the atomic concept that is the subject of the initiative query. As a result, we obtain several such explications that should be further evaluated. To this end, we apply the method of association rules. The system organises constituents of the obtained molecular concepts into an incident matrix. The rows of the matrix represent particular explications, and the columns represent the concepts of properties mentioned in those explications. We use a twovalued Boolean matrix. Next step consists in extracting association rules. Each rule is of form $A \Rightarrow B$ where $A$ is the antecedent and $B$ the succedent of the rule, and $A, B$ are sets of concepts. The sense of such a rule is this. If a given explication contains all the concepts from $A$ then it is to a certain degree probable that it also contains concepts from $B$. The so-called minimal confidence of an association rule, i.e. conditional probability of occurrences of concepts from $B$ provided there are concepts from $A$ is defined by a user. By computing the rules that are valid at least with this user-defined minimal confidence, the algorithm then proposes other textual sources that might be relevant as well.

The rest of the paper is organised as follows. Section 2 briefly summarises the main principles of Transparent Intensional Logic and introduces TIL constructions that serve as a concept defining formalism. In Section 3, we summarise previous results on the topic, namely the system of seeking relevant textual resources as presented in [12]. In Section 4, we introduce the theory of association rules, while in Section 5, the whole algorithm of their computing is described. Concluding remarks and further research proposals can be found in Section 6 .

\section{Foundations of TIL}

In TIL, expressions encode algorithmically structured procedures as their meanings. These procedures produce extensional or intensional entities, or even lower-order procedures, as their products. This approach has summarised by an algorithmic turn in semantics and advocated, for instance, by Moschovakis in [14]. Yet much earlier, in the early 1970s, Pavel Tichý defined six kinds of such meaning procedures that he coined TIL constructions as the centre-piece of his system, see [6] or [17].

The syntax of TIL is a hyperintensional, typed $\lambda$-calculus of partial functions. However, TIL $\lambda$-terms do not denote functions; rather, they denote procedures (constructions 
in TIL terminology) that produce functions or functional values as their products. A linguistic sense of an expression is an abstract procedure detailing how to arrive at an object (if any) of a particular logical type denoted by the expression.

There are two kinds of TIL constructions, atomic and molecular. Atomic constructions (Variables and Trivialisations) do not contain any other constituent but itself; they supply objects (of any type) on which compound constructions operate. Variables $x, y$, $p, q, \ldots$ construct objects dependently on a valuation; they $v$-construct. To each type, countably many variables are assigned, which $v$-constructs elements of the assigned type; we also say that variables range over that type. Trivialisation of an object $X$ (of any type, even a construction), in TIL symbolism ' $X$, refers to or displays the object $X$ without the mediation of any other construction. In order to operate on $X$, the object must be grabbed first; Trivialisation is such a simple grabbing mechanism.

There are two dual molecular constructions, namely Composition and Closure. Composition $\left[F A_{1} \ldots A_{\mathrm{n}}\right]$ is the procedure of applying a function $f$ ( $v$-constructed by the first constituent $F$ ) to a tuple argument $a$ (v-constructed by the constituents $\left.A_{1}, \ldots, A_{\mathrm{n}}\right)$. Composition $v$-constructs the value of $f$ at $a$, if the function $f$ is defined at $a$, otherwise the Composition is $v$-improper, i.e., it fails to $v$-construct anything. To produce a function rather than its value, there is $(\lambda-)$ Closure $\left[\lambda x_{1} \ldots \lambda x_{n} X\right]$. It is a procedure of $v$-constructing a function by abstracting over the values of variables $x_{1}, \ldots, x_{n}$ in the ordinary manner of $\lambda$-calculi. Finally, higher-order constructions producing lower-order constructions can be executed twice over. This is achieved by a fifth construction called Double Execution, ${ }^{2} X$, that behaves as follows: If $X v$-constructs a construction $Y$, and $Y v$-constructs an entity $Z$, then ${ }^{2} X v$-constructs $Z$; otherwise ${ }^{2} X$ is $v$-improper by failing to produce anything.

TIL constructions, as well as the entities they construct, all receive a type within a ramified hierarchy of types. Thus, the formal ontology of TIL is bidimensional; one dimension is made up of constructions of order $n \geq$ !, the other dimension encompasses non-constructions. On the ground level of the type-hierarchy, there are non-procedural entities unstructured from the algorithmic point of view belonging to a type of order 1 . Given a so-called epistemic (or 'objectual') base of atomic types (o-truth values, t-individuals, $\tau$-time moments/real numbers, $\omega$-possible worlds), the induction rule for forming functions is applied: where $\alpha, \beta_{1}, \ldots, \beta_{n}$ are types of order 1, the set of partial mappings from $\beta_{1} \times \ldots \times \beta_{n}$ to $\alpha$, denoted $\left(\alpha \beta_{1} \ldots \beta_{n}\right)$, is a type of order 1 as well. ${ }^{6}$ Constructions that construct entities of a type of order 1 are constructions of order 1 . They belong to a type of order 2 , denoted by $*_{1}$. This type $*_{1}$ together with atomic types of order 1 serves as a base for the induction rule of forming functions: any collection of partial mappings, type $\left(\alpha \beta_{1} \ldots \beta_{n}\right)$, involving $*_{1}$ in their domain or range is a type of order 2. Constructions that construct entities belonging to a type of order 1 or 2 are constructions of order 2 . They belong to a type of order 3 , denoted ${ }_{2}$; any collection of partial mapping involving $*_{2}$ in their domain or range is a type of order 3 . And so on ad infinitum.

Empirical sentences and terms denote (PWS-)intensions, functions with the domain of possible worlds $\omega$; they are frequently mappings from $\omega$ to chronologies of $\alpha$-objects, hence functions of types $((\alpha \tau) \omega)$, or $\alpha_{\tau}$, for short. Where variables $w, t$ range over possible worlds $(w \rightarrow \omega)$ and times $(t \rightarrow \tau)$, respectively, constructions of intensions are usually Closures of the form $\lambda w \lambda t[\ldots w \ldots t \ldots]$. We model sets and relations by their

${ }^{6}$ The above epistemic base $\{0,1, \tau, \omega\}$ was chosen, because it is apt for natural-language analysis, but the choice of the base depends on the area to be analysed. 
characteristic functions. Hence, while (or), (our) are types of a set of individuals and of a binary relation-in-extension between individuals, respectively, (o $)_{\tau \omega}$, (ou $)_{\tau \omega}$ are types of a property of individuals and a binary relation-in-intension between individuals, respectively. Quantifiers $\forall^{\alpha}, \exists^{\alpha}$ are type-theoretically polymorphic total functions of types $(\mathrm{o}(\mathrm{o} \alpha)$ ) defined as follows. Where $B$ is a construction that $v$-constructs a set of $\alpha$-objects, $\left[{ }^{0} \forall^{\alpha} B\right] v$-constructs $\mathbf{T}$ if $B v$-constructs the set of all $\alpha$-objects, otherwise $\mathbf{F} ;\left[^{0} \exists^{\alpha} B\right] v$ constructs $\mathbf{T}$ if $B v$-constructs a non-empty set, otherwise $\mathbf{F}$.

Notational conventions. That an object $X$ belongs to a type $\alpha$ is denoted as ' $X / \alpha$ '; that a construction $C v$-constructs an $\alpha$-object (provided not $v$-improper) is denoted by ' $C \rightarrow \alpha^{\prime}$ '. Instead of [ $\left.{ }^{\circ} \forall^{\alpha} \lambda x A\right],\left[{ }^{\prime} \exists^{\alpha} \lambda x A\right]$ we write ' $\forall x A$ ', ' $\exists x A$ ' whenever no confusion arises. If $C \rightarrow \alpha_{\tau}$ then the frequently used Composition [[Cw] $\left.t\right]$, aka extensionalization of the $\alpha$-intension $v$-constructed by $C$, is abbreviated as $C_{w t}$. We use classical infix notation without Trivialization for truth-value functions $\wedge$ (conjunction), $\vee$ (disjunction), $\supset$ (implication) and $\neg$ (negation). Also, identities $=^{\alpha}$ of $\alpha$-objects are written in the infix way without Trivialisation and the superscript $\alpha$ whenever no confusion arises.

Concepts are modelled as closed constructions in their normal form. The atomic concept of an object $a$ is its Trivialisation, ' $a$, while molecular concept of an object $a$ is its ontological definition, i.e. a closed molecular construction producing $a$. Unlike Frege and in compliance with Church, we deal with concepts of entities of any type, including concepts of propositions of type $\mathrm{O}_{\tau \omega}$ in an empirical vernacular and of truth-values in mathematics. $^{7}$

For a simple example, where Student/(o 1$)_{\tau \omega}$ is a property of individuals and John/ $/ \mathrm{an}$ individual, the sentence "John is a student" encodes as its meaning the concept of the proposition

$$
\lambda w \lambda t\left[{ }^{\prime} \text { Student }_{w t} \text { 'John }\right]
$$

The property Student must be extensionalized first, 'Student $t_{w t} \rightarrow$ (o $)$ and only then can it be applied to John, ['Student ${ }_{w t}$ 'John] $\rightarrow$ o, to obtain a truth value according as John belongs to the population of students in a world $w$ and time $t$ of evaluation. Abstracting over the values of variables $w, t$ the proposition of type $\mathrm{O} \tau \omega$ that John is a student is produced. The atomic concept 'Student of the property of being a student can be further explicated by a molecular concept, for instance of the property of being a person who attends a school.

$$
\lambda w \lambda w \lambda x\left[\left[{ }^{\prime} \text { Person }_{w t} x\right] \wedge\left[\text { ['Attend }{ }_{w t} x \text { 'School }\right]\right]
$$

This completes our brief introduction to the system of TIL and its theory of concepts.

\section{Explication of atomic concepts by machine learning}

Supervised machine learning is a method of predicting functional dependencies between input values and the output value. The supervisor provides an agent/learner with a set of training data. These data describe an object by a set of attribute values such that there is a functional dependency between these values. ${ }^{8}$

\footnotetext{
${ }^{7}$ For details on TIL theory of concepts see [6, § 2.2].

${ }^{8}$ In this section we briefly recapitulate the results as presented in [12].
} 
For instance, a house can be characterised by its size, locality, date of being built up, architecture style, etc., and its price. Obviously, the price of a house depends on its size, locality, date of building and architecture style. Hence, the price is called an output attribute, and the other attributes are input attributes. The goal of learning is to discover this functional dependency on the grounds of training data examples so that the agent can predict the value of the output attribute given the values of input attributes of a new instance. ${ }^{9}$

In our project of natural language processing and question answering, we decided to apply this method to learning concepts. To this end, we have to adjust the method a bit. First, instead of input/output attributes, we deal with concepts, that is closed constructions. The role of input attributes is played by the constituents of a hypothetic molecular concept, and instead of the output attribute, we deal with the atomic concept that the learner wants to learn by refining examples extracted from the textual documents. The hypothetic function is the relation of a requisite, or typical property or even a semantic association. Training data are natural-language texts, and the supervisor extracts from the text data positive and negative examples. The general framework of machine learning based on symbolic representation consists of the learning objectives, training data and heuristic methods for manipulating the symbolic representation of the data. For our purpose, we voted for an adjusted version of Patrick Winston algorithm [18, pp. 349-363] of supervised machine learning. This algorithm applies the principles of generalisation and specialisation to obtain a plausible hypothesis. Another adjustment of the algorithm is this. In addition to generalisation and specialisation, we also use the method of refinement. By refining a hypothetic concept, we insert new constituents into the molecular construction learned so far.

Generalisation usually consists in replacing one or more constituents of the hypothetic concept by a more general one, which is either extracted from agent's ontology or created from the chosen constituents by composing them in a disjunctive way. As a particular case, generalisation can also be applied to numerical values of attributes. For instance, if we obtain a piece of information that the in-heat period of a wild cat is two days and another positive example specifies eight days, we generalise it to the interval $2-8$ days.

Specialisation is triggered by negative examples. As a result, the negation of a property that does not belong to the essence of the hypothetic concept is inserted. Specialisation serves to distinguish the concept from similar ones. For instance, a wooden horse can serve as a negative example to the concept of a horse because a wooden horse is not a horse; rather, it is a toy horse though it may look like a genuine living horse.

For example, let the 'output' concept (to be learned) be that of a cat, i.e. 'Cat. The role of positive examples is played by ontological definitions of the property of being a cat, like "Cat is a predatory mammal that has been domesticated". The learner establishes a hypothesis that the property

$$
\lambda w \lambda t \lambda x\left[\left[[\text { 'Predatory 'Mammal }]_{w t} x\right] \wedge\left[\text { 'Domesticated }_{w t} x\right]\right]
$$

belongs to the essence of the property Cat. Negative examples delineate the hypothesis from other similar objects. For instance, the sentence "Dog is a domesticated predatory mammal that barks" can serve as a negative example for Cat. This triggers a specialisation of the hypothetic concept to the construction

${ }^{9}$ For details, see [13, pp. 20-28], [15, Ch. 7.8], [16, pp. 695-697]. 


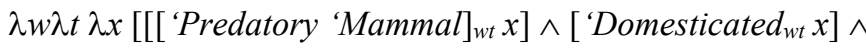

$$
\begin{aligned}
& \left.\neg\left[{ }^{\prime} \operatorname{Bark}_{w t} x\right] \wedge \neg\left[{ }^{\prime} \operatorname{Dog}_{w t} x\right]\right]
\end{aligned}
$$

Hence, given a positive example, the learner refines the hypothetic molecular concept by adding other constituents to the essence, while a negative example triggers specialisation of the hypotheses. The hypothetic concept can also be generalised. For instance, the learner can obtain the sentence "Cat is a wild feline predatory mammal" as another positive example describing the property Cat. Since the properties Wild and Domesticated are inconsistent, the agent consults his/her ontology for a more general concept. If there is none, the 'union' of the properties, Wild or Domesticated, is included. As a result, the learner obtains this hypothesis.

$$
\begin{aligned}
& \lambda w \lambda t \lambda x\left[[[\text { 'Feline ['Predatory 'Mammal }]]_{w t} x\right] \wedge \\
& {\left[\left[{ }^{\text {'Domesticated }}{ }_{w t} x\right] \vee\left[{ }^{\prime} \text { Wild }_{w t} x\right]\right] \wedge} \\
& \left.\neg\left[{ }^{\prime} \operatorname{Bark}_{w t} x\right] \wedge \neg\left[{ }^{\prime} \operatorname{Dog}_{w t} x\right]\right]
\end{aligned}
$$

Remark. Both Feline and Predatory are property modifiers of type $\left((\mathrm{o \imath})_{\tau \omega}(\mathrm{o})_{\tau \omega}\right)$, i.e. functions that given a root property return another property as an output. Since these two modifiers are intersective, the rules of left- and right-subsectivity are applicable here. ${ }^{10}$ In other words, the predatory mammal is a predator and is a mammal, similarly for a feline. If our agents have these rules in their knowledge base, the above Composition

can be further refined to

$$
\text { [['Feline ['Predatory 'Mammal } \left.]]_{w t} x\right]
$$

$$
\left[\left[\text { 'Feline }^{\mathrm{p}}{ }_{\text {wt }} x\right] \wedge\left[{ }^{\text {'Predator }}{ }^{\mathrm{p}}{ }_{w t} x\right] \wedge\left[{ }^{\prime} \text { Mammal }_{w t} x\right]\right] \text {, }
$$

where Feline $^{\mathrm{p}}$ and Predator $^{\mathrm{p}}$ are properties of individuals, i.e. objects of type (or) $)_{\tau \omega}$.

Heuristic methods of the original Winston algorithm work with examples that cover all the attributes of a learned object. Based on positive examples, the hypothesis is modified in such a way that the values of attributes are adjusted, or in case of a negative example, an unwanted attribute marked as Must-not-be is inserted. In our application, the sentences that mention the learned concept contain as constituents some but not all the requisites of this concept, and we build up a new molecular concept by adding new information extracted from positive or negative examples. Hence, we had to implement a new heuristic Concept-introduction for adding concepts of new requisites into a hypothetic concept. Negative examples trigger the method Negative-concept that inserts a concept of negated property into the hypothesis. Generalisation is realised by modules that introduce a concept of a more general property; to this end, we also adjusted the original heuristic Close-interval so that it is possible to generalise values of numeric concepts by the union of interval values.

\section{Description of the Explication algorithm}

Here is a brief specification of the algorithm.

Refinement.

1. Compare the model hypothesis (to be refined) and the positive example to find a significant difference

2. If there is a significant difference, then

${ }^{10}$ For details on and analysis of modifiers, see [3]. Details on the way of integrating such special semantic rules into a standard proof calculus can be found in [5], [4]. 
a) if the positive example contains as its constituent a concept that the model does not have, use the Concept-introduction

b) else ignore example

Specialisation.

1. Compare the model hypothesis (to be refined) and the near-miss negative example to find a significant difference

2. If there is a significant difference, then

c) if the near-miss example has a constituent of the concept that the model does not have, use the Negative-concept

d) else ignore example

Generalisation.

1. Compare the model hypothesis (to be refined) and the positive example to determine a difference

2. For each difference do

a) if a concept in the model points at a value that differs from the value in the example, then

i) if the properties in which the model and example differ have the most specific general property, use the General-concept

ii) else use Disjunctive-concept

b) if the model and example differ at an attribute numerical value or interval, use the Close-interval

c) else ignore example.

\section{Association rules}

The method of association rules extraction has been introduced in [1]. Yet ten years earlier a similar method has been described in $\left.{ }^{[8}\right]$. Basically, it is the process of looking for interesting relations among a large number of items. The method can be applied in various areas such as market survey or risk management, and a typical application is a market basket analysis. The goal is to discover associations between items occurring in a dataset that satisfy predefined minimum support and confidence.

The algorithm first extracts frequent item-sets, i.e. those item-sets whose occurrences exceed a predefined threshold $k$ called minimal support. Then confidence of associations among these frequent item-sets is computed and compared with predefined minimal confidence. Only those associations that exceed the predefined minimal support and confidence are then considered to be interesting results of the data mining method.

To put these ideas on more solid ground, here are the definitions. First, we need to define the support of a given set $A$ of items in a dataset. It is the probability of an occurrence of the set $A$ in the entire dataset.

Definition (support). Let $I=\left\{i_{1}, \ldots, i_{n}\right\}$ be a set of items and $D=\left\{T_{1}, \ldots, T_{m}\right\}$ a dataset of records such that each $T_{\mathrm{i}} \subseteq I$. Then support of a set of items $A \subseteq I$ in $D$ is

$$
\operatorname{supp}(A)=\frac{|\{t \in D: A \subseteq t\}|}{|D|}
$$


Remark. By $|S|$ we denote the cardinality of a set $S$. Since $|D|=m$, the support of a set $A$ is the ratio that compares the number of records containing all items from $A$ to the total number $m$ of records in the dataset. Hence, the support of $A$ is the probability of the occurrence of items from $A$ in the dataset.

Definition (association rule, confidence). Let $I=\left\{i_{1}, \ldots, i_{n}\right\}$ be a set of items and $D=$ $\left\{T_{1}, \ldots, T_{m}\right\}$ a dataset of records such that each $T_{\mathrm{i}} \subseteq I$. Farther, let $A, B \subseteq I$ such that $\operatorname{supp}(A \cup B) \geq k$, where $k$ is a predefined threshold. Then $A \Rightarrow B$ is an association rule iff $A \cap B=\varnothing$ and $A, B \neq \varnothing$. Confidence of the rule $A \Rightarrow B$ is

$$
\operatorname{conf}(A \Rightarrow B)=\frac{\operatorname{supp}(A \cup B)}{\operatorname{supp}(A)}
$$

Example. Let us have the following dataset of shopping transactions:

$\mathrm{D}=\{\mathrm{T} 1, \mathrm{~T} 2, \mathrm{~T} 3, \mathrm{~T} 4, \mathrm{~T} 5\}$

$\mathrm{T}_{1}=\{$ milk, butter $\}$,

$\mathrm{T}_{2}=\{$ bread, milk, butter $\}$,

$\mathrm{T}_{3}=\{$ milk $\}$,

$\mathrm{T}_{4}=\{$ bread, milk, butter $\}$,

$\mathrm{T}_{5}=\{$ bread, butter $\}$

Then the incident matrix is this.

\begin{tabular}{|c|ccc|}
\hline $\mathrm{D}$ & \multicolumn{3}{|c|}{ bread milk butter } \\
\hline $\mathrm{T}_{1}$ & 0 & 1 & 1 \\
\hline $\mathrm{T}_{2}$ & 1 & 1 & 1 \\
\hline $\mathrm{T}_{3}$ & 0 & 1 & 0 \\
\hline $\mathrm{T}_{4}$ & 1 & 1 & 1 \\
\hline $\mathrm{T}_{5}$ & 1 & 0 & 1 \\
\hline
\end{tabular}

Let $\min$-supp $=0.25$ and $\min$-conf $=0.75$. Then there are the following association rules meeting the thresholds:

$$
\begin{aligned}
& \operatorname{supp}(\{\text { milk, butter }\})=3 / 5 \\
& \operatorname{conf}(\{\text { milk }\} \Rightarrow\{\text { butter }\})=3 / 4 \\
& \operatorname{conf}(\{\text { butter }\} \Rightarrow\{\text { milk }\})=3 / 4 \\
& \operatorname{supp}(\{\text { bread, butter }\})=3 / 5 \\
& \operatorname{conf}(\{\text { bread }\} \Rightarrow\{\text { butter }\})=1 \\
& \operatorname{conf}(\{\text { butter }\} \Rightarrow\{\text { bread }\})=3 / 4 \\
& \operatorname{supp}(\{\text { bread, milk, butter }\})=2 / 5 \\
& \operatorname{conf}(\{\text { bread, milk }\} \Rightarrow\{\text { butter }\})=1
\end{aligned}
$$

As the example illustrates, the method can be applied for instance, in e-shops to recommend other products to be bought once a customer inserts into the shopping basket a given set of products. This feature inspired us to apply the method in our system in order 
to recommend other possible interesting explications of a given concept once a user votes for one of the obtained explications.

\section{Algorithm of text-source recommendations}

In this chapter, we summarise the whole system including the modules of supervised machine learning introduced in [10]. For the system outline see Fig. 1; the new functionality is incorporated in the very last part of the system, namely the Relevant Source Selection module.

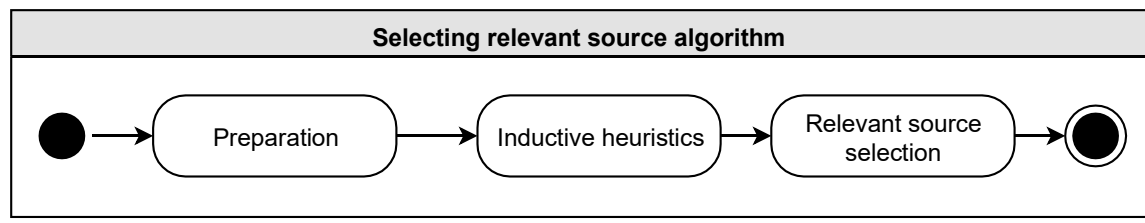

Fig. 1. Algorithm outline

First, we need to analyse textual resources to obtain the base of formalised TIL constructions. To this end, linguistic and logical analysis is applied. ${ }^{11}$ Then the set of relevant propositional constructions is selected; namely those where the concept to be explicated occurs.

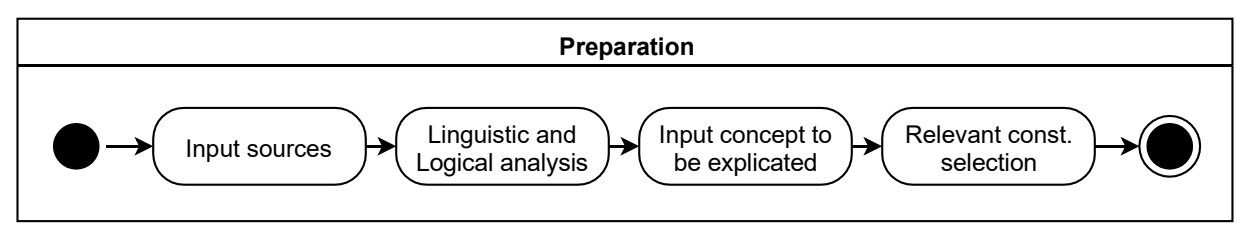

Fig. 2. Pre-processing and formalisation of textual resources

Next, the set of the selected constructions serves as an input for machine learning techniques, in particular, the Inductive heuristics module (see [12]), to obtain plausible hypotheses that explicate the given simple concept (Fig. 3). In this way, we obtain several explications, each of which corresponds to one of the input textual documents. ${ }^{11}$ Textual data are linguistically and logically processed so that TIL constructions are extracted
by the Natural Language Logical Analyzer algorithm [9]. 


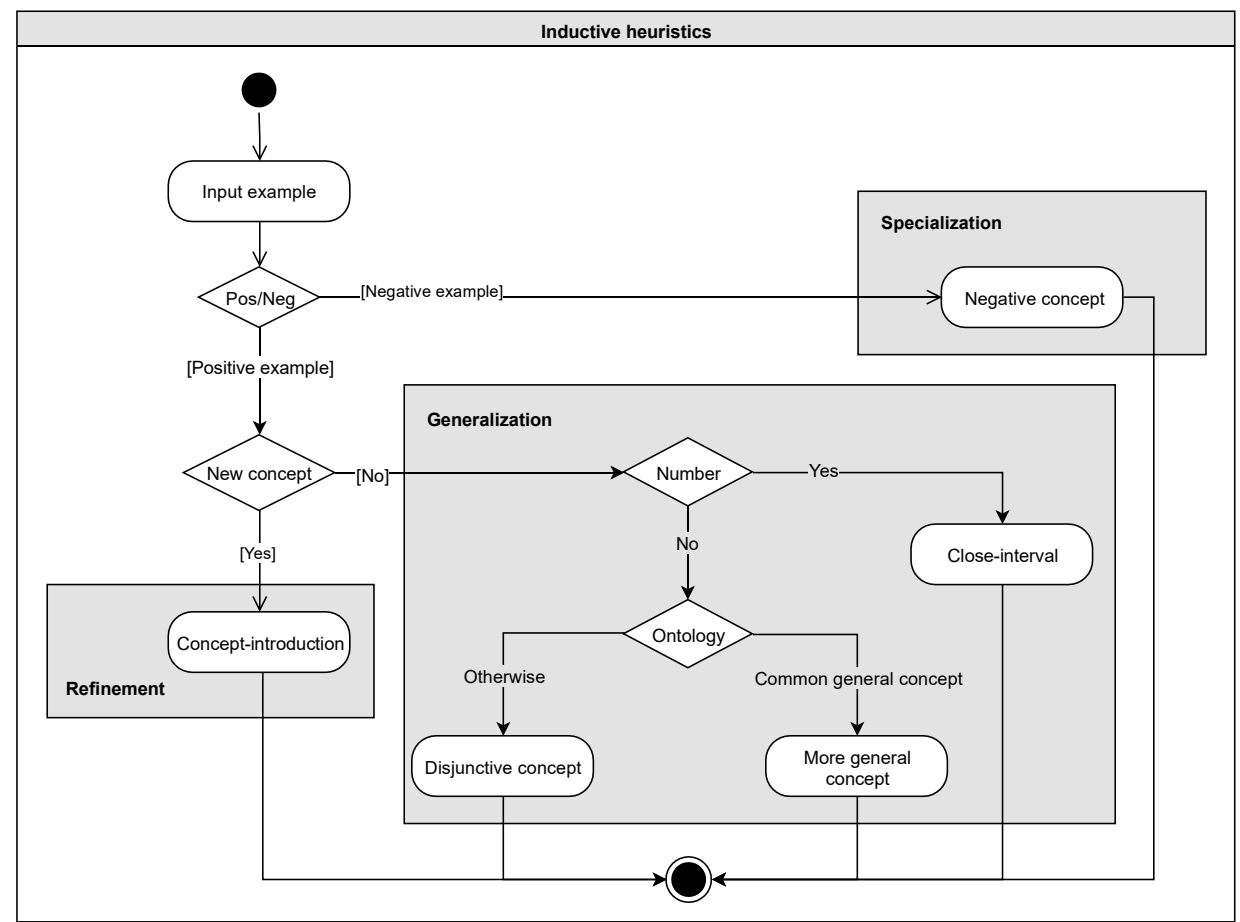

Fig. 3. Supervised machine learning

The last module of Relevant Source Selection (Fig. 4) is still work in progress. It is the module that deals with hypotheses processing and their evaluation. There are several functionalities that might be realised here. They include, inter alia, filtering out irrelevant sources according to the additional user-defined linguistic and logical criteria, search for inconsistencies among the hypotheses such as contrarieties and contradictions, looking for striking news that defies our intuitions and as such might be fake news, checking the reliability of resources.

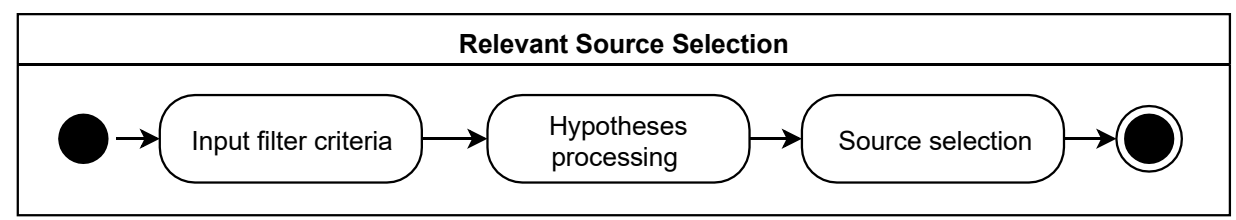

Fig. 4. Relevant source selection

Our recommendation system introduced in this paper is incorporated in the module Hypotheses processing, see Fig. 5. 


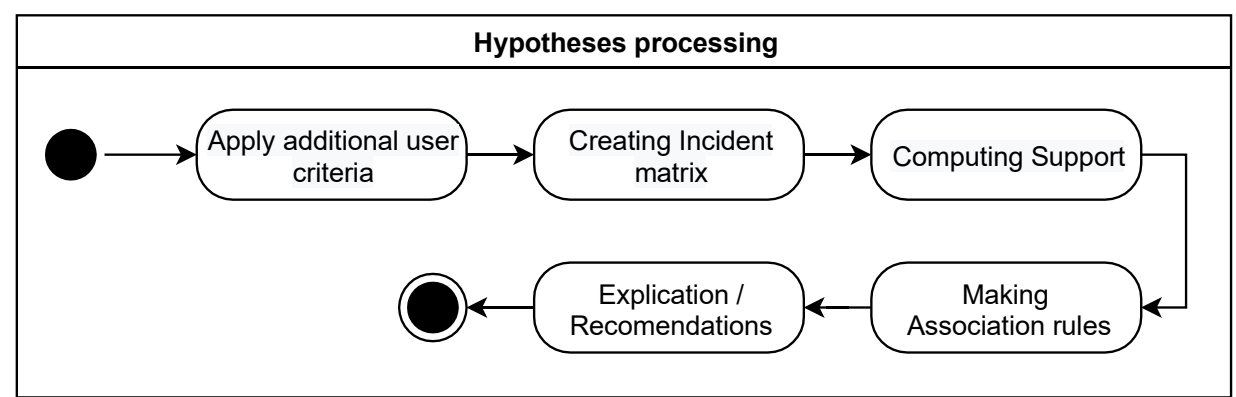

Fig. 5. Hypotheses processing

The input data to this module are a collection of closed constructions, i.e. the molecular concepts extracted from textual documents by the machine-learning module as explications of the simple concept $c$ asked for. The algorithm keeps the track of the document from which particular explications have been extracted. The algorithm extracts from each of these explications $e$ the concepts of properties $P_{\mathrm{j}} \rightarrow(\mathrm{o} \alpha)_{\tau \omega}$ such that these $P_{\mathrm{j}}$ are 'conjunctive constituents' of $e$. By 'conjunctive constituents' we mean those subconstructions that are connected in a conjunctive way. For instance, the conjunctive constituents of the molecular concept

are these:

$$
\begin{gathered}
\lambda w \lambda t \lambda x\left[[[\text { 'Feline ['Predatory 'Mammal }]]_{w t} x\right] \wedge \\
{\left[\left[\text { 'Domesticated }_{w t} x\right] \vee\left[{ }^{\prime} \text { Wild }_{w t} x\right]\right] \wedge} \\
\left.\neg\left[\text { 'Bark }_{w t} x\right] \wedge \neg\left[\text { 'Dog }_{w t} x\right]\right]
\end{gathered}
$$

$$
\begin{aligned}
& \left.\lambda w \lambda t \lambda x[[\text { 'Feline ['Predatory 'Mammal }]]_{w t} x\right] \text {, } \\
& \lambda w \lambda t \lambda x\left[\left[\text { 'Domesticated }_{w t} x\right] \vee\left[{ }^{\prime} \text { Wild }_{w t} x\right]\right] \text {, } \\
& \lambda w \lambda t \lambda x \neg\left[{ }^{\prime} \operatorname{Bark}_{w t} x\right] \text {, } \\
& \lambda w \lambda t \lambda x \neg\left[{ }^{\prime} \operatorname{Dog}_{w t} x\right]
\end{aligned}
$$

Let $I$ be the set of all the constituents extracted from the explications. The algorithm now computes binary incident matrix, rows of which represent explications and items of columns are constituents $i \in I$. Farther, association rules are computed from this incident matrix. Recall that association rule $A \Rightarrow B$ represents an association between disjoint sets of sufficiently frequent non-empty sets of items in a given dataset. Our dataset is now a set of records (rows of incident matrix) extracted from particular explications, see Table 1 below.

The user selects one of the input explications that is the closest one to his/her intuitive idea explaining the simple concept $c$ asked for. The goal is to find other explications (and thus text documents as well) which concern the concept $c$ and might be potentially interesting for the user. Association rules that serve as those recommending other explications are computed with respect to these criteria.

1. Antecedent contains only those constituents which occur in the selected explication $e$.

2. Succedent contains only the remaining constituents from $I$ which do not occur in the selected explication $e$.

3. Support and confidence of the rule are greater or equal to the value of the predefined criteria min-supp and min-conf. 
Formally, these criteria are defined as follows.

Definition: Let $A \Rightarrow B$ be an association rule, $E=\left\{e_{1}, \ldots, e_{n}\right\}$ the set of all explications, $e \in E$ the user-selected explication, and let $\operatorname{Prop}(x)$ be the set of all constituents occurring in an explication $x$. Then the rule $A \Rightarrow_{e} B$ is a rule of recommendation generated by the selected explication $e$ iff:

$$
\begin{gathered}
A \subseteq \operatorname{Prop}(e) \\
B \subseteq\left(\bigcup_{i=1}^{n} \operatorname{Prop}\left(e_{i}\right)\right) \backslash \operatorname{Prop}(e) \\
\operatorname{supp}(A \cup B) \geq \min \text {-supp } \\
\operatorname{conf}(A \Rightarrow B) \geq \min \text {-conf }
\end{gathered}
$$

Remark. Obviously, to each explication $e$ there can be more than one rule of recommendation generated by $e$.

Having computed the rules of recommendation, we want to recommend other documents dealing with the input concepts $c$. Thus, we define:

Definition: Let $A \Rightarrow_{e} B$ be a rule of recommendation generated by the selected explication $e$. Let $\exp (d, c)$ be an explication of an input simple concept $c$ extracted from a textual document $d$. Then the recommended sources dealing with the concept $c$ according to the rule $A \Rightarrow_{e} B$ is a set of text-sources $R S$ such that

$$
R S=\{d:(A \cup B) \subseteq \operatorname{Prop}(\exp (d, c))\}
$$

Moreover, weakly recommended sources explaining the concept $c$ is a set of text-sources WRS such that

$$
W R S=\{d: B \subseteq \operatorname{Prop}(\exp (d, c))\}
$$

\subsection{Case study example}

In our case study, we had eight documents, i.e. text-sources, dealing with the concept of a wild cat. From each document, the algorithm selected those sentences where 'wild cat' receives mention. These sentences have been formalised as TIL constructions explicating the concept 'wild cat'.

Remark. In the constructions below, we use two relations between properties, namely $R e q$ (for a requisite) and Typ-p (typical property). Though the differentiation between $R e q$ and $T y p-p$ is irrelevant for the purposes of this paper, we briefly explain. The first one obtains between two properties $P$ and $Q$ necessarily. Hence, $[$ Req $P Q]$, i.e. $P$ is a requisite of $Q$, should be understood like this: necessarily, if an individual $a$ happens to be a $Q$ then $a$ is a $P$. On the other hand, [Typ-p $P Q$ ], i.e. $P$ is typical for $Q$, is to be read as follows: Typically, if an individual $a$ happens to be a $Q$ then $a$ is a $P$. Note that both these sentences should be read de dicto. They talk about properties (intensions) rather 
than about a particular individual. Hence, that having a fur is a requisite of the property of being a wild cat does not exclude the possibility that this or that cat lost its fur.

\section{Source 1 .}

- The weight of a wild cat is between 1.2 and 11 kilograms.

- Wild cats are mammals.

- Wild cats have fur.

- The body length of wild cats is from 47 to $80 \mathrm{~cm}$.

- The average skull capacity of wild cats is $41.25 \mathrm{~cm}^{3}$.

- The average height of wild cats at the withers is $37.6 \mathrm{~cm}$.

Exp (Source 1, 'Wild-cat).

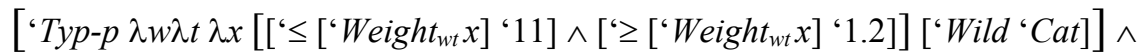

['Req 'Mammal ['Wild 'Cat $]] \wedge[$ ['Req 'Has-fur ['Wild 'Cat $]$ ^

['Typ-p $\lambda w \lambda t \lambda x\left[\left[{ }^{\circ} \leq\left[[\text { 'Average 'Body-Length }]_{\mathrm{wt}} x\right]\right.\right.$ '80]

$$
\wedge\left[{ }^{\circ} \geq\left[\left[{ }^{\prime} \text { Average 'Body-Length }\right]_{w t} x\right] \text { '47]] ['Wild 'Cat }\right] \text { ^ }
$$

$\left[{ }^{\prime}\right.$ Typ-p $\lambda w \lambda t \lambda x\left[{ }^{\prime}=\left[\left[{ }^{\prime} \text { Average 'Skul-Size }\right]_{\mathrm{wt}} x\right]\right.$ '41.25] ['Wild 'Cat $]$ ^

['Typ-p $\lambda w \lambda t \lambda x\left[{ }^{\prime}=\left[\left[{ }^{\prime} \text { Average 'Height }\right]_{\mathrm{wt}} x\right]\right.$ '37.6] ['Wild 'Cat $\left.]\right]$

Source 2 .

- The typical occurrence of wild cats is mixed or deciduous forests.

- The size of the territory of a wild cat is greater than 50 ha.

- Wild cat marks its territory with its claws, urination and droppings.

$\operatorname{Exp}$ (Source 2, 'Wild-cat).

['Typ-p $\lambda w \lambda t \lambda x\left[\right.$ ['Live-in ${ }_{w t} x \lambda w \lambda t \lambda y\left[\left[[\text { ['Mixed 'Forest }]_{w t} y\right]\right.$

$\left.\vee\left[\left[{ }^{\circ} \text { Decidious 'Forest }\right]_{w t} y\right]\right][$ ['Wild 'Cat $\left.]\right] \wedge$

['Typ-p $\lambda w \lambda t \lambda x\left[\right.$ [' $\geq\left[\right.$ 'Territory-Size $\left.{ }_{w t} x\right]$ '47] ['Wild 'Cat $\left.]\right] \wedge$

$\left[\right.$ ['Typ-p $\lambda w \lambda t \lambda x\left[\left[{ }^{\circ}\right.\right.$ Ter-Marking ${ }_{w t} x$ 'Clawing $] \vee\left[\right.$ ['Ter-Marking ${ }_{w t} x$ 'Urinating $] \vee$

['Ter-Marking ${ }_{w t} x$ 'Droppings]] ['Wild 'Cat]]

Source 3.

- The in-heat period of the wild cat is $2-8$ days.

- The wild cat is looking for a mate with a loud meow.

- The pregnancy period of a wild cat is 65 days.

- The size of the litter of wild cats is $3-4$ kittens.

Exp (Source 3, 'Wild-cat). 
['Typ-p $\lambda w \lambda t \lambda x\left[\left[{ }^{\circ} \leq\left[{ }^{\prime}\right.\right.\right.$ In-Heat-Period $\left.{ }_{w t} x\right]$ '8]

$\wedge\left[{ }^{\circ} \geq\left[{ }^{\prime}\right.\right.$ In-Heat-Period $\left.{ }_{w t} x\right]$ '2 2$][$ ['Wild 'Cat $\left.]\right] \wedge$

['Typ-p $\lambda w \lambda t \lambda x\left[{ }^{\prime}\right.$ Seek $_{w t} x$ 'Mate ['Loud 'Meow] $[$ ['Wild 'Cat $\left.]\right] \wedge$

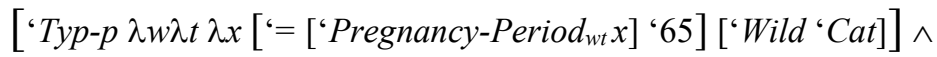

['Typ-p $\lambda w \lambda t \lambda x\left[\left[{ }^{\prime} \leq\left[{ }^{\prime}\right.\right.\right.$ Litter-Size $\left._{w t} x\right]$ '4 4$] \wedge\left[{ }^{\circ} \geq\left[{ }^{\prime}\right.\right.$ Litter-Size $\left._{w t} x\right]$ '3 3$\left.]\right][$ ['Wild 'Cat $\left.]\right]$

Source 4.

- Wild cats are mammals.

- Wild cats have fur.

- The average skull capacity of a wild cat is $41.25 \mathrm{~cm}^{3}$.

- Wild cats mark their territory with claws, urination, droppings.

- The pregnancy period of a wild cat is 65 days.

- The size of the litter of wild cats is up to 4 kittens.

Exp (Source 4, ' Wild-cat).

['Req 'Mammal ['Wild 'Cat $]$ ^['Req 'Has-fur ['Wild 'Cat] $] \wedge$

$\left[{ }^{' T y p}-p \lambda w \lambda t \lambda x\left[{ }^{'}=\left[\left[{ }^{\prime} \text { Average 'Skul-Size }\right]_{\mathrm{wt}} x\right]\right.\right.$ '41.25] ['Wild 'Cat $]$ ^

$\left[{ }^{\prime}\right.$ Typ-p $\lambda w \lambda t \lambda x\left[\left[{ }^{\prime}\right.\right.$ Ter-Marking ${ }_{w t} x$ 'Clawing $] \vee\left[{ }^{\prime}\right.$ Ter-Marking ${ }_{w t} x$ 'Urinating $] \vee$ ['Ter-Marking $w_{t} x$ 'Droppings] ['Wild 'Cat $] \wedge$

['Typ-p $\lambda w \lambda t \lambda x\left[{ }^{\circ}=\left[{ }^{\prime}\right.\right.$ Pregnancy-Period $\left._{w t} x\right]$ '65] ['Wild 'Cat $\left.]\right] \wedge$

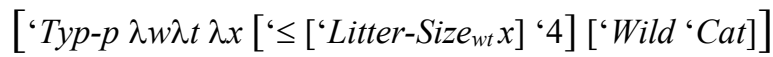

Source 5 .

- The average body length of a wild cat is $47 \mathrm{~cm}$ or more.

- Wild cats mark their territory with claws, urination, droppings.

- The pregnancy period of a wild cat is 65 days.

- The size of the litter of wild cats is up to 4 kittens.

$\operatorname{Exp}$ (Source 5, 'Wild-cat).

['Typ-p $\lambda w \lambda t \lambda x\left[{ }^{\circ} \geq\left[\left[{ }^{\prime} \text { Averige 'Body-Length }\right]_{\mathrm{wt}} x\right]{ }^{\prime} 47\right]\left[{ }^{\prime}\right.$ Wild 'Cat $\left.]\right] \wedge$

$\left[\right.$ 'Typ-p $\lambda w \lambda t \lambda x\left[\left[{ }^{\circ}\right.\right.$ Ter-Marking ${ }_{w t} x$ 'Clawing $] \vee\left[{ }^{\prime}\right.$ Ter-Marking ${ }_{w t} x$ 'Urinating $] \vee$ ['Ter-Marking ${ }_{\text {wt }} x$ 'Droppings $\left.]\right]\left[{ }^{\prime}\right.$ Wild 'Cat $\left.]\right] \wedge$

$\left[{ }^{\prime}\right.$ Typ-p $\lambda w \lambda t \lambda x\left[{ }^{\prime}=\left[{ }^{\prime}\right.\right.$ Pregnancy-Period $\left.{ }_{w t} x\right]$ ' 65$]\left[{ }^{\prime}\right.$ Wild 'Cat $\left.]\right] \wedge$

['Typ-p $\lambda w \lambda t \lambda x\left[{ }^{\circ} \leq\left[{ }^{\prime}\right.\right.$ Litter-Size $w t$ $\left.x\right]$ '4] ['Wild 'Cat $]$ 
Source 6 .

- The body length of wild cats is $47 \mathrm{~cm}$ or more.

- Wild cat marks its territory with its claws, urination, droppings.

- Wild cats seek their mate by a loud meow.

- The size of the litter of wild cats is up to 4 kittens.

Exp (Source 6, 'Wild-cat).

['Typ-p $\lambda w \lambda t \lambda x\left[\right.$ [' $\left.\geq\left[{ }^{\prime} \text { Averige 'Body-Length }\right]_{\mathrm{wt}} x\right]$ '47] ['Wild 'Cat $\left.]\right] \wedge$

['Typ-p $\lambda w \lambda t \lambda x\left[\left[{ }^{\prime}\right.\right.$ Ter-Marking ${ }_{w t} x$ 'Clawing $] \vee\left[{ }^{\prime}\right.$ Ter-Marking ${ }_{w t} x$ 'Urinating $] \vee$ ['Ter-Marking ${ }_{w t} x$ 'Droppings $\left.]\right]$ ['Wild 'Cat $\left.]\right] \wedge$

['Typ-p $\lambda w \lambda t \lambda x\left[\right.$ ['Seek ${ }_{w t} x$ 'Mate ['Loud 'Meow] ['Wild 'Cat]]^

['Typ-p $\lambda w \lambda t \lambda x\left[{ }^{\prime} \leq\left[{ }^{\prime}\right.\right.$ Litter-Size $\left._{w t} x\right]$ '4] ['Wild 'Cat $\left.]\right]$

Source 7.

- Wild cats are mammals.

- The weight of a wild cat is up to 11 kilograms.

- Wild cats usually live in mixed or deciduous forests.

- Wild cat marks its territory with its claws, urination, droppings.

- Wild cat looks for a mate with a loud meow.

- The pregnancy period of a wild cat is 65 days.

- Wild cat has fur.

$\operatorname{Exp}$ (Source 7, 'Wild-cat).

['Req'Mammal ['Wild'Cat $]] \wedge$

['Typ-p $\lambda w \lambda t \lambda x\left[{ }^{\prime} \leq\left[{ }^{\prime}\right.\right.$ Weight $\left._{w t} x\right]$ ' 11$][$ ['Wild 'Cat $\left.]\right] \wedge$

['Typ-p $\lambda w \lambda t \lambda x\left[\right.$ ['Live-in ${ }_{w t} x \lambda w \lambda t \lambda y\left[\left[\left[{ }^{\circ} \text { Mixed 'Forest }\right]_{w t} y\right]\right.$

$\left.\vee\left[\left[{ }^{\prime} \text { Decidious 'Forest }\right]_{w t} y\right]\right][$ ['Wild 'Cat $\left.]\right] \wedge$

['Typ-p $\lambda w \lambda t \lambda x\left[\left[{ }^{\prime}\right.\right.$ Ter-Marking ${ }_{w t} x$ 'Clawing $] \vee\left[{ }^{\prime}\right.$ Ter-Marking ${ }_{w t} x$ 'Urinating $] \vee$ ['Ter-Marking ${ }_{w t} x$ 'Droppings $\left.]\right]$ ['Wild'Cat $\left.]\right] \wedge$

['Typ-p $\lambda w \lambda t \lambda x\left[\right.$ 'Seek ${ }_{w t} x$ 'Mate ['Loud'Meow] ['Wild 'Cat $]$ '

$\left[{ }^{\prime} T y p-p \lambda w \lambda t \lambda x\left[\right.\right.$ '= ['Pregnancy-Period $\left.{ }_{w t} x\right]$ '65] ['Wild 'Cat $\left.]\right] \wedge$

['Req 'Has-fur ['Wild 'Cat]

Source 8 .

- The body length of wild cats is up to $80 \mathrm{~cm}$.

- The size of the territory of a wild cat is greater than 50 ha.

- The size of the litter of wild cats is up to 4 kittens. 
$\operatorname{Exp}$ (Source 8, 'Wild-cat).

['Typ-p $\lambda w \lambda t \lambda x\left[{ }^{\circ} \leq\left[\left[{ }^{\prime} \text { Average 'Body-Length }\right]_{\mathrm{wt}} x\right]\right.$ ' 80$][$ ['Wild 'Cat $\left.]\right] \wedge$

['Typ-p $\lambda w \lambda t \lambda x\left[{ }^{\circ} \geq\left[{ }^{\prime}\right.\right.$ Territory-Size $\left.w t x\right]$ '50] ['Wild 'Cat $\left.]\right] \wedge$

['Typ-p $\lambda w \lambda t \lambda x\left[{ }^{\circ} \leq\left[{ }^{\prime}\right.\right.$ Litter-Size $w t$ x] '4] ['Wild 'Cat $]$

Types:

Wight, Body-Lenght, Height, Skull-Size, Territory-Size, Litter-Size,

In-Heat-Period,Pregnancy-Period $/(\tau \iota)_{\tau \omega}$ : attributes

Average $/\left((\tau \iota)_{\tau \omega}(\tau \iota)_{\tau \omega}\right)$ : attribute modifier

Mammal, Cat, Has-Fur, Forests, Clawing, Urinating, Droppings,

Mate, Meow $/(o \iota)_{\tau \omega}$ : properties

Wild, Loud, Mixed, Deciduous $/\left((o \iota)_{\tau \omega}(o \iota)_{\tau \omega}\right)$ : property modifiers

$x \rightarrow \iota$

Live-in, Ter-Marking $/\left(o l(o l)_{\tau \omega}\right)_{\tau \omega}$

Seek/(ol(ol) $\left.)_{\tau \omega}(o l)_{\tau \omega}\right)_{\tau \omega}$

$T y p-p /\left(o(o l)_{\tau \omega}(o l)_{\tau \omega}\right)$

$\operatorname{Req} /\left(o(o \iota)_{\tau \omega}(o \iota)_{\tau \omega}\right)$

Table 1 is the incident matrix computed from these explications.

Table 1. Incident matrix. Explications/properties

\begin{tabular}{|c|c|c|c|c|c|c|c|c|c|c|c|c|c|c|c|c|c|}
\hline & 1 & 2 & 3 & 4 & 5 & 6 & 7 & 8 & 9 & 10 & 11 & 12 & 13 & 14 & 15 & 16 & 17 \\
\hline $\mathrm{e}_{1}$ & 1 & 1 & 1 & 1 & 1 & 1 & 1 & 1 & 0 & 0 & 0 & 0 & 0 & 0 & 0 & 0 & 0 \\
\hline $\mathrm{e}_{2}$ & 0 & 0 & 0 & 0 & 0 & 0 & 0 & 0 & 1 & 1 & 1 & 0 & 0 & 0 & 0 & 0 & 0 \\
\hline $\mathrm{e}_{3}$ & 0 & 0 & 0 & 0 & 0 & 0 & 0 & 0 & 0 & 0 & 0 & 1 & 1 & 1 & 1 & 1 & 1 \\
\hline $\mathrm{e}_{4}$ & 1 & 1 & 0 & 0 & 0 & 0 & 1 & 0 & 0 & 0 & 1 & 0 & 0 & 0 & 1 & 1 & 0 \\
\hline $\mathrm{e}_{5}$ & 0 & 0 & 0 & 0 & 1 & 0 & 0 & 0 & 0 & 0 & 1 & 0 & 0 & 0 & 1 & 1 & 0 \\
\hline $\mathrm{e}_{6}$ & 0 & 0 & 0 & 0 & 1 & 0 & 0 & 0 & 0 & 0 & 1 & 0 & 0 & 1 & 0 & 1 & 0 \\
\hline $\mathrm{e}_{7}$ & 1 & 1 & 1 & 0 & 0 & 0 & 0 & 0 & 1 & 0 & 1 & 0 & 0 & 1 & 1 & 0 & 0 \\
\hline $\mathrm{e}_{8}$ & 0 & 0 & 0 & 0 & 1 & 0 & 0 & 0 & 0 & 1 & 0 & 0 & 0 & 0 & 0 & 1 & 0 \\
\hline
\end{tabular}

1. 'Mammal

2. 'Has-fur

3. $\lambda w \lambda t \lambda x\left[{ }^{\prime} \leq\left[{ }^{\prime} \text { Weight }_{w t} x\right]^{\prime} 11\right]$

4. $\lambda w \lambda t \lambda x\left[{ }^{\prime} \geq\left[{ }^{\prime} \text { Weight }_{w t} x\right]^{\prime} 1.2\right]$

5. $\left.\lambda w \lambda t \lambda x\left[{ }^{\prime} \geq\left[\left[^{\prime} \text { 'Average 'Body-Length }\right]_{w t} x\right]\right]^{\prime} 47\right]$

6. $\lambda w \lambda t \lambda x\left[{ }^{\prime} \leq\left[\left[^{\prime} \text { 'Average 'Body-Length }\right]_{w t} x\right]\right.$ '80]

7. $\left.\lambda w \lambda t \lambda x\left[{ }^{\prime}=\left[{ }^{\prime} \text { 'Average 'Skul-Size }\right]_{w t} x\right]^{\prime} 41.25\right]$

8. $\lambda w \lambda t \lambda x\left[{ }^{\prime}=\left[\left[^{\prime} \text { Average 'Height }\right]_{w t} x\right]^{\prime} 37.6\right]$ 
9. $\lambda w \lambda t \lambda x\left[{ }^{\prime}\right.$ Live-in $_{w t} x\left[\lambda w \lambda t \lambda y\left[\left[\left[^{\prime} \text { Mixed 'Forrest }\right]_{w t} y\right] \mathrm{V}\right.\right.$ [['Deciduous 'Forrest $\left.\left.\left.\left.]_{w t} y\right]\right]\right]\right]$

10. $\lambda w \lambda t \lambda x\left[{ }^{\prime} \geq\left[{ }^{\prime} \text { Territory-Size }{ }_{w t} x\right]^{\prime} 50\right]$

11. $\lambda w \lambda t \lambda x\left[\left[{ }^{\prime}\right.\right.$ Ter-Marking ${ }_{w t} x{ }^{\prime}$ Clawing $] \mathrm{V}$ ['Ter-Marking ${ }_{w t} x$ 'Urinating ] v ['Ter-Marking ${ }_{w t} x^{\prime}$ Leaves-Droppings ]

12. $\lambda w \lambda t \lambda x\left[{ }^{\prime} \leq\left[{ }^{\prime} \text { In-Heat-Period }{ }_{w t} x\right]^{\prime} 8\right]$

13. $\lambda w \lambda t \lambda x\left[{ }^{\prime} \geq\left[{ }^{\prime} \text { In-Heat-Period }{ }_{w t} x\right]^{\prime} 2\right]$

14. $\lambda w \lambda t \lambda x\left[{ }^{\prime}{ }^{2}\right.$ ee $k_{w t} x$ 'Mate ['Loud 'Meow ]]

15. $\lambda w \lambda t \lambda x\left[{ }^{\prime}=\left[{ }^{\prime}\right.\right.$ Pregnancy-Period $\left.{ }_{w t} x\right]$ '65]

16. $\lambda w \lambda t \lambda x\left[{ }^{\prime} \leq\left[{ }^{\prime} \text { Litter-Size }_{w t} x\right]^{\prime} 4\right]$

17. $\lambda w \lambda t \lambda x\left[{ }^{\prime} \geq\left[^{\prime} \text { Litter-Size }_{w t} x\right]^{\prime} 3\right]$

Min-supp $=0.25$

Min- $\operatorname{conf}=0.66$

Assume that the user has chosen the first explication as the basic one. Hence, the concepts corresponding to the columns 1-8 can occur only in the antecedents of the recommendation rules. The remaining concepts occur only in rule consequents.

Rules:

Confidence $=0.66 ; \boldsymbol{R S}=\{\mathbf{s} 4, \mathrm{~s} 7\}$

$\left\{{ }^{\prime}\right.$ Mammal, 'Has-fur $\} \Rightarrow_{e 1}$

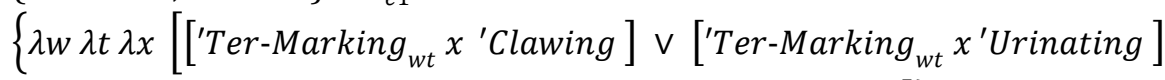
$\vee\left['\right.$ Ter-Marking ${ }_{w t} x^{\prime}$ Leaves-Droppings $\left.\left.]\right]\right\}$

Confidence $=0.66 ; \boldsymbol{R S}=\{\mathrm{s} 4, \mathrm{~s} 7\}$

$\left\{{ }^{\prime}\right.$ Mammal,'Has-fur $\} \Rightarrow_{e 1}$

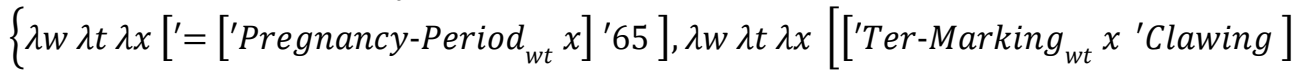

$\vee\left[{ }^{\prime}\right.$ Ter-Marking ${ }_{w t} x^{\prime}$ Urinating $] \vee\left[{ }^{\prime}\right.$ Ter-Marking ${ }_{w t} x^{\prime}$ Leaves-Droppings $\left.\left.]\right]\right\}$

Confidence $=0.66 ; \boldsymbol{R S}=\{\mathbf{s} 4, \mathrm{~s} 7\}$

$\left\{{ }^{\prime}\right.$ Mammal, 'Has-fur $\} \Rightarrow_{e 1}$

$\left\{\lambda w \lambda t \lambda x\left[{ }^{\prime}=\left[{ }^{\prime}\right.\right.\right.$ Pregnancy-Period $\left.{ }_{w t} x\right]$ '65 6$\}$

Confidence $=0.75 ; \boldsymbol{R S}=\{\mathbf{s 5}, \mathbf{s 6}, \mathbf{s} 8\}$

$\left\{\lambda w \lambda t \lambda x\left[^{\prime} \geq\left[\left[^{\prime} \text { Average 'Body-Length }\right]_{w t} x\right]^{\prime} 47\right]\right\} \Rightarrow_{e 1}$

$\left\{\lambda w \lambda t \lambda x\left[{ }^{\prime} \leq\left[{ }^{\prime} \text { Litter-Size }{ }_{w t} x\right]^{\prime} 4\right]\right\}$

Based on the first explication $e_{1}$, the algorithm proposes textual resources as being relevant for the concept of wild cat. According to the first three rules, the algorithm proposes sources No. 4 and 7 because these documents contain information on mammals, those 
that have fur, on territory marking and pregnancy period. The last rule is a recommendation for the documents No. 5, 6 and 8; these sources contain information on average body length and litter size.

If the algorithm computed also weakly recommended documents (WRS) then it would not take into account the properties of being a mammal, having a fur and average body length, and thus it would recommend much more documents containing information on, for instance, territory marking.

\section{Conclusion}

In this paper we described the proposal of exploration of the data mining method of 'association rules' for the search of relevant textual documents. The goal is the selection of information sources that contain information relevant for dealing with or explaining or answering the initiative query on a simple concept $C$. The paper broadens our previous results on explication of simple concepts by means of molecular concepts extracted from textual documents by supervised machine learning methods. By applying these methods to textual resources, we obtain several explications of the simple input concept, which are further evaluated and processed. We introduced an algorithm that computes associations of the concepts occurring in these explications with other concepts from other resources. In this way the algorithm discovers hidden associations that might be relevant with respect to the query on the simple input concept; as a result, it recommends other textual resources that might be overlooked in the huge amount of input documents and thus ignored. Future research will concentrate on optimisation of this method, in particular on an effective generating of association rules from a large dataset obtained from a huge number of textual documents.

Concerning the entire project on natural language processing and question answering of which this system is a component, we will concentrate on improvement of the methods introduced here. In particular, molecular concepts that explicate a simple input concept and that are obtained from several textual resources should be checked for inconsistencies that contradict each other or yield paradoxes. Another promising idea seems to be checking the concepts of propositions for striking news that go against our common sense and intuitions. In this way, we can signalise fake news coming from unreliable Internet sources.

Acknowledgements. This research has been supported by the Grant Agency of the Czech Republic, project No. GA18-23891S, "Hyperintensional Reasoning over Natural Language Texts", and by The Ministry of Education,Youth and Sports of the Czech Republic from the National Programme of Sustainability (NPU II) project IT4Innovations Excellence in Science, LQ1602.

\section{References}

1. Agrawal, R., Imielinski, T., and Swami, A. N. (1993): Mining association rules between sets of items in large databases. In Proceedings of the 1993 ACM SIGMOD International Conference on Management of Data, pp. 207-216.

2. Carnap, R. (1947): Meaning and necessity. Chicago: Chicago University Press. 
3. Duží, M. (2017): Property modifiers and intensional essentialism. Computación y Sistemas, vol. 21, No. 4, pp. 601-613. DOI: 10.13053/CyS-21-4-2811.

4. Duží, M., Fait, M., Menšík, M. (2019): Adjustment of goal-driven resolution for natural language processing in TIL. In Recent Advances in Slavonic Natural Language Processing, RASLAN 2019, Horák A., Rychlý P., Rambousek, A. (eds.), pp. 71-82.

5. Duží, M., Fait, M.: Integrating special rules rooted in natural language semantics into the system of natural deduction. In the proceedings of ICAART 2020, the 12th International Conference on Agents and Artificial Intelligence, Ana Rocha, Luc Steels, Jaap van der Herik (eds.), vol.1, pp. 410-421, Malta, Valletta.

6. Duží, M., Jespersen, B., Materna, P. (2010): Procedural Semantics for Hyperintensional Logic. Foundations and Applications of Transparent Intensional Logic. Berlin: Springer.

7. "Globalisation: Threat or Opportunity?". International Monetary Fund, 12 April 2000. Retrieved 28 January 2020.

8. Hájek P., Havránek T., Chytil M.K. (1983): Metoda GUHA - automatická tvorba hypotéz. (In Czech. GUHA method; automatic creation of hypotheses). Academia Praha.

9. Medved', M., Šulganová, T., Horák, A. (2017): Multilinguality Adaptations of Natural Language Logical Analyzer. In Proceedings of the Eleventh Workshop on Recent Advances in Slavonic Natural Language Processing, RASLAN 2017. Brno: Tribun EU, pp. 51-58.

10. Menšík, M., Duží, M., Albert, A., Patschka, V., Pajr, M. (2019): Seeking relevant information sources. In Informatics '2019, IEEE $15^{\text {th }}$ International Scientific Conference on Informatics, Poprad, Slovakia, pp. 271-276.

11. Menšík, M., Duží, M., Albert, A., Patschka, V., Pajr, M. (2020): Machine learning using TIL. In Frontiers in Artificial Intelligence and Applications, vol. 321: Information Modelling and Knowledge Bases XXXI, A. Dahanayake, J. Huiskonen, Y. Kiyoki, B. Thalheim, H. Jaakkola, N. Yoshida (eds.), pp. 344-362, Amsterdam: IOS Press.

12. Menšík, M., Duží, M., Albert, A., Patschka, V., Pajr, M. (2020): Refining concepts by machine learning. Computación y Sistemas, Vol. 23, No. 3, pp. 943-958; doi: 10.13053/CyS-23-3-3242

13. Mitchell T. M. (1997): Machine Learning. New York: McGraw-Hill, 1997.

14. Moschovakis, Y. N. (1994): Sense and denotation as algorithm and value. In Lecture Notes in Logic, eds. J. Väänänen and J. Oikkonen, vol. 2, pp. 210-249. Berlin: Springer.

15. Poole D. L., Mackworth A. K. (2010): Artificial Intelligence: Foundations of Computational Agents. 2nd pub. Cambridge: Cambridge University Press.

16. Russell S. J., Norvig P.(2014): Artificial intelligence: a modern approach. 2nd ed. Harlow: Pearson Education, 2014. ISBN 978-1-29202-420-2.

17. Tichý, P. (1988): The Foundations of Frege's Logic. Berlin, New York: De Gruyter.

18. Winston P. H.(1992): Artificial intelligence. $3^{\text {rd }}$ ed., Mass.: Addison-Wesley Pub. Co., 1992. 


\title{
Contentual Characteristics of Concepts
}

\begin{abstract}
In traditional approach, i.e. in the Port Royal Logic, the rule of inverse relation between the extension and the conceptual content of a concept holds. However, Bolzano was able to show the rule invalid, and thus his conception of conceptual content of a concept differed from the traditional one. It is shown that Leibniz's conception of intensional content of concepts differs from the traditional one as well as Bolzano's conception. Thus, it is now possible to distinguish between the intensional and the conceptual content of concepts as well as the extension of concepts.
\end{abstract}

Keywords: Concepts, concept theory, extension, intension, intensional logic, relational concepts.

A famous logic text, the Port Royal Logic, composed by two leaders of the Port Royal movement Antoine Arnauld and Pierre Nicole in 1662, made a distinction between the comprehension [compréhension] and the extension [étendue or extension] of an idea. The comprehension of an idea consists of "the attributes which it includes in itself, and which cannot be taken away from it without destroying it," (Arnauld and Nicole, 1996, I, 6; II, 17). The extension of an idea consists of "the subjects with which that idea agrees," or which contain it. Both the comprehensions of ideas and the extensions of ideas are used in the Port Royal Logic in justifying the basic rules of traditional logic, (ibid., II, 17-20).

Leibniz, in turn, distinguished these two types in terms of ideas [secundum ideas or per ideas] on one hand, and in terms of instances [secundum individua or per exempla subjecta] or individuals belonging to the terms [per individuis terminorum] on the other hand, (Adams 1994, 59). Nowadays this distinction is usually made in terms of "the intension of a concept" and "the extension of a concept".

In the Port Royal Logic "the extension of an idea" constituted both the species and the individuals that fall under it, whereas in Leibniz the extensional treatment is almost always in terms of individuals that fall under the idea, (Kauppi 1960, 43). Now "the 
extension of a concept" is taken to be a class (or a set) of all those individuals which fall under it. However, nowadays there are at least two different ways to interpret "the comprehension of an idea", i.e. either as "the intension of a concept" or as "the conceptual content of a concept". However, these two things are to be distinguished as well, which will be shown in this paper below.

\section{Limits of the Traditional Conceptual Content of a Concept}

In traditional approach the conceptual content and the extension of a concept can be defined as follows:

I The conceptual content of a concept consists of all those attributes, i.e. concepts, which are contained in it.

II The extension of a concept consists of all those objects which fall under it.

From these two definitions the rule of inverse relation between the extension and the conceptual content of a concept follows:

\# The lesser the extension of a concept, the greater is its conceptual content, and vice versa.

However, Bernard Bolzano in his Wissenschaftslehre $(1837, \S 120)$ gives the following examples in order to show that the rule (\#) is not always the case:

1. 'A man, who understands every European language',

and

2. 'a man, who understands every living European language'.

The conceptual content of the concept (1) is lesser than the conceptual content of the concept (2), for the concept (2) has in addition the concept of 'living' as its conceptual content. Also, the extension of the concept (1) is also lesser than the extension of the 
concept (2), for there are fewer people who understand every European language (including e.g. Latin) than who understand every living European language. Thus, according to Bolzano, the concepts (1) and (2) contradicts the rule (\#).

A reason why Bolzano was able to show the rule (\#) invalid was that his concept of conceptual content of a concept differed from the traditional one. On the other hand, under the traditional definition of conceptual content of a concept, where (I) is constrained only on the conjunctive form of the conceptual content of concepts, the rule (\#) is still valid. ${ }^{1}$

\section{The Non-Traditional Conceptual Content of a Concept}

For Bolzano the conceptual content of a concept is the sum of all simple concepts, which are parts of the concept. Since the "conceptual content of a concept" is only the "sum" of its parts, and the conjunctive is not the only way of holding these parts together, Bolzano is not to be classified with traditional logicians. Especially, it is now possible to distinguish between the conceptual content of a concept and the concept itself, where different concepts may have the same conceptual content, cf. Bolzano's examples $(1837, \S 56)$ :

3. 'A learned son of a non-learned father' $v s$. 'a non-learned son of a learned father',

and

4. '5 $5^{3,} v s .3^{5}$,

where the conceptual content of concepts in (3) consists of concepts: 'son', 'learned',

\footnotetext{
${ }^{1}$ However, the conceptual content of a concept can be defined so that the traditional definition will be the special case of the general one including Bolzano's conception. It is worthy of note that Formal Concept Analysis (FCA) follows basically this traditional approach to concepts as well, see Ganter \& Wille 1998, and Ganter, Stumme \& Wille eds. 2005. Another theory of concepts, i.e. Transparent Intensional Logic, TIL, is actually a concept theory at all, since it presupposes already conceptualized ideas and represented in meaningful natural language. That natural language expressions is then analyzed and turned in "procrustean bed", i.e. TIL, see e.g. Tichý 1988, and Duží, Jespersen \& Materna 2010 .
} 
'father', 'non', and ' $x$ is a father of $y$ ', and the conceptual content of concepts in (4) consists of concepts: ' 5 ', ' 3 ', and ' $x$ is power of $y$ '. Accordingly, two different concepts may have the same conceptual content. However, the reason why these concepts are to be considered as different is left open by Bolzano. ${ }^{2}$

\section{The Intensional Content of a Concept}

In a letter to Arnauld 14 July 1786 Leibniz wrote, (Leibniz 1997, 62):

"[I]n every affirmative true proposition, necessary or contingent, universal or singular, the notion of the predicate is contained in some way in that of the subject, praedicatum inest subjecto [the predicate is included in the subject]. Or else I do not know what truth is."

This view may be called the conceptual containment theory of truth, (Adams 1994, 57), which is closely associated with Leibniz's preference for an "intensional" as opposed to an "extensional" interpretation of categorical propositions. Leibniz worked out a variety of both intensional and extensional treatments of the logic of predicates, i.e. concepts, but preferring the intensional approach, (Kauppi 1960, 220, 251, 252). ${ }^{3}$

Now, given the Bolzano's 'counter-examples' (1) and (2), the intensional content of the concept (1) is greater that the intensional content of the concept (2). That is, because the man, who understands every European language, understands also every living European language, whereas the man, who understands every living European language, does not necessarily understand every European language. Hence, provided that the conceptual content of concepts is now understood as the intensional content of concepts, the rule (\#) holds.

\footnotetext{
${ }^{2}$ A logical analysis of Bolzano's examples (1) - (4) reveal also that he is using not only monadic concepts and other logical connectives than just conjunctions, but also quantifiers and relational concepts, e.g. 'every language', ' $x$ understands $y$ ', and ' $x$ is power of $y$ '.

${ }^{3}$ Raili Kauppi (1920-1995), influenced by Leibniz's logic, developed an intensional concept theory in (1967). This intensional concept theory, denoted by KC, is presented in a first-order language $L$ that contains individual variables $a, b, c, \ldots$, which range over the concepts, and one non-logical 2-place intensional containment relation, denoted by " $\geq$ ". When $a \geq b$, we say that a concept $b$ is intensionally
} 
It is now possible to distinguish between the intensional and the conceptual content of concepts as well as the extension of concepts. These differences are illustrated by means of Bolzano's 'counter-examples' (1) and (2) as follows: Firstly, the intensional content of the concept (1) is greater than the intensional content of the concept (2), for the man, who understands every European language, understands also every living European language, whereas the man, who understands every living European language, does not necessarily understand every European language. Secondly, the conceptual content of the concept (1) is smaller than the conceptual content of the concept (2), for the concept (2) has in addition the concept of 'living' as its conceptual content. Thirdly, the extension of the concept (1) is smaller than the extension of the concept (2), for there are fewer people who understand every European language than who understand every living European language.

\section{Conclusion}

In traditional approach, i.e. in the Port Royal Logic, the rule of inverse relation between the extension and the conceptual content of a concept holds. However, by an example Bolzano was able to show that rule invalid, for his own conception of conceptual content of a concept differed from the traditional one. On the other hand, it was shown that Leibniz's conception of intensional content of concepts differs from the traditional one as well as Bolzano's conception. Hence, it is now possible to distinguish between the intensional and the conceptual content of concepts as well as the extension of concepts.

contained in a concept $a$, or that the intension of concept $a$ contains the intension of concept $b$. This intensional concept theory is further studied e.g. in Palomäki 1994, and 2014. 


\section{References}

Adams, R. M., 1994: Leibniz: Determinist, Theist, Idealist. New York, Oxford: Oxford University Press.

Arnauld, A. and Nicole, P., 1996: Logic or the Art of Thinking. Trans. J. V. Buroker. Cambridge: Cambridge University Press.

Bolzano, B., 1837: Wissenschaftslehre I. Sulzbach.

Duží, M, Jespersen, B, \& Materna, P, 2010: Procedural Semantics for Hyperintensional Logic. Foundations and Applications of TIL. Springer.

Ganter, B. \& Wille, R. 1998: Formal Concept Analysis: Mathematical Foundations, Transl. by C. Franzke. Berlins: Springer-Verlag.

Ganter, B., Stumme, G., \& Wille, R., eds. 2005: Formal Concept Analysis: Foundations and Applications. Lecture Notes in Artificial Intelligence, no. 3626, Berlin: Springer-Verlag.

Kauppi, R., 1960: Über die Leibnizsche Logic mit besonderer Berücksichtigung des Problems der Intension und der Extension. Acta Philosophica Fennica, Fasc. XII. Helsinki: Societas Philosophica Fennica.

Kauppi, R., 1967: Einführung in die Theorie der Begriffssysteme. Acta Universitatis Tamperensis. Ser. A. Vol. 15. Tampere: Tampereen yliopisto.

Leibniz, G. W., 1997: Philosophical Writings. Ed. G. H. R. Parkinson. Trans. M. Morris and G. H. R. Parkinson. London: The Everyman Library.

Palomäki, J., 1994: From Concepts to Concept Theory: Discoveries, Connections, and Results. Acta Universitatis Tamperensis, ser. A, vol. 416. Tampere: Tampereen yliopisto, 1994.

Palomäki, J. 2014: “An Axiomatic Approach to Relational Concepts". Information Modelling and Knowledge Bases, XXVI. Eds. B. Thalheim, H. Jaakkola, Y. Kiyoki, and N. Yoshida. Amsterdam, Berlin, Oxford, Tokyo, Washington, DC.: IOSPress, 355-360.

Tichý, P., 1988: The Foundations of Frege's Logic. De Gruyter, Berlin and New York. 


\title{
Smart Cities through the Lens of News: A Comparative Content Analysis of Japanese and Slovenian Newspapers
}

\author{
Yukiko SATO ${ }^{\mathrm{a}, 1}$ and Stefan BRÜCKNER ${ }^{\mathrm{a}, 2}$ and Maja PUŠNIK ${ }^{\mathrm{b}, 3}$ \\ ${ }^{a}$ Keio University, Graduate School of Media and Governance \\ ${ }^{\mathrm{b}}$ University of Maribor, Faculty of Electrical Engineering and Computer Science
}

\begin{abstract}
The realisation of smart cities has attracted much attention in recent years from private and governmental actors, as a means to make cities more efficient, climate friendly and socially inclusive through the use of modern technology. However, few studies examine how smart cities are framed and understood within the public sphere. The aim of this study is to compare how domestic smart city initiatives are reported in the news of their respective countries, and to clarify the differences and similarities in media content. In this paper, we present the initial findings of our planned long-term comparative news content analysis. As a first step, we analysed national newspaper articles published between 2011 and 2019 in Japan and Slovenia. Our corpus consists of 41 Japanese and 20 Slovenian articles, written in relation to domestic smart city initiatives. In total, we identified 14 themes, five of which were common in both countries, while the remaining nine appeared exclusively in the news of one country. Our conclusions indicate that the news in both countries differ in what application domains of Smart Cities are discussed (e.g. natural resources and energy, transportation and mobility). We establish a procedure for further cross-cultural analyses, necess ary to understand how smart cities are framed in the public sphere. Thereby, we contribute to further discussion on the nature and definition of smart cities and how they are communicated.
\end{abstract}

Keywords. Smart cities, news media, cross-cultural newspaper content analysis

\section{Introduction}

According to the United Nations Department of Economic and Social Affa irs [1], the urban population in the world has grown rapidly from 751 million in 1950 to 4.2 billion in 2018 . By $2050,68 \%$ of the global population is projected to live in urban a reas. In conjunction, the number of cities with more than 10 million inhabitants will increa se by ten, from currently 33 to 43 by 2030 . This development brings with it problems, such

\footnotetext{
${ }^{1}$ Yukiko Sato, Keio University, Graduate School of Media and Governance, Endo 5322 Fujisawa, Kanagawa, 252-0882 Japan; E-mail: yukisato@sfc.keio.ac.jp.

${ }^{2}$ Stefan Brückner, Keio University, Graduate School of Media and Governance, Endo 5322 Fujisawa, Kanagawa, 252-0882 Japan; E-mail: bruckner@sfc.keio.ac.jp.

${ }^{3}$ Maja Pušnik, University of Maribor, Faculty of Electrical Engineering and Computer Science, Koroška cesta46, 2000 Maribor, Slovenia; E-mail: maja.pusnik@um.si.
} 


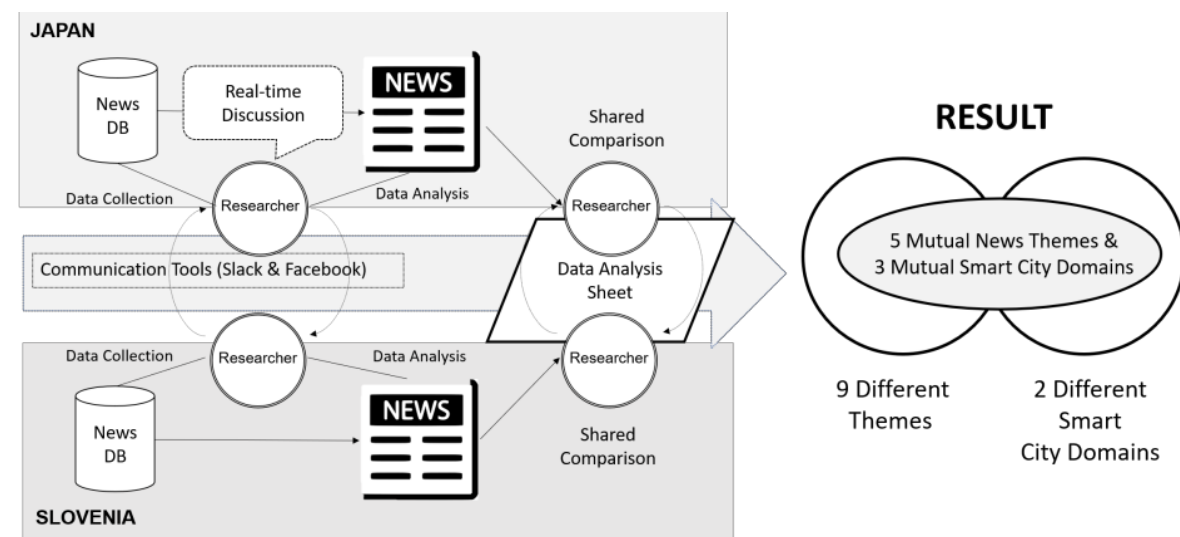

Figure 1. Overview of research design

as insufficient housing capacity and a lack of job opportunities, leading to an increase in poverty. It a lso poses challenges to inhabitants' health and the environment [2]. To mitigate such challenges, the use of modem Information and Communications Technology (ICT) to design and build economically and socially sustainable "smart cities" has received widespread attention $[3,4]$.

In general, smart cities are presented as a complex and digital ecosystem that includes a network of govemmental, academic and business institutions, and citizens; however, a universal definition of smart cities does not exist. Mora and Deakin [5] sumise, "Researchers seem to agree in picturing the smart city as an urban environment in which an ICT-driven approach to urban susta inability is activated". However, the concrete understanding of what constitutes a smart city differs among researchers, governmental institutions, the industry and among citizens [5], and indeed from city to city and country to country [6]. We have, therefore, no clear and concise way to define what exactly smart cities are or how they should be realised. Research on smart cities has, so far, been focused on economic and technological aspects, disregarding the social, cultural and environmental dimensions necessary for ICTrela ted urban innovation [7].

This study examines how smart cities are portrayed in the public sphere in different localities by analysing Japanese and Slovenian newspaper articles on domestic smart city initiatives (Figure 1). We thereby hope to provide insights into what kind of information on smart cities the general public is exposed to, and how public discussions are shaped in relation to this topic. Citizens themselves are core stakeholders of smart city development, but the public discourse, especially outside Western Europe and the US, has so far been neglected in prior research.

Based on a content analysis of 41 Japanese and 20 Slovenian daily national newspaper articles, we identified 14 thematic structures in articles related to smart cities. Five of those were found in both Japan and Slovenia, while 9 themes were found exclusively in only one of the countries. We a lso examined which constitutive elements of a smart city were mentioned concretely in each article. The results of this study are the first step towards a systematic examination of the public discourse on smart cities in different localities. Such an examination can provide valuable input into discussions on what does, and what should, constitute smart cities, and on how such concepts are communicated to the public. 


\section{Background}

Since the first publications on the topic in 1992, research into how to realise smart cities has gradually intensified and grown in scope [7]. Searching for research papers including the terms of "smart city" or "smart cities" in academic databases, such as Google Scholar, ACM, IEEE, it is obvious that research in this field has developed since the end of the millennium, currently reaching the highest number of research activities in the past two years. As Gupta et al. [8] closly classified the popular research areas on smart cities, they found that themes such as design and management of smart cities, innovation and technology and citizens' engagenemnt have been extensively studied, whilst the focus on the social impact on society is moderate. Especially in this field, the lack of a common definition for smart cities is noted by several researchers $[9,10]$. In their litera ture review, Mora et al. [7] came to the conclusion that researchers generally agree that a smart city is an urban environment utilising an ICT-driven approach to urban sustainability, but also point out thematic and definitional differences between the academic literature [11-13], pursuing a holistic view, and business reports, focusing on the technological dimension of smart cities $[14,15]$. Despite the lack of a common definition of smart cities, researchers have conducted cross-cultural a nalysis of smart city concepts on the regional and global levels.

On the regional level, Vanolo [16] compares how smart cities are outlined conceptually in policy papers produced by the Italian government and municipalities, to documents from the Commission of the European Union. Similarly, Haarstad [17] conducted a comparative text analysis, focusing on sustainability, in documents from the European Union and Norway. On a global level, Joss et al. [18] analysed documents retrieved on the 27 cities with the highest hit-count for "smart city" worldwide, using co-occurrence and concordance analysis. These approaches showcase a focus on official policy papers to examine the current discourses on smart cities, and are limited to material available in English; however, systematic reviews on how the public conceives smart cities are rarely seen.

Therefore, in this paper, we conduct a comparative content analysis aimed at clarifying how smart cities are framed in the public sphere, by investigating news articles in national newspapers. Newspapers are still a premier source of information for a broad range of people, and highly influential in shaping public discourse. By comparing news articles from two countries, we can draw conclusions on how and what different aspects are seen in smart city discourses across country borders. This paper is envisioned as a first step in a larger project towards a comparative analysis of the discourses on smart cities. The focus on Japan and Slovenia allows us to examine two countries that face similar structural problems, such as an a geing population [19], and have similar incentives towards the rea lisation of smart cities [20]. The outcomes of this project contribute to further constructive discussions on how smart cities should be communicated to the public.

\section{Method}

In this paper, we present the results of a content analysis of Japanese and Slovenian newspaper articles. Content analysis is a widely used methodological approach for studying (textual or audio-visual) communication artifacts [21]. First, we selected the research subject (i.e. newspaper articles as the primary unit of ana lysis), and conducted 
initial analyses by examining a small sub-set of the data. Based on this step, we established the code system for this study and proceeded with the ma in analysis. Table 1 depicts the variables we gathered for each article, based on Lynch and Peer's guide to newspaper content a nalysis [22].

Table 1. Variables for the newspaper content analysis

\begin{tabular}{|l|l|}
\hline Variable Name & Explanation \\
\hline Paper ID & 6-digit abbreviation of newspaper name, date, article number \\
\hline Year & Year of the produced article \\
\hline Month, Date & Month and date of the article \\
\hline Page Number & Page number of the article \\
\hline Section & $\begin{array}{l}\text { Section or theme in which the story appears } \\
\text { (e.g., politics, business, science) }\end{array}$ \\
\hline Source of Story & Author of the article (news service, journalist, reader, unknown) \\
\hline Treatment & $\begin{array}{l}\text { The way in which the news story is written } \\
\text { (general news, feature, opinion, others) }\end{array}$ \\
\hline Main Theme & The most prominent main theme of the article \\
\hline Sub-theme & The main application domain of smart cities focused on in the article \\
\hline No. of Words & The total number of words of the article \\
\hline
\end{tabular}

For the comparison of Japanese and Slovenian articles, we use a dual system of inductive and deductive codes. The inductive codes were developed through a close reading of the text material and discussion in the research team. They were used to signify the overall thematic frame of each article. In addition, we also coded each article based on which application domains of smart cities are discussed within [23]. To identify the application doma ins, we used the conceptual framework developed based on a litera ture review by Anthopoulos [24] (see Table 2).

Table 2. Overview of application domains of smart cities, based on [23]

\begin{tabular}{|l|l|}
\hline Application Domain & Example \\
\hline Natural Resources and Energy & $\begin{array}{l}\text { Natural resources, natural environment, energy, smart environment, water } \\
\text { management, waste management }\end{array}$ \\
\hline $\begin{array}{l}\text { Transportation } \\
\text { and Mobility }\end{array}$ & $\begin{array}{l}\text { Mobility, transport, activities, walkability, green transportations, smart } \\
\text { mobility }\end{array}$ \\
\hline Urban Infrastructure & $\begin{array}{l}\text { Buildings, urban planning, architecture, urban design, housing, facility } \\
\text { management }\end{array}$ \\
\hline Living & $\begin{array}{l}\text { Healthcare, safety, security, education, people, density, quality of life, } \\
\text { technology, smart living, entertainment, pollution control, culture, welfare, } \\
\text { hospitality, public spaces management }\end{array}$ \\
\hline Government & E-government, e-democracy procurement, transparency \\
\hline Economy & $\begin{array}{l}\text { Economy, institutions, urban openness, partnership, smart economy, } \\
\text { cultural heritage managem ent, innovation and entrepreneurship, human } \\
\text { capital management, digital education }\end{array}$ \\
\hline Coherency & Society, social, connectivity, social integration, social connectedness \\
\hline
\end{tabular}

Table 3 depicts the national newspapers from which articles were drawn for this study, the Japanese Asahi Shimbun and Yomiuri Shimbun and the Slovenian Delo and Večer. They were chosen to represent different political leanings. In the first step, we collected all articles published up to December 2019 from the respective databases that 
included the term "smart city". The first such article was originally published in 2011. In the second step, we identified relevant articles that focused thematically on domestic smart city initia tives through close rea ding.

Table 3. Sources for research

\begin{tabular}{|l|l|l|l|}
\hline Country & Name & Type/Orientation & $\begin{array}{l}\text { Distributed } \\
\text { (Base) Region }\end{array}$ \\
\hline \multirow{2}{*}{ Japan } & Asahi Shimbun & $\begin{array}{l}\text { National } \\
\text { daily/Liberal }\end{array}$ & All Japan \\
\cline { 2 - 4 } & Yomiuri Shimbun & $\begin{array}{l}\text { National } \\
\text { daily/Conservative }\end{array}$ & All Japan \\
\hline \multirow{2}{*}{ Slovenia } & Delo (Labour) & $\begin{array}{l}\text { National } \\
\text { daily/Liberal }\end{array}$ & All Slovenia \\
\cline { 2 - 4 } & Večer (Evening News) & $\begin{array}{l}\text { National } \\
\text { daily/Conservative }\end{array}$ & All Slovenia \\
\hline
\end{tabular}

\section{Results}

In total, our data set consisted of 61 articles, 41 from Japan (Asahi Shimbun: 20, Yomiuri Shimbun: 21) and 20 from Slovenia (Delo: 10, Većer: 10). In this section, we show the following results from our comparison of Japanese and Slovenian newspaper articles: 1) The number of articles published from 2011 to 2019,2) The distribution of articles in newspaper sections, 3) The type of articles, 4) The distribution of main themes, and 5) The mentioned application domains for smart cities.

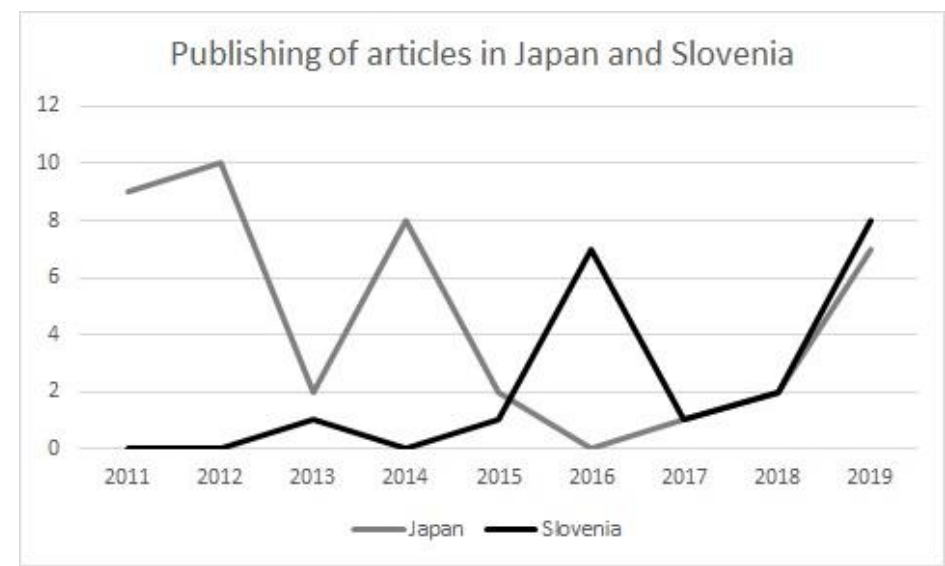

Figure 2. Number of articles from 2011 to 2019

Our findings show that Japanese articles began reporting on domestic smart city initiatives in 2011, while the first Slovenian article dates to 2013. Although the total number of articles per year fluctuated until 2016, a steady rise in the number of articles can be seen in both countries since 2017 (see Figure 2). Japanese articles originally peaked in 2012, while the first Slovenian article was found in 2013, with a peak of seven articles in 2016 and a further growth to 8 in 2019. 
Table 4. Distribution of newspaper articles per section

\begin{tabular}{|l|l|l|l|}
\hline Section & All & Japan & Slovenia \\
\hline Business & 28 & 22 & 6 \\
\hline Special & 14 & 9 & 5 \\
\hline General & 14 & 5 & 9 \\
\hline Politics & 2 & 2 & 0 \\
\hline Opinion & 2 & 2 & 0 \\
\hline Front Page & 1 & 1 & 0 \\
\hline
\end{tabular}

Table 4 shows the distribution of the articles in both countries per newspaper section. 28 (Japan 22, Slovenia 6) of the 61 articles were published in the business section. More than half of all Japanese articles were published here. This was followed by the special section, with nine Japanese and five Slovenian articles respectively, as well as the general section, in which nine of the 20 Slovenian articles were published. The remaining Japanese articles were published in the politics section (2), the opinions section (2) and the frontpage (1).

Table 5. Distribution of article types

\begin{tabular}{|l|l|l|l|}
\hline Type of Articles & All & Japan & Slovenia \\
\hline News Report & 40 & 28 & 12 \\
\hline Feature Article & 9 & 4 & 5 \\
\hline Opinion Piece & 6 & 3 & 3 \\
\hline Event Report & 6 & 6 & 0 \\
\hline
\end{tabular}

In both the Slovenian and Japanese newspapers, news reports, written by joumalists of the respective paper, were the most common form of article found. This was followed by feature articles, which were the second most common type in the Slovenian newspapers, focusing mostly on social problems. Three Japanese and Slovenian articles were opinion pieces on smart city initiatives, written by academics. In Japan, six articles reported on events sponsored by the newspapers involving the discussion of smart city concepts.

Table 6. Comparison of news themes

\begin{tabular}{|l|l|l|l|}
\hline Category & All & Japan & Slovenia \\
\hline Smart City - Business & 24 & 17 & 7 \\
\hline Smart City - Concept & 14 & 6 & 8 \\
\hline Smart City - Development & 4 & 4 & 0 \\
\hline Reconstruction after 3.11 & 4 & 4 & 0 \\
\hline Energy & 3 & 3 & 0 \\
\hline Personal information & 2 & 1 & 1 \\
\hline Artificial Intelligence & 2 & 1 & 1 \\
\hline Foreign Relations & 2 & 1 & 1 \\
\hline Overseas Expansion & 1 & 1 & 0 \\
\hline Society 5.0 Movement & 1 & 1 & 0 \\
\hline Government Project & 1 & 1 & 0 \\
\hline Depopulation & 1 & 1 & 0 \\
\hline Industry 4.0 Concept & 1 & 0 & 1 \\
\hline Smart City - Education & 1 & 0 & 1 \\
\hline
\end{tabular}


A total of 14 thematic categories were identified through the inductive coding analysis of the articles (see Table 6). Five of those were found in Japanese and Slovenian articles, while another seven themes in the Japanese and two in the Slovenian articles were exclusive to those countries. Most articles were focused either on smart city initiatives initiated by private corporations (Smart City - Business), or on the general concept, purpose and benefits of smart cities (Smart City - Concept). 38 of the 61 articles fell into one of those categories. However, despite their thematic similarity, the focus of those articles differed between the Japanese and Slovenian newspapers. Japanese articles coded as "Smart City - Business" were mostly announcements of major companies engaging in new smart city ventures, focused predominantly on infrastructure, while articles coded as "Smart City - Concept" tended to discuss the ideal form of smart cities, often in relation to elements of "smart living". In Slovenia, articles labelled as "Smart City - Business" included various concrete efforts of local and foreign companies to invest or support cities and municipa lities in Slovenia, while "Smart City - Concept" addressed more abstract strategies of smart cities, discussing whether or not citizens are ready for such solutions, and providing introspective insights into whether they were a ware of the benefits.

Although not appearing frequently, concerns regarding the handling of "Personal Information", or expectations towards the use of "AI" in smart cities and how smart city establishment affects "Foreign Relations", were shared in both countries. On the other hand, more reports about the country's own "Smart City Development" projects were evident in Japan, in both liberal and conservative newspapers, whereas the Slovenian media focused more on social issues regarding smart city establishment. In Japan, the progress of the Kashiwa-no-ha smart city initiative in Chiba prefecture, Japan, has been discussed frequently since 2014. Furthemore, the Japanese articles tended to portray the establishment of smart cities as a vision and symbol of hope for the cities affected by the March 2011 earthquake, tsunami and Fukushima nuclear catastrophe. Aside from this, the Japanese articles also reported on govemmental initiatives a imed at the realisation of a "Society 5.0" and the "Overseas Expansion" of domestic companies. The realisation of smart cities was also discussed as a possible counter to "Depopulation" in Japan, ba sed on its ageing population. In Slovenia, one further article was focused on "Smart City - Education", highlighting successful smart city solutions around the globe and how they can be used to adapt best practices in Slovenia.

Table 7. Comparison of application domains discussed in the articles

\begin{tabular}{|l|l|l|l|}
\hline Domain & All & Japan & Slovenia \\
\hline Natural Resources and Energy & 21 & 21 & 0 \\
\hline Urban Infrastructure & 11 & 11 & 0 \\
\hline Transportation and Mobility & 10 & 1 & 9 \\
\hline Economy & 10 & 1 & 9 \\
\hline Living & 9 & 7 & 2 \\
\hline Government & 0 & 0 & 0 \\
\hline Coherency & 0 & 0 & 0 \\
\hline
\end{tabular}

Table 7 shows what kind of application doma ins of smart cities the articles focused on. In total, five domains were evident in the examined articles. Out of those, three were present in the articles in both countries, while two were only observed in the Japanese articles. Seven Japanese and two Slovenian articles were sorted into the category "Living", reporting on how smart cities can enhance health, safety and 
security, through the use of ICT and data management. The doma ins "Economy" and "Transportation and Mobility" were also observed in both countries, but appeared more frequently in Slovenia, with nine articles each. "Economy" subsumed articles reporting on the economic benefits that investment into smart cities would have on municipa lities, while "Transportation and Mobility" included articles that mentioned the potential to shorten commuting time in smart cities. In the Japanese newspapers, the most frequently found doma in was "Natural resources and energy", aga in, in relation to the discourse on the 3.11 catastrophe and its implications on energy issues in Japan. This was followed by articles focusing on "Urban Infrastructure", discussing ways to utilise innovative ICT to improve infrastructure.

To summa rise our findings, despite their low overall number, articles on smart city initiatives have increased gradually since 2017 . The most common form of articles on smart city initiatives was news reports (Japanese newspapers: 68\%, Slovenian: 60\%). Articles were placed in similar sections in both countries, usually business, general news or special feature, although more than half of the Japanese articles were published in the business section (54\%), while Slovenian articles were found most frequently in the general section (45\%). Japan and Slovenia shared the thematic complexes of "Smart City - Business" and "Sma rt City - Concept", although the former was more prevalent in Japan (41\%) than in Slovenia $(35 \%)$, whereas the latter was more frequently seen in Slovenia (40\%), and less so in Japan (15\%). In the Japanese context, smart cities were often linked to the 3.11 disa ster, with $10 \%$ of the articles focusing on reconstruction efforts in the aftermath. The issues of "Artificial Intelligence (AI)", "Persona P' information and "Foreign Relations" were discussed in both countries. Comparing the smart city application doma ins, while Japan showed a focus on "Natural resources and energy" (51\% of the articles), "Industry" (27\%), and "Living" (17\%), "Economy" (45\%) and "Transportation and Mobility" (45\%) were most common in the Slovenian media.

\section{Discussion}

Our examination of Japanese and Slovenian newspaper articles on smart city initiatives revea led similarities in the way smart cities are framed in both countries. Articles were based mainly on information provided by governmental or corporate entities. Smart city initiatives were also not only discussed to improve liveability and susta inability in cities, but also as part of new business opportunities, with chances to partake in increasing revenues [25]. Govemmental initiatives on the Intemet of Things (IoT), AI, robots and data management a re becoming more visible, and a re reported on in relation to smart cities, rela ted closely to the Ja panese goal of realising "Society 5.0" [26].

The differences in the examined articles can be explained most easily by the differing stages of smart city realisation in both countries. While several projects, such as the "Ka shiwa-no-ha Smart City", are currently under development in Japan, spurred by the experience of the 3.11 catastrophe and perceived as an altemative way for the Japanese reconstruction efforts $[27,28]$. In Slovenia, the articles reflected a different stage of smart city realisation in the country, discussing mostly business opportunities and model cases for the establishment of best practices. While the application domains discussed in Japan are influenced greatly by the 3.11 cata strophe and resulting concerns on energy and susta inability, Slovenia is more focused on benefits to transportation and mobility, aside from concrete business expectations. This reflects the current priorities 
of the Slovenian govemment to solve traffic problems and increase people's wellbeing in a susta inable manner [29].

Our analysis suggests that the discourse on smart cities in both countries is currently dominated largely by govemmental and industrial actors, highlighting the benefits of such initiatives. This brings with it potential problems in relation to an imba lance of information, and an underrepresentation of academic or civil voices in the discourse, that aga in results in a lack of reports on the application domains "Government" and "Coherency" in the examined articles.

As this comparison of Japanese and Slovenian articles related to the theme of smart cities is a preliminary step in our research, a iming to form the base for our crossregional comparative newspaper analysis, the numbers of analysed resources are limited. In order to enhance the generalisability of this research, further articles, as well as literature, are planned to be examined. This paper contributes to discovering major diversities in discourses regarding smart cities, as well as providing a cross-cultural analysis foundation for future work.

\section{Conclusion}

In this paper, we examined 61 Japanese and Slovenian newspaper articles on smart city initiatives through a content analysis. We identified 14 thematic complexes, of which five were found in both countries: 1) Smart City - Business, 2) Smart City - Concept, 3) Personal Information, 4) AI and 5) Foreign Relations. Slovenian newspapers tended to focus more on educating the public about smart city initiatives, and discussing potential benefits towards transportation and mobility, while Japanese articles were focused on the business dimension, but a lso influenced heavily by the 3.11 cata strophe and resulting energy issues.

One motivation for this research was to establish a methodological approach to study newspaper content from a cross-cultural perspective. This paper shows an example of a comparative text analysis conducted across regional and cultural borders between researchers located in different time zones. Through this study, we contribute towards a clearer picture of the underlying structure of current discourses on smart cities, and how their definition and the way they are communicated changes across countries. As these are the first outcomes of our study, the results are still limited by the small number of articles examined. We plan to extend the number of articles for further analysis by including more media outlets into our corpus.

\section{References}

[1] United Nations Department of Economic and Social Affairs Population Division. World Urbanization Prospects. New York: United Nations. 2019.

[2] National Geographic. Urban Threats. 2019. [Accessed 2019 December 21]. https://www.nationalgeographic.com/environment/habitats/urban-threats/.

[3] Silva BN, Khan M, Han K. Towards Sustainable Smart Cities: A Review of Trends, Architectures, Components, and Open Challenges in Smart Cities. Sustainable Cities and Society. 38 (2018), 697-713.

[4] Arasteh H, Hosseinnezhad V, Loia V, Tommasetti A, Troisi O, Shafie-Khah M, Siano P. Iot-Based Smart Cities: A Survey. Paper presented at: 2016 IEEE 16th International Conference on Environment and Electrical Engineering (EEEIC). IEEE; 2016 June 7-10; Florence, Italy. 
[5] Mora L, Deakin M. Untangling Smart Cities: From Utopian Dreams to Innovation Systems for a Technology-Enabled Urban Sustainability. Amsterdam, Netherlands: Elsevier. 2019.

[6] Smart Cities Mission. What Is Smart City. 2018. [Accessed 2019 December 31]. http://smartcities.gov.in/upload/uploadfiles/files/What\%20is\%20Smart\%20City.pdf.

[7] Mora L, Bolici R, Deakin M. The First Two Decades of Smart-City Research: A Bibliometric Analysis. Journal of Urban Technology. 24 (2017), 3-27.

[8] Gupta P, Chauhan S, Jaiswal M. Classification of Smart City Research-a Descriptive Literature Review and Future Research Agenda. Information Systems Frontiers. 21 (2019), 661-685.

[9] Hollands RG. Will the Real Smart City Please Stand Up? Intelligent, Progressive or Entrepreneu rial? City. 12 (2008), 303-320.

[10] Chourabi H, Nam T, Walker S, Gil-Garcia JR, Mellouli S, Nahon K, Pardo TA, Scholl HJ. Understanding Smart Cities: An Integrative Framework. Paper presented at: 2012 45th Hawaii International Conference on System Sciences. IEEE; 2012 January 4-7 Maui, Hawaii.

[11] Caragliu A, Del Bo C, Nijkamp P. Smart Cities in Europe. Journal of Urban Technology. 18 (2011), 6582.

[12] Giffinger R, Fertner C, Kramar H, Meijers E. City-Ranking of European Medium-Sized Cities. Cent Reg Sci Vienna UT. (2007), 1-12.

[13] Schaffers H, Komninos N, Pallot M, Trousse B, Nilsson M, Oliveira A. 2011. Smart Cities and the Future Internet: Towards Cooperation Frameworks for Open Innovation. Paper presented at: The Future Internet Assembly. Springer.

[14] Dirks S, Keeling M. A Vision of Smarter Cities: How Cities Can Lead the Way into a Prosperous and Sustainable Future. New York: USA: IBM. 2009.

[15] Washburn D, Sindhu U, Balaouras S, Dines RA, Hayes N, Nelson LE. Helping Cios Understand "Smart City" Initiatives. Growth. 17 (2009), 1-17.

[16] Vanolo A. Smartmentality: The Smart City as Disciplinary Strategy. Urban Studies. 51 (2014), 883-898.

[17] Haarstad H. Constructing the Sustainable City: Examining the Role of Sustainability in the 'Smart City'discourse. Journal of Environmental Policy \& Planning. 19 (2017), 423-437.

[18] Joss S, Sengers F, Schraven D, Caprotti F, Dayot Y. The Smart City as Global Discourse: Storylines and Critical Junctures across 27 Cities. Journal of Urban Technology. 26 (2019), 3-34.

[19] Daimon S. Slovenia Faces Similar Fiscal Challenges to Japan, Finance Minister Andrej Bertoncelj Says. 2019. [Accessed 2019 December 24].

https://www.japantimes.co.jp/news/2019/06/20/business/slovenia-faces-similar-fiscalchallenges-japan-finance-minister-andrej-berton celj-says/\#.XgGnWi3APaY.

[20] Ministry of Foreign Affairs of Japan. Japan-Slovenia Foreign Ministers' Meeting. 2019. [Accessed 2019 December 25]. https://www.mofa.go.jp/erp/c_see/si/page6e_000206.html.

[21] Macnamara JR. Media Content Analysis: Its Uses, Benefits and Best Practice Methodology. Asia Pacific Public Relations Journal. 6 (2005).

[22] Lynch S, Peer L. 2002. Analyzing Newspaper Content: A How-to Guide. Readership Institute: Media Management Center at Northwestern University. [accessed 2019 December 25]. https://www.orau.gov/cdcynergy/erc/Content/activeinformation/resources/NewspaperContentA nalysis.pdf.

[23] Neirotti P, De Marco A, Cagliano AC, Mangano G, Scorrano F. Current Trends in Smart City Initiatives: Some Stylised Facts. Cities. 38 (2014), 25-36.

[24] Anthopoulos LG. Understanding the Smart City Domain: A Literature Review. Transforming City Governments for Successful Smart Cities. Springer. p. 9-21. 2015.

[25] Maddox T. Smart City Iot Revenue to Explode from \$25b to \$62b in 2026. 2018. [Accessed 2020 Janurary 4]. https://www.techrepublic.com/article/smart-city-iot-revenue-to-explode-from-25bto-62b-in-2026/.

[26] Cabinet Office Government of Japan. Society 5.0. 2019. [Accessed 2019 December 25]. https://www8.cao.go.jp/cstp/society5_0/index.html.

[27] Mitsui Fudosan. Kashiwa-No-Ha Smart City. 2014. [Accessed 2020 January 4]. https://www.kashiwanoha-smartcity.com/en/concept/whatssmartcity.html.

[28] Tsuchiya Y, Nakabayashi I, Otagiri R. Current Conditions and Issues of Reconstructed-Type Smart City in Disaster Area of the Great East Japan Earthquake. Paper presented at: Institute of Social Safety Science. 2016 November 4-5; Shizuoka, Japan.

[29] European Commission. Smat Specialisation Platform: Slovenia. 2018. [Accessed 2020 Janurary 10]. https://s3platform.jrc.ec.europa.eu/regions/SI. 


\title{
Visitor Journey Application Development For Omni-Channels
}

\author{
Bakhtiyor ESANOV ${ }^{\mathrm{a}, 1}$ and Ajantha DAHANAYAKE ${ }^{\mathrm{a}}$ \\ ${ }^{\mathrm{a}}$ Lappeenranta-Lahti University of Technology LUT
}

\begin{abstract}
The primary purpose of conducting this research is to determine how campus journey application development is progressing. As a result, this research proposes a conceptual model for visitor journey application development. The study included 100 top ranking educational institutes and additionally included Finnish and Estonian universities. 39 virtual campus tour applications and 38 visitor journey applications are benchmarked in total for this study. Provides an example of visitor journey mapping with features, complexities, and best practices that are influential for improving visitor experience during visitor journey application development.
\end{abstract}

Keywords. Omni-channel, visitor experience, visitor journey, touchpoints

\section{Introduction}

The number of smartphone users continue to increase daily. By 2021, 1 in 2 people worldwide is projected to use the smartphone for their daily use [1]. Companies, enterprises, and organizations in retail and marketing are shifting their strategies for reaching out to public from multi-channel to omni-channel strategy. Channel is a medium to reach out to visitors. For example in e-commerce, channels are divided into two types: physical and digital. Physical channels are mail, catalogs, showrooms, and brick and motor stores. Digital channels are websites, email, mobile applications, web applications, social media, e-marketplace, virtual, and mixed reality applications.

Multi-channel is a strategy where channels are heterogeneous, separated, and not integrated. The visitors cannot trigger channel interactions, and the enterprise cannot control channel interaction and integration [2]. Figure 1a presents the visitor's interaction in the multi-channel environment, and channels are not integrated. However, in omnichannel strategy channels are integrated, visitors can trigger channel interaction and have a seamless visitor experience. The enterprise has full control over channel integration and interaction [2].

The omni-channel's primary purpose is to present a single view to the visitor in a consistent manner to supply the visitor's needs. Omni-channels originated as a retail and marketing concept to improve customer visits and their experience. Therefore, in this respect, we consider customer visiting a channel as a visitor and it is slightly different from a user as defined computer science and human computer interaction domain. The challenge of software developers is to provide the visitors that seamless experience so

\footnotetext{
${ }^{1}$ Corresponding Author, Bakhtiyor Esanov, School of Engineering Science, Lappeenranta-Laht University of Technology LUT, Yliopistonkatu 34, 53850, Lappeenranta, Finland; E-mail: bakhtiyor.esanov@student.lut.fi.
} 
that they can interact whenever, wherever, and however, they want across all the channels, and with multiple touchpoints integrated. Figure $1 \mathrm{~b}$ presents channel integration in omni-channel strategy.

Understanding visitor's expectations is another aspect for providing a seamless, consistent experience across all channels and touchpoints.

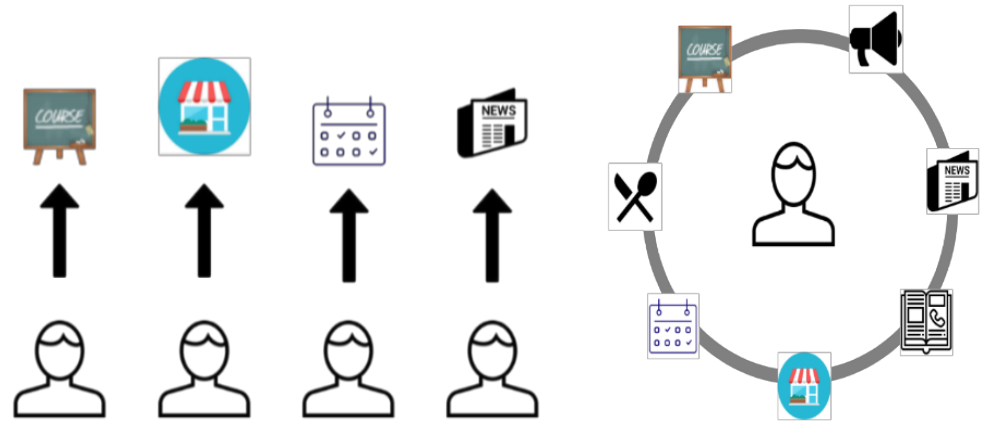

Figure 1.a) multi-channel, b) omni-channel

The recent developments in technology are virtual reality, augmented reality, mixed reality, and the internet of things. These developments bring challenges to visitor journey definitions in retail as well as university and other environments that want to improve the visitor experience of their brand. A visitor journey is similar to visiting a brick and motor store in the virtual space through web application or web site. Many universities have built their visitor journey applications. For example Harvard, Massachusetts Institute of Technology (MIT), Stanford University, and University Jyvaskyla have their visitor journey applications.

Visitor journey applications are very important for brand identity, service improvements, brand experience, giving the right service to the right person, and interaction between brand and customers.

In this research, we investigate visitor journey applications, types of visitor journey applications, overall investigation of how channels are integrated, and features of the visitor journey. This study has a limitation. Mainly, this paper focuses on university visitor journey applications. In the end, we present the functionality and requirements for building visitor journey applications.

There are various types of visitor interactions in visitor journey applications. These users can be students, staff, parents, and other visitors in an education environment. Omni-channel is not a strategy only for retail industry. However, it can be used in various industries to build integrated, seamless visitor experience.

This study aims to investigate various types of visitor journey applications for omnichannel visitor journey application development. We discuss the importance of customized and personalized visitor experience for such application development. In this research, we use benchmarking to identify best practices for the improvement of visitor experience while using mobile applications. We investigate the world's top 100 universities [3] mobile applications(apps), and web apps. Surprisingly most of the universities do not own their visitor journey applications.

The structure of the paper is as follows. The second section presents an overview of the research methods used in this research. The third section lists previous studies about 
campus journey applications. The fourth section of the paper reports the findings of the benchmarking of campus journey applications. The fifth and sixth chapters of the paper introduces the research results from campus journey applications. The sixth chapter summarizes the paper.

\section{Research Approach}

\subsection{Systematic Literature Review}

In order to accomplish research goals, the research must be driven in a theoretical and analytical approach. In the first step of this research work, we address recent papers on visitor journey applications by conducting a systematic literature review [4]. Within the systematic literature review, we have focused on investigating into applications of successful visitor journey applications and visitor's experience cases with the main interest in architectures used, seeking to understand how omni-channel environments can integrate visitor's touchpoints through a full range of functionality integration for optimum visitor engagement and satisfaction.

The literature review helped the identification and classification of omni-channel architectures as the first step, and this study firstly reviews the characteristics of omnichannel environments in visitor journey applications.

Search keywords used for the systematic literature review are omni-channel, customer/visitor journey, user experience, virtual campus, campus journey, touchpoint, journey planning.

The systematic literature review is selected as part of the research approach for this study[4]. Table 1 provides the selected articles for review. After the literature study, we conducted benchmarking of university visitor journey applications to arrive at gathering data for building the research objectives.

Table 1.List of reviewed studies

\begin{tabular}{|l|l|}
\hline Paper Title & Focused Keyword \\
\hline $\begin{array}{l}\text { Omni-Channel Product Distribution Network Design by Using the } \\
\text { Improved Particle Swarm Optimization Algorithm[5] }\end{array}$ & $\begin{array}{l}\text { Omni-channel, user } \\
\text { experience }\end{array}$ \\
\hline Enhanced Visitor Experience Through Campus Virtual Tour[6] & $\begin{array}{l}\text { Virtual campus, } \\
\text { campus journey }\end{array}$ \\
\hline $\begin{array}{l}\text { System Architecture for Virtual Team Campus on Cloud to Support } \\
\text { Internal Quality Assurance of Rajamangala University of } \\
\text { Technology[7] }\end{array}$ & Virtual Campus \\
\hline $\begin{array}{l}\text { User Experience in Mobile Augmented Reality: Emotions, } \\
\text { Challenges, Opportunities and Best Practices[8] }\end{array}$ & User experience \\
\hline $\begin{array}{l}\text { Mapping Learner-Data Journeys: Evolution of a Visual Co-Design } \\
\text { Tool[9] }\end{array}$ & User journey \\
\hline $\begin{array}{l}\text { Learning Multi-touch Conversion Attribution with Dual-attention } \\
\text { Mechanisms for Online Advertising[10] }\end{array}$ & Touchpoint \\
\hline $\begin{array}{l}\text { Key Factors for In-Store Smartphone Use in an Omnichannel } \\
\text { Experience: Millennials vs. Nonmillennials[11] }\end{array}$ & $\begin{array}{l}\text { Omni-channel, user } \\
\text { experience }\end{array}$ \\
\hline $\begin{array}{l}\text { Virtual reality interactive media for universitas sumatera utara - a } \\
\text { campus introduction and simulation[12] }\end{array}$ & $\begin{array}{l}\text { Virtual campus, } \\
\text { campus journey }\end{array}$ \\
\hline
\end{tabular}




\begin{tabular}{|l|l|}
\hline Journey Mapping the User Experience[13] & $\begin{array}{l}\text { User journey, user } \\
\text { experience }\end{array}$ \\
\hline T2*-Personalized Trip Planner[14] & touchpoint \\
\hline The Case for Graph-based Recommendations[15] & User experience \\
\hline $\begin{array}{l}\text { Understanding Mobile Phone Activities via Retrospective Review of } \\
\text { Visualizations of Usage Data[16] }\end{array}$ & User experience \\
\hline Challenges on the Journey to Co-Watching YouTube[17] & $\begin{array}{l}\text { User journey, } \\
\text { journey planning }\end{array}$ \\
\hline $\begin{array}{l}\text { Development Plan for Research on Omni-Channel Shopping to } \\
\text { Purchase Intention[18] }\end{array}$ & Omni-channel \\
\hline $\begin{array}{l}\text { Context-Aware User Modeling Strategies for Journey Plan } \\
\text { Recommendation[19] }\end{array}$ & User journey \\
\hline $\begin{array}{l}\text { VICTour 1.1: Introducing virtual learning environments and } \\
\text { gamification[20] }\end{array}$ & $\begin{array}{l}\text { User journey, virtual } \\
\text { campus }\end{array}$ \\
\hline $\begin{array}{l}\text { An Application of Game Technology to Virtual University Campus } \\
\text { Tour and Interior Navigation[21] }\end{array}$ & Virtual campus \\
\hline Just Browsing? Understanding User Journeys in Online TV[22] & User journey \\
\hline $\begin{array}{l}\text { Mobility Crowdsourcing: Toward Zero-Effort Carpooling on } \\
\text { Individual Smartphone[23] }\end{array}$ & User journey \\
\hline $\begin{array}{l}\text { Customer Experience Modeling: Designing Interactions for Service } \\
\text { Systems[24] }\end{array}$ & User experience \\
\hline $\begin{array}{l}\text { Redefining Touchpoints: An Integrated Approach for Implementing } \\
\text { Omnichannel Service Concepts[25] }\end{array}$ & $\begin{array}{l}\text { Touchpoints, user } \\
\text { experience }\end{array}$ \\
\hline The mobile university: from the library to the campus[26] & Virtual campus \\
\hline Which platform do our users prefer: website or mobile app?[27] & User journey \\
\hline A framework for evaluating university mobile websites.[28] & User experience \\
\hline $\begin{array}{l}\text { Customer experience evaluation in the omnichannel environment[29] } \\
\text { User experience, } \\
\text { omni-channel }\end{array}$ \\
\hline
\end{tabular}

\section{State of visitor journey applications}

\subsection{Omni-Channel}

Omni-channel is the deep integration of all available physical and digital channels into one touchpoint [5] to create a seamless visitor experience across channels. The enterprises such as in retail industry offer visitors a wide range of possibilities to interact with their products. At present such means are: the physical store, catalogs, telephone, web app, and mobile app. Additionally, the visitor can trigger full interaction, and the enterprise controls full integration of all channels [2]. Föhr Janne et al. [29] groups the channels into online, offline, and complex channels. Complex channels are promotions, showroom, and advertisements.

A university visitor journey applications can be identified as a journey through services such as student services, university news services, sports facilities, email service, library services, courses and learning management systems, calendar services, booking room service, restaurants, and cafes and many more. 
Ana Mosquera et al. [11] identifies the key factors influencing visitor's intention to use smartphone devices while visiting the in-store and having an omni-channel experience. Those key factors are performance expectancy, effort expectancy, social influence, facilitating conditions, hedonic motivation, habit, price, and value. Behavior intention is the superset of these factors. Behavior intention, facilitating conditions, and habits directly and positively affects visitor's behavior. The physical store is preferred as the main channel for selling consumer goods or services to customers in retail. With the revolution of mobile technology, customers are ahead of retailers. The customer researches the online to find about the product before visiting the physical store. In the physical store, the customer expects a brand experience.

Some enterprises have already implemented this omni-channel approach successfully. Examples are:

Disney: Once a trip is booked, My Disney Experience tool leads to planning the entire trip, taking care of all details to locate the attractions the visitor wants to see, and the estimated wait time for each of them [30].

Virgin Atlantic: A customer service experience is shared, is a story of personalized user experience with a representative of Virgin Atlantic who reaches across the company's different marketing channels to give the customer personalized service experience [31].

Oasis: is a fashion retailer with a fancy e-commerce site, mobile app, and several brick-and-mortar locations. They fuse those channels to give a visitor a simple and great shopping experience [32].

\subsection{Touchpoint}

Kronqvist et al.[25] distinguishes touchpoints into physical touchpoints, digital touchpoints, and social touchpoints. Physical touchpoints are cards, signs, brochures, service desk, robots, etc. Digital touchpoints are web, mobile, tablets, Augmented/Virtual Reality, chat, artificial intelligence, etc. Social touchpoints are customer service, sales, reception, maintenance, etc. During the visitor journey, the visitor can use different devices, interact with a variety of touchpoints, and can choose a large number of distributed channels, websites, and apps [14].

Stein et al. [33] collected qualitative data obtained from interviews and analyzed thematically to identify elements of customer experience touchpoints. This analysis reveals seven elements of touchpoints: atmospheric, technological, communicative, process, employee-customer interaction, customer-customer interaction, product interaction. Atmospheric elements such as sensory factors, visual effects (paintings, colors), and story layouts are essential in visitor experience. Technological elements have a direct interaction with visitors and these technologies (kiosks, social media, mobile applications, websites etc.,) influence the brand experience. Communicative elements are one-way communication from the retailer to the customer and can be advertisements, informative messages, and promotions. Process elements are actions or steps of customer needs such as navigation, waiting time, and service process.

Orchestration and alignment of these various touchpoints in time and quality form a harmonious experience, and successful alignment result in positive visitor experiences [25]. 


\subsection{Visitor Journey}

Customer or visitor experience is dependent of the visitor journey. We consider the visitor journey as a synonym to the user journey or customer journey. The visitor journey is a virtual tour that a visitor navigates while visiting the virtual-space of the omnichannel application. During a visitor journey, the visitor navigates through touchpoints. These touchpoints are services offered to the visitor by the enterprise. Along the visitor journey, visitors must engage in impression management to consider how their choices might make them appear to others. Emily Sun et al.[17] explores the short-form video co-watching journey. The journey starts with searching for pre-watched content or taking the time to find new content, to negotiating or turn-taking to make a decision, to switching videos less when with another person. The video-sharing platform YouTube merge different user's recommended videos based on common interests and recommend to new users.

Journeys of watching TV Patterns Based on [22], the researchers inspect the journeys in each segment to investigate whether to generalize the patterns of user behavior in groups within each time segment and how patterns change over time.

Visitor journeys have starting and ending points. Visitor journey mapping highlights movement through space, track the time required, visitor's interaction with the brand, and pinch points that users might encounter while performing a task [34]. This mapping is integral to understand customer/visitor experience [35].

\subsection{Virtual Campus}

R F Rahmat et. al. [12] implemented the Unity 3D game engine for creating a virtual campus tour application for Universitas Sumatera Utara. In their research, they present steps of creating a virtual environment and end-user tests of the application. They state all end users agreed that the $3 \mathrm{D}$ virtual campus is helpful for new students.

Rizawati et al. [6] present the problem of representing campus, facilities, services to potential students with images and videos. However, potential students cannot participate at the intended on-campus information day time and venue. The virtual campus tour allows the potential students to visualize and explore campus environments virtually and have a significant impact on choosing campus for their studies.

\section{Benchmarking University visitor journey applications}

Camp [36] defines Benchmarking as a process of measuring, finding, comparing products, services with the companies, with the leaders in the same sector of the industry. One of the essential steps of the benchmarking process is searching for the best in set of companies or organizations [37].

After conducting a pilot search, testing, and examining different mobile applications in university mobile application segment, the criteria for comparing the visitor journey applications is identified. Figure 2 presents the steps of the benchmarking process. Different universities are compared according to the selected criteria. The list of 36 benchmarked university visitor journey applications are presented in Table 2. The benchmarking process is conducted between April 2019-July, 2019. Data collection for benchmarking is performed with iPhone 6, iPad 2, Samsung Galaxy J3 and Lenovo Yoga 510 devices. Findings are enumerated below. 


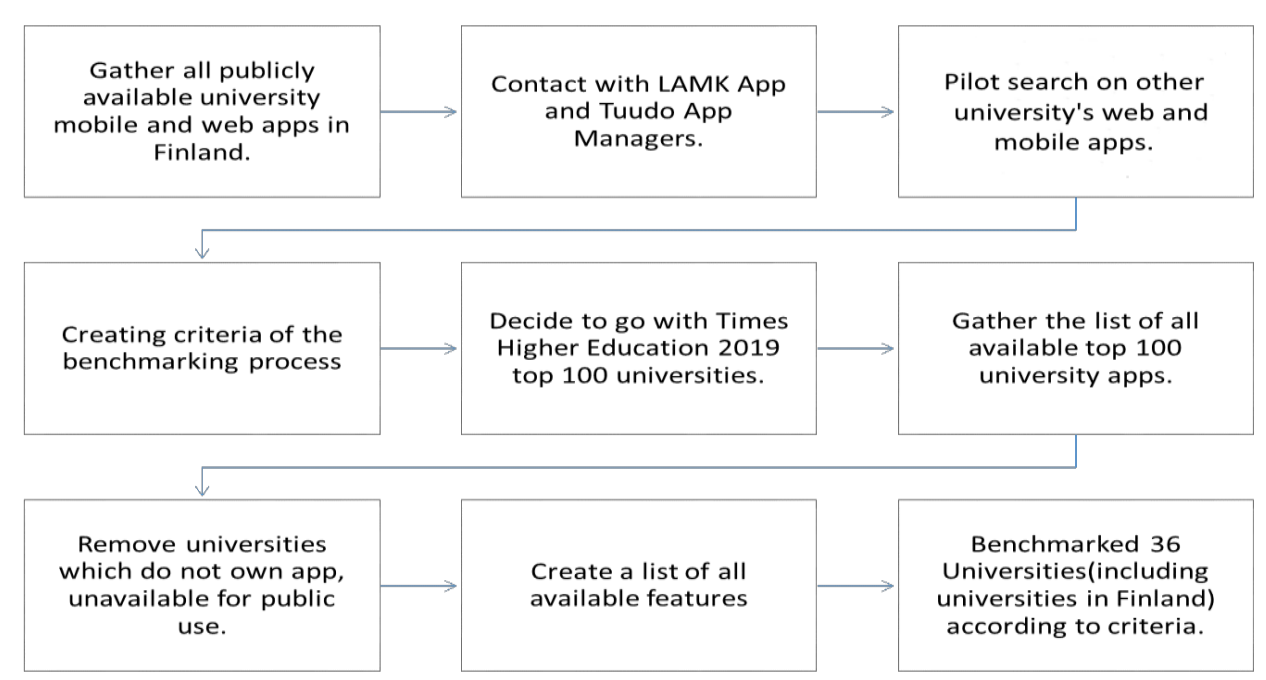

Figure 2. Process of Benchmarking

Table 2.List of Benchmarked Universities

\begin{tabular}{|c|c|c|c|c|c|c|}
\hline University Name & App Name & $\begin{array}{l}\text { Androi } \\
\text { d } \\
\text { Version } \\
\end{array}$ & $\begin{array}{l}\text { IOS } \\
\text { Versi } \\
\text { on }\end{array}$ & $\begin{array}{l}\text { Web App } \\
\text { version }\end{array}$ & \begin{tabular}{l|} 
Year of \\
First \\
Release \\
\end{tabular} & Country \\
\hline Stanford University & $\begin{array}{l}\text { Stanford } \\
\text { Mobile }\end{array}$ & Yes & Yes & Yes & 2016 & $\begin{array}{l}\text { United States of } \\
\text { America }\end{array}$ \\
\hline $\begin{array}{l}\text { Massachusetts } \\
\text { Institute } \\
\text { Technology }\end{array}$ & $\begin{array}{l}\text { MIT } \\
\text { Mobile }\end{array}$ & Yes & Yes & Yes & 2011 & $\begin{array}{l}\text { United States of } \\
\text { America }\end{array}$ \\
\hline $\begin{array}{l}\text { California Institute of } \\
\text { Technology }\end{array}$ & $\begin{array}{l}\text { CSR(Caltec } \\
\text { h Student } \\
\text { Resources) }\end{array}$ & Yes & Yes & No & 2018 & $\begin{array}{l}\text { United States of } \\
\text { America }\end{array}$ \\
\hline Harvard University & Harvard & Yes & Yes & Yes & 2012 & $\begin{array}{l}\text { United States of } \\
\text { America }\end{array}$ \\
\hline Princeton University & $\begin{array}{l}\text { Princeton } \\
\text { Mobile }\end{array}$ & Yes & Yes & Yes & 2013 & $\begin{array}{l}\text { United States of } \\
\text { America }\end{array}$ \\
\hline Yale University & Yale & Yes & Yes & No & 2018 & $\begin{array}{l}\text { United States of } \\
\text { America }\end{array}$ \\
\hline $\begin{array}{ll}\text { Imperial } & \text { College } \\
\text { London } & \end{array}$ & Imperial & Yes & Yes & Yes & 2011 & Great Britain \\
\hline ETH Zurich & ETH Zurich & Yes & Yes & No & 2012 & Switzerland \\
\hline $\begin{array}{l}\text { University of } \\
\text { California, Berkeley }\end{array}$ & Berkeley & Yes & Yes & No & 2018 & $\begin{array}{l}\text { United States of } \\
\text { America }\end{array}$ \\
\hline Duke University & $\begin{array}{l}\text { Dukemobil } \\
\text { e }\end{array}$ & Yes & Yes & Yes & 2011 & $\begin{array}{l}\text { United States of } \\
\text { America }\end{array}$ \\
\hline Cornell University & $\begin{array}{l}\text { Cornell } \\
\text { Connects }\end{array}$ & Yes & Yes & No & 2018 & $\begin{array}{l}\text { United States of } \\
\text { America }\end{array}$ \\
\hline University of Toronto & $\begin{array}{l}\text { U of T } \\
\text { Mobile }\end{array}$ & Yes & Yes & No & 2013 & Canada \\
\hline $\begin{array}{l}\text { Lahti University of } \\
\text { Applied Science }\end{array}$ & $\begin{array}{l}\text { LAMK } \\
\text { APP }\end{array}$ & Yes & Yes & No & 2017 & Finland \\
\hline $\begin{array}{l}\text { École Polytechnique } \\
\text { Fédérale de Lausanne }\end{array}$ & $\begin{array}{l}\text { EPFL } \\
\text { Campus }\end{array}$ & Yes & Yes & No & 2011 & Switzerland \\
\hline
\end{tabular}




\begin{tabular}{|c|c|c|c|c|c|c|}
\hline Jyvaskyla University & MyJYU & Yes & Yes & Yes & 2019 & Finland \\
\hline Tuudo App & Tuudo App & Yes & Yes & No & 2016 & Finland \\
\hline $\begin{array}{l}\text { University College } \\
\text { London(UCL) }\end{array}$ & UCL Go! & Yes & Yes & Yes & 2011 & Great Britain \\
\hline $\begin{array}{l}\text { Technical University } \\
\text { of Munich(TUM } \\
\text { Campus) }\end{array}$ & $\begin{array}{l}\text { TUM } \\
\text { Campus }\end{array}$ & Yes & Yes & No & 2011 & Germany \\
\hline $\begin{array}{l}\text { The University of } \\
\text { North Carolina at } \\
\text { Chapel Hill }\end{array}$ & $\begin{array}{l}\text { CarolinaG } \\
\mathrm{O}\end{array}$ & Yes & Yes & No & 2014 & $\begin{array}{l}\text { United States of } \\
\text { America }\end{array}$ \\
\hline $\begin{array}{lr}\text { University } & \text { of } \\
\text { California, } & \text { Santa } \\
\text { Barbara } & \\
\end{array}$ & $\begin{array}{l}\text { UCSB-UC } \\
\text { Santa } \\
\text { Barbara }\end{array}$ & Yes & Yes & No & 2016 & $\begin{array}{l}\text { United States of } \\
\text { America }\end{array}$ \\
\hline McGill University & McGill & Yes & Yes & No & 2014 & Canada \\
\hline $\begin{array}{l}\text { University of } \\
\text { Pennsylvania(Penn } \\
\text { Mobile) }\end{array}$ & $\begin{array}{l}\text { Penn } \\
\text { Mobile }\end{array}$ & Yes & Yes & No & 2015 & $\begin{array}{l}\text { United States of } \\
\text { America }\end{array}$ \\
\hline $\begin{array}{l}\text { The University of } \\
\text { Texas at Austin } \\
\text { (MyUT Texas) }\end{array}$ & $\begin{array}{l}\text { MyUT } \\
\text { Texas }\end{array}$ & Yes & Yes & No & 2018 & $\begin{array}{l}\text { United States of } \\
\text { America }\end{array}$ \\
\hline $\begin{array}{ll}\text { University } & \text { of } \\
\text { Wisconsin } & \\
\end{array}$ & Wisconsin & Yes & Yes & No & 2011 & $\begin{array}{l}\text { United States of } \\
\text { America }\end{array}$ \\
\hline $\begin{array}{l}\text { The University of } \\
\text { Sydney University }\end{array}$ & Sydney Uni & Yes & Yes & No & 2012 & Australia \\
\hline $\begin{array}{l}\text { Wageningen } \\
\text { University } \\
\text { Research }\end{array}$ & $\begin{array}{l}\text { WUR } \\
\text { W'App }\end{array}$ & Yes & Yes & No & $\begin{array}{l}\text { not } \\
\text { found }\end{array}$ & Netherlands \\
\hline $\begin{array}{l}\text { University } \\
\text { Amsterdam }\end{array}$ & My UvA & Yes & Yes & No & 2015 & Netherlands \\
\hline $\begin{array}{l}\text { The University of } \\
\text { Hong Kong }\end{array}$ & $\mathrm{HKU}$ & Yes & Yes & No & 2012 & China \\
\hline New York University & $\begin{array}{l}\text { NYU } \\
\text { mobile }\end{array}$ & Yes & Yes & No & 2012 & $\begin{array}{l}\text { United States of } \\
\text { America }\end{array}$ \\
\hline $\begin{array}{ll}\text { Université } & \text { PSL(Paris } \\
\text { Sciences \& Lettres - } \\
\text { PSL } & \text { Research } \\
\text { University) } & \\
\end{array}$ & My.PSL & Yes & Yes & No & 2017 & France \\
\hline $\begin{array}{l}\text { The University of } \\
\text { Melbourne }\end{array}$ & my.unimelb & Yes & Yes & No & 2014 & Austalia \\
\hline $\begin{array}{l}\text { University of British } \\
\text { Columbia }\end{array}$ & UBC & Yes & Yes & Yes & 2014 & Canada \\
\hline $\begin{array}{l}\text { The Chinese } \\
\text { University of Hong } \\
\text { Kong }\end{array}$ & $\begin{array}{l}\text { CUHK } \\
\text { Mobile }\end{array}$ & Yes & Yes & No & 2012 & Hong Kong \\
\hline $\begin{array}{ll}\text { King's } & \text { College } \\
\text { London } & \\
\end{array}$ & $\begin{array}{l}\text { King's } \\
\text { Mobile }\end{array}$ & Yes & Yes & Yes & 2012 & Great Britain \\
\hline $\begin{array}{l}\text { Nanyang } \\
\text { Technological } \\
\text { University } \\
\end{array}$ & $\begin{array}{l}\text { NTU } \\
\text { Mobile }\end{array}$ & Yes & Yes & No & 2011 & Singapore \\
\hline
\end{tabular}

\subsection{Mobile Apps}

Android is one of the most used mobile operating systems and holds more than $75 \%$ of the mobile devices market share worldwide [38]. Google Play is the largest app store for downloading the applications and installing on supported mobile devices. Google 
maintains the Google Play Store. Android has a large community of application developers and supported by Google. From the benchmarking data, we can clearly state that almost every mobile application has an Android version.

IOS is the second-largest [38] mobile operating system runs on Apple mobile devices such as iPhone, iPad tablets, iPod Touch. IOS applications can be downloaded from the App Store and installed on supported devices. Apple maintains the App Store and supports its developer community. IOS version is the same version of the Android version of the mobile application. During the benchmarking it is discovered, there is a minor platform-specific design difference between Android and IOS in the user interface of the application.

\subsection{Web Applications}

The web application is a compatible web-browser based version of the same mobile application. The web application does not require to install on the user desktop. Web Application runs on all available web browsers such as Safari, Google Chrome, Mozilla Firefox, Microsoft Edge, Yandex. Android and IOS mobile applications run on a specific operating system. However, the web application does not depend on any operating system. Mobile applications can be downloaded from the Google Play Store and App Store and installed on mobile devices.

On the other hand, experiencing a web application is simple, can access by URL address of the web application, and the application contents load to the user's browser. The user of the web application can experience the latest version of the mobile application without any update notice of mobile application. Table 3 shows the list of universities, which have a web application version of their same mobile applications. With the web application, organizations can reach all available audiences, and audiences are free to experiences the brand without devices and operating system dependency.

The visitor starts his/her journey with the mobile application and can continue his/her journey with the web application. With the combining of web and mobile applications, the visitor's experience is seamless, consistent, and integrated.

Table 3. List of Web applications

\begin{tabular}{|l|l|}
\hline Universities & Web Application URL \\
\hline Stanford University & $\underline{\text { htps://m.stanford.edu }}$ \\
\hline Massachusetts Institute of Technology & $\underline{\text { htps://mobi.mit.edu/ }}$ \\
\hline Harvard University & $\underline{\text { htps://m.harvard.edu/ }}$ \\
\hline Princeton University & $\underline{\text { https://m.princeton.edu }}$ \\
\hline Imperial College London & $\underline{\text { htps://mobile.imperial.ac.uk/ }}$ \\
\hline Duke University & $\underline{\text { htps://mobile.duke.edu/ }}$ \\
\hline Jyvaskyla University & $\underline{\text { https://myjyu.jyu.fi/ }}$ \\
\hline University College London(UCL) & $\underline{\text { htps://ucl.ombiel.co.uk/ }}$ \\
\hline University of British Columbia & $\underline{\text { htps://m.ubc.ca }}$ \\
\hline King's College London & $\underline{\text { https://kingsmobile.kcl.ac.uk }}$ \\
\hline
\end{tabular}


From the benchmarking data in Table 2, we discover that visitor journey applications appeared from the beginning of 2011. Year of the first release information is based on Android Version. The First-time release data is available only for the users of the Android version. App Store does not show details about the first release of the IOS version of mobile applications.

\subsection{Features}

Universities may have several campuses in different countries or cities. Furthermore, campuses located in different corners of cities. Every campus has its facilities and services. Campus selection automatically updates the touchpoints data source with selected campus's services and channels. The visitor journey application automatically updates its data with campus services. New York University (NYU Mobile) and Technical University of Munich (TUM Campus) have campus selection feature.

Visitor mode is an essential feature for visitor journey applications. In visitor mode, visitors can use the application without a login to the application. The visitor mode has limited features. In visitor mode, visitors can not $\log$ in to the system. During the benchmarking process, we removed applications from the list which do not have the visitor mode.

Table 4 shows the list of most user touchpoints by universities, which also include the visitor mode. Some touchpoints require authorization for accessing the system, and these touchpoints are designed for the specific user types. These specific users are students and staff.

The persona can be a student, staff, and authorized campus visitors. With a change persona feature, the experience of the visitor journey is personalized and customized. There are no restrictions for authorized users while using the application. Furthermore, touchpoints such as internal chat and messaging, calendar, personalized schedules, booking room, library, announcements, health services, study space availability, student identification card, jobs, finance, laundry info, parking are fully available for authorized users.

The customized navigation feature provides a more personalized visitor experience of the brand. Customized navigation helps to minimize and organize the touchpoints. The visitor can personalize the application according to his/her specifications and preferences. A visitor can create a list of favorite touchpoints and list appears at the top of the navigation menu and navigational home screen. The application gives the option to add new touchpoints to the list, changes, and adjusts the position of touchpoint to top, bottom, and removes from the list. This feature helps to reach touchpoints quicker.

Following Universities mobile applications have Customized Navigation Feature: Massachusetts Institute of Technology(MIT Mobile), University of California Berkeley(Berkeley), University of Toronto(U of Mobile), École Polytechnique Fédérale de Lausanne(EPFL Campus), University of Pennsylvania(Penn Mobile), University of Sydney University(Sydney Uni), Nanyang Technological University(NTU Mobile). 


\subsection{Customizing the Features}

For customizing the selected touchpoints, the University of Pennsylvania (Penn Mobile), EPFL, ETH Zurich, and Technical University of Munich(TUM Campus) have included these features.

Features may have an extensive range of services and channels. In the visitor journey application context, some features have many services. For example, news. University has a wide range of channels to provide news.

This feature helps to optimize the visitor experience. Moreover, provide a more specific part of the touchpoint. With customizing this feature, the user's experience will be more personalized. Table 4 below provides the most common features related to tuchpoints in visitor journey applications.

Table 4.List of most used Features related to Touchpoints

\begin{tabular}{|c|c|c|c|}
\hline Feature Name & Description & Visitor Mode & Staff/Student \\
\hline Directory & $\begin{array}{l}\text { Get the contact information for University students, faculty, and staff, as well } \\
\text { as for departments, offices, arts, athletic venues, libraries, and services. }\end{array}$ & Yes & Yes \\
\hline Map & $\begin{array}{l}\text { Navigate the campus by searching for buildings, departments, libraries, } \\
\text { athletic facilities, dormitories, and offices. Zoom in, zoom out or scroll in any } \\
\text { direction. Locations include details such as images, website links, and phone } \\
\text { numbers. }\end{array}$ & Yes & Yes \\
\hline Places & $\begin{array}{l}\text { Look up places around campus and the services they provide. Get detailed } \\
\text { information such as open hours, whether space is reserved, contact } \\
\text { information, and payment types accepted. Examples of places include } \\
\text { residential colleges, the digital print center, and recreation facilities. }\end{array}$ & Yes & Yes \\
\hline Dining/Restaurants & $\begin{array}{l}\text { Choose where and what user would like to eat. Look up both residential and } \\
\text { retail dining locations and check open hours, menus, and nutrition } \\
\text { information. Integrated with dining services }\end{array}$ & Yes & Yes \\
\hline Transit/Transportation & $\begin{array}{l}\text { Access transportation routes, schedules, and locations in real-time. Integrated } \\
\text { with the local transportation system. Read news and see transportation contact } \\
\text { information. Check/load the balance from the transportation system }\end{array}$ & Yes & Yes \\
\hline Events & $\begin{array}{l}\text { Browse the public and student event listings by day and category. Find the } \\
\text { event locations on the campus map. Access the academic calendar for } \\
\text { important dates and deadlines. }\end{array}$ & Yes & Yes \\
\hline News & Keep up to date with and share the latest University news and announcements. & Yes & Yes \\
\hline Accommodation & $\begin{array}{l}\text { Integrated with university dormitories website, Student/User can make } \\
\text { book/reservation for Washing Machine/Dryer/Sauna }\end{array}$ & Partly Yes & Yes \\
\hline Sports & $\begin{array}{l}\text { Check SportsHall timetables, and news. Book a sports hall for indoor games. } \\
\text { Users can create sports events with friends. Integrated with sports facilities } \\
\text { services }\end{array}$ & Yes & Yes \\
\hline Report & $\begin{array}{l}\text { Submit fault reports concerning campus facilities, outdoor and indoor areas, } \\
\text { report a defect in class, outdoor and indoor places. }\end{array}$ & Yes & Yes \\
\hline Courses & $\begin{array}{l}\text { Search or browse the course schedule to find descriptions, meeting days and } \\
\text { times, locations, instructors, and class size. Link to instructor contact } \\
\text { information and check locations on the campus map. }\end{array}$ & Partly Yes & Yes \\
\hline Library & $\begin{array}{l}\text { Connect to the university library services to search the catalog, see hours for } \\
\text { each library, and send a text message or check loan status, prolong the book, } \\
\text { order library card }\end{array}$ & No & Yes \\
\hline Emergency & $\begin{array}{l}\text { Get quick access to telephone numbers of emergency services in case of } \\
\text { emergency in the university campus }\end{array}$ & Yes & Yes \\
\hline Social & $\begin{array}{l}\text { University social media pages, groups, accounts, and profiles. Follow } \\
\text { University's social media postings on happenings around campus and } \\
\text { breaking news. }\end{array}$ & Yes & Yes \\
\hline Announcements & Student/Staff can share updates about the important events, conferences. & No & Yes \\
\hline Video/Multimedia & $\begin{array}{l}\text { See a wide variety of videos about the university, made by students, staff, and } \\
\text { faculty, and featuring faculty members, researchers, student life, and campus } \\
\text { beauty. }\end{array}$ & Yes & Yes \\
\hline $\begin{array}{l}\text { Customize } \\
\text { menu/navigation }\end{array}$ & $\begin{array}{l}\text { User can select his/her favorite features, and selected features appear on the } \\
\text { navigation }\end{array}$ & Yes & Yes \\
\hline
\end{tabular}




\begin{tabular}{|c|c|c|c|}
\hline Settings & User can choose the size of fonts, language settings & Yes & Yes \\
\hline Parking & Campus Parking places and information, free parking areas. & Yes & Yes \\
\hline Log in $/ \log$ out & $\begin{array}{l}\text { User logins to his/her account, personalized schedules/contents, notifications, } \\
\text { messages }\end{array}$ & No & Yes \\
\hline Chat & Internal chat, group conversations. & No & Yes \\
\hline Marketplace & $\begin{array}{l}\begin{array}{l}\text { Sell, lend and rent things, buy printer quota, buy university t- } \\
\text { shirts/shorts/caps/mugs }\end{array} \\
\end{array}$ & Yes & Yes \\
\hline Health Service & Health Service places/Phone number and contact information & No & Yes \\
\hline Study Space Availability & User can check available study/meeting space and can book it, & No & Yes \\
\hline $\begin{array}{ll}\begin{array}{l}\text { Student } \\
\text { card }\end{array} & \text { Identification } \\
\end{array}$ & Virtual student card verified identification of student. & No & Yes \\
\hline Campus News & Read the latest news from several university news sources & Yes & Yes \\
\hline Laundry Info & Check availability of washers and dryers located in campus facilities & No & Yes \\
\hline Jobs(Find Jobs) & See the latest open positions from the University employment system. & No & Yes \\
\hline Finance & Reminds/Shows the student's tuition fee & No & Yes \\
\hline
\end{tabular}

\section{Virtual Campus Tour and Touchpoint Services}

After completing the visitor journey application benchmarking, research continued with top-ranking 60 universities [3] and also included some universities from Finland and Estonia, which have virtual campus tour applications. From the process of benchmarking, 39 virtual campus tour applications are selected. Table 5 shows the list of virtual campus tour applications.

The virtual campus tour application's primary purpose is to promote key areas of both indoor and outdoor campus and attract the attention of prospective students, campus visitors, and prospective student's parents. Users of this application explore the campus facilities, services, locations from their supported devices.

The virtual experience of the campus is priceless. These kinds of applications are good for promoting university services in a good way.

Virtual tour applications increase the physical visits $27 \%$, interactive web content engagement by $38 \%$, and have resulted in $18 \%$ increase in inquires [39].

Map, Menu, Navigation, Photos, Panorama, Video, 360-degree video, voice-guide, Google map, interactive human guide, web app, mobile app, VR device are the supported features selected for comparison criteria. We consider these features are essential and core for building virtual campus tour applications.

Table 5. List of virtual campus tour applications and selection criteria

\begin{tabular}{|c|c|c|c|c|c|c|c|c|c|c|c|c|c|}
\hline $\begin{array}{l}\text { University } \\
\text { Name }\end{array}$ & $\begin{array}{l}\mathbf{M} \\
\text { ap }\end{array}$ & 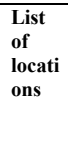 & $\begin{array}{l}\text { Navigation( } \\
\text { Controls) }\end{array}$ & $\begin{array}{l}\text { Ph } \\
\text { oto } \\
\text { s }\end{array}$ & $\begin{array}{l}\text { Pano } \\
\text { rama }\end{array}$ & $\begin{array}{l}\text { Vi } \\
\text { de } \\
\text { o }\end{array}$ & $\begin{array}{l}\text { 360d } \\
\text { egree } \\
\text { Vide } \\
\text { o }\end{array}$ & $\begin{array}{l}\text { Voi } \\
\text { ce- } \\
\text { gui } \\
\text { ded } \\
\text { tou } \\
\text { r }\end{array}$ & $\begin{array}{l}\text { Go } \\
\text { ogl } \\
\text { e } \\
\text { Ma } \\
\text { p }\end{array}$ & $\begin{array}{l}\text { Intera } \\
\text { ctive } \\
\text { huma } \\
\text { n } \\
\text { Guide }\end{array}$ & $\begin{array}{l}\mathbf{W} \\
\text { eb } \\
\text { Ap } \\
\text { p }\end{array}$ & $\begin{array}{l}\text { Mo } \\
\text { bile } \\
\text { Ap } \\
\text { p }\end{array}$ & $\begin{array}{l}\text { VR } \\
\text { Sup } \\
\text { port }\end{array}$ \\
\hline $\begin{array}{l}\text { University of } \\
\text { Oxford }\end{array}$ & $\begin{array}{l}\mathrm{Ye} \\
\mathrm{s}\end{array}$ & No & Yes & Yes & Yes & No & No & No & No & No & $\begin{array}{l}\mathrm{Ye} \\
\mathrm{s}\end{array}$ & No & No \\
\hline $\begin{array}{l}\text { University of } \\
\text { Cambridge }\end{array}$ & $\begin{array}{l}\mathrm{Ye} \\
\mathrm{s}\end{array}$ & Yes & Yes & Yes & Yes & No & No & No & No & No & $\begin{array}{l}\mathrm{Ye} \\
\mathrm{s}\end{array}$ & No & No \\
\hline $\begin{array}{l}\text { Stanford } \\
\text { University }\end{array}$ & $\begin{array}{l}\text { ye } \\
\text { s }\end{array}$ & no & yes & Yes & No & No & No & No & No & No & $\begin{array}{l}\mathrm{Ye} \\
\mathrm{s}\end{array}$ & No & No \\
\hline
\end{tabular}




\begin{tabular}{|c|c|c|c|c|c|c|c|c|c|c|c|c|c|}
\hline $\begin{array}{l}\text { Massachuset } \\
\text { ts Institute of } \\
\text { Technology }\end{array}$ & $\begin{array}{l}\mathrm{Ye} \\
\mathrm{s}\end{array}$ & Yes & No & Yes & No & No & No & No & No & No & $\begin{array}{l}\mathrm{Ye} \\
\mathrm{s}\end{array}$ & No & Yes \\
\hline $\begin{array}{l}\text { California } \\
\text { Institute of } \\
\text { Technology }\end{array}$ & $\begin{array}{l}\mathrm{Ye} \\
\mathrm{s}\end{array}$ & Yes & No & Yes & No & No & No & No & Yes & No & $\begin{array}{l}\mathrm{Ye} \\
\mathrm{s}\end{array}$ & No & No \\
\hline $\begin{array}{l}\text { Harvard } \\
\text { University }\end{array}$ & $\begin{array}{l}\mathrm{Ye} \\
\mathrm{s}\end{array}$ & Yes & Yes & Yes & Yes & $\begin{array}{l}\mathrm{Ye} \\
\mathrm{s}\end{array}$ & Yes & Yes & Yes & Yes & $\begin{array}{l}\mathrm{Ye} \\
\mathrm{s}\end{array}$ & Yes & Yes \\
\hline $\begin{array}{l}\text { Princeton } \\
\text { University }\end{array}$ & $\begin{array}{l}\mathrm{Ye} \\
\mathrm{s}\end{array}$ & Yes & Yes & Yes & Yes & $\begin{array}{l}\mathrm{Ye} \\
\mathrm{s}\end{array}$ & Yes & Yes & Yes & Yes & $\begin{array}{l}\mathrm{Ye} \\
\mathrm{s}\end{array}$ & Yes & Yes \\
\hline $\begin{array}{l}\text { Yale } \\
\text { University }\end{array}$ & $\begin{array}{l}\mathrm{Ye} \\
\mathrm{s}\end{array}$ & Yes & Yes & Yes & Yes & $\begin{array}{l}\mathrm{Ye} \\
\mathrm{s}\end{array}$ & Yes & Yes & Yes & Yes & $\begin{array}{l}\mathrm{Ye} \\
\mathrm{s}\end{array}$ & Yes & Yes \\
\hline $\begin{array}{l}\text { Imperial } \\
\text { College } \\
\text { London }\end{array}$ & $\begin{array}{l}\mathrm{Ye} \\
\mathrm{s}\end{array}$ & Yes & Yes & No & Yes & No & No & No & Yes & No & $\begin{array}{l}\mathrm{Ye} \\
\mathrm{s}\end{array}$ & No & No \\
\hline ETH Zurich & $\begin{array}{l}\mathrm{Ye} \\
\mathrm{s}\end{array}$ & Yes & Yes & Yes & Yes & $\begin{array}{l}\mathrm{Ye} \\
\mathrm{s}\end{array}$ & No & Yes & No & Yes & $\begin{array}{l}\mathrm{Ye} \\
\mathrm{s}\end{array}$ & Yes & No \\
\hline $\begin{array}{l}\text { Johns } \\
\text { Hopkins } \\
\text { University }\end{array}$ & $\begin{array}{l}\mathrm{Ye} \\
\mathrm{s}\end{array}$ & Yes & Yes & Yes & Yes & $\begin{array}{l}\mathrm{Ye} \\
\mathrm{s}\end{array}$ & Yes & Yes & Yes & Yes & $\begin{array}{l}\mathrm{Ye} \\
\mathrm{s}\end{array}$ & Yes & Yes \\
\hline $\begin{array}{l}\text { University of } \\
\text { Pennsylvania }\end{array}$ & $\begin{array}{l}\mathrm{Ye} \\
\mathrm{s}\end{array}$ & Yes & No & Yes & No & No & No & No & Yes & No & $\begin{array}{l}\mathrm{Ye} \\
\mathrm{s}\end{array}$ & No & No \\
\hline $\begin{array}{l}\text { UCL(Univer } \\
\text { sity College } \\
\text { London) }\end{array}$ & No & No & Yes & No & No & No & Yes & No & No & No & No & No & No \\
\hline $\begin{array}{l}\text { Columbia } \\
\text { University }\end{array}$ & $\begin{array}{l}\mathrm{Ye} \\
\mathrm{s}\end{array}$ & Yes & Yes & Yes & Yes & $\begin{array}{l}\mathrm{Ye} \\
\mathrm{s}\end{array}$ & Yes & Yes & Yes & Yes & $\begin{array}{l}\mathrm{Ye} \\
\mathrm{s}\end{array}$ & Yes & Yes \\
\hline $\begin{array}{l}\text { University of } \\
\text { California, } \\
\text { Los Angeles }\end{array}$ & $\begin{array}{l}\mathrm{Ye} \\
\mathrm{s}\end{array}$ & Yes & Yes & Yes & Yes & $\begin{array}{l}\mathrm{Ye} \\
\mathrm{s}\end{array}$ & Yes & Yes & Yes & No & $\begin{array}{l}\mathrm{Ye} \\
\mathrm{s}\end{array}$ & Yes & Yes \\
\hline $\begin{array}{l}\text { Duke } \\
\text { University }\end{array}$ & $\begin{array}{l}\mathrm{Ye} \\
\mathrm{s}\end{array}$ & Yes & Yes & Yes & Yes & $\begin{array}{l}\mathrm{Ye} \\
\mathrm{s}\end{array}$ & Yes & Yes & Yes & Yes & $\begin{array}{l}\mathrm{Ye} \\
\mathrm{s}\end{array}$ & Yes & Yes \\
\hline $\begin{array}{l}\text { Cornell } \\
\text { University }\end{array}$ & $\begin{array}{l}\mathrm{Ye} \\
\mathrm{s}\end{array}$ & Yes & Yes & No & Yes & No & No & No & Yes & No & $\begin{array}{l}\mathrm{Ye} \\
\mathrm{s}\end{array}$ & No & No \\
\hline $\begin{array}{l}\text { University of } \\
\text { Michigan }\end{array}$ & No & No & No & Yes & No & No & No & No & No & No & No & No & No \\
\hline $\begin{array}{l}\text { Tsinghua } \\
\text { University }\end{array}$ & $\begin{array}{l}\mathrm{Ye} \\
\mathrm{s}\end{array}$ & No & No & No & No & No & No & No & No & No & No & No & No \\
\hline $\begin{array}{l}\text { Carnegie } \\
\text { Mellon } \\
\text { University }\end{array}$ & No & Yes & Yes & Yes & Yes & $\begin{array}{l}\mathrm{Ye} \\
\mathrm{s}\end{array}$ & Yes & Yes & No & No & $\begin{array}{l}\mathrm{Ye} \\
\mathrm{s}\end{array}$ & Yes & Yes \\
\hline $\begin{array}{l}\text { Northwester } \\
\text { n University }\end{array}$ & $\begin{array}{l}\mathrm{Ye} \\
\mathrm{s}\end{array}$ & Yes & Yes & Yes & Yes & $\begin{array}{l}\mathrm{Ye} \\
\mathrm{s}\end{array}$ & Yes & Yes & Yes & Yes & $\begin{array}{l}\mathrm{Ye} \\
\mathrm{s}\end{array}$ & Yes & Yes \\
\hline $\begin{array}{l}\text { London } \\
\text { School of } \\
\text { Economics } \\
\text { and Political } \\
\text { Science }\end{array}$ & No & Yes & Yes & Yes & No & $\begin{array}{l}\mathrm{Ye} \\
\mathrm{s}\end{array}$ & No & No & No & No & $\begin{array}{l}\text { ye } \\
\text { s }\end{array}$ & No & No \\
\hline $\begin{array}{l}\text { University of } \\
\text { Edinburg }\end{array}$ & No & Yes & Yes & Yes & Yes & $\begin{array}{l}\mathrm{Ye} \\
\mathrm{s}\end{array}$ & No & No & No & No & No & No & Yes \\
\hline $\begin{array}{l}\text { University of } \\
\text { California } \\
\text { San Diego } \\
\text { (UC San } \\
\text { Diego) }\end{array}$ & $\begin{array}{l}\mathrm{Ye} \\
\mathrm{s}\end{array}$ & Yes & Yes & Yes & Yes & $\begin{array}{l}\mathrm{Ye} \\
\mathrm{s}\end{array}$ & Yes & Yes & Yes & No & $\begin{array}{l}\mathrm{Ye} \\
\mathrm{s}\end{array}$ & Yes & Yes \\
\hline $\begin{array}{l}\text { Georgia } \\
\text { Institute of } \\
\text { Technology }\end{array}$ & $\begin{array}{l}\mathrm{Ye} \\
\mathrm{s}\end{array}$ & Yes & Yes & Yes & Yes & $\begin{array}{l}\mathrm{Ye} \\
\mathrm{s}\end{array}$ & Yes & Yes & Yes & Yes & $\begin{array}{l}\mathrm{Ye} \\
\mathrm{s}\end{array}$ & Yes & Yes \\
\hline $\begin{array}{l}\text { École } \\
\text { Polytechniqu } \\
\text { e Fédérale de } \\
\text { Lausanne }\end{array}$ & $\begin{array}{l}\mathrm{Ye} \\
\mathrm{s}\end{array}$ & Yes & Yes & Yes & No & $\begin{array}{l}\mathrm{Ye} \\
\mathrm{s}\end{array}$ & No & No & No & No & $\begin{array}{l}\mathrm{Ye} \\
\mathrm{s}\end{array}$ & No & No \\
\hline $\begin{array}{l}\text { University of } \\
\text { British } \\
\text { Columbia }\end{array}$ & $\begin{array}{l}\mathrm{Ye} \\
\mathrm{s}\end{array}$ & Yes & Yes & Yes & Yes & $\begin{array}{l}\mathrm{Ye} \\
\mathrm{s}\end{array}$ & Yes & Yes & Yes & Yes & $\begin{array}{l}\mathrm{Ye} \\
\mathrm{s}\end{array}$ & Yes & Yes \\
\hline $\begin{array}{l}\text { University of } \\
\text { Texas at } \\
\text { Austin }\end{array}$ & $\begin{array}{l}\mathrm{Ye} \\
\mathrm{s}\end{array}$ & Yes & Yes & Yes & Yes & No & No & No & No & No & $\begin{array}{l}\mathrm{Ye} \\
\mathrm{s}\end{array}$ & No & No \\
\hline $\begin{array}{l}\text { Heidelberg } \\
\text { University }\end{array}$ & No & Yes & Yes & Yes & Yes & No & No & No & No & No & $\begin{array}{l}\text { Ye } \\
\mathrm{s}\end{array}$ & No & No \\
\hline KU Leuven & No & Yes & Yes & Yes & No & No & No & Yes & No & No & No & No & No \\
\hline $\begin{array}{l}\text { Australian } \\
\text { National } \\
\text { University }\end{array}$ & No & Yes & Yes & Yes & yes & yes & Yes & no & no & no & $\begin{array}{l}\text { ye } \\
\mathrm{s}\end{array}$ & No & No \\
\hline $\begin{array}{l}\text { University of } \\
\text { Illinois at }\end{array}$ & No & No & Yes & Yes & No & No & No & No & No & No & No & No & No \\
\hline
\end{tabular}




\begin{tabular}{|c|c|c|c|c|c|c|c|c|c|c|c|c|c|}
\hline $\begin{array}{l}\text { Urbana- } \\
\text { Champaign }\end{array}$ & & & & & & & & & & & & & \\
\hline $\begin{array}{l}\text { University of } \\
\text { California, } \\
\text { Santa } \\
\text { Barbara }\end{array}$ & $\begin{array}{l}\mathrm{Ye} \\
\mathrm{s}\end{array}$ & Yes & Yes & Yes & Yes & no & no & yes & yes & yes & $\begin{array}{l}\text { ye } \\
\text { s }\end{array}$ & yes & yes \\
\hline $\begin{array}{l}\text { Brown } \\
\text { University }\end{array}$ & $\begin{array}{l}\text { Ye } \\
\text { s }\end{array}$ & Yes & Yes & Yes & Yes & $\begin{array}{l}\mathrm{Ye} \\
\mathrm{s}\end{array}$ & Yes & Yes & Yes & Yes & $\begin{array}{l}\mathrm{Ye} \\
\mathrm{s}\end{array}$ & Yes & Yes \\
\hline $\begin{array}{l}\text { University of } \\
\text { North } \\
\text { Carolina at } \\
\text { Chapel Hill }\end{array}$ & $\begin{array}{l}\mathrm{Ye} \\
\mathrm{s}\end{array}$ & Yes & Yes & Yes & Yes & $\begin{array}{l}\mathrm{Ye} \\
\mathrm{s}\end{array}$ & Yes & Yes & No & No & $\begin{array}{l}\mathrm{Ye} \\
\mathrm{s}\end{array}$ & Yes & Yes \\
\hline $\begin{array}{l}\text { University of } \\
\text { California, } \\
\text { Davis }\end{array}$ & $\begin{array}{l}\mathrm{Ye} \\
\mathrm{s}\end{array}$ & Yes & Yes & Yes & Yes & no & no & Yes & Yes & Yes & $\begin{array}{l}\mathrm{Ye} \\
\mathrm{s}\end{array}$ & yes & yes \\
\hline $\begin{array}{l}\text { University of } \\
\text { Sydney }\end{array}$ & No & Yes & Yes & Yes & Yes & $\begin{array}{l}\mathrm{Ye} \\
\mathrm{s}\end{array}$ & Yes & Yes & No & No & $\begin{array}{l}\mathrm{Ye} \\
\mathrm{s}\end{array}$ & Yes & Yes \\
\hline $\begin{array}{l}\text { Tallinn } \\
\text { University of } \\
\text { Technology }\end{array}$ & $\begin{array}{l}\mathrm{Ye} \\
\mathrm{s}\end{array}$ & Yes & Yes & Yes & Yes & $\begin{array}{l}\mathrm{Ye} \\
\mathrm{s}\end{array}$ & Yes & Yes & Yes & No & $\begin{array}{l}\mathrm{Ye} \\
\mathrm{s}\end{array}$ & No & Yes \\
\hline $\begin{array}{l}\text { Lahti } \\
\text { University of } \\
\text { Applied } \\
\text { Science }\end{array}$ & $\begin{array}{l}\text { Ye } \\
\mathrm{s}\end{array}$ & Yes & Yes & Yes & Yes & No & No & No & No & No & $\begin{array}{l}\text { Y } \\
\text { Es }\end{array}$ & No & No \\
\hline
\end{tabular}

\section{Conceptualization}

From the findings of benchmarking during the research, the following conceptual model for the visitor journey application is proposed. Figure 3 shows the conceptual model for the visitor journey application. The rationale behind the construction of the conceptual model is as follows: The connection between channels and services is twodirectional. Data flow between channels and touchpoints also two-directional. The visitor journey starts with interacting with touchpoints. Each journey of visitor is recorded to the system during the data collecting process. Understanding the visitor helps to identify how, from where, and which touchpoints visitor interacts with the brand. The software analyzes the collected data from the visitor journey with models, scales, and techniques. The software creates visitor journey mapping. In each visitor journey, the visitor experiences a personalized visitor journey, and the visitor continues the journey from where he/she left each time revisits the application. The process of creating a visitor journey is iterative.

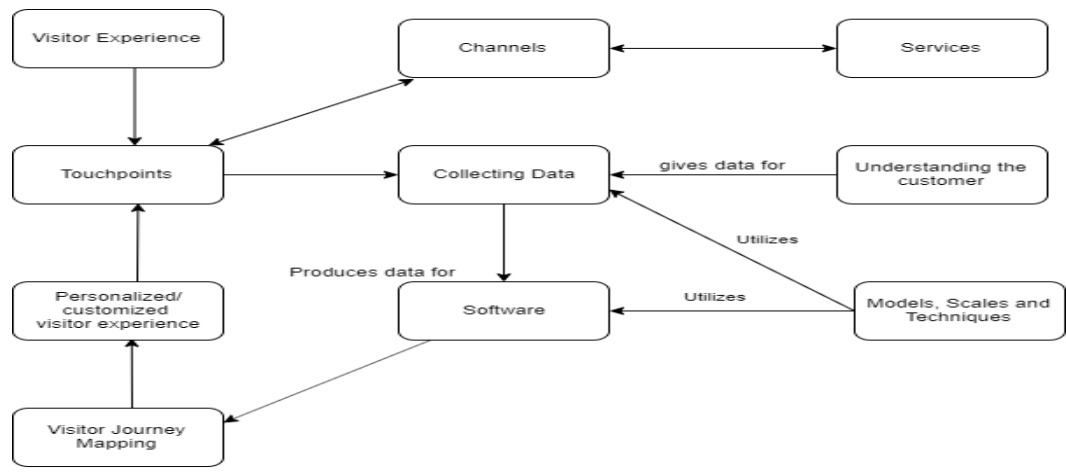

Figure 3.Conceptual model for Visitor Journey Application 
Figure 4 presents the visitor journey process created from the conceptual model for the visitor journey application. A visitor interacts with touchpoints, and each interaction is recorded to the system and creates a list of visitor journeys. The system creates a visitor journey map from the list of visitor journeys and provides a personalized visitor experience. The personalized visitor experience depends on the number of journeys. As the number of journeys increases, personalized visitor experience optimizes, the interaction of the visitor improves.

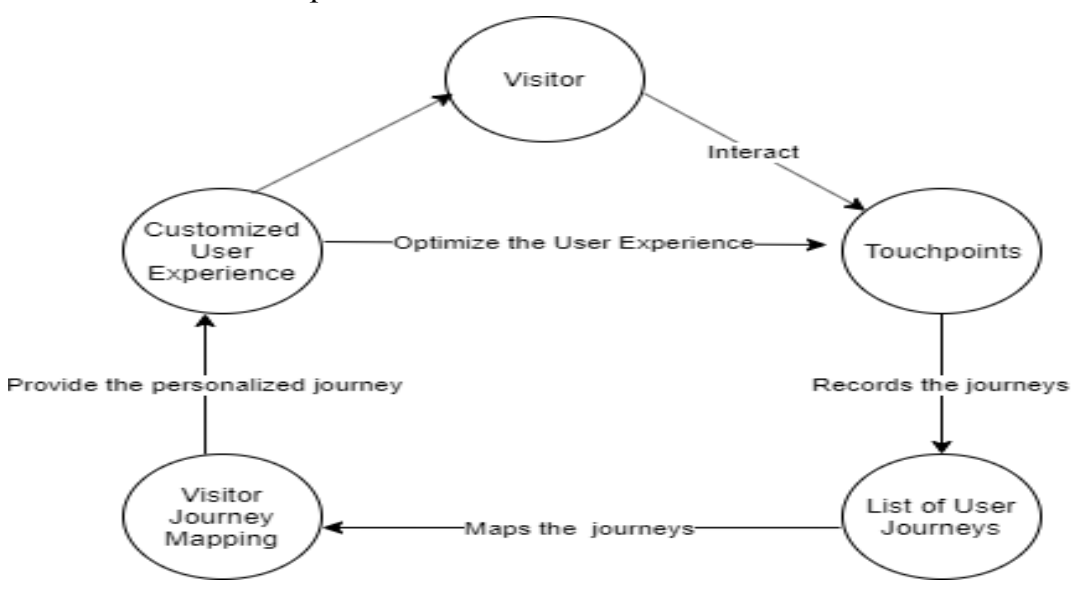

Figure 4.Visitor Journey Application process

\section{Visitor journeys and touchpoints mapping}

Table 6 presents the visitor journey across the touchpoints. A visitor journey starts from any touchpoint and ends in any touchpoint. In Table 6 visitor journey starts from touchpoint (T1) 1 and ends in touchpoint $\mathrm{T}$. $\mathrm{T} 1=>\mathrm{T} 2=>\mathrm{T} 5=>\mathrm{T} 3=>\mathrm{T} 10=>\mathrm{T} 11=>\mathrm{T} 8=>\mathrm{T} 3$ here the simple visitor journey of figure 4 .

Table 6:User journey across the touchpoints

\begin{tabular}{|c|c|c|c|c|c|c|c|c|}
\hline & 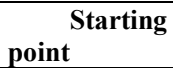 & $2^{\text {nd }}$ & $3^{\text {rd }}$ & $4^{\text {th }}$ & $5^{\text {th }}$ & $6^{\text {th }}$ & $8^{\text {th }}$ & $9^{\text {th }}$ \\
\hline \multirow{8}{*}{ 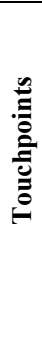 } & $\mathrm{T} 1$ & $\mathrm{~T} 2$ & T1 & $\mathrm{T} 1$ & T1 & $\mathrm{T} 1$ & T1 & $\mathrm{T} 1$ \\
\hline & $\mathrm{T} 2$ & $\mathrm{~T} 3$ & T3 & $\mathrm{T} 2$ & $\mathrm{~T} 2$ & $\mathrm{~T} 2$ & $\mathrm{~T} 2$ & $\mathrm{~T} 2$ \\
\hline & T3 & $\mathrm{T} 4$ & $\mathrm{~T} 4$ & T3 & T3 & T3 & T3 & T3 \\
\hline & $\mathrm{T} 4$ & T5 & T5 & $\mathrm{T} 4$ & $\mathrm{~T} 4$ & $\mathrm{~T} 4$ & $\mathrm{~T} 4$ & $\mathrm{~T} 4$ \\
\hline & T5 & T6 & T6 & T6 & T5 & T5 & T5 & T5 \\
\hline & T6 & $\mathrm{T} 7$ & $\mathrm{~T} 7$ & $\mathrm{~T} 7$ & T6 & T6 & T6 & $\mathrm{T} 6$ \\
\hline & T7 & $\mathrm{T} 8$ & T8 & T8 & T7 & T7 & T7 & $\mathrm{T} 7$ \\
\hline & T8 & T9 & T9 & T9 & $\mathrm{T} 8$ & T8 & T8 & T9 \\
\hline
\end{tabular}




\begin{tabular}{|l|l|l|l|l|l|l|l|r|}
\hline & T9 & T10 & T10 & T10 & T9 & T9 & T9 & T10 \\
\cline { 2 - 9 } & T10 & T11 & T11 & T11 & T10 & T11 & T10 & T11 \\
\hline & T11 & & & & & & & \\
\hline
\end{tabular}

\begin{tabular}{|l|l|}
\hline Journeys & Route of Journeys \\
\hline Journey 1 & $\mathrm{T} 1=>\mathrm{T} 2=>\mathrm{T} 5=>\mathrm{T} 3=>\mathrm{T} 10=>\mathrm{T} 11=>\mathrm{T} 8=>\mathrm{T} 3$ \\
\hline Journey 2 & $\mathrm{T} 2=>\mathrm{T} 7=>\mathrm{T} 5=>\mathrm{T} 3=>\mathrm{T} 2=>\mathrm{T} 11=>\mathrm{T} 8=>\mathrm{T} 5$ \\
\hline Journey 3 & $\mathrm{T} 3=>\mathrm{T} 10=>\mathrm{T} 11=>\mathrm{T} 8=>\mathrm{T} 3$ \\
\hline Journey $\mathrm{n} .$. & $\ldots \ldots \ldots . \mathrm{n}$ \\
\hline
\end{tabular}

Table 7: Number of nodes

\begin{tabular}{|l|l|l|l|}
\hline Journey 1 & Journey 2 & Journey 3 & Journey $\mathrm{n} .$. \\
\hline $\mathrm{T} 3=2$ & $\mathrm{~T} 3=3$ & $\mathrm{~T} 3=5$ & $\ldots \mathrm{n}$ \\
\hline $\mathrm{T} 1=1$ & $\mathrm{~T} 5=3$ & $\mathrm{~T} 5=3$ & $\ldots \mathrm{n}$ \\
\hline $\mathrm{T} 2=1$ & $\mathrm{~T} 2=3$ & $\mathrm{~T} 2=3$ & $\ldots \mathrm{n}$ \\
\hline $\mathrm{T} 5=1$ & $\mathrm{~T} 11=2$ & $\mathrm{~T} 8=3$ & $\ldots \mathrm{n}$ \\
\hline $\mathrm{T} 8=1$ & $\mathrm{~T} 8=2$ & $\mathrm{~T} 11=2$ & $\ldots \mathrm{n}$ \\
\hline $\mathrm{T} 10=1$ & $\mathrm{~T} 10=1$ & $\mathrm{~T} 10=2$ & $\ldots \mathrm{n}$ \\
\hline $\mathrm{T} 11=1$ & $\mathrm{~T} 1=1$ & $\mathrm{~T} 1=1$ & $\ldots \mathrm{n}$ \\
\hline
\end{tabular}

A significant number of visitor journeys help to build visitor journey maps. With machine learning algorithms, it is possible to detect the most used touchpoints; most used combinations of touchpoints, and the duration of the visitor journey. With this information, the user/visitor will have a personalized experience. Analytics can be involved to record each step of the visitor while experiencing the brand. End of the current journey analytic tools draw the visitor's journey network. With the help of the software, new personalized visitor experience will be created dynamically. The visitor can continue the journey where he/she left in the last session. End of each journey analytic tools merge the new visitor journey map with the old one. Here the number of nodes between the touchpoints are important. The high number of nodes will be shown first. In Table 7 the number of nodes connected to the touchpoints are given and calculated. Figure 5 illustrates an example of the above mentioned visitor journey and touchpoints mapping. 

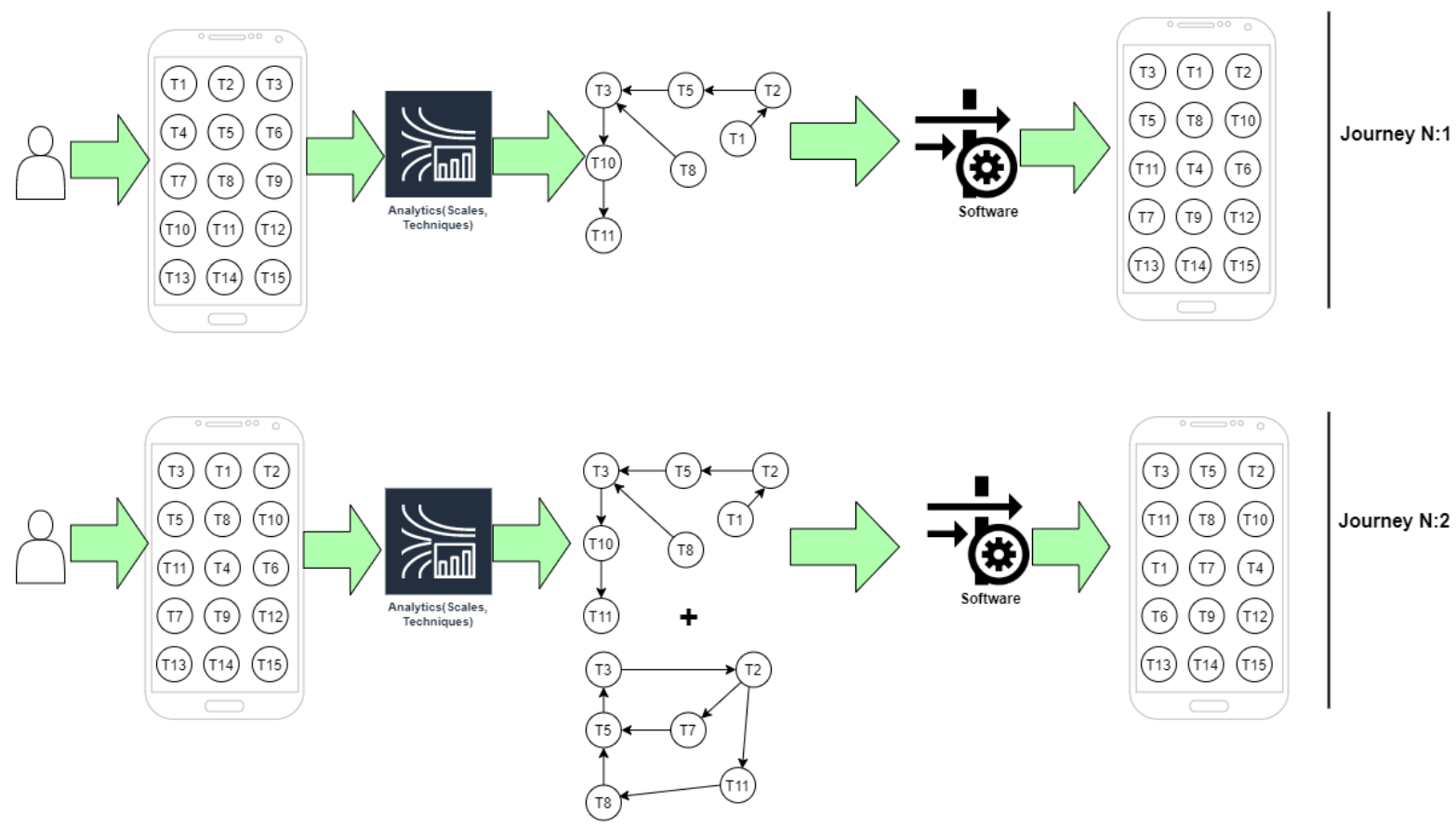

Figure 5: Simulation of the visitor journey mapping

\section{Concluding remarks}

The primary purpose of conducting this research is to determine how campus journey application development is progressing. Campus virtual tour applications mostly run on a web browser, and almost every benchmarked visitor journey application of this research have a web app. Campus visitor journey applications mostly run on mobile devices and few of them have web application versions. Some universities, for example, Sydney University, combines virtual campus tour and visitor journey application in one application. From the research findings, we recommend combining the virtual tour with campus journey applications. This task may be challenging. However, there is the advantage of it in allocating resources. Further, this research provided insight into visitor journey application development, how they are used in the present, the features that are necessary for introducing seamless visitor journey mapping, and involving touchpoints of visitor journey applications for improving the customer/visitor experience of omni channels.

\section{References}

[1] "Number of smartphone users worldwide 2014-2020." [Online]. Available: https:/www.statista.com/statistics/330695/number-of-smartphone-users-worldwide/. [Accessed: 01-Nov-2019].

[2] N. Beck and D. Rygl, "Categorization of multiple channel retailing in Multi- , Cross- , and Omni Channel Retailing for retailers and retailing,” J. Retail. Consum. Serv., vol. 27, pp. 170-178, 2015. 
[3] "World University Rankings." [Online]. Available: https://www.timeshighereducation.com/worlduniversity-rankings/2019/world-ranking\#!/page/0/length/25/sort_by/rank/sort_order/asc/cols/stats. [Accessed: 01-Nov-2019].

[4] B. Kitchenham, O. P. Brereton, D. Budgen, M. Turner, J. Bailey, and S. Linkman, "Systematic literature reviews in software engineering - A systematic literature review," Inf. Softw. Technol., vol. 51, no. 1, pp. 7-15, 2009.

[5] S. Zhang, H. Zhu, X. Li, and Y. Wang, "Omni-Channel Product Distribution Network Design by Using the Improved Particle Swarm Optimization Algorithm,” vol. 2019, 2019.

[6] M. R. B. P. Rizawati Binti Rohizan, Daniel Mago Vistro, "Enhanced Visitor Experience Through Campus Virtual Tour,” J. Phys. Conf. Ser., vol. 1228, 2019.

[7] V. Kankaew and P. Wannapiroon, "System Architecture for Virtual Team Campus on Cloud to Support Internal Quality Assurance of Rajamangala University of Technology,” vol. 15, no. 7, pp. 99-110, 2019

[8] T. H. L. Amir Dirin, "User Experience in Mobile Augmented Reality: Emotions, Challenges, Opportunities and Best Practices," Computers, vol. 7, 2018.

[9] C. G. Prieto-alvarez, R. Martinez-maldonado, and S. B. Shum, "Mapping Learner-Data Journeys : Evolution of a Visual Co-Design Tool," Proc. 30th Aust. Conf. Comput. Interact., pp. 205-214, 2018.

[10] Kan Ren, Yuchen Fang, Weinan Zhang, Shuhao Liu, Jiajun Li, Ya Zhang, Yong Yu, Jun Wang, "Learning Multi-touch Conversion Attribution with Dual-attention Mechanisms for Online Advertising," Proc. 27th ACM Int. Conf. Inf. Knowl. Manag., pp. 1433-1442, 2018

[11] A. Mosquera, E. Juaneda-ayensa, C. Olarte-pascual, and J. Pelegrín-borondo, "Key Factors for InStore Smartphone Use in an Omnichannel Experience : Millennials vs . Nonmillennials," Complexity, vol. Volume 201, 2018.

[12] R F Rahmat, Anthonius, M A Muchtar, A Hizriadi and M F Syahputra, "Virtual reality interactive media for universitas sumatera utara - a campus introduction and simulation," J. Phys. Conf. Ser. vol. 978, 2018.

[13] S. Sue, G. Kim, and A. Adrienne, “Journey Mapping the User Experience,” $C \& R L$, vol. 78, pp. 459471, 2017.

[14] Joao C.Ferreira, Ana Lucia Martins, Jorge Vieira da Silva, João Almeida, "T2 * - Personalized Trip Planner," Adv. Intell. Syst. Comput., vol. 615, pp. 167-175, 2018.

[15] Iulia Popescu, Kurt Portelli, Christos Anagnostopoulos, Nikos Ntarmos, "The Case for Graph-Based Recommendations," 2017 IEEE Int. Conf. Big Data (Big Data), pp. 4819-4821, 2017.

[16] Y. Bhavnani, K. Rodden, L. C. Guarnotta, M. T. Lynn, S. Chizari, and Laura Granka, "Understanding Mobile Phone Activities via Retrospective Review of Visualizations of Usage Data," MobileHCI '17 Proc. 19th Int. Conf. Human-Computer Interact. with Mob. Devices Serv., 2017.

[17] E. Sun, R. De Oliveira, and J. Lewandowski, "Challenges on the Journey to Co-Watching YouTube," CSCW 2017, pp. 783-793, 2017

[18] D. B. Viany Utami Tjhin, Bahtiar S.Abbas, Raymond Kosala, "Development Plan for Research on Omni-Channel Shopping to Purchase Intention,” 2016 Int. Conf. Inf. Manag. Technol., pp. 229-234, 2016

[19] V. Codina, J. Mena, and Luis Oliva, "Context-Aware User Modeling Strategies for Journey Plan Recommendation," UMAP 2015 User Model. Adapt. Pers., pp. 68-79, 2015.

[20] C. L.Maines, S. Tang, and David Llewellyn-Jones, "VICTour 1 . 1 : Introducing virtual learning environments and gamification," 2015 Int. Conf. Dev. E-Systems Eng., pp. 159-164, 2015.

[21] C. Maines and S. Tang, "An Application of Game Technology to Virtual University Campus Touring and Interior Navigation," 2015 Int. Conf. Dev. E-Systems Eng., pp. 341-346, 2015.

[22] Y. Elkhatib, R. Killick, M. Mu, and N. Race, "Just Browsing? Understanding User Journeys in Online TV Initial observations,” pp. 965-968.

[23] N. Liu, Y. Feng, F. Wang, B. Liu, and J. Tang, "Mobility Crowdsourcing: Toward Zero-Effort Carpooling on Individual Smartphone," vol. 2013, 2013.

[24] J. Teixeira, L. Patrício, N. J. Nunes, and L. Nóbrega, "Customer Experience Modeling : Designing Interactions for Service Systems," pp. 136-143, 2011.

[25] J. Kronqvist and T. Leinonen, "Redefining Touchpoints : An Integrated Approach for Implementing Omnichannel Service Concepts," in Springer, Cham, pp. 279-288.

[26] S. Wilson and G. Mccarthy, "The mobile university : from the library to the campus," Ref. Serv. Rev., vol. 38 No. 2, pp. 214-232, 2010.

[27] S. Han and R. Wong, "Which platform do our users prefer : website or mobile app ?," vol. 40, no. 1, pp. $103-115,2012$.

[28] H. S. Al-khalifa, "A framework for evaluating university mobile websites," Emerald Gr. Publ. Ltd., vol. 38 No. 2, pp. 166-185, 2012.

[29] Föhr Jann, Hietanen Hannes, Khvatov Artem, Lepistö Toni, Liukka, Niko, Dahanayake Ajantha, 
"Customer experience evaluation in the omnichannel environment," in Frontiers in Artificial Intelligence and Applications, 2017, vol. 301, 2018, pp. 21-34.

[30] "Disney Parks.” [Online]. Available: https://disneyparks.disney.go.com/. [Accessed: 27-Nov-2018].

[31] Fransgaard, "Getting an omni-channel customer service experience as a Virgin advocate." [Online]. Available: http://fransgaard.com/getting-an-omni-channel-customer-service-as-a-virgin-advocate/. [Accessed: 28-Nov-2019]

[32] “Oasis." [Online]. Available: https://www.oasis-stores.fi/. [Accessed: 26-Nov-2019].

[33] A. Stein and B. Ramaseshan, "Towards the identification of customer experience touch point elements,” J. Retail. Consum. Serv., vol. 30, pp. 8-19, 2016.

[34] J. J. Marquez, A. Downey, and R. Clement, "Walking a Mile in the User' s Shoes : Customer Journey Mapping as a Method to Understanding User Experience," Internet Ref. Serv. Q., vol. 20, no. 3-4, pp. $135-150,2015$.

[35] G. C. R. Mark S Rosenbaum, Mauricio Losada Otalora, "How to create a realistic customer journey map," Bus. Horiz., vol. 60, no. 1, pp. 143-150, 2017.

[36] R. C. Camp, "Business Process Benchmarking: Finding and Implementing Best Practices," Milwaukee ASQC Qual. Press, 1995.

[37] H. L. Richardson, "Improve Quality Through Benchmarking," Transp. Distrib., no. Oct 1992; 33, pp. 32-37, 1992.

[38] "Mobile Operating System Market Share Worldwide," StatCounter, 2019. [Online]. Available: https://gs.statcounter.com/os-market-share/mobile/worldwide. [Accessed: 09-Dec-2019].

[39] "YouVisit," 2020. [Online]. Available: https://www.youvisit.com/. [Accessed: 10-Jan-2020]. 


\title{
Augmented Reality for Human Needs: an Ontology
}

\author{
Manal A. YAHYA ${ }^{\mathrm{a}, 1}$ and Ajantha DAHANAYAKE ${ }^{\mathrm{a}}$ \\ ${ }^{a}$ Lappeenranta University of Technology, Lappeenranta, Finland
}

\begin{abstract}
Augmented Reality is a display and interaction method of future computing. It augments digital information in real environments in text, audio, image, or video formats. Augmented reality can be more effective if supported by knowledge about human needs. Basic human needs are finite in number and with the right methods, they are detectable or predictable. To support efforts to develop a robust conceptual model, an ontology is developed that describes the structure and relations between the elements that combine the concepts of augmented reality, context information, and human needs. Ontology development is a knowledgedriven approach to represent data and reasoning. This paper focuses on linking the aforementioned concepts to enable correct data representation and reasoning. The research approach, process used, and the evaluation of the ontology is presented as well.
\end{abstract}

Keywords. Human needs, augmented reality, context-awareness, ontology, satisfiers, conceptual model

\section{Introduction}

An Ontology is a notion that has origins in Latin (onto + logy) which means the science of being or existence. The term is borrowed from philosophy and used in the field of computer science. Thomas Gruber set its definition to be "a specification of a representational vocabulary for a shared domain of discourse- definitions of classes, relations, functions, and other objects- is called an ontology" [1]. Ontologies are commonly used in semantic web technologies to ease computers' understanding and manipulation of internet data. The semantic web is built on a set of languages and technologies: The Extensible Markup Language (XML) provides the syntax. The Resource Description Framework (RDF) represents information about resources. The RDF schema enables a taxonomical organization, and the Web Ontology Language (OWL) provides expressive constructs to extend the RDF schema [2].

This paper presents an ontology that describes the use of Augmented Reality in the satisfaction of human needs.

\footnotetext{
${ }^{1}$ Corresponding Author, Manal Yahya; E-mail: manal.yahya@student.lt.fi.
} 


\subsection{Augmented Reality}

An Augmented Reality (AR) system embodies the following properties [3]:

- Enhances real environments by adding virtual objects.

- Works in real-time and provides interactivity.

- Provides the correct placement of virtual objects within the environment.

AR has many application areas such as education and learning [4], entertainment and gaming [5], food and beverage industry [6], health care [7], manufacturing [8], museums [9], space exploration [10], and tourism [11].

\subsection{Human Needs}

There is a difference between needs, desires, and satisfiers [12]:

- $\quad$ Need: the most fundamental requirement and the base for desires and satisfiers.

- Desire: personal and intentional, two possible desires may fulfill a certain need, however, it is a personal preference to choose one over the other.

- Satisfier: objects or states that fulfill needs or desires.

To keep the case general to all users, this research focuses on needs and satisfiers rather than desires. There are many theories of human needs. The goal of using MaxNeef's model [13] in this research is to provide a base for recognizing human needs rather than claiming that it is the only valid model.

\subsection{Problem Definition and Motivation}

The study of human needs has roots in psychology, economics, philosophy, sociology, anthropology, and social sciences; which indicates the importance of needs, and their satisfaction in human life. Despite that fact, there is still a shortage of incorporating human needs in information systems and tools [14]. Augmented reality is a growing field that may benefit from the utilization of human needs. A.K. Dey [15] defines context awareness as "A system that uses context to provide relevant information and/or services to the user, where relevancy depends on the user's task". This concept allows augmented reality applications to be adaptive [16]; nevertheless, we argue that incorporating the concept of human needs leverages the pervasiveness of context-aware systems. This creates a motive to study the development of augmented reality applications with a concentration on human needs detection and satisfaction. Previous studies focused on the development of a conceptual model to address this motive [17] [18]. The characteristics of conceptual models according to [19] are:

- Conceptual models have conceptual semantics

- They are ontologically grounded

- They make an ontological commitment

This reflects the base in which ontologies provide support for the development of conceptual models and the use of a foundational ontology to advance conceptual modeling [20].

From another perspective, some of the reasons that drive the effort of ontology development are [21]:

- Providing a shared understanding of the structure of information between people or software agents.

- Analyzing domain knowledge and separating it from operational knowledge. 
- Allowing domain knowledge reuse.

- Explicitly defining domain assumptions.

Based on the above reasoning, this paper looks into the development of an ontology that combines the concepts of augmented reality, human needs' satisfaction, and the detection of those needs from context and user information to support conceptual modeling. Previous studies on ontologies included a representation of needs as they are found in various theories of human needs [14], an ontology focusing only on Max-Neef's model of human needs [22], context modeling [23], and developing augmented reality based on ontological context awareness [16].

The contribution and novelty of this research involve developing a vocabulary that conceptualizes the relationship between humans, their needs, existing satisfiers and their possible representation in augmented reality; with the ultimate goal of providing augmented reality experiences to users according to their detected needs. This ontology should enhance and enrich our conceptual model, and enable the development of models and systems based on it.

In addition to the representation of the needs, this research requires building relations with many other elements affecting them such as the trigger of a need, the context in which it occurs, and the possible services that satisfy those needs.

To solve such a problem, a bottom-up approach in defining the cycle of needs is followed. The cycle starts with a balanced state, a trigger elicits a need which is then detected, a satisfier is matched with the need detected, which is then accepted or rejected, and feedback is provided by the user at the end of the cycle and a state of balance is retained. All the elements in this cycle must be represented clearly in the ontology under development.

This paper is divided as follows: Section 2 explains the research approach used in the development of the ontology. Section 3 discusses related work. Section 4 details the steps of ontology development and the outcomes. Then, section 5 presents the evaluation of the ontology. Finally, the discussion and the conclusion are in sections 6 and 7.

\section{Research Approach}

To develop the Augmented Reality for human needs ontology the methodologies described in [24] and [21] are chosen and combined. Those research papers describe detailed steps in ontology development. 
In [24] Methontology (a term coined by the researchers), the ontology lifecycle is defined by some states and activities (Fig. 1), these activities represent the method to develop the ontology. Below is a list of activities and important notes for each activity.

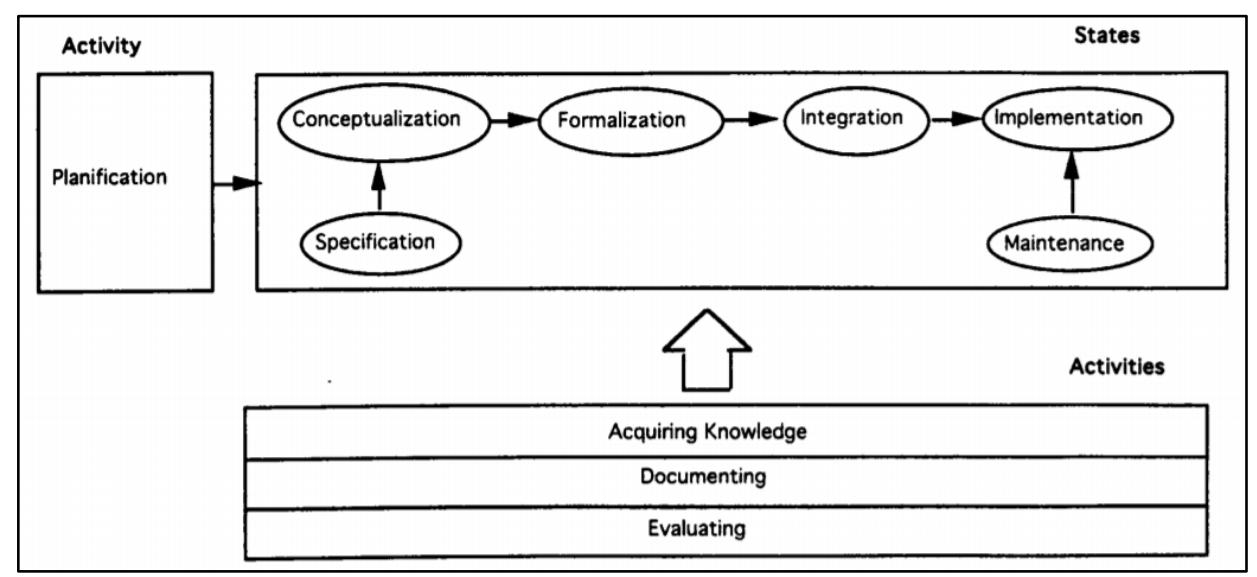

Figure 1. States and Activities of Ontology development [24]

\section{Methontology Phases [24]}

1. Specification: In this phase, the developers produce an ontology specification document that includes the purpose, level of formality, and the scope of the ontology under development.

2. Knowledge Acquisition: This step may be performed parallel to the specification phase and continues until not needed, Knowledge can be collected from many sources including books, experts, and research papers.

3. Conceptualization: this phase requires the development of a conceptual model based on the information from the specification document. The model should demonstrate the problem and its solution. To aid the development of the model, the authors suggest creating a Glossary of Terms (GT) including concepts, properties, instances, and verbs.

4. Integration: the authors suggest using previously built ontologies to derive terms and their definitions and descriptions. They also recommend building an integration document that describes the meaning of each term, the ontology it was derived from, and its name in the conceptual model.

5. Implementation: this activity requires the utilization of an ontology development environment or editor to build the complete ontology.

6. Evaluation: the ontology must be evaluated through verification and validation following predefined techniques based on the specification document.

7. Documentation: in addition to the publication resulting from building the ontologies, thorough documentation aids in keeping the ontology, maintaining, and reusing it.

The research on ontology development in [21] defines the following steps for the ontology development process:

1. Determine the domain and scope of ontology.

2. Consider reusing existing ontologies. 
3. Enumerate important terms in the ontology.

4. Define the classes and class hierarchy.

5. Define the properties of classes-slots.

6. Define the facets of the slots.

7. Create instances.

\section{Related Works}

This section reviews previous research on the topics at the intersection of augmented reality, human needs, context awareness, and ontology development.

The research presented in [16] studies adding adaptiveness to augmented reality by using ontologies to represent and analyze context. The defining factor in creating adaptivity is user activity. Four main elements compose the structure of the context model ontology: user ontology, device ontology, physical environment ontology, and service ontology. The developed AR application provides the required information in a three steps process:

1. The mobile device reads the URI object reference.

2. The device requests the relevant information from the server that returns an RDF describing the location of all relevant elements.

3. The user views a relevant augmented reality scene based on the situation.

The researchers developed a prototype that enables reading of Quick Response (QR) codes and the use of Near-Field Communication (NFC) technology. They provide an Ambient Assisted Living (AAL) scenario to evaluate the proposed system.

In [22], Dsouza presents the Fundamental Human Needs (FHN) ontology based on Max-Neef's Fundamental Human Needs theory [13]. The research defines the main entities in the conceptual model as Agent, Role, Need, Satisfier, and Existential Category. As a method to further describe the satisfier, he portrayed the existential category to be a categorization of the satisfier rather than the need. The author used the Methontology [24] method to develop the ontology and provided a verification and validation approach to evaluate it.

An ontology-based model for human activity representation in smart homes [25], proposes a model developed using the $\mathrm{NeON}$ methodology [26] and it consists of connected ontologies that fall in one of three categories: user ontologies, smart home context ontologies, and Activity of Daily Life (ADL) ontologies. The authors provided a case describing a scenario of an elder person living in a smart home environment; they applied the developed ontology on the case scenario for evaluation. The researchers identified two main approaches to model activity in the smart home environment:

- Data-Driven approach:

Learns activity models using data mining and machine learning methods.

- Advantages: good at processing uncertain knowledge, and is able to handle long-term temporal data.

- Disadvantages: suffers from the cold start problem; and lacks the reusability in mixed systems.

- Knowledge-Driven approach (including Ontology development) Uses knowledge engineering methods and representation formalisms.

- Advantage: effective representation abilities and reasoning methods for heterogeneous data. 
A study in [27] describes the use of cultural heritage ontology to provide location information in certain cultural sites. The researchers developed an application that provides contextual information in mobile augmented reality. They used the Korea Cultural Heritage Data Model (KCHDM) to collect and display contextual information. The KCHDM has five super-classes: actor, event, thing, time-span, and place. It also has eighty-four properties that connect the classes.

The researchers in [14] pinpointed the limited use of human needs concepts in information systems and the lack of needs assessment tools in computing. They developed an ontology called OPENEED and incorporated need theories and need assessment methods. OPENEED is composed of a core ontology (Fig. 2) that describes basic human needs theories, and extensions that describe adjectives to describe needs, and assessment methods. To validate the developed ontology, the researches performed a need assessment study in a Viennese district local community. The collection of ontologies in OPENEED has a set of rules in the Semantic Web Rule Language (SWRL)[28]. The authors identified five main applications of the ontology:

1. Enables publishing of related studies as "self-contained datasets".

2. Allows querying the results from single or multiple studies that make comparative analysis possible.

3. Enriches the data collected in studies by using SWRL rules.

4. Enables tracking the origin of assessments and evaluations whether by individuals, experts, or communities.

5. Allows representation of different sizes of data due to the modular structure of the ontology.

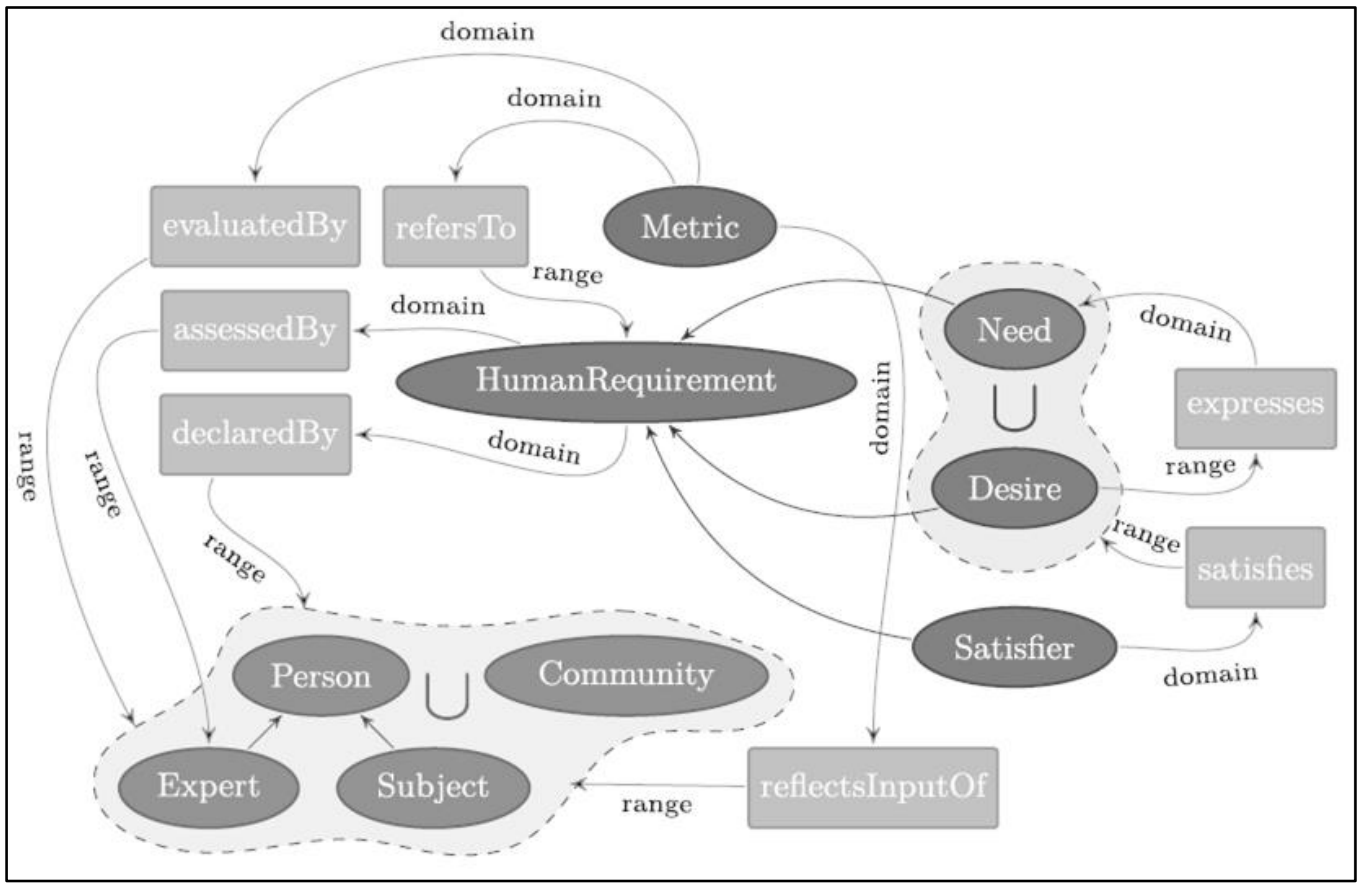

Figure 2 Outline of Core-OPEENEED Ontology [14] 


\section{Ontology Development}

This section provides details on creating an ontology that is capable of representing the information of a user, his/her needs, context, AR experiences as services and the relationship between these concepts.

\subsection{Specification: Determine the Domain and Scope of the Ontology}

To determine the domain and scope, several questions must be answered [21]:

- "What is the domain that the ontology will cover?

- For what we are going to use the ontology?

- For what types of questions, the information in the ontology should provide answers?

- Who will use and maintain the ontology?"

The domain defined for the "Augmented Reality for Human Needs" ontology includes the information needed to detect human needs, the representation of those needs, and elements of augmented reality experiences that represent the satisfiers or aids in the satisfaction of those needs. This ontology may be used for applications that match AR satisfiers with human needs when they arise. The ontology is used and maintained initially by the authors of this research.

As an additional help to answer the third question, the following competency questions are proposed so that a system using this ontology should be able to answer:

- Which human need is currently active for a user?

- What augmented reality experience can satisfy a certain need?

- What is the current internal state of the user?

An important task in the ontology development process is to create an Ontology Requirement Specification Document (ORSD) which describes the purpose of the ontology, its uses and users, and the requirements it should realize [29].

Table 1 Ontology Requirement Specification Document

AR for Human Need reference Ontology Requirements Specification Document

1 Purpose

The purpose of creating the ontology is to provide a knowledge model for the augmented reality domain that responds to various human needs and fulfills them.

2 Scope

The ontology should focus on the application development and representation part of the described system.

3 Implementation Language $\mathrm{RDF} / \mathrm{OWL}$ 

$4 \quad$ Intended End-Users
User 1. Application developer aiming to develop a system that detects human needs and matches it with AR satisfiers.
User 2. The Person who uses the application to find needs satisfiers.
User 3. The Company providing services as satisfiers.
User 4. Health care provider monitoring a user's change in health

5 Intended Uses

Use 1. Develop an application interface and background that reflect the ontology and match needs and satisfiers.

Use 2. Create a profile, monitor needs' triggers and view possible satisfiers

Use 3. Create AR satisfiers for various human needs

Use 4. Provide monitored health information

6 Ontology Functional Requirements

FR 1. The Ontology proposed must support user modeling. Static information, as well as dynamic context information about the user, must be supported.

FR 2. The ontology must support a human needs theory model, in this case, Max-Neef's model of human needs.

7 Pre-Glossary Terms

Augmented Reality, Human needs, satisfaction, balance, triggers, feedback

\subsection{Consider Reusing existing Ontologies}

After a deliberate search for ontologies that encompass the concepts of augmented reality and human needs, below are research papers that provide similar ideas and inspire the development of our proposed ontology:

1. FHN- Cloud-based Ontology solution for conceptualizing human needs. [22].

2. OpeNeed- Ontology for Representing Human Needs [14].

3. GUMO- The General User Model Ontology [30]

4. A Context Model based on Ontological Languages [23]

The user modeling part in this study is created based on the General User Model Ontology (GUMO)[30] [31]. It provides a detailed description of the user including physiological state, characteristics, personality, demographics, and emotional state.

The context ontology section is derived from the work of Hervás, Bravo and Fontecha [23]. Concepts from the device model ontology, the environment model ontology, and the information visualization ontology are incorporated in the developed ontology. The Human Needs section is derived from the FHN [22], and OpeNeed Ontologies [14].

\subsection{Conceptualization}

Conceptual Models are important instruments to represent engineering scenarios. They reflect our understanding of the world and aid in the "development of knowledge about 
these worlds" [32]. The model presented in Fig. 3 is application-dependent; it reflects the idea of a system capable of recognizing human needs and presenting augmented reality experiences that function as satisfiers or means for a satisfier for those needs. This model is based on previous research presented in [17] [18].

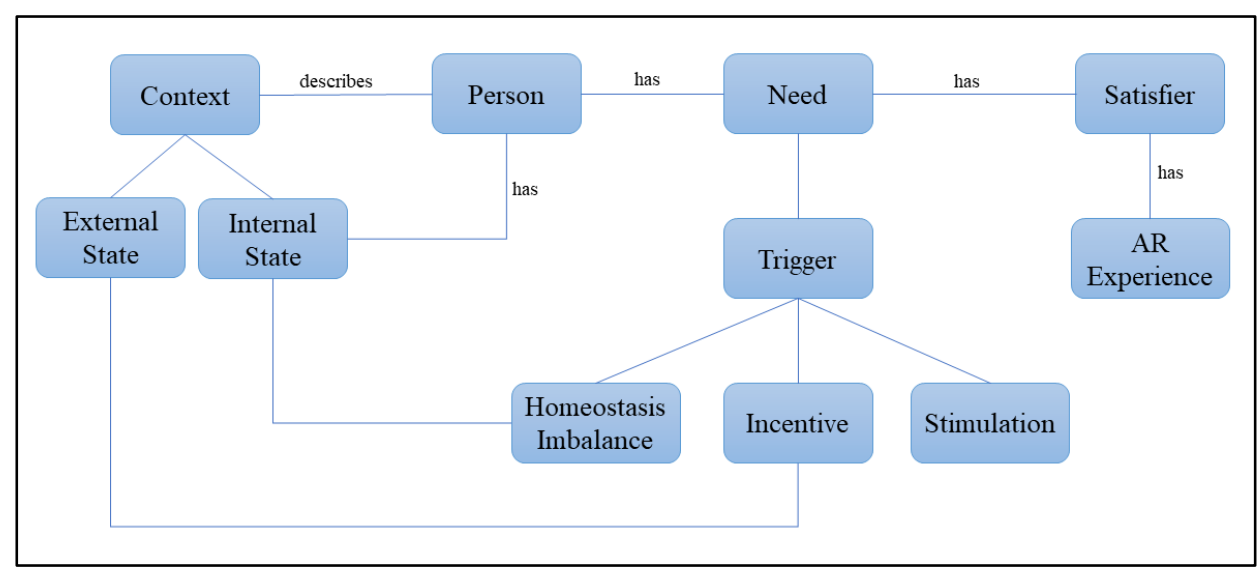

Figure 3. Conceptual Model of Augmented Reality for Human Needs Satisfaction

The main concepts in this model are:

- Person: the user of the system who has a profile and carries sensors to detect his/her basic needs.

- Context: describes the setting/ situation on which a person resides at a certain point in time. Context is divided into external and internal states.

○ External State: describes the environment, and all information related to it at a certain time including temperature, location, activity, social setting.

- Internal State: it portrays the internal state of a person, the change in this state may reflect the occurrence of a need. The main element to be checked in the internal state is the body's homeostasis.

- Need: is one of the basic needs in Max-Neef's model of fundamental human needs. The need is a combination of existential and axiological categories, having a format of Subsistence/Being need, or Subsistence/Having need, etc.

- Trigger: a trigger is a factor that activates a need. There are three types of triggers in psychology [33]. These are Homeostasis imbalance, incentive, and stimulation.

- Homeostasis Imbalance: is the internal state that reflects a malfunction in the body processes resulting in a rise of a need.

- Incentive: is an external positive or negative environmental stimulus that motivates a person.

- Stimulation: is an activity that causes excitement or pleasure.

- Satisfier: an object, idea, or social structure that contributes to the satisfaction of a need. 
- Augmented reality experience: is an experience that is developed to function as a satisfier of a basic human need or aid in the satisfaction of the need.

These concepts describe the problem and the possible solution for it. The ontology development activity of this research is based on this model to discover all related terms and expand the knowledge in this system domain.

\subsection{Enumerated Important Terms in the Ontology}

In addition to the pre-glossary terms identified in the ORSD in section 4.1, further concepts and terms are discovered by creating a Glossary of Terms (GT) that includes concepts, verbs, instances, and properties [24].

Table 1 below shows the most important terms to develop the Ontology within the subjects of Human, Need, Context, Augmented Reality. The list does not reflect the relations between the terms, it just lists them.

Table 1. Important terms in the ontology

\begin{tabular}{crrr}
\hline Human & Need & Context & \multicolumn{1}{c}{$\begin{array}{c}\text { Augmented } \\
\text { Reality }\end{array}$} \\
\hline User & Subsistence & Environment & Aatisfier \\
Profile & Protection & AR experience \\
Internal State & Affection & Object \\
Hemostasis Imbalance & Understanding & Incentive & Placement \\
& Participation & Stimulation & System \\
& Leisure & & Direct Satisfier \\
& Creation & & Indirect Satisfier \\
& & & Satisfaction \\
\hline
\end{tabular}

\subsection{Define the classes and the class hierarchy}

To define the classes and class hierarchy, the Protégé open-source ontology editor [34] is used. To develop the class hierarchy, a combination of top-down and bottom-up approaches are used for creating classes and connecting them in relations from top-level concepts to details and other times from middle concepts to more general ones (Fig. 4).

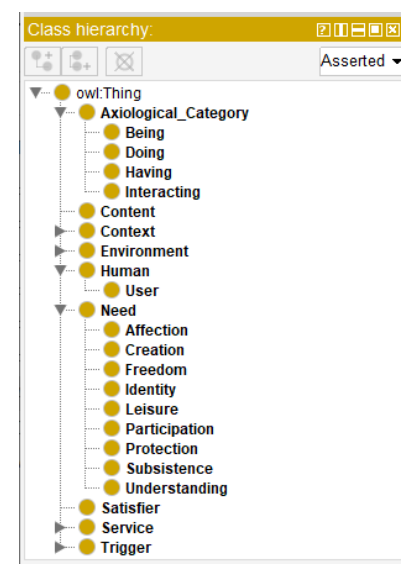

Figure 4 Protégé Class Hierarchy 


\subsection{Define the Properties and facets of Classes-Slots}

The properties of each class describe its structure. In the Protégé editor, there are two types of properties: objects properties that describe the relation between classes and data properties that describe the instances.

Classes can have many facets that describe their features. These include slot cardinality, slot-value type, and domain and range of a slot. For each of the classes identified, defined are the various facets of the properties in the Protégé tool. Each property is then linked to the class it describes (Fig. 5).

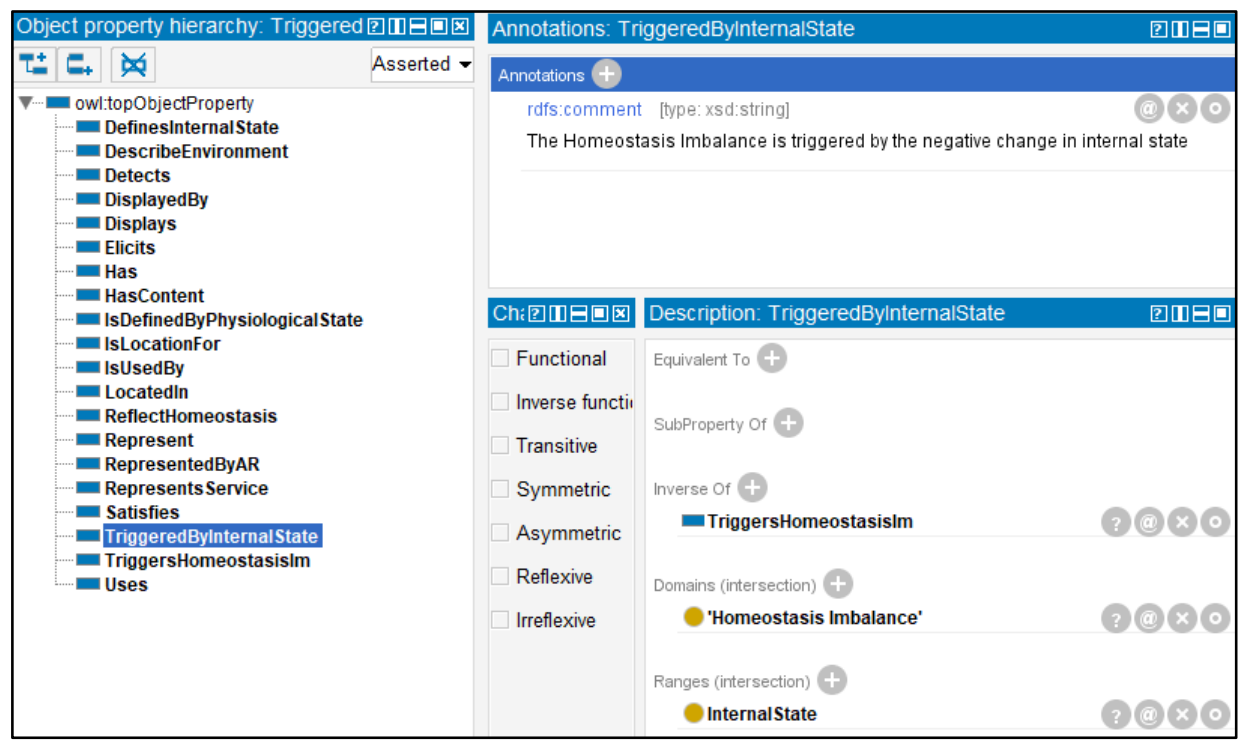

Figure 5 Define Properties and Facets of Classes

\subsection{Create Instances}

The creation of instances helps in answering the many queries the ontology must answer. An important role of instances is to validate the correct function of the ontology. In Web Protégé, the concept of instances is called individuals. To enhance the ontology, several instances are created for each of these classes: Human, Need, Satisfier, Augmented Reality experience, and Context for ontology evaluation.

\subsection{Ontology Visualization}

To better view the structure and relations in the developed ontology, a visualization is provided (Fig.6) using a web-based visualization tool for ontologies WebVOWL ${ }^{2}$.

${ }^{2}$ http://vowl.visualdataweb.org/webvowl.html 


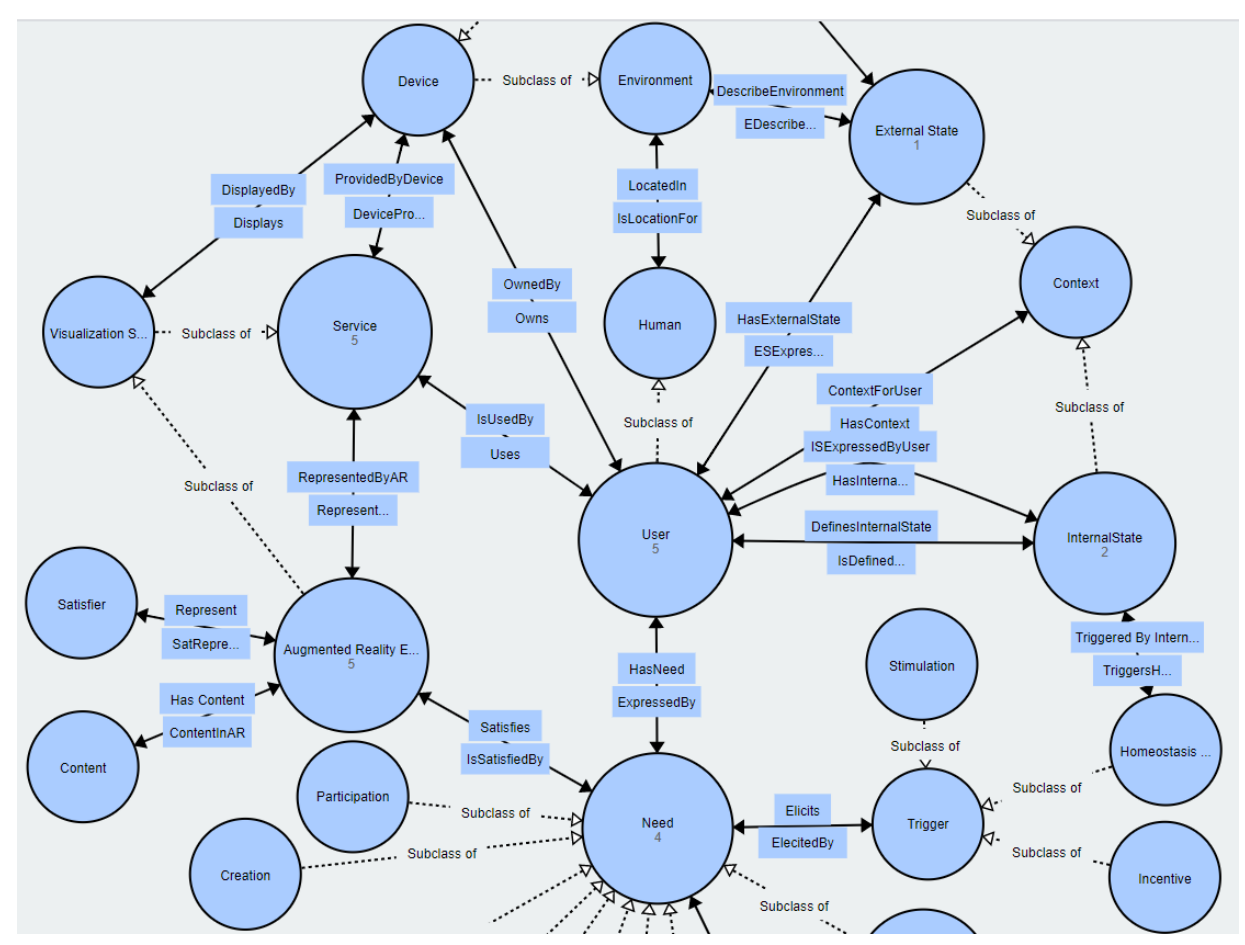

Figure 6 Augmented Reality for Human Needs Ontology Visualization

\section{Evaluation}

Many methods and metrics are developed for ontology evaluation. All these methods fall into one of two evaluation categories: verification, and validations. While verification is concerned with the correctness of the ontology, validation is concerned with developing the correct ontology [35].

For the evaluation of the AR for Human Needs ontology (AR-HN), the OOPS! Ontology Pitfall Scanner developed by Poveda-Villalon, Suarez-Figueroa, and GomezPerez [36] is used. The OOPS! Ontology Pitfall Scanner is a web-based tool that verifies ontologies' correctness by detecting possible errors. The developers of OOPS! provide a catalog of common pitfalls ${ }^{3}$ that are categorized into the following classification lists [37]:

\section{Evaluation Classification by Dimension:}

- Structural Dimension

- Functional Dimension

- Usability Profiling Dimension

Evaluation Classification by Criteria:

- Consistency

- Conciseness

${ }^{3} \underline{\mathrm{http}: / / o o p s . l i n k e d d a t a . e s / c a t a l o g u e . j \mathrm{sp}}$ 
- Completeness

The list of pitfalls possible to be detected by OOPS! Ontology Pitfall Scanner according to the abovementioned classification is presented in Fig.7 and Fig.8 as extracted from [37].

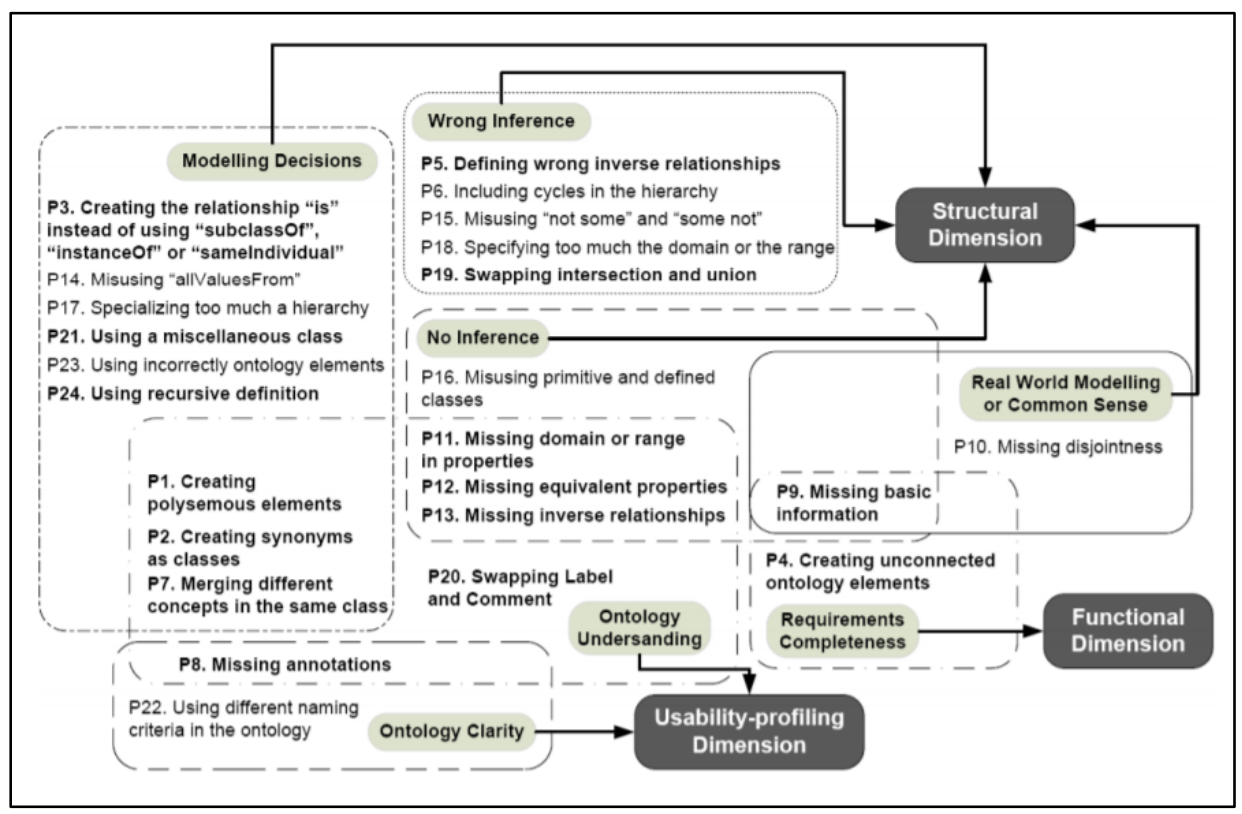

Figure 7 Classification of Pitfalls according to Dimensions [28]

\begin{tabular}{|c|c|c|}
\hline $\begin{array}{l}\text { Consistency } \\
\text { P1. Creating polysemous elements } \\
\text { P5. Defining wrong inverse relationships } \\
\text { P6. Including cycles in the hierarchy } \\
\text { P7. Merging different concepts in the same } \\
\text { class } \\
\text { P14. Misusing "allValuesFrom" } \\
\text { P15. Misusing "not some" and "some not" } \\
\text { P18. Specifying too much the domain or the range | } \\
\text { P19. Swapping intersection and union } \\
\text { P24. Using recursive definition }\end{array}$ & $\begin{array}{l}\quad \text { Completeness } \\
\text { P4. Creating unconnected } \\
\text { ontology elements } \\
\text { P9. Missing basic information } \\
\text { P10. Missing disjointness } \\
\text { P11. Missing domain or range in } \\
\text { properties } \\
\text { P12. Missing equivalent properties } \\
\text { P13. Missing inverse relationships } \\
\text { P16. Misusing primitive and defined } \\
\text { classes }\end{array}$ & $\begin{array}{l}\text { Conciseness } \\
\text { P2. Creating synonyms } \\
\text { as classes } \\
\text { P3. Creating the relationship "is" } \\
\text { instead of using "subclassOf", } \\
\text { "instanceOf" or "samelndividual" } \\
\text { P17. Specializing too much a } \\
\text { hierarchy } \\
\text { P21. Using a miscellaneous } \\
\text { class }\end{array}$ \\
\hline
\end{tabular}

Figure 8 Classification of Pitfalls according to Criteria [28] 


\subsection{Evaluating Correctness of the Ontology: Verification}

Ontology evaluation is an on-going process throughout the design and development stages. In this section, the correctness of the ontology is verified by comparing the results of the scans performed on the Augmented Reality for Human Needs ontology using the OOPS! Ontology Pitfall Scanner. The results of the scan done after the complete development of the ontology are presented as "AR-HNOnto Previous" in the charts. Alternatively, the results of the scan after addressing the issues detected by the first scan are represented as "AR-HNOnto Final".

The advanced options in OOPS! allows for general and specific scanning by dimension or criteria. For comparisons with the developed ontology, the scanner is being run on two developed ontologies that are named Good Ontologies by the world wide web consortium $\mathrm{W}^{3} \mathrm{C}^{4}$ :

- The Marine Ontology

- The Good Relations Ontology

The Marine, Good Relations, and Augmented Reality for Human Needs ontologies are comparable since the size difference is reasonable (Table 2).

Table 2 Sizes of the Compared Ontologies

\begin{tabular}{|l|l|l|}
\hline Ontology & Class Count & Logical Axiom Count \\
\hline Marine & 106 & 267 \\
\hline Good Relations & 38 & 450 \\
\hline Augmented Realty for Human Needs & 32 & 260 \\
\hline
\end{tabular}

\subsubsection{Verification by Dimensions Classification}

This step presents the results of the AR-HN ontology before and after corrections based on the structural, functional, and usability dimensions. The results are compared with the results obtained from the Marine and Good Relations ontologies.

${ }^{4}$ https://www.w3.org/wiki/Good Ontologies 


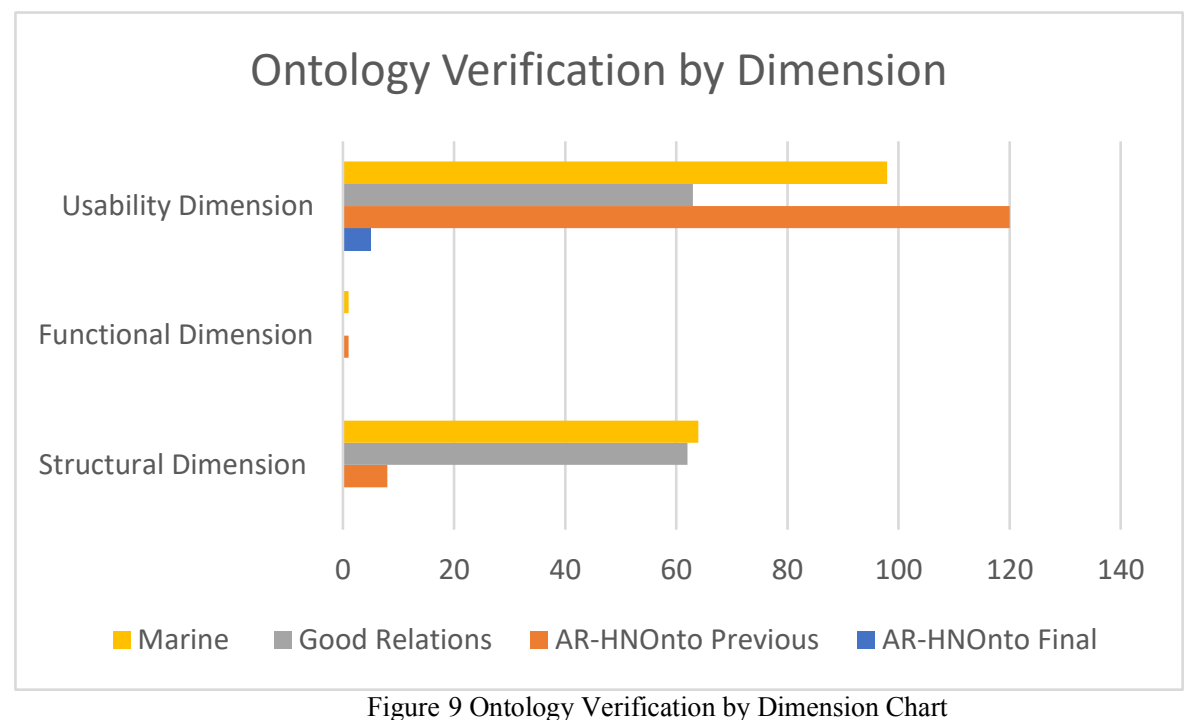

The high number of pitfalls (120 pitfalls) in the usability dimension is due to missing annotations which aid in the human readability of the ontology. After providing proper annotations in the form of labels and comments, the number of pitfalls decreased significantly.

\subsubsection{Verification by Criteria Classification}

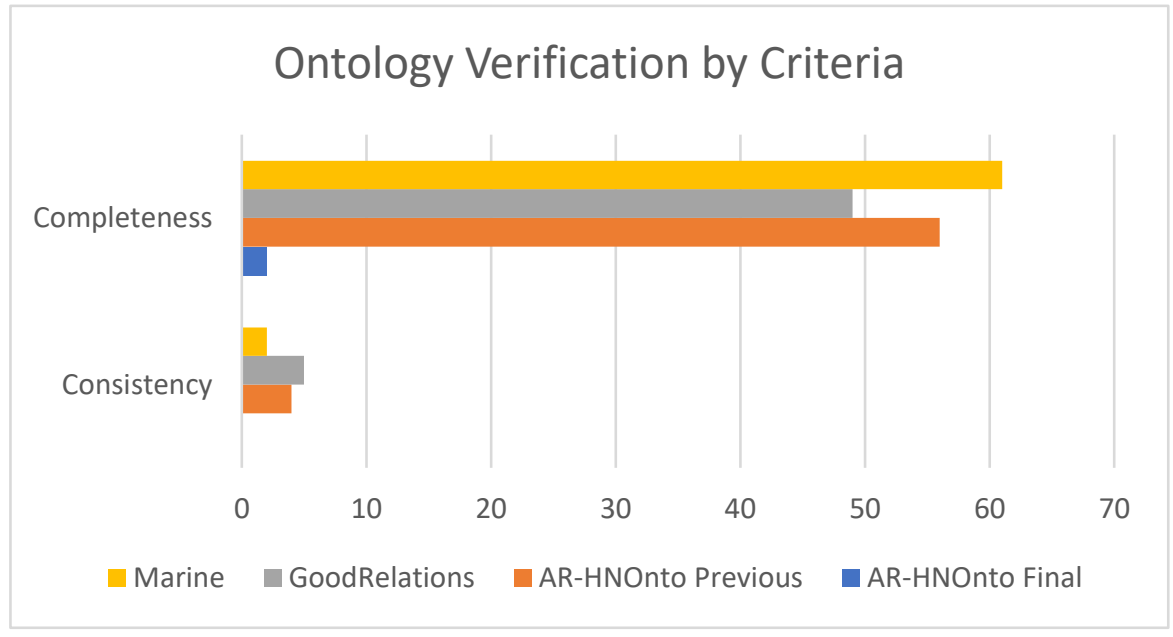

Figure 10 Ontology Verification by Criteria Chart

The results of the Conciseness criteria, which represent the bad practice in the ontology, returned zero pitfalls for all ontologies. Therefore, it is not included in the verification by criteria chart. The consistency result of the AR-HNOnto Previous is four pitfalls, and after correcting it AR-HNOnto Final has zero consistency pitfalls. 


\subsection{Ontology Evaluation via Competency Questions}

The importance of competency questions resides in ensuring the correct performance of the ontology-based on the defined requirements. This section evaluates the ontology based on the competency questions (initially presented in section 4.1):

- Which human need is currently active for a user?

- What augmented reality experience can satisfy a certain need?

- What is the current internal state of the user?

A manual approach is followed to evaluate using the competency questions as shown in [22]. For that purpose, test data for various class individuals are provided, in addition to a list for expected answers for the competency questions to perform the evaluation.

The first step in this evaluation is to set up the test data; 17 individuals are created as follows:

- Five User Individuals: Adam, Brad, Emma, Kareem, Maya

- Three Context Individuals: InternalContextBalanced, InternalContextImbalanced, ExternalConextImbalanced

- Four Need Individuals: SubsistenceNeed, AffectionNeed, LeisureNeed, UnderstandingNeed

- Five AR Individuals: ARFoodService, ARHealth Service, AREntertainmentService, ARTransportationService, AREduationalService Then, by setting a total of 14 asserted facts on the individuals. The facts are random and they reflect a relationship between the major classes. Table 3 presents the test data, the asserted facts and the total facts per individual.

Table 3 Test Data Individuals, Asserted, and Inferred Facts

\begin{tabular}{|c|c|c|c|}
\hline Individual & Asserted Facts & $\begin{array}{c}\text { No. of } \\
\text { Asserted } \\
\text { Facts }\end{array}$ & $\begin{array}{c}\text { No. of } \\
\text { Inferred } \\
\text { Facts }\end{array}$ \\
\hline Adam & $\begin{array}{l}\text { HasInternalState }=\text { InternalContext } \\
\text { Imbalanced } \\
\text { HasNeed= SubsistenceNeed } \\
\text { NeedActive = Active }\end{array}$ & 3 & \\
\hline Brad & $\begin{array}{l}\text { HasInternalState }=\text { InternalContext } \\
\text { Balanced }\end{array}$ & 1 & \\
\hline Emma & HasNeed = LeisureNeed & 1 & \\
\hline \multicolumn{4}{|l|}{ Kareem } \\
\hline Maya & $\begin{array}{l}\text { HasNeed }=\text { AffectionNeed } \\
\text { HasNeed }=\text { UnderstandingNeed }\end{array}$ & 2 & \\
\hline InternalContextBalanced & Internal State Balanced & 1 & 1 \\
\hline InternalContextImbalanced & Internal State Imbalanced & 1 & 2 \\
\hline \multicolumn{4}{|l|}{ ExternalContextImbalanced } \\
\hline SubsistenceNeed & NeedActive $=$ Active & 1 & 3 \\
\hline AffectionNeed & & & 1 \\
\hline LeisureNeed & $\begin{array}{l}\text { Is Satisfied } B y= \\
\text { AREntertainmentService }\end{array}$ & 1 & 1 \\
\hline UnderstandingNeed & & & 2 \\
\hline ARFoodService & Satisfies $=$ SubsistenceNeed & 1 & \\
\hline ARHealthService & Satisfies $=$ SubsistenceNeed & 1 & \\
\hline
\end{tabular}




\begin{tabular}{|l|r|r|c|}
\hline AREntertainmentService & & & 1 \\
\hline AREducationalService & Satisfies= UnderstandingNeed & 1 & \\
\hline ARTransportationService & & & \\
\hline & Total & 14 & 11 \\
\hline
\end{tabular}

The next step is to translate the competency questions into Description Logic (DL) queries to be executed against the ontology. Returning the expected answer for each of the competency questions indicates that the ontology meets the requirement.

To execute the DL Queries, the Pallet (Incremental) reasoner is used to classify the ontology. Afterward, running the DL queries to retrieve the results.

Competency Question 1: Which human need is currently active for a user?

DL Query: Need and ExpressedBy value Maya

Expected Answer: a list of needs for a specific user

Result:

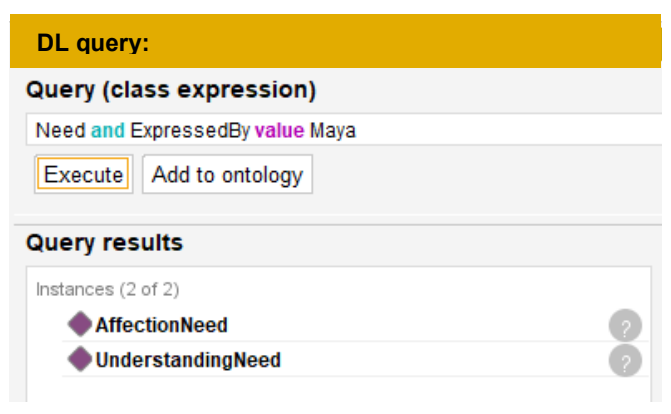

Figure 11 Active needs query Execution in Protégé

Competency Question 2: What augmented reality experience can satisfy a certain need?

DL Query: AugmentedRealityExperience and Satisfies value SubsistenceNeed

Expected Answer: a list of augmented reality services that satisfy the subsistence needs

Result:

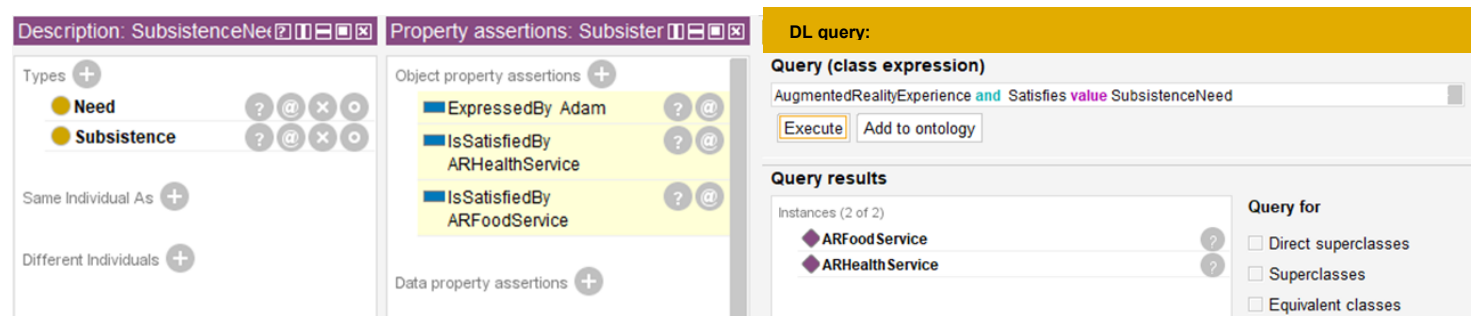

Figure 12 AR experience query execution in Protégé

Competency Question 3: What is the current internal state of the user?

DL Query: InternalState and ISExpressedByUser value Emma

Expected Answer: the internal state of the user 
Result:

\begin{tabular}{l} 
DL query: \\
Query (class expression) \\
InternalState and ISExpressedBy User value Emma \\
Execute Add to ontology \\
Query results \\
Instances (1 of 1) \\
InternalContextImbalanced \\
\hline
\end{tabular}

Figure 13 Internal state query execution in Protégé

\section{Discussion}

Section 5, provides the evaluation of the Augmented Reality for Human Needs ontology for its correctness and completeness using the OOPS! Ontology Pitfall Scanner. Also presented is the evaluation of the ontology via the competency questions to check whether it provides answers to the queries it is meant to answer. The evaluations show positive results. Using the OOPS! Ontology Pitfall Scanner, the tool pinpointed minor, important, and critical pitfalls which are fixed to improve the ontology. The charts in section 5.1, which compare the AR-HN ontology to major ontologies, show that it scored well in the Structural, and Functional dimensions. The usability dimension scan detected a high number of pitfalls caused by missing annotations. The completeness criteria contained many pitfalls as well. The consistency criteria returned one pitfall which is fixed. Addressing the pitfalls, the final ontology is much improved.

Section 5.2 present the evaluation of competency questions. 17 individuals are created and 14 facts are declared; the Pallet (Incremental) reasoner returned 11 inferred facts. For such a small number of asserted facts, a total of 11 inferred facts is a promising result. The query results of the competency questions reflect correct inference and outcome, which proves that the ontology satisfies the related requirement.

\section{Conclusion and Future work}

This research presents the conceptualization of an OWL vocabulary that defines the Augmented Reality for Human Needs class structure and relationships. The research details the development of the ontology based on the Methontology [24] and the ontology development guide [21]. The evaluation process for the verification and validation of the ontology is also presented. The augmented reality for human needs ontology shows promising evaluation results. It provides the vocabulary and concepts of relationships for further development of the conceptual model. As a future work and for additional development of the Augmented Reality for Human Needs ontology, the integration with a foundational ontology, to increase interoperability and reusability, is considered. 


\section{References}

[1] T.R. Gruber, "'A translation approach to portable ontology specifications," Knowledge acquisition, vol. 5, no. 2, pp. 199-220, 1993.

[2] W3C, "'W3C Semantic Web Vocabulary,", 2015.

[3] R. Azuma, Y. Baillot, R. Behringer, S. Feiner, S. Julier and B. MacIntyre, "'Recent advances in augmented reality," IEEE Comput.Graphics Appl., vol. 21, no. 6, pp. 3447, 2001.

[4] M. Dunleavy and C. Dede, "'Augmented reality teaching and learning,", pp. 735745, 2014.

[5] J.M. Patricio, M.C. Costa, J.A. Carranca and B. Farropo, "'SolarSystemGO-An augmented reality based game with astronomical concepts,", pp. 1-3, 2018.

[6] G. Waltner, M. Schwarz, S. Ladstätter, A. Weber, P. Luley, H. Bischof, M. Lindschinger, I. Schmid and L. Paletta, "'Mango-mobile augmented reality with functional eating guidance and food awareness,", pp. 425-432, 2015.

[7] W.S. Khor, B. Baker, K. Amin, A. Chan, K. Patel and J. Wong, "'Augmented and virtual reality in surgery - the digital surgical environment: applications, limitations and legal pitfalls," Annals of translational medicine, vol. 4, no. 23, 2016.

[8] M.A. Frigo, E.C. da Silva and G.F. Barbosa, "'Augmented Reality in Aerospace Manufacturing: A Review."," Journal of Industrial and Intelligent Information, vol. 4, no. 2, 2016.

[9] R. Hammady, M. Ma and N. Temple, "'Augmented reality and gamification in heritage museums,", pp. 181-187, 2016.

[10] G.A. Boy and D. Platt, "'A situation awareness assistant for human deep space exploration,", pp. 629-636, 2013.

[11] D. Han, M.C. tom Dieck and T. Jung, "'User experience model for augmented reality applications in urban heritage tourism," Journal of Heritage Tourism, vol. 13, no. 1, pp. 46-61, 2018.

[12] A. Kaiser and F. Kragulj, "'Bewextra: Creating and inferring explicit knowledge of needs in organizations," Journal of Futures Studies, vol. 20, no. 4, pp. 79-98, 2016.

[13] M.A. Max-Neef, "'Human scale development,", 1991.

[14] S. Human, F. Fahrenbach, F. Kragulj and V. Savenkov, "'Ontology for Representing Human Needs,", pp. 195-210, 2017.

[15] A.K. Dey, "'Understanding and using context," Personal and ubiquitous computing, vol. 5, no. 1, pp. 4-7, 2001.

[16] R. Hervás, J. Bravo, J. Fontecha and V. Villarreal, "'Achieving adaptive augmented reality through ontological context-awareness applied to aal scenarios," Journal of Universal Computer Science, vol. 19, no. 9, pp. 1334-1349, 2013. [17] M.A. Yahya, "'A Context-Aware Personalization Model for Augmented Reality Applications", Master's Thesis in Software Engineering, February, 2016. 
[18] M. Yahya and A. Dahanayake, "'A Needs-Based Personalization Model for Context Aware Applications," Frontiers in Artificial Intelligence and Applications, vol. 292, pp. 63-82, 2016.

[19] N. Guarino, G. Guizzardi and J. Mylopoulos, "'On the Philosophical Foundations of Conceptual Models,", 2019.

[20] G. Guizzardi, G. Wagner, J.P.A. Almeida and R.S. Guizzardi, "'Towards ontological foundations for conceptual modeling: The unified foundational ontology (UFO) story," Applied ontology, vol. 10, no. 3-4, pp. 259-271, 2015.

[21] N.F. Noy and D.L. McGuinness, "'Ontology development 101: A guide to creating your first ontology,", 2001.

[22] S.D. Dsouza, "'Cloud-based Ontology Solution for Conceptualizing Human Needs,", 2015.

[23] R. Hervás, J. Bravo and J. Fontecha, "'A Context Model based on Ontological Languages: a Proposal for Information Visualization." J.UCS, vol. 16, no. 12, pp. 1539-1555, 2010.

[24] M. Fernández-López, A. Gómez-Pérez and N. Juristo, "'Methontology: from ontological art towards ontological engineering,", 1997.

[25] Q. Ni, Pau de la Cruz, Iván and A.B. García Hernando, "'A foundational ontologybased model for human activity representation in smart homes," Journal of Ambient Intelligence and Smart Environments, vol. 8, no. 1, pp. 47-61, 2016.

[26] NeOn Project, "'NeOn Toolkit,", vol. 2020, no. 11 Jan, 2014.

[27] H. Kim, T. Matuszka, J. Kim, J. Kim and W. Woo, '"An ontology-based augmented reality application exploring contextual data of cultural heritage sites,", pp. 468-475, 2016.

[28] I. Horrocks, P.F. Patel-Schneider, H. Boley, S. Tabet, B. Grosof and M. Dean, "'SWRL: A semantic web rule language combining OWL and RuleML," W3C Member submission, vol. 21, no. 79, pp. 1-31, 2004.

[29] M. Suárez-Figueroa, A. Gomez-Perez and B. Villazon Terrazas, "'How to Write and Use the Ontology Requirements Specification Document,", vol. 5871, 2009.

[30] D. Heckmann, E. Schwarzkopf, J. Mori, D. Dengler and A. Kröner, "'The user model and context ontology gumo revisited for future web 2.0 extensions," Contexts and Ontologies: Representation and Reasoning, pp. 37-46, 2007.

[31] D. Heckmann, "'Ubiquitous User Modeling,", ed. 297, 2006.

[32] B. Thalheim, "'Conceptual Models and Their Foundations,", pp. 123-139, 2019.

[33] D.G. Myers, "'Psychology,", ed. Tenth edition, international edition, 2013.

[34] Anonymous "'Protege,", vol. 2020, no. 11 Jan.

[35] A. Gomez-Perez, M. Fernández-López and O. Corcho, "'Ontological Engineering: with examples from the areas of Knowledge Management, e-Commerce and the Semantic Web,", 2006.

[36] M. Poveda-Villalón, M.C. Suárez-Figueroa and A. Gómez-Pérez, "'Validating ontologies with oops!", pp. 267-281, 2012.

[37] M. Poveda Villalon, M.C. Suárez-Figueroa and A. Gómez-Pérez, "'A double classification of common pitfalls in ontologies,", 2010. 


\title{
Cross-Cultural Religious Tourism with Impression Distance Search System
}

\author{
Piyaporn NURARAK ${ }^{\mathrm{a}, 1}$, Shiori SASAKI ${ }^{\mathrm{a}}$, Irene Erlyn Wina RACHMAWAN ${ }^{\mathrm{a}}$ and \\ Yasushi KIYOKI ${ }^{a}$
}

${ }^{\mathrm{a}}$ Graduate School of Media and Governance, Keio University, Japan

\begin{abstract}
Cross-cultural religious tourism is computational to promote crosscultural communication and understanding according to impression distance. Our motivation to implement semantic search with an emotion-oriented context into the proposed system is to realize global tourism recommendations expressed in different cultures. The objectives of this paper are (1) to find the religious places by using the tour-user's emotional distance, (2) to find similar religious places not only in the same culture but also in the different cultures with the tourist's emotional distance calculations. Experimental results demonstrate the feasibility and applicability of this method.
\end{abstract}

Keywords. Cross-cultural, Impression distance, Religious, Tourism.

\section{Introduction}

Nowadays, from a quantitative perspective, and in general terms, Tourism Industry is one of the economic sectors that is growing increasingly all over the world, according to the United Nations World Tourism Organization (UNWTO)[1]. Low-cost travels make new tourism industry. In several globalized social phenomena, people across the world can easily exchange ideas and values whether they are environmental, technological, cultural, or economic settings. Consequently, this exchange has enabled the circulation of massive knowledge amongst people to understand one another better and this has hugely boosted tourism.

We aim to recognize an emotional context in religious places. We provide a function for tourists to find impressions for tourist places. Numerous studies have proposed various methods for information retrieval using "Kansei" and semantic computing. Semantic computing is an important approach to analysis and information retrieval. The function using emotion finds the essential features related to an impression context, assisting tourists to find the desired places. The described of the Mathematical Model of Meaning (MMM) [2] [3] for emotion-based information retrieval and emotional based retrieval system. MMM has proposed a meta-database knowledge system with a new search method of semantic associative [2] [3]. In those studies, semantic-searching in sensibility and measuring the impression were realized in semantic spaces. In the Mathematical Model of Meaning [2] [3] [4] [5], an orthogonal semantic space is created

\footnotetext{
${ }^{1}$ Corresponding Author, Graduate School of Media and Governance, Keio University, Shonan Fujisawa Campus, 5322 Endo, Fujisawa, Kanagawa, 252-0882, Japan; Email: pnurarak@sfc.keio.ac.jp.
} 
for semantic associative search. In the impression distance in semantic search system, our method is using a vector space and realizes calculations on relationships between place-data mapped in semantic space and emotion.

This paper is organized as follows: Section 2 shows the overview of our impression distance search system for cross-cultural religious tourism and its key technologies. Section 3 shows the implementation of a prototype system. Section 4 presents our experimental results of the implemented prototype system, and the conclusion and future work in section 5 .

\section{Impression Distance Search System}

This paper proposes cross-cultural religious tourism, with computations for promoting cross-cultural communication and understanding according to impression distance. Our motivation to implement a semantic search with emotion-oriented contexts is to make global tourism recommendations expressed in different cultures. The objectives of this paper are (1) to find the religious places by using the tourist's emotional distance, (2) to find similar religious places not only in the same culture but also in the different cultures that have the same tourist's emotion.

\subsection{Creation of Kansei Spaces}

A Kansei space of religious tourism is connected for impression distance. A Kansei vector space $\mathrm{K}$ is created as $\mathrm{p}+4$ dimensions by the impression which expresses religious place name, image, latitude, longitude, and $\mathrm{p}$ impression words of religious tourism as shown in Figure 1. The matrix is defined as Kense $i$ vector space $\mathrm{K}_{\mathrm{i}}$, when i define cultural (i=1,2,3,..m). In the row, vector data $T_{i j}(j=1,2, \ldots, n)$ composed and their impressions are registered.

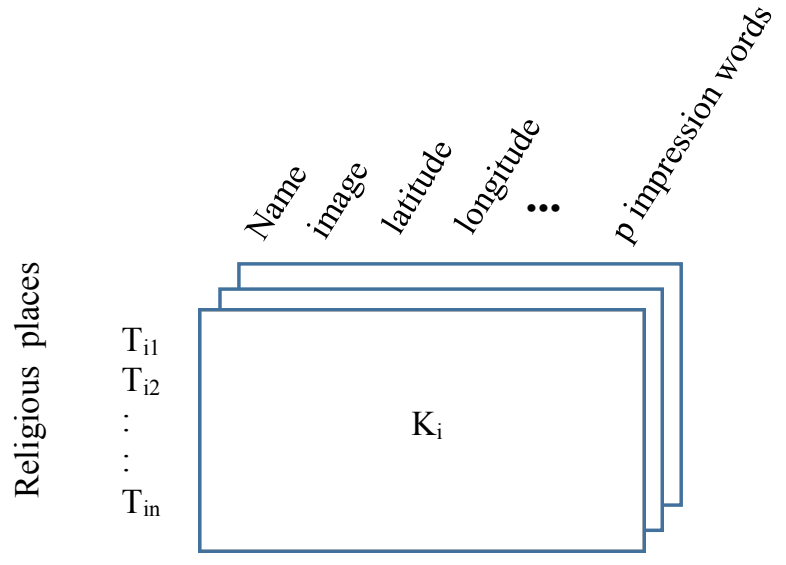

Figure 1. A Kansei-Tour Vector Space 


\subsection{Cosine Similarity}

Cosine similarity measures the cosine of the angle between two non-zero vectors of an inner product space. This similarity measurement is particularly concerned with orientation, rather than magnitude. In short, two cosine vectors that are aligned in the same orientation will have a similarity measurement of 1 , whereas two vectors aligned perpendicularly will have a similarity of 0 . If two vectors are diametrically opposed, meaning they are oriented in exactly opposite directions (i.e. back-to-back), then the similarity measurement is -1 . Often, however, cosine similarity is used in positive space, between the bounds 0 and 1 . Cosine similarity is not concerned, does not measure, differences is the magnitude and is only a representation of similarities in orientation ([6] [7]).

The Cosine similarity measurement begins by finding the cosine of the two non-zero vectors. This can be derived using the Euclidean dot product formula which is written as:

$$
A \cdot B=\|A\|\|B\| \cos \theta
$$

Then, given the two vectors and the dot product, the cosine similarity is defined as:

$$
\text { Cosine Similarity }=\cos \theta=\frac{A \cdot B}{\|A\|\|B\|}=\frac{\sum_{i=1}^{n} A_{i} B_{i}}{\sqrt{\sum_{i=1}^{n} A_{i}^{2}} \sqrt{\sum_{i=1}^{n} B_{i}^{2}}}
$$

In other words, the cosine of the angle between two vectors is the inner product of the vectors, after they have been normalized to unit length. If A and B are frequency vectors for impressions, a higher relate impression will have a long vector and a rarely relate impression will have a short vector, yet the impression might be synonyms. Cosine captures the idea that the length of the vectors is irrelevant; the important thing is the angle between the vectors. The cosine ranges from -1 when the vectors point in opposite directions ( $\theta$ is 180 degrees) to +1 when they point in the same direction ( $\theta$ is 0 degrees). When the vectors are orthogonal ( $\theta$ is 90 degrees), the cosine is zero. With raw impression vectors, which necessarily cannot have negative elements, the cosine cannot be negative, but weighting and smoothing often introduce negative elements.

\section{Implementation of a prototype system}

To recognize an emotion context, the impression distance search system realizes a function for the users to express their impressions, such as interesting, clam, elegant, etc., for religious tourism places in the same culture or different cultures. This function finds the most essential features related to emotion contexts, given as the user's impression of 
the religious place queries. The overview of this system is shown in Figure 2. This system consists of 3 processes: (a) Data aggregation, (b) Space creation and integration and (c) Data retrieval and querying. The procedure of proposed idea is represented in Figure 2.

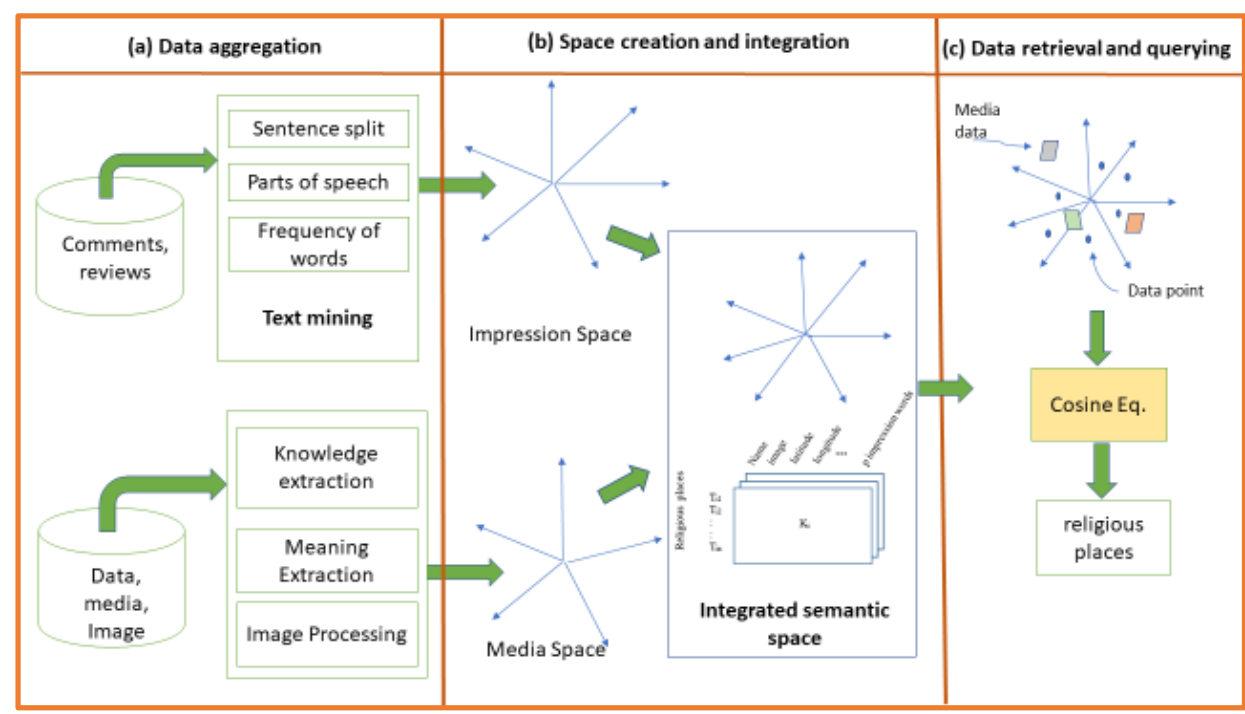

Figure 2. System overview and operating procedure.

\section{(a) Data aggregation}

In the process, we apply information on the religious places by using digital documents and traveler reviews from 48 travel websites. Then we using text mining, such as tf-idf to filtering and frequently words, to find impressions of the places. Moreover, the images of the places have been used to find the meaning of the places.

\section{(b) Space creation and integration}

After that, we select useful data to create impression space and media space and integrated both spaces into semantic space.

\section{(c) Data retrieval and querying}

We use cosine similarity to find the places that have the same impression or close to in the same culture or different culture by ranking..

\section{Experiment results}

To clarify the feasibility and applicability of our method, we have implemented our impression distance search system for temple datasets. In experimental study, we use 10 
Thailand temples located in Bangkok area, 10 Japan Temples located in Tokyo area,10 Finland churches located in Helsinki area and 13 emotion contexts as dataset. We set retrieved result by ranking that can be (1) show the Thailand temples/Japan temples /Finland churches by using emotion context, (2) show the temples in the same religiouscultures that has the same emotion context and (3) show the temples in the other two different religious-cultures that have the same emotion context by queries.

\subsection{Experiment 1}

10 Thailand temples were collected and people were asked to judge the implied emotion of each temples. Table 1 is the result of impression words were extracted for Thailand temples. We also calculated the precision and recall to evaluate the retrieval performance of our method (illustrated in Table 2). The total precision is $63 \%$ and the total recall is $72 \%$.

Table 1. Experimental results of emotion detection for 10 Thailand temples

\begin{tabular}{|c|c|c|c|c|c|c|c|c|c|c|c|c|c|}
\hline \multirow{2}{*}{ id } & \multicolumn{13}{|c|}{ Detected Emotion } \\
\hline & $\mathrm{C} 1$ & $\mathrm{C} 2$ & $\mathrm{C} 3$ & $\mathrm{C} 4$ & $\mathrm{C} 5$ & $\mathrm{C} 6$ & $\mathrm{C} 7$ & $\mathrm{C} 8$ & $\mathrm{Cg}$ & $\mathrm{C} 10$ & $\mathrm{C} 11$ & $\mathrm{C} 12$ & $\mathrm{C} 13$ \\
\hline 1 & 0.3 & 0.2 & 0.2 & 0.8 & 0.4 & 0.7 & 0.4 & 0.5 & 0.5 & 0.8 & 0.4 & 0.3 & 0.4 \\
\hline 2 & 0.6 & 0.4 & 0.2 & 0.8 & 0.2 & 0.8 & 0.5 & 0.6 & 0.8 & 0.8 & 0.1 & 0.8 & 0.1 \\
\hline 3 & 0.5 & 0.4 & 0.1 & 0.3 & 0.5 & 0.4 & 0.6 & 0.8 & 0.3 & 0.8 & 0.1 & 0.8 & 0.1 \\
\hline 4 & 0.3 & 0.3 & 0.1 & 0.4 & 0.6 & 0.3 & 0.3 & 0.3 & 0.3 & 0.3 & 0.2 & 0.3 & 0.4 \\
\hline 5 & 0.2 & 0.1 & 0.3 & 0.3 & 0.6 & 0.1 & 0.4 & 0.3 & 0.3 & 0.4 & 0.6 & 0.3 & 0.2 \\
\hline 6 & 0.6 & 0.5 & 0.1 & 0.4 & 0.4 & 0.7 & 0.6 & 0.8 & 0.3 & 0.5 & 0.3 & 0.2 & 0.1 \\
\hline 7 & 0.4 & 0.2 & 0.3 & 0.3 & 0.5 & 1 & 0.6 & 0.6 & 0.9 & 0.5 & 0.2 & 0.8 & 0.1 \\
\hline 8 & 0.4 & 0.1 & 0.4 & 0.3 & 0.6 & 0.1 & 0.3 & 0.3 & 0.3 & 0.5 & 0.7 & 0.2 & 0.2 \\
\hline 9 & 0.1 & 0.1 & 0.2 & 0.3 & 0.4 & 0.4 & 0.2 & 0.1 & 0.3 & 0.6 & 0.4 & 0.1 & 0.4 \\
\hline 10 & 0.7 & 0.5 & 0.1 & 0.7 & 0.4 & 0.1 & 0.9 & 0.9 & 0.8 & 0.4 & 0.2 & 0.7 & 0.1 \\
\hline
\end{tabular}

$\mathrm{C} 1=$ interesting, $\mathrm{C} 2=$ mysterious, $\mathrm{C} 3=$ rural-like, $\mathrm{C} 4=$ relaxed, $\mathrm{C} 5=$ calm, $\mathrm{C} 6=$ unique, $\mathrm{C} 7=$ elegant, $\mathrm{C} 8=$ precious, $\mathrm{C} 9=$ faithful, $\mathrm{C} 10=$ historical, $\mathrm{C} 11=$ quiet, $\mathrm{C} 12=$ bustling, $\mathrm{C} 13=$ commonplace.

Detected emotion means implied emotion of religious places which were detected by our system

Table 2. Retrieval performance for Thailand temples

\begin{tabular}{ccccccccccccccc}
\hline & $\mathrm{C} 1$ & $\mathrm{C} 2$ & $\mathrm{C} 3$ & $\mathrm{C} 4$ & $\mathrm{C} 5$ & $\mathrm{C} 6$ & $\mathrm{C} 7$ & $\mathrm{C} 8$ & $\mathrm{C} 9$ & $\mathrm{C} 10$ & $\mathrm{C} 11$ & $\mathrm{C} 12$ & $\mathrm{C} 13$ & Average \\
\hline $\begin{array}{c}\text { Precision } \\
(\%)\end{array}$ & 100 & 75 & 42.9 & 62.5 & 50 & 100 & 50 & 80 & 50 & 60 & 57.1 & 57.1 & 40 & 63.43 \\
Recall (\%) & 85.7 & 60 & 75 & 100 & 75 & 100 & 50 & 66.7 & 33.3 & 75 & 66.7 & 80 & 66.7 & 71.85 \\
\hline
\end{tabular}

To start with Thailand culture, we select impression keyword as "calm", similarity places as "Wat Arun", Similarity Thai to Japanese as "Wat Pho" and Similarity Thai to Finland as "Wat Suthat", shown in Figure 3.

The impression distance search system shows the result of Thai temples that relate to this impression by queries. As shown in Figure 4, the closest to the impression of "calm" are "Wat Benjamabhopit", "Wat Traimit" and "Wat Suthat" that has 0.6 in similarity. On the other hand, "Wat Pho" has 0.2 that means this temple is not good place to visit if the tourist wants to calm. 


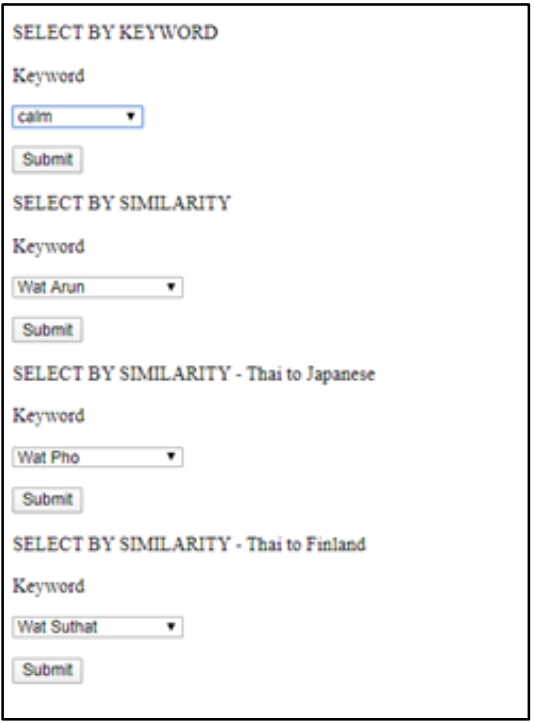

Figure 3. Selected impression and Thailand temples.

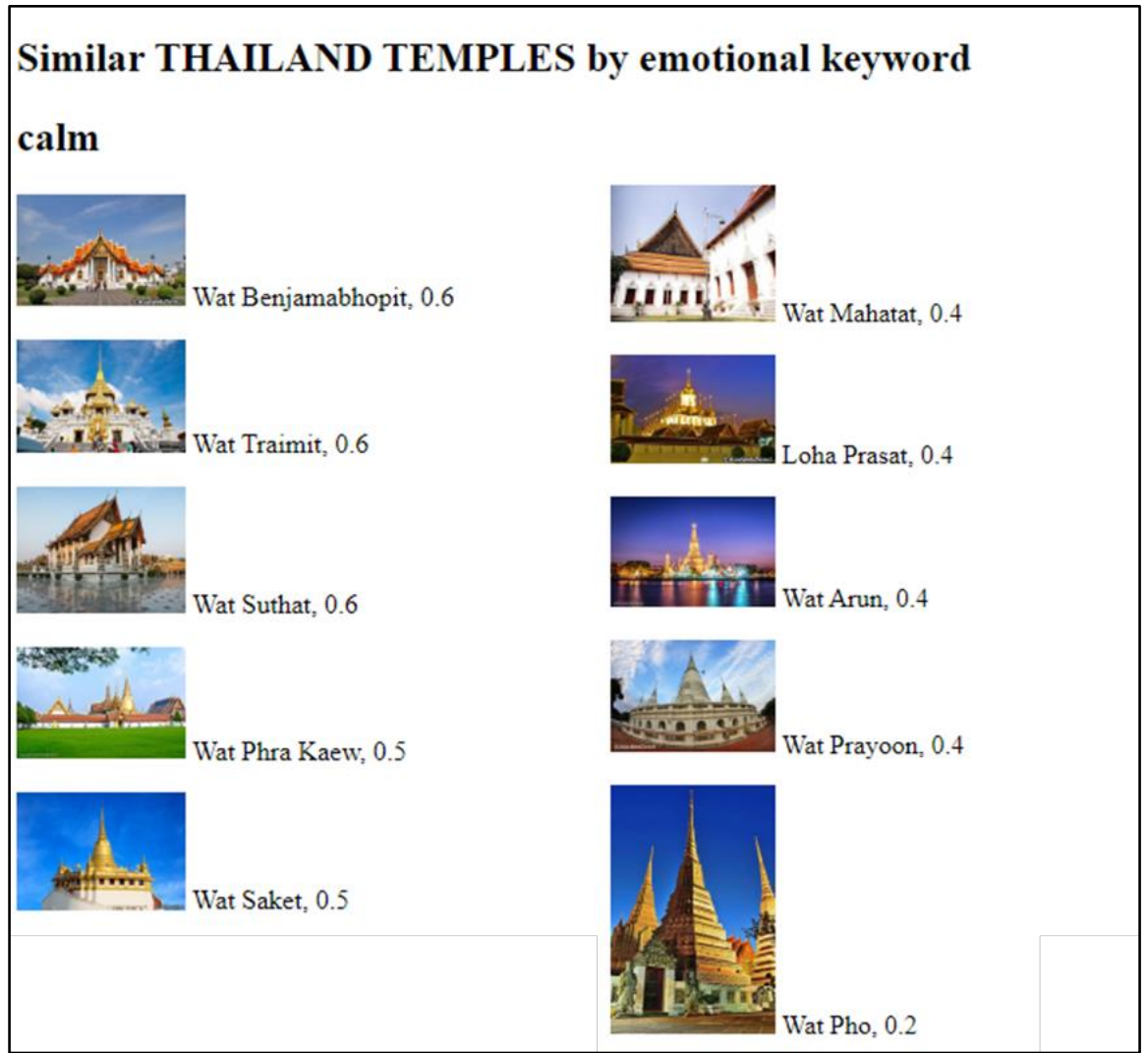

Figure 4.Thailand temples by "clam" impression. 
Figure 5 shown Thailand temples that give the tourist feeling the same emotion as "Wat Arun" that means if tourist knows "Wat Arun" well and want to find the other temples in Bangkok that given the same impression with, this system give you the answer. Figure 6 presents Japan temples that give the tourists feeling same as "Wat Pho" in Thailand.

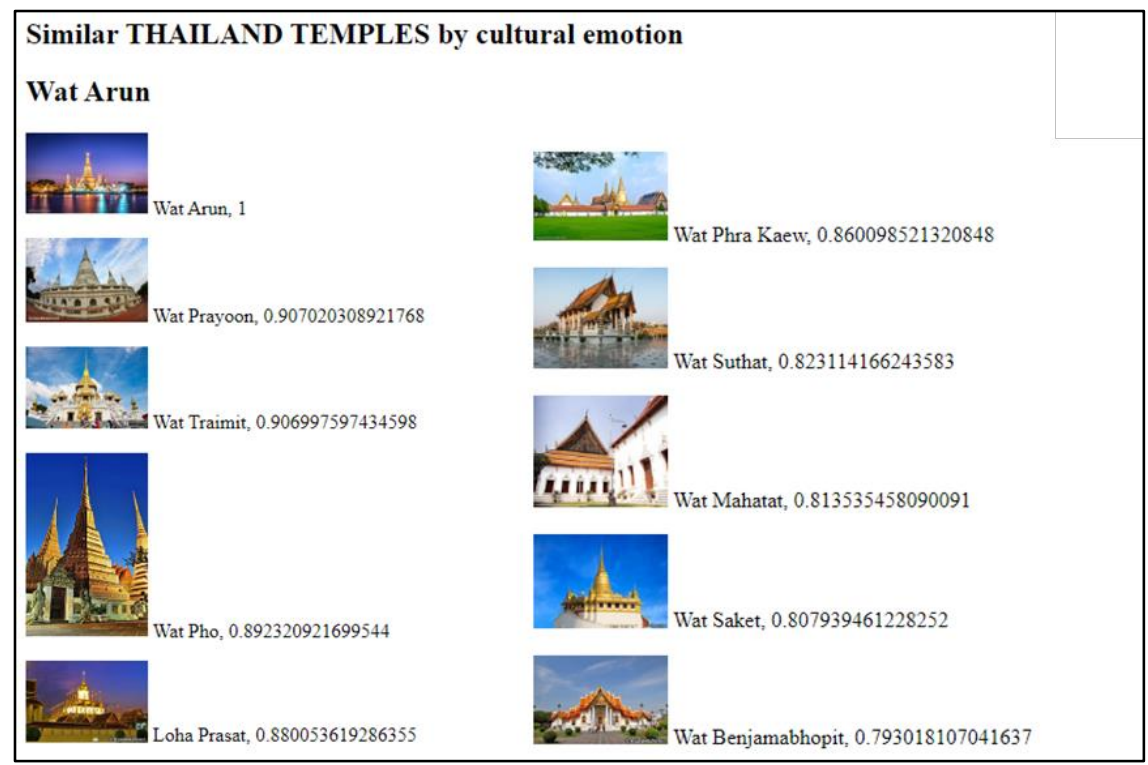

Figure 5. Search results of Thailand temples by similar to "Wat Arun" impression.

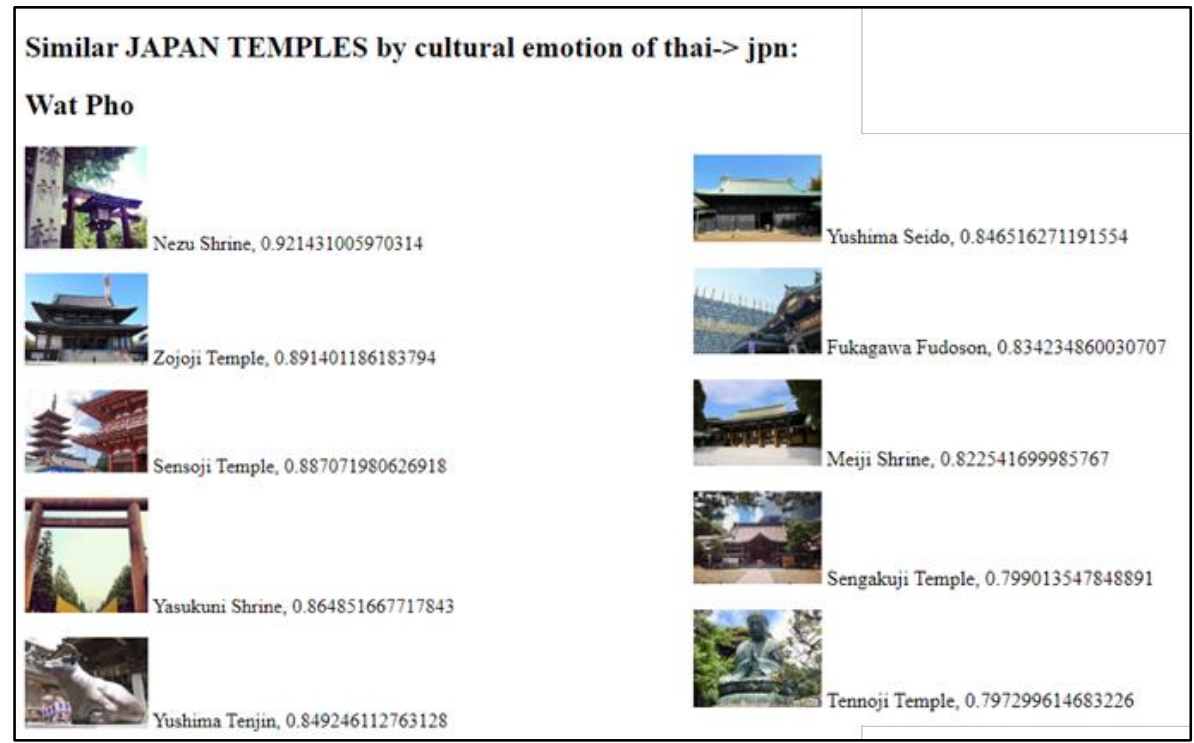

Figure 6. Japan temples by similar to "Wat Pho" impression. 
Figure 7. shows Finland Churches that have the same impression as "Wat Suthat". Even though Finland churches and Thailand temples are not the same religion, this system can find the emotional distance by using context.

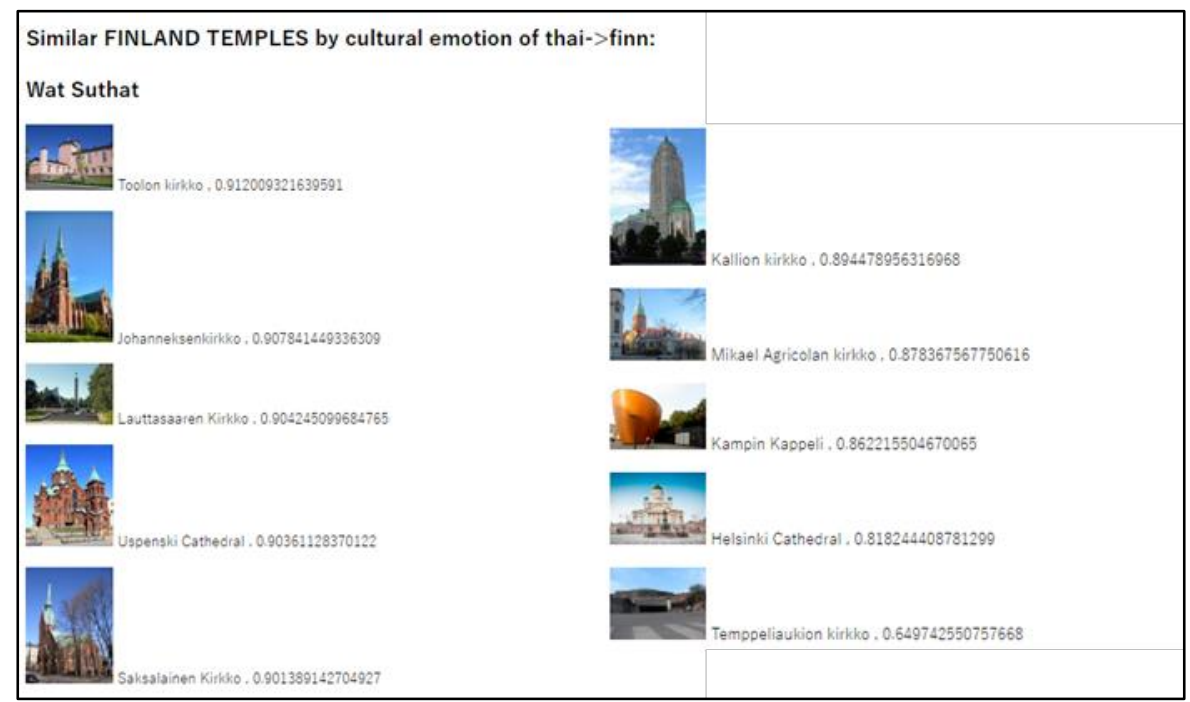

Figure 7. Search results of Finland churches in the same impression as "Wat Suthat".

\subsection{Experiment 2}

In a similar way, an experiment was done with Japan temples. The detail results of temples retrieval are shown in Table 3 . The precision and recall of information retrieval are shown in Table 4 . The total precision is $70 \%$ and the total recall is $68 \%$.

Table 3. Experimental results of emotion detection for 10 Japan temples

\begin{tabular}{cccccccccccccc}
\hline id & \multicolumn{1}{c}{} & \multicolumn{10}{c}{ Detected Emotion } \\
& $\mathrm{C} 1$ & $\mathrm{C} 2$ & $\mathrm{C} 3$ & $\mathrm{C} 4$ & $\mathrm{C} 5$ & $\mathrm{C} 6$ & $\mathrm{C} 7$ & $\mathrm{C} 8$ & $\mathrm{C} 9$ & $\mathrm{C} 10$ & $\mathrm{C} 11$ & $\mathrm{C} 12$ & $\mathrm{C} 13$ \\
\hline 1 & 0.6 & 0.3 & 0.5 & 0.2 & 0.6 & 0.1 & 0.2 & 0.4 & 0.6 & 0.6 & 0.4 & 0.6 & 0.1 \\
2 & 0.3 & 0.1 & 0.1 & 0.2 & 0.1 & 0.5 & 0.2 & 0.3 & 0.6 & 0.2 & 0.1 & 0.8 & 0.3 \\
3 & 0.4 & 0.1 & 0.3 & 0.6 & 0.3 & 0.2 & 0.3 & 0.3 & 0.6 & 0.7 & 0.3 & 0.4 & 0.1 \\
4 & 0.5 & 0.1 & 0.7 & 0.6 & 0.8 & 0.9 & 0.2 & 0.3 & 0.8 & 0.5 & 0.8 & 0.3 & 0.2 \\
5 & 0.6 & 0.4 & 0.7 & 0.3 & 0.5 & 0.3 & 0.3 & 0.4 & 0.5 & 0.7 & 0.4 & 0.4 & 0.1 \\
6 & 0.2 & 0.1 & 0.3 & 0.4 & 0.1 & 0.3 & 0.1 & 0.1 & 0.5 & 0.2 & 0.1 & 0.1 & 0.3 \\
7 & 0.1 & 0.2 & 0.1 & 0.2 & 0.2 & 0.7 & 0.1 & 0.1 & 0.5 & 0.3 & 0.3 & 0.5 & 0.3 \\
8 & 0.4 & 0.1 & 0.5 & 0.6 & 0.7 & 0.3 & 0.3 & 0.2 & 0.6 & 0.5 & 0.2 & 0.5 & 0.1 \\
9 & 0.4 & 0.2 & 0.3 & 0.3 & 0.4 & 0.3 & 0.2 & 0.2 & 0.6 & 0.5 & 0.4 & 0.4 & 0.1 \\
10 & 0.3 & 0.5 & 0.5 & 0.3 & 0.2 & 0.3 & 0.1 & 0.3 & 0.5 & 0.6 & 0.5 & 0.1 & 0.1 \\
\hline \hline
\end{tabular}

$\mathrm{C} 1=$ interesting, $\mathrm{C} 2=$ mysterious, $\mathrm{C} 3=$ rural-like, $\mathrm{C} 4=$ relaxed, $\mathrm{C} 5=$ calm, $\mathrm{C} 6=$ unique, $\mathrm{C} 7=$ elegant, $\mathrm{C} 8=$ precious, $\mathrm{C} 9=$ faithful, $\mathrm{C} 10=$ historical, $\mathrm{C} 11=$ quiet, $\mathrm{C} 12=$ bustling, $\mathrm{C} 13=$ commonplace.

Detected emotion means implied emotion of religious places which were detected by our system

Table 4. Retrieval performance for Japan temples

\begin{tabular}{ccccccccccccccc}
\hline & $\mathrm{C} 1$ & $\mathrm{C} 2$ & $\mathrm{C} 3$ & $\mathrm{C} 4$ & $\mathrm{C} 5$ & $\mathrm{C} 6$ & $\mathrm{C} 7$ & $\mathrm{C} 8$ & $\mathrm{C} 9$ & $\mathrm{C} 10$ & $\mathrm{C} 11$ & $\mathrm{C} 12$ & C13 & Average \\
\hline $\begin{array}{c}\text { Precision } \\
(\%)\end{array}$ & 100 & 40 & 40 & 100 & 100 & 100 & 33.33 & 28.57 & 100 & 75 & 60 & 75 & 60 & 70.15 \\
Recall (\%) & 66.67 & 40 & 50 & 100 & 62.5 & 100 & 25 & 50 & 75 & 60 & 50 & 100 & 100 & 67.63 \\
\hline \hline
\end{tabular}


This experiment from the Japanese site, shows the Japanese temples by using an emotion context and using Japan temples. In addition, by select Japan temples will show Thailand temples and Finland churches that have the same impression distance. Figure 8 shows the selected emotion and Japan temples. Figure 9 show the result of "elegant" impression.

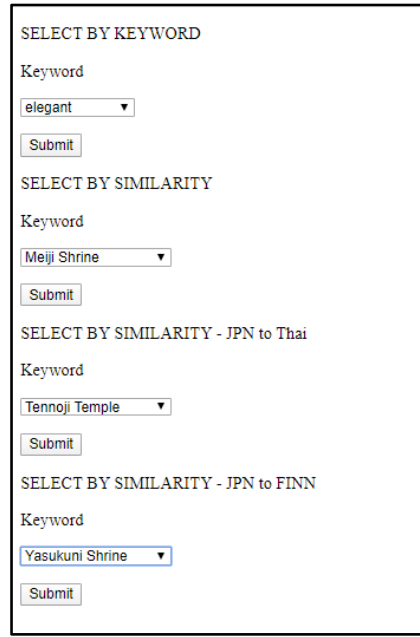

Figure 8. Selected impression and Japan temples.

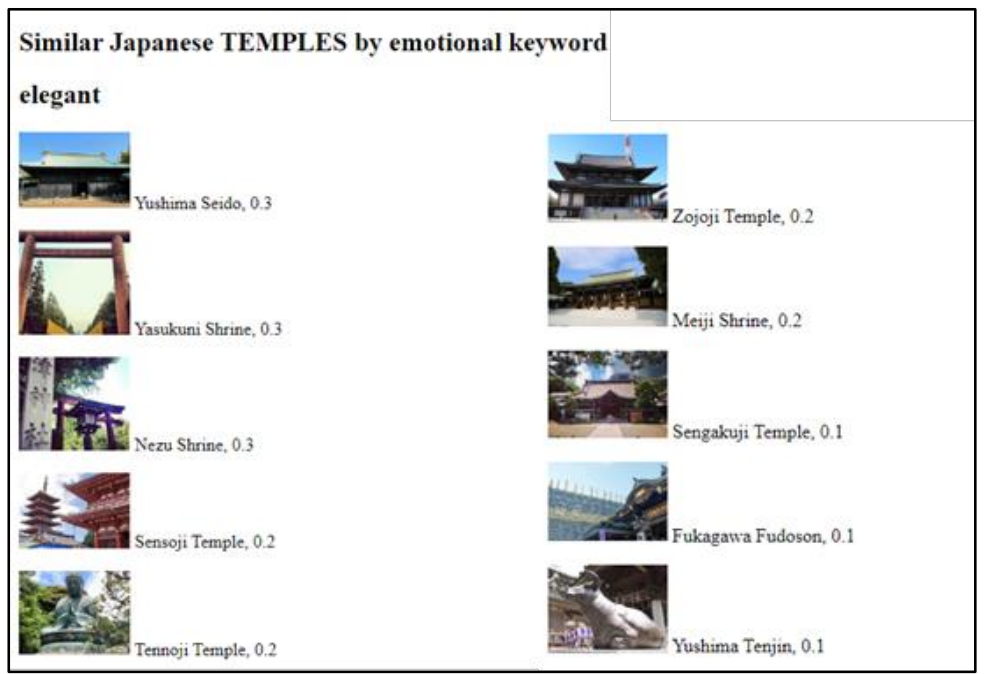

Figure 9. Japan temples with "elegant" impression.

Figure 10 shows Japan Temples that have the same emotion context with "Meiji shrine" and Thailand temples that have the same impression as "Tennoji temple" represent in Figure 11 


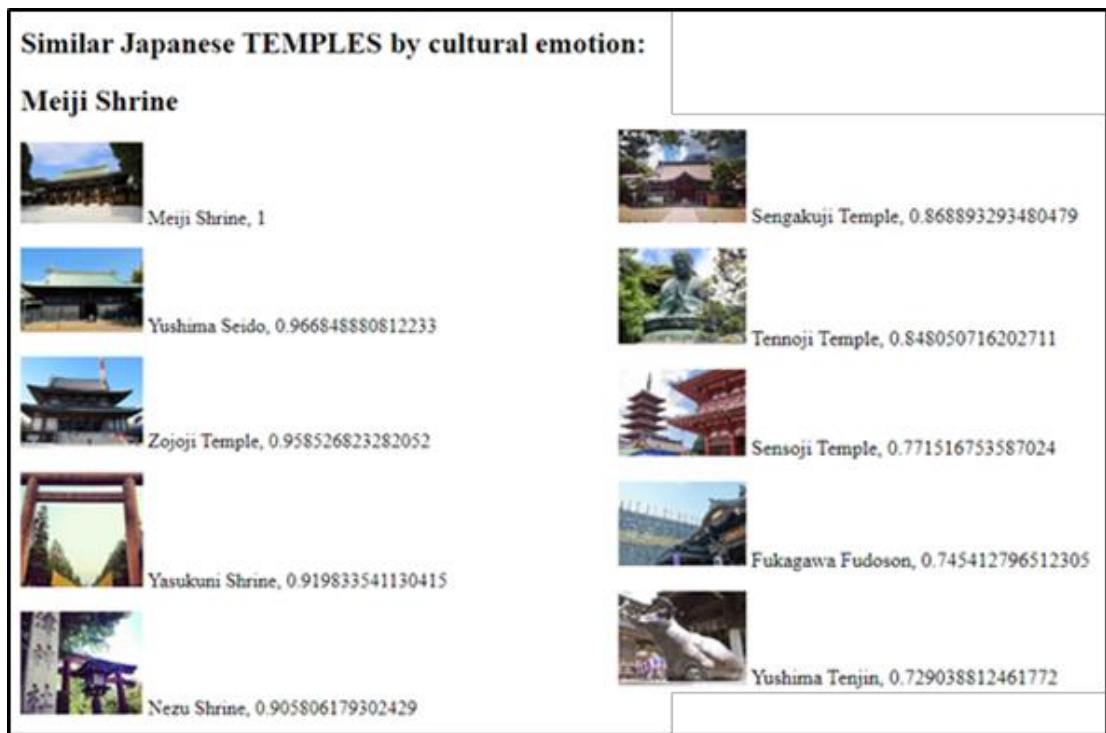

Figure 10. Search results of Japan temples with "Meiji shrine".

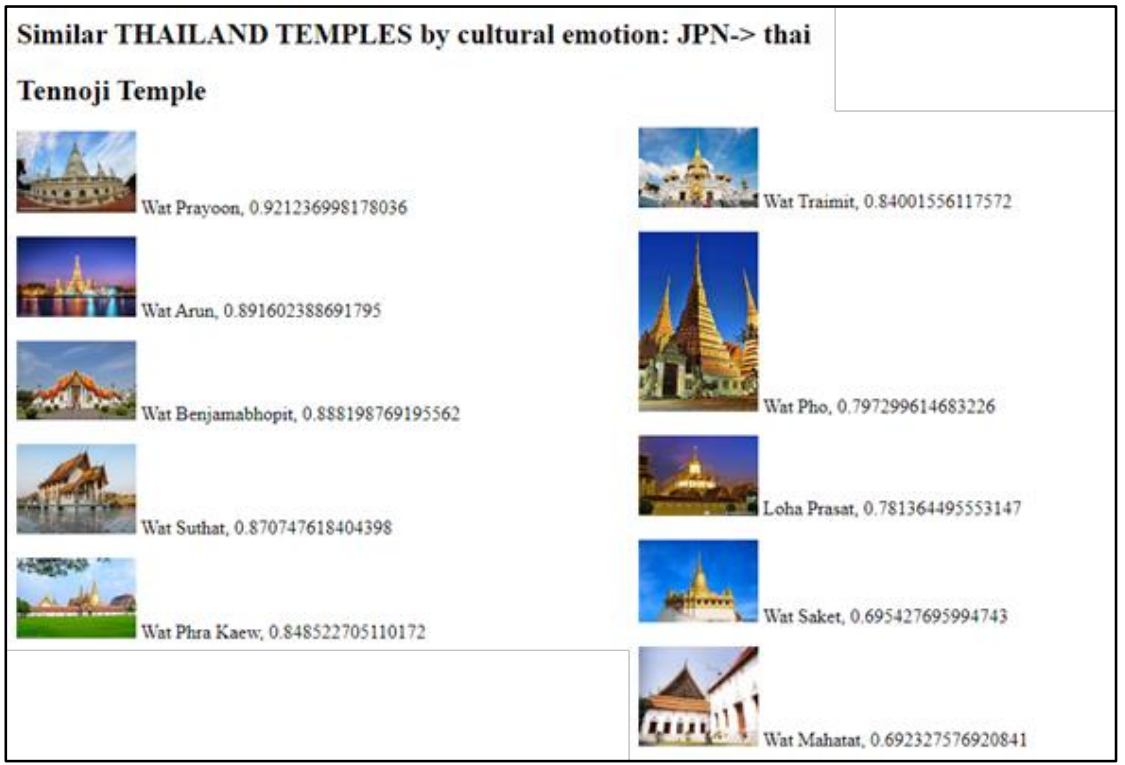

Figure 11. Thailand temples similarity with "Tennoji temple".

Figure 12 shows Finland churches that have impression distance close to "yasukuni shrine". 


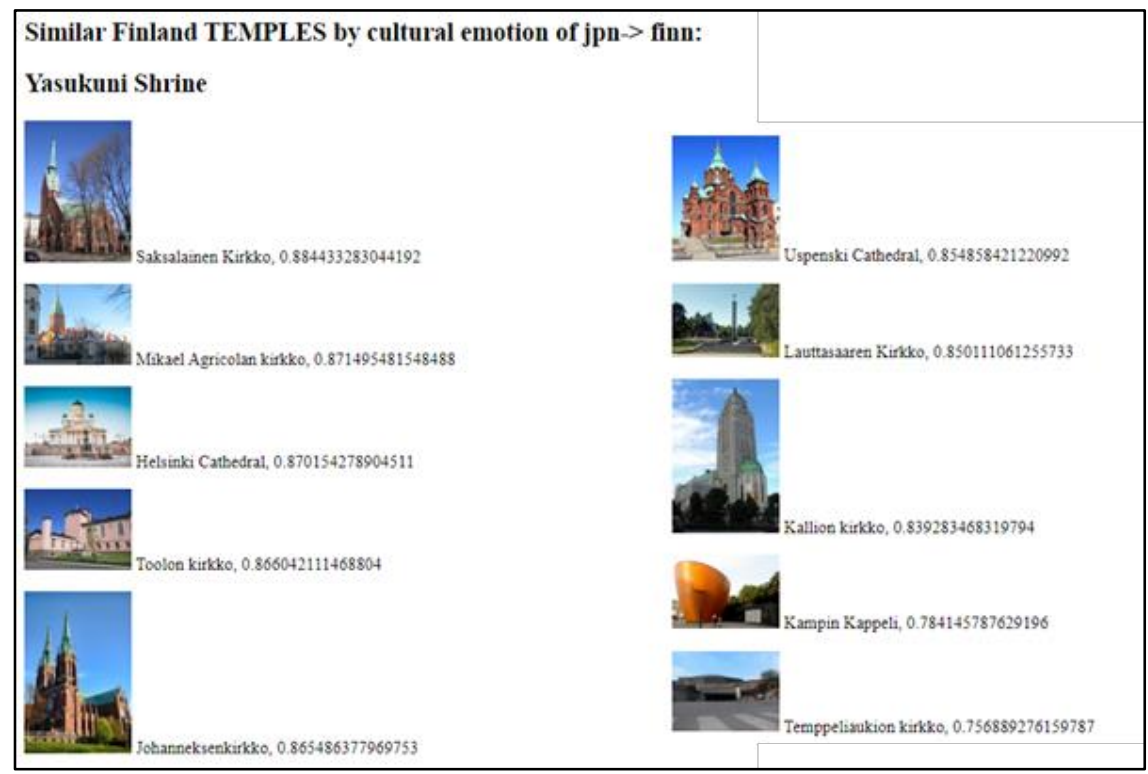

Figure 12. Finland churches similarity with "Yasukuni shrine".

\subsection{Experiment 3}

The similar tasks were done with Finland Churches as well. The detail results of emotion detection are shown in Table 5. In addition, the precision and recall were calculated as Table 6 . The total precision is $61 \%$ and the total recall is $75 \%$.

Table 5. Experimental results of emotion detection for 10 Finland churches

\begin{tabular}{cccccccccccccc}
\hline \multirow{2}{*}{ id } & \multicolumn{10}{c}{} & \multicolumn{10}{c}{ Detected Emotion } \\
& C1 & C2 & C3 & C4 & C5 & C6 & C7 & C8 & C9 & C10 & C11 & C12 & C13 \\
\hline 1 & 0.5 & 0.1 & 0.1 & 0.2 & 0.6 & 0.3 & 0.6 & 0.6 & 0.7 & 0.7 & 0.5 & 0.3 & 0.1 \\
2 & 0.5 & 0.1 & 0.1 & 0.5 & 0.5 & 0.9 & 0.6 & 0.6 & 0.8 & 0.6 & 0.4 & 0.5 & 0.1 \\
3 & 0.7 & 0.4 & 0.1 & 0.3 & 0.3 & 0.7 & 0.1 & 0.3 & 0.4 & 0.1 & 0.2 & 0.3 & 0.2 \\
4 & 0.3 & 0.2 & 0.2 & 0.3 & 0.4 & 0.3 & 0.6 & 0.6 & 0.7 & 0.6 & 0.5 & 0.4 & 0.1 \\
5 & 0.6 & 0.3 & 0.1 & 0.2 & 0.8 & 0.8 & 0.4 & 0.5 & 0.5 & 0.3 & 0.8 & 0.2 & 0.2 \\
6 & 0.5 & 0.2 & 0.1 & 0.3 & 0.4 & 0.3 & 0.5 & 0.5 & 0.4 & 0.3 & 0.4 & 0.2 & 0.2 \\
7 & 0.3 & 0.1 & 0.2 & 0.3 & 0.3 & 0.3 & 0.4 & 0.3 & 0.7 & 0.3 & 0.4 & 0.2 & 0.3 \\
8 & 0.2 & 0.1 & 0.3 & 0.3 & 0.3 & 0.3 & 0.3 & 0.2 & 0.5 & 0.2 & 0.5 & 0.2 & 0.4 \\
9 & 0.2 & 0.2 & 0.2 & 0.3 & 0.4 & 0.3 & 0.4 & 0.3 & 0.7 & 0.4 & 0.4 & 0.2 & 0.3 \\
10 & 0.4 & 0.1 & 0.1 & 0.2 & 0.4 & 0.3 & 0.4 & 0.4 & 0.5 & 0.4 & 0.4 & 0.2 & 0.2 \\
\hline \hline
\end{tabular}

$\mathrm{C} 1=$ interesting, $\mathrm{C} 2=$ mysterious, $\mathrm{C} 3=$ rural-like, $\mathrm{C} 4=$ relaxed, $\mathrm{C} 5=$ calm, $\mathrm{C} 6=$ unique, $\mathrm{C} 7=$ elegant , $\mathrm{C} 8=$ precious, $\mathrm{C} 9=$ faithful, $\mathrm{C} 10=$ historical, $\mathrm{C} 11=$ quiet, $\mathrm{C} 12=$ bustling, $\mathrm{C} 13=$ commonplace .

Detected emotion means implied emotion of religious places which were detected by our system

Table 6. Retrieval performance for Finland churches

\begin{tabular}{ccccccccccccccc}
\hline & $\mathrm{C} 1$ & $\mathrm{C} 2$ & $\mathrm{C} 3$ & $\mathrm{C} 4$ & $\mathrm{C} 5$ & $\mathrm{C} 6$ & $\mathrm{C} 7$ & $\mathrm{C} 8$ & $\mathrm{C} 9$ & $\mathrm{C} 10$ & $\mathrm{C} 11$ & $\mathrm{C} 12$ & $\mathrm{C} 13$ & Average \\
\hline $\begin{array}{c}\text { Precision } \\
(\%)\end{array}$ & 80 & 60 & 50 & 42.9 & 75 & 80 & 80 & 66.7 & 40 & 66.7 & 42.9 & 50 & 60 & 61.09 \\
Recall (\%) & 66.7 & 75 & 100 & 100 & 60 & 100 & 80 & 80 & 50 & 66.7 & 75 & 50 & 75 & 75.26 \\
\hline
\end{tabular}

Figure 13 shown the keyword in impressions and Finland churches that have been selected. The result of "historical" impression shown in Figure 14. 


SELECT BY KEYWORD
Keyword
Sistorical
Seywword
Temppeliaukion kirkko -
Submit
SELECT BY SIMILARITY - Finland to Thailand
Keyword
Kallion kirkko
Submit
SELECT BY SIMILARITY - Finland to Japan
Keyword
Saksalainen Kirkko -
Submit

Figure 13. Impression and Finland churches

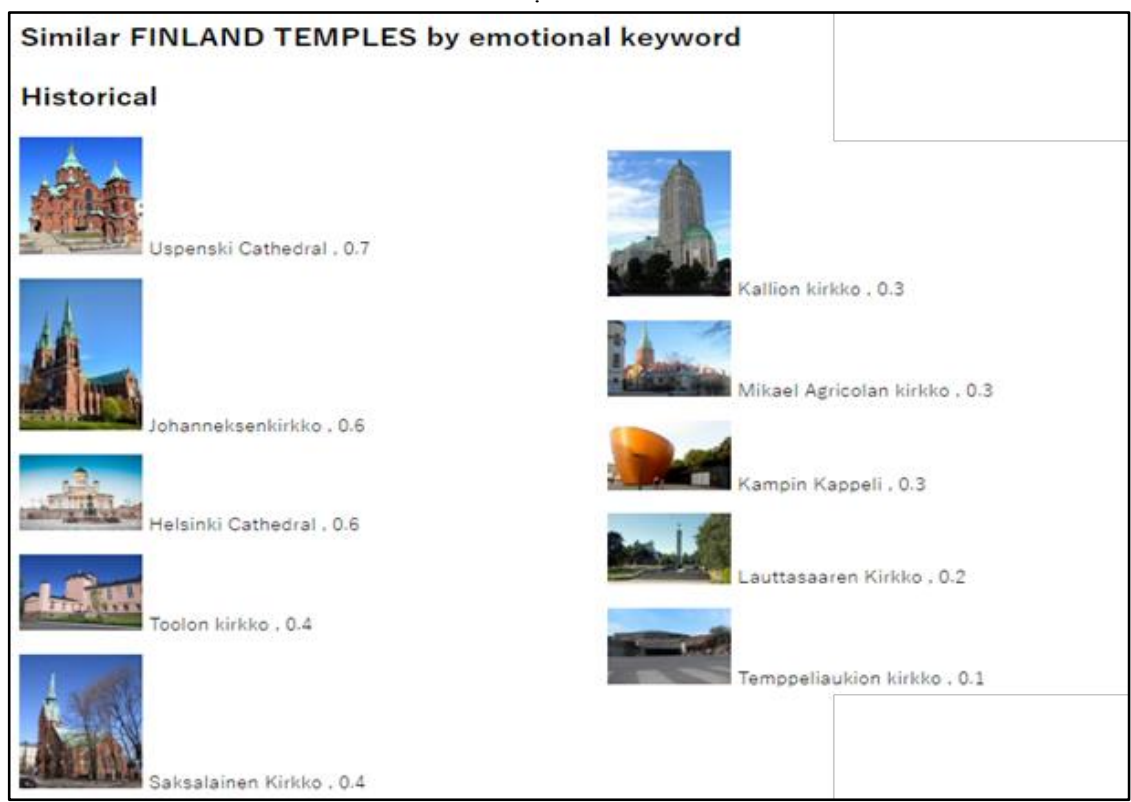

Figure 14. Finland Churches with "historical" impression.

Finland Churches that have the same impression with "Temppeliaukion kirkko" shown in Figure 15 and Figure 16 shows the Thailand temples that have the same emotion distance as "Kallion kirkko" and Figure 17 Show Japan temples that have the same impression with "Saksalainen kirkko". 


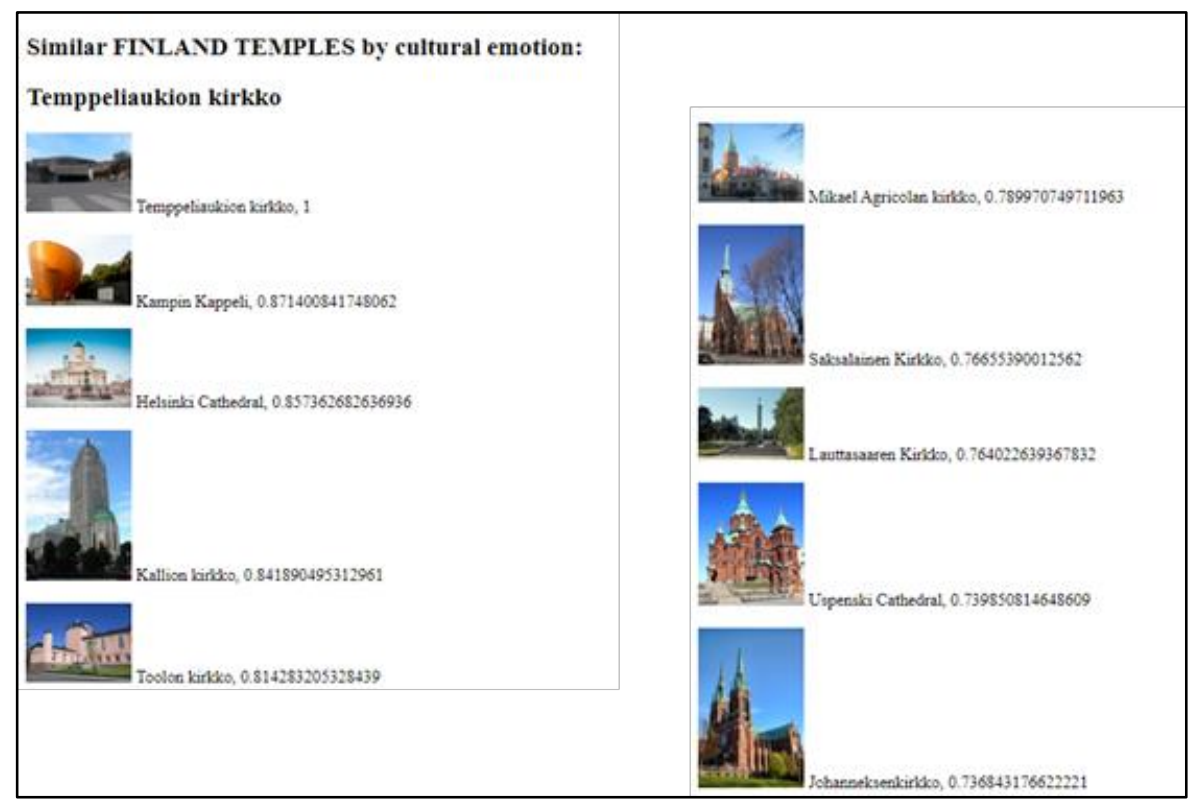

Figure 15. Finland churches similar with "Temppeliaukion kirkko".

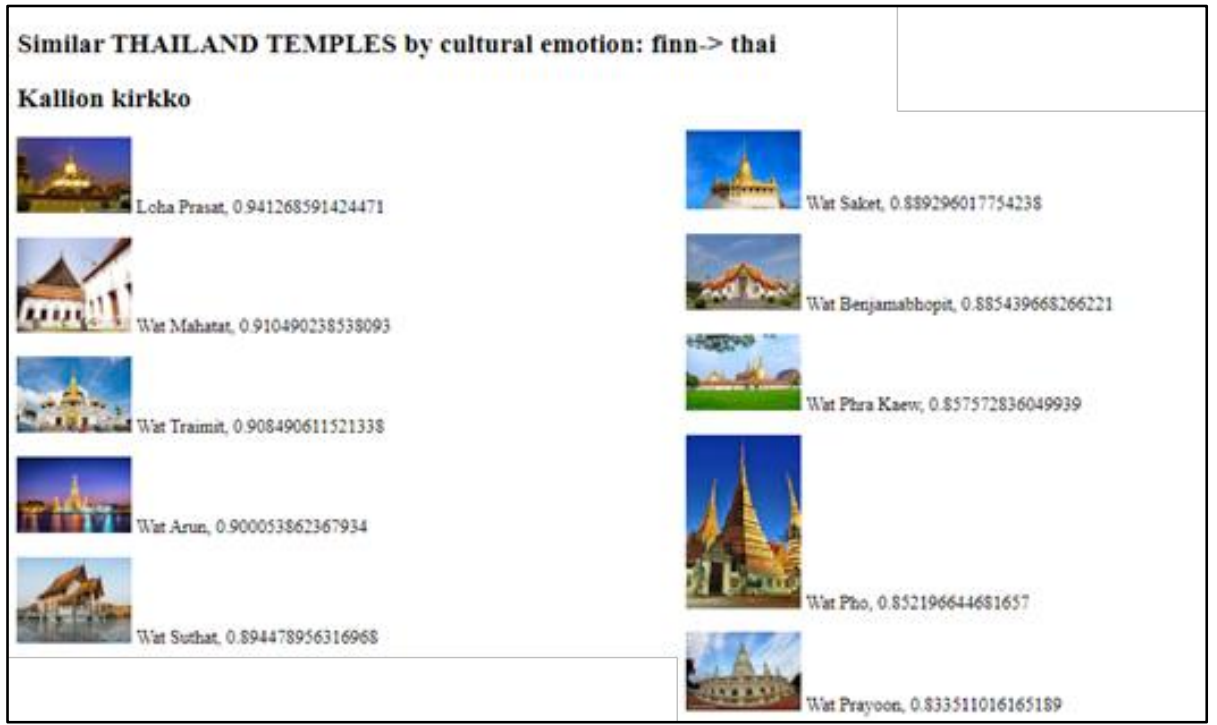

Figure 16. Thailand temples with emotion distance as "Kallion kirkko". 


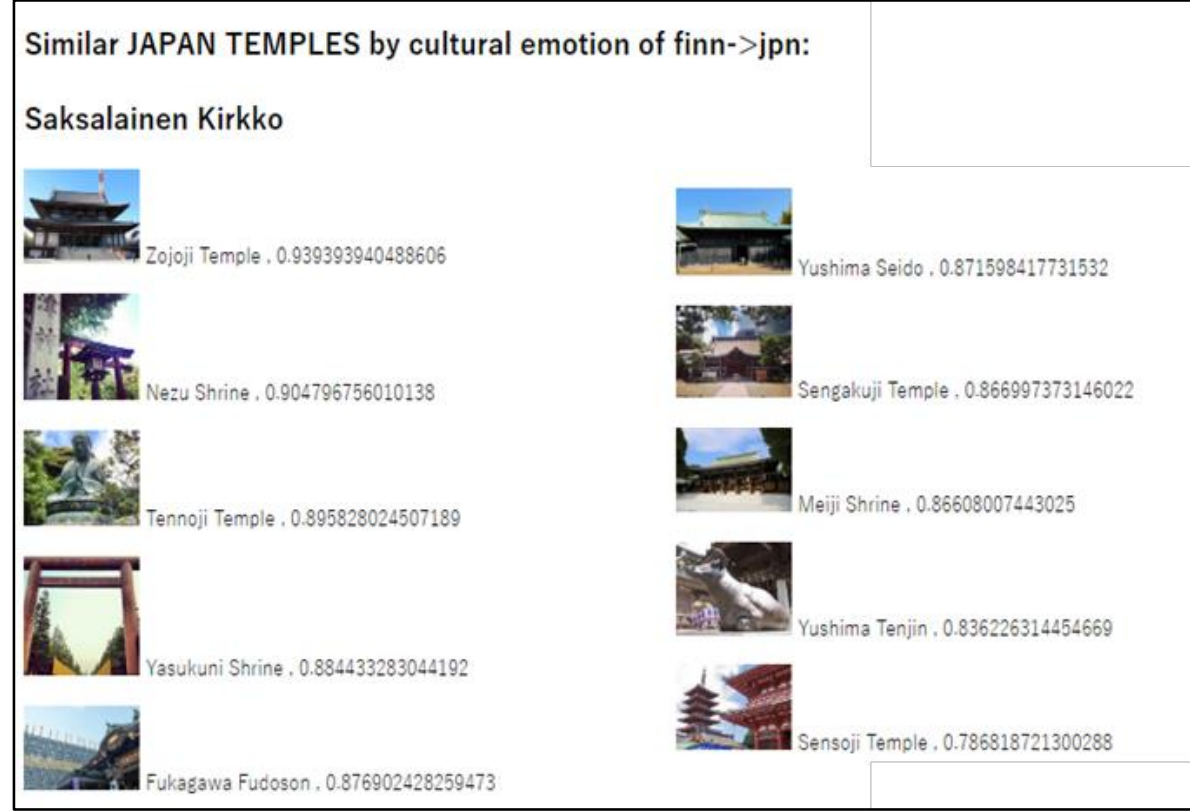

Figure 17. Japan temples with emotion distance as "Saksalainen kirkko".

\section{Conclusion}

This paper proposed the impression distance search system for cross-cultural religious tourism by presenting a variety of emotional responses from different cultures. We have described the main features of our research: (1) a culture-dependent metadata extraction method function for cross-cultural religious analysis and search. (2) a cross-cultural computing mechanism to represent the variety of emotional tourism interpretations from different religious-cultural with impression. As future work, we will extend the number of various application systems integration with symbolic essences database to improve cross-cultural tourism experiences with cultural color-analysis methodology [8].

\section{Acknowledgement}

This work is supported by Multimedia Database Laboratory (MDBL), Graduate School of Media and Governance, Keio University. We thank the MDBL members for their valuable comments and suggestions

\section{References}

[1] The World Tourism Organization. Tourism and the Sustainable Development Goals - Journey to 2030. US: UNWTO Publications; 2018. 114 p.

[2] Kiyoki Y, Kitagawa T, Hayama T. A Metadatabase System for Semantic Image Search by a Mathematical Model of Meaning. SIGMOD Record. 1994 Jan 12;23(4):34-41. 
[3] Y. Kiyoki and M. Kawamoto. Semantic Associative Search and space Integration Methods Applied to semantic Metrics for Multiple Medical Fields. Information Modelling and Knowledge Bases XVIII. 2007. p. $120-135$.

[4] Kitagawa, T. Kiyoki, Y. A mathematical model of meaning and its application to multidatabase systems. Proceedings of 3rd IEEE International Workshop on Research Issues on Data Engineering: Interoperability in Multidatabase system. 1993 April 19-20; Vienna, Austria: IEEE; c1993. p. 130-135.

[5] Kiyoki Y, Kitagawa T, Hitomi Y. A Fundamental Framework for Realizing Semantic Interoperability in a Multidatabase Environment. Integrated Computer-Aided Engineering. 1995 Jan;2(1):3-20.

[6] Potts C. From frequency to meaning: vector space models of semantics. Journal of Artificial Intelligence Research. 2010;37:141-188.

[7] Itabashi, Y., Sasaki, S. and Kiyoki, Y. Cross-cultural Image Computing with Multiple Color-Space Transformation. Journal of EMITTER. 2011;2(2):182-192. 


\title{
Conceptualization of Aviation Cabin Crew's Fatigue Risk Assessment
}

\author{
Çevik Irem ${ }^{1}$, Bam Bibek, Dahanayake Ajantha and Elfvengren Kalle \\ School of Engineering Science, Lappeenranta-Lahti University of Technology, \\ Lappeenranta, Finland
}

\begin{abstract}
Airlines are of great importance to the transportation sector. With the increase in commercial air travel, airlines require extra flight crews. Aviation industry's cabin crewmembers are faced with working overtime, working in shifts and long working hours. The shift system causes fatigue for flight crews. Fatigue is of critical importance in the aviation industry. Depending on the physical and psychological fatigue, explicit or implicit results appear. There are a number of approaches in the aviation industry to prevent fatigue. When previous studies are examined, there are few studies examine in the general, and aviation crew's fatigue treat both pilots and cabin crew alike. The relationship between cabin crew's fatigue-to-fatigue risk management systems, key fatigue-causing factors, tools to alarm fatigue, and outcome assessments are non-existent. However, various difficulties are encountered in measuring the cabin crews fatigue levels and measurements and are often subjective and not reliable. Therefore, the aim of this study is to create a concept map to be integrated into the aviation cabin crew's fatigue risk assessment application design and implementation in order to arrive at a comprehensive fatigue risk assessment tool for the aviation industry.
\end{abstract}

Keywords. Fatigue, Airline Cabin Crew, Fatigue Risk Management System,

FRMS, Fatigue Assessment, Fatigue Assessment Tools

\section{Introduction}

Fatigue is among the workplace hazards that affect employee health and safety [1]. The International Civil Aviation Organization (ICAO) defines fatigue as "A physiological state of diminished mental or physical performance potential, from a sleep loss, extended alertness, circadian phase, and / or workload relating to mental and / or physical activity that can damage a person's alertness and capability to appropriately perform safety related operational duties [2].

Fatigue affects the aviation industry as well as all business areas. As one of the safest transport routes, the aviation industry needs to actively manage the hazards that affect safety [3]. Various organizations have made some recommendations for fatigue management. Flight duty time limitations (FTL) are officially released by international authorities in order to reduce and control risk of fatigue. Similarly, some guidelines are published by ICAO to support and contribute to the aviation industry. New rules have also been proposed by the Federal Aviation Administration (FAA) and the European Aviation Safety Agency (EASA) [4].

\footnotetext{
${ }^{1}$ Corresponding Author, İrem Çevik, School of Engineering Science, Lappeenranta-Lahti University of Technology, Lappeenranta, Finland; E-mail: irem.cevik@student.lut.fi
} 
More than $70 \%$ of aviation accidents are caused by human errors [5]. Various accidents in the aviation world have defined fatigue-related factors as one of the main causes of aviation accidents. With 24/7 flight operations, pilot and flight crew surveys show that fatigue is an important problem and causes at least 4-8\% setbacks in this sector [6].

There are many factors that cause fatigue, including social and family situations leading to sleep and circadian processes affecting sleep propensity and alertness [7]. Although cabin crew and pilots are evaluated in a similar way within the aviation sector, work and workload varies significantly between cabin crew and pilots. While the main workload of a pilot is the take-off and landing stages, the workload of the cabin crew continues during the flight. The fatigue risk related to cabin crew has not gained the needed attention in research [8].

In this context, this research pays attention to examine the fatigue risk management of aviation cabin crews. This research explores Fatigue and Fatigue Risk Management System (FRMS) in aviation sector, effects of fatigue and types of risks it creates for cabin crews, strategies and approaches available for reducing the cabin crew fatigue, and models available for fatigue assessment in flight crew (pilots and cabin crew). Finally, this research presents a fatigue assessment model specifically developed for fatigue risk management of aviation cabin crew. Figure 1 describes briefly the research approach followed in this research.

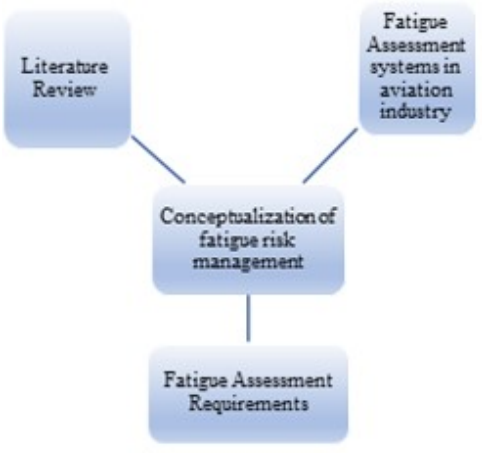

Figure 1: Research method

\section{Related studies}

\subsection{Definition of fatigue}

Fatigue is a state of feeling which can be physical, mental or both and it is considered as a warning that a person is not able to characterize but feels lackadaisical, exhausted, and tired. It is considered as the lack of energy and motivation in individuals and is not able to perform activities during work. It is described as the need for sleep. Fatigue can also include shortness of breath and weakness in muscles. It is considered as a complaint and it is necessary for an individual to consider fatigue as a symptom and it 
is not a disease [15]. In complaint of fatigue, many illnesses can result, as it is a symptom of many diseases.

\subsection{Types and factors of fatigue}

Fatigue includes the factors which are categorized into three factors: lifestyle factors, physical health conditions, and mental health issues. The lifestyle of fatigue includes experiencing fatigue because of activities like physical exertion, lack of physical activities, boredom, emotional stress, overweight, and using alcohol on regular basis. In case of physical health, condition causes anemia, arthritis, fibromyalgia, eating disorders, cancer, diabetes and many more. Mental health conditions also lead to fatigue and the common symptoms of fatigue are anxiety, depression and seasonal affective disorder [13]. These factors of fatigue result in not enough sleep, sleep apnea, anemia, depression, hypothyroidism, overloaded caffeine, and diabetes as some common examples.

Fatigue people may have three major disorder; however, each person's situation may vary. May lack motivation or the ability to start an activity, After the activity started, the person is easily tired; and the person is mentally tired or has difficulty concentrating and remembering to start or complete an activity. It is thought that the cause of fatigue may be in various forms depending on the nature. Fatigue can be classified as mental and physical fatigue $[5,6,9]$.

Mental Fatigue- Anyone who can be under stress for a long time may experience mental exhaustion. It can make you feel overwhelmed and mentally exhausted, and it seems that your responsibilities and problems cannot be handled properly. Mental fatigue affects cognitive performance. In addition, sleep deprivation and other health factors affect mental fatigue [29]. Mental fatigue symptoms include the physical as well as emotional symptoms [14]. The emotional signs are stress, depression, anxiety, emotionlessness, dread feeling, lack of motivation, difficulty concentrating and hopelessness feeling.

Physical signs include body aches, stomachaches, change in appetite, loss or gain of weight, increase in illness. Physical Fatigue is also muscle fatigue. It includes the physical inability of muscles that are not able to perform optimally for a temporary period [28]. It depends on the level of individual's physical fitness [27]. The symptoms of physical fatigue include body ache, headache, and tiredness. When an individual is not able to perform the task and continue the work, it is a symptom of fatigue. The most common symptoms include mental disorders, lack of motivation, irritability, eating disorder, stress or loss of appetite and insomnia [14]. Mental fatigue can affect you for short-term or long-term. This is reversed with appropriate rest [28].

\subsection{Effects of fatigue}

Fatigue effects in many different ways at the workplace, it affects employee in a negative way. Lack of sleep unable a person to complete the work on time. Fatigue has an effect on physical and cognitive functions of an individual. The fatigue progression is continuous and involves important physiological changes that occur before and during mechanical failure. Cognitive function disorder is a growing public health problem. Physical exercise is known to be good for health and helps to reduce the risk of many cardiovascular and lung diseases [10]. 
In the transport industry like aviation, moving heavy items during flights cause physical as well as metal fatigue to an individual. It impacts on the work performed, because of fatigue and productivity can decrease. Continuous working times cause fatigue as well as working in night shift or working overtime hours may decrease work performance. Insufficient sleep can lead to a decline in productivity. In fact, this may cost employers thousands of dollars more each year [11]. Insomnia itself causes billions of dollars in damage and sleep disorders each year, which can lead to higher medical costs. Fatigue can affect a person's physical and mental ability to perform a given task. Especially those who strive to push themselves to the limit are also common among people who work hard. The onset of fatigue can be rapid, or it can be the result of cumulative effects after weeks or months of manual labor. Factors taken into consideration in aviation industry in this respect are:

- Age: It is an important factor for age-related dynamic tasks. In the aviation industry, flight crews tend to fall asleep late with age. This is particularly common for male flying teams [3][55].

- Caffeine, nicotine, and alcohol: The effect of caffeine in many foods and beverages such as coffee, chocolate, tea, energy drinks vary from person to person. The effect of caffeine, which is known to have an effect on attention, is very effective for some, while for others it is negligible. Caffeine stimulates the brain. Alcohol makes you feel sleepy. Nicotine is a major factor in continuous attention [3][56].

- Sleep disorders: Sleep quality is an important factor affecting fatigue. Sleep disorder can contribute to the formation of fatigue. Flight crews are at special risk of sleep disorders due to shift work system [3][28].

- Environmental Factors: Many factors such as light, noise and resting areas affect sleep. Sleeping in the dark room is easier. Resting places are very important for flight crews. For example, radio stations around the hotel may cause noise, which may affect rest during a stay in a hotel after a flight [3].

\section{Fatigue risk management in aviation industry}

Air transport is a complex system that includes a complex, interconnected, distributed network of human operators, procedures and technological systems [38]. Multiple causes of known and sometimes unknown risk factors related to aviation should be analyzed in an integrated systematic manner [37]. Flight and duty limitations and associated resolution conditions are traditional ways to manage fatigue. Conventional regulations do not take into account the interaction between sleep loss and circadian rhythms. New methods need to be developed to understand the causalities of the predecessors leading to serious events and accidents [40]. In ultra-secure systems such as commercial aviation operations, security-critical errors and incidents are unpredictable, uncommon, and are not a source of routine monitoring data.

Fatigue Risk Management System (FRMS) is defined as data-driven and scientifically based, which identifies organizational procedures and procedures for controlling fatigue risk in aviation operations and enables continuous monitoring and management of security risks associated with errors [3]. In FRMS, they need to be analyzed to determine if flight crew fatigue is a contributing factor [53]. In the 
literature, flight fatigue is a significant and long-term problem in the aviation transport industry [54]. To minimize the effect of fatigue-related errors, a controller is made, and measurements are evaluated periodically [22].

\subsection{The principles for fatigue management}

For high-risk industries such as aeronautics and medicine, 24-hour uninterrupted work is mandatory in some cases where work is necessary, and these industries are known to carry higher security risks associated with fatigue [15]. As stated in the ICAO definition, there are four basic scientific principles to be managed [16]:

- Scientific principle 1: Sleep

Fatigue is associated with lack of sleep or sleep deprivation [20]. It is known that good quality sleep has a significant impact on human health and employee performance depending on the amount of sleep, working hours and order [26]. Especially shift workers are more vulnerable to performance and health effects such as sleep deprivation, misalignment of circadian rhythms [27]. According to neurophysiological conditions, there are two types of sleep: Rapid eye movement (REM) and Non-Rapid eye movement (NREM) [22].

$\checkmark$ Rem: Rapid eye movement (REM) sleep accounts for approximately $25 \%$ of sleep time [9]. REM sleep is known to be associated with long-term apneas, hypopneas, and severe hypoxemia [25].

$\checkmark$ Non-Rem: Non-REM sleep is divided into three stages depending on the characteristics of brain waves. Stage 1 represents lighter sleep and occurs as the transition from burn to sleep.

This stage is generally the result of frequent arousal caused by sleep disorders such as sleep apnea, periodic movement in sleep, or snoring. Stage 2 is known as moderate sleep and dominates the sleep stages with $50 \%$ of the total sleep time. Stage 3, known as deep sleep, is called slow-wave sleep (SWS) [23][24].

\section{- Scientific principle 2: Sleep loss and recovery}

Effects such as cognitive reasoning, attention, memory and increased response time and error rates due to sleep deprivation pose a great risk especially for the aviation and medical sectors. Workers in the aviation industry are threatened by fatigue from sleep loss. Risks are greater both in terms of cost and in terms of passenger carrying capacity. The melting at the Three Mile Island nuclear power plant in 1979; The Chernobyl nuclear disaster in 1986; the 1986 explosion of the space shuttle Challenger; Exxon Valdez oil spill in 1989; Korea Air 801 is known to have been partly responsible for fatigue in major accident that killed more than 200 people in a 1997 crash. In these accidents, it is clear that determining the sleep requirement of employees can save people's lives and institutions can save money [28-30]. In the research of the 401 Army aviator and aircraft crew, $72 \%$ of the pilots reported that they flew under high numbness [18].

\section{- Scientific principle 3: Circadian effects}


The term "Circadian", introduced by Halberg in 1959, is a fluctuation controlled by the body's 24-hour biological clock [34]. It affects long working hours and circadian rhythms in aviation operations [35]. Circadian rhythm includes extensive body functions include body temperature, hormone secretion, digestion, physical and mental performance, mood and others [40].

The effects of Circadian rhythms, whose effects on alertness, physiology, and subjective fatigue experience are known to peak in the afternoon (day) and rise in the morning or early morning (at night) [33].

\section{- Scientific principle 4: Workload}

The International Air Transport Association (IATA) reveals that current trends in air transport can increase the number of passengers to 8.2 billion by 2037 . Many occupational groups in the aviation sector need long-term training. Therefore, the personnel working in the aviation industry are working overtime. Employees may feel tired due to the increased workload [4]. Fatigue, which adversely affects the probability of people producing safe performances and actions, has been found to be a contributing factor to accidents, injuries, and death [3]. ICAO defines workload as mental and physical activity. Employees tend to experience physical and mental fatigue due to irregular working hours, long flight times, pressure changes, and tasks that crewmembers must complete within a certain period of time [39].

\subsection{Fatigue management approaches for aviation cabin crew}

Fatigue management refers to methods by which Service Providers and operational personnel address the security effects of fatigue. In general, FAA (Federal Aviation Administration), NTSB (National Transportation and Safety Board), ICAO Standards and Recommended Practices (SARPs) and care organizations support different approaches to identify and prove the role of fatigue in aviation incidents and accidents, for example:

- First of all, the industry complies with the flight and duty time limits in accordance with the rules set by the regulator and manages fatigue hazards using existing Safety Management System (SMS) operations to identify other types of hazards and express risks

- Fatigue is defined as a legal hazard in the industry, a FRMS approved by the regulator is developed and implemented

- The flight crew is informed about this subject and related trainings are organized

- $\quad$ The effects can be measured by developing fatigue assessment techniques, and companies can perform cost-benefit analysis [4][44][45][46].

In relation to these approaches, FRMS shares some key features for employees. First, operational experience is supported by scientific approaches: 
- Vigilance that affects physical, mental performance and social life should be examined in all aspects and conditions, and conditions can be provided for adequate sleep

- Mental and physical activities and sleep management (falling asleep and dormancy) with circadian clock effect can be monitored [31][32][33]

- Due to the fatigue of the workload, it can be monitored whether the employee has decreased in physical and mental performance

- It can be expressed as the security risk posed by team members due to fatigue $[42][43][44]$.

Second, because fatigue is affected by all waking activities (not just job demands), fatigue management should have a common responsibility among regulators, operators and crew members.

\subsection{Fatigue assessment for cabin crew}

FRMS involves measuring the fatigue, sleep, performance and workload of the cabin crew. Performance, psychophysiological and subjective measurement techniques are used [44][45].

\subsubsection{Assessment methods}

There is no single method for determining and measuring fatigue risk. Because there are many factors associated with fatigue, and furthermore, new ways to determine the effects of fatigue and to measure sleep continue to be developed based on scientific research. Measurement tools can be divided into subjective and objective (such as performance tests and psychophysiological tests). Each type of measurement has its strengths and weaknesses. [44][45]

- Subjective measurement: Depending on the programs and operations, flight crews make reports about fatigue or fatigue related errors, incidents and accidents, and accordingly, reports can be transmitted through programs. The collected data are evaluated, and meaningful results are obtained.

$\checkmark$ Transmission of fatigue by cabin crew fatigue reports (online fatigue reporting system or written statements)

$\checkmark$ Surveys for cabin crew

$\checkmark$ Determination of subjective fatigue and sleepiness levels (Karolinska sleepiness scale and status control of the Samn Perelli team) [46].

- Objective measurement: Performance and psychophysiological tests are applied to cabin crews to determine the risk associated with fatigue. Tests such as Psychomotor Vigilance Task (PVT), Multi-Attribute Test Battery (MATB), and Operator Vehicle Interface Task (OVI) are used to characterize fatigue [44]. The PVT test is a high-signal load response - time test that produces various performance measures against sleep deprivation. Firstly, the deceleration of sleep deprivation and sleep-related responses, PVT results by measuring the employee's contribution to work and task reveal the interaction with the circadian system and sleep processes [44][45][46][47][48][49]. 


\subsection{Modeling}

Organizations and regulators need the analysis of data to identify the root cause of fatigue. Because they follow a more sophisticated method to better define 24/7 uptime regulations (WTAs), potentially increase safety, reduce risk levels, and increase operational flexibility and efficiency. Various modeling methods are used for best results. Computer modeling can be used to monitor the effects of the flight crew and contribute to the changes in performance. There are following commercial biomathematical models (BMMs) used for fatigue sleep deprivation:

- $\quad$ Fatigue Avoidance Scheduling Tool (FAST)

- The Sleep, Activity, Fatigue and Task Effectiveness (SAFTE)

- $\quad$ Fatigue Index Tool (FIT)

- System for Aircrew Fatigue Evaluation (SAFE).

For example, Fatigue Audit InterDyne (FAID) and System for Aircrew Fatigue Evaluation (SAFE) can provide insight into the way the sleep regulator works, depending on the duration of sleep and the time to start sleeping [47][48].

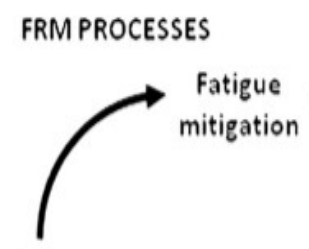

Risk Assessment
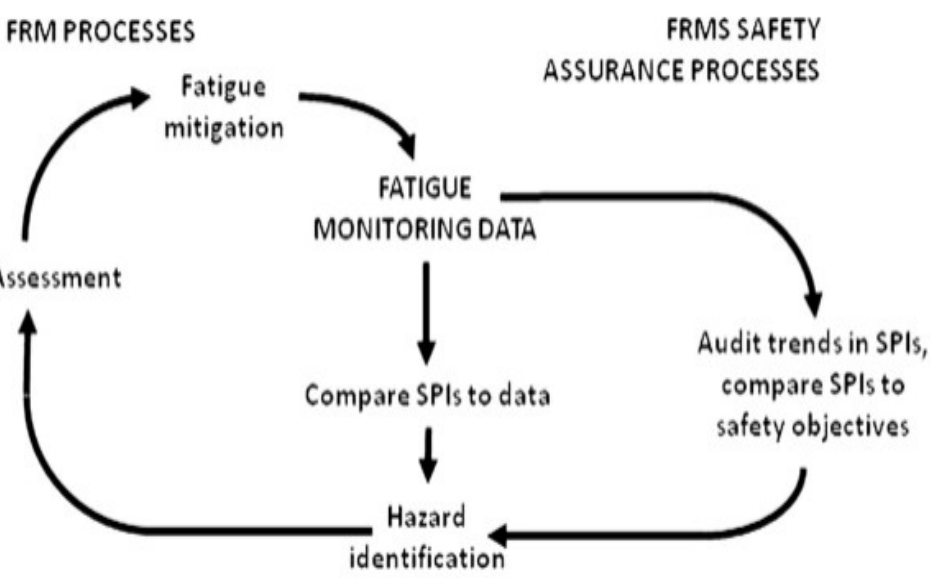

Figure 2: Analysis of fatigue [47]

\subsection{Fatigue risk management and mitigation}

Fatigue, seen as an increasing security risk by the society, needs regulation by governments and operators. Rule-of-service hours (HOS) are one of the most common control processes, and due to the weak scientific support and less flexibility, a broader security approach is emerging, the Security management system (SMS).The aerospace 
industry, which is at an important and critical level in terms of safety, imposes common safety responsibility for regulators, practitioners, and employees [50][51].

\begin{tabular}{|l|l|l|}
\hline \multicolumn{1}{|c|}{ Challenges } & \multicolumn{2}{c|}{ Solutions } \\
\hline Industry Culture & Fatigue Education \\
\hline Fatigue Assessment & 2 & FRMS \\
\hline Scheduling Practices & 3 & Fatigue Research \\
\hline Government Inaction & 4 & Assessment \\
\hline Fatigue Education & 5 & Hours of Service Limits \\
\hline Work Life Balance & 6 & Safety Culture \\
\hline Fatigue Research & 7 & Collaboration \\
\hline Root Cause Analysis & 8 & Public Relations \\
\hline
\end{tabular}

Figure 3: Fatigue challenges and solutions [46]

\subsection{Regulatory Responsibility and Industry Responsibility}

Regulatory means providing the frame; operators to manage fatigue risks that is responsible for achieving an acceptable applicable safety and security level. Operators are responsible for monitoring and managing fatigue hazards, providing fatigue management training, and planning and implementing cabin crews and pilots to perform their duties safely [50][51]. Operators should be responsible for:

- Making sure that cabin crews are informed and introduced about FRMS

- Creating an appropriate working environment

- Establishment of an appropriate reporting mechanism and encouraging flight crew to report on fatigue related issues such as not fit to fly, etc.

- Marking sure that roster of crew is based on international aviation organizations and civil aviation organization regulations

- Make sure that indicators for any exceedance are tracked via FRMS

- Making promotion and encouraging cabin crews to contribute to FRMS.

Individual responsibility

Cabin crews should be responsible for

- Following the fatigue risk management policies determined by the industry and operator

- Determining sleep and rest management based on shift and duty duration,

- Managing their own fatigue levels

- Participating in training and education related to fatigue risk management provided by the operator

- Reporting of any issues and occurrence related to fatigue via the company reporting system [3][4][50][51]. 


\subsection{Fatigue assessment systems and tools in aviation industry}

Following are few visual examples of tools and systems that are in current use in the aviation industry for fatigue assessment and analysis. Figure 4 is the representation of FAST interface taking into consideration the consequences of longer work hours against the amount of sleep, resulting in sleep deprivation and fatigue.

Figure 5 - similar to figure 4, represents fatigue assessment and avoidance by analyzing sleep patterns and showing when performance might be lower.

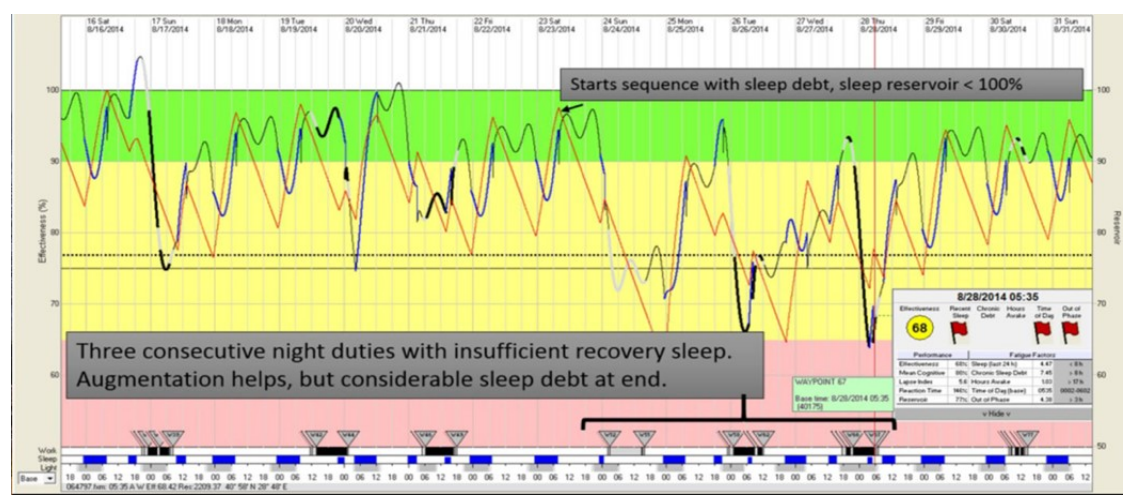

Figure 4: visual FAST interface [57]

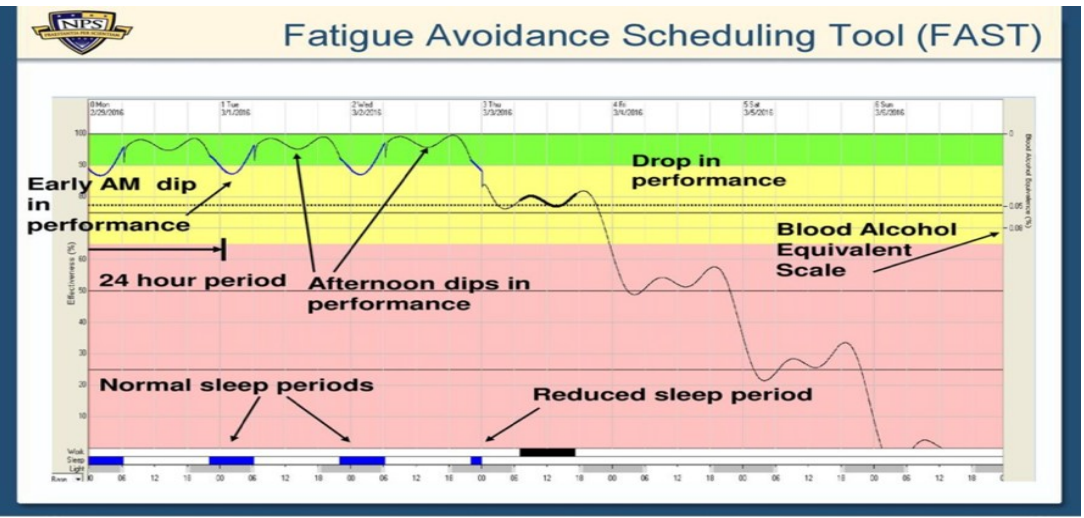

Figure 5: FAST system interface 
Figure 6 - represents a fatigue calculator which helps to self-evaluate sleep fatigue level and counter measures for each level.

Figure 7 - an app available on store. This uses user input of work time and rest period to determine fatigue seriousness and respective counter measure.

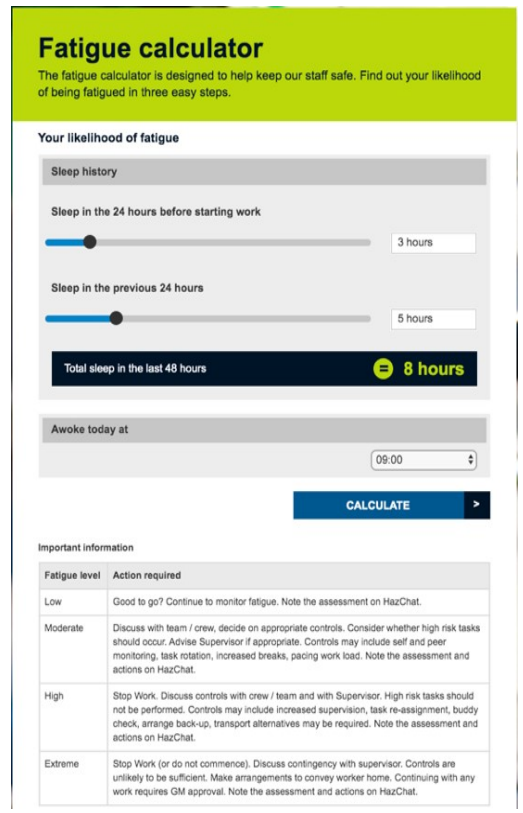

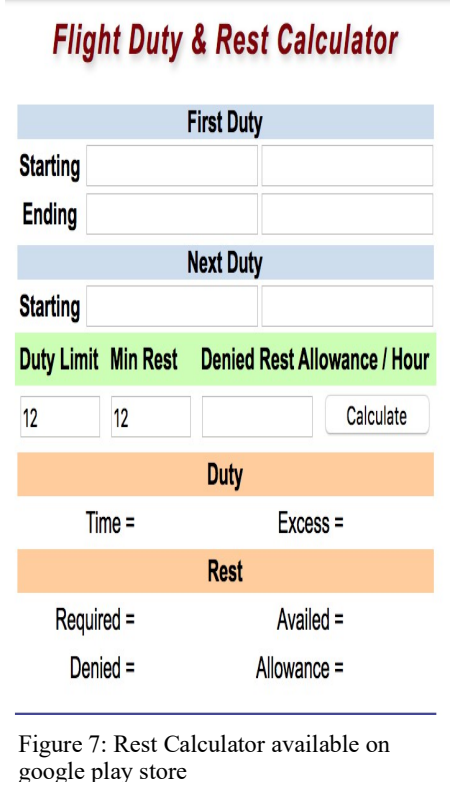

Figure 6: Fatigue Calculator by Energex [58]

Figure 8 - EU flight time calculator available on the app store. Similar to the Flight duty and rest calculator this app takes flight time and rest time as input to determine the level of fatigue and whether personnel are fit for duty.

Figure 9 - crew alert app available on app store takes in duty roster, sleep algorithm, time variation in different cities during travel as data to provide relevant analysis and result for crew members. 


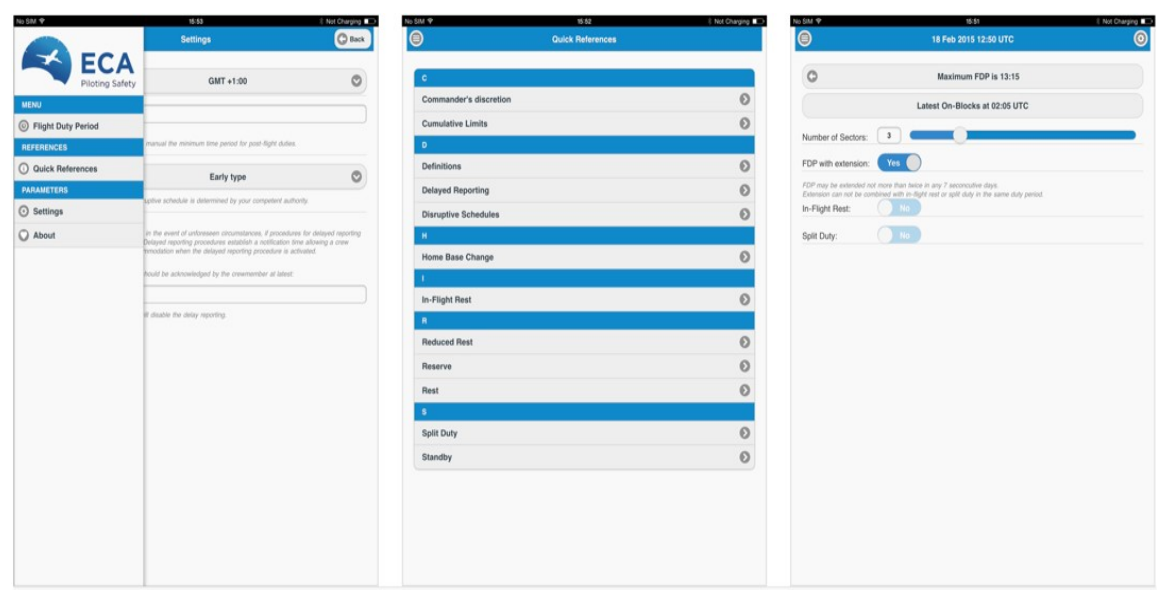

Figure 8: EU FLT Calculator, Available on apple app store
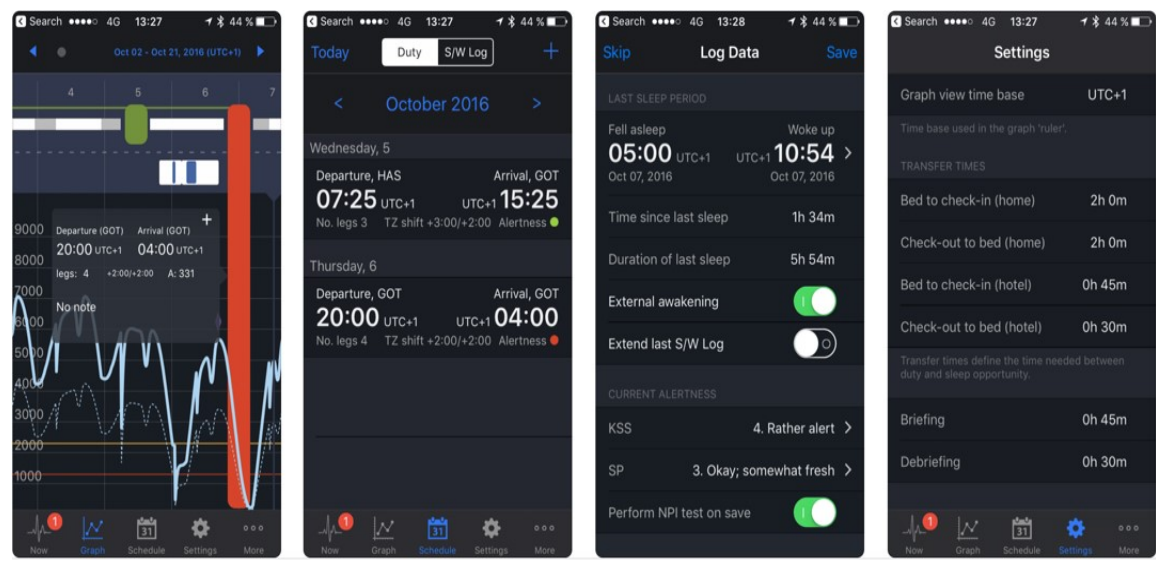

Figure 9: Crew Alert lite app on Apple app 
Table 1. Fatigue Measurement Map

\begin{tabular}{|c|c|c|c|c|c|c|}
\hline $\begin{array}{c}\text { Fatigue } \\
\text { Categories }\end{array}$ & $\begin{array}{l}\text { HOW: Influencing } \\
\text { Factors }\end{array}$ & WHICH: Symptoms & $\begin{array}{l}\text { WHEN: Indicators of } \\
\text { Cabin Crew }\end{array}$ & WHAT: To Measure & WHAT: Data needed & WHAT: Technologies/tools available \\
\hline \multirow{5}{*}{ Lifestyle } & regular alcohol use & drowsy eyes, tiredness & less attentive & $\begin{array}{l}\text { high blood pressure, } \\
\text { irregular heartbeat }\end{array}$ & $\begin{array}{c}\text { blood sample, urine sample, } \\
\text { breath analysis }\end{array}$ & $\begin{array}{c}\text { use of breathalysers, urine and blood } \\
\text { test }\end{array}$ \\
\hline & lack of physical activity & quick tiredness, physical pain & Quick exhaustion & $\begin{array}{l}\text { muscular tension, } \\
\text { breathlessness }\end{array}$ & $\begin{array}{l}\text { electrical synapsis of the } \\
\text { nerves }\end{array}$ & $\begin{array}{l}\text { motor and sensory reflex test of } \\
\text { muscles, self-reports }\end{array}$ \\
\hline & emotional stress & mood swings, unstable mood & emotional breakdown & $\begin{array}{l}\text { irregular brain/nerval } \\
\text { activity }\end{array}$ & heart rate, breathing interval & $\begin{array}{l}\text { different PDAs, use of ECG (electro } \\
\text { cardio graph) }\end{array}$ \\
\hline & overweight & body structure change, & $\begin{array}{l}\text { prone to diseases, self- } \\
\text { loathing }\end{array}$ & $\begin{array}{c}\text { standard mass index to } \\
\text { current state }\end{array}$ & body weight, height & BMI (Body Mass Index) \\
\hline & physical exertion & tiredness, low interest & $\begin{array}{l}\text { less attentive, quick } \\
\text { exhaustion }\end{array}$ & $\begin{array}{l}\text { self-report, other medical } \\
\text { factors as heart rate, } \\
\text { breathing status }\end{array}$ & $\begin{array}{l}\text { electrical synapsis of the } \\
\text { nerves, tension in muscles } \\
\text { and ligaments }\end{array}$ & $\begin{array}{l}\text { motor and sensory reflex test of } \\
\text { muscles, self-reports }\end{array}$ \\
\hline \multirow{5}{*}{$\begin{array}{l}\text { Physical Health } \\
\text { conditions }\end{array}$} & anaemia & pale skin, insomnia, dizziness & tiredness, less attentive, & $\begin{array}{l}\text { haemoglobin and iron count } \\
\text { in blood }\end{array}$ & $\begin{array}{c}\text { haemoglobin count, mean } \\
\text { corpuscular volume, iron } \\
\text { count }\end{array}$ & CBC (complete blood count) test \\
\hline & arthritis & $\begin{array}{l}\text { decreased range of motion, } \\
\text { pain, stiffness }\end{array}$ & $\begin{array}{l}\text { functionality, job duties } \\
\text { hinderance, tiredness }\end{array}$ & $\begin{array}{l}\text { swollenness of joints, loss of } \\
\text { motion, blood test }\end{array}$ & $\begin{array}{l}\text { physical deformity, motion } \\
\text { data, blood test data }\end{array}$ & physical examination, $x$-ray, blood test \\
\hline & eating disorder & rapid weight loss/gain, & $\begin{array}{l}\text { prone to diseases, issues } \\
\text { with self }\end{array}$ & $\begin{array}{c}\text { eating attitude test, other } \\
\text { psychological behaviour and } \\
\text { tests }\end{array}$ & $\begin{array}{c}\text { height, weight, heart rate, } \\
\text { blood pressure, data on skin } \\
\text { and nails }\end{array}$ & $\begin{array}{l}\text { Rating of Anorexia and Bulimia } \\
\text { (RAB) test, psychological aptitude test }\end{array}$ \\
\hline & diabetic & $\begin{array}{l}\text { frequent urination, blurry } \\
\text { vision, extreme fatigue }\end{array}$ & prone to other diseases, & $\begin{array}{l}\text { sugar level in blood, glucose } \\
\text { tolerance }\end{array}$ & $\begin{array}{l}\text { glucose and sugar level, } \\
\text { BMI (body mass index) }\end{array}$ & $\begin{array}{l}\text { blood test for sugar and glucose level, } \\
\text { Glycated haemoglobin (A1C) test, }\end{array}$ \\
\hline & allergies & $\begin{array}{l}\text { rashes, irritation, shortness of } \\
\text { breath }\end{array}$ & $\begin{array}{c}\text { transferrable, causes } \\
\text { hindrance in duties and work } \\
\text { area }\end{array}$ & skin test, blood test & $\begin{array}{l}\text { IgE (immunoglobulin E test) } \\
\text { level, antibody count in } \\
\text { body }\end{array}$ & blood and skin test \\
\hline \multirow{3}{*}{ Mental Issue } & anxiety & $\begin{array}{c}\text { nervousness, hyperventilation, } \\
\text { weakness }\end{array}$ & $\begin{array}{l}\text { tiredness, breathlessness, } \\
\text { emotional instability }\end{array}$ & $\begin{array}{c}\text { self-report, psychometric } \\
\text { data }\end{array}$ & $\begin{array}{c}\text { reports, Q\&A, } \\
\text { characteristics of patients }\end{array}$ & $\begin{array}{l}\text { mental and physical test, blood and } \\
\text { urine test, behavioural aptitude test }\end{array}$ \\
\hline & depression & fatigue, melancholy, sadness & $\begin{array}{l}\text { emotional instability, trust } \\
\text { issue }\end{array}$ & $\begin{array}{l}\text { self-report, psychometric } \\
\text { data }\end{array}$ & $\begin{array}{l}\text { primary care/physician data, } \\
\text { medications in use }\end{array}$ & $\begin{array}{l}\text { mental } / \text { physical test, blood/ urine test, } \\
\text { behavioural aptitude test }\end{array}$ \\
\hline & Medication & nausea, upset stomach & various & contents of medication & prescriptions, dosage of use & standard dosage Vs prescribed dosage \\
\hline
\end{tabular}




\section{Fatigue Measurement Concept Map}

Software development is a complex enduvear. When modeling for future business needs, the model that best meets the needs and is suitable for time and budget constraints is selected. Two situations are important when developing a process model: current state and future state. It is vital to determine the scope of the model. A detailed measurement fatigue map is made as the first step to define the limits of the concepts to be modeled. Table 1 provides a comprehensive measurement map of fatigue including factors, symptoms, risks posed on cabin crew, measuring technique, data needed for measurement. It has been developed using the $\mathrm{W}^{*} \mathrm{H}$ model. Another important step is to create a change management process on how to move from the current state to the future state. $[59][60][61][62][63][64][65][66][67][68][69][70][71][75][76]$.

\subsection{Process model for fatigue measurement}

The process model is a simplified view of the events with details. The organization is expanded by detailing the layer by layer until the details are at the desired level. It is expected that the current difficulties will be well understood and the model to be designed will overcome these difficulties. Business process modeling is useful for defining, analyzing and demonstrating the desired processes of the operational performance of tasks. From various tools that are available to model business processes, Bizagi Modeler is selected for this study [71[75].

A process model has been created in order to find out under which conditions fatigue occurs. Additionally, the process also helps to determine the risk level of fatigue, to reveal what are the available measurements, and to identify various strategies and methods to mitigate risks if needed. The most important factor in this process model is the selection of the appropriate measurement method

Process view: BizAgi Process Modeler (www.bizagi.com) is used for graphic representation of the workflow process. BizAgi Modeler provides fast process automation thanks to its graphical and dynamic environment. BizAgi Process Modeler is a program that allows you to create work flow diagrams (schemes) that allow you to increase your productivity. BizAgi supports the entire business process lifecycle through different components. It provides many operations such as compliance with Business Process Model and Notation (BPMN) standards, opening to teamwork, and previewing the schema process with its simulation feature [72][75]. 


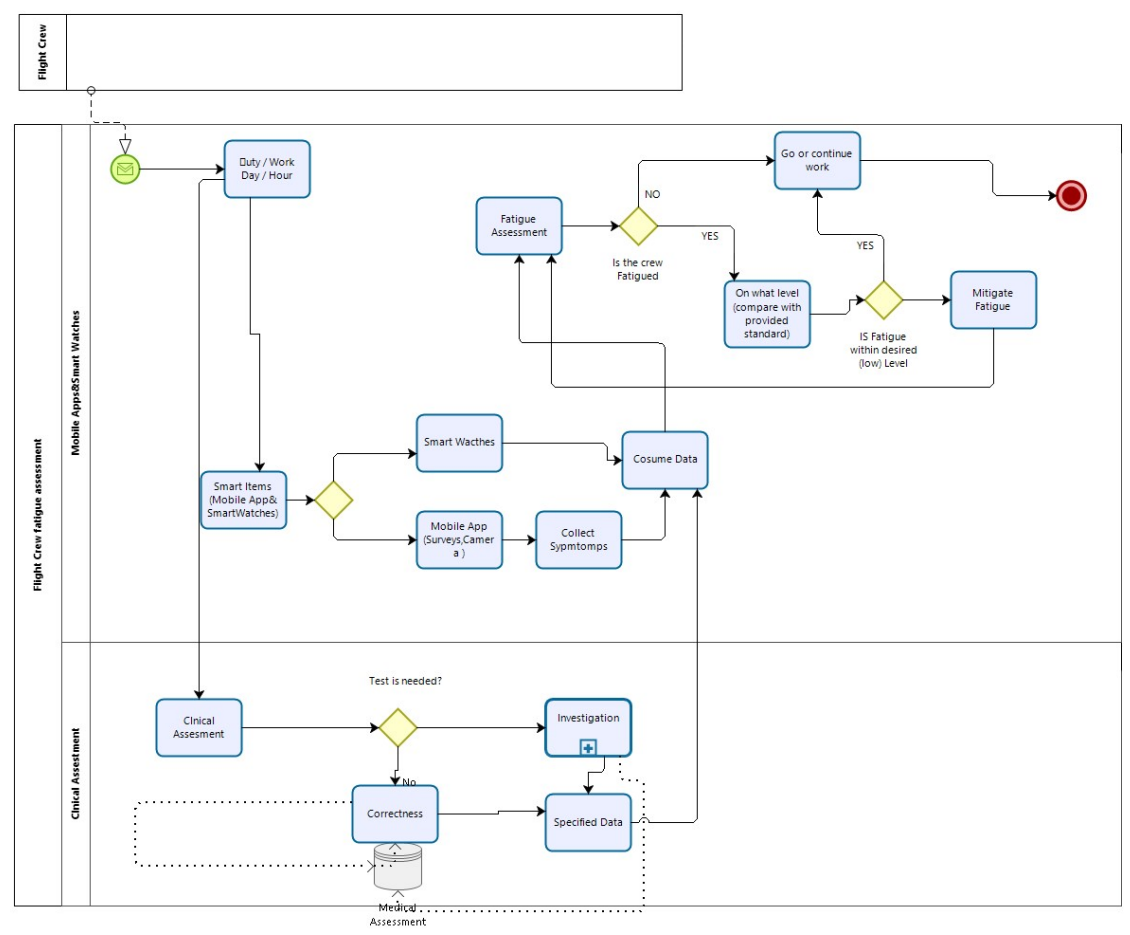

Figure 10: BPMN diagram representing FRMS assessment methods

There is no single measurement method for measuring fatigue. In order to perform fatigue measurements of the flight crew, objective tests are planned based on performance tests and physical impressions. As shown in Figure 10, this process is divided into three different levels. At each level, it is to be designed as to include different devices and techniques to gather performance data with regards to assigned tasks and to transmit the data to the next level or to create a direct output process. An example of such a sub process view is illustrated in Figure 11.

Data sources are needed for FRMS monitoring. Intelligent devices and medical proficiency testing can be used for this purpose. In the first stage, it is aimed to be able to track the data via mobile applications and wearable watches, during and after the flight. Wearable technology, which is expressed for all kinds of objects containing the technology we wear, is an intelligent machine with computing capabilities. Wearable technology incorporating electronic components such as sensors, cameras, microphones and keyboards has an important field of applications in many sectors [73]. Smart watches are expected to become even more popular in the near future. With the new features expected in smartwatches, personalized data can be transformed into a reliable clinical tools for improved health monitoring. Heart rate, blood oxygenation, deep sleep monitoring and reflex measurement can be done with smart devices and applications. In the second stage, medical proficiency tests such as blood test, urine test 
and height-weight index can be applied from the periodic mandatory medical tests of the cabin crew [17][74].

In the second stage, it is planned to make measurements of medical proficiency tests such as blood test, urine test and height-weight index and to access the recorded data as needed. Figure 12 presents the comprehensive fatigue risk assessment methodology and the system to be supported in the future for aviation cabin crews.

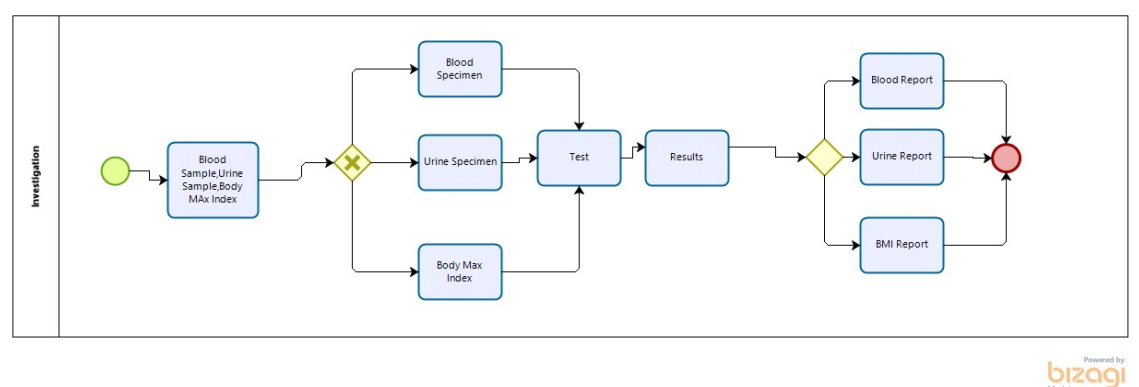

Figure 11: BPMN diagram representing Sub-Process

Risk management is one of the pillars of safety management systems. Data collection and analysis are important for assessing the risk of fatigue. In Figure 12, a comprehensive fatigue risk assessment methodology is proposed, combining datadriven analysis and assessment techniques. There are three main parts of this methodology: collecting various information and data, evaluating the results and calculating the risk value.

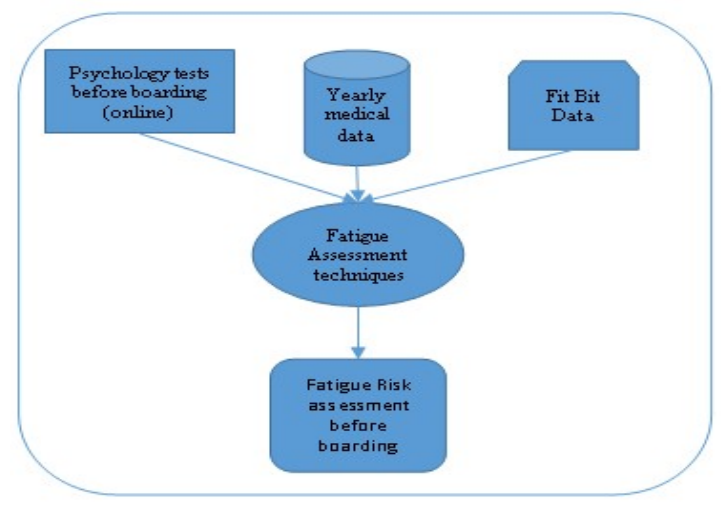

Figure 12: A Comprehensive fatigue risk assessment methodology

\section{Conclusion}

Safety plays an important role in the aviation world. In relation to this, airlines need a safety management system. Fatigue is a big problem for the flight crew. There are 
many factors that cause fatigue and fatigue symptoms vary. Fatigue risk assessment and management is not addressed in a comprehensive manner for cabin crew members.

In this study, firstly, the definition of fatigue is explained, and related concepts are clarified. Factors affecting fatigue, types of fatigue, causes of fatigue, why fatigue risk management is needed, methods of measuring fatigue, and the responsibilities of institutions and employees to reduce fatigue risk are discussed.

According to the explored researches there is no comprehensive and integrated mechanism which comprise physical, psychological and social parameters to determine cabin crew's fatigue level. Therefore, a concept map is created to reach a comprehensive risk assessment tool. While creating the workflow chart, wearable technology will be provided to the cabin crew team to access real-time information of the personnel to realize their organized functions and to obtain reliable data. In the future this technology will be developed to ensure that all data is accurate and that the measurements are saved for reducing the risks of fatigue with acceptable objective measurements.

In summary, this study has provided the first step towards achieving a major contribution to flight safety for designing feedback and measuring mechanisms, In the future research a risk assessment method will be designed and implemented as a mobile app for a comprehensive fatigue risk assessment system and steps to improve fatigue risk management of aviation cabin crew.

\section{References}

[1] Sadeghniiat-Haghighi, Khosro, and Zohreh Yazdi. "Fatigue Management in the Workplace." Industrial Psychiatry Journal 24, no. 1 (2015): 12-17.

[2] Doc 9966 Manual for the Oversight of Fatigue Management Approaches-2016 Second Edition

[3] Fatigue Management Guide For Airline Operators 2nd edition 2015

[4] Cabon, Philippe, Stephane Deharvengt, Jean Yves Grau, Nicolas Maille, Ion Berechet, and Régis Mollard. "Research and Guidelines for Implementing Fatigue Risk Management Systems for the French Regional Airlines." Accident Analysis and Prevention 45, no. sS (2012): 41-44.

[5] Yen, Jin-Ru, Chiung-Chi Hsu, Hsuan Yang, and Hero Ho. "An Investigation of Fatigue Issues On Different Flight Operations." Journal of Air Transport Management 15, no. 5 (2009): 236-240.

[6] Caldwell, John A. "Fatigue in Aviation." Travel Medicine and Infectious Disease 3, no. 2 (2005): 85-96.

[7] Lee, Seungyoung, and Jin Ki Kim. "Factors Contributing to the Risk of Airline Pilot Fatigue." Journal of Air Transport Management 67 (2018): 197-207.

[8] Li, Wei. A Cabin Crew Fatigue Risk Comprehensive Evaluation Model. 2015.

[9] Boksem, Maarten A.S, and Mattie Tops. "Mental Fatigue: Costs and Benefits." Brain Research Reviews 59, no. 1 (2008): 125-139.

[10] Boksem, Maarten A.S, Theo F. Meijman, and Monicque M. Lorist. "Mental Fatigue, Motivation and Action Monitoring." Biological Psychology 72, no. 2 (2006): 123-132

[11] Ellemberg, Dave, and Mathilde St-Louis-Deschênes. "The Effect of Acute Physical Exercise On Cognitive Function During Development." Psychology of Sport \& Exercise 11, no. 2 (2010): 122-126.

[12] Marcora, Samuele M., Walter Staiano, and Victoria Manning. "Mental Fatigue Impairs Physical Performance in Humans." Journal of Applied Physiology (Bethesda, Md. : 1985) 106, no. 3 (2009): 857.

[13] Mizuno, Kei, et al. "Antifatigue Effects of Coenzyme Q10 During Physical Fatigue." Nutrition 24, no. 4 (2008): 293-299.

[14] Walker, Ross F., and Michio Kumagai. "Image Analysis As a Tool for Quantitative Phycology: A Computational Approach to Cyanobacterial Taxa Identification." Limnology 1, no. 2 (2000): 107-115.

[15] Ventikos, N. P., Lykos, G. V., \& Vagias, N. A. "Human Fatigue: Evaluation with the Usage ofBayesian Networks." In Computational Intelligence Systems in Industrial Engineering, no. 6 (2012): 651-676 .

[16] Thomas, Matthew J. W.; Ferguson, Sally A. "Aviation, Space, and Environmental Medicine." Aerospace Medical Association, no. 2 (2010): 665-670.

[17] Godfrey, A., Hetherington, V., Shum, H., Bonato, P., Lovell, N. \& Stuart, S. (2018). From A to Z: 
[18] Mitler, M.M., M.A Carskadon, C.A Czeisler, W.C Dement, D.F Dinges, and R.C Graeber. "Catastrophes, Sleep, and Public Policy: Consensus Report." Sleep 11, no. 1 (1988): 100-109.

[19] Chang, Yu-Hern, Hui-Hua Yang, and Wan-Jou Hsu. "Effects of Work Shifts On Fatigue Levels of Air Traffic Controllers." Journal of Air Transport Management 76 (2019): 1-9.

[20] Nozaki, Satoshi, et al. "Mental and Physical Fatigue-related Biochemical Alterations." Nutrition 25, no. 1 (2009): 51-57.

[21] Cheng, Yi Han, Gregory D. Roach, and Renee Ma Petrilli. "Current and Future Directions in Clinical Fatigue Management: An Update for Emergency Medicine Practitioners." Emergency Medicine Australasia 26, no. 6 (2014): 640-644.

[22] Williamson, Ann, David A. Lombardi, Simon Folkard, Jane Stutts, Theodore K. Courtney, and Jennie L. Connor. "The Link between Fatigue and Safety." Accident Analysis and Prevention 43, no. 2 (2011): 498-515.

[23] Cai, Zi-Jian. "The Functions of Sleep: Further Analysis." Physiology \& Behavior 50, no. 1 (1991): 5360.

[24] Javaheri, Sogol, and Susan Redline. "Sleep, Slow-Wave Sleep, and Blood Pressure." Current Hypertension Reports 14, no. 5 (2012): 442-448.

[25] Shrivastava, Deepak, Syung Jung, Mohsen Saadat, Roopa Sirohi, and Keri Crewson. "How to Interpret the Results of a Sleep Study." Journal of Community Hospital Internal Medicine Perspectives 4, no. 5 (2014).

[26] Ratnavadivel, Rajeev, Nuy Chau, Daniel Stadler, Aeneas Yeo, R Doug Mcevoy, and Peter G. Catcheside. "Marked Reduction in Obstructive Sleep Apnea Severity in Slow Wave Sleep." Journal of Clinical Sleep Medicine: JCSM: Official Publication of the American Academy of Sleep Medicine 5, no. 6 (2009): 519

[27] Joint Commission Health care worker fatigue and patient safety. 2011 Retrieved from http://www.pwrnewmedia.com/2011/joint commission/fatigue/downloads/printfriendly.pdf.

[28] Kolla, Bp, and Rr Auger. "Jet Lag and Shift Work Sleep Disorders: How to Help Reset the Internal Clock." Cleveland Clinic Journal Of Medicine 78, no. 10 (2011): 675-684.

[29] Caldwell, John A. "Crew Schedules, Sleep Deprivation, and Aviation Performance." Current Directions in Psychological Science 21, no. 2 (2012): 85-89.

[30] Tarman, D. [Recent Evidence On Fatigue and Sleep Deprivation in Both the Medical and Military Aviation Professions]. 2013.

[31] Owens, Rachel. "The Science of Sleep." Library Journal 143, no. 14 (2018): 28.

[32] Tran, Tuan, Kimberly R. Raddatz, Elizabeth T. Cady, Bradford Amstutz, Pete D. Elgin, Christopher Vowels, and Gerald Deehan. "Effects of Extreme Sleep Deprivation On Human Performance." Idaho Academy of Science Symposium and Meeting,University Place, Idaho Falls, ID 2007.

[33] Caldwell, J., and S. Gilreath. "A Survey of Aircrew Fatigue in a Sample of U.S. Army Aviation Personnel." Aviation, Space, and Environmental Medicine 73, no. 5 (2002): 472-480

[34] Arendt, J., S. Deacon, J. English, S. Hampton, and L. Morgan. "Melatonin and Adjustment to Phase Shift." Journal Of Sleep Research 4 (1995): 74-79.

[35] McCallum, M., Sanquist, T., \& Mitler, M. "Commercial Transportation Operator Fatigue Management Reference." U.S. Department of Transportation Research and Special Programs Administration., (2003).

[36] Brandt, Summer L., John A. Caldwell, Lucia Arsintescu, Patrick M. Chapman, Charlie W. Deroshia, Raymond L. Oyung, Dinah D. Reduta-Rojas, Laura M. Colletti, and Melissa M. Mallis. The Effects of Ultra-Long-Range Flights On the Alertness and Performance of Aviators - NASA/TM-2006-213484. 2006.

[37] Caldwell, John A., J. Lynn Caldwell, Lauren A. Thompson, and Harris R. Lieberman. "Fatigue and Its Management in the Workplace." Neuroscience and Biobehavioral Reviews 96, no. C (2019): 272-289.

[38] Luxhoj, J. T., \&amp; Coit, D. W. " Modeling low probability/high consequence events: anaviation safety risk model. In RAMS\&\#39;06." Annual Reliability and Maintainability Symposium, (2006). 215221.

[39] Netjasov, Fedja, and Milan Janic. "A Review of Research On Risk and Safety Modelling in Civil Aviation." Journal of Air Transport Management 14, no. 4 (2008): 213-220.

[40] Armentrout, J. J., Holland, D. A., O'Toole, K. J. \& Ercoline, W. R. (2006). Fatigue and related human factors in the near crash of a large Armentrout, Jeffrey J., Dwight A. Holland, Kevin J. O'Toole, and William R. Ercoline. "Fatigue and Related Human Factors in the Near Crash of a Large Military Aircraft." Aviation, Space, and Environmental Medicine 77, no. 9 (2006): 963.

[41] Miyagi, M. "Serious Accidents and Human Factors - Breaking the Chain of Events Leading to an Accident: Lessons Learned from the Aviation Industry." Reston, VA: AIAA, (2005)

[42] Samel, Alexander, R. Curtis Graeber, David F. Dinges, and Mark R. Rosekind. Principles and Guidelines for Duty and Rest Scheduling in Commercial Aviation - NASA/TM-1996-110404. 1996. 
[43] Wesensten, Nancy Jo, Gregory Belenky, David R. Thorne, Mary A. Kautz, and Thomas J. Balkin. "Modafinil Vs. Caffeine: Effects on Fatigue During Sleep Deprivation." Aviation, Space, and Environmental Medicine 75, no. 6 (2004): 520.

[44] Mehta, Ranjana, and Michael Agnew. "Influence of Mental Workload On Muscle Endurance, Fatigue, and Recovery During Intermittent Static Work." European Journal of Applied Physiology 112, no. 8 (2012): 2891-2902.

[45] Wilson, G.F., J.A Caldwell, and C.A Russell. "Performance and Psychophysiological Measures of Fatigue Effects On Aviation Related Tasks of Varying Difficulty." International Journal of Aviation Psychology 17, no. 2 (2007): 219-247.

[46] Avers, Katrina E., William B. Johnson, Joy O. Banks, Darin Nei, and Elizabeth Hensley. Fatigue Solutions for Maintenance: From Science to Workplace Reality. 2011.

[47] Gander, Philippa H., Jim Mangie, Margo J. Van Den Berg, A Alexander T. Smith, Hannah M. Mulrine, and T Leigh Signal. "Crew Fatigue Safety Performance Indicators for Fatigue Risk Management Systems." Aviation, Space, and Environmental Medicine 85, no. 2 (2014): 139.

[48] Dawson, Drew, Y. Ian Noy, Mikko Härmä, Torbjorn Åkerstedt, and Gregory Belenky. "Modelling Fatigue and the Use of Fatigue Models in Work Settings." Accident Analysis and Prevention 43, no. 2 (2011): 549-564

[49] Dawson, Drew, David Darwent, and Gregory D. Roach. "How Should a Bio-mathematical Model Be Used Within a Fatigue Risk Management System to Determine Whether or Not a Working Time Arrangement Is Safe?" Accident Analysis and Prevention 99, no. Pt B (2017): 469-473.

[50] Honn, Kimberly A., Brieann C. Satterfield, Peter Mccauley, J. Lynn Caldwell, and Hans P.A Van Dongen. "Fatiguing Effect of Multiple Take-offs and Landings in Regional Airline Operations." Accident Analysis and Prevention 86, no. C (2016): 199-208.

[51] Dawson, Drew, and Kirsty Mcculloch. "Managing Fatigue: It's About Sleep." Sleep Medicine Reviews 9, no. 5 (2005): 365-380.

[52] Gander, P., L. Hartley, D. Powell, P. Cabon, E. Hitchcock, A. Mills, and S. Popkin. "Fatigue Risk Management: Organizational Factors at the Regulatory and Industry/company Level." Accident Analysis And Prevention 43, no. 2 (2011): 573-590.

[53] Gander, Philippa H., Hannah M. Mulrine, Margo J. Berg, A. Alexander T. Smith, T. Leigh Signal, Lora J. Wu, and Gregory Belenky. "Effects of Sleep/wake History and Circadian Phase On Proposed Pilot Fatigue Safety Performance Indicators." Journal of Sleep Research 24, no. 1 (2015): 110-119.

[54] Lei, W., and S. Ruishan. "Analysis on Flight Fatigue Risk and the Systematic Solution." Lecture Notes in Computer Science (including Subseries Lecture Notes in Artificial Intelligence and Lecture Notes in Bioinformatics) 6779 (2011): 88-96.

[55] Avin, Keith G., and Laura a Frey Law. "Age-related Differences in Muscle Fatigue Vary By Contraction Type: A Meta-analysis." Physical Therapy 91, no. 8 (2011): 1153.

[56] Moore, David J., Edmund Keogh, and Christopher Eccleston. "Identifying Experimental Methods to Determine the Effect of Pain On Attention: A Review of Pain, Caffeine, Alcohol and Nicotine Studies." Human Psychopharmacology: Clinical and Experimental 24, no. 8 (2009): 601-618.

[57] Saftefast.com. (2019). SAFTE-FAST | FRMS | SAFTE-FAST CONSOLE. [online] Available at: https://www.saftefast.com/frms-visual-fast.html [Accessed 8 Nov. 2019].

[58] Energex.com.au. (2019). Fatigue Calculator - Energex. [online] Available at: https://www.energex.com.au/home/fatigue-calculator [Accessed 9 Nov. 2019].

[59] Shattuck, N. (2017). Scheduling A Clear Mind, A Combat Edge.

[60] Zhu, Y., R.R Jankay, L.C Pieratt, and R.K Mehta. "Wearable Sensors and Their Metrics for Measuring Comprehensive Occupational Fatigue: A Scoping Review." Proceedings of the Human Factors and Ergonomics Society 61, no. 1 (2017): 1041-1045.

[61] Gleaves, David H., Crystal A. Pearson, Suman Ambwani, and Leslie C. Morey. "Measuring Eating Disorder Attitudes and Behaviors: A Reliability Generalization Study." Journal of Eating Disorders 2 , no. 1 (2014): 6.

[62] Julian, Laura J. "Measures of Anxiety: State-Trait Anxiety Inventory (STAI), Beck Anxiety Inventory (BAI), and Hospital Anxiety and Depression Scale-Anxiety (HADS-A." Arthritis Care \& Research 63, no. S11 (2011): S467-S472.

[63] Garner, D M., P E. Garfinkel, and D P. Bonato. "Body Image Measurement in Eating Disorders." Advances in Psychosomatic Medicine 17 (1987): 119.

[64] Nevonen, Lauri, Anders G. Broberg, David Clinton, and Claes Norring. "A Measure for the Assessment of Eating Disorders: Reliability and Validity Studies of the Rating of Anorexia and Bulimia Interview Revised Version (RAB-R." Scandinavian Journal of Psychology 44, no. 4 (2003): 303-310. 
[65] Mayoclinic.org. (n.d.). Allergy skin tests - Mayo Clinic. [online] Available at: https://www.mayoclinic.org/tests-procedures/allergy-tests/about/pac-20392895 [Accessed 16 Nov. 2019].

[66] Mayoclinic.org. (n.d.). Diabetes - Diagnosis and treatment - Mayo Clinic. [online] Available at: https:/www.mayoclinic.org/diseases-conditions/diabetes/diagnosis-treatment/drc-

20371451\#: :targetText=A\%20fasting\%20blood $\% 20$ sugar $\% 20$ level $\% 20$ from $\% 20100 \% 20$ to $\% 20125 \%$ 20mg,blood\%20sugar\%20level\%20is\%20measured. [Accessed 17 Nov. 2019].

[67] Mieli.fi. (n.d.). Depression | The Finnish Association for Mental Health. [online] Available at: https://mieli.fi/en/home/mental-health/mental-disorders/depression [Accessed 17 Nov. 2019].

[68] Spettell, Claire M., Terry C. Wall, Jeroan Allison, Jaimee Calhoun, Richard Kobylinski, Rachel Fargason, and Catarina I. Kiefe. "Identifying Physician-Recognized Depression from Administrative Data: Consequences for Quality Measurement." Health Services Research 38, no. 4 (2003): 1081-1102.

[69] Sylvia, Louisa G., Emily E. Bernstein, Jane L. Hubbard, Leigh Keating, and Ellen J. Anderson. "Practical Guide to Measuring Physical Activity." Journal of the Academy of Nutrition and Dietetics 114, no. 2 (2014): 199-208.

[70] Thalheim, B., and A. Dahanayake. "A Conceptual Model for Services." Lecture Notes in Computer Science (including Subseries Lecture Notes in Artificial Intelligence and Lecture Notes in Bioinformatics) 9382 (2015): 51-61

[71] Nurcan, Selmin, Anne Etien, Rim Kaabi, Iyad Zoukar, and Colette Rolland. "A Strategy Driven Business Process Modelling Approach." Business Process Management Journal 11, no. 6 (2005): 628649.

[72] Garcia, F., A. Vizcaino, and C. Ebert. "Software Technology Process Management Tools." Ieee Software 28, no. 2 (2011): 15-18.

[73] Park, Yong, and Marko Skoric. "Personalized Ad in Your Google Glass? Wearable Technology, Hands-Off Data Collection, and New Policy Imperative." Journal of Business Ethics 142, no. 1 (2017): 71-82.

[74] Quesada-González, Daniel, and Arben Merkoçi. "Mobile Phone-based Biosensing: An Emerging "diagnostic and Communication" Technology." Biosensors and Bioelectronics 92 (2017): 549-562.

[75] Garcia, Felix, Aurora Vizcaino, and Christof Ebert. "Process Management Tools." IEEE Software 28, no. 2 (2011): 15-18.

[76] Chinosi, Michele, and Alberto Trombetta. "BPMN: An Introduction to the Standard." Computer Standards \& Interfaces 34, no. 1 (2012): 124-134 


\title{
A Mental Health Database Creation Method with Neuroscience-inspired Search Functions
}

\author{
Venera RANEVA $^{1}$ and Yasushi KIYOKI ${ }^{2}$ \\ ${ }^{1}$ Graduate School of Media and Governance, Keio University, Japan \\ ${ }^{2}$ Graduate School of Media and Governance, Keio University, Japan
}

\begin{abstract}
Mental health, an essential factor for maintaining a high quality of life, is determined by one's nutritional, physical, and psychological situations. Since mental health is influenced by multiple factors, a multidisciplinary approach is effective. Due to the complexity of this mechanism, most non-specialists have little knowledge and access to the related information. There are multiple factors that influence one's mental health, such as nutrition, physical activities, daily habits, and personal cognitive characteristics. Because of this complexity, it can be hard for non-specialists to find and implement appropriate methods for improving their mental health. This paper presents the 2-Phase Correlation Computing method for interpreting the characteristics of each emotion/mental state, nutrients, exercises, life habits with a vector space. The vector space reflects the roles of neurotransmitters. The 2-Phase Correlation Computing extracts the information expected to be most relevant to the user's request. In this method, expert knowledge, characteristics of emotions, and mental states are defined in the "Requests" Matrix, and each stimulus into "Nutrients", "Exercises", and "Life Habits" Matrixes. "Nutrients", "Exercises", and "Life Habits" are expressed and computed to as "Stimuli". In short, this method introduces logos to the chaotic world of decision making in mental health.
\end{abstract}

Keywords. Mental health, combinatorial vector, 2-Phase Correlation Computing, neurotransmitters

\section{Introduction}

Mental health is a significantly important factor in the quality of life. It strongly affects one's performance and subjective well-being. In 2015, the World Health Organization announced the Sustainable Development Goals, which are "a universal call to action to end poverty, protect the planet and ensure that all people enjoy peace and prosperity" [1]. There are 17 goals, and Goal3; Good Health and Well-being is one of them. The World Health Organization has stated the importance of promoting mental health worldwide as well [2].

In this paper, we apply expert knowledge of mental health to our 2-Phase Correlation Computing. The fields of expert knowledge are neuroscience [3], psychiatry, and nutrition science [4]. We also present a knowledge base and database for realizing a

\footnotetext{
${ }^{1}$ Venera Raneva, Graduate School of Media and Governance, Keio University, 5322 Endo, Fujisawa-shi, Kanagawa, Japan; E-mail: venny@sfc.keio.ac.jp.
} 
comprehensive ranking of various types of mental health solutions. The concept of the Mathematical Model of Meaning was applied for creating the metadata [5,6]. In the database, there are 3 types of mental health solutions based on expert knowledge, "Nutrients", "Exercises", and "Life Habits". Based on neuroscience knowledge, we created the "Requests" matrix that enables comprehensive solution discovery from these multidisciplinary methods. The vector spaces of each matrix realize a semantic search function, expressing characteristics of the mental state numerically. The vector spaces are created based on previous studies showing the relations of stimuli and neurotransmitters. The solution ranking is based on scores that reflect the expected effectiveness of the information.

Mental disorders are caused by brain dysfunction, which is triggered by the imbalance of neurotransmitters in the brain $[3,4,7]$. The brain can function normally if their balance is restored. Internal medicine has been used for this purpose. Selective Serotonin Reuptake Inhibitors (SSRIs) such as Paxil, Prozac, and Zoloft suppress depression symptoms by eliminating the serotonin deficiency in the brain. Benzodiazepine anti-anxiety agents such as Librium, Barium, and Xanax inhibit excessive excitement of the brain by promoting the function of gamma-aminobutyric acid (GABA) in the brain. GABA is an inhibitory neurotransmitter [4].

The effectiveness of medicine has been proved. However, they have serious sideeffects, and long-term use develops tolerance, which can lead to addiction. To treat mental disorders, we have to restore the balance of neurotransmitters, and there are methods to realize this naturally without internal medicine [4].

Orthomolecular medicine describes the practice of treating and preventing diseases by providing the body with optimal amounts of endogenous and naturally occurring substances such as vitamins, minerals, and amino acids. Within orthomolecular medicine, there is orthomolecular psychiatry, which is the use of orthomolecular medicine for treating mental disorders [8].

Neuroscience is the scientific study of the nervous system. Numerous researches have proved the effectiveness of exercising in balancing brain functions, including neurotransmitters. The effectiveness of exercising in learning, managing stress, anxiety, depression, attention deficit disorder, addiction, hormonal changes, and aging has been proved [6]. There are also other life habits and activities that affect neurotransmitter secretion, such as insolation, meditation, taking naps, setting a deadline, and making a new challenge [3].

Many technologies support the adoption of each type of solution in daily life. For example, many nutrition management applications, exercise supporting applications, and habit tracking applications have been developed. However, a holistic approach that restores the balance of the whole body is more effective for the improvement of a condition. This is difficult because of the complexity of related information. There has not been a calculation method that ranks the effectiveness of information on nutrients, exercises, and life habits comprehensively.

This research realizes a novel calculation method that reflects the functions of neurotransmitters and provides solution ranking that helps the user adopt a holistic approach. 


\section{The Methodology of Database Creation}

There are three steps for creating the database. Firstly, a vector space that reflects moods and mental states is created. This is the "Requests" Matrix. Secondly, vector spaces that reflect the effects of the stimulus on the mental state are created. They are the "Nutrients", "Exercises", and "Life Habits" Matrixes. Thirdly, a vector space that reflects the contents of websites that include information about stimuli is created. This is the "URLs" Matrix. The basic rules of this database are as follows:

\subsection{Vector Space Structure}

\subsubsection{Origin Point}

The origin point in each stimulus matrix is where the stimulus has no significant relation with the neurotransmitter.

\subsubsection{Vector Normalization of Correlation Computing for Mental Health Analysis}

The goal of the vector normalization in this research is to express the actual mental health situation and make it possible to apply correlation computing to the normalized vector. In this method, 2-norm is used for the normalization with the minimum value, -2 , and the maximum value 2 .

The method of creating stimuli vectors is inspired by concepts of neuroscience, especially the roles of neurotransmitters on mental health. The value 0 means no significant relation. Negative values mean negative relation in mental health situations, and positive values mean positive relation. One key aspect of this research is to create a method of this normalization for neurotransmitter levels associated with different mental conditions. This realizes a semantic space reflecting expert knowledge such as neuroscience, medicine, and nutritional science.

The distance computing in this method consists of two phases. The first phase is calculating the inner product between the assumed neurotransmitter balance of particular mental states and stimuli. The second phase is calculating the inner product of information contained in websites and stimuli necessary for achieving the selected request. The value of this method is that it combines findings of various fields related to neurotransmitters and utilizes this metadata to find valuable information.

\subsection{System Structure}

A processing method with two inner product calculations is conducted in our system, which we refer to as "2-Phase Correlation Computing." Figure 1 shows the process of 2-Phase Correlation Computing in the system. The final calculation result of this process shows the scores of website URLs containing relevant information on effective solutions. 


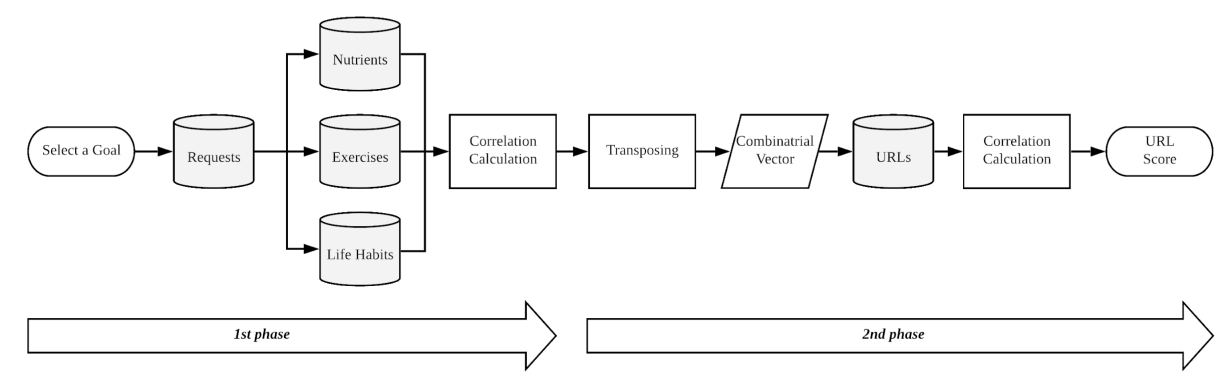

Figure 1. The Algorithm of 2-Phase Correlation Computing

As an input, the goal of the user is selected as a request. The "Requests" matrix consists of relations that are vectors reflecting the characteristics of each mental state. Characteristics of the mental states were numerically defined based on neuroscience knowledge and the concept of the Mathematical Model of Meaning (MMM) [5]. Semantic Search for finding personalized mental health information is realized with this method.

Next, the inner product of the requested mental state and each stimuli matrix ("Nutrients", "Exercises", and "Life Habits") is calculated. The calculation results are combined together and are transposed to form the combinatorial vector. The score of this calculation can be used to extract General Solutions. The first phase ends here.

Finally, the inner product of the combinatorial vector and "URLs" matrix is calculated. The "URLs" matrix consists of vectors reflecting the information content of each website. The URLs include general information, service information, products, and instructions. Various kinds of media that help the user understand or implement each solution (stimuli) have been selected. The correlation value is the score. Finally, based on the score, the website URLs expected to be effective in achieving the selected request are shown as a ranking.

Figure 2 shows the structure of each matrix. The method of vector creation in each matrix is explained after Figure 2. 


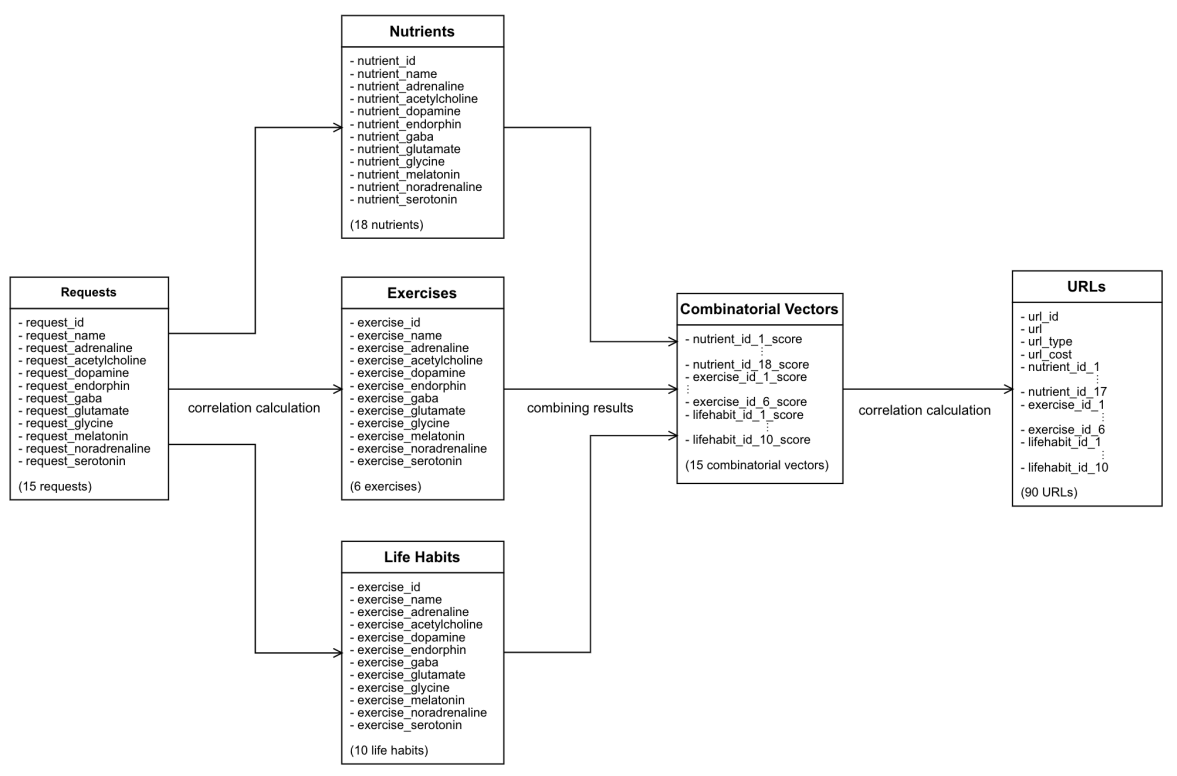

Figure 2. Database Structure

\subsubsection{The "Requests" Matrix}

The Requests matrix is meta-data that corresponds to the relation of each mental state and neurotransmitters that cause it. The relation is defined based on descriptions in a publication on neuroscience [3].

The Requests matrix consists of 10 dimensions. They are adrenaline, acetylcholine, dopamine, endorphin, gamma-aminobutyric acid (GABA), glutamine, glycine, melatonin, noradrenaline, and serotonin. " 0 " means that the state has no relation with the secretion of the corresponding neurotransmitter. " 1 " means that the state has a relation with the secretion of the corresponding neurotransmitter, and " 2 " means that it has a significant correlation. " -1 " means that it has a negative correlation with the secretion of the corresponding neurotransmitter. These values are interpretations of statements from expert knowledge on neuroscience [3]. "Defensive motivation" is a motivation triggered by the defensive system of the brain, and "intrinsic motivation" is a motivation that occurs as a result of the reward system of the brain. An example of a Semantic Search with "intrinsic motivation" as a request is shown in chapter 4.

\subsubsection{The "Nutrients", "Exercise", and "Life Habits" Matrixes (Stimuli Matrixes)}

Each stimulus matrix consists of 10 dimensions. They are identical to the dimensions of the "Requests" matrix. The values reflect the relation of each stimulus and the secretion of neurotransmitters. Vector values of nutrients [4,10,11,12], exercises [7], and life habits $[3,13,14,15,16]$ are determined based on previous studies.

In the "Exercises" matrix, based on a control experiment that studied the effect of exercising on depression patients, the exercise "aerobics (high dose)" means that the sum of the kilocalories consumed in a week is [17.6× (kilogram weight)]. The exercise named "aerobics (low dose)" means that the sum of the kilocalories consumed in a 
week is [17.6× (kilogram weight $) \times 0.45]$. Intense exercise that raises the heart rate is proved to be more effective in preventing anxiety [7].

\subsubsection{The "URLs" Matrix}

URL matrix is a metadata of the websites that would be shown as suggestions. 90 websites are registered. Websites that include information on the stimuli were chosen manually. The URL matrix has the information of each stimulus in "Nutrients", "Exercises" and "Life Habits". Various types of media are included so that the user can choose based on his/her needs. This matrix is a meta-data showing what stimuli data the websites include. The cost is also included. The type "g-information" means that the website has detailed information on the stimulus itself, and "s-information" means that the website has information on services that realize the acquisition of the stimulus.

For example, if the user is not sure what kind of media would be useful for him/her but prefers strongly related and affordable information, results excluding the types "product" and "s-information" can be extracted.

\subsection{The 2-Phase Correlation Computing}

The first phase and the second phase are both correlation calculations. The first phase has 10 factors (adrenaline, acetylcholine, dopamine, endorphin, gamma-aminobutyric acid, glutamine, glycine, melatonin, noradrenaline, and serotonin). The second phase can be freely determined by the preference of the URL or user's context.

In the second phase, the combinatorial vector that includes all the inner product calculation results of the selected request and each stimulus is generated. This realizes a calculation process that can evaluate the expected effectiveness of different types of websites containing different types of stimuli all at once. The mathematical expression of the process is as shown below.

First, the values in each matrix are converted into a numerical matrix as follows. The Request matrix, Nutrients matrix, Exercise matrix, and Life Habits matrix are each expressed as $R, N, E$, and $L$. Eq. (1), (2), (3), and (4) are the corresponding equations.

$$
\begin{aligned}
& R=\left(\left(r_{(1,1)} \cdots r_{(1,10)}\right), \cdots,\left(r_{(15,1)} \cdots r_{(15,10)}\right)\right) \\
& N=\left(\left(s_{(1,1)} \cdots n_{(1,10)}\right), \cdots,\left(s_{(18,1)} \cdots s_{(18,10)}\right)\right) \\
& E=\left(\left(s_{(1,1)} \cdots s_{(1,10)}\right), \cdots,\left(s_{(6,1)} \cdots s_{(6,10)}\right)\right) \\
& L=\left(\left(s_{(1,1)} \cdots s_{(1,10)}\right), \cdots,\left(s_{(10,1)} \cdots s_{(10,10)}\right)\right)
\end{aligned}
$$

The first row of the URL matrix consists of all the stimuli from Nutrients, Exercises, and Life Habits. The stimuli names listed in columns are converted into a row. The order is identical. The URL matrix is expressed as U, as shown in Eq. (5).

$$
U=\left(\left(u_{(1,1)} \cdots u_{(1,34)}\right), \cdots,\left(u_{(90,1)} \cdots u_{(90,34)}\right)\right)
$$


Second, the inner product of request and each stimulus is calculated, as shown in Eq. (6). The first phase ends here.

$$
a=R \cdot N, b=R \cdot E, c=R \cdot L
$$

Third, the results of this calculation are combined into one matrix, as shown in Eq. (7). The order is $a, b$, and finally $c$. Next, this matrix is transposed. This is the combinatorial vector. The order of the values in the combinatorial vector is identical to the order of all the stimuli in the URL matrix. The combinatorial vector is expressed as $\mathrm{V}$, as shown in Eq. (8).

$$
\begin{aligned}
& a \oplus b \oplus c=\left(\left(a_{1}, a_{18}\right), \cdots,\left(b_{1} \cdots b_{6}\right), \cdots,\left(c_{1} \cdots c_{10}\right)\right) \\
& V=\left(\left(a_{1} \cdots a_{18}\right),\left(b_{1} \cdots b_{6}\right),\left(c_{1} \cdots c_{10}\right)\right)
\end{aligned}
$$

Finally, the inner product of the combinatorial vector and URL Matrix is calculated, as shown in Eq. (9). The URL score is expressed as $Z$.

$$
Z=V \cdot U
$$

\section{Prototype System Implementation}

\subsection{Semantic Search for "Intrinsic Motivation"}

We have implemented the experimental system by using MATLAB [17]. The innerproduct values in the combinatorial vector show the effectiveness of each stimulus. Effective means that they are expected to lead to the mental state selected by the user. This is defined as the score of General Solutions for the request. General Solutions can be calculated after the first-phase correlation computing, as shown in Figure 3. In this case, "Intrinsic Motivation" is selected as a goal. The stimuli with a score higher than 0 are considered as General Solutions. The stimuli with a score lower than 0 are expected to cause the opposite effect. General Solutions show all the stimuli except those with score 0 .

These are the stimuli expected to be effective in achieving "intrinsic motivation". In this case, "aerobics (high dose)" was ranked with the highest score 2. This result matches with the findings that such exercise highly promotes motivation and prevents anxiety and depression [7]. There were 14 other stimuli with score 1 . They also matched with the expert knowledge included in the stimuli matrixes. In total, there were 3 exercises, 10 nutrients, and 2 life habits.

The score indicates that a high-dose aerobic exercise has the most significant influence in achieving this goal. This is because high-dose aerobic exercise influences more neurotransmitters than the other stimuli. Stimuli with score 1 trigger the production of a single neurotransmitter in the brain. This result shows that the expert knowledge included in the three stimuli matrixes are effectively integrated and can be extracted according to the user's request. 


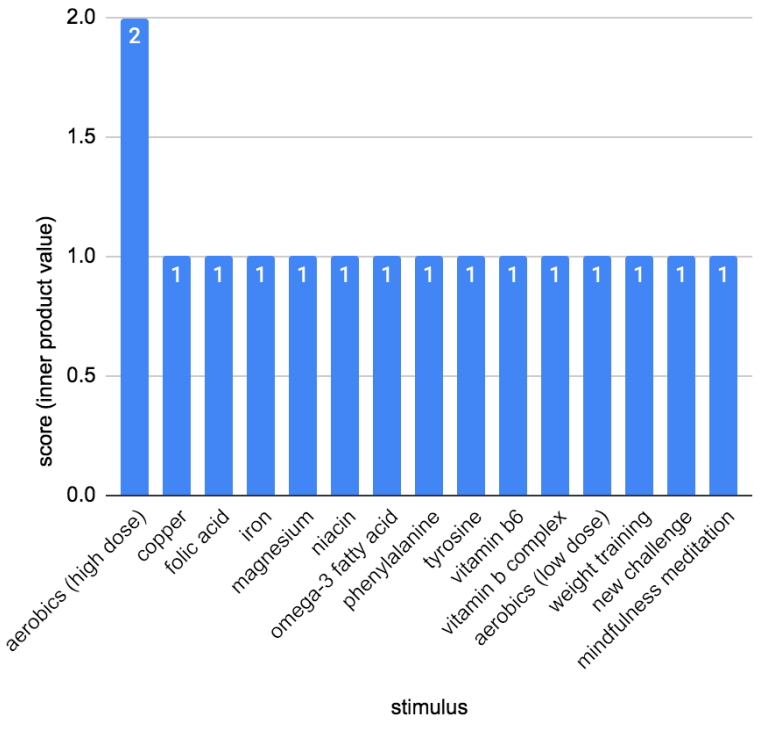

Figure 3. General Solutions for Intrinsic Motivation Ordered by Score (Inner Product Value)

As a result of the second-phase correlation computing, website URLs containing information on general solutions, and their scores are extracted. The score is defined as the inner-product value. Due to this nature, websites that include information of multiple stimuli tend to have higher results. The distribution of their score is shown in Figure 4.

By accessing any website with scores higher than 0 , the user can acquire detailed information on effective stimuli or purchase an effective product. The results with score 1 are websites with information or an online shop page for 1 stimulus. They are simpler and more targeted.

For example, all information on effective stimuli except "new-challenge" (a method to make a new challenge in life) can be found on the website with the highest score (https://www.webmd.com/). If the user wishes to acquire information, he/she can access the webpage ranked no.11 with score 1 , which has the instructions on making a new challenge ("https://www.wikihow.com/Do-Something-New").

There are many patterns in combining information for the user. The user can either start with accessing websites with relatively higher scores, or filter results based on their preferences. They can be filtered by stimuli types, information types, or cost. 


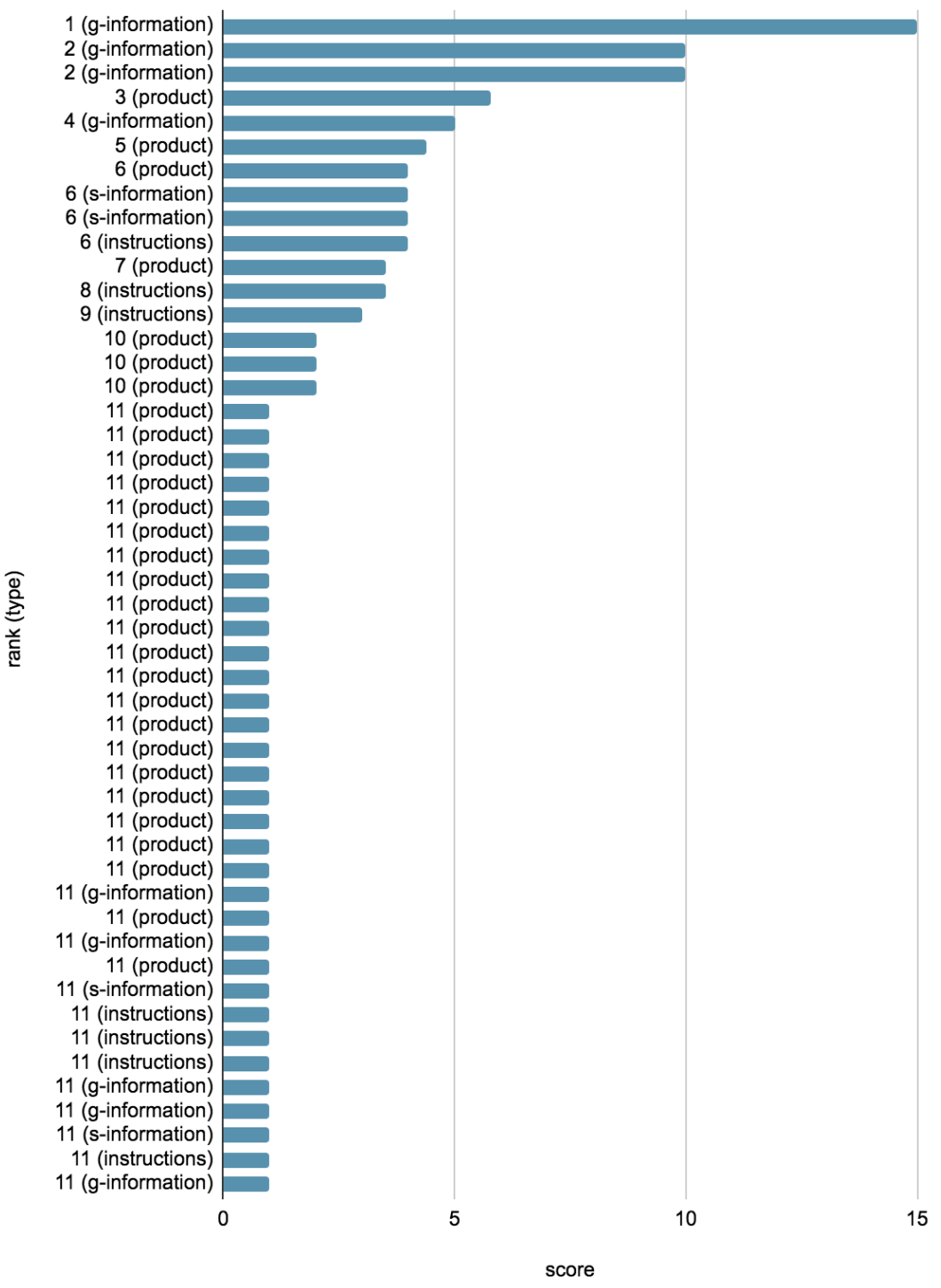

Figure 4. URL Score Distribution for Intrinsic Motivation (Ranking Ordered by Inner Product Value)

Next, with an assumption that the user only wishes to find instructions on acquiring the effective stimuli, the result can be filtered, as shown in Table 1. The webpage ranked no. 1 has information on high-dose aerobics, low-dose aerobics, and weight training. Thus, a wider variety of information can contribute to a higher score.

The websites with lower scores have information on fewer stimuli. However, it can mean their content is more concise if the score is bigger than 1. Using information with a score higher than 1, the user can implement actions that regulate their neurotransmitter levels to achieve the goal state. 
Table 1. Filtered URL Ranking for Intrinsic Motivation

\begin{tabular}{|c|c|c|c|}
\hline rank & url & score (inner product) & type \\
\hline 1 & $\frac{\text { https://www.bodybuilding.com/ }}{\text { index.html }}$ & 4 & instructions \\
\hline 2 & $\begin{array}{l}\text { https://www.youtube.com/result } \\
\text { s?search query=hiit }\end{array}$ & 3.5 & instructions \\
\hline 3 & $\begin{array}{l}\text { https://www.youtube.com/result } \\
\text { s?search query=cardio }\end{array}$ & 3 & instructions \\
\hline 4 & $\begin{array}{l}\underline{\text { https://www.youtube.com/result }} \\
\text { s?search query=how }+ \text { to }+ \text { squat }\end{array}$ & 1 & instructions \\
\hline 4 & $\begin{array}{l}\text { https://www.wikihow.com/Do- } \\
\text { Something-New }\end{array}$ & 1 & instructions \\
\hline 4 & $\frac{\text { https://hobbylark.com/misc/Ho }}{\text { w-to-Choose-a-Hobby }}$ & 1 & instructions \\
\hline 4 & ttps://youtu.be/yMz UagXkFk & 1 & instructions \\
\hline
\end{tabular}

\subsection{Semantic Search for "Calmness"}

When "calmness" is the selected goal, there were 27 general solutions calculated. They are shown in Figure 5. Unlike the previous case with the goal "intrinsic motivation", there are 9 general solutions with negative scores. This indicates that these stimuli lead to the opposite state of "calmness".

Calmness is promoted when inhibitory neurotransmitters are produced in the brain. The nutrients phenylalanine and tyrosine are necessary for the production of adrenaline and noradrenaline, which are excitatory neurotransmitters. They lead to excitement and agitation, which are the opposite states of calmness. Weight training, setting a deadline, and excess copper intake also promotes this. The nutrients folic acid, iron, and niacin act as coenzymes when producing the excitatory neurotransmitters. However, without tyrosine and phenylalanine, they have a neutral effect on neurotransmitters. There should be a function to exclude such nutrients.

In the case of "calmness", the solution with the highest score was mindfulness meditation. It promotes the synthesis of neurotransmitters that lead to calmness and prevents the synthesis of neurotransmitters that lead to the opposite state $[13,14,15,16]$.

As in the case with the Semantic Search for intrinsic motivation, when fewer neurotransmitters are associated with the stimulus, the score is lower. Again, stimuli shown matched with the expert knowledge included in the stimuli matrixes. The scores calculated with 2-Phase Correlation Computing can be used for discovering priority in mental health management and preventing non-favorable stimuli. 


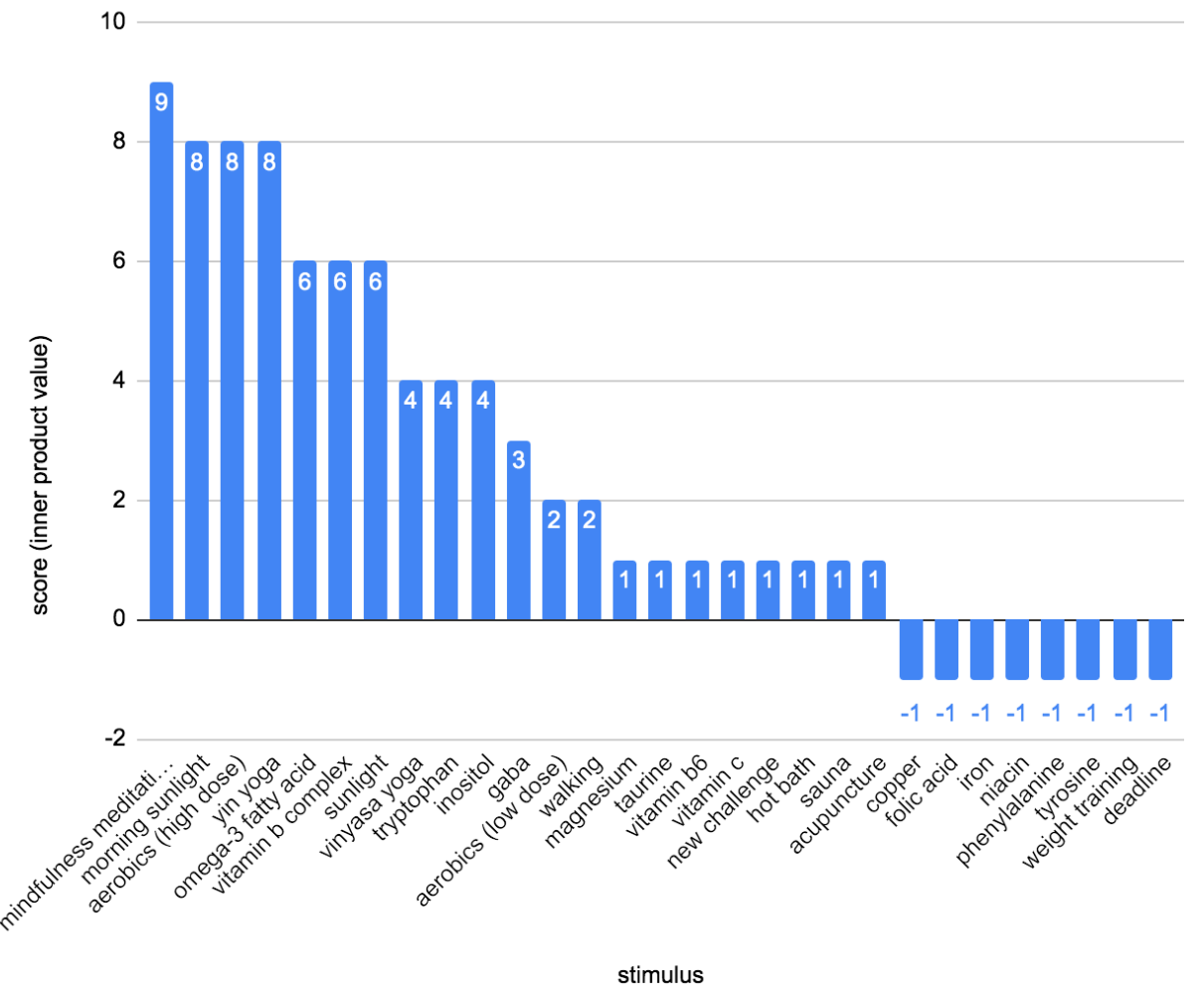

Figure 5. General Solutions for Calmness Ordered by Score (Inner Product Value)

Figure 6 shows the website score distribution of results after the second phase. Similar to the semantic search for "intrinsic motivation", the websites that include information on more stimuli tend to have a higher score. All information necessary for acquiring effective stimuli can be found.

This time, rank No.1 to 7 were all either general information or service information websites. Products that include multiple effective nutrients and single nutrients necessary for the production of multiple neurotransmitters had relatively high scores. This means that the priority of the information is assessed effectively. Discovering priority among multidisciplinary solutions is complex. However, it is automatically calculated and numerically expressed with our processing method. 


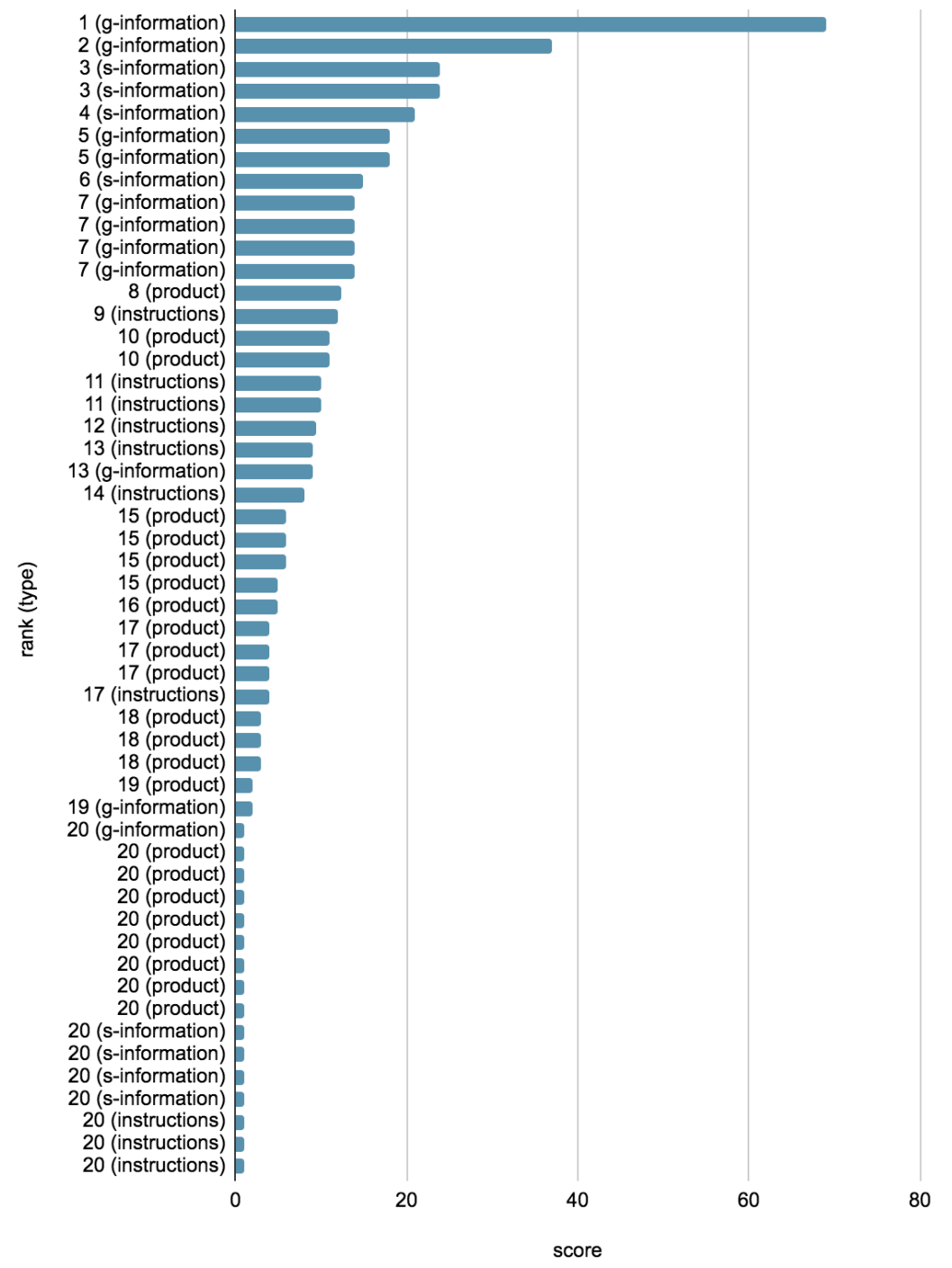

Figure 6. URL Score Distribution for Calmness (Ranking Ordered by Inner Product Value)

\subsection{Evaluation of the Prototype Implementations}

As Figure 7 shows high rates of recall and precision rates for each request, it can be said that 2-Phase Correlation Computing has a high performance in integrating multidisciplinary knowledge related to mental health. 


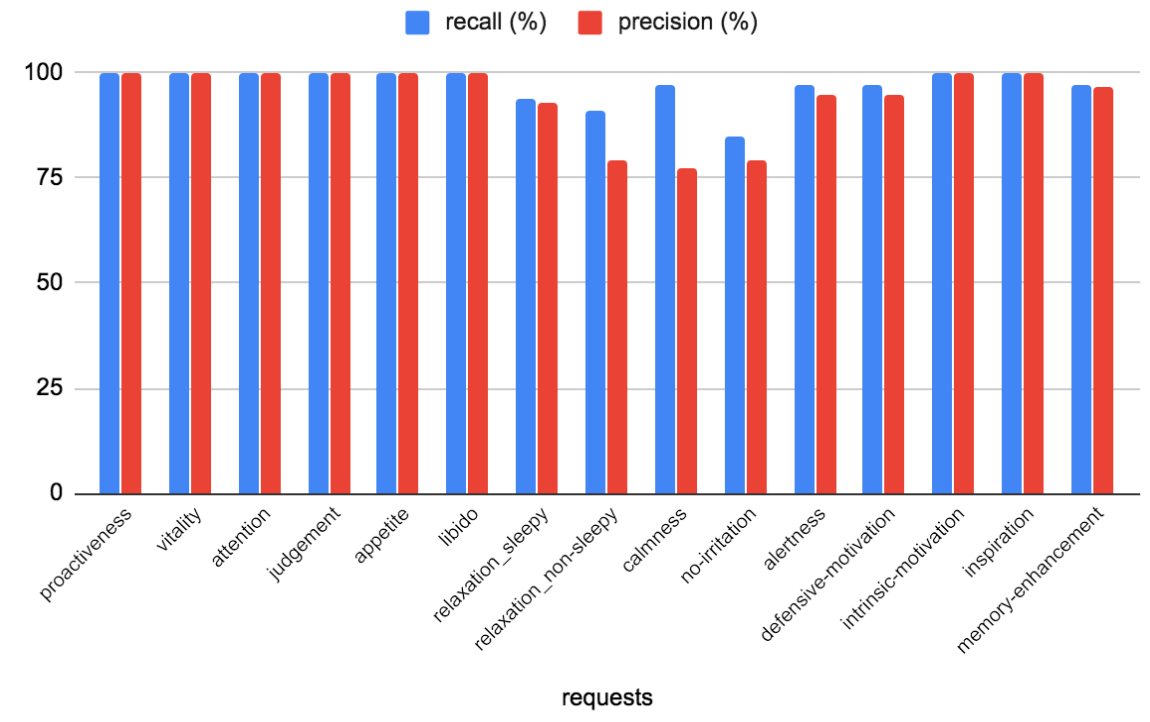

Figure 7. Recall \& Precision Rate after 2-Phase Correlation Computing

The results of the first-phase correlation computing showed General Solutions that reflect specialists' decisions on achieving each mental state. The results having minus values in the request had relatively low recall and precision rates.

The results of the second-phase correlation computing also showed scores of effective URLs that reflect specialists' decisions. URLs with score 1 have information or a product of a single stimulus, whereas higher scores were observed in general information, service information websites, products, and instructions that include multiple stimuli.

When filtering the results based on their types or costs, the differences in the score decreased. This indicates that specific types of information tend to show similar values.

\section{Conclusion}

In the prototype implementation, the notable features stated below were observed. They were realized with 2-Phase Correlation Computing. The main contribution of this research is the new information processing method for the integration of multidisciplinary knowledge on mental health. 2-Phase Correlation Computing can be a core function of a meta-level architecture for a mental health database. The current characteristics of the database are as stated below:

First, in this system, the user can choose the desired goal simply by the state name, and in the database, it is automatically converted into numbers.

Second, 2-Phase Correlation Computing allows finding multidisciplinary methods easily. This processing is dynamic and flexible. In this processing method, new parameters can easily be added, which means it has high scalability.

Third, the solutions are shown as a table. Currently, actuation is done within the database. Creating a web interface will allow many people to access this system. 
"A Mental Health Creation Method with Neuroscience-inspired Search Functions" realizes a highly accurate and comprehensive information discovery for improving mental health. This is realized with its unique information processing, 2-Phase Correlation Computing. This original method enables a comprehensive evaluation of various types of information on mental health. Utilizing the combinatorial vector, the user can discover effective solutions from multidisciplinary fields related to mental health in the first phase. In the second phase, concrete URLs containing information or products that include effective solutions are extracted. One of the advantages of this method is that both General Solutions and URLs can be extracted based on the user's preference. The information type can also be specified. This flexibility enables the user to find solutions that match his/her needs.

The novelty in this research is that it provides an information processing method to the field of mental health management. Sensing, processing, and actuation are three key elements for a system with an input and an output. The knowledge for improving mental health is usually patterns of the symptoms and treatments. On the other hand, our system offers interactive and dynamic processing for finding mental health solutions. By choosing a mental health goal as an input, customized information on effective solutions is shown as an output. The key achievement of this database creation method is processing. Through automatic curation, many types of people who are eager to increase their mental well-being or performance could benefit. The 2-Phase Correlation Computing showed a good performance in the universal evaluation of information related to mental health. Creating symbolic filter functions and an automatic patternmaking system for information will enable a more sophisticated information delivery.

In the future, processing could be improved to reflect expert knowledge on mental health more elaborately, including parameters other than neurotransmitters. For example, the endocrine system or brainwave balance can be reflected in creating a semantic space. With a new vector space creation that includes expert knowledge on health, it can also be applied to all health information. Enriching sensing and actuation functions will increase the usability of this research as well. Sensing can be improved to reflect the user's mental state more precisely, and actuation can be improved to offer information in more practical ways. Also, if vector space creation and website information retrieval from the Internet can be automated, the 2-Phase Correlation Computing can become an even more powerful platform for searching and analyzing health information.

\section{Acknowledgments}

We would like to express our sincere gratitude to Dr. Yasuhiro Hayashi, who provided us with valuable advice regarding system implementation.

\section{References}

[1] Sustainable Development Goals [Internet]. UNDP. [cited 2020January25]. Available from: http://www.undp.org/content/undp/en/home/sustainable-development-goals.html

[2] Mental health included in the UN Sustainable Development Goals [Internet]. World Health Organization. World Health Organization; 2016 [cited 2020January25]. Available from: http://www.who.int/mental_health/SDGs/en/ 
[3] Kabasawa S. Nō o saitekika sureba nōryoku wa nibai ni naru: shigoto no seido to sokudo o nōkagakuteki ni ageru hōhō (English translation: Human abilities can be enhanced through optimization of the brain). Bunkyōsha; 2016.

[4] Ikuta S. Kokoro no yamai wa shokuji de naosu (English translation: Mental diseases can be treated with food). Tōkyō: PHP Kenkyūjo; 2005.

[5] Kiyoki, Y. and Kitagawa, T., "A mathematical model of meaning and its application to multidatabase systems," Proceedings RIDE-IM' '93: Third International Workshop on Research Issues in Data Engineering: Interoperability in Multidatabase Systems, Vienna, Austria, pp. 130-135, 1993.

[6] Kiyoki, Y. Kitagawa, T. and Hayama, T., "A metadatabase system for semantic image search by a mathematical model of meaning," ACM SIGMOD Record, vol. 23, no. 4, pp.34-41, 1994.

[7] Ratey, JJ., Hagerman, E. and Spark, "The revolutionary new science of exercise and the brain," New York: Little, Brown; 2013.

[8] Zell, M. and Grundmann, O., "An orthomolecular approach to the prevention and treatment of psychiatric disorders," Adv Mind Body Med. 2012 Fall;26(2):14-28. Review.

[9] Murray, MT., "The encyclopedia of nutritional supplements," Rocklin, CA: Prima; 2002.

[10] Davidson, JRT. and Connor KM., "Herbs for the mind: what science tells us about nature's remedies for depression, stress, memory loss, and insomnia," New Delhi: Health Harmony; 2002.

[11] Braverman, ER., "The healing nutrients within. North Bergen," NJ: Basic Health Publications; 2003.

[12] Leitner, Z., "Nutritional studies in a mental hospital,” The Lancet. 1956;267(6922):565-7.

[13] Esch, T., "The neurobiology of meditation and mindfulness," Meditation-Neuroscientific Approaches and Philosophical Implications. 2013Dec;153-73.

[14] Krishnakumar, D., Hamblin, MR. and Lakshmanan, S., "Meditation and Yoga can modulate brain mechanisms that affect behavior and anxiety- A modern scientific perspective," Ancient Science. 2015Jan;2(1):13.

[15] Bujatti, M., and Biederer, P., "Serotonin, noradrenaline, dopamine metabolites in transcendental meditation-technique," Journal of Neural Transmission. 1976;39(3):257-67.

[16] Solberg, EE., Holen, A., Ekeberg, Ø., Østerud, B., Halvorsen, R., Sandvik, L., "The effects of long meditation on plasma melatonin and blood serotonin," Med Sci Monit 2004. 2004Mar;10(3)(CR96-101).

[17] MATLAB [Internet]. MathWorks. [cited 2020January25]. Available from: https://www.mathworks.com/products/matlab.html 


\title{
Q-Learning as Failure
}

\author{
Kei TAKAHATA, and Takao MIURA \\ Dept.of Advanced Sciences, HOSEI University \\ Kajinocho 3-7-2, Koganei, Tokyo, Japan \\ Email:kei.takahata.6a@stu.hosei.ac.jpmiurat@k.hosei.ac.jp
}

\begin{abstract}
Reinforcement Learning allows us to acquire knowledge without any training data. However, for learning it takes time. In this work, we propose a method to perform Reverse action by using Retrospective Kalman Filter that estimates the state one step before. We show an experience by a Hunter Prey problem. And discuss the usefulness of our proposed method.
\end{abstract}

Keywords. Reinforcement Learning, Q-Learning, Kalman Filter, Retrospective Kalman Filter, Reverse Action Learning

\section{Introduction}

Reinforcement Learning (RL) [1] [2] is a learning method in which any agent learns from interaction with its environment. It allows us to acquire knowledge without any training data. However, for learning it takes time. In a case of a robot, it takes heavy time to perform many experiences. Therefore, various methods aiming at reducing the amount of learning experiences have been proposed [4] [5]. These put focus on how to acquire quality knowledge efficiently. Unfortunately, there are few methods to improve knowledge proposed so far by using failure. For example in Fig.1, suppose that the agent acts twice to the left from the center position in the initial state and gets a reward of -100 where the circles in Figure 1 represent states, and the numbers represent rewards. Is it possible to improve knowledge by other choices? By the word "Reverse Action Learning(RAL)", we mean a learning method that agents select a reverse action and receive reverse rewards. In the case of Fig.1, the number of states and actions is small, therefore it could be possible to examine all the choices.However, in the case of complex tasks, it is not practical to keep all information. Therefore, the goal of this work is to propose RAL.

Retrospective behavior could have two interpretation, "compensation behavior" or "reverse behavior". "Compensation"[WIKI] refers to a type of defense mechanism in which people overachieve in one area to compensate for failures in another. So one can cover up, consciously or unconsciously, weaknesses, frustrations, desires, or feelings of inadequacy or incompetence in one life area through the gratification or (drive towards) excellence in another area. On the other hand, "reverse action" means a type of recovery mechanism whereby people can restore almost all the status back into the previous one to keep the environment consistently in any means of the situation before these actions. 


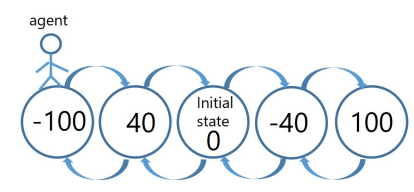

Figure 1. Example of state transition and rewards

Very often they differ from each other since compensation could be made by similar actions without any consideration of environment. The story looks like theory of transaction in databases [6]. We'd better say "roll back" for "reverse action". Here we stick to the situation "reverse" (or "roll back") behaviors since we might take strategic decisions every time we have to decide.

In this work, we model a method to perform RAL by using a Retrospective Kalman filter that estimates the state one step before. We discuss an experience by a Hunter Prey problem to see we show the usefulness of our proposed method.

The rest of the paper is organized as follows. In section 2 we give a denition of Reinforcement Learning. Section 3 discusses a background of Kalman filters for our learning. In section 4 we propose our approach of and section 5 concerns about experimental results to see how effective our approach works. In section 6 we conclude this investigation.

\section{Reinforcement Learning and Q-Learning}

\subsection{Reinforcement Learning}

Reinforcement learning (RL) is a learning method in which the agents obtains knowledge from interaction with its environment. Agents perform state perception and make a dicision. In RL, we don't give explicit correct answers and agents learn from rewards under the environment. Here, rewards include positive and negative. Agents goal is to find a policy that maximizes the total rewards.

An agent perceives its current state (or position at time $t$ ), selects an action for its behavior, to obtains reward, and then changes itself to the next state. When an agent takes an action $a_{t}$ at time $t$, the next state $s_{t+1}$ and the reward $r_{t+1}$ at time $t+1$ depend on all the previous states and all the rewards in the agent history. Let $s^{\prime}$ and $r$ be one of the possible next states and the rewards, then $s_{t+1}$ and $r_{t+1}$ can be described by means of conditional probabilities:

$$
\operatorname{Pr}\left\{s_{t+1}=s^{\prime}, r_{t+1}=r \mid s_{t}, a_{t}, r_{t}, s_{t-1}, \cdots, r_{1}, s_{0}, a_{0}\right\}
$$

Markov probability model is a stochastic framework to model randomly changing systems. Here next state depends only on the current state, not on the previous states nor actions before. This is called Markov property. Generally this assumption enables reasoning and computation with the model efficient. In our case we must have:

$$
\operatorname{Pr}\left\{s_{t+1}=s^{\prime}, r_{t+1}=r \mid s_{t}, a_{t}\right\}
$$

Assuming current state $s_{t}$ and an action $a_{t}$ under the assumption of Markov property, we can estimate the next state $s_{t+1}$ and the reward $r_{t+1}$ in a probabilistic manner as shown 
in a formula (2). Repeating this process of (2), we may obtain all the future states, the actions and the whole rewards. In RL, it is possible to say that behaviors and the value functions (described later) depend only on current state.

Let us define a notion of policy that consists of all the pairs $(a, p(a))_{s}$ of action $a$ and its probability $p(a)$ to each state $s$. The main goal of agents in RL is to obtain a policy $\pi$ to solve issues of interests efficiently, or, in our case, to maximize the whole rewards in its life-time. To do that, we introduce value functions. Since we discuss Q-Learning (QL) [8], we define action-value functions called the Q-value. Q-value represents the expected value of whole rewards under the certain policy $\pi$.

\subsection{Q-Learning}

$\mathrm{QL}$ is a represemtative learning method of $\mathrm{RL}$. By $Q(s, a)$, let us define the Q-value (the expected all the sum of rewards) at a state $s$ with an action $a$. Formally let $r=r(s, a)$ be a reward at $(s, a), s^{\prime}=N \operatorname{ext}(s, a)$ a next state to $s$. Also let $a^{\prime}$ be a next action at $s^{\prime}$ of the maximim $Q\left(s^{\prime}, a^{\prime}\right)$, that is, $a^{\prime}=\max _{a^{\prime} \in A\left(s^{\prime}\right)} Q\left(s^{\prime}, a^{\prime}\right)$ where $A\left(s^{\prime}\right)$ a set of possible actions at $s^{\prime}$. Then let $\alpha$ be learning rate $(0 \leq \alpha \leq 1)$, which means how large one learning step improves Q-values, and $\gamma$ be discount rate which means the effect of valuing rewards received earlier higher than those received later. This may also be interpreted as the probability to succeed (or survive).

At every learning step, we keep updating Q-values as follows:

$$
Q(s, a) \leftarrow Q(s, a)+\alpha\left[r+\gamma \max _{a^{\prime} \in A\left(s^{\prime}\right)} Q\left(s^{\prime}, a^{\prime}\right)-Q(s, a)\right]
$$

Note that we update the Q-values so as to make $Q(s, a)$ close to $r+\gamma \max _{a^{\prime} \in A\left(s^{\prime}\right)} Q\left(s^{\prime}, a^{\prime}\right)$.

Watkins [8] has shown the convergence of the Q-values if both learning rate and discount rate satisfy some constraints.

\subsection{Reverse Action Learning}

Let us discuss a case in which an agent receives a large negative reward. We assume that the reverse action allows us to improve the current situation. Under this assumption, we update efficiently the Q-value by Reverse Action Learning(RAL). If an agent recieves a large negative reward, an agent reduce the value taken to the negative reward actions by normal learning process. Moreover, an agent could improve the value of reverse actions by RAL process, that is, we expect that an agent learn how to avoid these actions.

\section{Kalman Filter}

Kalman filter (KF) is one of the well-known algorithm that estimates the state of a system from observation data with some noises. During KF process, whenever a new (temporal) observation data comes in, we improve estimation of state immediately. We estimate a priori estimate of a state $X_{k}$ at time $k$, denoted by $\hat{X}_{k}^{-}$, by examining $X_{k-1}, X_{k-2}, \ldots$ Similarly we estimate a posteriori estimate of $X_{k}$, denoted by $\hat{X}_{k}$, by examining $Y_{k}, X_{k-1}, X_{k-2}, \ldots$ Given a covariance matrix $P$ of state errors, we can think about a 
priori estimate $P_{k-1}^{-}$and a posteriori estimate $P_{k}$. Let us note that by minimizing $P_{k}$ (using minimum mean-square error), we can improve the precision of the estimation.

Now assume we have Kalman gain matrix $G_{k}$ at $k$, there happen filtering processes:

\begin{tabular}{ll}
\hline EstimateStep & \\
$\hat{X}_{k}^{-}$ & $=A \hat{X}_{k-1}$ \\
$P_{k}^{-}$ & $=A P_{k-1} A^{T}+B Q B^{T}$ \\
\hline FilteringStep & \\
$G_{k}$ & $=P_{k}^{-} C^{T}\left(C P_{k}^{-} C^{T}+R\right)^{-1}$ \\
$\hat{X}_{k}$ & $=\hat{X}_{k}^{-}+G_{k}\left(Y_{k}-C \hat{X}_{k}^{-}\right)$ \\
$P_{k}$ & $=\left(I-G_{k} C\right) P_{k}^{-}$ \\
\hline
\end{tabular}

Let us remark that we have to give initialization values of $\hat{X}_{k-1}$ initial states, $P_{k-1}$ initial a priori errors covariance, and $Q, R$ noises covariance.

The KF improves the accuracy of state estimate by reducing the error covariance. Kalman gain represents the rate at which the state is updated from observations. For example, when a priori estimate covariance is large (a priori state estimate is not reliable) and when the observation noise is small (the observed value is reliable), a priori state estimate is largely updated because the observed value is more reliable. Therefore, the Kalman gain also increases. Meanwhile, when a priori estimate covariance is small and the observation noise is large, the Kalman gain is small because the state transition is more reliable than the observed values.

KTD [13] [12] has been proposed as a method for estimate parameters to RL over continuous states. KTD has a problem that it depends on initial parameters. Our proposed method uses Kalman filter for agent action selection, so it is fundamentally different from KTD.

\section{Proposed method}

\subsection{Retrospective Kalman Filter}

Now let us propose a new method to learn efficiently by reverse actions due to the Qvalue update. We put a Failure Condition on our agents. Whenever the learning system detects Failure Condition due to the amount of Negative rewards, the system initiates RAL process. Otherwise the agent keeps learning. Let us discuss how to restore (recover) the original state of the agent. If the agent keeps both all transition histories and action histories, agent could put all the statues back to the original. However, because of memory limitation, we introduce Retrospective Kalman Filter(RKF) to recover any state one step before with few memory. Whenever we use KF, we can estimate a posteriori state estimate $\hat{X}_{k-1}$ and a posteriori estimate covariance $P_{k-1}$ from a priori state estimate and a priori estimate covariance.

KF uses posteriori state estimate one step before $\hat{X}_{k-1}$ and posteriori estimate covariance one step before $P_{k-1}$ in order to estimate a current a posteriori state estimate $\hat{X}_{k}$ and posteriori estimate covariance $P_{k}$. In RKF, we use a current a posteriori state estimate $\hat{X}_{k}$ and posteriori estimate covariance $P_{k}$ in order to estimate posteriori state estimate one step before $\hat{X}_{k-1}$ and posteriori estimate covariance one step before $P_{k-1}$.

We can get a posteriori estimate covariance $P_{k}$ from a priori estimate covariance $P_{k-1}^{-}$ and Kalman gain $G_{k}$ using Kalman Filter. Therefore, we can not get a priori estimate co- 
variance $P_{k-1}^{-}$analytically from a posteriori estimate covariance $P_{k}$. We note that agents retain some priori estimate covariance $P_{k-1}^{-}$when learning and that they take reverse action. The number retaining a priori estimate covariance matrix is a hyperparameter. This is equal to the number of times that RAL can be performed. We define a retrospective Kalman filter below:

\begin{tabular}{ll}
\hline RetrospectiveFilteringStep & \\
\begin{tabular}{ll}
$G_{k}$ & $=P_{k}^{-} C^{T}\left(C P_{k}^{-} C^{T}+R\right)^{-1}$ \\
$\hat{X}_{k}^{-}$ & $=\left(I-G_{k} C\right)^{-1}\left(\hat{X}_{k}-G_{k} Y_{k}\right)$ \\
\hline RetrospectiveEstimateStep & \\
$P_{k-1}$ & $=A^{-1}\left(P_{k}^{-}-B Q B^{T}\right)\left(A^{T}\right)^{-1}$ \\
$\hat{X}_{k-1}$ & $=A^{-1} \hat{X}_{k}^{-}$
\end{tabular} \\
\hline
\end{tabular}

\section{2. $O L R K F$}

By the word "QLRKF", we mean a learning method that agents perform RAL. We use RKF to return agent's states. Agents keep learning by QLKF [9] unless agents do obey a Failure Condition. In QLKF, agents take an action under KF and provability $\varepsilon$, and greedy action with probability $(1-\varepsilon)$. If agents follow a Failure Condition, agents take a reverse action using the estimate by RKF with probability $\varepsilon$, and a reverse greedy action with probability $(1-\varepsilon)$.

\section{Experiment}

\subsection{Hunter Prey Problem}

In this work, we deal with the Hunter Prey problem which is a standard task of RL. Traditionally in the problem we discuss a discrete $2 \mathrm{D}$ space but here we assume a continuous 2D space of $m \times m, 0 \leq x, y \leq m$ instead of the grid space. We assume one hunter and one prey in the space, the former agent always pursues (chases) the latter agent. The agents can't go outside the 2D space. Initially we put the two agents randomly in such a way that they keep at some distance off. We say the prey is captured when they stand closely with each other (say, less than $\rho$ ). Whenever the prey is captured, the hunter gets positive reward and the prey gets negative. Otherwise, the hunter gets negative and the prey positive.

There have been several kinds of assumption in hunter-prey games, but very often these preys take actions randomly without any knowledge. Here we assume both the agents might know positions of all the agents in the space, then we examine both cases of knowledgeable prey (i.e., the prey learns autonomously) and non-knowledgeable prey (actions are randomly selected). We assume the hunter learns by QL for the purpose of own Q-values improvement, considering prey's relative positions as states: based on $\mathrm{RL}$, we assume discrete states, although these states are continuous. As for the prey, we assume the prey may learn by QL, or we assume take actions randomly without any knowledge.

Let us describe how we construct 2D space in a discrete manner. Assume a hunter stands at a position considered as a center relatively, and we divide the $2 \mathrm{D}$ space into 8 areas where each area is further divided into two areas, a close area and the farther, as well as a center area. So there are 17 (relative) areas in total. Let us illustrate an example 


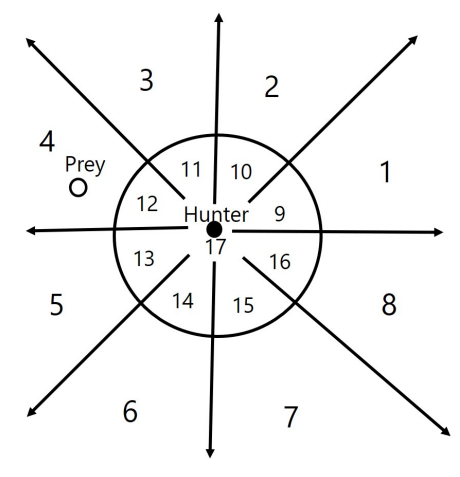

Figure 2. Position Area

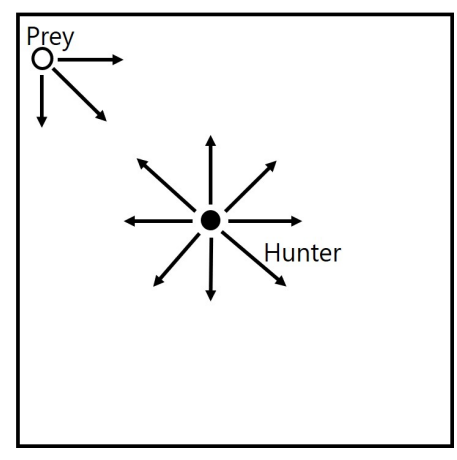

Figure 3. Actions of Hunter and prey

in a figure 2 where a hunter look at a prey in its area 4 . There are 9 actions in the problem as shown in a figure 3:8 directions and stay. For example, when a hunter stands at a center and a prey stands at the left upper corner, the hunter can select one of 9 actions. However, the prey can't move up or left any more because of the field boundary and can select one of 5 choices ${ }^{1}$.

Let us summarize the movement of the agent:

(a) put a hunter and a prey in a space initially.

(b) a prey perceives a hunter and selects an action.

(c) a hunter perceives a prey and selects an action.

(d) both get rewards according to their results.

(e) a prey perceives a hunter and learn.

(f) a hunter perceives a prey and learn.

(g) if a hunter captures a prey, go to (a). Go to (b) otherise. We keep utilizing both the Q-values and the Kalman gain in any case.

In our proposed method, if the hunter has moved a certain number, but has not been able to catch the prey, hunter perform RAL. Let us note a certain number is hyperprameter and equal the number that hunter can perform reverse action. Let us describe the flow during RAL:

(a) a hunter perceives a prey and selects an action.

(b) a prey perceives a hunter and selects an action.

(c) a hunter and a prey get rewards.

(d) a hunter perceives a prey and learn.

(e) a prey perceives a hunter and learn.

\subsection{Preliminaries}

Here we mention our experimental results to see how well our approach works. We examine a Hunter Prey problem with one hunter and one prey. We discuss two cases in which the prey dose not learn and prey learns. In each case, hunter learns with $\varepsilon$-greedy (comparison method 1), QLKF (comparison method 2), and QLRKF (proposed method)

\footnotetext{
${ }^{1}$ The prey can select one of right, right down, down and stay.
} 
and evaluates. We evaluate the number of capture steps. We consider that the number of capture steps indicates the quality of knowledge, and that the smaller the number of steps being better learning method.

When the hunter learns with QLKF (comparison method 2) and QLRKF (proposal method), the hunter uses a Kalman filter that estimates the position of the prey. The hunter takes an action aproaching the estimated position by KF with probability $\varepsilon$.

The field consists of a continuous $2 \mathrm{D}$ space $[0,1] \times[0,1]$. In the learning process, we assume the distance of 0.8 between the two agents initially, and the prey can be captured within distance $\rho=0.1$. The reward 100 is given to the hunter when the hunter captures the prey, and -1 otherwise. Hunters receive a reward of 1 during RAL by our proposed method. Note this reward is the reverse of normal learning. The hunter performes RAL on condition that the hunter do not captures the prey. Therefore, we do not consider the reverse reward at capture. On the contrary, the prey gets the reward - 80 when the prey is captured and 1 otherwise. As well as the hunter, the prey receive a -1 reward during the hunter performes RAL by our proposed method.

Here we assume $\alpha=0.1$ (learning rate) and $\gamma=0.9$ (discount rate) of QL for both agents. And we assume $\varepsilon=0.1$ for both agents. We set the number that holds $P_{k-1}^{-}$of our proposed method to 50 .

For KF processing, we initialize covariance matrices: $P_{0}=10^{4} I$ a covariance matrix of state estimation error, $V_{0}=0.05 I$ a covariance matrix of process noises and $0.9 \times$ $0.999^{\text {learningcount }} I$ a covariance matrix of observation noises. We set the covariance matrix of the observation noise as $0.9 \times 0.999^{\text {learningcount }} I$, so that the error decreases as the number of learning increases.

When a hunter likes to estimate the next state $x_{t+1}, y_{t+1}$ of the prey at time $t$ by KF, it observes the state $x_{t}, y_{t}$ of the prey by position sensors. Let us note the hunter estimates the current state using the previous observation using KF framework with process noise $V_{t}$ and observation noise $W_{t}$ like a state equation 3 and an observation equation 4:

$$
\begin{gathered}
\left(\begin{array}{l}
x_{t+1} \\
y_{t+1}
\end{array}\right)=\left(\begin{array}{ll}
1 & 0 \\
0 & 1
\end{array}\right)\left(\begin{array}{l}
x_{t} \\
y_{t}
\end{array}\right)-\left(\begin{array}{ll}
1 & 0 \\
0 & 1
\end{array}\right)\left(\begin{array}{l}
h v_{x t} \\
h v_{y t}
\end{array}\right)+\left(\begin{array}{ll}
1 & 0 \\
0 & 1
\end{array}\right) V_{t} \\
\left(\begin{array}{l}
x_{t} \\
y_{t}
\end{array}\right)=\left(\begin{array}{ll}
1 & 0 \\
0 & 1
\end{array}\right)\left(\begin{array}{l}
x_{t} \\
y_{t}
\end{array}\right)+W_{t}
\end{gathered}
$$

Here $h v_{x t}, h v_{y t}$ represent velocity of the hunter at time t.

\subsection{Evaluation Criteria}

Let us discuss evaluation criteria, and capturing process. First in this experiment, we say one step when both agents take every actions (behaviors), and one interval for 100 steps. This means, during one interval in the learning process, the hunter learns (updates Q-values) 100 times. We also say one episode for 100 intervals, or equivalently 10,000 steps. We examine a capturing steps in every interval. We initialize Q-values at the beginning of each episode (10,000 steps). We examine 10 episodes for every experiment and take the average steps for capturing of 10 episodes.

In this experiment, we examine both learning and capturing processes in an interleaved manner. That is, we do learning in one interval then capturing the prey 10 times 
Table 1. Total number of Capturing Steps

\begin{tabular}{|c|c|c|c|}
\hline Learning & QL & QLKF & QLRKF \\
\hline (only Hunter learned) & 19467 & $14916(0.77)$ & $12695(0.65)$ \\
\hline (Both learned) & 19868 & $9308(0.47)$ & $7806(0.39)$ \\
\hline
\end{tabular}

using the Q-values, and we continue learning further. At each interval, we stop learning and execute the capturing: the hunter chases the prey 10 times. Everytime we take counts of the steps ${ }^{2}$ until the hunter captures the prey, and take an average count as capturing step of this interval. The chasing manner in the capturing process is the same as in the learning process. However, during the capturing process of our proposed method, the hunter does not take a reverse action, and take an action based on QLKF. That is, when learning with $\varepsilon$-greedy during learning process, the capturing process is also $\varepsilon$-greedy. (Hereafter, in the case of an agent takes an action based on $\varepsilon$-greedy, we denote as QL.)

Note that we should have some discussion of how to evaluate results of learning and capturing. Clearly the smaller steps we need for capturing, the better knowledge we have. Also, the less steps we need to get convergence (described later), the better parameters we have for learning. The former concerns about capturing quality while the latter about learning efficiency. Then we also introduce a notion of harmonic average $h$ as a combined criteria of both learning and capturing where $h=2 p q /(p+q), 1 p$ means a earning step and $q$ a capturing step. The less $h$ goes, the better performance we have.

\subsection{Results}

In tables 2 and 3, we show the results of capturing steps depending on "who learn by QL, QLKF and QLRKF". We also illustrate overviews of the tables in figures 4 and 5 respectively.

It is clear that our proposed approach (QLRKF) outperforms the comparison methods (QL, QLKF), ie, we have less steps (the better efciency). A table 1 contains the comparison of capturing steps in QL, QLKF, QLRKF cases. In the total number of capturing steps of only Hunter learned, we see an improvement by using QLRKF during learning

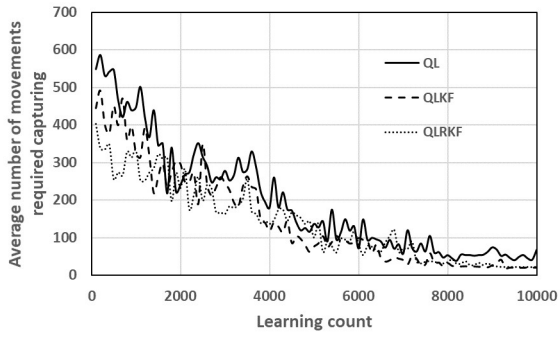

Figure 4. Relationship between learning count and capture step number (When only hunter is learning)

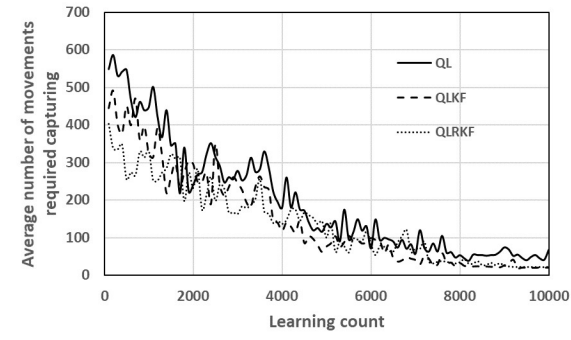

Figure 5. Relationship between learning count and capture step number (When both hunters and prey are learning)

${ }^{2} \mathrm{Up}$ to 1,000 steps. 
process to 65\% than QL, and 85\% than QLKF. As for the case of both hunter and prey learned, we see an improvement to $40 \%(\mathrm{QL})$ and $84 \%(\mathrm{QLKF})$.

Let us illustrate the detailed comparison using the harmonic averages in tables 4 and 5. In the averages of only Hunter learned, QLRKF becames to 75\% of QL and 97\% of QLKF. As for the case of both hunter and prey learned, QLRKF becames to $40 \%(\mathrm{QL})$ and $85 \%(\mathrm{QLKF})$. Let us see the result the standard deviation of the harmonic averages. In the standard deviation of only Hunter learned, we see an improvemed to $70 \%$. Moreover, as for the case of both hunter and prey learned, we see an improvement to $50 \%$ than QL and 75\% than QLKF. This result means that learning progresses stably.

\subsection{Discussion}

We change the number of times that the hunter can perform revers actions with QLRKF to 20,50, and 80, and examine the results of capturing steps. In tables 6 and 7, we show the results of capturing steps. We also illustrate overviews of the tables in figures 6 and 7 respectively. Let us compare the total number of capturing steps to 50 times and the others (20, 80 times). When the prey does not learn, it deteriorates to $120 \%$ in the case of the hunter perform RAL 20 times, and improves to $75 \%$ in the case of the learning is 80 times. As for the case of both hunter and prey learned, it deteriorated to $220 \%$ in the case of 20 times, and improved to $93 \%$ in the case of 80 times. We see that our process goes better everytime..

Let us illustrate the detailed comparison using the harmonic averages in tables 8 and 9. Similar to the total number of capturing steps, we compare in the case of 50 times and the others. In the case of only Hunter learned, it deteriorates to $126 \%$ in the case of 20 times, and improves to $70 \%$ in the case of the learning is 80 times. As for the case of both hunter and prey learned, it deteriorated to $235 \%$ in the case of 20 times, and improved to $92 \%$ in the case of 80 times. From these results, it can be seen that the more times the RAL can be performed, the better the results. Let us see the result the standard deviation of the harmonic averages. In the case of only Hunter learned, the standard deviation is $66 \%$ with 20 times, and $94 \%$ with 80 times. As for the case of both hunter and prey learned, the standard deviation is $134 \%$ with 20 times, and $106 \%$ with 80 times. Therefore, we cannot say that the standard deviation is affected by the number of RAL.
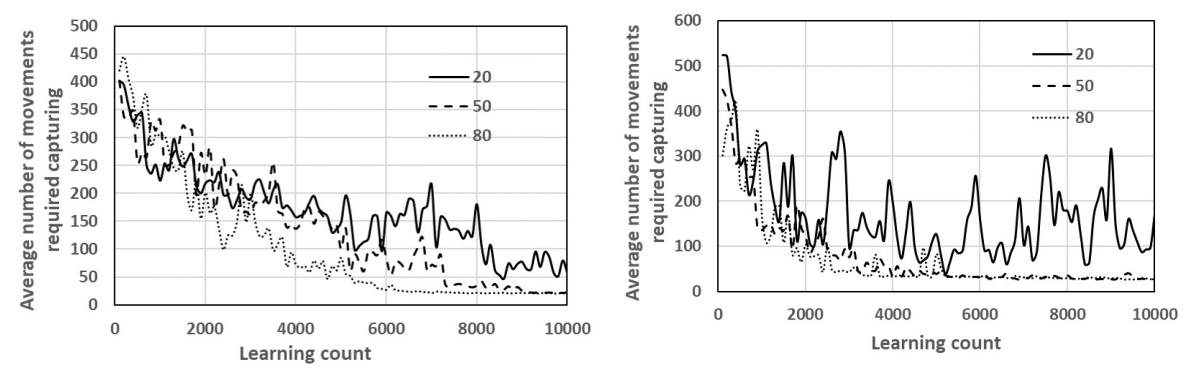

Figure 6. Capturing Steps with respect to the number of RAL (only Hunter learned)

Figure 7. Capturing Steps with respect to the number of RAL (Both learned) 


\section{Conclusion}

In this work, we have proposed a new method for reverse action by using Retrospective Kalman Filter that estimates the state one step before. In the total number of capturing steps of only Hunter learned, we see an improvement by using QLRKF during learning process to 65\% than QL, and 85\% than QLKF. As for the case of both hunter and prey learned, we see an improvement to $40 \%$ than QL and 84\% than QLKF. In the the arithmetic averages of the harmonic averages of only Hunter learned, we see an improvemement by using QLRKF during learning process to 75\% than QL, and 97\% than QLKF. Moreover, as for the case of both hunter and prey learned, we see an improvement to 40\% than QL and 85\% than QLKF.

\section{References}

[1] Sutton, Richard S., and Andrew G. Barto. Reinforcement learning: An introduction. Vol. 1. No. 1. Cambridge: MIT press, 1998.

[2] Leslie Pack Kaelbling, Michael L. Littman, Andrew W. Moore. "Reinforcement Learning: A Survey." CoRR cs.AI/9605103 (1996)

[3] Hado van Hasselt. "Double Q-learning.” NIPS 2010: 2613-2621

[4] Marco A, Wiering, and Hado van Hasselt. "Ensemble Algorithms in Reinforcement Learning." IEEE Trans. Systems, Man, and Cybernetics, Part B 38(4): 930-936 (2008)

[5] Vukosi Ntsakisi Marivate, Michael L. Littman. "An Ensemble of Linearly Combined ReinforcementLearning Agents.” AAAI (Late-Breaking Developments) 2013

[6] Raghu Ramakrishnan and Johannes Gehrke, Database Management Systems 3rd Edition. 2002.

[7] Benjamin Eysenbach, Shixiang Gu, Julian Ibarz, Sergey Levine. "Leave no Trace: Learning to Reset for Safe and Autonomous Reinforcement Learning." CoRR abs/1711.06782 (2017)

[8] Watkins, Christopher JCH, and Peter Dayan. "Q-learning." Machine learning 8.3-4 (1992): 279-292.

[9] Kei Takahata, Takao Miura. "Reinforcement Learning using Kalman Filters." IEEE International Conference on Cognitive Informatics and Cognitive Computing(ICCICC) 2019

[10] Takadama, K.:MultiAgent Learning, Corona-Sha, 2004 (in Japanese)

[11] Adati, S. and Maruta, I. : Fundamentals of Kalman Filter, Tokyo Denki University Press, 2012 (in Japanese)

[12] Takehiro Kitao, Masato Shirai, and Takao Miura: "Model Selection Based on Kalman Temporal Differences Learning." CIC 2017: 41-47

[13] Matthieu Geist, Olivier Pietquin: "Kalman Temporal Differences.” J. Artif. Intell. Res. 39: 483-532 (2010) 
Table 2. Capturing Steps (only Hunter learned)

\begin{tabular}{|c|c|c|c|}
\hline Learning & QL & QLKF & QLRKF \\
\hline 500 & 546.4 & 451.1 & 342.4 \\
\hline 1000 & 446.3 & 326.1 & 312.6 \\
\hline 1500 & 345.2 & 262.6 & 290.2 \\
\hline 2000 & 240.6 & 296.8 & 192.6 \\
\hline 2500 & 314.8 & 350.1 & 109.4 \\
\hline 3000 & 277.9 & 245.8 & 178.9 \\
\hline 3500 & 281.9 & 263.8 & 139.4 \\
\hline 4000 & 179.8 & 118.5 & 154.9 \\
\hline 4500 & 172.4 & 86.6 & 147.9 \\
\hline 5000 & 137.1 & 77.1 & 118.8 \\
\hline 5500 & 96.0 & 105.7 & 86.9 \\
\hline 6000 & 71.8 & 99.8 & 92.7 \\
\hline 6500 & 90.2 & 46.4 & 93.9 \\
\hline 7000 & 57.4 & 41.3 & 43.6 \\
\hline 7500 & 63.8 & 29.4 & 51.3 \\
\hline 8000 & 53.7 & 32.9 & 38.0 \\
\hline 8500 & 54.1 & 23.3 & 28.4 \\
\hline 9000 & 74.5 & 27.2 & 39.6 \\
\hline 9500 & 40.3 & 20.8 & 26.0 \\
\hline 10000 & 68.1 & 20.8 & 40.5 \\
\hline
\end{tabular}

Table 6. Capturing Steps with respect to the number of RAL (only Hunter learned)

\begin{tabular}{|c|c|c|c|}
\hline Learning & 20 & 50 & 80 \\
\hline 500 & 340.87 & 255.06 & 317.13 \\
\hline 1000 & 222.79 & 331.42 & 302.41 \\
\hline 1500 & 248.24 & 321.37 & 274.67 \\
\hline 2000 & 217.9 & 231.54 & 200.91 \\
\hline 2500 & 195.94 & 197.54 & 124.38 \\
\hline 3000 & 189.51 & 162.41 & 198.83 \\
\hline 3500 & 205.96 & 255.78 & 97.06 \\
\hline 4000 & 157.18 & 135.94 & 71.78 \\
\hline 4500 & 173.24 & 166.76 & 64.27 \\
\hline 5000 & 147.86 & 99.51 & 83.88 \\
\hline 5500 & 112.45 & 59.87 & 39.15 \\
\hline 6000 & 165.12 & 82.91 & 26.41 \\
\hline 6500 & 190.25 & 62.12 & 23.03 \\
\hline 7000 & 215.91 & 71.73 & 20.62 \\
\hline 7500 & 136.16 & 35.29 & 21.14 \\
\hline 8000 & 180.38 & 34.06 & 21.15 \\
\hline 8500 & 52.85 & 26.38 & 20.57 \\
\hline 9000 & 76.67 & 24.82 & 21 \\
\hline 9500 & 94.36 & 21.37 & 20.4 \\
\hline 10000 & 56.83 & 22.29 & 19.39 \\
\hline & & & \\
\hline 500 & & \\
\hline
\end{tabular}

Table 3. Capturing Steps (Both learned)

\begin{tabular}{|c|c|c|c|}
\hline Learning & QL & QLKF & QLRKF \\
\hline 500 & 581.7 & 419.4 & 295.6 \\
\hline 1000 & 340.4 & 271.3 & 136.2 \\
\hline 1500 & 488.5 & 173.9 & 135.3 \\
\hline 2000 & 415.7 & 64.3 & 121.0 \\
\hline 2500 & 328.2 & 149.8 & 92.0 \\
\hline 3000 & 274.7 & 124.2 & 75.9 \\
\hline 3500 & 160.7 & 71.2 & 49.4 \\
\hline 4000 & 125.2 & 68.9 & 34.0 \\
\hline 4500 & 107.4 & 35.8 & 35.2 \\
\hline 5000 & 119.1 & 68.1 & 38.7 \\
\hline 5500 & 96.8 & 31.5 & 29.2 \\
\hline 6000 & 88.6 & 30.8 & 31.7 \\
\hline 6500 & 71.1 & 30.5 & 32.1 \\
\hline 7000 & 83.6 & 30.7 & 29.9 \\
\hline 7500 & 59.5 & 30.8 & 32.5 \\
\hline 8000 & 134.0 & 29.7 & 35.2 \\
\hline 8500 & 155.8 & 29.4 & 30.1 \\
\hline 9000 & 148.5 & 30.5 & 27.2 \\
\hline 9500 & 91.0 & 31.9 & 33.2 \\
\hline 10000 & 42.1 & 30.4 & 27.3 \\
\hline
\end{tabular}

Table 7. Capturing Steps with respect to the number of RAL (Both learned)

\begin{tabular}{|c|c|c|c|}
\hline Learning & 20 & 50 & 80 \\
\hline 500 & 280.8 & 295.63 & 234.62 \\
\hline 1000 & 324.7 & 136.22 & 165.73 \\
\hline 1500 & 284.99 & 135.28 & 107.87 \\
\hline 2000 & 162.72 & 120.99 & 104.12 \\
\hline 2500 & 195.17 & 92.05 & 100.71 \\
\hline 3000 & 98.18 & 75.89 & 42.75 \\
\hline 3500 & 124.92 & 49.44 & 34.61 \\
\hline 4000 & 195.77 & 33.96 & 32.73 \\
\hline 4500 & 98.25 & 35.18 & 35.22 \\
\hline 5000 & 126.89 & 38.71 & 83.36 \\
\hline 5500 & 84.8 & 29.23 & 34.4 \\
\hline 6000 & 158.09 & 31.69 & 33.55 \\
\hline 6500 & 106.94 & 32.08 & 32.6 \\
\hline 7000 & 102.99 & 29.85 & 31.17 \\
\hline 7500 & 301.55 & 32.48 & 29.73 \\
\hline 8000 & 178.92 & 35.21 & 29.42 \\
\hline 8500 & 64.19 & 30.1 & 31.41 \\
\hline 9000 & 316.93 & 27.18 & 30.14 \\
\hline 9500 & 135.52 & 33.18 & 26.17 \\
\hline 10000 & 172.26 & 27.26 & 25.55 \\
\hline
\end{tabular}


Table 4. Harmonic Average (only Hunter learned)

\begin{tabular}{|c|c|c|c|}
\hline Learning & QL & QLKF & QLRKF \\
\hline 100 & 169.1 & 163.3 & 162.9 \\
\hline 500 & 522.2 & 474.3 & 406.5 \\
\hline 1000 & 617.2 & 491.8 & 476.3 \\
\hline 1500 & 561.3 & 446.9 & 486.4 \\
\hline 2000 & 429.5 & 516.9 & 351.4 \\
\hline 2500 & 559.2 & 614.2 & 209.7 \\
\hline 3000 & 508.7 & 454.3 & 337.7 \\
\hline 3500 & 521.7 & 490.6 & 268.1 \\
\hline 4000 & 344.1 & 230.2 & 298.3 \\
\hline 4500 & 332.1 & 169.9 & 286.4 \\
\hline 5000 & 266.8 & 152.0 & 232.1 \\
\hline 5500 & 188.7 & 207.4 & 171.1 \\
\hline 6000 & 141.8 & 196.4 & 182.7 \\
\hline 6500 & 177.9 & 92.1 & 185.1 \\
\hline 7000 & 113.8 & 82.1 & 86.6 \\
\hline 7500 & 126.5 & 58.6 & 101.9 \\
\hline 8000 & 106.8 & 65.6 & 75.6 \\
\hline 8500 & 107.6 & 46.5 & 56.5 \\
\hline 9000 & 147.7 & 54.3 & 78.9 \\
\hline 9500 & 80.2 & 41.5 & 51.8 \\
\hline 10000 & 135.3 & 41.6 & 80.6 \\
\hline (Average) & 310.2 & 240.6 & 213.9 \\
\hline (StdDev) & 184.5 & 178.0 & 127.0 \\
\hline
\end{tabular}

Table 8. Harmonic Averages with respect to the number of RAL (only Hunter learned)

\begin{tabular}{|c|c|c|c|}
\hline Learning & 20 & 50 & 80 \\
\hline 500 & 405.4 & 337.8 & 388.1 \\
\hline 1000 & 364.4 & 497.8 & 464.4 \\
\hline 1500 & 426.0 & 529.3 & 464.3 \\
\hline 2000 & 393.0 & 415.0 & 365.1 \\
\hline 2500 & 363.4 & 366.1 & 237.0 \\
\hline 3000 & 356.5 & 308.1 & 372.9 \\
\hline 3500 & 389.0 & 476.7 & 188.9 \\
\hline 4000 & 302.5 & 262.9 & 141.0 \\
\hline 4500 & 333.6 & 321.6 & 126.7 \\
\hline 5000 & 287.2 & 195.1 & 165.0 \\
\hline 5500 & 220.4 & 118.5 & 77.7 \\
\hline 6000 & 321.4 & 163.6 & 52.6 \\
\hline 6500 & 369.7 & 123.1 & 45.9 \\
\hline 7000 & 418.9 & 142.0 & 41.1 \\
\hline 7500 & 267.5 & 70.2 & 42.2 \\
\hline 8000 & 352.8 & 67.8 & 42.2 \\
\hline 8500 & 105.0 & 52.6 & 41.0 \\
\hline 9000 & 152.0 & 49.5 & 41.9 \\
\hline 9500 & 186.9 & 42.6 & 40.7 \\
\hline 10000 & 113.0 & 44.5 & 38.7 \\
\hline (Average) & 297.1 & 235.0 & 165.7 \\
\hline (StdDev) & 100.1 & 151.4 & 143.5 \\
\hline
\end{tabular}

Table 5. Harmonic Averages(Both learned)

\begin{tabular}{|c|c|c|c|}
\hline Leaning & QL & QLKF & QLRKF \\
\hline 500 & 537.7 & 456.2 & 371.6 \\
\hline 1000 & 507.9 & 426.8 & 239.8 \\
\hline 1500 & 737.0 & 311.7 & 248.2 \\
\hline 2000 & 688.4 & 124.6 & 228.2 \\
\hline 2500 & 580.1 & 282.6 & 177.6 \\
\hline 3000 & 503.3 & 238.4 & 148.0 \\
\hline 3500 & 307.3 & 139.5 & 97.5 \\
\hline 4000 & 242.7 & 135.6 & 67.3 \\
\hline 4500 & 209.8 & 71.1 & 69.8 \\
\hline 5000 & 232.6 & 134.3 & 76.8 \\
\hline 5500 & 190.3 & 62.6 & 58.2 \\
\hline 6000 & 174.6 & 61.3 & 63.0 \\
\hline 6500 & 140.6 & 60.8 & 63.8 \\
\hline 7000 & 165.2 & 61.2 & 59.4 \\
\hline 7500 & 118.1 & 61.4 & 64.7 \\
\hline 8000 & 263.6 & 59.2 & 70.1 \\
\hline 8500 & 305.9 & 58.6 & 60.0 \\
\hline 9000 & 292.2 & 60.8 & 54.2 \\
\hline 9500 & 180.2 & 63.5 & 66.1 \\
\hline 10000 & 83.8 & 60.6 & 54.4 \\
\hline (Average) & 311.2 & 148.5 & 125.6 \\
\hline (StdDev) & 185.0 & 126.4 & 94.2 \\
\hline
\end{tabular}

Table 9. Harmonic Averages with respect to the number of RAL (Both learned)

\begin{tabular}{|c|c|c|c|}
\hline Learning & 20 & 50 & 80 \\
\hline 500 & 359.6 & 371.6 & 319.4 \\
\hline 1000 & 490.2 & 239.8 & 284.3 \\
\hline 1500 & 479.0 & 248.2 & 201.3 \\
\hline 2000 & 301.0 & 228.2 & 197.9 \\
\hline 2500 & 362.1 & 177.6 & 193.6 \\
\hline 3000 & 190.1 & 148.0 & 84.3 \\
\hline 3500 & 241.2 & 97.5 & 68.5 \\
\hline 4000 & 373.3 & 67.3 & 64.9 \\
\hline 4500 & 192.3 & 69.8 & 69.9 \\
\hline 5000 & 247.5 & 76.8 & 164.0 \\
\hline 5500 & 167.0 & 58.2 & 68.4 \\
\hline 6000 & 308.1 & 63.0 & 66.7 \\
\hline 6500 & 210.4 & 63.8 & 64.9 \\
\hline 7000 & 203.0 & 59.4 & 62.1 \\
\hline 7500 & 579.8 & 64.7 & 59.2 \\
\hline 8000 & 350.0 & 70.1 & 58.6 \\
\hline 8500 & 127.4 & 60.0 & 62.6 \\
\hline 9000 & 612.3 & 54.2 & 60.1 \\
\hline 9500 & 267.2 & 66.1 & 52.2 \\
\hline 10000 & 338.7 & 54.4 & 51.0 \\
\hline (Average) & 296.0 & 125.6 & 116.4 \\
\hline (StdDev) & 126.4 & 94.2 & 100.4 \\
\hline
\end{tabular}




\section{EJC 2020}

\section{0th International Conference}

\section{on Information Modelling and Knowledge Bases}

June 8-9, 2020, Hamburg, Germany

The series of European Japanese Conference on Information Modelling and Knowledge Bases (EJC) originally started as a co-operation initiative between Japan and Finland in 1982. The practical operations were then organized by professor Ohsuga in Japan and professors Hannu Kangassalo and Hannu Jaakkola in Finland (Nordic countries). Geographical scope has expanded to cover Europe and also other countries. Workshop characteristic-discussion, enough time for presentations and limited number of participants (50) / papers (30)is typical for the conference. Suggested topics include, but are not limited to:

- Conceptual modelling

- Knowledge and information modelling and discovery

- Linguistic modelling

- Cross-cultural communication and social computing

- Environmental modelling and engineering

- Multimedia data modelling and systems

The Kiel Computer Science Series (KCSS) is published by the Department of Computer Science of the Faculty of Engineering at Kiel University. The scope of this open access publication series includes dissertations, habilitation theses, and textbooks in computer science.

ISSN 2193-6781 (print version) ISSN 2194-6639(electronic version)

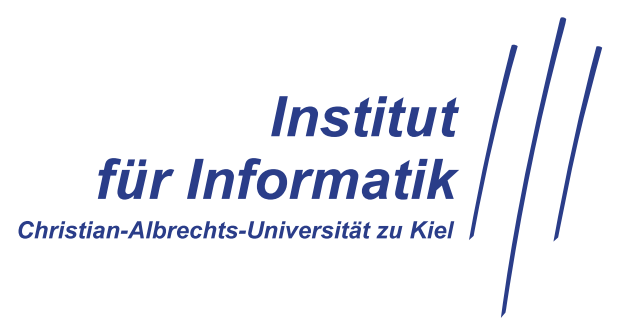

Information Systems Engineering Group 